\title{
POSTIRRADIATION EXAMINATION AND EVALUATION OF PEACH BOTTOM FUEL TEST ELEMENT FTE-6
}

by

C. F. WALLROTH, J. F. HOLZGRAF, and D. D. JENSEN

Prepared under

Contract EY-76-C-03-0167

Project Agreement No. 17

for the San Francisco Operations Office

U.S. Energy Research and Development Administration

DATE PUBLISHED: SEPTEMBER 1977 


\section{DISCLAIMER}

This report was prepared as an account of work sponsored by an agency of the United States Government. Neither the United States Government nor any agency Thereof, nor any of their employees, makes any warranty, express or implied, or assumes any legal liability or responsibility for the accuracy, completeness, or usefulness of any information, apparatus, product, or process disclosed, or represents that its use would not infringe privately owned rights. Reference herein to any specific commercial product, process, or service by trade name, trademark, manufacturer, or otherwise does not necessarily constitute or imply its endorsement, recommendation, or favoring by the United States Government or any agency thereof. The views and opinions of authors expressed herein do not necessarily state or reflect those of the United States Government or any agency thereof. 


\section{DISCLAIMER}

Portions of this document may be illegible in electronic image products. Images are produced from the best available original document. 


\section{NOTICE}

This report was prepared as an account of work sponsored by the United States Government. Neither the United States nor the United States Energy Research and Development Administration, nor any of their employees, nor any of their contractors, subcontractors, or their employees, makes any warranty, express or implied, or assumes any legal liability or responsibility for the accuracy, completeness or usefulness of any information, apparatus, product or process disclosed, or represents that its use would not infringe privately owned rights.

Printed in the United States of America Available from

National Technical Information Service

U.S. Department of Commerce

5285 Port Royal Road

Springfield, Virginia 22161

Price: Printed Copy $\$ 10.75$; Microfiche $\$ 3.00$ 


\section{POSTIRRADIATION EXAMINATION AND EVALUATION OF PEACH BOTTOM FUEL TEST ELEMENT FTE-6}

by

C. F. WALLROTH, J. F. HOLZGRAF, and D. D. JENSEN

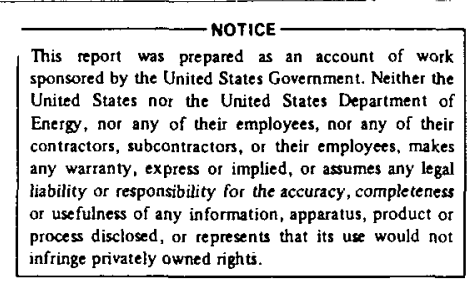

Prepared under

Contract EY-76-C-03-0167

Project Agreement No. 17

for the San Francisco Operations Office

U.S. Energy Research and Development Administration

GENERAL ATOMIC PROJECT 3224

DATE PUBLISHED: SEPTEMBER 1977 
$\theta$

-

$\theta$ 


\section{FOREWORD}

This report is prepared with all text appearing first, followed by appendices that contain all the referenced figures and tables. Figures for the entire report appear in Appendix A, and all tables are presented in Appendix B. Each figure and table is identified by the reference number applied to it in the text. A table of contents is included as a part of each appendix. 
$\ominus$

•

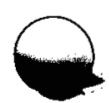


ABSTRACT

Fuel test element FTE- 6 was irradiated in the Peach Bottom hightemperature gas-cooled reactor (HTGR) for 645 equivalent full power days. Four fuel varieties, contained in H-327 graphite bodies, were tested.

A primary result of this test has been to demonstrate acceptable performance even with calculated high stresses in the graphite bodies. Heterogeneous fuel loadings in the element caused local power peaking and azimuthal power variations, deforming the graphite fuel bodies and thereby causing bowing nearly five times as large as the diametral clearance within the sleeve. The axial stresses resulting from interference between the fuel bodies and sleeve were estimated to have reached $45 \%$ of the ultimate material strength at the end of the irradiation. Residual stresses from differential contraction within the fuel body resulted in probable in-plane stress levels of $130 \%$ of the material strength at the end-of-life shutdown and of up to $150 \%$ of the strength at shutdown during the irradiation cycle. The high in-plane stresses are local peaks at the corners of a sharp notch in the element, which may account for the stresses failing to cause damage. The lack of observable damage, however, indicates that the methods and data used for stress analysis give results that are either fairly accurate or conservative.

The irradiation history has been modeled with HTGR design codes. An extensive measurement program has been carried out to determine the nuclear isotopic distribution within the element via gamma scanning at the reactor site and at General Atomic hot cells. The decalibration effects of the thermocouples have been measured. The dimensional strain characteristics of the graphite and fuel rod components have been determined. The isotopic composition of the fuel was evaluated by six different 
methods. The diffusion characteristics of mobile fission products were measured from center spine samples. The fuel performance has been verified through application of LHTGR fuel performance models.

Results from earlier postirradiation examinations done for sister elements FTE- 3 and FTE- 4 and some first results from the forthcoming evaluation of FTE-5 have been included in the analysis and are reported here for comparison with the FTE- 6 findings. The collected data are regarded as valuable confirmation and improvement of the engineering data base for HTGR fuel element designs.

The nuclear, thermal, structural, fuel, and fission product performances of the test element have been verified, and areas of improvement for measurement methods and applied models have been identified using rigorous statistical methods. The methods and all essential numerical data have been documented in this report. 
CONTENTS

FOREWORD . . . . . . . . . . . . . . . . . . . . .

ABSTRACT . . . . . . . . . . . . . . . . . . . . $\mathrm{v}$

1. INTRODUCTION ...................... . . . . . . . 1-1

2. EXPERIMENT DESCRIPTION . . . . . . . . . . . . . . 2-1

References . . . . . . . . . . . . . . . 2-5

3. IRRADIATION CONDITIONS . . . . . . . . . . . . . . 3-1

3.1 Irradiation History . . . . . . . . . . . . . . 3-1

3.2 Fluences .................... . . . 3-3

3.2.1 Fluence Calculations . . . . . . . . . 3-3

3.2.2 Fluence Measurements . . . . . . . . . 3-6

3.3 Temperatures .... . . . . . . . . . . 3-12

3.3.1 Temperature Calculations .......... 3-12

3.3.2 Temperature Measurements . . . . . . . . 3-15

3.4 Burnup . . . . . . . . . . . . . . . . . 3-21

3.4.1 Burnup Calculations . . . . . . . . . . . 3-21

3.4.2 Burnup Measurements ........... 3-23

3.5 Stress Analysis ............. . . . . . . . . . .

3.5.1 Method and Material Properties........ 3-33

3.5.2 Initial Thermal Stress . . . . . . . . . 3-34

3.5.3 Peak Stress During Life . . . . . . . . 3-37

3.5.4 Peak Stress at EOL . . . . . . . . . . . 3-39

3.5.5 Stress Analysis Conclusions . . . . . . . 3-41

3.6 Irradiation Summary . . . . . . . . . . . . 3-42

References ....... . . . . . . . . . . 3-42

4. POSTIRRADIATION EXAMINATION AND EVALUATION . . . . . . . . 4-1

4.1 Data Evaluation Procedure . . . . . . . . . . . . 4-1

4.1.1 Error Analysis . . . . . . . . . . . . 4-1

4.1.2 Stratification . . . . . . . . . . . . 4-4

4.1.3 Comparison of Measured and Predicted Values . . 4-8

4.1.4 Axial Variation Test . . . . . . . . . . . 4 4-13 
4.1.5 Regression Analysis ........ . . . . 4-15

4.1 .6 Interpolation. . . . . . . . . . . . 4-18

4.1.7 Normalization. . . . . . . . . . . . 4-19

4.2 Nuclear Measurements. . . . . . . . . . . 4-22

4.2.1 Fuel Gamma Scan at Peach Bottom ........ 4-22

4.2.2 Fuel Gamma Scan Analysis . . . . . . . . . 4-26

4.2.3 Delayed Neutron Dose Measurements . . . . . . 4-44

4.2.4 Nuclear Measurement Summary . . . . . . . . 4-46

4.3 Disassembly and Visual Inspection . . . . . . . . . 4-47

4.4 Dimensional Change Evaluation . . . . . . . . . . 4-49

4.4.1 Fuel Element and Sleeve ........... 4-50

4.4.2 Fuel Bodies ............... . . 4-52

4.4.3 Sleeve-Fuel Body Gaps ............ 4-62

4.4.4 Fuel Rods ................. . 4-64

4.4.5 Fuel Body - Fuel Rod Gaps ... . . . . . . 4-70

4.4.6 Dimensional Performance Summary ... . . . . 4 4-71

4.5 Fuel Rod Irradiation Performance . . . . . . . . . 4-73

4.5.1 Visual Examination .. . . . . . . . . 4-73

4.5.2 Fission Gas Release Measurements ... . . . . 4-74

4.5.3 Fuel Rod Metallography ........... 4-75

4.5.4 Fuel Rod Gamma Scanning .......... . 4-77

4.5.5 Fission Product Distributions in the Graphite
Fuel Bodies . . . . . . . . . 4-80

4.5.6 Fuel Performance Prediction . . . . . . . . . 4-85

4.6 Thermal Stability Spine Samples . . . . . . . . . . 4-87

4.6.1 Visual Examination . . . . . . . . . . . . 4-88

4.6.2 Fission Gas Release Measurements . . . . . . . 4-88

4.6.3 Metallography ............... 4-88

4.6.4 Spine Sample Gamma Scans ............ . 4-88

4.7 Fuel Performance Summary . . . . . . . . . . . . 4-89

4.8 Metallic Diffusion Spine Samples . . . . . . . . . 4-90

4.8.1 Test Description ............... 4-90

4.8.2 Experimental Procedure ........... . 4-92

4.8.3 Analysis and Results for Strontium, Europium,

Barium, and Cesium ... . . . . . . . . 4-93

4.8.4 Summary ............... . . 4-101

References ..................... . 4-101 
5. DISCUSSION AND CONCLUSIONS ............ . . 5-1

6. ACKNOWLEDGEMENT . . . . . . . . . . . . . . . . . . 6-1

APPENDIX A: FIGURES .................... . . A-1

APPENDIX B: TABLES . . . . . . . . . . . . . . . . B-1 


\section{INTRODUCTION}

Fuel test element FTE- 6 belongs to a group of 33 test elements that were irradiated in the Peach Bottom high-temperature gas-cooled reactor (HTGR). FTE-6 was part of a test program designed to study the behavior of HTGR fuels at the correctly combined conditions of time, fluence, temperature, coolant impurity level, and cycling frequency of a power reactor. The main test objectives were to do the following:

1. Demonstrate performance of various types of HTGR fuels over a wide range of operating conditions and life times.

2. Confirm the integrity of the compound fuel rod structural graphite system (or alternative concepts) from statistically representative samples.

3. Provide experimental data on fuel thermal stability; metallic fission product diffusion rates; and dimensional, structural, and chemical stability of fuel rods, graphite, and absorber materials.

4. Provide HTGR design code validation with respect to (1) fluence and depletion, (2) thermal performance, and (3) thermal and Wigner strain and stress fission product behavior predictions.

This report presents the preirradiation measurements, the irradiation history, the postirradiation examination, and the evaluation of the experimental data. Where applicable, $95 \%$ confidence limits have been estimated and experimental results have been compared with HTGR design code predictions. 


\section{EXPERIMENT DESCRIPTION}

FTE-6 is the third of four nearly identical test element experiments being analyzed at General Atomic. FTE-3 and FTE-4 (Refs, 2-1 and 2-2) are completed; FTE-5 is undergoing examination. The generic design of FTE-6 is described in Refs. 2-1 through 2-5, and specific design details are given in Refs. 2-6 and 2-7. Reference 2-6 gives the as-built General Atomic assemb1y and detail drawings that are 1isted in Table 2-1. Reference 2-7 contains the fuel rod metrology data. Figures 2-1, 2-2, and 2-3 show typical axial and radial views of the test element assembly. A comparison of the graphite materials used in test and standard driver elements is given in Table 2-2.

The active portion of the test element consisted of three identical H-327 graphite fuel bodies with an eight-hole teledial configuration. H-327 graphite is a needle core graphite manufactured by Great Lake Carbon Corporation (GLCC) and was used for the initial core of the Fort St. Vrain HTGR. The composite stack length of all three bodies at beginning of life (BOL) was $2370.4 \mathrm{~mm}$ (93.323 in.). The bottom body was $631.2 \mathrm{~mm}$ ( 24.85 in.) above the core support plate. The active core height began $660.4 \mathrm{~mm}$ (26.00 in.) above the core support plate and was used as a reference for all thermal and nuclear calculations ${ }^{*}$ of standard fue 1 elements in the core. The fuel in FTE-6 started at $664.2 \mathrm{~mm}$ (26.15 in.) above the core support plate, i.e., $3.8 \mathrm{~mm}$ ( $0.15 \mathrm{in.})$ above active core height or $33.0 \mathrm{~mm}(1.30 \mathrm{in.})$ above the bottom end of the fuel body. Detailed metrology is reported in Section 4.4.2.

FTE-6 was instrumented with thermocouples. One was a Chromel-Alumel (C/A) type for the graphite fuel body temperature, and the other was a *Active core height started at $660.4 \mathrm{~mm}$ (26.00 in.) above core support plate (= start of total core height) and ended at $2.946 \mathrm{~m}$ (116.00 in.), covering a 2.286-m (90.00-in.) length of fuel (see also Fig. 2-1). Active core height and total core height are used interchangeably in this report. 
tungsten-rhenium type for fuel temperature measurements at an active core height of $1257.3 \mathrm{~mm}$ (49.5 in.).

Axial and radial locations are indicated in Figs. 2-1 and 2-3, respectively. Bodies 1 and 2 were pinned together by the two thermocouples. Body 3 was subject to azimuthal movement relative to bodies 1 and 2 .

FTE-6 contained 336 fuel rods that were carbonized in $\mathrm{Al}_{2} \mathrm{O}_{3}$ beds. These rods consisted of a closely packed bed of blended fissile and fertile coated fuel particles bonded together in a carbonaceous matrix. The matrix was a blend of $27 \%$ to $30 \%$ natural flake graphite flour and $70 \%$ to $73 \%$ coal-tar pitch. The irradiation performance of these materials is similar to present HTGR reference materials which consist of LONZA graphite flour and petroleum coke pitch. The matrix was prepared by hotmixing the ingredients to form a homogeneous blend, cooling to room temperature, and grinding the solid matrix into granules suitable for use in the fuel rod injection equipment. The impurities in the matrix were kept in the low ppm range.

Both the fuel particles and fuel rods were made in production equipment using the available production processes and quality control techniques. The hot matrix was injected into the particle bed with a prototype injection machine similar to those built for the manufacture of Fort St. Vrain initial core fuel. After cooling, the fuel rods were removed from the injection die; at this point, the rods were referred to as "green" rods. The green rods were packed into $\mathrm{Al}_{2} \mathrm{O}_{3}$ beds and subjected to a carbonization treatment.

The carbonization cycle consisted of heating the fuel rods in a flowing nitrogen atmosphere for $2 \mathrm{hr}$ to $750^{\circ} \mathrm{C}$ and holding at that temperature for $0.5 \mathrm{hr}$. The fuel rods were then heat treated by passing them through a furnace at $1800^{\circ} \mathrm{C}$ and at a rate of $25.4 \mathrm{~mm}$ ( 1 in.) per minute. An argon atmosphere was maintained in the furnace, and the hot 
zone was $1892 \mathrm{~mm}$ (72 in.) 1ong. The cured rods were measured for length and diameter (Ref. 2-7) and loaded into the three graphite bodies in groups of 14 per fuel hole. Four different fuel blends were loaded:

\begin{tabular}{|c|c|c|c|c|c|}
\hline Holes & FTE-3 & FTE-4 & $E-6$ & Fue1 Composition & \\
\hline and 2 & 5.8 & 77.6 & $26.2: 73.8$ & $\mathrm{UO}_{2}$ TRISO, $\mathrm{ThO}_{2}$ BISO & \\
\hline ad 4 & 85 & 78 . & 92 & $(2.75 \mathrm{Th}: \mathrm{U}) \mathrm{C}_{2}$ TRISO, $\mathrm{ThC}_{2}$ & IsO \\
\hline$d 6$ & $85.8: 14.2$ & 78.6 & 3 & $(2.75 \mathrm{Th}: \mathrm{U}) \mathrm{C}_{2}$ TRISO, $\mathrm{ThC}_{2}$ & TRTS \\
\hline nd 8 & 4.0 & 35.9 & 10 & $\mathrm{UC}_{2}$ TRISO, $\mathrm{ThC}_{2}$ BISO & \\
\hline
\end{tabular}

Fuel types were identical for FTE-3, -4 , and -6 fuel rods; fuel loadings varied slightly. The coated particle attributes summarized in Table 2-3 were taken from a data storage system (Ref. 2-8). Fuel rod attributes were retrieved from Quality Control records (Ref. 2-9) and are presented in Table 2-4. All heavy metal loading information is nominal data obtained during the fuel rod manufacture by weighing the amount of fissile and fertile fuel for each fuel rod. Fuel was blended by hand, perhaps causing the fuel inhomogeneity found in some varieties (see Section 4.5).

Nitrogen impurities were not measured specifically on FTE-6 fue1 rods, but levels less than $1000 \mathrm{ppm}$ were representative for measurements taken on coated particles from similar manufacturing runs. The outer pyrocarbon layer was deposited using nitrogen gas as diluent for the propylene coating gas. This was the most probable source of nitrogen in the fuel particles, which would be subject to $\mathrm{C}-14$ conversion through a neutron activation process during irradiation.

FTE- 6 contained 36 spine samples, as summarized below:

$$
\begin{aligned}
& 9 \text { metallic diffusion samples } \\
& 12 \text { fission product release samples } \\
& 3 \text { advanced fuel rods }
\end{aligned}
$$

\footnotetext{
* Carbon 14 activation levels measured by ORNL for two fuel rods from FTE- 4 confirm impurity levels of less than $1000 \mathrm{ppm}$ nitrogen per particle (Ref. 2-10).
} 
7 thermal stability samples

5 boronated graphite samples.

Each of the spine sample types is described in detail in Ref. 2-11. Preirradiation data for Type II thermal stability spine samples are presented in Table 2-5. All spine samples and their time-integrated or time-averaged irradiation parameters (fast and thermal fluence, burnup, and temperature) are tabulated in Tables 2-6(a) and 2-6(b).

The element was purged in the same way as standard Core 2 elements. Purge gases were collected by the main purge system and were not measured individually for fission gas release as was done for other fuel test elements. The purge gas entered the element through a porous plug at the top; the purge flow rate was determined from the pressure drop to be $0.5 \mathrm{~kg} / \mathrm{hr}(1.1 \mathrm{lb} / \mathrm{hr})$. The cross-sectional area of the purge channels did not limit the purge flow.

The downward ${ }^{*}$ flowing purge gas passed through the element along the annular gap between the sleeve and fuel body, was collected via radial grooves at the top face of the bottom reflector, and was transported through the stand-off pin into the main manifold. A polyurethane foam spacer, burned off during initial startup of the experiment, was positioned between the top fuel body and the top reflector assembly. This area provided a plenum for differential shrinkage of the fuel body stack as well as a mixing chamber for the entering purge gas. The purge flow characteristics for FTE-6 are summarized in Table 2-7. The table gives BOL and $\mathrm{EOL}^{* *}$ annular flow areas for each individual fuel body, as well as for the total element. This may be useful in the fission product transport evaluation.

Component manufacturing and final assembly of the experiment were carried out by Genera1 Atomic in San Diego. The element was shipped to Peach Bottom, and irradiation began on July 11, 1971.

* The coolant gas flow in Peach Bottom was upward.

** Beginning of life (BOL) and end of life (EOL). 
REFERENCES

2-1. Wa1lroth, C. F., N. L. Baldwin, C. B. Scott, and L. R. Zumwalt, "Postirradiation Examination of Peach Bottom Fuel Test Element FTE-3," ERDA Report GA-A13004, General Atomic Company, August 15, 1974.

2-2. Wallroth, C. F., J. F. Holzgraf, D. D. Jensen, and L. R. Zumwa1t, "Postirradiation Examination and Evaluation of Peach Bottom Fuel Test Element FTE-4," ERDA Report GA-A13452, General Atomic Company, May 1977.

2-3. Scheffel, W. J., and C. B. Scott, "Irradiation Experience with HTGR Fuels in the Peach Bottom Reactor," General Atomic Report GA-A12897, November 1, 1974.

2-4. Wallroth, C. F., F. McCord, R. J. Meyer, J. J. Saurwein, "Postirradiation Examination and Evaluation of Peach Bottom Molded Fuel Test Element FTE-18," General Atomic Report GA-A13699, June 1, 1976.

2-5. "Peach Bottom Atomic Power Station Unit 1 Test Fuel Element Design and Operational Evaluation - Proposed Facility Change and Technical Specification Change (No. 12)," Philadelphia Electric Company, April 1970.

2-6. Genera1 Atomic "As Built Assembly Drawing 11497" and additiona1 drawings as listed in Table 2-1 of this report.

2-7. Vrouwes, J.H.L., "Phase 2 Test Element Program, Pre-Irradiation Data Collected by Fuel Design Branch," General Atomic International Documentation, February 14, 1972.

2-8. Harmon, D. P., "Fuel Materials Branch Data Retrieval System," General Atomic Company, private communication, May 1976.

2-9. Disselhorst, B. F., General Atomic Company, private communication, May 1976.

2-10. Vaughen, V. C. A., Oak Ridge National Laboratories, private communication, May 1976.

2-11. Steward, K. P., "Spine Samples in the Phase I and Phase II Peach Bottom Test Elements," General Atomic Company, unpublished data, September 30, 1971. 


\section{IRRADIATION CONDITIONS}

This section presents calculated irradiation parameters, and where applicable compares them with the measurements. A detailed method is described in Section 4.1, with emphasis on statistical analyses.

\subsection{IRRADIATION HISTORY}

Fuel test element FTE- 6 began irradiation in the Peach Bottom reactor in core position CO2-01 on Ju1y 11, 1971, at 252.4 EFPD. $*$ It was irradiated in this position for 644.9 EFPD of Core 2 operation, and irradiation terminated on October 31,1974 at 15.35 hour. The time-averaged power over 792.0 days of operation (real time) was $111.54 \mathrm{~kW}$, and the energy production was $88.3 \mathrm{MWd}\left(7.63 \times 10^{12} \mathrm{~J}\right)$.

The nuclear and thermal irradiation history has been simulated with the following HTGR design codes:

GAUGE (Ref. 3-1), a two-dimensional four-group diffusiondepletion program. The Peach Bottom core was collapsed into one layer; the nuclide densities are calculated as a function of time and radial core location ( $X$ - Y geometry).

FEVER (Ref. 3-2), a one-dimensional multigroup diffusiondepletion program for analyzing a fuel region or subregion in the axial direction. (The GAUGE-calculated collapsed-nuclide densities are stretched over the axial fuel height.)

BUG-2 (Ref. 3-3), a two-dimensional multigroup burnup code for

*An EFPD is defined as an equivalent full power day of $115.0 \mathrm{MW}(t)$, which has been used for Cores 1 and 2 . The power was generated from 804 fuel elements. 
modeling the influence of partially-inserted control rods ( $\mathrm{R}-\mathrm{Z}$ geometry).

TREVER (Ref. 3-4), a one-dimensional heat transfer code, ca1culating the axial temperature distribution for several specific points along the radial cross section of the fuel test element. The code was modified for Peach Bottom test element application.

TAC2D (Ref. 3-5), a two-dimensional finite-element heat transfer code, calculating radial or axial heat transfer characteristics for specific geometries.

GTEPC-2D (Ref. 3-6), a two-dimensional finite element code for heat transfer and viscoelastic stress analysis of graphite.

The reactor history of Core 2 has been modeled via a step function in several time intervals. The detailed reactor operating history is given in Ref. 3-7. The operating parameters, as used for the TREVER simulation, are displayed in Fig. 3-1. The reactor power history is given in Figs. 3-2 and 3-3, which present thermal balance (Ref. 3-7) and TREVER simulation data (Ref. 3-8), respectively. Figure 3-4 gives the power history for FTE-6, which has been used for GAUGE calculations. A summary of the power, flow, and four-group flux history is given in Table 3-1.

The time-averaged ${ }^{*}$ values are:

\begin{tabular}{|c|c|c|c|c|}
\hline Power History & Mean & & $\underline{\mathrm{RMS}}$ & \\
\hline Irradiation time, equivalent & 644.9 & & - & (EFPD) \\
\hline Irradiation time, real & 792.0 & & - & (d) \\
\hline E1ement power & 111.54 & \pm & 12.34 & $(\mathrm{~kW})$ \\
\hline Element power factor ${ }^{* *}$ & 0.986 & \pm & 0.098 & $(-)$ \\
\hline Coolant power factor ${ }^{* *}$ & 1.024 & \pm & 0.117 & $(-)$ \\
\hline
\end{tabular}




$\begin{array}{clll}\text { Power History } & \frac{\text { Mean }}{\text { RMS }} & \\ \text { Coolant flow for one tricusp channel } & 0.031 \pm 0.002 & (\mathrm{~kg} / \mathrm{s}) \\ \text { F1ux, fast }(\mathrm{E} \leq 29 \mathrm{fJ}) & 3.38 \pm 0.35 & \left(10^{17} \mathrm{n} / \mathrm{m}^{2} / \mathrm{s}\right) \\ \text { Fast }(13.9 \mathrm{fJ} \leq \mathrm{E} \leq 29 \mathrm{fJ}) & 0.60 \pm 0.06 & \left(10^{17} \mathrm{n} / \mathrm{m}^{2} / \mathrm{s}\right) \\ \text { Epithermal }(2.8 \mathrm{aJ} \leq \mathrm{E}<13.9 \mathrm{fJ}) & 7.06 \pm 0.87 & \left(10^{17} \mathrm{n} / \mathrm{m}^{2} / \mathrm{s}\right) \\ \text { Epithermal }(0.38 \mathrm{aJ} \leq \mathrm{E}<2.8 \mathrm{aJ}) & 1.15 \pm 0.14 & \left(10^{17} \mathrm{n} / \mathrm{m}^{2} / \mathrm{s}\right) \\ \text { Thermal }(0 \leq \mathrm{E}<0.38 \mathrm{aJ}) & 4.37 \pm 0.94 & \left(10^{17} \mathrm{n} / \mathrm{m}^{2} / \mathrm{s}\right)\end{array}$

The coolant power factor represents the equivalent heat given into an equivalent coolant channel by accounting for different power levels from neighboring elements. The time-weighted root-mean-square (RMS) deviation (in Section 3.2.1, Eq. 3-1b, and Section 4.1) represents 1ongterm time variation information coming from the GAUGE- and TREVERselected time intervals. The GAUGE-derived fast flux group was multiplied by 0.85 to convert from a cutoff energy of $13.9 \mathrm{fJ}$ used in GAUGE to one of $29 \mathrm{fJ}$ as used for HTGR spectra. Consequently, the 0.15 fraction of the fast flux falls into the energy range between $13.9 \mathrm{fJ}$ and $29 \mathrm{fJ}$.

\subsection{FLUENCES}

\subsubsection{Fluence Calculations}

The fast and thermal fluences have been calculated by multiplying the GAUGE-derived fast flux values with the applicable real time at operation. The GAUGE data represent the average values for a specific core location. The axial distribution is given by applying the FEVERderived fast and thermal flux distribution of Fig. 3-5. FEVER was used to calculate the unrodded or fully inserted control rod case; BUG-2 was applied to evaluate the partially rodded situation. All test elements were at least two locations away from a control rod position where no control rod influence on the axial profile was assumed.

The calculated fluence values for FTE- 6 are: 


\begin{tabular}{|c|c|c|c|c|c|}
\hline EOL F1uences & Peak & Mean & & $\underline{\mathrm{RMS}}$ & $\begin{array}{c}\text { Mean/Gradient }{ }^{*} \\
\text { Fue1 Body Sequence }\end{array}$ \\
\hline $\begin{array}{l}\text { Fast fluence } \\
\qquad\left(10^{25} \mathrm{n} / \mathrm{m}^{2}, \mathrm{E}>29 \mathrm{fJ}\right)\end{array}$ & 2.88 & 2.30 & \pm & 0.59 & $213 / 231$ \\
\hline $\begin{array}{l}\text { Thermal fluence } \\
\qquad\left(10^{25} \mathrm{n} / \mathrm{m}^{2}, \mathrm{E}<0.38 \mathrm{aJ}\right)\end{array}$ & 3.92 & 2.99 & \pm & 0.84 & $231 / 231$ \\
\hline
\end{tabular}

Detailed fluence information for each of the three fuel bodies and 14 fuel rod positions per body are given in Table 3-2. The RMS deviations used in Table 3-2 and above are derived via

$$
\operatorname{RMS}=\left\{\frac{1}{n} \sum\left(x_{i}-\bar{x}\right)^{2}\right\}^{1 / 2}
$$

where $\mathrm{n}=$ number of calculations, combined in one stratum,

$$
\begin{aligned}
x_{i} & =\text { calculation within one stratum, } \\
\bar{x} & =\text { strata mean. }
\end{aligned}
$$

A weighted RMS variation can be determined from

$$
\text { RMS }=\left\{\frac{1}{\Sigma n_{i}} \Sigma n_{i}\left(x_{i}-\bar{x}\right)^{2}\right\}^{1 / 2},
$$

where $\mathrm{n}_{i}=$ number (or length of interval) of observations in stratum $i$. RMS terms within an interval, RMS ${ }_{j}$, and along all considered intervals, $\underline{\mathrm{RMS}}$, can also be combined by the following equation:

$$
\begin{aligned}
\text { RMS }= & \left\{\frac{1}{\sum n_{j}} \sum n_{j} R_{j}{ }_{j}^{2}+\frac{1}{\sum n_{j}} \sum n_{j}\left(\bar{x}_{j}-\overline{\bar{x}}\right)^{2}\right\}^{1 / 2}=\left\{\overline{R M S}_{j}^{2}+\right. \\
& \left.\underline{\operatorname{RMS}}^{2}\right\}^{1 / 2} .
\end{aligned}
$$

* Example 213/231: Highest to lowest ranking for average value along body sequence $2,1,3$ / lowest to highest ranking for gradient or RMS data along body sequence $2,3,1$. 
The number of calculations $\mathrm{n}$ is arbitrarily selected. In the presented case, $n$ was determined by the number of fuel rods in an axial arrangement per body; the fluence data in turn were determined by linear interpolation between the 30 nodal points used in the TREVER calculations.

It can be concluded from Table 3-2 that body 2 received the highest fast and thermal fluence exposure, body 3 the lowest fast fluence, and body 1 the lowest thermal fluence. The RMS is proposed as a measure of the fluence gradients experienced in the fuel test element or its fuel bodies. The ranking from lowest to highest fluence gradient, fast and thermal, follows the sequence 231 (i.e., body 2 , body 3 , and body 1 ).

The core height information in Table 3-2 is based on BOL measurements for fuel body and fuel stack length and can be interchanged with EOL measurements, if required. It should be noted that all axial distributions are normalized over the full axia1 core height of the Peach Bottom core. In cases like FTE-6, where the accumulative ${ }^{*}$ fueled length of the element is less than the active length of the core $[(2.079 \mathrm{~m}$ (81.85 in.) at BOL; $2.036 \mathrm{~m}$ (80.18 in.) at EOL rather than $2.286 \mathrm{~m}$ (90.00 in.)], the axial profiles have been applied to the actual fuel length and all unfueled zones along the axis have been ignored, i.e., given the same flux levels as the adjacent fuel zones. This may be correct for determining local FIMA and power values, where the integration should equal the GAUGE-derived values. In the case of the fast flux distribution, this assumption is slightly arbitrary; however, it is believed to be acceptable in view of the overall accuracy of $\pm 10 \%$ for this kind of nuclear analysis, where two sets (radial and axial) of two-dimensional nuclear calculations are combined instead of using more accurate, but also more expensive, three-dimensional models.

* In Section 4.2.2.6, a discrimination between accumulative fueled length (sum of individual fuel rod lengths) and composite fueled length (sum of fuel rod stack lengths) is described. The latter results in slightly shorter stack dimensions, i.e., $2073 \mathrm{~mm}$ at BOL, $2033 \mathrm{~mm}$ at EOL (31.60 in. and 80.05 in., respectively). 


\subsubsection{F1uence Measurements}

Direct means of fluence measurements for FTE-6 were not available, i.e., no flux dosimetry was included in the experiment. However, gamma spectroscopy can be used as an indirect measure of integrated flux profiles. Long-life isotopes are employed in this comparison so that the decay during times of operation and shutdown is small and can be accounted for. Cs-137, having a 30-year half-life, is an ideal monitor as long as it is contained in the fuel. Cs-134, with a two-year half-life, is a second choice, but its shorter half-life makes corrections more important for reactor operation and shutdown times.

Cs-137 is a direct-yield isotope from the fission of U-235 and U-233, and its formation follows the thermal fluence profile linearly. Cs-134 is formed indirectly from fission through the decay of $\mathrm{Xe}-133$ by a ( $\beta-$ ) reaction to Cs-133, followed by neutron activation. Therefore, Cs-134 is formed in a quadratic correlation with the fluence and the Cs-134/ Cs-137 ratio should follow the fluence in a linear correlation.

A relative comparison between calculated thermal fluence profiles and measured cesium profiles has been attempted by the process of normalization (see Section 4.1.6). The cesium activities in counts per minute (CPM), which were back-decayed from the time of counting to the actual time of final reactor shutdown, have been used. No correction was made for reactor operation and interim shutdown times. In Fig. 3-6(a), a comparison is made between the normalized Cs-137 and Cs-134/Cs-137 profiles. The profiles are in agreement with each other within the range of the measurement uncertainties. The combination of profiles (via arithmetic average) is compared in Fig. 3-6(b) with the GAUGE/FEVER derived thermal fluence. The measured profile is significantly different from the predicted one. In absolute terms, the differences are sma11 and explainable by the uncertainties of the predictions. (A detailed description of the fluence identity test is given in Section 4.2.2.5). Table 3-2 contains the calculated fast and thermal fluence data and the 
measured thermal fluence profile. ${ }^{*}$ For the convenience of direct comparison, the latter has been multiplied by the mean calculated thermal fluence. It should be noted that the comparison is relative only. The absolute amount of thermal fluence experienced in FTE- 6 has not been verified through this exercise. The fuel burnup verification will allow indirect conclusions about the absolute level of thermal (and fast) fluence.

To clarify the evaluation procedure, the applied algorithm is presented in Eqs. 3-2 through 3-9. A generic description is given in Section 4.1.6.

Typically, two adjacent fixed-length segments of a fuel rod were scanned to determine the cesium activity of the fuel rod in CPM. These two segments represented up to $80 \%$ of the fuel rod and did not include the end zones. For each segment the scan was made continuously along the rod axis for seven minutes. In general, if $\mathrm{n}$ consecutive segments in a fuel rod $j$ are scanned, the true cesium activity, $x_{j}$, can be estimated by the mean of the counts $y_{1}, y_{2}, \ldots, y_{n}$ :

$$
x_{j}=\frac{1}{n} \Sigma y_{i}
$$

The standard deviations $\mathrm{s}_{\mathrm{yi}}$ of the counting errors are then combined according to

$$
s_{x_{j}}=\left(\frac{1}{n^{2}} \sum s_{y i}\right)^{2 / 2}
$$

If only one segment was scanned, an additional error arises because of the incomplete sampling. In this case,

$$
s_{x_{j}}=\left(s_{y 1}^{2}+\hat{s}^{2}\right)^{1 / 2}
$$

\footnotetext{
* The fuel body average values of the measured fluence profile in Table 3-2 are slightly different from the data reported in Section 4.2.2.5, because measurements and statistical tests were made for every other fuel rod along a fuel stack, whereas Table 3-2 contains interpolation values for every fuel rod.
} 
Where $\quad \hat{s}=\left\{\frac{1}{m} \Sigma_{j} \hat{s}_{j}^{2}\right\}^{1 / 2}$

is the RMS variation between segments in a single fuel rod for which $n_{j}>1$ segments are scanned,

$$
\hat{s}_{j}^{2}=\frac{1}{n_{j}} \sum\left(y_{i}-x_{j}\right)^{2}
$$

This intra-rod variability is averaged over $m$ rods (Eq. 3-4a), similar to the rod for which only one segment was scanned, and then is used in Eq. 3-3b.

It was further assumed that all rods contained the same amount of heavy metal prior to irradiation and that any variation from the specified loading was systematical and hence the same for each rod.

The activity estimates for the cesium distribution were normalized by dividing the individual rod activity by the mean activity of all rods measured:

$$
u_{j}=\frac{x_{j}}{\bar{x}}
$$

The standard deviation of $u_{j}$ can be estimated by progressing the standard deviations of $x_{j}$ and $\bar{x}$ (see Section 4.1.6):

$$
s_{u_{j}} \cong u_{j}\left\{\left(\frac{s_{j}}{x_{j}}\right)^{2}-\left(\frac{s_{\bar{x}}}{\bar{x}}\right)^{2}\right\}^{1 / 2} .
$$

The standard deviation of the grand mean $\bar{x}$ is determined from $k$ fuel rods of the analyzed fuel type

$$
s_{\bar{x}}=\frac{1}{k}\left\{s_{x_{j}}\right\}^{1 / 2}
$$


A typical counting error $(1 \sigma)$ was $s_{y_{i}} / y_{i}=1 \%$ of the counts (see Section 4.2.1), the standard deviation along a fuel rod was, e.g., $\hat{s}_{j} / x_{j}=$ $1.5 \%$, and the number of fuel rods analyzed was $k=21$, which gives the following typical values for the Cs-137 profile analysis:

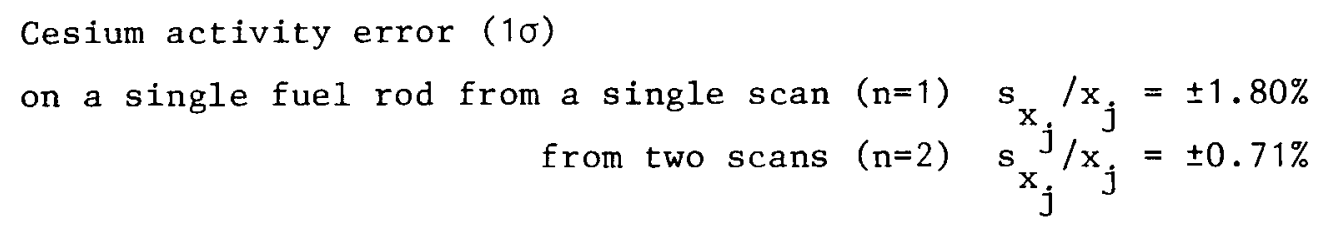

The normalization error is slightly lower than the uncertainty for the activity of a single fuel rod, i.e., by a factor $\sqrt{(k-1) / k}$ for the given example.

The evaluation of the $\mathrm{Cs}-134 / \mathrm{Cs}-137$ ratios for $\mathrm{n}$ scans, representing a single fuel rod, follows the same algorithm as given above by substitution into $\mathrm{Eq} \cdot 3-2$ of

$$
y_{i}=\left(\frac{N_{4}}{N_{7}}\right)_{i}
$$

where $\mathrm{N}_{4}=\mathrm{Cs}-134$ activity (CPM),

$$
\mathrm{N}_{7}=\text { Cs-137 activity (CPM). }
$$

The standard deviation of $y_{i}$ is determined by substitution into Eq. 3-3 of

$$
s_{y_{i}}=y_{i}\left\{\left(\frac{s_{4}}{N_{4}}\right)_{i}^{2}+\left(\frac{s_{7}}{N_{7}}\right)_{i}^{2}\right\}^{1 / 2}
$$


where $\frac{s_{4}}{N_{4}}=$ relative counting error of $\mathrm{Cs}-134$,

$$
\frac{s_{7}}{N_{7}}=\text { relative counting error of } \mathrm{Cs}-137 \text {. }
$$

Using the cesium counting errors from above, the following typical values can be derived for the Cs $-134 / \mathrm{Cs}-137$ ratio analysis:

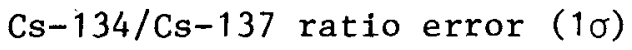

on a single fuel rod $\left(n=2\right.$ scans) $\quad s_{x_{j}} / x_{j}= \pm 1.00 \%$

Variation on normalized ratio

profile $(1 \sigma)$

$$
s_{u_{j}} / u= \pm 0.98 \%
$$

The error on the ratio is $\sqrt{2}$ times larger than on the individual cesium measurements (assuming the same counting error for both cesium isotopes).

Stratification of the measurements and separation of RMS variation from uncertainty information follows the algorithm as detailed in section 4.1.

A comparison has been made for the TRISO/TRISO fuel rod variety and for one TRISO/BISO fuel rod variety, both of which showed very little fuel failure. A numerical comparison between calculated and measured profiles is made in Table 3-2, from which the following comparable data can be drawn (within the limitations stated earlier) and sumarized below: 
Mean/Grad-

ient Fue1

Relative

Body

Thermal Fluence Comparison $\quad \underline{\text { Peak }} \pm$ Error Mean \pm RMS \pm Error Sequence

\begin{tabular}{|c|c|c|c|c|c|c|}
\hline $\begin{array}{l}\text { Calculated fluence (Table } \\
\text { 2-6) } \\
\qquad\left(10^{25} \mathrm{n} / \mathrm{m}^{2}, \mathrm{E}<2.38 \mathrm{eV}\right)\end{array}$ & 3.92 & - & 2.99 & 0.84 & - & $213 / 231$ \\
\hline ormalized Cs-137 profile & & & & & & \\
\hline TRISO/TRISO fuel & 3.74 & 0.05 & 2.99 & 0.62 & 0.05 & $213 / 231$ \\
\hline TRISO/BISO fuel & 3.70 & 0.06 & 2.99 & 0.63 & 0.05 & $213 / 231$ \\
\hline $\begin{array}{l}\text { Normalized } \mathrm{Cs}-134 / \mathrm{Cs}-137 \\
\text { profile }\end{array}$ & & & & & & \\
\hline TRISO/TRISO fue1 & 3.69 & 0.10 & 2.99 & 0.68 & 0.05 & $213 / 231$ \\
\hline TRISO/BISO fuel & 3.73 & 0.11 & 2.99 & 0.69 & 0.05 & $213 / 231$ \\
\hline $\begin{array}{l}\text { Combined measurements } \\
\text { (Table 2-6) }\end{array}$ & 3.70 & 0.04 & 2.99 & 0.65 & 0.05 & $213 / 231$ \\
\hline Bias $\hat{b}=\mathrm{calc} / \mathrm{m}$ & 6.04 & 1.11 & $-1 \cdot 30^{*}$ & 9.35 & 0.39 & $321 / 2$ \\
\hline
\end{tabular}

A11 measured values are relative values that have been multiplied by the mean calculated thermal fluence. Fuel rod end effects are not included in the measurements and corrections for reactor operation and interim shutdown times have not been made.

The following conclusions can be drawn from this comparison:

1. No significant difference can be detected for the TRISO/TRISO and TRISO/BISO fuel varieties.

2. The Cs-137 distribution and Cs-134/Cs-137 distribution agree within their $95 \%$ confidence bands for the majority of the fuel element. The bottom and top ends of the element show some difference (see Fig. 3-6(a)).

3. The measured fluence distribution is flatter ( 1.24 peak versus 1.31 predicted for body 2) than the calculated distribution

* Because of mode of stratification, the bias $\hat{b}$ for the total element is $\neq 0$ in Table $2-6$. 
and is slightly shifted towards the top end of the fuel element (see Fig. 3-6(b) and Table 3-2).

Further details about utilizing fuel gamma scan data in comparison with predicted fluence profiles are given in Section 4.2.

\subsection{TEMPERATURES}

\subsubsection{Temperature Calculations}

Temperature calculations were carried out with the TREVER program for 30 axial nodal points and seven radial locations by applying the power profile in Fig. 3-5; radial distribution and axial effects toward the fuel body ends were tuned with the TAC-2D code (Ref. 3-4). The axial temperature distribution was linearly interpolated to arrive at temperature statements for each midplane of the 42 fuel rods along the fuel stack ( 14 per fuel body). A typical radial temperature distribution is given in Fig. 3-7, which indicates the seven radial locations for which the following temperatures were determined:
A: bulk coolant temperature
COOLANT
B: minimum sleeve temperature
MIN SLV
C: maximum sleeve temperature
MAX SLV
D: minimum graphite fuel body temperature
MIN BODY
E: median graphite fuel body temperature
MAX BODY
F: minimum fuel rod temperature
MIN FUEL
G: maximum fue1 rod temperature
MAX FUEL

- The maximum temperature of the graphite fuel body is about the same as the center spine sample temperature, which has been determined from TAC-2D calculations to reach $85 \%$ of the temperature rise in the fue 1 $\operatorname{rod}(\operatorname{Ref} .3-4)$ :

$$
\mathrm{T}_{\mathrm{W} / \mathrm{Re}} \equiv \mathrm{T}_{\text {spine }}=0.85\left(\mathrm{~T}_{\text {fuel max }}-\mathrm{T}_{\text {fuel min })}+\mathrm{T}_{\text {fuel min. }}(3-10)\right.
$$


This temperature a1so approximates the temperature of the W/Re thermocouple, which was located between the spine and graphite fuel body (see Fig. 2-3). The temperature of the C/A thermocouple is determined from TAC-2D calculations to be:

$$
\mathrm{T}_{\mathrm{C} / \mathrm{A}}=1.018 \mathrm{~T}_{\max } \text { body } \quad \text {. }
$$

The median fuel body temperature, MAX BODY, is the representative upper graphite temperature for the shortest heat transfer path from the fuel to the sleeve.

Volume average temperatures have been determined for

$\begin{array}{ll}\text { Average sleeve temperature } & \text { SLV AVG } \\ \text { Average fuel body temperature } & \text { BODY AVG } \\ \text { Average fuel rod temperature } & \text { FUEL AVG }\end{array}$

These are arithmetic means between maximum and minimum (e.g., sleeve) temperatures, because the integration over the volume and temperature profile gave only slightly different results. The average temperatures have been used to determine the material properties (thermal expansion, thermal conductivity, and Wigner strain) for the different components with the exception of the fuel body where the median body temperature was used as reference for thermal and Wigner strain. The material properties used in the thermal evaluation are presented together with the stress analysis in Section 3.5 .

Al1 temperatures have been time averaged, based on the real time at operation used in the TREVER simulation. Time variation RMS information is available from these calculations. It is not reported, however, except for a comparison between measured and calculated temperatures at the thermocouple location. In addition to the above temperatures, a search program has been used to identify the maximum and minimum temperature for the fuel rod, the fuel body, and the sleeve. 
Envelope temperatures, as we11 as time and time-volume average temperatures, are tabulated in Tables 3-3(a) through 3-3(d) for the four fuel varieties. The TRISO/BISO fuel content varied from 0 to 74 vol \% BISO, which is the controlling parameter in the interrelated fuel rod strain, fuel rod - fuel body gap, heat transfer, and temperature predictions.

The maximum difference at peak in time (envelope temperature) between the four groups is about $30^{\circ} \mathrm{C}$, with the fuel rod variety in holes 1 and 2 being the highest because of the higher BISO fuel content and the consequently higher shrinkage of these fuel rods.

The axial distributions of the time and time-volume-average temperatures are given in Fig. 3-8, the axial temperature envelope distribution is shown in Fig. 3-9, and the axial temperature distributions for each of the TREVER time intervals are presented in Figs. 3-10 through 3-24. All graphically displayed temperatures are for the fuel rod variety in holes 7 and 8 . Temperature calculations are based on a uniform azimuthal temperature distribution.

Using the thermal calculation as a basis, the following fuel rods can be identified as having experienced the highest irradiation temperatures.

\section{Highest Irradiation Temperature}

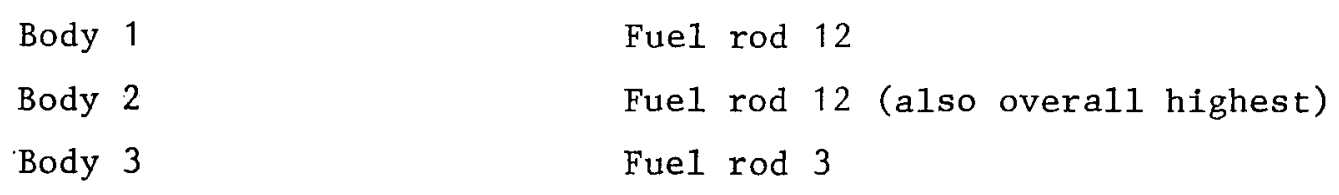

This is based on time-weighted temperatures. Highest envelope conditions were experienced by rod 4 in body 3 . The temperature performance is summarized in Section 3.3.2. 
Irradiation temperatures for the spine samples are listed in Table 2-6 together with spine sample burnups and fluences. It should be noted that the thermal analysis did not account for any fission heat generation from spine samples or purge flow heat transfer effects except for gamma heating, which was assumed to be $10 \%$ of the element power. Spine sample temperatures have been averaged by two different time weighting methods:

$$
\begin{aligned}
& \bar{T}=\Sigma_{i}\left(W_{i} T_{i}\right) \\
& R M S \frac{T}{T}=\frac{1}{\sum W_{i}} \sum W_{i}\left(T_{i}-\bar{T}\right)^{2},
\end{aligned}
$$

where $W_{i}=$ weighing factor for time interval $\Delta t_{i}$.

(a) Linear weighting:

$$
w_{i}=\frac{\Delta t i}{\sum \Delta t_{i}}
$$

(b) Exponential weighting:

$$
W_{i}=\frac{\Delta t_{i} \exp \left(-Q / R T_{i}\right)}{\sum \Delta t_{i} \exp \left(-Q / R T_{i}\right)}
$$

where $Q=$ activation energy or equivalent,

$\mathrm{R}=$ gas constant .

The first weighting procedure is appropiate for general fuel and material performance correlations. The latter procedure is applicable for any kind of kinetic effect (e.g., amoeba, SiC attack, fission product diffusion).

\subsubsection{Temperature Measurements}

FTE-6 was assembled with the following two thermocouples at $1.918 \mathrm{~m}$ (75.5 in.) total core height (i.e., $1.257 \mathrm{~m}$ ( $49.5 \mathrm{in.}$ ) active core height). See Figs. 2-1 and 2-3 for detailed axial and radial location. 
TC-A: W/Re type thermocouple near spine sample.

TC-B: C/A type thermocouple near outer sleeve location.

Readings were recorded on a weekly basis and had to be corrected for the following:

1. Changes in cold junction temperature (standoff pin temperature) for both types; the correction was done automatically at the reactor site.

2. Adjustment in calibration equipment for the $W /$ Re type, for which readings were $22^{\circ} \mathrm{C}\left(40^{\circ} \mathrm{F}\right)$ too high because of change from the $\mathrm{W}-5 \% \operatorname{Re} / \mathrm{W}-26 \%$ Re type to the $\mathrm{W}-3 \% \operatorname{Re} / \mathrm{W}-25 \% \operatorname{Re}$ type between Core 1 and Core 2 .

3. Decalibration of the W/Re type only for effect on emf output by neutron bombardment and transmutation.

The decalibration can be correlated with the thermal fluence as we11 as with probable fluence and temperature gradient effects. Recalibration has been carried out on a group of Peach Bottom test elements. FTE-6 was not included because of thermocouple damage during unloading.

Recalibration results (Ref. 3-9) are presented in Fig. 3-25, which plots the actual temperature $\mathrm{T}_{a}$ against the indicated temperature $\mathrm{T}_{i}$. The recalibration results have been analyzed via linear regression by the method of least squares under the assumption of $0^{\circ} \mathrm{C}$ as the cold junction temperature:

$$
\mathrm{T}_{\mathrm{a}}=\mathrm{T}_{\mathrm{o}}+\mathrm{K} \cdot \mathrm{T}_{\mathrm{i}},
$$

with the regression constants being

$$
\mathrm{T}_{\mathrm{o}}=\frac{1}{\mathrm{n}} \Sigma \mathrm{T}_{\mathrm{a}}-\frac{\mathrm{K}}{\mathrm{n}} \Sigma \mathrm{T}_{\mathrm{i}}
$$


and

$$
\mathrm{K}=\frac{\sum \mathrm{T}_{i_{\mathrm{a}}} \mathrm{T}_{\mathrm{a}}-\frac{\sum \mathrm{T}_{\mathbf{i}} \Sigma \mathrm{T}_{\mathrm{a}}}{\mathrm{n}}}{\sum \mathrm{T}_{i}{ }^{2}-\frac{\left(\sum \mathrm{T}_{i}\right)^{2}}{\mathrm{n}}}
$$

The correlation coefficient was determined via

$$
r^{2}=k \cdot \frac{\sum T_{i}^{2}-\frac{\left(\Sigma T_{i}\right)^{2}}{n}}{\sum T_{a}{ }^{2}-\frac{\left(\Sigma T_{a}\right)^{2}}{n}} .
$$

The correlation coefficient was in all cases very close or equal to 1.0 (see Fig. 3-25), which indicated very good linear correlation for the covered temperature range $\left(680^{\circ}\right.$ to $980^{\circ} \mathrm{C}$ for $\left.\mathrm{T}_{\mathrm{a}}\right)$. This represents irradiation temperatures of $1020^{\circ}$ to $1320^{\circ} \mathrm{C}$, based on a standoff pin temperature of $340^{\circ} \mathrm{C}$. For thermocouple FPTE-3B the range was further extended downward to $420^{\circ} \mathrm{C}$ (i.e., $760^{\circ} \mathrm{C}$ irradiation temperature), which gave similar results. It was therefore concluded that the recalibration was sufficient for extrapolation towards higher temperatures as we11. (These temperatures were not achievable during the recalibration because of a furnace limitation.) However, most of the experienced irradiation temperatures are covered by this recalibration exercise.

The regression constants $\mathrm{T}_{0}$ and $\mathrm{K}$ were correlated with the thermal fluence $\Psi$. For the intercept $T_{0}$, a second order fit was attempted, and a first order fit was attempted for the slope K (see Fig. 3-26(a) and 3-26b)). It was found that the thermocouples from FTE-18 and FTE-5 were following a similar pattern, whereas the FPTE-3 thermocouples had to be treated separately. This finding may be based on the fact that FPTE-3 carried the hot junctions within the fuel rather than in the graphite or matrix components. (FTE-18 was a molded block experiment using a molded graphite matrix rather than machined graphite bodies as structural material; see Ref. 2-4.) The thermocouples in FPTE-3 were of different lengths, which resulted in different fluence and temperature gradients. 
The following recalibration formula was determined for FTE-18 and FTE-5, which gave agreement within $\pm 100^{\circ} \mathrm{C}$ by application to FTE-4 (see Ref. 2-2: time-averaged calculated temperature was $+100^{\circ} \mathrm{C}$ above timeaveraged and decalibration-compensated thermocouple reading) and to FTE-18 (see Ref. 2-4: calculated temperature mean was $-80^{\circ} \mathrm{C}$ below the mean thermocouple temperature).

$$
\mathrm{T}_{\mathrm{a}}=31.638 \Psi+2.1181 \Psi^{2}+(1+0.12172 \Psi)\left(\mathrm{T}_{i}-\mathrm{T}_{\mathrm{s}}\right)+\mathrm{T}_{\mathrm{s}},(3-16)
$$

where $\mathrm{T}_{\mathbf{a}}=$ actual irradiation temperature (corrected signal), ${ }^{\circ} \mathrm{C}$,

$\mathrm{T}_{i}=$ indicated temperature (uncorrected signal), ${ }^{\circ} \mathrm{C}$,

$\mathrm{T}_{\mathrm{S}}=$ standoff pin temperature (cold junction), ${ }^{\circ} \mathrm{C}$,

$\Psi=$ thermal fluence in $\left(10^{25} \mathrm{n} / \mathrm{m}^{2}, E<38 \mathrm{aJ}\right)$, dimensionless.

The mean standoff pin temperature for $\mathrm{FTE}-6$ was $\mathrm{T}_{\mathrm{S}}=341^{\circ} \mathrm{C}$, which has been used when no standoff temperature was reported with the thermocouple readings. Where available, the actual value for $\mathrm{T}_{\mathrm{S}}$ was used. Since an uncertainty evaluation of the thermocouple is not available, no uncertainty limits can presently be stated.

The C/A thermocouple was short-circuited shortly after BOL at a core location which was estimated to coincide with fuel rod 4 position of the bottom body. (One partial recovery occurred at 552 EFPD, which was omitted in the evaluation.) The $\mathrm{W} / \mathrm{Re}$ thermocouple was functional for the whole irradiation period. A time history of the uncorrected and corrected thermocouple readings is given in Figs. 3-27(a) and 3-27(b) in actual and stratified form together with the TREVER-calculated temperature. Tables 3-4(a) and 3-4(b) tabulate the TREVER temperatures together with the measured and recalibrated temperature readings. The thermocouple readings have been combined for each of the TREVER intervals on a real-time-average basis. The RMS deviation of these periods can be used as medium-term (over a weekly period) time variation information. These week-long variations range between $\pm 8^{\circ}$ and $\pm 129^{\circ} \mathrm{C}$ for the $\mathrm{W} / \mathrm{Re}$ 
type and between $\pm 5^{\circ}$ and $\pm 80^{\circ} \mathrm{C}$ for the $\mathrm{C} / \mathrm{A}$ type. The TREVER and thermocouple readings have been averaged over the whole period of irradiation, resulting in the following mean and time variant information:

\section{Temperature Comparison}

W/Re thermocouple at $1.918 \mathrm{~m}$

icore height (Table 3-4(a))

TREVER-predicted $\mathrm{T}_{\mathrm{A}}\left({ }^{\circ} \mathrm{C}\right)$

Recalibrated thermocouple

${ }^{\circ} \mathrm{T}_{\mathrm{C}}\left({ }^{\circ} \mathrm{C}\right)$

C/A thermocouple at $837 \mathrm{~mm}$

core height (Table $3-4(\mathrm{~b})$ )

TREVER-predicted $\mathrm{T}_{\mathrm{A}}\left({ }^{\circ} \mathrm{C}\right)$
Thermocouple $\mathrm{T}_{\mathrm{C}}\left({ }^{\circ} \mathrm{C}\right)$

Peak $\quad$ Mean $\quad \begin{array}{cc}\text { Long Term } \\ \text { RMS }\end{array} \quad \begin{gathered}\text { Medium Term } \\ \text { RMS }\end{gathered}$

1293

1173

$\pm 98$

N.D.

$1336^{*} 1200$

$\pm 62$

$\pm 87$

$737 \quad 663 \quad \pm 50 \quad$ N.D.

$758^{*} \quad 665 \quad \pm 34 \quad \pm 52$

For the W/Re thermocouple, the TREVER-predicted long-term RMS of $\pm 98^{\circ} \mathrm{C}$ is about $37^{\circ} \mathrm{C}$ higher than the RMS from the stratified thermocouple readings and about $11^{\circ} \mathrm{C}$ higher than the time-averaged medium-term RMS. It therefore can be concluded that the time variation is overpredicted by the TREVER simulation. This is consistent with FTE-18 (Ref. 2-4), where the long-term RMS was overpredicted by $40^{\circ} \mathrm{C}$ and was $21^{\circ} \mathrm{C}$ higher than the measured medium-term RMS, and with FTE-4 (Ref. 2-2), where the RMS was overpredicted by $12{ }^{\circ} \mathrm{C}$ for the long-term RMS only but the measured medium-term RMS exceeded the prediction by $8^{\circ} \mathrm{C}$. For the $\mathrm{C} / \mathrm{A}$ thermocouple in FTE-6, the same tendency of overprediction of the long-term variation (by $16^{\circ} \mathrm{C}$ ) can be seen; the medium-term RMS exceeds the prediction by $2^{\circ} \mathrm{C}$.

The agreement for the time-weighted mean values is within $27^{\circ} \mathrm{C}$ and 'is partially related to a compensation of periods of overpredictions by periods of underpredictions. As stated earlier, the agreement was within $\pm 100^{\circ} \mathrm{C}$ for FTE-4 and FTE-18.

*Highest (recalibrated) read-out during irradiation, which occurred at 614 EFPD for W/Re thermocouple and at 800 EFPD for C/A thermocouple. The peak TREVER temperatures were calculated for the intervals 610 to 701 EFPD and 835 to 858 EFPD, respectively (see Tables 3-5(a) and 3-5(b)). 
The thermal evaluation can be summarized with the values listed below (see also Table 3-3(a)). Mean temperatures are time averages of real time at operation. Maximum and minimum fuel temperatures for the total element (calculated temperatures) or for the time history (thermocouple comparison) have been selected for the two extreme cases within the TREVER simulation at the radial location of maximum and minimum fuel temperature respectively. Time-averaged fuel temperatures at locations of maximum and minimum fuel temperature are also included in Table 3-3, and should not be confused with time maxima and minima of a specific 1ocation. The RMS deviation on the calculated mean temperature represents axial variation information for the time-weighted mean fuel element temperature and temporal time variation information for the thermocouple comparison.

Calculated Temperatures (Holes $1 \& 2$ )

Fuel average $\left({ }^{\circ} \mathrm{C}\right)$

(time and volume)

Fue1 maximum $\left({ }^{\circ} \mathrm{C}\right)$

(time and location)

Fuel minimum $\left({ }^{\circ} \mathrm{C}\right)$

(time and location)
Mean/Gradient

Peak Mean \pm RMS Axial Fuel Body Sequence

$1161 \quad 1024 \quad 141$

$231 / 231$

$321 / 231$

$1429 \quad 1242 \quad 175$

96

$231 / 231$

The following conclusions can be drawn from the thermal evaluation:

1. The time-averaged thermocouple temperature is $+27^{\circ} \mathrm{C}$ higher than the TREVER predictions, which gives credibility to the applied thermocouple recalibration as well as to the thermal calculation.

2. The power history simulation for FTE-6 may be questioned with regard to actuality of the true history, although it appears to be approximately correct for the time average.

3. Calculated values for time variation effects are larger than determined from weekly thermocouple readings. 
4. Body 2 experienced the highest temperatures and lowest temperature gradients.

5. The conclusions drawn are consistent with earlier findings for FTE-4 and FTE-18.

3.4. BURNUP

\subsubsection{Burnup Calculations}

Burnup is defined as fissions per initial metal atom (FIMA) and can be related to fissile, fertile, or composite or mixed heavy metal atoms. The algorithm for determining burnup values from GAUGE calculations is given in Ref. 2-2. GAUGE calculations are integrated values over the total length of the element, the axial distribution of which is determined from FEVER calculations as a function of residence time in the reactor. Figure 3-28 gives the normalized axial distribution of fissile FIMA, Fig. 3-29 gives fertile FIMA, and Fig. 3-30 gives composite or mixed FIMA for a Th:U atomic ratio of $\mathrm{N}=5.5$. For other composite burnups, the following formula can be applied:

$$
F_{C}=\frac{1}{1+N}\left(F_{5}+N \cdot F_{3}\right)
$$

where $\mathrm{N}=$ Th: U atomic ratio,

$$
\begin{aligned}
& \mathrm{F}_{5}=\text { fissile FIMA, } \\
& \mathrm{F}_{3}=\text { fertile FIMA. }
\end{aligned}
$$

Mass spectrometry allows the comparison with specific isotopes by atomic ratios, or in the case of isotopic diluted mass spectrometry (IDMS) by concentration. Calculated isotopic distributions for seven individual nuclides and three different duration times (300, 600, and 900 EFPD) in Peach Bottom are given in Figs. 3-31 and 3-32. The actual form factor for a specific interval should be obtained by interpolation. 
Fuel loadings for FTE-6 are given in Table 3-5 for the four different fuel blends, with one blend per two holes. Blends varied by fertile fuel loading, not by fissile loading; the fertile fuel had been used to shim the fuel rods up to $62 \%$ volume packing fraction (see Table 2-4). Additional heavy metal was located in the center spine samples. EOL total element loading had been taken from the GAUGE calculation, for which linear corrections had to be made for the Th loading, which happened to be $5.11 \%$ too low for BOL in the calculation against the asloaded figures.

The following peak and mean values and axial RMS, which were taken from Table 3-6, can be stated for FTE-6:

$\begin{array}{lrrrrr}\text { Burnup Calculation } & \text { Peak } & \text { Mean } & \pm \text { RMS } & \begin{array}{c}\text { Mean/Gradient } \\ \text { Fuel Body Sequence }\end{array} \\ \text { Fissile FIMA (\%) } & 45.60 & 38.20 & 7.09 & 213 / 231 \\ \text { Fertile FIMA (\%) } & 1.56 & 1.03 & 0.45 & 213 / 231 \\ \text { Mixed FIMA (\%) } & 13.30 & 10.94 & 1.75 & 213 / 231 \\ \quad(2.75 \text { Th:U) } & & & & \\ \text { Composite FIMA (\%) } & & & & 213 / 231 \\ \text { Holes 1 and 2 } & 6.62 & 5.31 & & 213 / 231 \\ \text { Holes 3 and 4 } & 12.54 & 10.30 & & 213 / 231 \\ \text { Holes 5 and 6 } & 12.79 & 10.52 & & 213 / 231 \\ \text { Holes 7 and 8 } & 17.57 & 14.54 & & \\ \text { Tota1 element } & 10.20 & 8.32 & 1.75 & \end{array}$

The composite FIMA values are calculated using Eq. 3-17, the fissile and fertile FIMA values in Table 3-6, and the following $\mathrm{N}$ values for the different fuel blends:

$\begin{array}{lc}\frac{\text { Holes }}{1 \text { and } 2} & \frac{N}{7.69} \\ 3 \text { and } 4 & 3.01 \\ 5 \text { and } 6 & 2.92 \\ 7 \text { and } 8 & 1.75 \\ \text { Total element } & 4.09\end{array}$




\subsubsection{Burnup Measurements}

Four different burnup measurement methods have been applied to FTE-6:

1. Radiochemistry (Refs. 3-10 and 3-11)

2. Mass Spectrometry (Refs. 3-10 and 3-12)

3. Isotopic Diluted Mass Spectrometry (Ref, 3-13)

4. Gamma Spectroscopy (Ref. 3-14).

Methods 1, 2, and 3 were employed for loose particles, irradiated in two spine positions of body 3 . No loose particle samples were contained in bodies 1 and 2, which limited these examinations to cross method checks and did not allow any burnup profile examination. Method 4 was used for every other fuel rod along the axial length of the fuel element and for each of the four fuel blends. Axial fluence (see Section 3.2.1) and burnup profile information, as well as fuel failure information (see Section 4.5.4), were obtained from this analysis. Methods are described in Ref. 2-2 together with analysis functions and associated error ana1ysis. The nuclear constants and uncertainty limits are given in Table 3-7. They have been taken from Refs. 3-15 through 3-18. Results of analysis from Ref. 3-19 have been analyzed further in this report for associated errors. Two loose fuel particle samples from different spine locations of body 3 were analyzed in duplicate or triplicate by each method. One sample was a UC 2 TRISO fissile fuel particle, the other a fertile fuel $\mathrm{ThC}_{2}$ TRISO particle.

\subsubsection{Isotopic Analysis. Mass spectrometric results are contained} in Table $3-8$ as relative atomic concentration (atom \%); isotopic diluted mass spectrometry results are shown in Table 3-9 as absolute concentration (ppm). Table 3-8 includes a comparison of the mass spectrometric 
results with GAUGE/FEVER calculations, and Table 3-10 compares IDMSdetermined uranium content per analyzed fuel particle with colorimetric results.

The following conclusions can be drawn from Tables 3-8, 3-9, and 3-10 for the mass spectrometric and colorimetric laboratory data in comparison with each other and with FEVER/GAUGE calculations:

1. Mass spectrometry and IDMS agree very well for isotopic concentrations.

2. Contrary to common knowledge, the uncertainties for the IDMS concentrations are larger than for the mass spectrometry results. The validity of the error statements for the mass spectrometry data as stated by the laboratory is questioned.

3. When progressing the IDMS uncertainties for the individual nuclides in Table 3-10 via

$$
s=\left(\sum s_{i}^{2}\right)^{1 / 2}
$$

the error statement associated with the total uranium concentration is smaller than the value quoted by the laboratory. It is concluded that there is a slight conservatism for the IDMS error statements, which appear to be the sum of the errors of the single measurements.

4. A comparison of the uranium content determined by IDMS and by colorimetry for each of the four particles (two fissile, two fertile) analyzed gives a $-4.3 \%$ lower colorimetric value with a standard deviation of $\pm 7.5 \%$ (see Table 3-10).

5. A comparison of the mass spectrometric data (combination of mass spectrometry and IDMS results, Table 3-9) with the 
GAUGE/FEVER calculated isotopic concentrations has been made for fissile and fertile fuel:

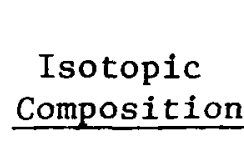

Fissile Fuel

$\mathrm{U}-234$

$\mathrm{U}-235$

$\mathrm{U}-236$

$\mathrm{U}-238$

Fertile Fuel

U-233

$\mathrm{U}-234$

$\mathrm{U}-235$

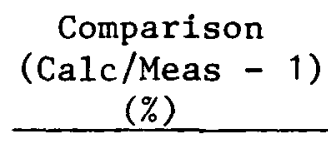

$-26.8$

$+3.3$

$-14.8$

$-5.5$

$-1.3$

$+16.0$

$+34.6$
Error

(1б)

(\%)

$\pm 0.4$

$\pm 0.1$

$\pm 0.3$

$\pm 0.5$

$\pm<0.1$

$\pm 0.6$

$\pm 7.4$

No calculated nuclide composition was available from GAUGE ca1culations for U-236 and U-238 bred fuel, although measured.

Al1. error statements result from the measurement errors only. No specific knowledge is presently available for the nuclear calculations, which are commonly stated to be accurate within $\pm 10 \%$ (Ref. 3-20).

3.4.2.2. Fissile and Fertile FIMA Analysis. A comparison has been made for fissile and fertile FIMA values in Table 3-11, as determined by the different methods and as calculated from GAUGE/FEVER data. Mass spectrometry results have been employed by three different ASTM analysis functions (Ref. 3-12; see Ref. 2-2 for detailed application and error progression):

ASTM Equation 1

$$
\mathrm{F}_{5}=\mathrm{N}_{5}^{\mathrm{O}}+\mathrm{N}_{6}^{\mathrm{O}}-\frac{\mathrm{N}_{8}^{\mathrm{O}}}{\mathrm{N}_{8}} \cdot\left(\mathrm{N}_{5}+\mathrm{N}_{6}\right) \text {. }
$$


ASTM Equation 2

$$
\mathrm{F}_{5}=\left(\mathrm{N}_{5}^{\mathrm{o}} \cdot \mathrm{N}_{6}-\mathrm{N}_{6}^{\mathrm{o}} \cdot \mathrm{N}_{5}\right) /\left(\mathrm{N}_{6}+\mathrm{N}_{6} \cdot \alpha_{5}+\mathrm{N}_{5} \cdot \alpha_{5}\right) \text {. }
$$

ASTM Equation 3

$$
\mathrm{F}_{5}=\left(\mathrm{N}_{5}^{\mathrm{o}}-\mathrm{N}_{5} \cdot \frac{\mathrm{N}_{8}^{\mathrm{o}}}{\mathrm{N}_{8}}\right) /\left(1+\alpha_{5}\right)
$$

where $\mathrm{N}_{i}^{0}=$ heavy metal atom percent of uranium isotope $i$ (last digit of mass number used) related to the total uranium atoms, in the preirradiated fuel,

$\mathrm{N}_{\mathbf{i}}=$ the same as above for the irradiated fuel,

$\alpha_{5}=$ effective ratio of $U-235(n, \gamma)$ capture-to-fission cross section,

$\mathrm{F}_{5}=$ fissile FIMA.

$\left(\mathrm{F}_{3}=\right.$ fertile FIMA.)

The $\alpha_{5}$ value is a function of core location and duration time and can be deduced from the GAUGE depletion calculation. For FTE-6,

$$
\alpha_{5}=0.242 \pm 0.009
$$

has been used for the FIMA determination. Mass spectrometry and IDMS results have both been evaluated with Eqs. 3-19 through 3-21. IDMS uranium values have further been employed to reduce the error of the radiochemistry values.

The following conclusions can be drawn from Table 3-11, and are in line with the conclusions of the isotopic analysis:

1. Mass spectrometry and IDMS-derived FIMA values are in very good agreement. 
2. The uncertainties associated with the mass spectrometric FIMA values are lower than for the IDMS values. This is contrary to experience and related to doubtful error statements. IDMS values are believed to be the most accurate ones.

3. Application of the IDMS uranium values improved the radiochemistry results significantly; however, they are still inferior to the mass spectrometric data.

4. The use of a capture-to-fission ratio of $\alpha_{5}=0.242 \pm 0.009$ agrees with the results obtained from all three ASTM analysis functions. This confirms the selection of $\alpha=0.242$.

5. A numerical comparison between the measured and calculated FIMA values can be made for the two reference locations in body 3 .

Burnup

Comparison

Fissile FIMA

(2684-mm core

height)

Mass Spec.

Error $(1 \sigma)$

IDMS

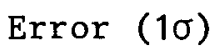

Radiochem. Cs-137

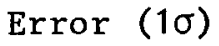

Radiochem. $\mathrm{Zr}$

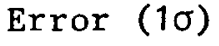

Radiochem./IDMS

$$
\mathrm{Cs}-137
$$

Error $(1 \sigma)$

Radiochem./IDMS Zr

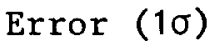

GAUGE/FEVER Ca1c.

Error

\begin{tabular}{ccc}
$\begin{array}{c}\text { Sample } \\
\text { Mean }\end{array}$ & $\begin{array}{r}\text { Sample } \\
\text { Mean }\end{array}$ & $\frac{\text { Meas. }}{\text { Meas.* }}-1 \frac{\text { Calc. }}{\text { Meas. }}-1$ \\
$(\%)$ & $(\%)$ & $(-)$ \\
\hline
\end{tabular}

$\begin{array}{llcc}34.80 & 2 & -0.0046 & -0.128 \\ \pm 0.31 & & \pm 0.0127 & \pm 0.008 \\ 35.12 & 2 & +0.0046 & -0.136 \\ \pm 0.52 & & \pm 0.0175 & \pm 0.013 \\ 35.81 & 2 & +0.0243 & -0.152 \\ \pm 1.72 & & \pm 0.0501 & \pm 0.041 \\ 30.69 & 2 & -0.122 & -0.011 \\ \pm 3.71 & & \pm 0.106 & \pm 0.119 \\ & & & \\ 33.64 & 2 & -0.038 & -0.098 \\ \pm 1.32 & & \pm 0.039 & \pm 0.035 \\ 28.67 & 2 & -0.180 & 0.0586 \\ \pm 3.20 & & \pm 0.092 & \pm 0.118 \\ 30.35 & 1 & -- & --\end{array}$




\begin{tabular}{|c|c|c|c|c|}
\hline $\begin{array}{c}\text { Burnup } \\
\text { Comparison } \\
\end{array}$ & $\begin{array}{c}\text { Sample } \\
\text { Mean } \\
(\%) \\
\end{array}$ & $\begin{array}{l}\text { Sample } \\
\text { Number }\end{array}$ & $\begin{array}{c}\frac{\text { Meas. }}{\text { Meas.** }}-1 \\
(-)\end{array}$ & $\begin{array}{c}\frac{\text { Meas. }}{\text { Meas.* }}-1 \\
(-) \\
\end{array}$ \\
\hline$\frac{\text { Fertile FIMA }}{\begin{array}{c}\text { (2589-mm core } \\
\text { height })\end{array}}$ & & & & \\
\hline $\begin{array}{l}\text { Radiochem. Cs-137 } \\
\text { Error }(1 \sigma)\end{array}$ & $\begin{array}{l}0.88 \\
\pm 0.026\end{array}$ & 5 & $\begin{array}{l}+0.011 \\
\pm 0.093\end{array}$ & $\begin{array}{l}-0.159 \\
\pm 0.025\end{array}$ \\
\hline $\begin{array}{l}\text { Radiochem. Zr-95 } \\
\text { Error }(1 \sigma)\end{array}$ & $\begin{array}{l}0.74 \\
\pm 0.028\end{array}$ & 5 & $\begin{array}{l}-0.149 \\
\pm 0.081\end{array}$ & $\begin{array}{r}0.000 \\
\pm 0.038\end{array}$ \\
\hline $\begin{array}{l}\text { Radiochem/IDMS } \\
\quad \text { Cs- } 137 \\
\text { Error }(1 \sigma)\end{array}$ & $\begin{array}{c}0.87 \\
\pm 0.076\end{array}$ & 2 & $\begin{array}{l}-0.000 \\
\pm 0.000\end{array}$ & $\begin{array}{l}-0.149 \\
\pm 0.074\end{array}$ \\
\hline $\begin{array}{l}\text { Radiochem/IDMS } \mathrm{Zr} \\
\text { Error }(1 \sigma)\end{array}$ & $\begin{aligned} & 0.76 \\
\pm & 0.045\end{aligned}$ & 2 & $\begin{array}{l}-0.126 \\
\pm 0.092\end{array}$ & $\begin{array}{l}-0.026 \\
\pm 0.058\end{array}$ \\
\hline $\begin{array}{l}\text { GAUGE/FEVER calc. } \\
\text { Error }\end{array}$ & 0.74 & 1 & -- & -- \\
\hline
\end{tabular}

All error statements result from the measurements only. The combined mass spectrometric and IDMS values (Meas.*) have been used to compare methods as well as calculations for finite FIMA. Fertile FIMA measurements were compared with radiochemistry results using Cs-137 as burn-up monitor and IDMS-determined uranium content (Meas.**).

6. The fissile and fertile FIMA for a specific core location show deviations between calculated and measured burnup by $-0.132 \pm 0.079$ $(1 \sigma)$ and $-0.149 \pm 0.033$ for fissile and fertile FIMA, respectively, when compared to what is believed the most reliable measurement method (Meas.* and Meas.**).

3.4.2.3. Composite Burnup Analysis. Every other fuel rod along the fuel stack length and each of the four fuel blends was subjected to multiisotopic gamma scanning. The Cs-137 activity was employed to determine the composite burnup and to provide for a comparison of activity verus the calculated fluence profile (see Section 3.2.2) and for fuel failure analysis (Section 4.5.4). Analysis functions and error treatment are given in Ref. 2-2. The results of this analysis are presented in Table 
3-12 together with the GAUGE/FEVER derived composite burnup values. A visual display is given in Fig. 3-33. All available burnup measurements have been collected in Table 3-13 for a comparison between methods and calculated burnup statements on the basis of fuel rods closest to the spine locations from which the burnup samples were taken. No mixed particles had been submitted for analysis; therefore, the composite burnup was deduced from the fissile and fertile values according to Eq. 3-17 and the following assumptions:

1. The location of the fissile fuel particles from spine sample TS6-5 has been used as the core height reference for the comparison.

2. The fertile fuel particles from spine sample TS18-3 were $95 \mathrm{~mm}$ from the fissile fuel spine location. Measured burnup has therefore been modified using the FEVER Form Factor FFF(x) from Fig. 3-17:

$$
\mathrm{F}_{3}\left(\mathrm{x}_{2}\right)=\mathrm{F}_{3}\left(\mathrm{x}_{1}\right) \cdot \frac{\mathrm{FFF}\left(\mathrm{x}_{2}\right)}{\mathrm{FFF}\left(\mathrm{x}_{1}\right)}
$$

3. Composite burnup values were calculated via Eq. 3-17 using the following combinations:
a. Radiochemistry, Cs-137 method.
b. Radiochemistry, $\mathrm{Zr}$ method.
c. Radiochemistry plus IDMS-determined uranium, Cs-137 method.

d. Radiochemistry plus IDMS-determined uranium, $\mathrm{Zr}$ method.

e. Mass spectrometry for fissile FIMA as a mean of all three
ASTM Eqs. 3-19, 3-20, and 3-21, fertile FIMA from c. above. 


\section{f. IDMS for fissile FIMA as a mean of all three ASTM equa- tions, fertile FIMA from c. above.}

4. Selection of the most accurate burnup value and method was based on the associated error statement. This burnup value was used for comparison with the gamma spectroscopically-determined and GAUGE/FEVER calculated fuel rod burnups.

5. The comparable gamma spectroscopic burnup value was determined for the reference location from the neighboring fuel rods for which linear regression could be visualized in Fig. 3-33 (up to 6 rods away from the reference location, and as little as 1 rod away where fuel failure and cesium loss were determined). The linear regression

$$
y=y+k(x-\bar{x})
$$

with the regression constant $\mathrm{K}$ (Eq. 3-14), allows an unbiased estimate of the standard deviation via

$$
s_{u}^{2}(x)=\frac{n}{n-2}\left(s_{y}^{2}-k^{2} \cdot s_{x}^{2}\right)\left\{\frac{1}{n}+\frac{(x-\bar{x})^{2}}{\sum\left(x_{i}-\bar{x}\right)^{2}}\right\},
$$

where $s_{y}{ }^{2}$ and $s_{x}{ }^{2}$ are the variances of the observed $y$ values (burnup) and of the observed $x$ values (core height), respectively. A detailed description of the regression analysis is given in Section 4.1.5.

The following conclusions, which follow the conclusions of the fissile and fertile burnup analysis, can be drawn from the composite burnup analhsis.

1. Radiochemical burnup employing the stable $\mathrm{Zr}$ isotopes is the least accurate method with regard to its error statement, which was significant1y improved by use of IDMS-determined uranium contents. This is followed by the radiochemical burnup based on $\mathrm{Cs}-137$ activity counts, which became further improved by use of IDMS uranium data. 
2. Mass spectrometry fissile FIMA combined with radiochemical/ IDMS fertile FIMA gave the most accurate composite burnup statement, which was again superior to the IDMS case (and is believed to be explainable by doubtful error statements for the mass spectrometry case).

3. When combining the six semi-independent methods in Table 3-11 to combined estimates, the two mass spectrometry methods result in the most accurate estimate, which has been used for further data comparison.

4. A numerical comparison between the combined mass spectrometric estimate with the gamma spectroscopic and GAUGE/FEVER calculated burnup can be made for each of the four fuel blends at the reference location (see Table 3-13 and Fig. 3-21).

\begin{tabular}{|c|c|c|c|c|c|}
\hline $\begin{array}{c}\text { Composite FIMA } \\
(2684 \mathrm{~mm} \\
\text { core height })\end{array}$ & $\begin{array}{c}\text { Combined } \\
\text { IDMS+ } \\
\text { Mass Spec. } \\
\text { (A) } \\
\text { (atom \%) }\end{array}$ & $\begin{array}{c}\text { Gamma Spec. } \\
\text { (B) } \\
\text { (atom } \%)\end{array}$ & $\begin{array}{c}\frac{B}{A}-1 \\
(-)\end{array}$ & $\begin{array}{l}\text { Calc. } \\
\text { (atom } \\
\% \text { ) }\end{array}$ & $\begin{array}{l}\frac{\mathrm{Calc}}{\mathrm{B}}-1 \\
(-)\end{array}$ \\
\hline \multicolumn{6}{|c|}{ Fuel stacks $1 \& 2$} \\
\hline Mean & 4.79 & 4.36 & -0.090 & 4.09 & -0.146 \\
\hline Error $(1 \alpha)$ & \pm 0.04 & \pm 0.11 & \pm 0.024 & & \pm 0.007 \\
\hline \multicolumn{6}{|c|}{ Fuel stacks $3 \& 4$} \\
\hline Mean & 9.17 & 10.33 & +0.126 & 8.07 & -0.120 \\
\hline Error $(1 \alpha)$ & \pm 0.10 & \pm 0.39 & \pm 0.044 & & \pm 0.010 \\
\hline \multicolumn{6}{|c|}{ Fue1 stacks $5 \& 6$} \\
\hline Mean & 9.53 & 10.37 & +0.088 & 8.24 & -0.135 \\
\hline Error $(1 \alpha)$ & \pm 0.10 & \pm 0.33 & \pm 0.036 & & \pm 0.009 \\
\hline \multicolumn{6}{|c|}{ Fuel stacks $7 \& 8$} \\
\hline Mean & 13.24 & 13.30 & -0.003 & 11.46 & -0.134 \\
\hline Error $(1 \alpha)$ & \pm 0.13 & \pm 0.13 & \pm 0.014 & & \pm 0.009 \\
\hline
\end{tabular}

All error statements are related to the measurements only. 
5. Based on the above proven reliability of the fuel gamma spectroscopy, a comparison between measured and calculated fuel rod burnup versus axial core height appears meaningful, as detailed in Table 3-12 and Fig. 3-21. The following bias estimates can be stated (in relative percentages) on a fuel element average basis.

Bias Estimate (calc./meas. - 1) \pm 10 Error

\begin{tabular}{|c|c|c|c|c|}
\hline $\begin{array}{l}\text { Composite Burnup } \\
\text { Rel. Comparison }\end{array}$ & $\begin{array}{c}\text { Body } 1 \\
\text { Mean } \pm \text { Error }(10) \\
(\%) \\
\end{array}$ & $\begin{array}{c}\text { Body } 2 \\
\text { Mean } \pm \text { Error (16) } \\
\end{array}$ & $\begin{array}{c}\text { Body } 3 \\
\text { Mean } \pm \text { Error (10) } \\
(\%) \\
\end{array}$ & $\begin{array}{c}\text { Element } \\
\text { Mean } \pm \text { Error }(1 \sigma) \\
(\%)\end{array}$ \\
\hline Fuel stacks $1 \& 2$ & $-3.0 \pm 6.2$ & $+10.7 \pm 10.2$ & $+3.5 \pm 13.7$ & $+3.7 \pm 11.7$ \\
\hline Fue 1 stacks $3 \& 4$ & $-14.6 \pm 5.1$ & $-18.8 \pm 2.0$ & $-19.0 \pm 4.2$ & $-17.5 \pm 4.4$ \\
\hline Fuel stacks $5 \& 6$ & $-11.7 \pm 6.8$ & $-18.3 \pm 2.9$ & $-18.7 \pm 2.4$ & $-16.3 \pm 5.4$ \\
\hline Fuel stacks $7 \& 8$ & $-8.3 \pm 3.5$ & $-12.7 \pm 5.2$ & $-15.0 \pm 6.0$ & $-10.4 \pm 6.0$ \\
\hline
\end{tabular}

The fuel failure experienced with the fuel blends of stacks 1 and 2 was most pronounced in body 2 and masks any kind of burnup profile evaluation. For the other three varieties, it was concluded that there was an under-calculation of the burnup of between $-10 \%$ and $-18 \%$ for the element average, which can be as high as $-19 \%$ for a specific fuel body. The fuel rod mean relative error was determined analog to Eq. 3-6, and in accordance with Eq. 3-3a for fuel body and the total element mean.

6. The following measured and calculated data can be stated for FTE-6 fuel rod burnups. Mean and RMS information are related to the total element. The calculated mean and RMS values shown differ slightly from those shown in Table 3-12. This is caused by a different sampling procedure being used to obtain the values shown in Table 3-12 (every other rod) as compared with those shown in Table 3-5 (every rod). The measured RMS values for the three intact fuel blends (stacks 3 through 8 ) are somewhat higher than the calculated values. This difference results from a larger scatter in the measured values as compared with the calculated distribution. 


\begin{tabular}{|c|c|c|c|c|c|}
\hline $\begin{array}{c}\text { Burnup } \\
\text { Evaluation } \\
\end{array}$ & Peak & $\begin{array}{l}\text { Error } \\
(1 \sigma)\end{array}$ & Mean & $\begin{array}{l}\text { RMS } \\
(1 \sigma)\end{array}$ & $\begin{array}{l}\text { Error } \\
(1 \sigma)\end{array}$ \\
\hline \multicolumn{6}{|c|}{ Composite FIMA $(\%)$} \\
\hline \multicolumn{6}{|l|}{ Holes 1 and 2} \\
\hline Calculated & 6.62 & ND & 5.34 & \pm 1.15 & ND \\
\hline Measured & 6.43 & \pm 0.67 & 5.14 & \pm 1.08 & \pm 0.15 \\
\hline \multicolumn{6}{|l|}{ Holes 3 and 4} \\
\hline Calculated & 12.52 & ND & 10.31 & \pm 1.95 & ND \\
\hline Measured & 15.59 & \pm 1.59 & 12.53 & \pm 2.49 & \pm 0.29 \\
\hline \multicolumn{6}{|l|}{ Holes 5 and 6} \\
\hline Calculated & 12.77 & $\mathrm{ND}$ & 10.52 & \pm 2.10 & ND \\
\hline Measured & 15.77 & \pm 1.58 & 12.61 & \pm 2.51 & \pm 0.25 \\
\hline \multicolumn{6}{|l|}{ Holes 7 and 8} \\
\hline Calculated & 17.55 & ND & 14.57 & \pm 2.80 & ND \\
\hline Measured & 20.31 & \pm 2.12 & 16.45 & \pm 3.35 & \pm 0.40 \\
\hline
\end{tabular}

\subsection{STRESS ANALYSIS}

\subsubsection{Method and Material Properties}

Stress calculations have been carried out to determine the following:

1. The distribution of thermal and irradiation-induced operating stresses as a function of time, fluence, and axial position.

2. The maximum operating and shutdown stresses for the irradiation period, as well as for BOL and EOL.

Temperature and stress distributions at the midplane of each fuel body were calculated using GTEPC-2D (Ref. 3-6), a computer program for viscoelastic analysis of graphite. GTEPC uses for stress calculations a standard viscoelastic solid, where the coefficients of the springs and dashpots are functions of temperature and fluence. 
The finite element model of the fuel body and sleeve was a 22.5-deg sector of unit thickness taken at the midplane of each of the three eighthole teledial fuel bodies. The finite element mesh, consisting of 223 nodes and 368 elements, is shown in Fig. 3-34. The full mesh system was used for the thermal calculations, whereas stresses were calculated in the graphite fuel body only. Thermal boundary conditions were determined from the one-dimensional TREVER (see Ref. 3-4) calculations. GTEPC temperature field calculations have been verified with TAC2D (see Ref. 3-5) and with TREVER calculations to within $\pm 40^{\circ} \mathrm{C}$.

Material properties used in the stress analysis as well as in the thermal analysis of the H-327 graphite bodies were taken from Ref. 3-21; irradiation-induced creep data were taken from Ref. 3-22. The thermal conductivity and irradiation strain for the $\mathrm{H}-381 / 2$ sleeve graphite were taken from Ref. 3-23, and the coefficient of thermal expansion for this graphite came from Ref. 3-24.

\subsubsection{Initial Thermal Stress}

A summary of the initial thermal stress results is given on the first lines of Tables 3-14(a), 3-14(b), and 3-14(c) for each midplane position of the three fuel bodies together with maximum temperature differences within each plane at BOL (6624 hr of reactor operation). This temperature difference is an indicator of the general level of initial stresses to be found at startup.

Maximum initial tensile stresses occurred at the midplane of body 2 at element position 288 (Fig. 3-34). This is on the periphery of the fuel hole adjacent to the slot cut into the inside of the element. The slot was a safety feature, designed to confine any graphite breakage due to swelling of fuel rods to the inside diameter of the element. 
Maximum compression stresses are located in the region at the tip of the safety slot (i.e., at element position 359). Comparison of the stress results with the ultimate tensile strength (UTS) and ultimate compressive strength (UCS) values for $\mathrm{H}-327$ graphite in Table 3-15 shows that initial thermal stresses are less than the ultimate strength values. The in-plane tensile and compressive stresses are $22 \%$ and $18 \%$ of the corresponding strength. The strength is obtained by correcting the initial strength at room temperature for the effects of temperature and fast neutron irradiation according to Ref. 3-21.

$$
\mathrm{UTS}_{\mathrm{t}}=\mathrm{UTS}_{\mathrm{o}}\left(\mathrm{E}_{\mathrm{t}} / \mathrm{E}_{\mathrm{o}}\right)^{\mathrm{n}}
$$

where $\mathrm{n}=0.4$ for radial direction, $\mathrm{n}=0.67$ for axia1 direction for $\mathrm{H}-327$ graphite, $\mathrm{E}_{\mathrm{O}}=$ elastic modulus at $\mathrm{BOL}$ and room temperature $(298 \mathrm{~K})$, $E_{t}=$ elastic modulus at time $t$ as a function of fluence and irradiation temperature with an additional correction for elevated temperatures.

$$
E_{t}=E_{o}\left(1+1.5 \times 10^{-4} \Delta T\right)
$$

where $\Delta \mathrm{T}=$ the difference between the operating temperature and $298 \mathrm{~K}$.

The initial tensile strength at room temperature is obtained from Table 3-15 for the midlength edge of the 1og. The midlength edge location was selected because the test element bodies were known to have been machined from the outer portion of the H-327 graphite $10 \mathrm{~g}$. This may overestimate the strength somewhat, since some of the test elements were taken from a position intermediate between the outer portion of the $10 \mathrm{~g}$ (designated as edge) and the central portion of the log. On the other hand, the test elements were machined from logs that are likely to be somewhat stronger than the mean. The tensile strengths of the specimens taken from 
the midlength center of each of the logs from which bodies in FTE- 6 were machined were measured to be 12.6 to $14.8 \mathrm{MPa}$, well above the mean strength of all logs of $11.2 \mathrm{MPa}$. In a11, the midlength edge strength is believed to be an unbiased best estimate of tensile strength at the midplanes of the test element bodies. For the initial compressive strength, which has not been characterized as a function of position in the $10 \mathrm{~g}$, a conservative minimum value of $27.6 \mathrm{MPa}$ (4000 psi) was used. Compressive strength changes with fluence and temperature were calculated according to Eqs. $3-25 a$ and $3-25 b$.

Compressive stress values have been found to be very small in comparison with the UCS values.

The following peak initial thermal stresses can be stated for FTE-6:

\begin{tabular}{|c|c|c|c|c|}
\hline Initial Thermal Stress & Peak & Mean $^{*}$ & $\pm \mathrm{RMS}^{*}$ & Fue1 Body Sequence \\
\hline \multicolumn{5}{|l|}{ Tensile stress (MPa) } \\
\hline In-plane (element 288) & 2.32 & 1.76 & 0.40 & 231 \\
\hline Stress-strength ratio & 0.24 & 0.18 & 0.04 & 231 \\
\hline Axial (element 297) & 1.59 & 1.17 & 0.31 & 231 \\
\hline Stress-strength ratio & 0.16 & 0.12 & 0.03 & 231 \\
\hline \multicolumn{5}{|l|}{ Compression stress (MPa) } \\
\hline In-plane (element 359) & -5.07 & -3.84 & 0.89 & 231 \\
\hline Stress-strength ratio & 0.18 & 0.13 & 0.03 & 231 \\
\hline Axial (element 20) & -2.84 & -2.10 & 0.54 & 231 \\
\hline Stress-strength ratio & 0.10 & 0.07 & 0.02 & 231 \\
\hline
\end{tabular}

Body 2 experiences the highest BOL stresses; the stress level is well below the ultimate strength values.

*The mean and RMS are used to describe the variation of calculated peak stresses between the three fuel body midplanes and are not a representative statement for mean body stresses nor for uncertainties in calculations. 
Temperature and stress contour plots for startup at BOL are presented in Figs. 3-35 through 3-37 for the center region of each fuel body (in MPa). Figure 3-38 gives the stress direction in each plane, which is the same for all three bodies.

\subsubsection{Peak Stress During Life}

The stress distribution changes rapidly with time because of increased irradiation strain in the hotter areas of the fuel body. The higher temperature areas reverse from initial compressive stress to a state of tensile stress, while the cooler areas near the sleeve go into compression. This stress reversal is predicted by the code at all three axial locations.

In genera1, peak EOL tensile stress occurs in the hot positions toward the center of the body, while peak EOL compressive stresses are found in the low temperature areas near the outer edge of the element.

Tables 3-14(a), 3-14(b), and 3-14(c) contain the peak operating stresses found during the irradiation simulation. The time points (Core 2 reactor hours at operation) and finite elements, where the peak stresses occur, are also included. Time points refer to TREVER simulation intervals, and element locations to the finite element mesh in Fig. 3-34. Temperature and stress contour plots for EOL operation are presented in Figs. 3-39 through 3-41, and stress directions are shown in Fig. 3-42 for al1 three planes. Figures 3-43 through 3-45 give the history of the elements with the maximum and minimum temperature and show maximum and minimum tensile stress (in-plane and axia1) during operation and shutdown of the midplane of each body. Finite elements with peak in time condition have been selected for the presentation.

The following peak operational and shutdown stress values during the irradiation life of FTE-6 can be stated: 


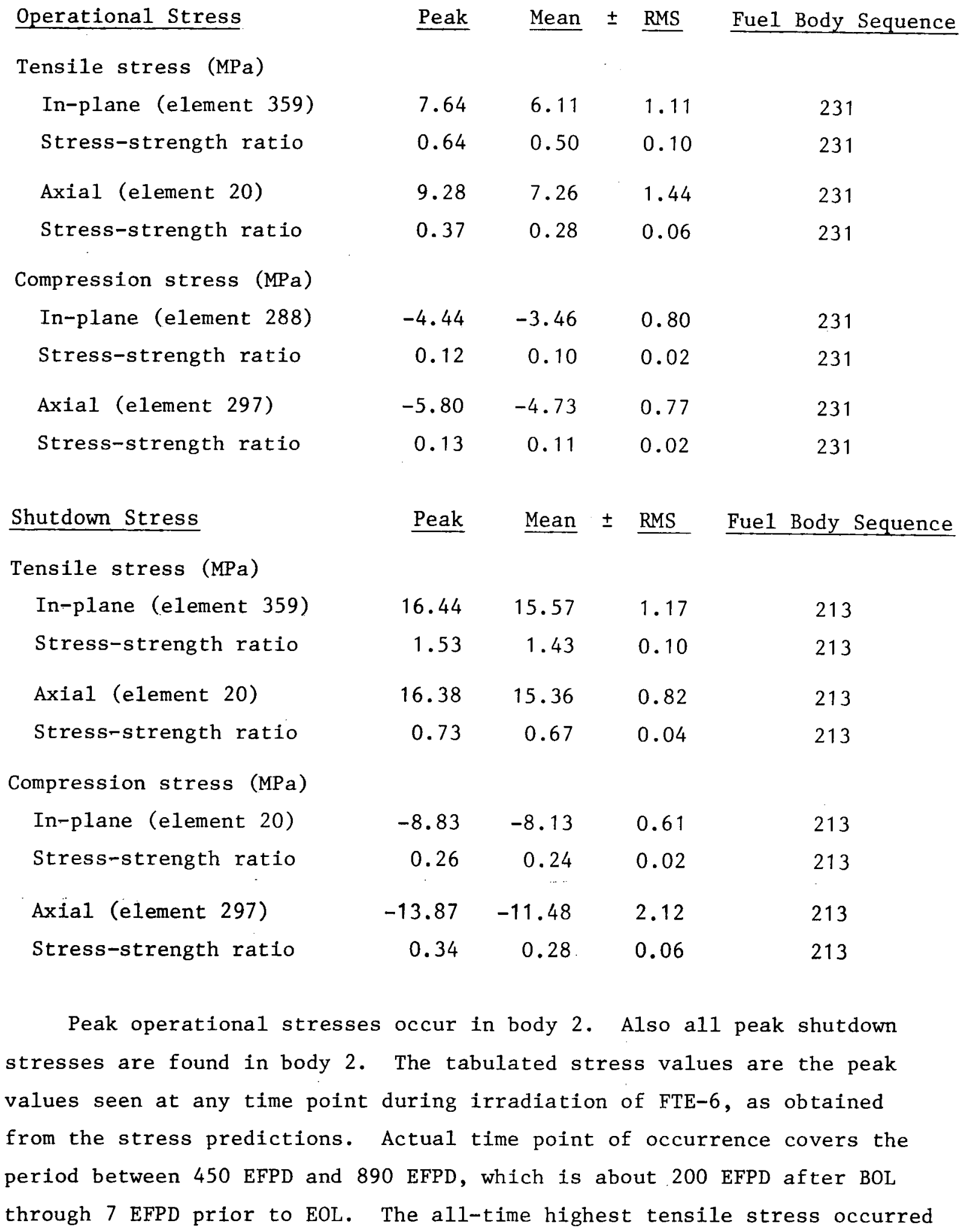


strength ratio of 0.73 . The highest stress-strength ratio of 1.53 is calculated for a shutdown at 450 EFPD for the inner safety slot location. Although the predicted stress exceeds the predicted strength of the irradiated material, no defects have been detected during destructive PIE. It should be noted that the stress at the edge of the safety slot is not calculated reliably by the present models or methods. The stress has been shown (Ref. 3-25) to be sensitive to the fineness of the mesh used at this point, and is also expected to be sensitive to the exact shape of the notch, which is modeled as a sharp corner but will actually have a finite radius of curvature. The results would also be expected to be sensitive to the shape of the stress-strain curve for graphite which has been approximated in the present calculations by a straight line.

\subsubsection{Peak Stress at EOL}

Peak EOL operation and shutdown stresses have been determined and are included in Tables 3-14(a), 3-14(b), and 3-14(c) for each of the three bodies. The finite element locations are also presented. As mentioned earlier, an inversion took place between BOL and EOL for the peak stress locations: The highly compressed areas became subject to tensile stresses and vice versa. On EOL shutdown these tensile stresses and compression stresses increased nearly threefold in both cases, compared with the EOL operating stresses.

Temperature and stress contour plots for shutdown stresses are presented in Figs. 3-46 through 3-48. Shutdown temperature is $20^{\circ} \mathrm{C}$; for better comparison, the temperature field prior to shutdown has been included in these figures, which are also presented in the contour plots for EOL operation (Figs. 3-39 through 3-41). Figure 3-49 contains the EOL shutdown stress directions, which vary slightly from body to body.

The following values can be summarized for the peak EOL stresses. 
EOL Operational Stress

Tensile stress (MPa)

In-plane (element 359)

Stress-strength ratio

Axia1 (element 20)

Stress-strength ratio

Compression stress (MPa)

In-plane (element 288)

Stress-strength ratio

Axial (element 297)*

Stress-strength ratio

EOL Shutdown Stress

Tensile stress ( $\mathrm{MPa})$

In-plane (element 359)

Stress-strength ratio

Axial (element 20)

Stress-strength ratio

Compression stress (MPa)

In-plane (element 91$)^{* * *}$

Stress-strength ratio

Axial (element 297)

Stress-strength ratio $\underline{\text { Peak }} \underline{\text { Mean }} \pm \underline{\text { RMS }}$ Fuel Body Sequence

$\begin{array}{llll}5.42 & 4.37 & 0.81 & 231\end{array}$

$\begin{array}{llll}0.45 & 0.36 & 0.07 & 231\end{array}$

$\begin{array}{llll}7.69 & 6.20 & 1.05 & 213\end{array}$

$\begin{array}{llll}0.30 & 0.24 & 0.04 & 213\end{array}$

$\begin{array}{llll}-2.17 & -1.86 & 0.23 & 231\end{array}$

$\begin{array}{llll}0.06 & 0.05 & 0.01 & 231\end{array}$

$\begin{array}{llll}-4.73 & -3.36 & 1.10 & 231\end{array}$

$\begin{array}{llll}0.11 & 0.08 & 0.03 & 231\end{array}$

Peak $\quad \underline{\text { Mean }} \pm \underline{\text { RMS }} \quad \underline{\text { Fuel Body Sequence }}$

$\begin{array}{rrrl}14.33 & 12.18 & 1.52 & 132 \\ 1.28 & 1.11 & 0.12 & 132 \\ 13.98 & 13.05 & 0.86 & 123 \\ 0.58^{* *} & 0.56 & 0.03 & 213^{* *}\end{array}$

$\begin{array}{rrrr}-6.07 & -5.54 & 0.68 & 132 \\ 0.18 & 0.16 & 0.02 & 123 \\ -12.38 & -10.28 & 1.78 & 123 \\ 0.30 & 0.25 & 0.05 & 213\end{array}$

Peak operational stresses all occur in body 2 at EOL and are always lower than the strength. Tensile shutdown stresses, however, are significant for the inner edge of the safety slot, where the in-plane stressstrength ratio reaches 1.28 for body one, and for the inner location of

${ }^{*}$ Element 322 for body 1.

** Body 2 has lower stress (13.26 MPa), but also has lower increase in elastic modulus because of higher irradiation temperature, which results in a higher stress factor (0.581) than for body $1(0.578)$. ネネネ

Element 359 for body 1 . 
the fuel hole surface, where the axial stress-strength ratio is determined to be 0.58 . No structural damage has been found during destructive PIE.

\subsubsection{Stress Analysis Conclusions}

1. FTE-6 was a high stress experiment, reaching shutdown stresses up to $153 \%$ of the UTS. The maximum stresses occurred during shutdowns at about $1 / 3$ and $2 / 3$ of the irradiation cycle. Compressive stresses were small in comparison with the ultimate compressive strength of the material.

2. Highest in-plane stresses were found for the inner edge of the safety slot for operation $(64 \%)$ and shutdown $(153 \%)$. For the fuel hole surface closest to the center, highest axial stresses

- were found during shutdown (73\%).

3. Residual shutdown stresses at EOL are calculated to have reached $128 \%$ and $58 \%$ in the radial and axial direction, respectively, when compared with BOL nominal UTS values compensated for fluence and temperature.

4. Visual examination of the fuel bodies and several slices taken during PIE did not reveal any structural damage of the graphite, although the predicted stresses are significantly higher than measured strength values of the $\mathrm{H}-327$ material. The in-plane stresses at the safety slot, in particular, were predicted to be more than $50 \%$ above the ultimate strength. The fact that these stresses did not cause damage is probably due to the relatively low notch sensitivity of graphite (Ref. 3-26) and the very small area near the slot that was highly stressed. It should also be noted that the actual stresses near the slot may be significantly different than the calculated stresses, as discussed in Section 3.5.3. 
5. FTE-6 experienced significant constrained bowing, as evidenced from disassembly and postirradiation examination (see Section 4.4.2). The effects of bowing were not evaluated in the present analysis, in which azimuthal symmetry was assumed. A more detailed nuclear and thermal analysis for flux and temperature gradients would be required to treat the effects of bowing.

6. Because of the relatively high stress levels in FTE-6 combined with the in-pile bowing, some specimens have been identified as potential samples for destructive stress examination for residual stresses as well as for stress margins (Ref. 3-27).

\subsection{IRRADIATION SUMMARY}

The computer-simulated irradiation history of FTE-6 is summarized in Table 3-16 for the parameters fluence, temperature, burnup, stress, and irradiation-induced strain. Where applicable, measured parameters are added for comparison. The standard deviation on the mean values is stated for axial variability information (or temporal variability information, for measured temperature only); the fuel body sequence is stated for decreasing body mean and increasing gradient within the fuel body, where applicable. Error statements $(1 \sigma)$ are added where available. Temperature statements are time-weighted volume averages and maximum or minimum values at envelope conditions. Anisotropy was determined from axial versus radial strain.

\section{REFERENCES}

3-1; Wagner, R. M., "GAUGE, A Two-Dimensional Few Group Neutron Diffusion Depletion Program for a Uniform Triangular Mesh," General Atomic Report GA-8307, March 1968.

3-2. Todt, F. W., and L. J. Todt, "FEVER/M1, a One-Dimensiona1 Depletion Program for Reactor Fuel Cycle Analysis," General Atomic Report GA-9780, October 22, 1969. 
3-3. Dorsey, J. P., R. Froelich, and F. Todt, "BUG-2/BUGTRI, Two Dimensiona1 Multigroup Burnup Codes for Rectangular and Hexagonal Geometry," USAEC Report GA-8272, Gulf General Atomic, August 22, 1969.

3-4. Saurwein, J. J., "Peach Bottom Test Element Thermal Analysis with TREVER Code," General Atomic Company, unpublished data, April 19, 1976.

3-5. Peterson, J. F., "TAC2D, A General Purpose Two-Dimensional Heat Transfer Computer Code," USAEC Report GA-8868, Gulf General Atomic, September 6, 1969.

3-6. Tzung, F. K., "GTEPC-2D, A Computer Program for Two-Dimensional Graphite Thermal-Elastic-Plastic-Creep Analysis User's Manual," Genera1 Atomic Report GA-A13532, January 31, 1976.

3-7. Scheffel, W. J., N. L. Baldwin, and R. W. Tomlin, "Operating History Report for the Peach Bottom High Temperature Gas-Cooled Reactor," GRDA Report GA-A63907, Vols. I and II, General Atomic Company, August 31, 1976.

3-8. Saurwein, J. J., and F. McCord "FTE-6 Data Compilation of Nuclear, Thermal and Strain Evaluation," General Atomic Company, unpublished data, August 20, 1976.

3-9. Wallroth, C. F., and J. McNair, "Recalibration of Tungsten Rhenium and Chromel-Alumel Thermocouples Irradiated in the Peach Bottom High Temperature Reactor," General Atomic report, to be published.

3-10. Crockett, T. B., "Atom Percent Fission in Fissile and Fertile Fuel Particles," General Atomic procedure, unpublished data, October 8 , 1975.

3-11. "Standard Method of Test for Atom Percent Fission in Uranium Fuel (Radiochemical Method)," ASTM Procedure E219-69, March 21, 1969.

3-12. "Standard Method of Test for Atom Percent Fission in Uranium and Plutonium Fuel (Mass Spectrometric Method)," ASTM Procedure E244-69, March 21, 1969.

3-13. Jones, J. J. (editor), and T. D. Morgan, "Mass-Spectrometric Isotopic Analysis of Uranium in Irradiated-Fuel Dissolver Solutions using the Isotope-Dilution Technique," Method 2.505 in Selected Measurement Methods for Plutonium and Uranium in the Nuclear Fuel Cycle, TID 7029, 1963, p. 279. 
3-14. Holzgraf, J. F., and T. L. Emrick, "BETSY, a Computer Program to Evaluate Fuel Rod Gamma Spectroscopy for Fission Product Inventory and Burnup," General Atomic Company, unpublished data, March 1976.

3-15. "Evaluated Nuclear Data File Library, ENDF/B, Version IV," National Cross Section Center, Brookhaven National Laboratories, 1974.

3-16. Meek, M. E., and B. F. Rider, "Compilation of Fission Product Yields," Vallecitos Nuclear Center Report No. NEDO-12154-1, 1974.

3-17. Martin, M. J., and P. H. Blichert-Toft, Nuclear Data Tables, Vol. $\underline{8}$, October 1970.

3-18. Merrit, J. S., and J. G. V. Taylor, "Decay of Cs-137 Determined by Absolute Counting Methods," Ana1 Chem 37, No. 3 (1965).

3-19. Crockett, T. B., and D. M. Fleishman, "FTE-6 Burnup Determination," General Atomic Company, unpublished data, May 26, 1976.

3-20. "Reactor Burnup Physics," in Proceedings of a Panel on Reactor Burnup Physics, a conference organized by the International Atomic Energy Agency and held in Vienna, July 12-16, 1971, IEAE, Vienna, 1973.

3-21. Beavan, L. A., "H-327 Graphite, Design Data Package," General Atomic Company, unpublished data, December 17, 1975.

3-22. Price, R. J., "Review of Irradiation-Induced Creep in Graphite Under HTGR Conditions," General Atomic Report GA-A12332, November 1, 1972. Price, R. J., and A. L. Beavan, "Graphite Data Package for Analysis of Peach Bottom Test Elements," General Atomic Company, unpublished data, November 26, 1974.

3-24. Koyoma, K., "Therma1 Expansion of Peach Bottom II Sleeve No. 2466," General Atomic Company, unpublished data, May 27, 1970.

3-25. "HTGR Fuels and Core Development Program, Quarterly Progress Report for the Period Ending August 31, 1976," ERDA Report GA-A14046, Genera1 Atomic Company, September 24, 1976, p. 11-11.

3-26. Brockelhurst, J. E., and R. G. Brown, "Fatigue Notch Sensitivity, and Work of Fracture Studies on Isotropic Graphite," UKAEA Report TRG 2513(s), August 1974.

3-27. Miller, C. M., "Test Plan for Destructive Stress Examination of Peach Bottom Fuel Test Elements," General Atomic Company, unpublished data, June 8, 1976. 


\section{POSTIRRADIATION EXAMINATION AND EVALUATION}

\subsection{DATA EVALUATION PROCEDURE}

\subsubsection{Error Analysis}

A certain property $x$, e.g., fuel rod diameter, has usually been measured several times in a given population. It is desired to estimate the true mean $\mu$ and to obtain error limits for this estimate. The following error model is assumed:

$$
x=\mu+k_{1}+k_{2}+k_{3},
$$

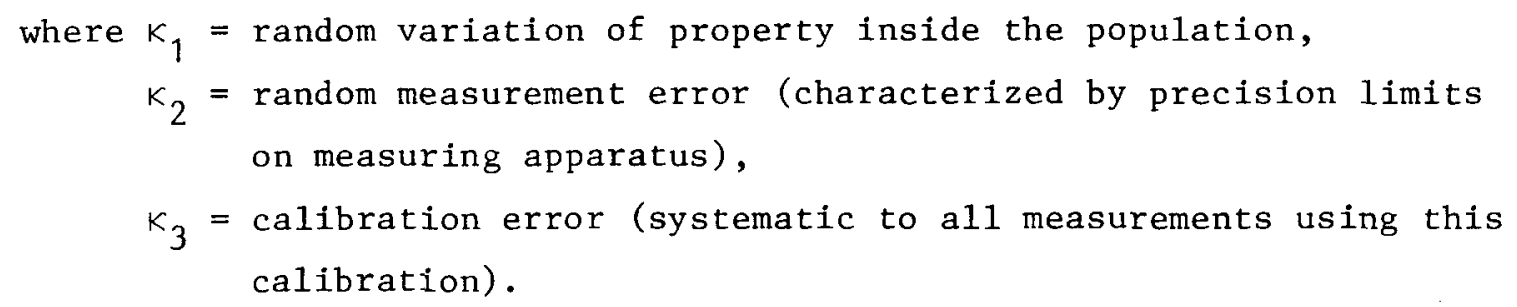

These errors have means of 0 and standard deviations $\sigma_{1}, \sigma_{2}$, and $r$, respectively. Any systematical error has been detected by proper calibration and has been accounted for before any further data reduction. The true mean $\mu$ is estimated by the arithmetic average

$$
\overline{\mathrm{x}}=\frac{1}{\mathrm{n}} \sum \mathrm{x}_{\mathrm{i}}
$$


of all measurements $x_{i}$. The sample standard deviation ${ }^{*}$ is

$$
s_{x}=\left\{\frac{1}{n-1} \sum\left(x_{i}-\bar{x}\right)^{2}\right\}^{1 / 2}
$$

Then $\mathrm{s}_{\mathrm{x}}^{2}$ is an unbiased estimate of $\sigma_{1}{ }^{2}+\sigma_{2}{ }^{2}$. The standard deviation of the random measurement error is estimated from the precision e, which is the finest graduation on the readout scale, accurately read within $\pm 1 / 2$ e of the scale mark, as

$$
\sigma_{2}=\frac{\mathrm{e}}{\sqrt{12}}
$$

It represents a lower bound on $s$ (i.e., $s \geq \sigma_{2}$ ). The standard deviation of the mean $\bar{x}$ can then be estimated by

$$
s_{x}=\frac{s_{x}}{\sqrt{n}},
$$

and the combined standard deviation of all random errors $\varepsilon$ is estimated by

$$
\varepsilon_{\bar{x}}=\left\{s \frac{2}{\mathrm{x}}+\mathrm{r}^{2}\right\}^{1 / 2}
$$

Results from several fuel rods or samples can be combined in a stratum, representing, for example, a group of rods at the same reactor core position, or a batch of fuel rods tested in a fuel element. For each stratum $j$ consisting of $n_{j}$ elements, a mean $\bar{x}_{j}$ is available. If the strata are logically grouped into an ensemble, then the ensemble mean is estimated by

The following different standard deviations of a property $z$ are used in the presented procedure (see also further definition in the fo'llowing pages):

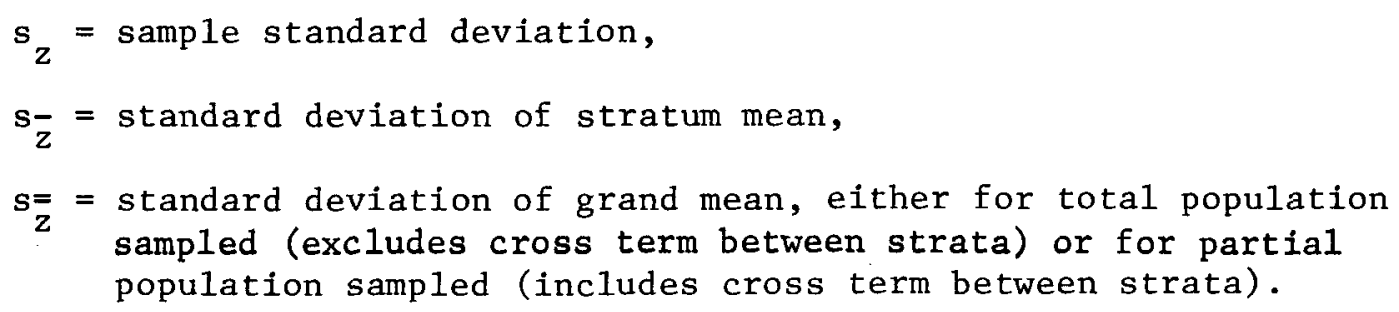
sampled (excludes cross term between strata) or for partial population sampled (includes cross term between strata). 


$$
\overline{\bar{x}}=\frac{1}{\sum n_{j}}\left(\sum n_{j} \bar{x}_{j}\right)
$$

and the estimated standard deviation of this ensemble mean is given by

$$
s_{x}^{=}=\left\{\frac{1}{\left(\sum n_{j}\right)^{2}} \sum n_{j}{ }^{2} s_{x_{j}}^{2}\right\}^{1 / 2} .
$$

Assuming a common calibration, ${ }^{*}$ the combined standard deviation of the ensemble mean is

$$
\varepsilon=\left(s \stackrel{2}{x}+r^{2}\right)^{1 / 2}
$$

If each indivjdual measurement in all the strata in the ensemble is regarded as a random sample from a single population, then the standard deviation of this population can be estimated from

$$
s_{x}=\left\{\frac{1}{\sum n_{j}-1}\left[\sum\left(n_{j}-1\right) s_{x_{j}}^{2}+\sum n_{j}\left(\bar{x}_{j}-\overline{\bar{x}}\right)^{2}\right]\right\}^{1 / 2}
$$

This approach has been employed in STAT (Ref. 4-1), a computer code for statistical analysis of metrology data from the test elements.

Measurements prior (index 1) and after (index 2) irradiation are applied to derive strain information using the strain analysis function

$$
\overline{\mathrm{u}}=\frac{\overline{\mathrm{x}}_{2}}{\overline{\mathrm{x}}_{1}}-1
$$

Axial (index $y$ ) and radial (index $x$ ) measurements can be combined in an arithmetic average

*For a different calibration per stratum:

$$
\mathrm{r}^{2}=\frac{1}{\left(\sum \mathrm{n}_{\mathrm{j}}\right)^{2}} \sum \mathrm{n}_{\mathrm{j}}^{2} \mathrm{r}_{\mathrm{j}}^{2}
$$




$$
\bar{v}=\frac{1}{2}\left(\bar{u}_{y}+\bar{u}_{x}\right)
$$

Additionally, axial and radial strain valves can be compared using the anisotropy analysis function

$$
\overline{\mathrm{w}}=\overline{\mathrm{u}}_{\mathrm{y}} / \overline{\mathrm{u}}_{\mathrm{x}}
$$

In genera1, error estimates (standard deviations) for an analysis function $\mathrm{f}\left(\mathrm{x}_{1}, \mathrm{x}_{2}\right)$ are given by ${ }^{*}$

$$
s_{f}=\left\{\left(\frac{\partial f}{\partial x_{1}} s_{x_{1}}\right)^{2}+\left(\frac{\partial f}{\partial x_{2}} s_{x_{2}}\right)^{2}\right\}^{1 / 2} .
$$

A formula summary is given in Table 4-1, and a more detailed description of the error analysis is given in Ref. 2-2. Equations 4-1 through 4-14 represent the statistical basis of the algorithm used in the STAT code for the case where all observations are equally weighted random samples to be used in the estimation of the underlying population mean and standard deviation.

\subsubsection{Stratification}

Quite often the knowledge of a single measurement $u_{j}$ may not be particularly useful or even available for purposes of data analysis. Instead, comparisons are made between equally weighted groups or between

* This form tandard error Otherwise,

$$
\varepsilon_{\mathrm{f}}=\left\{\left(\frac{\partial \mathrm{f}}{\partial \mathrm{x}_{1}} \varepsilon_{\mathrm{x}_{1}}\right)^{2}+\left(\frac{\partial \mathrm{f}}{\partial \mathrm{x}_{2}} \varepsilon_{\mathrm{x}_{2}}\right)^{2}+2 \frac{\partial f}{\partial \mathrm{x}_{1}} \frac{\partial f}{\partial \mathrm{x}_{2}} \operatorname{cov}\left(\mathrm{x}_{1}, \mathrm{x}_{2}\right)\right\}^{1 / 2}
$$

If $\mathrm{x}_{1}$ and $\mathrm{x}_{2}$ are linear measurements on which the calibration corrections were made,

$$
\operatorname{cov}\left(x_{1}, x_{2}\right)=r^{2} \quad
$$


strata which may consist of unequal numbers of observations. Here the information on each stratum $j$ is condensed into a mean $\vec{u}_{j}$ and an estimated standard deviation of the mean $s_{u_{j}}$. Additionally, a sample standard deviation $s_{\mathbf{u}_{j}}$ within the stratum $j$ may be known.

The grand mean of the combined strata is estimated by

$$
\overline{\bar{u}}=\frac{1}{m} \sum \bar{u}_{j}
$$

and the estimated standard deviation of the mean is given by

$$
s_{u}=\left\{\frac{1}{m^{2}} \sum s \frac{2}{u}_{j}\right\}^{1 / 2},
$$

and the combined standard deviation under the assumption of a common calibration* is

$$
\varepsilon=\left\{s \frac{2}{u}+r^{2}\right\}^{1 / 2}
$$

This standard error estimate does not include a cross term between strata because of the sampling characteristic, which represents the total population. If the sample standard deviation estimates $s_{u_{j}}$ of the individual strata are known, then the sample standard deviation estimate is

$$
s_{u}=\left\{\frac{1}{m} \sum s_{u_{j}}{ }^{2}+\frac{1}{m} \sum\left(\bar{u}_{j}-\overline{\bar{u}}\right)^{2}\right\}^{1 / 2} .
$$

The combined standard deviation is estimated ${ }^{* *}$ by

$$
\begin{gathered}
\text { *For a different calibration per stratum: } \\
\qquad r^{2}=\frac{1}{m^{2}} \sum r_{j}^{2} . \\
{ }^{* *} \text { For a different calibration per stratum: } \\
r^{2}=\frac{1}{m} \sum r_{j}{ }^{2} .
\end{gathered}
$$




$$
\varepsilon_{u}=\left\{s_{u}^{2}+r^{2}\right\}^{1 / 2}
$$

The first term in Eq. 4-18 represents the standard deviation inside a given stratum averaged for a group of m strata:

$$
\bar{s}_{u}=\left\{\frac{1}{m} \sum s_{u_{j}}^{2}\right\}^{1 / 2}
$$

The average combined standard deviation ${ }^{*}$ is defined by:

$$
\bar{\varepsilon}_{u}=\left\{\bar{s}_{u}^{2}+r^{2}\right\}^{1 / 2}
$$

Analogously to Eq. 4-20, an average standard deviation of the stratum mean can be established for a group of $m$ strata:

$$
\bar{s}_{\bar{u}}=\left\{\frac{1}{m} \sum s_{\bar{u}_{j}}^{2}\right\}^{1 / 2}
$$

The combined form with the calibration standard error ${ }^{*}$ is

$$
\bar{\varepsilon}-\bar{u}=\left\{\bar{s} \bar{u}^{2}+r^{2}\right\}^{1 / 2}
$$

The second term in Eq. 4-18 represents the standard deviation between strata means

$$
\operatorname{RMS}=\left\{\frac{1}{\mathrm{~m}} \sum\left(\overline{\mathrm{u}}_{\mathrm{j}}-\overline{\overline{\mathrm{u}}}\right)^{2}\right\}^{1 / 2}
$$

The formulas Eq. 4-15 through Eq. 4-18 represent the estimated mean, the standard deviation of the mean, and the sample standard deviation for the population which consists of the combined strata from which the samples were taken. If, however, the strata were selected from a very much larger ${ }^{*}$ For a different calibration per stratum:

$$
\mathrm{r}^{2}=\frac{1}{\mathrm{~m}} \sum \mathrm{r}_{\mathrm{j}}^{2}
$$


population which included many strata that were not sampled, then the estimated grand mean of this population is

$$
\overline{\bar{u}}=\frac{1}{m} \sum \bar{u}_{j}
$$

and the standard deviation of the mean is

$$
s_{u}=\left\{\frac{1}{m^{2}} \sum s_{u_{j}}{ }^{2}+\frac{1}{m^{2}} \Sigma\left(\bar{u}_{j}-\overline{\bar{u}}\right)^{2}\right\}^{1 / 2} .
$$

The combined standard deviation, under the assumption of a common calibration, ${ }^{* *}$ is

$$
\varepsilon_{\bar{u}}=\left\{s \underline{u} \underline{\underline{u}}+r^{2}\right\}^{1 / 2}
$$

Equation 4-26 differs from Eq. 4-16 only in the term which accounts for the additional uncertainty about the mean of the entire population that arises from the fact that only $m$ strata are sampled.

Occasionally, strata information is very limited, e.g., for FTE-6, fuel rod length was determined from a single measurement only; so $\bar{u}_{j}=$ $u_{j}$. Therefore, sample random variation had to be taken from historical knowledge as gained with FTE-4 (Ref. 2-2). The estimate for the standard deviation of the mean is then

$$
\underset{\mathrm{u}}{\mathrm{u}}=\left\{\frac{\overline{\mathrm{s}}_{\mathrm{u}}^{2}}{\mathrm{~m}}+\frac{1}{\mathrm{~m}^{2}} \sum\left(\mathrm{u}_{j}-\overline{\bar{u}}\right)^{2}\right\}^{1 / 2} .
$$

$\overline{\mathrm{s}}_{\mathrm{u}}$ is determined via Eq. 4-20. It is called the "default" value when 'taken from historical knowledge and applied to a property or analysis for which no variation information is available. Table 4-2 shows the average sample standard deviations resulting from or used in the FTE-6

\footnotetext{
*For a different calibration per stratum:
}

$$
r^{2}=\frac{1}{m^{2}} \sum r_{j}^{2}
$$


analysis. The formulations in Eqs. 4-15 through 4-28 are included in the GAP and STRAIN codes (Refs. 4-2 and 4-3), whereas STAT (Ref. 4-1) uses the algorithm of Section 4.1.1.

\subsubsection{Comparison of Measured and Predicted Values}

4.1.3.1. Analysis. Suppose an irradiation parameter $u$ for a test element is measured for m strata. The following four distinct values are useful in describing the parameter distribution in the elements:

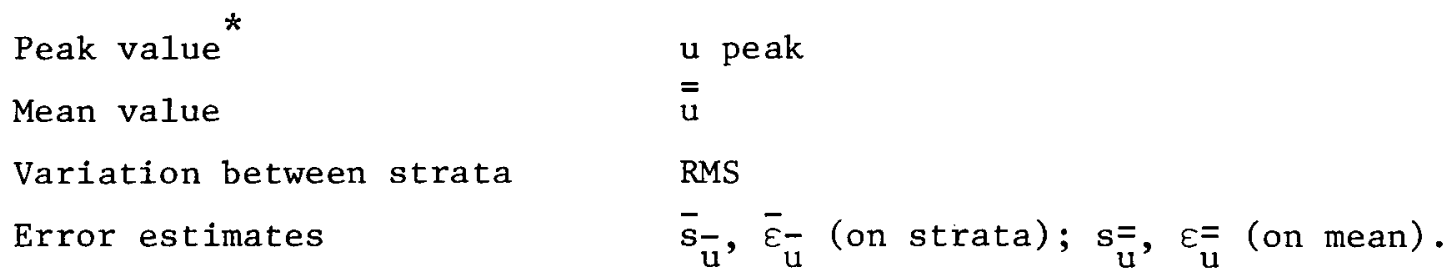

Typical parameters are graphite and fuel rod strain, burnup, and irradiation temperature. Stratification can be done either on a spatial basis (e.g., by axial grouping) or on a temporal basis (by dividing the reactor history into periods of some similar characteristic, such as control rod pattern, as was done for the GAUGE and TREVER calculations). The distribution of a parameter does not necessarily represent a random event, but will most likely follow a distinct distribution originated by the buckling of the neutron flux, the coolant flow, physical property changes under irradiation, or the reactor power history. The description of the axial distribution and variation with time of a certain parameter by a few values lacks information, for example, about where and when a peak event occurred. This information can be partially maintained by subdividing the test elements into sections, e.g., fuel bodies, and by reporting the sequence of fuel bodies along which a parameter decreases or inćreases. The timepoint and location of a peak event can also be reported.

If a calculational model exists for predicting the values listed above, then comparison between measured and predicted values can be made

* Peak value is usually described by the maximum value along the length and/or time axis of the element. Minimum values can also be of interest. 
quantitatively based on the actual values as well as through a fractional difference analysis function:

$$
z=\frac{C}{M}-1,
$$

where $\mathrm{C}=$ calculated value,

$M=$ measured value.

The propagated error is

$$
\varepsilon_{z}=(z+1)\left\{\left(\frac{\varepsilon_{C}}{C}\right)^{2}+\left(\frac{\varepsilon_{M}}{M}\right)^{2}\right\}^{1 / 2},
$$

where $\varepsilon_{C}$ and $\varepsilon_{M}$ are estimated standard deviations for $C$ and $M$, respectively. In most cases, calculations are based on mean material properties and sensitivity analyses to determine calculational uncertainties have not yet been done. As a preliminary test of the calculational model, however, this error will be ignored and comparisons will be made on the assumption that

$$
\varepsilon_{z}=(z+1) \frac{\varepsilon_{M}}{M}
$$

With this assumption, statistical tests can be made to determine if differences between calculated and measured values are small enough to be attributed to the measurement error alone. Test procedures are described in Sections 4.1.3.2 and 4.1.4.

Meaningful comparison between calculated and predicted values within and between test elements requires that the experiment be uniformly sampled, e.g., every other fuel rod along the axis of the fuel element may be used for the described tests. Inadequate sampling can be overcome via regression analysis between associated measurements within the neighborhood of the specific location (see Section 4.1.4).

The variation term RMS describes how much spatial or temporal variation a certain parameter has around its mean value. In case of no 
variation, RMS equals 0 . When comparing two individual axial distributions or time variations, the higher RMS value describes the more pronounced distribution or variation, the lower one the flatter distribution or less pronounced time variation. Counter-current distributions or variations may result in the same peak, mean, and RMS values; therefore, a visual examination of the distribution or variation is still required via graphical display and sequential ranking between subsections. *

The described formulation has been included in the GAP (Ref. 4-2) and STRAIN (Ref. 4-3) codes, which compare calculated and measured heat transfer gaps and material strain characteristics, respectively. This formulation applies also to other areas in this report, where comparison is made between two or more methods of analysis and prediction, e.g., for burnup, fluence, and thermocouple temperature analysis.

4.1.3.2. Statistical Tests. Suppose a calculational model exists for predicting the value of some parameter $u$. It is desired to test the degree of agreement between a set of calculated values $\mathrm{C}_{1}, \mathrm{C}_{2}, \ldots, \mathrm{C}_{\mathrm{m}}$ and a set of measured values $M_{1}, M_{2}, \ldots, M_{m}$. A typical example consists of strain measurements for $m$ successive axial rod positions in a fuel body. For these tests, it is assumed that the measured values are independent random variables.

The comparison is based on the fractional difference analysis function Eq. 4-29;

$$
z_{j}=\frac{C_{j}-M_{j}}{M_{j}}=\frac{C_{j}}{M_{j}}-1 \text {. }
$$

Let $\varepsilon_{z_{j}}$ be the propagated estimated standard deviation of $z_{j}$.

${ }^{*}$ A numerical description can be made by a mean cubic deviation:

$$
s^{3}=\frac{1}{m} \sum\left(\bar{u}_{j}-\bar{u}\right)^{3},
$$

which should differ in sign for the described case. 
Test 1a: Group Agreement Test. This test is designed to determine if there is any overall bias between the calculated and measured values taken as a group. The null hypothesis is that there is no difference, i.e.,

$E z_{j}=0$ (read: expected value of $z_{j}$ is zero)

for $j=1,2, \ldots, m$.

Let

$$
\begin{aligned}
& \mathrm{d}_{j}=\frac{z_{j}}{\varepsilon_{z}}, \\
& \bar{d}=\frac{1}{m} \sum d_{j} .
\end{aligned}
$$

Under the nu11 hypothesis,

$$
\begin{aligned}
& E_{d_{j}}=0 \\
& \left.s_{d_{j}}=1 \text { (standard deviation of all } d_{j}\right) .
\end{aligned}
$$

Then $\sqrt{\mathrm{m}} \overline{\mathrm{d}}$ has the approximate distribution $\mathrm{N}(0,1)$. The hypothesis is then accepted at the 0.05 significance level if

$$
|\sqrt{\mathrm{m}} \overline{\mathrm{d}}| \leq 1.96
$$

If the hypothesis is rejected, the conclusion is that the probability is less than 0.05 that differences between calculated and measured values are due to measurement errors alone.

If there is a constant non-zero bias

$$
\mathrm{b}=\mathrm{E} \mathrm{z}_{\mathbf{j}},
$$

then a simple estimate of $b$ is given by 


$$
\hat{b}=\frac{1}{m} \sum z_{j},
$$

with the standard deviation*

$$
\left.\varepsilon_{\hat{b}}=\left\{\frac{1}{m^{2}} \sum \varepsilon_{z}\right\}^{2}\right\}^{1 / 2} .
$$

Test 1b: Individual Values Agreement Test. This test is designed to determine if the difference between a particular calculated and measured value can be reasonably attributed to measurement errors.

For an individual fractional difference $z_{j}$, the nu11 hypothesis is $E z_{j}=0$. The distribution of $z_{j}$ will be assumed to be well approximated by $N\left(0, \varepsilon_{z_{j}}{ }^{2}\right)$. The nu1l hypothesis is accepted at the 0.05 significance level if

$$
\left|\frac{z_{j}}{\varepsilon_{z_{j}}}\right| \leq 1.96
$$

Values which fail the test should be flagged and examined as a group for any pattern that would indicate where the calculational model needs improvement.

Test 2a: Group Goodness-of-Fit Test. Even if no overall bias is found by test $1 \mathrm{a}$, the difference between calculated and measured values may be significantly larger than that which can be accounted for by chance. This can be determined by test $1 \mathrm{~b}$ for individual values. The following test can be used when the values are considered as a group.

\footnotetext{
*In case of common calibration errors $r_{M}$ and $r_{C}$, propagated via $E q$. 4-30 or 4-31 as $r_{z_{j}}$, the standard deviation is estimated by$$
\varepsilon_{\hat{b}}=\left\{\frac{1}{m^{2} \sum s_{z_{j}}}{ }^{2}+\frac{1}{m} \sum r_{z_{j}}\right\}^{1 / 2} .
$$ 
As in test $1 \mathrm{~b}$, the null hypothesis is that $z_{j}$ has the distribution $\mathrm{N}\left(0, \varepsilon_{z_{j}}{ }^{2}\right)$ for $j=1,2, \ldots, \mathrm{m}$. Then

$$
\sum \mathrm{d}_{j}{ }^{2}=\Sigma\left(\frac{z_{j}}{\varepsilon_{z_{j}}}\right)^{2}
$$

is a chi-squared random variable with $m$ degrees of freedom. The null hypothesis is accepted at the 0.05 significance level if

$$
\frac{1}{m} \sum\left(\frac{z_{j}}{\varepsilon_{z}}\right)^{2} \leq \chi_{.95}^{2} / \mathrm{m},
$$

where $\chi_{.95}^{2}$ is the upper 95 percentile point for this chi-squared distribution with $m$ degrees of freedom. If the hypothesis is rejected, the interpretation is that the total variance of all values of $z_{j}$ is larger than that which can be accounted for by measurement errors alone.

For calculational purposes, the following approximation (Ref. 4-4) can be used for large values of degrees of freedom:

$$
x_{\alpha}^{2} / \mathrm{m}=\left(1-\frac{2}{9 m}+z_{\alpha} \sqrt{\frac{2}{9 m}}\right)^{3},
$$

where $\mathrm{m}=$ degree of freedom,

$$
\begin{aligned}
z_{\alpha} & =\text { normal deviate for the upper } \alpha \text { percentile point, } \\
z_{.95} & =1.645 .
\end{aligned}
$$

\subsubsection{Axial Variation Test}

Let $\bar{x}_{1}, \bar{x}_{2}, \ldots, \bar{x}_{m}$ be strata means of a given property (or the difference of this property determined by two different means) for successive axial positions in a test element. Let $s_{\mathrm{x}_{j}}$ be the standard deviation of the measurement error for $\bar{x}_{j}$. Define the root mean square axial variation 


$$
\operatorname{RMS}=\left\{\frac{1}{\mathrm{~m}} \Sigma\left(\bar{x}_{j}-\overline{\bar{x}}\right)^{2}\right\}^{1 / 2},
$$

where

$$
\overline{\bar{x}}=\frac{1}{m} \sum \bar{x}_{j}
$$

It is desired to test whether or not the axial variation RMS can be accounted for by measurement error alone. If not, the implication is that there is an additional, possibly systematic, source of axia1 variation of the property.

The null hypothesis is that the axial measurements $\bar{x}_{j}$ are independent random variables with a common mean $\mu$. This implies that the observed value of RMS is due to measurement error alone. It will be assumed that the measurement errors are normally distributed with mean 0 and common standard deviation $\sigma$. Thus this test is only applicable when the estimated standard deviations $\varepsilon_{\mathrm{x}_{j}}$ are all approximately equal. In this case
$\sigma$ will be estimated ${ }^{*}$ by

$$
\sigma=\left\{\frac{1}{m} \sum \varepsilon_{\bar{x}_{j}}^{2}\right\}^{1 / 2}
$$

The test statistic is

$$
\mathrm{q}=\frac{\mathrm{m} \mathrm{RMS}^{2}}{\sigma^{2}} .
$$

The null hypothesis is accepted if

$$
\frac{\mathrm{m} \mathrm{RMS}^{2}}{\mathrm{df} \sigma^{2}} \leq x_{.95}^{2} / \mathrm{df},
$$

\footnotetext{
* In case of a common calibration error $r$, the standard deviation is
} estimated by

$$
\sigma^{2}=\left\{\frac{1}{m} \sum s_{x_{j}}^{2}+r^{2}\right\}^{1 / 2}
$$


where $x_{.95}^{2}$ is the 95 percentile point of the chi-square distribution with df degrees of freedom. For this case:

$$
\mathrm{df}=\mathrm{m}-1
$$

Eq. 4-42 can be used for large values of df to determine $\chi^{2} / \mathrm{df}$.

If the null hypothesis is rejected, then there is less than a $5 \%$ chance that the observed RMS axial deviation could be due to measurement error alone.

\subsubsection{Regression Analysis}

An irradiation parameter u, e.g., burnup, may vary systematically with core height $x$. A correlation between available observations of $u_{j}$ and $x_{j}$ by least squares regression can be used to estimate values of $u$ in the absence of measurements. The regression is usually valid only for a small range of the test element. In this range, the regression equation is

$$
u(x)=\bar{u}+b(x-\bar{x})=a+b x
$$

The least squares estimates for the regression constants are

$$
b=\frac{\sum\left[\left(x_{j}-\bar{x}\right)\left(u_{j}-\bar{u}\right)\right]}{\sum\left(x_{j}-\bar{x}\right)^{2}}=\frac{\sum\left(x_{j} u_{j}\right)-\sum x_{j} \sum u_{j} / m}{\sum x_{j}^{2}-\left(\sum x_{j}\right)^{2} / m}
$$

and

$$
a=\bar{u}-b \bar{x}=\frac{1}{n} \sum u_{j}-\frac{b}{n} \sum x_{j}
$$

An estimate of the standard deviation about the regression line is

$$
s=\left\{\frac{1}{n-2} \sum\left[u_{j}-\bar{u}-b\left(x_{j}-\bar{x}\right)\right]^{2}\right\}^{1 / 2}
$$


or the algebraically equivalent formula

$$
s=\left\{\frac{n-1}{n-2}\left(s_{u}^{2}-b^{2} s_{x}^{2}\right)\right\}^{1 / 2}
$$

where

$$
s_{u}=\left\{\frac{1}{n-1} \Sigma\left(u_{j}-\bar{u}\right)^{2}\right\}^{1 / 2}
$$

and

$$
s_{x}=\left\{\frac{1}{n-1} \sum\left(x_{j}-\bar{x}\right)^{2}\right\}^{1 / 2}
$$

Then the estimated standard deviation of $u(x)$ under the linearity assumption is

$$
s_{u}(x)=s\left\{\frac{1}{n}+\frac{(x-\bar{x})^{2}}{\sum\left(x_{j}-\bar{x}\right)^{2}}\right\}^{1 / 2}=s\left\{\frac{1}{n}+\frac{(x-\bar{x})^{2}}{D}\right\}^{1 / 2}
$$

The above analysis requires at least three data points for the regression and assumes that the axial positions $x_{j}$ are known (nonrandom) values. A linear interpolation could be used if only two data points are available.

The standard error along the slope can be determined via

$$
s_{b}=\left\{\frac{1}{\sum\left(x_{i}-\bar{x}\right)}\right\}^{1 / 2} \quad s=\frac{s}{\sqrt{D}} .
$$

The $95 \%$ confidence statement interval for the true slope is given by

$$
b \pm t_{n-2}, 0.975^{s},
$$

where $t_{n-2}, 0.975=97.5$ percentile of the student $t$ distribution at $n-2$ degree of freedom. The standard error on the intercept is

$$
s_{a}=\left\{\frac{\sum x_{i}^{2}}{n \sum\left(x_{i}-\bar{x}\right)^{2}}\right\}^{1 / 2} s=s\left\{\frac{D / n-\bar{x}^{2}}{D}\right\}^{1 / 2} .
$$


The $95 \%$ confidence interval of the intercept is

$$
a \pm t_{n-2,0.975} \quad s_{a}
$$

The described regression analysis requires at least three data points for the regression and assumes that the axial positions $x j$ are known (nonrandom) values.

The more general analysis for two regression constants $a$ and $b$ and any two functions of $\mathrm{x}$ along the regression equation

$$
f(x)=a \cdot g(x)+b \cdot h(x)
$$

results in

$$
s^{2}(x)=s^{2} \frac{[\gamma g(x)+\beta h(x)] g(x)+[\alpha h(x)-\beta g(x)] h(x)}{\gamma \alpha-\beta^{2}},(4-62)
$$

where

$$
\begin{aligned}
\alpha & =\sum g^{2}\left(x_{i}\right) \\
\beta & =S g\left(x_{i}\right) h\left(x_{i}\right) \\
\gamma & =\sum h^{2}\left(x_{i}\right) \\
s^{2} & =\frac{\sum\left[x_{i}-f\left(x_{i}\right)\right]^{2}}{n-2},
\end{aligned}
$$

The term $s$ is the standard deviation about the regression (see Eq. 4-52). For $g(x)=x^{0}$ and $h(x)=x^{1}$, Eq. 4-62 is identical with Eq. 4-56. 


\subsubsection{Interpolation}

The linear interpolation between the two points $\left(x_{1}, y_{1}\right)$ and $\left(x_{2}, y_{2}\right)$ gives at any intermediate point $x$

$$
\begin{aligned}
& y(x)=y_{1}+\frac{y_{2}-y_{1}}{x_{2}-x_{1}} x \\
& y(x)=a \cdot y_{1}+b \cdot y_{2},
\end{aligned}
$$

where

$$
\begin{aligned}
& a=\frac{x_{2}-x_{1}-x}{x_{2}-x_{1}} \\
& b=\frac{x}{x_{2}-x_{1}}
\end{aligned}
$$

The standard error of $y(x)$ is then

$$
s_{y(x)}=\left(a^{2} s_{y_{1}}^{2}+b^{2} s_{y_{2}}^{2}\right)^{1 / 2} .
$$

Linear interpolation has to be used where no more than two neighboring measurements are available, whereas at least three points are required for a linear regression analysis. Linear interpolation employs the standard errors $s_{y_{1}}$ and $s_{y_{2}}$ of the neighboring measurements to establish the standard error for the intermediate point. A linear regression determines the error from the least square distance of the individual measurements from the regression line (see Eq. 4-62). 


\subsubsection{Normalization}

Qualitative results can be derived from a measured parameter $u$ along the axis of the test element via normalization. This in turn allows comparing a measured with a predicted profile in accordance with the test procedures of Section 4.1.3.

Assume $u_{1}, u_{2}, \ldots, u_{m}$ are independent and were determined in intervals of equal distance* along the core height $x$ of the element. The analysis function for normalization is

$$
f\left(x_{j}\right)=\frac{u_{j}}{\bar{u}},
$$

with

$$
\overline{\mathrm{u}}=\frac{1}{\mathrm{~m}} \sum \mathrm{u}_{\mathrm{j}}
$$

The progressed standard deviation of $f\left(x_{j}\right)$ is

$$
s_{f}=\left\{\left(\frac{\partial f}{\partial u_{j}} s_{u_{j}}\right)^{2}+\left(\frac{\partial f}{\partial \bar{u}} s_{\bar{u}}\right)^{2}+2 \frac{\partial f}{\partial u_{j}} \frac{\partial f}{\partial \bar{u}} \operatorname{cov}\left(u_{j}, \bar{u}\right\}^{1 / 2},(4-69\right.
$$

where the partial derivatives

$$
\begin{aligned}
& \frac{\partial f}{\partial u_{j}}=\frac{1}{\bar{u}} \\
& \frac{\partial f}{\partial \bar{u}}=-\frac{u_{j}}{\bar{u}^{2}} .
\end{aligned}
$$

* This is guaranteed when tests are done for a stack of fuel rods. Continuous fuel gamma scans at Peach Bottom were done for a constant life time with variable dead times, which resulted in some variation of the scanned intervals. This can be compensated via linear regression or by weighing via $\Delta x_{j} / \overline{\Delta x}$. For FTE-6, this correction has been neglected. 
The covariance is

$$
\begin{aligned}
& \operatorname{cov}\left(u_{j}, \bar{u}\right)=\sum_{k}^{\sum} \operatorname{cov}\left(u_{j}, \frac{u_{k}}{m}\right), \\
& \operatorname{cov}\left(u_{j}, \bar{u}\right)=\operatorname{cov}\left(u_{j}, \frac{u_{j}}{m}\right), \\
& \operatorname{cov}\left(u_{j}, \bar{u}\right)=\frac{1}{m} \operatorname{var} u_{j}, \\
& \operatorname{cov}\left(u_{j}, \bar{u}\right)=\frac{1}{m} s_{u_{j}}^{2} .
\end{aligned}
$$

This gives for Eq. 4-59

$$
s_{f}=\frac{u_{j}}{\bar{u}}\left\{\left[\left(\frac{s_{j}}{u_{j}}\right)^{2}+\left(\frac{s_{-}}{\bar{u}}\right)^{2}-\frac{2}{m} \frac{s_{j}}{u_{j} u}\right]\right\}^{1 / 2},
$$

which can be simplified to

$$
s_{f} \cong \frac{u_{j}}{\bar{u}}\left\{\left(\frac{s_{j}}{u_{j}}\right)^{2}-\left(\frac{s_{-}}{\bar{u}}\right)^{2}\right\}^{1 / 2}
$$

when assuming the following approximations

$$
\frac{s_{u_{j}}}{m u_{j}} \cong \frac{1}{m} \sum\left(\frac{s_{u_{j}}^{2}}{m u_{j}}\right) \cong \frac{\sum s_{u_{j}}}{m \sum u_{j}}=\frac{s-\frac{{ }^{2}}{\bar{u}}}{.}
$$

The standard deviation on the mean is determined via Eq. 4-16 as

$$
s_{u}=\left\{\frac{1}{m^{2}} \quad \sum s_{u_{j}}^{2}\right\}^{1 / 2}
$$


If $u_{j}$ was determined as the mean of $n$ independent tests, then Eq. 4-26 applies for determining the standard deviation

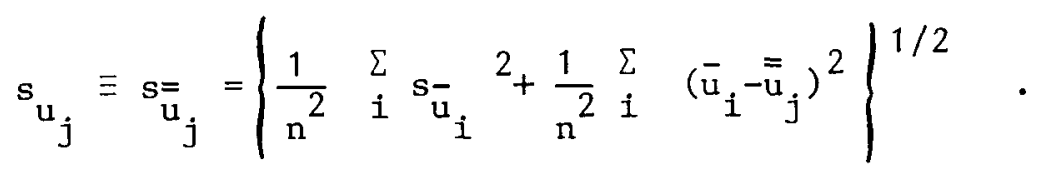

If there is a common calibration error with the standard deviation $r$, the progression of the combined standard errors $\varepsilon$ follows from Eq. 4-59:

$$
\varepsilon_{f}=\frac{u_{j}}{\bar{u}}\left\{\left(\frac{\varepsilon_{u_{j}}}{u_{j}}\right)^{2}+\left(\frac{\varepsilon_{\bar{u}}}{\bar{u}}\right)^{2}-\frac{2}{m} \frac{\varepsilon_{u_{j}}^{2}}{u_{j} \bar{u}}-2 \frac{r^{2}}{u_{j} \bar{u}}\right\}^{1 / 2},
$$

where

$$
\begin{aligned}
& \varepsilon_{u_{j}}^{2}=s_{u_{j}}^{2}+r^{2} \\
& \varepsilon_{u}^{2}=s_{u}^{2}+r^{2} .
\end{aligned}
$$

A specific application of the presented algorithm is described in Section 3.2.1, where fluence profiles have been determined via normalization of Cs-137 and Cs-134/Cs-137 activity measurements.

With $\mathrm{m}=1$ and consequently $\overline{\mathrm{u}}=\varepsilon_{\mathrm{u}}$, the presented algorithm can be used (with the exception of the simpliflcation via Eqs. 4-64 and 4-65) to describe conovsion from absolute to relative concentrations, as applied to burnup evaluations from 10MS measurements in Section 3.4.2. 


\subsection{NUCLEAR MEASUREMENTS}

FTE-6 was gamma scanned at the reactor site shortly after final shutdown to determine short-lived isotopes; 15 months later gamma scanning was carried out in the GA hot cell in a disassembled form to determine long-lived isotopes and to permit component evaluation (fuel particles, fuel rods, graphite parts). Both gamma scanning data sets have been analyzed to establish a direct comparison and a consistency between methods. This will assist evaluation of other fuel test elements which were scanned with only one method. The scans have been analyzed to verify the nuclear, thermal, fuel, and fission product performance predicted with HTGR design codes. Specific interest focused on power and fluence profiles. EOL power distribution was estimated from the distribution of the short-1ived isotope La-140 and compared with the medium-1ived isotope $\mathrm{Zr}-95$. The latter can still be measured after a year or two in a hot cell. Cesium profiles were compared with fluence and burnup profiles. Fortunately, one fuel blend had generated a high enough fuel failure so that the scans could be analyzed for detection of fuel failure and fission product mobility (cesium, cerium, ruthenium).

Whenever possible, results from both scanning methods were compared with each other and against independent destructive measurement methods and design code predictions. All tests were performed on a statistical basis and analyzed for statistical significance.

The delayed neutron distribution of FTE- 6 was measured within the shipping cask and in an unshielded configuration as part of an effort to determine neutron shielding requirements for HTGR shipping casks. This was done under a separate program and only the axial neutron distribution has been analyzed for consistencies (one portion along the element showed a defect in neutron source strength) within the context of this report.

\subsubsection{Fue1 Gamma Scan at Peach Bottom}

On November 25, 1974, FTE-6 was removed from the reactor and axially 
scanned at the Peach Bottom site. ${ }^{*}$ The procedure was to lower the element in the charge machine slowly past a collimator which was in line with a high resolution $\mathrm{Ge}(\mathrm{Li})$ gamma spectrometer (Ref. 4-4). Continuous plots for the isotopes $\mathrm{Pa}-233, \mathrm{Zr}-95$, and La-140 were taken via single-channel analyzers (see Fig. 4-1). Concurrently, multichannel spectra were collected at about 1-minute intervals, which covered distances of about 80 $\mathrm{mm}$ along the element.

Actual time information was stored for each scan, from which the axial core location was calculated via an average scanning speed. The scanning speed was determined from single analyzer strip charts (see Fig. 4-1), which carried accurate information about traveled length (recorded via signals from the hoist mechanism in the charge machine).

Slight variations of the scanning speed during the approximately 45 minutes of continuous scanning were observed because of voltage fluctuations. It should be noted that the charge machine was not designed for the described application, but rather had to be modified via an additional gearbox to accommodate these low travel speeds (i.e., $1.27 \mathrm{~mm} / \mathrm{min}$.).

Core location parameters were adjusted for actual length between the beginning and the end of the total fuel stack as determined from PIE metrology. The nominal BOL value of $664 \mathrm{~mm}$ (26.15 in.) was used as a reference point for the beginning of the fuel. This point was assumed to fall within the scanned interval of spectrum 201 (in Table 4-3) at a fraction proportional to the recorded counts in comparison with the next fuel scan spectrum 202. This resulted in $3 \mathrm{~mm}$ (0.13 in.) of fuel length in spectrum 201. End of fuel was $2914 \mathrm{~mm}$ (113.19 in.), which was assumed to coincide with spectrum 227 in Table 4-4. The average scanning speed

* The Peach

The Peach Bottom gamma-scan exercise was made possible by free equipment lease and technical assitance from Intelcom Rad Tech, San Diego, by Dr. V. J. Orphan, Dr. V. C. Rodgers, and staff.

$* *$

The counting lifetime was kept constant at $60 \mathrm{~s}$; the system's dead time, however, varied as a function of the intensity of the gamma rays, which resulted in variable distances covered for each scan. An interval of about $6 \mathrm{~s}$, during which no scanning took place, was required for data transfer between scans. 
was corrected to cover the total fuel distance within the recorded time interval.

Nonlinearity of the scanning speed can be recognized for spectrum 210 , which still would account for about $6 \mathrm{~mm}$ ( 0.26 in.) of fuel, but does not record for any nuclide isotope. A check of the stripcharts (Fig. 4-1) indicates that the fueled length of body 1 may have coincided with the end of spectrum 209. Adding a traveled distance of about $5 \mathrm{~mm}$ (0.21 in.) during data transfer, the maximum observable mismatch in fuel length and spectrum distance is determined to be $11 \mathrm{~mm}$ ( $0.47 \mathrm{in}$.) for the FTE-6 Peach Bottom scan locations. This length is about $15 \%$ of the traveled distance for one spectrum and is rated small in comparison with the total fuel length of $22.50 \mathrm{~mm}$ ( $88.59 \mathrm{in.})$. In order to avoid interference from partially fueled spectra in the isotopic analysis of the fuel zones, spectra at the end of fuel stacks have been truncated from further evaluation. These details of analysis are reported in order to stress the importance of accurate location information, which is essential for precision gamma scanning and should be included in the data acquisition by direct means. Time and equipment limitations did not allow this for the Peach Bottom gamma scan, which makes the data analysis very labor intensive.

Using a special data reduction program, the following isotopes and their associated $2 \sigma$ counting errors were identified from the Peach Bottom EOL fuel gamma scanning and from fuel rod gamma scans for FTE-6:

\begin{tabular}{|c|c|c|c|}
\hline Isotope & $\begin{array}{l}\text { Energy } \\
(\mathrm{KeV})\end{array}$ & $\begin{array}{l}\text { Peach Bottom EOL, } \\
2 \sigma \text { Error } \\
\text { 1-Min. Lifetime }\end{array}$ & $\begin{array}{l}\text { Fue1 Rod Scan, } \\
2 \sigma \text { Error } \\
\text { 7-Min. Lifetime }\end{array}$ \\
\hline \multirow[t]{2}{*}{$\mathrm{Ce}-144$} & 133 & $\pm 64 \%$ & N.D. \\
\hline & 696 & N.D. & $\pm 12.8 \%$ \\
\hline $\mathrm{Ce}-141$ & 145 & $\pm 16 \%$ & N.D. \\
\hline $\mathrm{Pa}-233$ & 312 & $\pm 3.0 \%$ & N.D. \\
\hline$I-131$ & 364 & $\pm 31 \%$ & N.D. \\
\hline$R u-103$ & 497 & $\pm 8.1 \%$ & N.D. \\
\hline
\end{tabular}




\begin{tabular}{|c|c|c|c|}
\hline Isotope & $\begin{array}{l}\text { Energy } \\
(\mathrm{KeV})\end{array}$ & $\begin{array}{l}\text { Peach Bottom EOL, } \\
2 \sigma \text { Error } \\
\text { 1-Min. Lifetime }\end{array}$ & $\begin{array}{l}\text { Fuel Rod Scan, } \\
2 \sigma \text { Error } \\
\text { 7-Min. Lifetime }\end{array}$ \\
\hline$R u-106$ & 512 & N.D. & $\pm 13.4 \%$ \\
\hline \multirow[t]{2}{*}{$\mathrm{Cs}-134$} & 605 & N.D. & $\pm 2.45 \%$ \\
\hline & 796 & $\pm 22 \%$ & N.D. \\
\hline Cs -137 & 662 & $\pm 26 \%$ & $\pm 1.91 \%$ \\
\hline $\mathrm{Zr}-95$ & 724 & $\pm 5.4 \%$ & $\pm 18.2 \%$ \\
\hline $\mathrm{La}-140$ & 1596 & $\pm 4.8 \%$ & N.D. \\
\hline
\end{tabular}

Counting errors are averages determined via Eq. 4-20 from spectra selected for further evaluation by following the truncation procedure described above. The Peach Bottom EOL scans were taken 25 days after shutdown with a lifetime of 1 minute per spectrum; the fuel rod scans were made during a period of three weeks about 15 months after shutdown with a 7-minute lifetime per spectrum. This explains the differences in isotopes, energy levels, and counting errors. Different isotopes of the same element or different energy levels of the same isotope were detected for the two time points of scanning. Therefore, comparisons have to be made on an element rather than an isotope basis for cerium and ruthenium. $\mathrm{Pa}-233$, I-131, and La-140 were decayed away by the time of the second scanning exercise. Lower counting errors were achieved for cerium and cesium, and larger errors were found for ruthenium and zirconium at the second time point.

The as-measured Peach Bottom EOL fuel gamma scan 1 results are plotted in Figs. 4-2 through 4-5. The Cs -134 and $\mathrm{Cs}-137$ profiles plus the Cs137/Cs-134 ratio are plotted in Fig. 4-2 as counts per minute (CPM) backdecayed to shutdown, versus axial core height. Figure 4-3 contains the Ce-141 and Ru-103 distribution, Fig. 4-4 presents the Pa-233, Zr-95, and La-140 profiles, and Fig. 4-5 gives the $\mathrm{Zr}-95 / \mathrm{Cs}-137$, Ru-103/Zr-95, and $\mathrm{Ce}-141 / \mathrm{Zr}-95$ ratios. Heterogeneous fuel loading in the axial direction and loss of cesium in some areas can be concluded from these profiles. A detailed analysis is described in the next section. 
Figure 4-6 details the azimuthal configuration for the Peach Bottom gamma scanning. A11 of the spine sample cross sections and the equivalent of 3.5 out of 8 fuel holes were under surveillance with the following approximate fractions:

\begin{tabular}{|c|c|c|}
\hline Area & Fraction & under Surveillance (\%) \\
\hline Spine & 100 & \multirow{4}{*}{$\begin{array}{l}\text { front, } \\
21 \% \text { of total fuel rod area }\end{array}$} \\
\hline Hole 4 & 65 & \\
\hline Hole 5 & 100 & \\
\hline Hole 6 & 2 & \\
\hline Hole 8 & 78 & \multirow{3}{*}{$\begin{array}{l}\text { rear, } \\
23 \% \text { of total fuel rod area }\end{array}$} \\
\hline Hole 1 & 100 & \\
\hline Hole 2 & 6 & \\
\hline
\end{tabular}

The geometrical configuration is very sensitive to the exact position of the collimator with respect to the fuel element. A slight tilt or eccentricity of the collimator axis would give significantly different fractional fuel areas. It is concluded from the best estimate values above that $44 \%$ of the fuel and all of the spine sample were counted.

\subsubsection{Fuel Gamma Scan Analysis}

The fuel gamma scan results from Peach Bottom were compared with data taken from individual fuel rods. The evaluation procedure of the fuel rod scans is described in Section 3.2.2. Numerical results from fuel stacks 1, 3, and 5 are presented in this section in direct comparison with Peach Bottom scanning data. Detailed information on fuel rod performance is included in Section 3.4.2 for burnup and in Section 4.5.4 for fuel failure determination. Table $4-4$ reports the actual CPM activities, and Table 4-3 lists several nuclide CPM ratios and normalized profiles as determined from Peach Bottom scan 1. Table 4-5 contains the CPM information, and Table 4-6 contains the relevant nuclide ratios and normalized profiles for fuel stack 1 , which was composed of a failed $\mathrm{UO}_{2}$ TRISO fue1 variety. 
Several statistical tests have been carried out to establish an evaluation scheme for fuel gamma scan information. The numerical results for these tests have been included into Tables 4-7 through 4-13 on a fuel body and fuel element average basis, in order to document the procedures used. Numerical information for specific axial locations can be retrieved through Table 4-3 and Table 4-6.

\subsubsection{Test for Identity of La-140 and $\mathrm{Zr}-95$ Normalized Profiles.}

Hypothesis: La-140, which is a daughter of $\mathrm{Ba}-140$, best resembles the EOL power profile because of an effective 12.8-day half-life. Zr-95, with a half-1ife of 65.5 days, is representative for the last 100 to 200 days of operation. It is hypothesized that the power profile did not change significantly over the last 200 days of reactor operation.

Test: La-140 and $\mathrm{Zr}-95$ have been tested for agreement by comparing the normalized profiles derived from the Peach Bottom EOL data.

Results: Numerical and graphical results are reported in Table 4-7 and Fig. 4-7, respectively. In 14 or 21 tests, the two isotope distributions agree at the 0.05 significance level. The group agreement test 1 [student $t$ test (see Section 4.1.3.2)] does not reveal differences for the three fuel bodies or for the total element. Only the goodness-of-fit test 2 ( $\chi^{2}$ test) indicates some disagreement. This is caused by the seven tests which failed the individual value agreement test. (Five out of these seven tests still show a partial overlap of the $95 \%$ confidence intervals. The test, however, is made on the difference between two values, and the error progression is a geometrical rather than an arith'metical combination.)

Summary:

1. The power distribution for FTE-6 did not significantly change over the last 100 to 200 days. 
2. Therefore, $\mathrm{Zr}-95$ is a good indicator for the EOL power profile for cases where La-140 has a1ready decayed away.

\subsubsection{Comparison of Calculated and Measured EOL Power Profiles for Composite Element.}

Hypothesis: Calculated and measured EOL power profiles agree.

Test: The GAUGE/FEVER calculated EOL power profile has been compared with the arithmetic mean of the La and $\mathrm{Zr}$ distribution.

Results: Results are presented in Table 4-7 and Fig. 4-8. In 15 of 21 tests, the axial power distributions do not agree. The group agreement test, however, does not show a difference for body 1 as a total, but this is rejected by the goodness-of-fit test. It is felt that underestimation at the bottom of the body is compensated by overprediction at the top part of body 1. Calculated and measured power distribution disagree between $+29 \%$ and $-24 \%$ locally. ${ }^{*}$ The axial variability is significant for all fuel bodies and the total element.

Discussion: The Peach Bottom gamma scan surveyed 44\% (estimated) of the fuel and $100 \%$ of the spine sample areas. FTE- 6 contained boronated graphite samples (resulting in flux depression), fission product release samples (no nuclear impact except for fission product distribution), advanced fuel rods (thorium-fueled, but flux increase from U-233 fission), two types of thermal stability samples, and several fission product retention samples with different fissile and fertile fuel loadings. The fissile loading of the spine samples at BOL represented $1.18 \%$ of the total fissile loading of the element; the thorium loading was $7.30 \%$. At EOL the U-233 and U-235 inventory of the spine samples was $2.11 \%$ of the total inventory, resulting in a relative increase of power production of a factor of 1.78 and an absolute increase of a factor of 1.04 (see Table

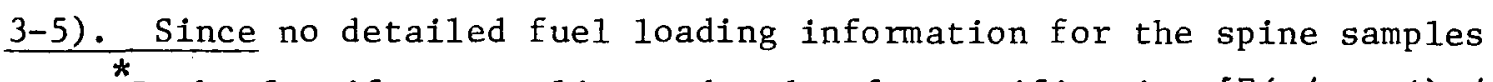
* Lack of uniform sampling and mode of stratification $[\Sigma(c / m-1) \neq$ $\mathrm{m} \Sigma \mathrm{c} / \Sigma \mathrm{m}-\mathrm{m}]$ resulted in a bias of $\mathrm{b}=-2.3 \%$ for the total element. The quoted data in the text have been corrected by this amount, compared with tabulated results in Table $4-7$. 
was available, an assessment of these effects had to be made.

The majority of the fissile material was included in the type 1 thermal stability spine samples in body 3 , which resulted in linear heat ratings at $B O L$ of about 0.5 to 0.7 compared with a fuel rod at the same location. At EOL it was about equivalent (factor 1.0 to 1.2) to the heat generation from a stack 1 fuel rod at the same leve1. This was concluded from Zr-95 CPM activity measurements. The type 2 spine samples in body 3 revealed about 10\% of the activity leve1 of the type 1 spine samples, which can be used as an estimate of the heat generation at BOL and EOL. The advanced fuel rods in body 2 were thorium fueled and generated about $30 \%$ of the power of a stack 1 fuel rod at the same location based on the $\mathrm{Zr}-95 \mathrm{CPM}$ activities. Some of the fission product release samples in body 2 may a1so have had $10 \%$ to $20 \%$ of the linear heat generation of a fuel rod. Spine sample gamma scan results are presented in Section 4.6.4.

The impact of the heat generation in the fueled spine samples can clearly be identified in Fig. 4-9. The location of the advanced fuel rods and the types 1 and 2 thermal stability samples can be positively determined. The position of the boronated graphite is close to an area of significant depression. The same depression shows up in Fig. 4-10 for a fuel stack profile, but with a shift of $100 \mathrm{~mm}$ towards the bottom end of the element, which is more coincidental with the boronated graphite location. (Self-compensating nonlinearity in the scanning speed at Peach Bottom within the fuel area can remain undetected because core height correlations were made only for fuel end zones.)

\section{Summary:}

1. Spine samples containing fissile and fertile fuel and burnable poison had a significant impact on the local axial power distribution. 
2. The axial profile from the Peach Bottom fuel scan is a superposition of gamma rays from the spine samples and between 2 and 3.5 fuel stacks. The lower number would be the parallel projection of the collimator; the upper limit is the geometrical projection shown in Fig. 3-40. The heat rating assessment supports a value toward the lower limit. Attenuation of gamma rays of the rear fuel rods could also be an explanation for the lower limit.

\subsubsection{Comparison of Calculated and Measured EOL Power Profiles for One Fuel Rod Variety.}

Hypothesis: Measured and calculated EOL power profiles agree.

Test: The GAUGE/FEVER calculated EOL power profile has been compared with the Zr-95 CPM distribution measured for one specific fuel rod variety with uniform fuel loading (stack 1).

Results: Numerical and graphical results are displayed in Table 4-8 and Fig. 4-9 respectively. In 18 of 20 tests, the measured and calculated power profiles agree. The locations of disagreement are at $13 \%$ and $99 \%$ relative core height. The latter location indicates a larger reflector effect than predicted; the former is adjacent to the area of significant flux depression, which concurs with a burnable poison spine sample position. Areas of some flux increases can also be realized; these coincide with spine sample locations, which contained notable amounts of fissile and fertile fuel. Some impact of rod-to-rod fuel loading variations may also be evident. The uncertainty of the $\mathrm{Zr}-95$ activity is much larger than for the Peach Bottom scan, which does not surpress the similarity in shape with Fig. 4-8.

Summary:

* In both cases, the lower $2 \sigma$ limits still match the predicted profile. The test, however, is made on the relative difference, which results in a quadratic rather than a linear error progression, as described earlier. 
1. The power distribution of a fuel stack with uniform loading is approximated by the predicted profile within the uncertainty range coming from the counting error.

2. Although inside the uncertainty band, the microstructure of the power profile can be detected, as imposed from neighboring fuel heterogeneities.

3. For detailed thermal analyses, the axial power distribution of spine samples and fuel rods have to be superimposed.

4. The tested fuel variety stack 1 experienced a high fuel failure during irradiation, which was not detrimental to any power distribution analysis.

5. The location of diffusion spine samples in body 1 shows a possible $11 \%$ power peak (at $14 \%$ relative core height in Fig. 4-9) above the predicted profile, which may have resulted from burnout of adjacent burnable poison material. Some variation of fuel rod loadings may also have been the cause.

\subsubsection{Nuclide Mobility Tests.}

Hypothesis: The ratio of direct-yield fission product isotopes is approximately constant along a fuel element for an unfailed and nondiffusive fuel variety (TRISO coated fuel). Alternatively, for failed TRISO fuel and for BISO fuel with sufficient burnup, time, and temperature, mobile species will show a defect when compared with nonmobile species.

\section{Tests:}

1. Determine the Cs-137/Zr-95 ratio for an unfailed fuel variety and test for significance. 
2. Compare the Cs-137/Zr-95 ratio of a failed fuel variety with an unfailed variety and establish the amount of lost cesium.

3. Compare the fuel rod scan with the composite scan of the element.

4. Test cerium for mobility by comparing composite scan results with fuel rod scan results.

5. Test ruthenium for mobility by the same comparison.

Resu1ts: Cesium Test: The Cs $-137 / \mathrm{Zr}-95$ ratio was determined from 48 TRISO/TRISO fuel rods which did not reveal fuel failure from metallographic examination and fission gas release measurements (fuel stacks 5 and 6$)$. The following mean and uncertainty values were achieved:

$$
\begin{aligned}
\overline{\mathrm{x}} & =0.1142, \\
\mathrm{~m} & =48, \\
\bar{s}_{\mathrm{x}} & =0.0189 \text { (mean counting error, progressed), } \\
\mathrm{s}_{\mathrm{x}} & =0.0030 \text { (composite error statement), } \\
\text { RMS } & =0.0080 \text { (rod-to-rod variability). }
\end{aligned}
$$

The axial variation test (see Section 4.1 .4 ) gives

$$
\frac{\mathrm{m} \mathrm{RMS}^{2}}{\mathrm{df} \sigma^{2}}=0.18
$$

and

$$
\frac{\chi^{2}}{d f}=1.36
$$

with $0.18<1.36, \mathrm{Cs}-137 / \mathrm{Zr}-95$ variation along the axis of the fuel element can be reasonably attributed to the measurement error. The best estimate for an intact ratio for fuel stacks 5 and 6 is 


$$
\left(\frac{\mathrm{Cs}-137}{\mathrm{Zr}-95}\right)_{I=5 \& 6, \text { intact }}=0.114 \pm 0.006(2 \sigma)
$$

Fission yields for U-235 and U-233 are different for both isotopes (see Table 3-7); so corrections would be required when comparing plain fissile and fertile fuel. The nuclide ratios are proportional to the fission yields

$$
\frac{\mathrm{Cs}}{\mathrm{Zr}} \sim \frac{\mathrm{Y}_{\mathrm{cs}}}{\mathrm{Y}_{\mathrm{zr}}}
$$

and in case of composite fuel varieties

$$
\left(\frac{\mathrm{Cs}}{\mathrm{Zr}}\right)_{\mathrm{c}} \sim \frac{\mathrm{Y}_{\mathrm{cs}, 5}+\frac{\mathrm{N}_{3} \cdot \sigma_{\mathrm{f}, 3}}{\mathrm{~N}_{5} \cdot \sigma_{\mathrm{f}, 5}} \cdot \mathrm{Y}_{\mathrm{cs}, 3}}{\mathrm{Y}_{\mathrm{zr}, 5}+\frac{\mathrm{N}_{3} \cdot \sigma_{\mathrm{f}, 3}}{\mathrm{~N}_{5} \cdot \sigma_{\mathrm{f}, 5}} \cdot Y_{\mathrm{zr}, 3}},
$$

$$
\text { where } \begin{aligned}
\sigma_{\mathrm{f}, 5} & =580 \mathrm{~b} \text { fission cross section for } \mathrm{U}-235, \\
\sigma_{\mathrm{f}, 3} & =525 \mathrm{~b} \text { fission cross section for } \mathrm{U}-233, \\
\mathrm{~N}_{5} & =\mathrm{U}-235 \text { atomic concentration, } \\
\mathrm{N}_{3} & =\mathrm{U}-233 \text { atomic concentration, } \\
\mathrm{Y}_{i, j} & =\text { fission yield of isotope } i \text { from U-23j fission. }
\end{aligned}
$$

Stationary reactor conditions are assumed. By application of the EOL uranium concentrations from Table 3-5 and the fission yields from Table 3-7, the following intact ratios can be determined for FTE-6 fuel varieties:

\begin{tabular}{ccc}
$\begin{array}{c}\text { FTE-6 } \\
\text { Fue1 Variety }\end{array}$ & $\left(\frac{(\mathrm{Cs}-137}{\mathrm{Zr}-95}\right)_{\text {intact }} \pm 20$ \\
\hline $1 \& 2$ & $0.1160 \pm 0.0060$ \\
$3 \& 4$ & $0.1142 \pm 0.0059$ \\
$5 \& 6$ & $0.1142 \pm 0.0059$ \\
$7 \& 8$ & $0.1137 \pm 0.0058$
\end{tabular}




$$
\begin{array}{ll}
\begin{array}{l}
\text { FTE-6 } \\
\text { Fue1 Variety }
\end{array} & \frac{\left(\frac{C s-137}{Z r-95}\right)_{\text {intact }} \pm 2 \sigma}{\text { Plain U-235 }} \\
\text { P1ain U-233 } & 0.1128+0.0058 \\
& 0.1271 \pm 0.0065
\end{array}
$$

With the exception of plain bred fuel, the differences are small between the fuel varieties.

In Table 4-9 and Fig. 4-10, the actual test has been carried out for fuel stack 1 , the fuel variety with large fuel failure ( $\mathrm{UO}_{2}$ TRISO fuel). The axial variation test does not reveal significant deviations within fuel bodies; however, the test carried out for the element as a whole shows significance.

Postulating a linear relationship between $\mathrm{Cs} / \mathrm{Zr}$ ratios and cesium loss, the following values can be stated from Table 4-9:

FTE-6

Fuel Element

Body 1 (bottom)

Body 2 (center)

Body 3 (top)

Total element

RMS

Test results (on

fue1 bodies)

\section{Cesium Depletion ${ }^{*} \pm 2 \sigma(\%)$} P.B. EOL Scan 1

Fuel Stack 1 Scan

0

$$
-17.1 \pm 8.8
$$$$
-22.6 \pm 8.6
$$$$
-14.9 \pm 5.4
$$$$
\pm 22.5
$$

Significant

\section{0}

$-19.7 \pm 4.4$

$-22.4 \pm 5.7$

$-16.0 \pm 3.2$

$\pm 16.6$

No cesium loss is detectable for body 1 ; bodies 2 and 3 show cesium depletion in the range of $20 \%$, with body 3 being slightly the higher.

Fission gas release measurements on two fuel rods from body 2 gave the following results:

The intact nuclide ratio for fuel variety stack 5 was used in Table 4-9 for the test. The quoted results have been corrected for the intact nuclide ratio for stack 1 . 
FTE-6

Fue1 Rods

Rod 2-1-7

Rod 2-2-7

$$
\begin{array}{r}
\text { Equivalent Fuel Failure } \\
\bar{x}-2 \sigma(\%)<\bar{x}(\%)<\bar{x}+2 \sigma(\%) \\
\hline
\end{array}
$$$$
8<21<53
$$$$
9<23<58
$$

Cesium Depletion * $\bar{x}(\%) \pm 2 \sigma(\%)$

$-21.4 \pm 10.0$

$-13.7 \pm 10.5$

The equivalent failure fraction determined from fission gas release agrees with the cesium depletion determined from two individual fuel rods and from composite fuel body or fuel stack measurements.

It should be noted that fission gas release measures pressure vesse1 failure as well as exposed contamination, whereas cesium loss includes loss from failure particles, BISO particles, and particles with defective or missing SiC layers. Cesium depletion and retention is also dependent on temperatures; therefore, cesium loss is not a direct measurement of fuel failure. The agreement noted above between cesium loss and fuel failure is explained by the relatively large uncertainties of the measurements.

Table 4-10 and Fig. 4-11 make a comparison of the cesium mobility determined for stack 1 and for the composite scan done at Peach Bottom. There is surprisingly good agreement (17 out of 21 tests in Table 3-16) between the two measurement methods which were scanning different fractions of the element. As discussed earlier, between 2 and 3.5 fuel stacks plus the spine samples were covered by the composite scanning method. The presence of fuel failure was established for stack 1, 2, and 8 (see Section 4.5.2), as was the survival of stacks 4, 5, and 6 . One would expect that the composite measurements would reveal only about half as much cesium loss as the individual scan of stack 1. A likely explanation for the good agreement is additional major fuel failure for most of the spine sample fuel, which fully masks the presence of unfailed fuel. This is well demonstrated by the Peach Bottom scan for the location of the advanced fuel rods, which gives three consecutive spectra with nuclide 4-9 for the test. The quoted results have been corrected for the intact nuclide ratio for stack 1 . 
ratios as low as 0.06 ; this is nearly identical with the nuclide ratios determined from individual scans for the advanced fuel rods $(0.066$, 0.073, and 0.075). Similar observations can be made for the two large type 2 thermal stability spine samples: The Peach Bottom scan gives readings as low as 0.075 , and the individual measurements are 0.088 and 0.089. Anticipating U-233 as the primary fissile material in these spine samples, fuel failure of between $30 \%$ and $48 \%$ can be established for these five spine fuel measurements.

Resu1ts: Cerium Test: Table 4-11 and Fig. 4-12 test cerium for mobility by the two methods. Because of different cerium isotopes detected during the Peach Bottom and the fuel rod scan, a comparison of the normalized $\mathrm{Ce} / \mathrm{Zr}$ ratios was attempted. The two methods show agreement in 17 out of 21 tests. Because of the normalization, an absolute loss, if any, cannot be determined; only a relative distribution within the element can be analyzed against the mean value of 1.0 .

FTE-6

Fuel Element

Body 1 (bottom) *

Body 2 (center)

Body 3 (top)

Tota1 element

RMS

Test results (on fuel bodies)

Re1. Cerium Variation (\%) $\pm 2 \sigma(\%)$

P.B. EOL Scan 1

$$
+15.4 \pm 7.1
$$$$
0.2 \pm 6.1
$$$$
-15.2 \pm 6.1
$$$$
0.8^{* *} \pm 3.7
$$$$
\pm 18.2
$$

Significant
Fue1 Stack 1 Scan

$$
\begin{gathered}
+11.9 \pm 16.1 \\
-4.2 \pm 7.3 \\
-6.9 \pm 7.8 \\
-0.3^{* *} \pm 6.1 \\
\pm 17.7
\end{gathered}
$$

Not significant

Both tests indicate a cerium level below average for the top body and above average for the bottom body. By application of the axial variation

\footnotetext{
*In reference to the cesium test, fuel rod 1-1-2, which gave very inaccurate activity readings, and Peach Bottom scans at $789 \mathrm{~mm}$ and $1208 \mathrm{~mm}$, which did not have $\mathrm{Cs}-137$ reading, were included in the numerical cerium and ruthenium tests. This biases the body 1 results toward the higher end.

** Per definition, the mean variation is zero, and the difference is caused by the mode of stratification.
} 
test, (see Table 4-11), it was determined that no significance can be given to the fuel rod scans. The same conclusion can be drawn from the large uncertainty limits, which by far exceed the determined effect. The Peach Bottom results show significant cerium mobility. Since cerium mobility could not be verified by the group of individual fuel rod scans, possible explanations are that cerium migration occurred as the consequence of high fuel failure, high temperature release from BISO fuel in the spine samples, or high spine temperatures (Ce is volatile above $1300^{\circ} \mathrm{C}$, Ref. 4-5). Evaluation of the advanced fuel rods and the type 2 spine samples resulted in an approximate $25 \%$ loss for two of the advanced fuel rods, the third one showing a $37 \%$ accumulation of additional cerium; and a $6 \%$ to $8 \%$ loss was determined for the two thermal stability samples. For the latter location, the Peach Bottom data show $30 \%$ to $50 \%$ cerium depletion, compared with $12 \%$ depletion at the fission product release sample and advanced fuel rod locations in body 2 and with up to $30 \%$ plateout in body 1. A probable explanation is cerium loss from fuel rods, which had not been part of the described fuel rod analysis, in conjunction with the reported release from spine samples. This explanation is supported by the fact that two fuel rods having a $15 \%$ cerium depletion were found in stack 2 , bodies 2 and 3 .

The case described above is an example of the value of a second independent method of investigation. In spite of thorough examination and evaluation, the mobility of cerium in FTE-6 might have gone undetected except for the second method.

Test: Ruthenium Mobility: Table 4-11 and Fig. 4-13 present the mobility test for ruthenium using the same procedure. No difference between the two methods with the exception of 1 test out of 20 can be detected. The test results are:

FTE-6

Fue1 Element

Body 1 (bottom) *
Re1. Ruthenium Variation (\%) $\pm 2 \sigma \quad(\%)$

P.B. EOL Scan 1 Fuel Stack 1 Scan

$$
+7.0 \pm 4.5 \quad+4.3 \pm 14.5
$$

\footnotetext{
* See single-asterisked footnote on previous page.
} 
FTE-6

Fue1 E1 ement

Body 2 (center)

Body 3 (top)

Total element

RMS

Test results (on

fuel bodies)
Re1. Ruthenium Variation $(\%) \pm 2 \sigma(\%)$

$$
\text { P.B. EOL Scan } 1
$$

$-5.6 \pm 3.5$

$0 \pm 7.3$

$-2.0 \pm 2.5$

$-3.7 \pm 7.6$

$0.1^{*} \pm 2.5$

$0 \pm 5.7$

$\pm 9.1$

$\pm 16.0$

Significant

Not significant

The fuel rod scan does not show sigificance; however, the Peach Bottom scan does, as in the case of cerium mobility. Ruthenium depletion for the center body and for part of the top body and the accumulation of ruthenium in the bottom body and the top part of the top body are indicated in Fig. 4-13.

Because of a factor of 1.85 difference in the fission yields of Ru103 for U-235 and U-233, the unfailed Ru-103/Zr-95 ratio for U-233 fuel is only $56 \%$ of the nuclide ratio for U-235 fuel (Eq. 4-71). This would lead to misleading conclusions in the case of heterogeneous thorium loadings, because areas with large amounts of thorium fuel would have a lower inventory of ruthenium caused by lower fission yields and not by any 1oss. This can clearly be seen in Fig. 4-13 for the axial locations of the advanced fuel rods, fission product release samples, and thermal stability spine samples in body 2 and 3 .

The individual spine samples have been analyzed for the Ru-106 isotopes (Ru-103 had already decayed at the time of measurement). The unfailed $\mathrm{Ru}-106 / \mathrm{Zr}-95$ ratio for $\mathrm{U}-233$ is $71 \%$ of the nuclide ratio for the fuel composition of stack 5 (68\% for plain U-235 fuel). This is exactly what was measured for the thermal stability spine samples. The advanced fuel rods, however, still show ruthenium depletion between $10 \%$ and $30 \%$, when compared with the unfailed $\mathrm{Ru}-106 / \mathrm{Zr}-95$ ratio for U-233. The measured effect is masked by the $2 \sigma$ values of the progressed counting

* Per definition, the mean variation is zero, and the difference is caused by the mode of stratification. 
errors. But because all three fuel rods show the same effect, some ruthenium loss cannot be excluded for these exotic spine samples in FTE-6, which also showed a high cesium loss (between $41 \%$ and $48 \%$ ) and high cerium mobility (between $-26 \%$ and $+37 \%$, i.e., there was an increased cerium inventory for one of the three rods above the expected value for unfailed fue1).

Summary:

1. The Cs $-137 / \mathrm{Zr}-95$ ratio is a valid measure for determining cesium variation and cesium loss.

2. The preferred method to accurately determine actual cesium loss requires the presence of unfailed and nonreleasing fuel to establish the $\mathrm{Cs}-137 / \mathrm{Zr}-95$ unfailed ratio. This ratio then has to be corrected for the fuel composition of the tested fuel to determine cesium depletion.

3. Cesium depletion can be used to establish qualitatively the fuel failure for FTE-6, as has been proven by fission gas release measurements and metallography. For fuel failure above 5\%, the cesium method is more accurate than fission gas release measurements. For fuel composites, only the overall cesium loss can be determined. Fissile fuel failure and fertile fuel failure have been separated by different means. Determining a true failure fraction is further dependent on the amount of cesium retention.

4. It was concluded that FTE-6 exhibited cesium mobility, since it was clearly detectable from the Peach Bottom scans. This mobility agreed in magnitude with independent values established from fuel rod gamma scan analyses, and was highest for hot spot locations originated by spine samples, with heat generating primarily from bred fuel. The quantitative agreement between 
the two methods was related to the fact that a high fuel failure in the spine samples compensated for unfailed fuel scanned simultaneously.

5. Cerium mobility was detected from analysis of the composite scan, but not by the analysis of a group of fuel rods with failed particles. Cerium mobility was then found from individual analyses of spine samples, which included one case of cesium accumulation, and in fuel rods which did not participate in the original fuel rod test. This stresses the importance of independent methods of analysis.

6. By application of the same procedure, ruthenium variability was detected from the Peach Bottom scan, but not from individual fuel rod scans. This variability, however, was caused by significantly different fission yields for $U-235$ and $U-233$. Therefore, with the possible exception of three fertile fuel rods, it was concluded that there was ruthenium stability for FTE-6.

\subsubsection{Test for Identity of Cesium Profiles with Thermal Fluence.}

Hypothesis: $\quad$ Cs-137 is a long-life direct-yield isotope (half-life 30.1 years), the formation of which is proportional to the thermal fluence. Cs-134 is a medium-1ife isotope (ha1f-life 2.06 years), which is indirectly formed by neutron reaction steps. Therefore, its distribution is a quadratic form of the fluence, which can be linearized by ratioing Cs-134/ Cs-137. It is hypothesized that the Cs-137 and the Cs-134/Cs-137 profiles do not differ significantly and that the combination of both profiles is a good representation of the thermal fluence profile.

Test: Normalized Cs-137 and Cs-134/Cs-137 CPM profiles have been tested for identity and the arithmetical mean of both have been compared with predicted thermal fluence. 
Results: The results have already been described in Section 3.2.2. Data have been taken from 84 fuel rods from stack 3 (IRISO/BISO fuel) and stack 5 (TRISO/TRISO fuel); this data showed very little fuel failure and no detectable cesium depletion. The two profiles are graphically compared in Fig. 3-6(a), and the numerical data are presented in Table 4-12. Agreement is found in 17 of 20 tests. Only the measurements at the top and bottom ends of the element plus one additional location within body 3 fail the value agreement test. At the top end, the $\mathrm{Cs}-13 \mathrm{~T}$ profile shows a more pronounced reflector effect than the Cs-137/Cs-134 profile. Very smal1 counting errors for the low activities at the bottom end lead to the statistical disagreement statement; both profiles are actually in parallel at this part of the element.

The combination of the two profiles is compared in Fig. 3-6(b) with the GAUGE/FEVER derived thermal fluence. The numerical data of this test are included in Table 4-12. The following results can be stated.

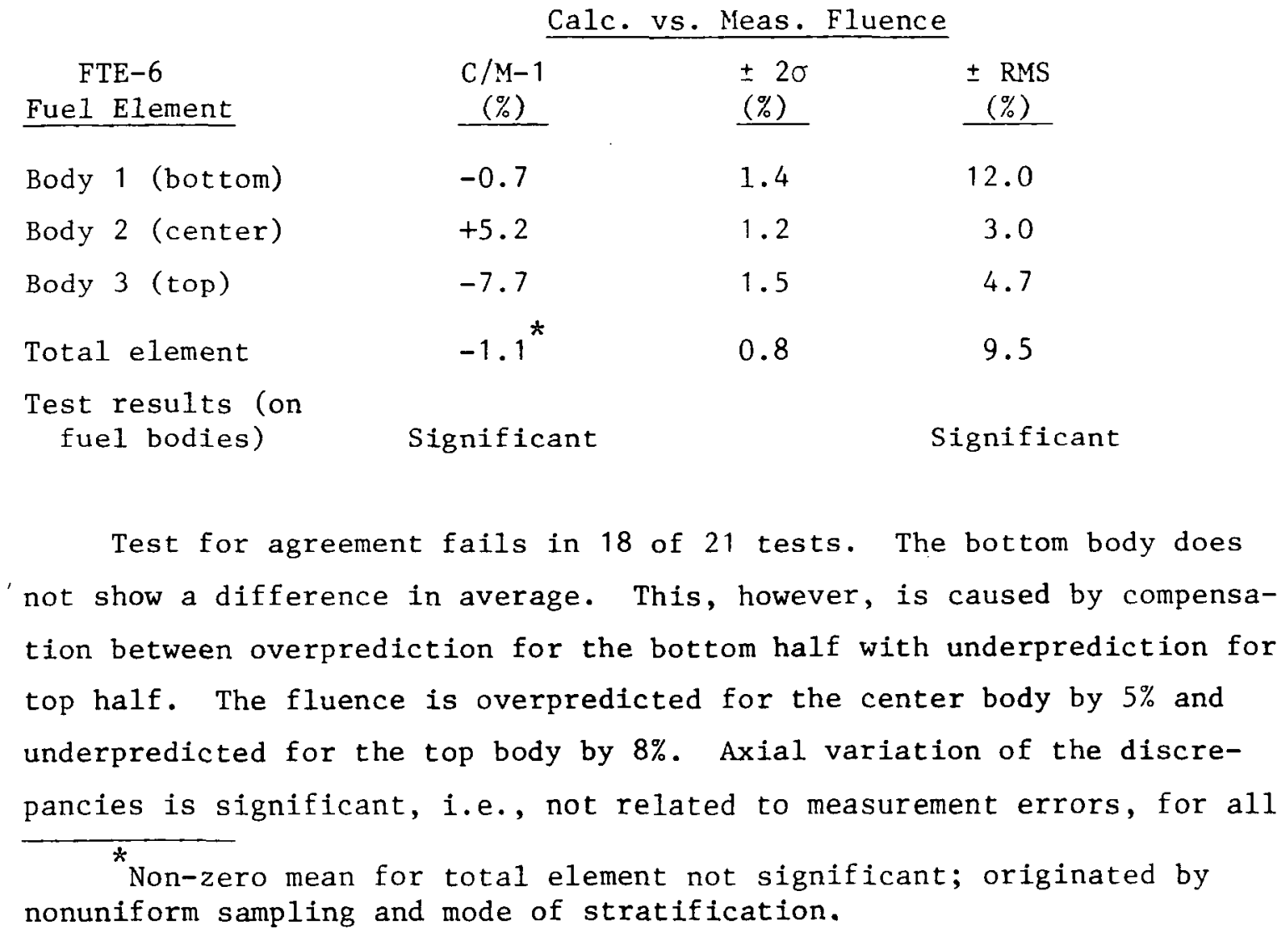


three fuel bodies as well as for the total element. The profile is flatter and slightly shifted toward the top end of the element as compared with the prediction. A location of flux depression, which had been identified earlier to coincide with a boronated graphite spine sample at the center of body 1 , shows up on the fluence profile. Undulations in relation to flux increases by fueled spine samples have not so strongly developed as for the EOL power profile (see Fig. 3-43). Some loading variations for the fuel rods are superimposed.

\section{Summary:}

1. Normalized $\mathrm{Cs}-137$ and $\mathrm{Cs}-134 / \mathrm{Cs}-137$ profiles are in close agreement and are good representations of the thermal fluence distribution of the experiment.

2. The actual fluence profile for FTE-6 is flatter (1.24 peak vs. 1.31 predicted) and slightly skewed toward the top end compared with the GAUGE/TREVER prediction. The uncertainties of the measured profile are small: $\pm 3.5 \%(2 \sigma)$. The comparison with the predicted profile, although judged statistically as significantly different, is still good in light of a generally stated uncertainty of $\pm 10 \%$ (probably $1 \sigma$ ) for nuclear predictions.

3. A fluence depression can be identified and related to a burnable poison spine location.

4. A possible way to improve thermal predictions is to use the measured fluence profiles as a correction term for thermal flux and power profiles during the irradiation history. The EOL power and thermal flux profile could be modified by the measured La or $\mathrm{Zr}$ profile. The first step should improve nuclear performance predictions for burnup and local temperature histories for diffusion spine samples. The latter step will be important in determining EOL shutdown stresses more accurately. 


\subsubsection{Fuel Stack Length Comparison.}

Hypothesis: Fuel stack length measurements for individual fuel bodies done by means of gamma scanning do not differ from metrology measurements. Fuel stack length determined for the composite fuel rod stacks does not differ from accumulated fuel stack length derived from individual fuel rod length measurements.

Test: Peach Bottom fuel stack length data, available from the singlechannel analyzer strip charts (see Fig. 4-1) are compared with PIE metrology data. Composite and accumulated length data are both derived from preirradiation and postirradiation metrology and are compared against each other.

Results: Results are tabulated in Table 4-13. The Peach Bottom and composite PIE stack length data agree within $\pm 0.4 \%$ or $2.5 \mathrm{~mm}$ over a length of $680 \mathrm{~mm}$. The progressed uncertainty is $\pm 1 \%(2 \sigma)$, which means that there is no significant difference. Comparisons are made on a fuel body and total element average basis. The comparison between composite and accumulated EOL stack length gives, on a fuel body average basis, +0.9 $\mathrm{mm}$ and $+1.4 \mathrm{~mm}$ larger stack length data $(0.13 \%$ to $0.20 \%)$ for the accumulated length with a progressed uncertainty of $0.4 \%(2 \sigma)$, which again puts the observed difference within the uncertainty of the measurements. An increase of $\pm 1.4 \mathrm{~mm}$ over 14 fuel rods would mean, if real, that on the average each fuel rod carries an out-of-squareness effect of $0.1 \mathrm{~mm}$ ( 4 mils), when measured between flat and parallel surfaces. This could be systematic end effects of the fuel rods, generated during the manufacturing process. Because of a systematic loading procedure into the fuel , bodies, these end effects could compensate each other.

The same test made for the BOL measurements reveals a fuel element average of $+2.2 \mathrm{~mm}(+0.3 \%)$ from 24 individual stack measurements with a progressed uncertainty of $\pm 0.1 \%(2 \sigma)$. This is significant and means a BOL out-of-squareness of $0.15 \mathrm{~mm} \pm 0.05 \mathrm{~mm}(2 \sigma)$ (0.006 in. $\pm 0.002 \mathrm{in.})$ 
per fuel rod. The EOL fuel element average is $0.08 \mathrm{~mm}(0.003 \mathrm{mil})$, which is within the measurement error of $\pm 0.12 \mathrm{~mm}(2 \sigma)$ ( $\pm 0.005 \mathrm{in.}$ ) as stated above.

\section{Summary:}

1. Fuel stack 1ength measurements made for FTE-6 at Peach Bottom via gamma scanning is verified by PIE metrology.

2. Comparison between composite and cumulative stack measurements indicates a possible out-of-squareness of $0.15 \mathrm{~mm}$ (0.006 in.) per fuel rod, which results in a shorter stack length because of systematic loading.

\subsubsection{Delayed Neutron Dose Measurements}

Neutron dose rates $(\mathrm{mrem} / \mathrm{hr}$ ) have been determined for the FTE- 6 element contained in the Hallam cask during May 23 and May 25, 1975, i.e., 207 days after shutdown, in order to determine neutron shielding requirements for HTGR shipping casks (Ref. 4-6). The measurements were repeated in the hot cell for the element in unshielded configuration prior to disassembly. The two neutron dose profiles are presented in Fig. 4-14. The $1 \sigma$ counting errors and the range of counted fuel length, i.e., length of the SNOOPY detector, are included.

Both profiles show a region of reduced neutron intensity for the upper part of the element. This is more pronounced for the measurements along the unshielded configuration. Scattering effects may have masked the effect for the shielded configuration (Hallam cask measurements). Some of the dose rates were measured repeatedly to test the source strength reduction in the unshielded case for significance. (These points can be recognized from smaller uncertainty bands.)

The in-situ positions of the FTE- 6 fuel bodies were determined afterward from PIE metrology and have been included in Fig. 4-14. The position 
of the Hallam cask was tilted during transportation of FTE-6 and at the time the measurements were taken. It is therefore concluded that the fuel bodies, fuel element, and inner shipping container were positioned toward the lower end of the Hallam cask, which coincided with the bottom end of FTE-6. The fuel element was free to move $23 \mathrm{~mm}(0.92 \mathrm{in}$.) within the inner container; the maximum axial movement of the fuel bodies was 51 mm (2.01 in.) within the fuel element, as determined from EOL PIE metrology. Bodies 1 and 2 carried two thermocouple assemblies; therefore, any axial movement other than that of the fuel element within the inner container during unloading of the Hallam cask and transfer into the hot cell were unlikely. Body 3, however, could have been moved up to $74 \mathrm{~mm}$ (2.93 in.) relative to a reference point at the bottom end of the container. The minimum and maximum ${ }^{*}$ possible axial displacements of the three fuel bodies are indicated in Fig. 3-40 for the unshielded case.

The area of significantly reduced neutron intensity coincides with the unfueled zone between bodies 2 and 3 , which could have ranged from one-half to three-quarters of the size of the detector length, i.e., $107 \mathrm{~mm}$ (4.23 in.) to $159 \mathrm{~mm}(6.24 \mathrm{in.)}$ versus $200 \mathrm{~mm}$ ( $8 \mathrm{in.)}$ for the unshielded configuration. The intersection between bodies 1 and 2 was also covered in part by the detector geometry, but without showing a significant reduction. This area was measured only once, compared with up to five measurements taken for some of the adjacent locations, which may explain the lack of the effect for this area.

Fuel gamma scanning along the element revealed fuel heterogeneity, which is traceable to three thorium-loaded advanced fuel rods in body 2 and to several loose particle capsules in the center spine sample location. The total amount of additional fissile material, added either by loading or by thorium conversion to U-233, and its contribution to delayed neutron activities is rated insignificant.

Summary: The reduced neutron intensity, observed for FTE-6, is rejected as being a depletion abnormality, but is explained rather as being caused by the absence of fuel between adjacent fuel bodies. This

* The maximum event is unlikely because of restrained bow (see Section $4.4 .2)$. 
was more pronounced for the unshielded configuration because of a lower scattering probability from adjacent fuel zones and because of a larger possible range of the unfueled zone between fuel bodies than for the shielded case.

\subsubsection{Nuclear Measurement Summary}

Gamma scanning of the assembled element at Peach Bottom and of individual fuel rods in the General Atomic hot cells provided information on power and fluence profiles, fuel failure, and fission product mobility in FTE-6. Highlights of the analysis are as follows:

- La-140, a short-lived isotope, and $\mathrm{Zr}-95$, a medium-lived isotope, had essentially the same activity profiles, which indicates that the power profile across the element did not change in the last 100 to 200 days. Comparison of the calculated and measured end-of-life profiles shows conclusively that fissile and fertile fuel as well as burnable poison experiments in the spine samples had an effect on local axial power distributions. The power profile determined from the fuel stack scans showed that the overall calculated profile was within the counting errors of the measured power profile. It was concluded that, to insure a proper thermal analysis, the effects of the spine sample in the local power must be considered.

- Using Cs-137/Zr-95, Ce/Zr-95 and Ru/Zr-95 isotope ratios, the mobility of cesium, cerium, and ruthenium was tested from the Peach Bottom and hot-cell gamma scans. There was an obvious loss of cesium from the fuel in stacks 1 and $2\left(\mathrm{UO}_{2}\right.$ TRISO $\mathrm{ThO}_{2}$ BISO). A $10 \%$ to $20 \%$ loss in two individual fuel rods from stacks 1 and 2 agreed well with a fission gas release measured at $20 \%$ of equivalent fuel failure. Cerium mobility was not detected in individual fuel scans but was significant in the Peach Bottom gamma scans of the assembled element. The detection of cerium mobility in the Peach Bottom scans was 
attributed to a loss of cerium in several spine sample experiments. Ruthenium did not show significant mobility in either the Peach Bottom or fue1 rod gamma scans.

- Using normalized Cs-137 and Cs-134/Cs-137 activity profiles, a comparison was made to the GAUGE/FEVER calculated thermal fluence. The measured fluence profile was within the assumed $\pm 10 \%$ uncertainty on the calculated case, and a measured fluence depression was identified with a burnable poison spine sample location.

- The fuel stack length measurement determined from single-channe1 gamma-scan strip charts was confirmed by the PIE metrology.

- Maximum neutron dose rates of FTE-6 after 207 days decay were between 0.8 and $0.9 \mathrm{mrem} / \mathrm{hr}$ in both a shielded and unshielded geometry. A neutron depression in the top of the element was attributed to the unfueled zone between bodies 2 and 3 .

\subsection{DISASSEMBLY AND VISUAL INSPECTION}

All disassembly operations were performed according to Ref. 4-7. The helium-filled aluminum canister containing FTE-6 was removed from the Hallam cask and injected into the high-level cell without incident. The canister was girdle-cut approximately $4.8 \mathrm{~mm}$ (3/16 in.) deep and $1219 \mathrm{~mm}$ (4 ft) from the top end, and the piece was removed. The element was pulled out until clear of the canister, thereby exposing the thermocouple contacts. The remaining portion of the canister was discarded. At this point, the identity of the element was checked and the test element was photographed (Fig. 4-15). A composite of the total element is shown in Fig. 4-16. Thermocouple measurements were then taken and recorded (Table 4-14). No preirradiation thermocouple measurements were available; however, the postirradiation measurements seemed to be in line with preirradiation measurements from other Peach Bottom instrumented test elements. 
In order to section the fuel element, it was supported horizontally by a series of V-rollers, which facilitated horizontal and rotational movement. A special extension drill was inserted through the purge gas inlet hole in the upper reflector, and a hole was drilled through the porous graphite plug. While the bodies were held down, the upper reflector was removed by plunge-cutting through the sleeve at a point about $51 \mathrm{~mm}$ (2 in.) below the sleeve reflector joint. This section was stored for later gamma scanning. A plunge cut was then made $51 \mathrm{~mm}$ ( 2 in.) from the bottom of the element and the small section was discarded. The sleeve was then girdle-cut approximately $9.4 \mathrm{~mm}$ (0.37 in.) deep without hitting the lower reflector. This was done to remove the thermocouples; however, there was difficulty in the removal, and the wire thermocouple had to be forcibly removed. Six inches of the outer sheathing was stripped from the end of the thermocouple and was lodged in the center body.

The intact element had appeared normal, but difficulty was encountered when the body removal process began. The fuel bodies could not be moved with the push rod per the normal procedure. Two 76-mm (3-in.) sections of the sleeve were removed from either end of the sleeve containing the bodies. After an unsuccessful attempt to free the bodies by twisting, it was decided to cut the sleeve longitudinally to recover the bodies. It was later found by metrology that a significant bow of the graphite body had occurred that exceeded the clearance within the graphite sleeve and which had therefore led to the restraint bow causing the unloading difficulty of the fuel bodies.

After the bodies were cut from the sleeve, they were photographed and visually inspected with the Kollmorgan periscope at high magnification (Fig. 4-17). No cracks or abnormalities were found on any of the surfaces of the fuel bodies.

Following visual inspection, bow measurements, and dimensional measurements, the fuel bodies were placed in a special holding fixture to facilitate the removal of the center samples. A hole was drilled through the bottom sample hole plug and a push rod was inserted. The upper sample 
hole plug was unscrewed, and the distances from the top of the spine samples to the edge of the holes were measured. The measurements are compared with preirradiation measurements in Table 4-15. The spine samples were pushed out one at a time, identified, and placed in a pan. These operations were carried out over a special pan that would catch any loose fuel particles from the drilled holes; none were found. No problems were encountered during the removal of spine samples. The spine samples are described in Table 2-6.

Following removal of the spine samples, holes were drilled in the bottom of the fuel bodies to enable the fuel to be pushed out using the special discharge fixture. The graphite plugs capping the fuel holes were easily removed. The distances from the top of the fuel rods to the edge of the fuel holes were measured. These measurements, as well as fuel stack lengths, are compared with preirradiation measurements in Table 4-16. Data may be used to make fine adjustments to fuel rod and spine sample locations, if required. Strain data determined from plenum measurement vary considerably from the values deduced from the composite spine sample stack measurements in Table 2-6, indicating a somewhat different in-situ spine sample arrangement than the actual stack length.

The fuel bodies were then placed in the mechanical push device with Dillon load gauges ranging from 0 to $113.4 \mathrm{~kg}$; the force required to start movement did not exceed $13.6 \mathrm{~kg}$ (see Table 4-17). The fuel rods were pushed onto the trough and visually examined (see Section 4.5.1). The stack length was measured (see Table 4-16).

After the fuel was removed, one 12.7-mm (0.5-in.) graphite slice was cut approximately $76.2-\mathrm{mm}$ ( 3 in.) from the top of body 2 for fission product migration analysis. (See Section 4.5.5.)

\subsection{DIMENSIONAL CHANGE EVALUATION}

The dimensional measurements made prior to and after irradiation of FTE- 6 have been rigorously analyzed by application of the methods presented 
in Section 4.1 and by the error analysis summary given in Table 4-1. The methods have been computerized (Refs. 4-1, 4-2, and 4-3) and the results of the analysis are taken from Refs. 3-8 and 4-8. Wherever possible, measured strain data are compared with HTGR design code predictions. The fuel body bow measurements and preliminary strain results have been reported earlier (Ref. 4-9) and are included in this report in modified form. Stress levels have been assessed for the observed fuel body deformations.

The evaluation was done to verify the design of an HTGR fuel element. The applied method is comparison of observed and predicted performance of the graphite and fue1 components.

Where applicable, comparison has been made with sister elements having same design but different exposure, i.e., FTE-3, FTE-4 and FTE-5.

\subsubsection{Fue1 Element and Sleeve}

The overall length of the element was measured with a steel rule and confirmed by traversing its length with a saw head, the horizontal movement of which can be determined within $0.8 \mathrm{~mm}(1 / 32 \mathrm{in.})$. Results are reported in Table 4-18. A length change comparison with other test elements can be made:

\begin{tabular}{|c|c|c|c|}
\hline $\begin{array}{l}\text { Total } \\
\text { e1 Element }\end{array}$ & $\begin{array}{c}\text { Strain } \\
(\%) \\
\end{array}$ & $\begin{array}{c}\text { Accuracy } \\
(\%) \\
\end{array}$ & $\begin{array}{c}\text { Exposure } \\
\text { (EFPD) } \\
\end{array}$ \\
\hline FTE-3 & +0.07 & \pm 0.04 & 133 \\
\hline FTE-4 & +0.35 & \pm 0.04 & 449 \\
\hline FTE-18 & -0.19 & \pm 0.04 & 512 \\
\hline FTE-6 & -0.62 & \pm 0.04 & 645 \\
\hline FTE- 5 & -0.39 & \pm 0.04 & 897 \\
\hline
\end{tabular}

Length changes are small. There appears to be a slight increase in length for the first 500 EFPD of exposure, which then becomes negative. FTE-5 length change indicates a second turnaround. 
Tab1e 4-18 also reports weight measurements for the three fuel bodies. Weight changes range from $-8 \mathrm{~g}$ to $+10 \mathrm{~g}$. The following is a comparison with measurements made for other test elements:

\begin{tabular}{lccc} 
& \multicolumn{2}{c}{ Re1. Fuel Body Weight Changes (\%) } \\
Fuel Element & Body 1 & $\underline{\text { Body 2 }}$ & Body 3 \\
FTE-3 & +0.20 & +6.38 & +0.18 \\
FTE-4 & -0.18 & -0.08 & +0.05 \\
FTE-6 & -0.16 & +0.19 & -0.03 \\
FTE-5 & 0 & -1.57 & -1.44
\end{tabular}

Results are inconsistent and range from $+314 \mathrm{~g}$ gain (FTE-3) to -77.5 $\mathrm{g}$ loss (FTE-5). Weights of $5000 \mathrm{~g}$ could be determined with accuracies of $\pm 1 \mathrm{~g}$, resulting in an error of $\pm 0.04 \%$ for the relative weight changes. Weight gain of $314 \mathrm{~g}$ lacks any explanation, and weight losses of $-77.4 \mathrm{~g}$ appear much too high, especially in light of the lack of corrosion found for the graphite bodies. These weight measurements are concluded to be unreliable.

Sleeve outside diameters (Table 4-19) were determined with calibrated snap-on dial indicator gauges; inside diameters were measured from two slices taken from the upper and lower ends of the sleeve. The longitudinal slitting operation to free the fuel bodies did limit the number of diameter measurements.

No preirradiation measurements were made for the sleeve except for quality inspection for manufacturing tolerances. However, some archive material has been retrieved and measured repeatedly; from this material representative preirradiation dimensions and uncertainty statements have been derived (Ref. 4-9). FTE-4 was analyzed prior to this exercise, and the analysis (Ref. 2-2) suggested a bias of $0.03 \mathrm{~mm}$ to $0.10 \mathrm{~mm}(0.0013$ in. to $0.0040 \mathrm{in.)}$ for the preirradiation nominal value, which interestingly enough was found to be $0.046 \mathrm{~mm}(0.0018 \mathrm{in.})$ for the outside diameter. 
Figure 4-18 presents a comparison between measured and TREVER-ca1culated sleeve strain. The observed agreement is reasonable for the limited amount of measurements available. Without the correction made for the outer sleeve diameter, the strain would have been over-predicted.

\section{Conc1usions:}

1. Total length of fuel elements shows a slight increase for the first part of the irradiation cycle, then a decrease, with a possible second turnaround occurring near the end of the Peach Bottom core 2 cycle.

2. Fuel body weight changes are inconclusive, and the reliability of weight measurements is questioned.

3. An earlier suspected bias for the nominal preirradiation outer diameter of the sleeve was verified through measurements taken from archive material.

4. With the described correction, sleeve strain data obtained for FTE-6 are in reasonable agreement with TREVER predictions.

\subsubsection{Fue1 Bodies}

After separation of the fuel bodies from the graphite sleeve via longitudinal sectioning, the fuel bodies were placed on a graphite surface plate and measured for bow with a special dial gauge device (Fig. 4-19). Extensive bow was observed for all three bodies. The bow was measured across each of the eight fuel holes to find the azimuthal location of maximum bow. The worst cases were observed in body 1 along holes 3 and 7 , in body 2 for holes 3 and 7 and holes 4 and 8 , and in body 3 along holes 4 and 8 . The bow was up to $1.1 \mathrm{~mm}$ (0.45 in.) over the 790-mm-1ong (31.1in.) fuel bodies as compared with an 0.3-mm (0.012-in.) diametrical nominal BOL clearance between the fuel body and sleeve. A sinusoidal bowing was observed along the plane of holes 2 and 6 . 
Figures 4-20 through 4-22 illustrate the bow across opposite holes of each body. The bow is similar for opposite holes. Results of the bow measurements are summarized in Table 4-20, which gives maximum arc-to-bow displacement and relative location along the fuel body. Maximum bows are observed at about one-third of the length from either end of the fuel body. Accuracy of the measurements is within $\pm 0.025 \mathrm{~mm}$ ( $\pm 0.001 \mathrm{in}$.$) .$ Some axial variation due to handling may be the explanation for reported discontinuities in the bow lines. Measurements will be repeated during destructive stress examination (Ref. 3-27) and tested for reproducibility.

Radial gamma scanning was done on a qualitative basis at one axial location for body 2 , which indicated power variations between fuel holes (Fig. 4-23) from the Zr-95 CPM activity. Quantitative GAUGE fuel loading calculations are given in Table 3-5, which reveal variation in the EOL fissile loading between $+14 \%$ (holes 1 and 2) and $-8 \%$ (holes 7 and 8 ) based on the average fuel rod loading. This means a ratio of 1.24 between maximum and minimum fuel loading, which happened to occur between holes 1 and 8 . The variation is caused by different thorium loadings in each of the four fuel blends, which resulted in different U-233 conversions.

The given variations are fuel element averages, which change from body to body. The changes result from a superposition of axial variations from the fuel rods themselves and from local perturbations from spine samples with additional heat generation as described in Section 4.2. These power and temperature gradients are the most likely explanations for the deformation of the elements.

In Table 4-21, an evaluation of the fuel body bow is attempted. By application of the fuel body-sleeve gap information of Section 4.4.3, the following values can be derived for the EOL configuration of the fuel bodies in the sleeve: 


\begin{tabular}{|c|c|c|c|c|}
\hline $\begin{array}{c}\text { FTE-6 } \\
\text { Fuel Element }\end{array}$ & $\begin{array}{c}\text { Location } \\
\text { (Holes) } \\
\end{array}$ & $\begin{array}{l}\text { Max. } \\
\text { Bow } \\
(\mathrm{mm}) \\
\end{array}$ & $\begin{array}{c}\text { Diametrical } \\
\text { Gap } \\
(\mathrm{mm})\end{array}$ & $\begin{array}{c}\text { Restraint } \\
\text { Bow } \\
(\mathrm{mm}) \\
\end{array}$ \\
\hline Body 1 Mean & $3 \& 7$ & 1.10 & 0.24 & 0.86 \\
\hline S.D. ${ }^{*}(1 \sigma)$ & & \pm 0.05 & \pm 0.04 & \pm 0.06 \\
\hline Body 2 Mean & $3 \& 7, \quad 4 \& 8$ & 0.74 & 0.35 & 0.39 \\
\hline S.D. ${ }^{*}(1 \sigma)$ & & \pm 0.08 & \pm 0.08 & \pm 0.11 \\
\hline Body 3 Mean & $4 \& 8$ & 0.49 & 0.22 & 0.27 \\
\hline S.D. ${ }^{*}(1 \sigma)$ & & \pm 0.14 & \pm 0.09 & \pm 0.17 \\
\hline
\end{tabular}

The center of maximum bow shifts slightly by about 45 degrees from body 1 to body 3 . This may be caused by varying azimuthal power variations along the fuel element (which should be highest for the center body with the highest Th-to-U-233 conversion) in addition to axial power variations plus systematic variations in the material properties within and along the fuel bodies. A diametrical macroflux gradient as explanation for the excessive bow is less likely because of the slim design of the element.

Body 1 saw the highest bow, which was followed by bodies 2 and 3 . Maximum bow was determined as an absolute average between the two opposite measurements taken. For body 2, two planes qualified for maximum bow, so al1 four measurements have been used. The averaging was believed to be justified by the fact that an ideal bowed beam should show the same absolute deflection, when measured from either side. Variations of the fuel body diameter because of varying strain along the length are superimposed onto the measurements and should cancel out through the averaging. The standard deviations of these averages have then been progressed to determine significance.

The maximum bow values have then been reduced by the diametrical clearance of the fuel bodies within the sleeve. The remaining amount of bow was at least partially restrained by the sleeve. Under the assumption

* Sample standard deviations $s_{x}$ are progressed rather than the standard deviation on the mean in order to cover local effects along the length of one fuel body. 
of no deflection of the sleeve, the maximum possible fibre stress was calculated for the fuel bodies by assuming a three-point bending situation:

$$
\sigma_{\max 1}=\frac{12 \mathrm{rf}_{1} \mathrm{E}}{1^{2}},
$$

where $r=$ fuel body radius,

$$
\begin{aligned}
\mathrm{E}_{1} & =\text { maximum restraint bow, } \\
\mathrm{E} & =\text { elastic modulus (at EOL), } \\
1 & =\text { fuel body length. }
\end{aligned}
$$

A beam loaded with a constant bending momentum will bend circular with a maximum arc-to-bow displacement (sagitta) of

$$
\mathrm{f}_{2}=\frac{\mathrm{M} 1^{2}}{8 \mathrm{EI}}
$$

(four-point bending formula with cord between two inner points),

$$
\text { where } \begin{aligned}
\sigma & =\frac{r \cdot M}{I}, \\
r & =\text { distance from neutron axis to fibre with stress } \sigma(y=r), \\
I & =\text { momentum of inertia. }
\end{aligned}
$$

The maximum stress will be

$$
\sigma_{\max 2}=\frac{8 r \mathrm{f}_{2} \mathrm{E}}{1^{2}} .
$$

\footnotetext{
* No measurements of the straightness of the sleeve were made prior to disassembly, because corrosion rather than bowing was suspected of causing the high resistance of the fuel bodies against unloading. Unsuccessful retrieval of the FTE- 6 thermocouples (the niobium shields broke off with part of the thermocouples left in the body) supported this assumption. However, no corrosion was found for the fuel bodies. The loss of the thermocouple was probably caused by a graphite reaction with the shield because of high spine temperatures in FTE- 6 .
} 
This value represents the maximum fibre stress which would exist if a constant bending moment along the fuel body would be restrained from deforming the body.

The following maximum fibre stresses can be quoted from Table 4-21. Stress factors were calculated by applying the proper corrections for increase of the UTS during irradiation. Nominal UTS values have been used as described in Section 3.5.2.

\begin{tabular}{|c|c|c|c|c|c|}
\hline $\begin{array}{c}\text { FTE-6 } \\
\text { Fue1 Element }\end{array}$ & $\begin{array}{r}\text { UTS } \\
\text { o } \\
(\mathrm{MPa}) \\
\end{array}$ & $\begin{array}{c}\text { Restraint Bow } \\
\text { Max. Stress } \\
\text { (MPa) }\end{array}$ & $\begin{array}{c}\text { 3-point } \\
\text { Stress-Strength } \\
\text { Ratio } \\
(-) \\
\end{array}$ & $\begin{array}{c}\text { Residual } \\
\text { Bow } \\
\text { Max. Stress } \\
\text { (MPa) } \\
\end{array}$ & $\begin{array}{c}\text { 4-point } \\
\text { Stress-Strength } \\
\text { Ratio } \\
(-) \\
\end{array}$ \\
\hline Body 1 (bottom) & 16.51 & 11.33 & 0.45 & 9.68 & 0.38 \\
\hline Variation $(1 \sigma)$ & & \pm 0.79 & \pm 0.03 & \pm 0.44 & \pm 0.02 \\
\hline Body 2 (center) & 16.51 & 4.80 & 0.20 & 6.08 & 0.25 \\
\hline Variation $(1 \sigma)$ & & \pm 1.35 & \pm 0.06 & \pm 0.66 & \pm 0.03 \\
\hline Body 3 (top) & 16.51 & 3.29 & 0.14 & 3.94 & 0.16 \\
\hline Variation $(1 \sigma)$ & & \pm 2.07 & \pm 0.09 & \pm 1.14 & \pm 0.05 \\
\hline
\end{tabular}

Uncertainty statements are determined by linear progression of the $1 \sigma$ metrology variability from the dimensional measurements information only.

The highest stress situation is calculated for the in-situ stress of body 1, restrained in the sleeve. The stress leve1 is $45 \%$ of the EOL UTS (the BOL UTS would give a stress leve1 of $69 \%$ ). The maximum bending stress calculation indicates that a stress level of $38 \%$ of the EOL UTS ( $59 \%$ of BOL UTS) is released through the deformation of the fuel body. The stress situations in bodies 2 and 3 are less pronounced, with $20 \%$ and $14 \%$ stress levels for in-situ restraint bow and $25 \%$ and $16 \%$ stress levels released through deformation. The uncertainties, as determined from the variation of the gap and bow measurements, are small for body 1 , where stresses are high, and became very large for body 3 , where stresses are lowest. This means that the higher stress levels are reasonably well established. 
The stress levels, as determined from bow measurements, indicate that the structural deformation of the FTE-6 fuel bodies was subcritical, because none of the considered cases reaches the UTS of the irradiated materia1.

However, the predicted residual stresses at EOL shutdown were $128 \%$ and 58\% (body 1), 102\% and 58\% (body 2) and 104\% and 52\% (body 3) for inplane and axial directions, respectively (see Section 3.5.4). These predictions are independent of the observed bow of the fuel bodies; the true stress situation, however, is a combination of both. More severe shutdown stress situations existed during the irradiation cycle, and were even more stringent because of restraint bowing of the fuel bodies.

Weight measurements of the fuel bodies are reported in Section 4.4.1.

The dimensional changes for longitudinal and transverse directions of the fuel bodies were measured; results are presented in Tables 4-22 through 4-25. It should be noted that these data are based on Eqs. 4-10 and 4-11, which follow a different analysis function than the stratification procedure with Eq. 4-15 and following, which is applicable to Table 4-26 and other comparison tabulations between measurements and predictions. The following results can be stated (the error statement contains axial variation as well as measurement uncertainties):

Graphite Strain Measurements

\begin{tabular}{|c|c|c|c|c|c|c|c|c|}
\hline $\begin{array}{c}\text { FTE-6 } \\
\text { Fue1 Element } \\
\end{array}$ & $\begin{array}{c}\text { Axial } \\
(\%) \\
\end{array}$ & $\begin{array}{r}2 \sigma \\
\pm \quad(\%) \\
\end{array}$ & $\begin{array}{l}\text { Radial } \\
(\%) \\
\end{array}$ & \pm & $\begin{array}{l}2 \sigma \\
(\%) \\
\end{array}$ & $\begin{array}{c}\text { Anistropy } \\
(\%) \\
\end{array}$ & \pm & $\begin{array}{l}2 \sigma \\
(\%) \\
\end{array}$ \\
\hline Body 1 (bottom) & -0.12 & 0.08 & -0.13 & & 0.04 & 94 & & 67 \\
\hline Body 2 (center) & -0.70 & 0.06 & -0.32 & & 0.05 & 222 & & 38 \\
\hline Body 3 (top) & -0.54 & 0.03 & -0.27 & & 0.05 & 200 & & 41 \\
\hline Total element & -0.45 & 0.22 & -0.24 & & 0.04 & 192 & & 98 \\
\hline
\end{tabular}

For comparison, the results from FTE-3, 4, 5, and 6 are given for the element averages in increasing order of irradiation exposure: 
Graphite Strain Measurements

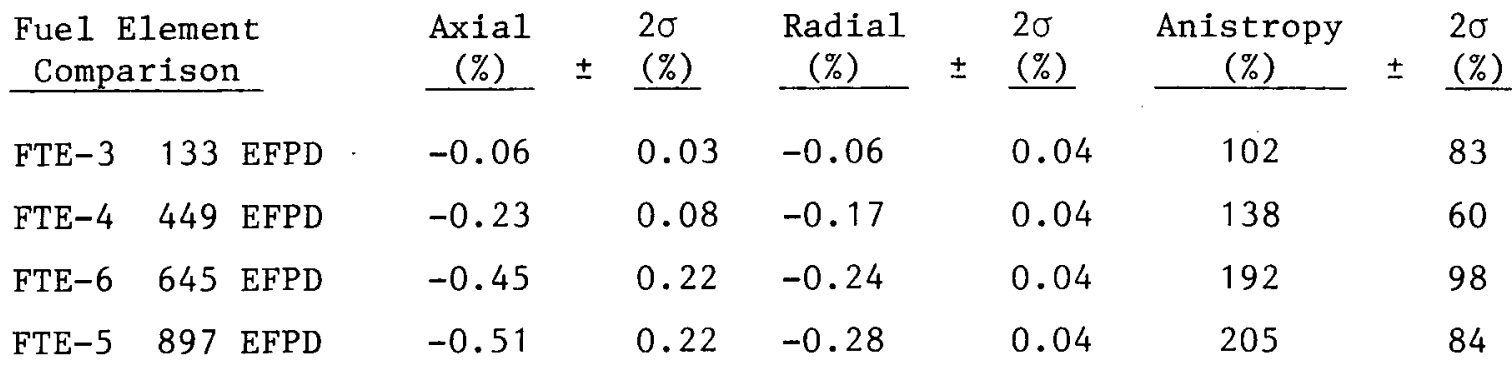

All three effects are steadily increasing with exposure. All uncertainty statements are true statistical statements (Eq. 4-10) and include al1 taken measurements and detected uncertainties for the fuel elements. Sampling was about uniform from element to element, and all elements consisted of the same design (three fuel bodies) and followed the same measurement procedure (diameter measurements were made in 51-mm (2-in.) intervals) and were exposed to similar flux distributions (at least two fuel element positions away from control rods).

An arbitrary rate can be determined for these irradiation parameters as strain percentage change over 100 EFPD exposure. Anisotropy change has been taken as the difference against 100\%, i.e., the BOL value.

\section{Fue1 Element Comparison}

FTE-3 133 EFPD

FTE-4 449 EFPD

FTE-6 645 EFPD

FTE-5 897 EFPD
Measured Graphite Strain Rate ( $\triangle \% / 100$ EFPD)

Axia1 Radia1 Anistropy -100

$-0.045$

$-0.045$

1.5

$-0.051$

$-0.038$

8.5

$-0.070$

$-0.037$

14.3

$-0.058$

11.7

Axial strain rate and differential anisotropy rate apparently have passed a peak situation with FTE-6; radial strain rate is continuously decreasing with exposure.

A comparison between measured and calculated radial fuel body strain data has been made in Table 4-30 for FTE-6. The data have been tested 
for significance using the statistical test methods described in Section 4.1. The following results can be reported:

Calculated Vs. Measured Radial Strain

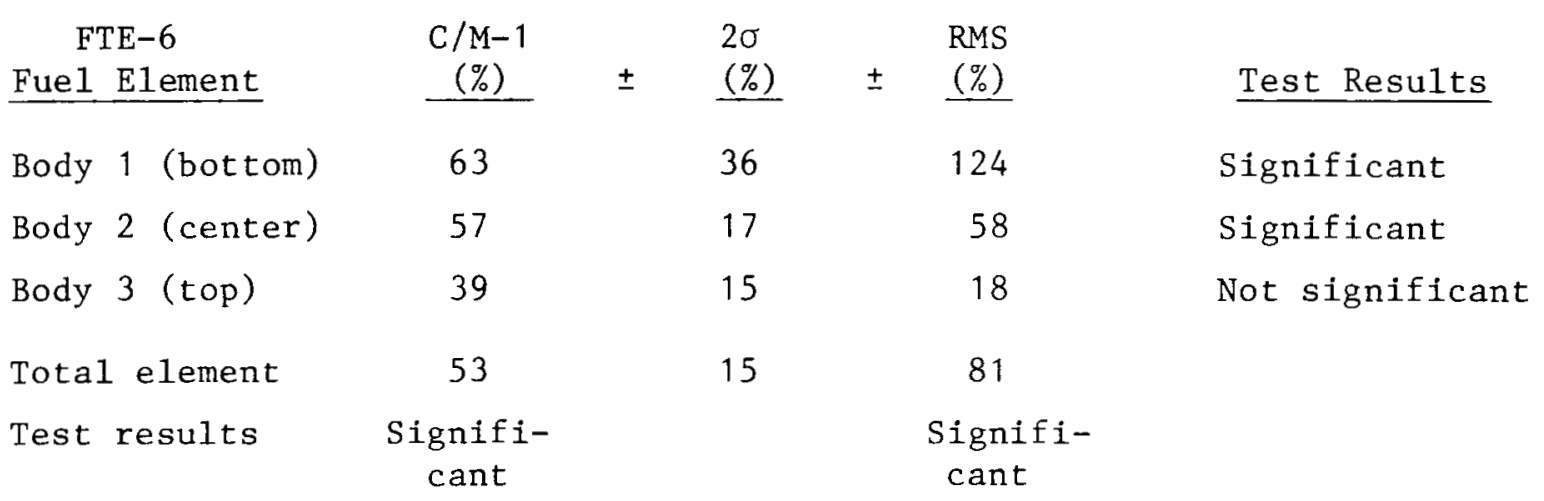

The $2 \sigma$ statement includes progressed measurement errors only (Eq. 4-12); the axial variability is given by the RMS statement (Eq. 4-24). The results clearly indicate that the strain is overpredicted. The largest bias of $63 \% \pm 36 \%(2 \sigma)$ appears for body 1 , the lowest for body 3. All three statistical test methods indicate significance of the disagreement, with one exception for body 3: The axial variation is not significantly different from the predicted one. The student $t$ test for individual samples is passed in 26 out of 54 tests, with 14 agreements within the uncertainty of the measurement being found for body 3 alone. The body average, however, still indicates disagreement.

A look at the visual display of the calculated and measured strain in Fig. 4-24 reveals that for three-quarters of fuel body 3 the measurements are in reasonable agreement with the prediction and that only for the last quarter (bottom side of the body) a significant decrease in strain, probably caused by end effects, leads to a deviation from the predicted performance. The applied prediction method does not include strain corrections for end effects. For body 2, the strain is overpredicted and end effects are again visible; for body 1, the curvature of the measured strain is the opposite of that predicted. This is coupled with large overprediction of the strain for three quarters and underpredictions at the bottom side. Large uncertainties in the strain data at the low 
temperature range is the explanation for the mismatch. This deviation and curvature inversion is also found for FTE-4. For comparison with FTE-6, the results of FTE- 4 are given:

\section{Calculated vs. Measured Radial Graphite Strain}

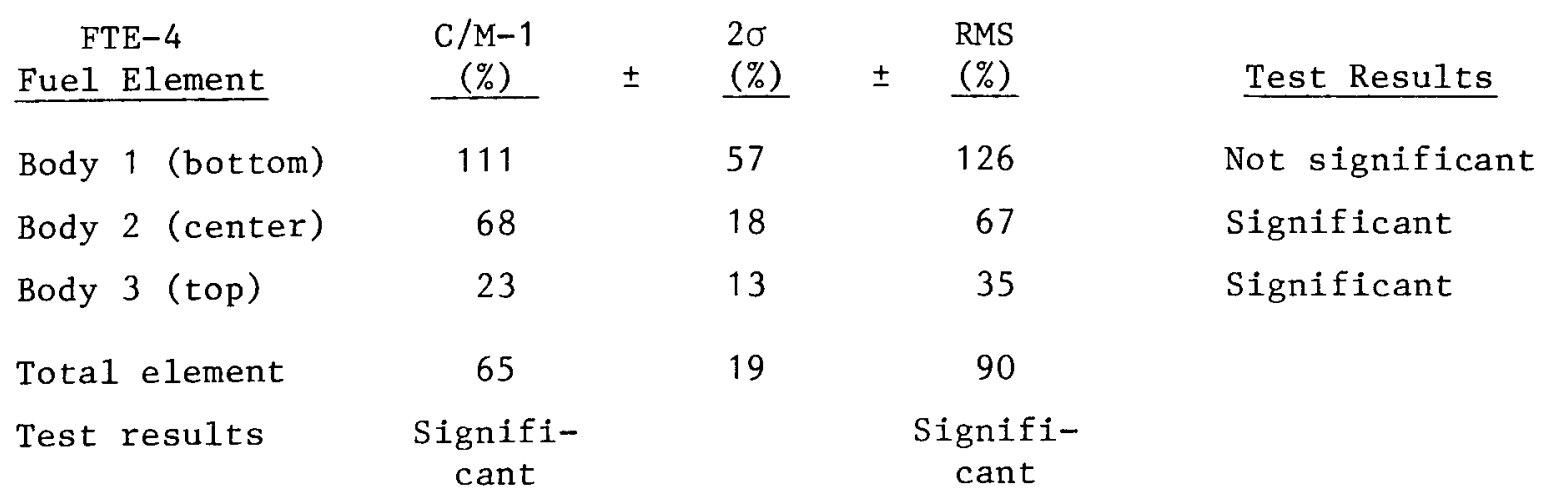

The same characteristic results as for FTE- 6 can be drawn from the FTE-4 comparison: very large overpredictions for body 1 , which decrease for bodies 2 and 3 . (The 1arge measurement uncertainties and the small absolute amount of strain for body 1 indicate insignificance between axial variation and measurement error; however, student t test and $x^{2}$ test clearly indicate disagreement.) The bias for overprediction of the strain is even slightly larger for the total element as compared with FTE-6. The top body strain for FTE-4 is in better agreement than for FTE-6. The bias is again generated because of inability of the model to predict end effects.

Comparisons between measured and predicted strain are not available for FTE-3 and FTE-5.

Based on the FTE- 4 and FTE-6 results, it can be said that the radial strain performance is overpredicted by about $60 \%$ on an element average.

\section{Conclusions:}

1. FTE-6 experienced a large degree of bowing for the fuel bodies, which was partially restrained in the graphite sleeve. This 
generated stresses up to $11.3 \mathrm{MPa}$, with stress levels of up to $45 \%$ of the EOL UTS. The bowing represents a maximum bending stress leve1 of $38 \%$ or $9.7 \mathrm{MPa}$. Body 1 experienced the highest deformation and stresses. Presented data are assessed for ambient temperature. They would be lower at power operation because of a reduction in bow (the hotter and more bowed zones will expand more) and because of an increase in UTS and elastic modulus with temperature.

2. The true stress situation within the fuel bodies is a combination of the restrained in-situ stresses and residual stresses, due to radial temperature gradients and differential shrinkage. The residual stresses have been predicted for EOL shutdown as high as $128 \%$ of the EOL UTS in the in-plane direction for body 1 and $58 \%$ in the axial direction for body 2 . Residual shutdown stresses during the reactor history were calculated to have reached $153 \%$ and $73 \%$ in the in-plane and the axial directions for body 2, which were further increased by the restrained bow of the fuel body.

3. The residual bow of the fuel bodies is attributed to azimuthal power variations within the element because of heterogeneous thorium loadings and consequently different Th-to-U-233 conversion. A shift in the center of the bow is observed, which indicates additional variables as material properties of the graphite and axial power variations.

4. The strain measurements for the three fuel bodies have been compared with average values determined for sister elements with different exposures. The observed quantitative amounts of axial and radial bow and anisotropy (on a fuel body basis) show a steady increase with exposure. The strain rates based on EFPD exposure reveal that the axial strain rate and the differential anisotropy rate have reached a peak situation with 
FTE-6: both were found to be lower for experiments with higher and lower exposure. The radial strain rate was found to decrease continuously with exposure.

5. Calculated and measured radial strain data have been compared for FTE-6 and FTE-4. In both elements an overprediction by $60 \%$ was found on an element average basis. For the bottom body the highest bias and an inversion of the strain curvature against predictions were found. The closest agreements were seen for the upper three-quarters of body 3 . The majority of the disagreement is attributed to thermal and nuclear end effects, resulting in much lower strain values than predicted except for the bottom end of the bottom body, where strains were underpredicted. The present model for predicting design performance of Peach Bottom FTE fuel bodies does not properly predict end effects and needs to be improved. Since axial strain predictions have not yet been carried out, a comparison with the measured axial strain behavior and fuel body anisotropy cannot be made.

\subsubsection{Sleeve-Fuel Body Gaps}

The sleeve and fuel body dimensions have been used to determine the heat transfer gap between the two components. Diameter measurements of the actual sleeve prior to irradiation were not available, only nominal values and tolerances. Archive material from the same manufacturing batch has been measured instead (Ref. 4-9) and the mean value and uncertainty information have been used as preirradiation measurements. Preirradiation dimensions of the fuel bodies were documented in the as-built assembly drawings (see Table 2-1); no variation was reported for these measurements.

Postirradiation measurements were ample for the fuel bodies, but again very limited for the sleeve. Diameter measurements were made at six different axial levels, two of which were inside diameter measurements, taken for the top and bottom end. The sleeve had to be slit longitudinally 
for removal of the fuel bodies, which prohibited further measurements.

The numerical data are given in Table 4-27. The following gaps can be stated:

\begin{tabular}{|c|c|c|c|c|c|c|c|}
\hline \multirow[b]{2}{*}{$\begin{array}{c}\text { FTE-6 } \\
\text { Fue1 Element } \\
\end{array}$} & \multicolumn{2}{|c|}{ BOL } & \multicolumn{2}{|c|}{ EOL } & \multirow[b]{2}{*}{$\begin{array}{c}\text { BOL / EOL-1 } \\
(\%)\end{array}$} & \multirow[b]{2}{*}{ \pm} & \multirow[b]{2}{*}{$\begin{array}{l}\text { S.D. } \\
(\%)\end{array}$} \\
\hline & $\begin{array}{l}\text { Gap } \\
(\mathrm{mm})\end{array}$ & $\begin{array}{r}\text { S.D. } \\
+\quad(\mathrm{mm}) \\
\end{array}$ & $\begin{array}{l}\text { Gap } \\
(\mathrm{mm})\end{array}$ & $\begin{array}{l}\text { S.D. } \\
(\mathrm{mm})\end{array}$ & & & \\
\hline Body 1 (bottom) & 0.155 & 0.012 & 0.121 & 0.028 & -22 & & 19 \\
\hline Body 2 (center) & 0.155 & 0.012 & 0.176 & 0.038 & 13 & & 26 \\
\hline Body 3 (top) & 0.155 & 0.012 & 0.109 & 0.044 & -29 & & 29 \\
\hline Total element & 0.155 & 0.012 & 0.140 & 0.053 & -9 & & 35 \\
\hline
\end{tabular}

For comparison, the FTE-4 values for the total element are given. (FTE-3 and FTE-5 values are not available.)

FTE-4

Total element $0.155 \pm 0.005 \quad 0.176 \pm 0.042+13 \pm 27$

In all cases because of a lack of multiple measurements for the sleeve, sample standard deviations are quoted that primarily consist of the variability found along the fuel bodies. This axial variability can be progressed when considering the uncertainties for restraint bow of the fuel bodies in the sleeve or for heat transfer calculations, although there is little impact for the latter uncertainties for the variability quoted. Restrained bow information are affected.

For FTE-6, there is a slight decrease (9\%) in gap dimensions during irradiation when averaged for the whole element; the opposite was found for FTE-4, with a $13 \%$ increase.

A comparison of the measured gaps versus TREVER-predicted gaps is made in Fig. 4-25. Only four of the six measurements cover the range of the fuel bodies. Two of these measurements are as predicted and two are lower than predicted by $40 \%$ to $50 \%$. When looking at the trend of the data, 
overprediction is the conclusion. Agreement was found to be somewhat better for FTE-4.

\section{Conclusions:}

The only conclusion that can be drawn from the very limited number of measurements is that measured and predicted gaps do not agree. The overprediction is in line with the earlier conclusion of overprediction of the fuel body strain (by $60 \%$ ). Looking backward, more sleeve metrology would have been justified. But residual bow of the fuel bodies was not anticipated or known at the time measurements were taken. In addition, no future HTGR application is considered for the sleeve graphite material.

A general conclusion for irradiation testing is the importance of multiple measurements, even for components believed to be unimportant. Modern data acquisition and data storage systems will make this much easier to achieve than it was in earlier years, when manual tools and manual data compilation were used.

\subsubsection{Fue1 Rods}

Radial and axial dimensions were determined for all 336 fuel rods prior to irradiation. The measurements consisted of one length and three diameter measurements at three axial locations per fuel rod. During PIE, 267 fuel rods were measured, with one length and six diameter measurements being taken at three axial locations and with an arbitrary 0-deg and 90-deg angle per fuel rod. An average sample standard deviation of 0.048 $\mathrm{mm}$ ( $1.9 \mathrm{mils})$ was assessed from FTE-4 length measurements, which consisted of two measurements per rod. This "default" value was used as uncertainty statement for al1 FTE-6 fuel rod length measurements in the STAT code (Ref. 4-1). Because STAT is based on explicit statistical formulations (Eq. 4-10), a fictitious number of $n \geq 2$ measurements had to be introduced.*

*Equation 4-28 presents a different treatment. A more precise average for FTE-4 fuel rod length variation is $0.046 \mathrm{~mm}(1.8 \mathrm{mils})$ as given in Table 4-2, which will be used in future error analyses together with Eq. 4-28 because Eq. 4-10 gives the lower uncertainty statement. 
The selected number of fictitious measurements was 2 .

Figures 4-26 through 4-29 present the measured and predicted fuel rod strain for holes 1 and 2, 3 and 4, 5 and 6 , and 7 and 8, respectively. The numerical comparison between measurement and prediction is made in Tables 4-27 through 4-31 for each of the four fuel blends. The strain summary for each blend is given on a fuel stack average basis in Tables 4-32 through 4-35. Table 4-35 also contains the average for the total element. The anisotropy test is made for each blend in Figs. 4-30 through 4-33.

This documentation contains all fuel rod strain information obtained in FTE-6 PIE in a form sufficient for data retrieval: Table 4-27 contains the numerical value of the average (between axial and radial) strain for a fuel rod from hole 1 or 2 (eventually average for both holes), the anisotropy of which can be found in Fig. 4-30, which then allows separation into axial and radial strain. Table 4-33 contains dimension and strain data on a fuel stack average basis, which allows conclusions about actual dimensions.

The TREVER-predicted strain data are based on FSV and LHTGR code subroutines, which calculate the fuel rod dimensional changes as the sum of the irradiation deformation of the different subcomponents within the fuel rod.

The pattern is the same for all three elements for each of the four fuel blends. The fuel blend of holes 1 and 2 always has the highest shrinkage, being followed by holes 7 and 8 , holes 3 and 4 , and holes 5 and 6 , with holes 5 and 6 having the lowest strain because of al1-TRISO fuel. The shrinkage clearly correlates with the amount of BISO fuel in the fuel rods. The ratio between TRISO and BISO fuel varies for a single blend from fuel element to fuel element (because of adjustments to fissile and fertile material loading); the variation, however, is so small that the relative ranking between blends does not change. 
The isotropic behavior of the fuel rods (see Figs. 4-30 through 4-33 and Tables 4-33 through 4-35) is established for the first three blends in all three test elements, all of which have the $100 \%$, i.e., isotropy, within their $2 \sigma$ band of the determined value. This means that anisotropic strain cannot be proven, although with two exceptions, all measured anisotropy values are slightly below $100 \%$. This indicates more pronounced shrinkage into radial direction.

The blend in holes 7 and 8 , however, demonstrates anisotropic strain performance in all three test elements. The radial strain definitely exceeds the axial strain. A different manufacturing process may be the cause, which gave some preorientation to the fuel rod.

Table 4-27 through 4-30 allow a comparison between calculated and measured strain. FTE-4 results have been added.

The following results can be quoted from Tables 4-32 through 4-34 for the fuel rod performance. For comparison, the data from FTE-3 and FTE-4 have been added:

\begin{tabular}{|c|c|c|c|c|c|c|c|}
\hline $\begin{array}{c}\text { FTE-6 } \\
\text { Fuel Rods }\end{array}$ & (645 EFPD $)$ & $\begin{array}{c}\text { Axial } \\
\text { Strain } \\
(\%) \\
\end{array}$ & $\begin{array}{r}2 \sigma \\
+\quad(\%) \\
\end{array}$ & $\begin{array}{c}\text { Radial } \\
\text { Strain } \\
(\%) \\
\end{array}$ & $\begin{array}{r}2 \sigma \\
\pm \quad(\%) \\
\end{array}$ & $\begin{array}{c}\text { Axial/ } \\
\text { Radial } \\
\text { Anisotropy } \\
(\%) \\
\end{array}$ & $\begin{array}{r}2 \sigma \\
\pm \quad(\%) \\
\end{array}$ \\
\hline Holes $1 \& 2$ & $\begin{array}{l}\text { TRISO/BISO } \\
26.2: 73.8\end{array}$ & -2.66 & 0.08 & -2.88 & 0.25 & 92.1 & 8.4 \\
\hline Holes $3 \& 4$ & $\begin{array}{l}\text { TRISO/BISO } \\
92.5: 7.5\end{array}$ & -1.86 & 0.07 & -1.97 & 0.25 & 94.2 & 12.3 \\
\hline Holes $5 \& 6$ & $\begin{array}{l}\text { TRISO/TRISO } \\
97.7: 7.3\end{array}$ & -1.75 & 0.07 & -1.86 & 0.24 & 94.2 & 12.8 \\
\hline Holes $7 \& 8$ & $\begin{array}{l}\text { TRISO/BISO } \\
80.2: 19.8\end{array}$ & -1.96 & 0.05 & -2.27 & 0.24 & 86.1 & 10.1 \\
\hline $\begin{array}{l}\text { Total ele- } \\
\text { ment }\end{array}$ & & -2.06 & 0.05 & -2.25 & 0.24 & 91.7 & 10.1 \\
\hline
\end{tabular}




\begin{tabular}{|c|c|c|c|c|c|c|c|c|}
\hline $\begin{array}{c}\text { FTE-4 } \\
\text { Fue1 Rods } \\
\end{array}$ & & (443 EFPD) & $\begin{array}{l}\text { Axial } \\
\text { Strain } \\
(\%) \\
\end{array}$ & $\begin{array}{r}2 \sigma \\
+\quad(\%) \\
\end{array}$ & $\begin{array}{c}\text { Radial } \\
\text { Strain } \\
(\%) \\
\end{array}$ & $\begin{array}{r}2 \sigma \\
\pm \quad(\%) \\
\end{array}$ & $\begin{array}{c}\text { Axial/ } \\
\text { Radial } \\
\text { Anisotropy } \\
(\%) \\
\end{array}$ & $\begin{aligned} & 2 \sigma \\
& \pm \quad(\%) \\
&\end{aligned}$ \\
\hline Holes $1 \& 2$ & 2 & $\begin{array}{l}\text { TRISO/BISO } \\
22.4: 77.6\end{array}$ & -2.57 & 0.11 & -2.63 & 0.25 & 97.7 & 10.2 \\
\hline Holes $3 \&$ & 4 & $\begin{array}{l}\text { TRISO/BISO } \\
78.1: 21.9\end{array}$ & -1.62 & 0.17 & -1.86 & 0.27 & 87.5 & 15.7 \\
\hline Holes $5 \& 6$ & 6 & $\begin{array}{l}\text { TRISO/TRISO } \\
78.6: 21.4\end{array}$ & -1.48 & 0.09 & -1.47 & 0.26 & 100.5 & 18.5 \\
\hline Holes $7 \& 8$ & 8 & $\begin{array}{l}\text { TRISO/BISO } \\
64.1: 35.9\end{array}$ & -1.73 & 0.16 & -2.10 & 0.27 & 82.5 & 13.2 \\
\hline $\begin{array}{l}\text { Total ele- } \\
\text { ment }\end{array}$ & & & -1.89 & 0.11 & -2.02 & 0.25 & 93.7 & 12.8 \\
\hline $\begin{array}{c}\text { FTE-3 } \\
\text { Fue1 Rods } \\
\end{array}$ & & $\underline{(133 \text { EFPD })}$ & & & & & & \\
\hline Holes $1 \&$ & 2 & $\begin{array}{l}\text { TRISO/BISO } \\
24.2: 75.8\end{array}$ & -1.58 & 0.14 & -1.55 & 0.26 & 102.3 & 19.0 \\
\hline Holes $3 \&$ & 4 & $\begin{array}{l}\text { TRISO/BISO } \\
85.4: 14.16\end{array}$ & -0.96 & 0.12 & -1.06 & 0.25 & 91.3 & 24.6 \\
\hline Holes $5 \&$ & 6 & $\begin{array}{l}\text { TRISO/TRISO } \\
85.8: 14.2\end{array}$ & -0.84 & 0.11 & -0.90 & 0.25 & 93.4 & 28.7 \\
\hline Holes $7 \&$ & 8 & $\begin{array}{l}\text { TRISO/BISO } \\
74.0: 26.0\end{array}$ & -0.97 & 0.09 & -1.26 & 0.25 & 76.9 & 17.0 \\
\hline $\begin{array}{l}\text { Total ele- } \\
\text { ment }\end{array}$ & & & -1.13 & 0.07 & -1.23 & 0.25 & 91.6 & 19.2 \\
\hline
\end{tabular}

Calculated vs. Measured Fuel Rod Strain

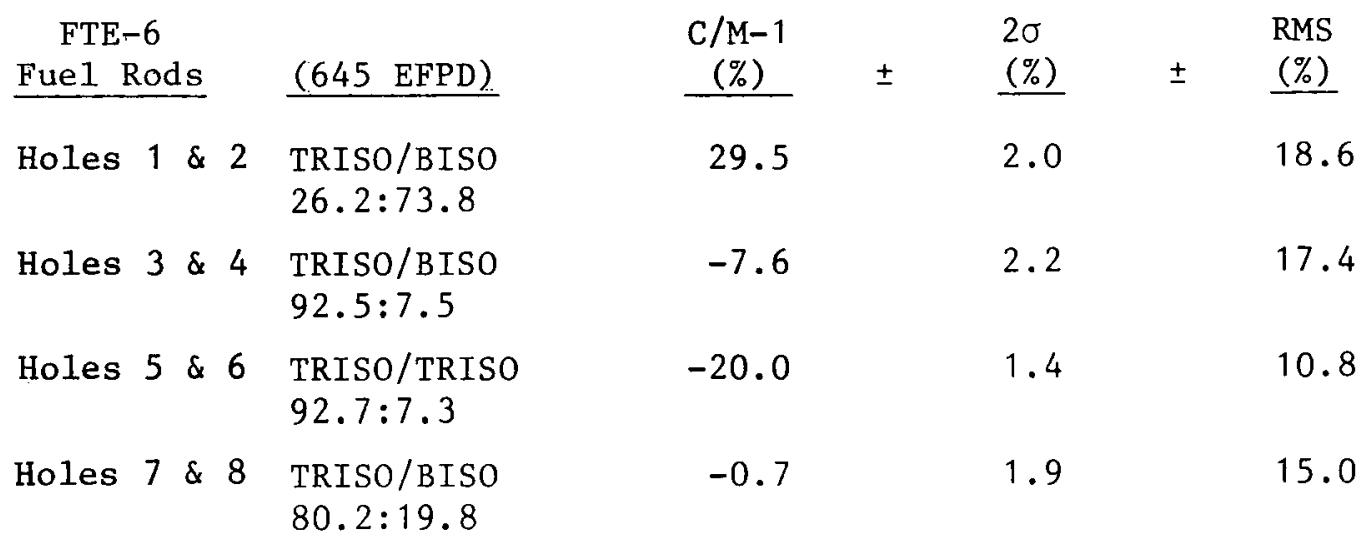


Calculated vs. Measured Fuel Rod Strain

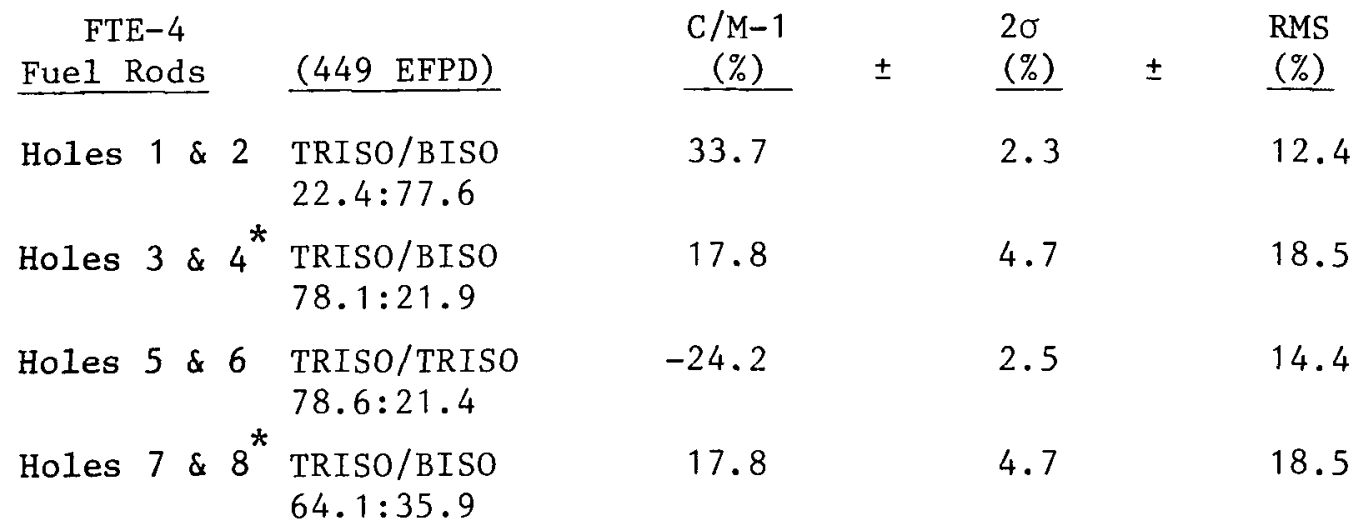

In all cases, with the exception of the fuel blend of holes 7 and 8 in FTE-6 the strain was either significantly overpredicted or underpredicted. The fuel element of holes 1 and 2 is apparently overpredicted by about $30 \%$ for both test elements. The all-TRISO fuel blend in holes 5 and 6 is underpredicted by about $20 \%$ for both test elements. The fuel blend of holes 3 and 4 is underpredicted for FTE- 6 and overpredicted for FTE-4. The fuel blend from holes 7 and 8 is properly predicted for FTE-6 and strong1y overpredicted for FTE-4.

The axial variation RMS indicates significance for both elements. Therefore, a comparison between fuel bodies is made. (The error and axial variation statements have been omitted for simplicity.)

Bias $(\mathrm{C} / \mathrm{M}-1)$

Calculated vs. Measured Fue1 Rod Strain

FTE-6

Fuel Rods per Fuel Body

Holes $1 \& 2$ TRISO/BISO

Holes $3 \& 4$ TRISO/BISO

Holes $5 \& 6$ TRISO/TRISO

Holes $7 \& 8$ TRISO/BISO

$\begin{array}{r}\text { Body } 1 \\ (\%) \\ \hline\end{array}$

50.0

18.1

$-9.6$

15.0

Body 2

(\%)

26.3

$-13.7$

$-18.2$

$-8.7$
Body 3

$(\%)$

12.2

$-19.7$

$-32.2$

$-11.6$
Total Element

29.5

$-7.6$

$-20.0$

$-0.7$

\footnotetext{
* Combined because of similar loading.
} 
Bias $(\mathrm{C} / \mathrm{M}-1)$

Calculated vs. Measured Fuel Rod Strain

\begin{tabular}{|c|c|c|c|c|}
\hline $\begin{array}{l}\text { FTE- } 4 \\
\text { Fuel Rods per Fuel Body }\end{array}$ & $\begin{array}{r}\text { Body } 1 \\
(\%) \\
\end{array}$ & $\begin{array}{r}\text { Body } 2 \\
(\%) \\
\end{array}$ & $\begin{array}{c}\text { Body } 3 \\
(\%) \\
\end{array}$ & $\begin{array}{c}\text { Total Element } \\
(\%)\end{array}$ \\
\hline Holes $1 \& 2$ TRISO/BISO & 46.8 & 32.9 & 23.1 & 33.7 \\
\hline Holes $3 \& 4$ TRISO/BISO & 38.3 & 12.5 & 2.7 & 17.8 \\
\hline Holes $5 \& 6$ TRISO/TRISO & -4.7 & -26.2 & -37.2 & -24.2 \\
\hline Holes $7 \& 8$ TRISO/BISO & 38.3 & 12.5 & 2.7 & 17.8 \\
\hline
\end{tabular}

The trend is clear in all cases: The effect is declining from bottom to top of the element, with a large positive bias becoming smaller or even negative and a negative bias becomes even more negative when moving from bottom to top. This indicates a systematic axial effect for generating the bias. A check of the plots (Figs. 4-26 through 4-29) shows a very flat distribution of the strain in the center of the element, which indicates a lack of proper temperature compensation for the strain correlations used. In nearly all cases the strain distribution within a fuel body shows end effects which again support a strong temperature correlation for the strain. TREVER predictions do not include proper adjustments for axial heat conductivity at the fuel body ends. (TREVER is a onedimensional code and can only be modified by predetermined form factors.)

\section{Conclusions:}

1. The strain measurements made for FTE-3, 4, and 6 clearly demonstrate the fluence dependence and composition dependence, which is primarily related to the amount of BISO fuel within the fuel rods.

2. It was concluded that there was isotropic performance for three of the four fuel blends tested for all three test elements within the $2 \sigma$ limits of the measurements. A slight preference towards a $5 \%$ to $20 \%$ larger radial strain versus axial strain is possible. 
3. One specific fuel blend showed anisotropic behavior in all three fuel experiments. Significantly different matrix characteristics are concluded to have given the fuel rod or one of the subcomponents a preorientation.

4. No fluence or temperature dependence can be detected for the anisotropy. The distribution is random within a test element and from test element to test element.

5. The applied fuel rod strain correlations are rejected as being representative for the fluence and temperature regime covered by the test elements. A stronger temperature dependence and probably a less accelerated fluence dependence for low fluence values are suggested.

\subsubsection{Fue1 Body - Fuel Rod Gaps}

The fuel-body/fuel-rod gaps have been calculated from actual fuel hole measurements at BOL, which have been prorated to EOL dimensions along the more precise fuel body dimensional measurements. The radial dimensional measurements of the fuel rods at BOL and EOL have been applied. The radial gap is primarily controlled by the fuel rod strain, which is an order of magnitude larger than the graphite strain.

Tables 4-36 through 4-39 present the results for each of the four fuel blends. A visual display of the measured and calculated gaps is shown in Figs. 4-34 through 4-37. Being composed of two sets of strain calculations, i.e., the graphite strain and fuel rod strain, both of which have been found to be improperly matched by the model, not much agreement with the measurements is expected.

The (indirectly) measured gaps range from $0.15 \mathrm{~mm}$ to $0.33 \mathrm{~mm}$ (6 mils to $13 \mathrm{mils}$ ) and the experienced underprediction and overpredictions range between $-0.03 \mathrm{~mm}$ to $0.09 \mathrm{~mm}(-1.1 \mathrm{mils}$ to $3.5 \mathrm{mils})$, which indicates 
reasonable agreement. The gap distributions of holes 1 and 2 and of holes 3 and 4 are consistently overpredicted. For the other holes, both overpredictions and underpredictions occur, i.e., overpredictions at the bottom end and underpredictions at the top end of the element.

\section{Conclusions:}

1. The gaps between fuel holes and fuel rods are predicted close enough to the measured gaps to guarantee reasonable fuel temperatures.

2. Gap contours will be a good measure to establish improvements for the graphite and fuel rod strain predictions.

\subsubsection{Dimensiona1 Performance Summary}

The dimensional analysis of FTE- 6 gave several conclusive results, the most impressive of which was the high stress levels found for the three fuel bodies of the element.

Stress was assessed for the fuel bodies' residual bows that resulted from relaxation of internal stresses. These residual bows represent maximum bending stresses of up to $38 \% \pm 2 \%(1 \sigma)$ of the EOL ultimate strength of the material. The idealized assumption used was circular bow under constant (momentum) bending moment.

Part of the bow was restrained by the sleeve, which could have reached $11.3 \mathrm{MPa}$ maximum tensile stresses. This represents a stress level of $45 \% \pm 3 \%(1 \sigma)$ at EOL shutdown. Material strength was measured prior to irradiation during manufacturing and was corrected for strength increase during irradiation.

Residual stress calculations revealed tensile stress-strength ratios up to $128 \%$ for EOL shutdown. Highest shutdown stresses during reactor operation reached $153 \%$ of the ultimate tensile strength (compensated for 
fluence and irradiation temperature). These residual stresses must be superimposed on the restraint bow of the fuel bodies. This makes FTE-6 an impressive stress experiment, since no graphite damage was found during destructive examination. Because of the absence of graphite failure, it is concluded that there was conservatism in the stress predictions and applied failure criteria.

Residual bow of the fuel bodies is attributed to azimuthal power variations between fuel blends. The fuel blends varied by a factor of 1.24 for the maximum-to-minimum power ratio at a radial plane. Gamma scan analysis revealed large axial power variations along the element because of U-233 and U-235 fissions in spine samples.

Evaluation of material strain data lead to the conclusion that based on element average the radial graphite strains are overpredicted by $53 \%$, partially because of lack of modeling end effects by the TREVER predictions.

Fuel rod strains are overpredicted by $30 \%$ for a blend containing $75 \%$ BISO fuel and are underpredicted by $20 \%$ for an a11-TRISO fuel blend. These prediction variations are related to lack of proper temperature and fluence correlation for the regime seen by the fuel test elements.

No fluence or temperature dependence was found for the anisotropy of the fuel rod strain. Three out of four blends were found to show consistently an isotropic behavior in FTE-3, FTE-4, and FTE-6. One blend showed consistent anisotropy with an axial-to-radial strain ratio of 0.8 . Manufacturing effects are believed to have caused this difference in behavior.

The predicted gaps between fuel rods and graphite body were found to be in reasonable agreement with the measurements because of the fact that the initial gap was relatively large compared with the fuel and graphite changes. The gap contour along the element was primarily controlled by 
the fuel rod strain, which is an order of magnitude greater than that for the graphite.

The predicted gap between graphite and sleeve was found to be overpredicted when compared with the measured evidence. This is in line with the overprediction of the fuel body strain. The small number of sleeve measurements limits the conclusiveness of this information.

Sleeve dimensional changes were found to be in agreement with the TREVER predictions. An earlier suspected bias for nominal preirradiation of the outer diameter of the sleeve was verified through measurements taken from archive material.

Fuel body weight changes were found to be inconclusive, and the reliability of weight comparisons over long time periods is questioned.

Total fuel element length showed a slight increase for the first part of the irradiation cycle, then decreased, and a possible turnaround was indicated near the end of the Peach Bottom Core 2 cycle. This is concluded from the length changes observed for FTE-3, FTE-4, FTE-6, and FTE-5.

\subsection{FUEL ROD IRRADIATION PERFORMANCE}

\subsubsection{Visual Examination}

After unloading the fuel rods from the graphite fuel bodies, composite Kollmorgan photographs were taken of 12 complete fuel stacks including various fuel blends from all three fuel bodies. These photographs are presented in Figs. 4-39 through 4-49. Random matrix end cap cracking and debonding were evident in all the fuel blends and in all three fuel bodies. Surface cracks and soot pockmarks (surface fuel failure) were more evident in the center region of the fuel element, which correlates with the higher temperatures and neutron fluences in this region. The 
majority of the soot marks caused by surface fuel failure were seen in rods from stacks 1 and 2 , which contained the $\mathrm{UO}_{2}$ TRISO particles that had high fuel failure (see section 4.5 .3 on fuel rod metallography). Unloading damage was also evident on some rods. This was caused by debonded fuel particles caught between the fuel rod and fuel hole periphery during unloading, which caused long striations and damaged fuel particles at the surface.

Fue1 rods from stacks 1 and 2 also showed significant bow of up to approximately $0.5 \mathrm{~mm}$ (0.020 in.) in their length (see Table 4-40 and Figs. 4-50 through 4-53). This was felt to be caused by inhomogeneous fuel loadings in the TRISO $\mathrm{UO}_{2}$ / BISO $\mathrm{ThO}_{2}$ blend, which would cause inhomogeneous fuel rod shrinkage. Single-channel gamma scans in Fig. 4-54 show that most of the bowed rods had an axial fuel inhomogeneity.

Several representative fuel rods which were chosen from body 2 for fission gas release analysis were examined and photographed with the stereomicroscope. Stereophotographs confirm the Kollmorgan photography results. Eight fuel rods are shown in Figs. 4-55 through 4-62 and a summary of the amount of surface fuel failure is shown in Table 4-41. In all cases there was cracking of the matrix end cap region of the rods and some small surface cracking. The soot marks left by the surface failed fuel are obvious in the stereoexamination of rods $2-1-7$ and $2-2-7$ (Figs. 4-55 and 4-56).

\subsubsection{Fission Gas Release Measurements}

Eight fuel rods were measured for $\mathrm{Kr}-85 \mathrm{~m}$ fission gas release at $1100^{\circ} \mathrm{C}$ in the TRIGA irradiation facility. Results are presented in Table 4-42. Using nominal heavy metal contamination and preirradiation fission gas release measurements the postirradiation fission gas release measurements were corrected using the techniques outlined in Ref. 4-10. Except for the fuel rods containing $\mathrm{UO}_{2} \mathrm{TRISO} / \mathrm{ThO}_{2}$ BISO particles, the fission gas release measurements indicate between $0.0 \%$ to $4.5 \%$ equivalent 
$\mathrm{U}-235$ exposed, if the release of $\mathrm{Kr}-85 \mathrm{~m}$ at $1100^{\circ} \mathrm{C}\left(\mathrm{R} / \mathrm{B}_{\mathrm{f}}\right)$ is assumed to be $0.0053 \pm 0.0027(1 \sigma)$ (Ref. 4-11). In rods 2-3-7, 2-6-7, and 2-7-8 the magnitude of fuel failure did not correlate well with the lower metallographically measured fuel failure. It is believed that this discrepancy is the result of the thorium-bearing fuel in these fuel rods hydrolyzing upon failure, which causes a higher $R^{\prime} B_{f}$ (Ref. 4-11).

Fuel rods $2-1-7$ and $2-2-7 \quad\left(\mathrm{UO}_{2}\right.$ TRISO/ThO 2 BISO) had fuel failures measured by fission gas release of $8<21<53$ and $9<23<58$ (2б), respectively, assuming an $\mathrm{R}_{\mathrm{f}} \mathrm{B}_{\mathrm{f}}$ of $5.3 \pm 2.7 \times 10^{-3}(1 \sigma)$. This compares with the $8 \%$ to $17 \%$ pressure vessel failure measured by metallography on these fuel rods (see Section 4.5.4). The error limits on failure from the fission gas release measurements are calculated assuming a log normal distribution in the fission gas release error. The $95 \%$ confidence limits on a fission gas release failure measurement $\mathrm{P}$ are $0.334 \mathrm{P}<\mathrm{P}<2.99$ (Ref. 4-12).

\subsubsection{Fue1 Rod Metallography}

Four fuel rods from the center of body 2, which had the highest fast neutron fluence and temperature, i.e., $2.84 \times 10^{25} \mathrm{n} / \mathrm{m}^{2}$ (E $\left.>29 \mathrm{fJ}\right)_{\mathrm{HTGR}}$ and $1250^{\circ} \mathrm{C}$, were chosen for metallography. These fuel rods represented each fuel blend tested in FTE-6. A summary of the postirradiation examination of these fuel rods is given in Table 4-41. A radial metallographic cross section and a representative photomicrograph of the matrix of each of these fuel rods are shown in Figs. 4-63 through 4-67. Representative photomicrography of the fuel particles in each of these fuel rods is shown in Figs. 4-68 through 4-76. A single-channel gamma scan plot of most of the fuel rods examined is shown in Fig. 4-77.

All the fuel particles examined in these fuel rods had $<1 \%$ pressure vessel failure except for the $\mathrm{UO}_{2}$ TRISO fissile particles in rods 2-1-7. These particles had approximately $20 \%$ to $30 \%$ pressure vesse1 failure due to migration of the $\mathrm{UO}_{2}$ kernels through the IPyC to the SiC inner surface and to subsequent mixed fission product attack and failure of the SiC 
coatings (Figs. 4-68, 4-69, and 4-70). In FTE-3, which had a low peak thermal neutron exposure $\left[\sim 0.6 \times 10^{25} \mathrm{n} / \mathrm{m}^{2}(\mathrm{E}>29 \mathrm{fJ})_{\mathrm{HTGR}}\right]$, the $\mathrm{UO}_{2}$ kernel had not really begun to deform plastically or to migrate (Ref. 2-1). FTE-4, which had an intermediate peak exposure of $1.9 \times 10^{25} \mathrm{n} / \mathrm{m}^{2}$ ( $\mathrm{E}>29$ fJ) ${ }_{\mathrm{HTGR}}$, showed significant $\mathrm{UO}_{2}$ kernel plasticity and migration up to the IPyC inner surface. FTE-6, with a peak exposure of $2.8 \times 10^{25} \mathrm{n} / \mathrm{m}^{2}$ (E $>29 \mathrm{fJ})_{\text {HTGR' }}$ reveals the final effect of the $\mathrm{UO}_{2}$ migration in the pressure vessel failure of the fuel particles. $\mathrm{UO}_{2}$ fissile particles have been previously rejected as candidate HTGR fuel based on similar poor irradiation performance observed in P13N and P13P (Ref. 4-12).

OPyC failure was low in most cases and was attributed mainly to polishing damage. The ThO 2 TRISO fuel in rod 2-6-7 showed the highest OPyC failure of approximately 9\% (Fig. 4-73). This is consistent with the approximate 5\% failure observed in FTE-4 (Ref. 2-2). Because of incomplete preirradiation data on this particular fuel particle, it is difficult to assess the reason for the OPyC failure. One possible explanation for this high failure is that this is the largest diameter TRISO-coated particle examined in FTE-6. Evidence discussed in Ref. 4-12 has shown that the larger the particle the higher the probability of failure. This is explained by Weibull statistics, in which the probability of finding a critical flaw in a stressed region is proportional to the area or volume under stress.

The only significant thermochemical effect in the fuel rods in FTE-6 was the $\mathrm{UO}_{2}$ kernel migration. $\mathrm{All}$ of the $\mathrm{UC}_{2}$ TRISO particles in rod 2-8-7 had graphitization of the IPyC layer, which indicates the presence of mixed fission products. One isolated $\mathrm{UC}_{2}$ particle showed what appeared to be contamination attack of the fuel particle (Fig. 4-76). All the fissile particles in FTE- 6 had buffer densification and debonding and, in some cases, IPyC debonding and IPyC failure. One isolated case of matrix-coating interaction was seen in rod $2-3-7$ on a (Th,U) $C_{2}$ TRISO particle (Fig. 4-72). The OPyC coating on this particle had been torn off by the differential shrinkage of the bonded matrix and the OPyC coating. 
Homogeneity of the fuel loadings was a further significant metallographic observation. Rods $2-1-7,2-3-7$, and $2-8-7$ all showed various forms of fuel inhomogeneity. Rods $2-3-7$ and 2-8-7 had significant skewing of the fertile particles toward the matrix end cap portion of the fuel rod. The single-channel gamma scan Zr-95 profile in Fig. 4-77 shows that this fertile inhomogeneity has little effect on the overall power profile in the fuel rod. On the other hand, rod 2-1-7, which showed a fissile particle inhomogeneity in metallography, had an equally great inhomogeneity in the activity profile in Fig. 4-77. Comparison of rods $2-1-7$ and 2-2-7, which showed good and bad fuel homogeneity, respectively, shows that the nonuniform fuel loadings had little effect on the $\mathrm{UO}_{2}$ TRISO and $\mathrm{ThO}_{2} \mathrm{BISO}$ fuel performance. In both cases the extent of the fuel failure and the degree of kernel migration and SiC attack were similar for the fissile fuel. This inhomogeneity may have caused bowing of the fuel rods, which was discussed previously in the section on the visual examination.

\subsubsection{Fuel Rod Gamma Scanning}

Fuel rods from all four fuel blends and from different axial locations in the element were gamma-scanned with a high-resolution Ge(Li) gamma spectrometer. The scanning geometry (Fig. 4-78) and calibration calculations are described in Ref. 2-2. These gamma scans give the following information:

1. Relative axial homogeneity of each fuel rod.

2. Fission product inventories of the most significant isotopes in each fuel rod.

3. Total burnup of each fuel rod.

The axial fuel homogeneity is shown in Fig. 4-77 for fuel rods used in the detailed examination and in Fig. 4-79 for a representative cross section of fuel rods in the element. In general, the fuel homogeneity was good except for several cases in holes $1,2,7$, and 8 . 
The fission product inventories and burnup of each fuel rod are presented in Table 4-43. The gamma-scan-calculated total burnup from the Cs-137 inventory is compared with the GAUGE/FEVER calculated values in Table 3-12 and plotted against the axial core location in Figs. 3-33(a), 3-33(b), 3-33(c), and 3-33(d). The shape of the total FIMA plots of the various fuel stacks is a direct comparison with the thermal neutron fluence and power profile of the test element (see Sections 3.2 and 4.2). For fue1 stacks from holes 3 through 8 , the curve is smooth and shows only a few anomalous points. Rods from stacks 1 and 2 show a large deviation from a smooth FIMA plot in Fig. 3-33(a). This was caused by the high failure of the $\mathrm{UO}_{2}$ fissile particles in these fuel rods, which allowed Cs-137 release, which in turn lowered the total FIMA value calculated from the Cs-137 inventory. Table 3-12 shows that in stacks 3 through 8 the gammaspectroscopy-measured mean total FIMA was systematically $10 \%$ to $20 \%$ higher than the GAUGE-calculated mean total FIMA. In stacks 1 and 2 the mean total FIMA was approximately 5\% lower than the GAUGE value, which is directly related to the $\mathrm{Cs}-137$ loss from the $\mathrm{UO}_{2}$ particle.

Using the measured and deduced theoretical Cs-137/Zr-95 ratios, the percent Cs-137 loss from the various fuel rods can be calculated. Figures 4-80 through 4-83 show the Cs $-137 / \mathrm{Zr}-95$ isotopic ratio for fuel rods from different fuel stacks along the axial length of the element. If there is no particle failure or diffusive loss of the volatile Cs-137, the Cs-137/ Zr-95 ratio should be nearly the same for all fuel rods in FTE-6. The mean deduced theoretical ratio was calculated by averaging the measured ratio in fuel rods from stacks 5 and 6 , where there was low fuel failure and no diffusive release because of the TRISO-TRISO fuel blend. This ratio was then corrected for other fuel blends with a knowledge of the isotopic yields from the fissile and fertile fuel and the fuel loadings (see Section 4.2.2.4). The deduced and measured mean Cs $-137 / 2 r-95$ ratios are compared below.

Cs-137/Zr-95 Ratio

Holes Intact Ratio $\pm \underline{2 \sigma} \quad \underline{\text { Measured Ratio }} \pm \underline{2 \sigma} \quad \underline{\text { Test for Significance }}$
$1 \& 2$
0.1160
0.0060
0.0975
0.0051 Significant diff. 
Cs-137/Zr-95 Ratio

\begin{tabular}{ccccccc} 
Holes & Intact Ratio & $\pm \underline{2 \sigma}$ & Measured Ratio & $\pm 2 \sigma$ & Test for Significance \\
\cline { 3 - 5 } $3 \& 4$ & 0.1142 & 0.0059 & 0.1140 & 0.0246 & Insignificant diff. \\
$5 \& 6$ & 0.1142 & 0.0059 & 0.1142 & 0.0059 & N.A. \\
$7 \& 8$ & 0.1137 & 0.0058 & 0.1182 & 0.0198 & Insignificant diff.
\end{tabular}

A11 fue1 stacks except 1 and 2, show insignificant differences between the measured and intact $\mathrm{Cs}-137 / \mathrm{Zr}-95$ ratio, which indicates little Cs-137 loss and low fuel failure. Stacks 1 and 2 show a significant Cs-137 loss, which has also been discussed in Section 4.2.2.4. Using the deduced intact and measured $\mathrm{Cs}-137 / \mathrm{Zr} / 95$ ratios, the percent $\mathrm{Cs}-137$ loss was calculated and is shown in Fig. 4-84 along the core height for stacks 1 and 2 . There is an obvious correlation of the Cs-137 loss with temperature. The Cs-137 loss was the lowest in body 1, which had the lowest temperature exposure, and the highest (up to $34 \%$ ) in the bottom part of body 3 , which experienced the highest temperatures. The Cs-137 loss can also be compared with fuel failure fractions determined by metallographic and fission gas release measurements. A comparison between rods $2-1-7$ and $2-2-7$ is given below.

\begin{tabular}{|c|c|c|c|c|}
\hline \multirow[b]{2}{*}{ Rod } & & \multicolumn{2}{|c|}{ Metallography } & Cs -137 \\
\hline & $\begin{array}{c}\text { Failure Measured by } \\
\text { Fission Gas Release } \\
\bar{x}-2 \sigma<\bar{x}<\bar{x}+2 \sigma \\
(\%) \\
\end{array}$ & $\begin{array}{r}\text { SiC Failure } \\
\overline{\mathrm{x}}-2 \sigma<\overline{\mathrm{x}}<\overline{\mathrm{x}}+2 \sigma \\
(\%) \\
\end{array}$ & $\begin{array}{c}\text { Pressure } \\
\text { Vesse1 Failure } \\
\overline{\mathrm{x}}-2 \sigma<\overline{\mathrm{x}}<\overline{\mathrm{x}}+2 \sigma \\
(\%) \\
\end{array}$ & $\begin{array}{l}\text { Loss From } \\
\text { Fue1 Rod } \\
\overline{\mathrm{x}} \pm 20 \\
\pm(\%) \\
\end{array}$ \\
\hline $1-7$ & $8<21<53$ & $13<18<26$ & $12<17<25$ & $-21 \pm 10$ \\
\hline$-2-7$ & $9<23<58$ & $14<20<27$ & $5<8<14$ & $-14 \pm 10$ \\
\hline
\end{tabular}

The following assumptions have been made:

1. $\mathrm{Kr}-85 \mathrm{~m}$ at $1100^{\circ} \mathrm{C} \mathrm{R} / \mathrm{B}_{\mathrm{f}}=5.3 \pm 2.7 \times 10^{-3}(1 \sigma)$ for failed fuel particles in a constrained geometry

2. Pressure vessel failure is failure of all structural coatings in the particle. 
3. Cs-137 loss is calculated from Cs $-137 / \mathrm{Zr}-95$ ratios.

4. Confidence limits are calculated with a cumulative binomial distribution for metallography, a $\log$ normal distribution for fission gas release and a normal distribution for Cs depletion.

Using the failure from fission gas release measurements and the fuel rod Cs-137 loss in rods $2-1-7$ and $2-2-7$, the average Cs-137 loss from a $\mathrm{UO}_{2}$ fuel failure in the constrained state (i.e., fuel rod) is calculated to be $33<81<100(2 \sigma)^{*}$. This is consistent with the high Cs-137 loss per failure measured in $P 13 R$ and $P 13 S$ fuel rods and particles (Ref. 4-13).

\subsubsection{Fission Product Distributions in the Graphite Fue1 Bodies}

Fission products that were released from the fuel rods because of fuel failure or diffusive release were found on the graphite fuel bodies. Cesium-134, Cs-137, and Eu-154 were the only isotopes that were detected with significant concentrations at the time of the analyses. Radial sectioning, gamma scanning, and autoradiography of selected graphite samples were used to characterize the fission product distribution in the graphite fuel bodies.

\subsubsection{Radia1 Meta1 Profile Determinations in Graphite Fuel Bodies.} As an adjunct to the work discussed in Section 4.8, diffusion profiles of Cs-134, Cs-137, and Eu-154 in the inner and outer webs of FTE-6 fuel body 2 have been measured. This work was performed to confirm that the sectioning techniques developed for the analysis of metallic diffusion samples could be adapted to actual fuel element bodies. In addition, the General Atomic reference computer code for predicting metallic release from fuel bodies, FIPERQ (Refs. 4-15 and 4-16), has been utilized to model the observed Cs-137 profile in the outer web.

A 15.90-mm (0.625-in.) thick cross-sectional graphite slice, irradiated at an active core height of $2.127 \mathrm{~m}$ ( $83.7 \mathrm{in.}$ ), was cut from the end of fuel body 2. Autoradiography of the slice revealed a highly

*Example: Rod 2-2-7 with $23 \%$ pressure vessle failure and $14 \%, \mathrm{Cs}-137$ loss has $\frac{14}{23}=0.61$ or $61 \% \mathrm{Cs}-137$ loss. 
contaminated web adjacent to fuel holes 1 and 2 (see Fig. 4-85 and autoradiography in section 4.5.5.3). This hole contained fuel rods with a $\mathrm{UO}_{2}$ TRISO/ThO 2 BISO particle blend in matrix material. After milling the slice to a thickness of $12.70 \mathrm{~mm}$ (0.500 in.), the graphite web was sectioned on a glove-box-enclosed milling machine. The resulting samples were then gamma counted to determine isotope loading.

Figures 4-86 through 4-90 present the profiles of Cs-134, Cs-137, and Eu-154 obtained via sectioning. The $2 \sigma$ error bars are shown according to the convention described in Section 4.8. [The uncertainty in the depth of cut, $2.5 \times 10^{-3} \mathrm{~mm}(0.001 \mathrm{in.})$ falls within the area shaded by the data point; as such, no horizontal error bars are shown.] The outer web profiles of Cs-134 (Fig. 4-86) and Cs-137 (Fig. 4-87) are virtually identical, with the concentration of $\mathrm{Cs}-134$ being an order of magnitude lower than that of Cs-137. The similarity of the profiles indicates that gaseous precursors are not making a significant contribution to the metal loading. This can be visualized after reviewing the decay and activation schemes for these isotopes:

$$
\begin{aligned}
\mathrm{Xe}-133(5.2 \mathrm{~d}) \rightarrow & \mathrm{Cs}-133(\text { stable })+\gamma \\
& \mathrm{Cs}-13.3+\mathrm{n} \rightarrow \mathrm{Cs}-134(2.06 \mathrm{y}) \\
\mathrm{Xe}-137(3.84 \mathrm{~m}) \rightarrow & \mathrm{Cs}-137(30 \mathrm{y})
\end{aligned}
$$

If the holdup time, $\mathrm{T}_{h}$, of xenon in graphite were $<30 \mathrm{~s}$, the decay of both isotopes would yield flat precursor profiles of differing magnitude $(\mathrm{Cs}-137>\mathrm{Cs}-134)$. Since the isotope profiles show similar decreasing concentrations toward the outer boundary of each web, the precursor contributions are seen to be insignificant. If a longer holdup time were to exist, $30 \mathrm{~s}<\mathrm{T}_{\mathrm{h}}<700 \mathrm{~s}$, the decay of Xe-137 would provide a precursor profile that was not flat but that decreased toward the outer web boundary; the Xe-133 precursor contribution would still be flat due to its much longer half-life. However, if Xe-137 decay did provide a measurable contribution to the profile, the $\mathrm{Cs}-137$ and $\mathrm{Cs}-134$ profiles would be disparate, a result not found during the sectioning. Analogous reasoning can be used to show that at $\mathrm{T}_{\mathrm{h}} \gtrsim 700 \mathrm{~s}$ the precursor contribution of $\mathrm{Xe}-133$ 
is likewise insignificant. Thus the combination of Cs-134 and Cs-137 profiles that are both identical and decreasing toward the outer web boundary indicates that metallic diffusion is the controlling release mechanism.

Having established that the profiles resulted from metallic diffusion, the Cs-137 profile in the outer web was fit with the FIPERQ code. (The similarity of the Cs-134 and Cs-137 profiles ensures that like values for the diffusion coefficients would be attained for both isotopes.) This code solves Eq. 4-77 to predict metallic fission product release from graphite fuel bodies:

$$
\begin{aligned}
\frac{\partial C_{i}(\vec{r}, t)}{\partial t} & =S_{i}(\vec{r}, t)-\lambda_{i} C_{i}(\vec{r}, t)-\vec{\nabla} \cdot \vec{J}_{i}(\vec{r}, t) \\
i & =1,2,3, \ldots, I,
\end{aligned}
$$

where $C_{i}(\vec{r}, t)=$ volume concentration of the $i$-th diffusing species,

$S_{i}(\vec{r}, t)=$ volumetric source of the $i-t h$ diffusing species,

$\lambda_{i}=$ radioactive decay constant of the $i-t h$ species,

$J_{i}(\vec{r}, t)=$ diffusion current of the $i-t h$ species.

For the purpose of this study, a constant source term was assumed. This is not entirely correct since time-dependent cesium release from the particles likely occurred. This simplification does not affect the code fitting since the Fickian diffusion coefficient (and thus the profile shape) used as input is assumed to be independent of concentration. The other major experimental inputs required for curve fitting are the graphite body temperatures (see Table 4-44) and dimensions. The former were obtained'from TREVER code thermal analysis calculations for body 2; the latter were derived from preirradiation metrology measurements. Other required inputs (i.e., diffusion coefficient, sorption isotherms) were taken from the General Atomic Design Data Manual (Ref. 4-17).

The measured and FIPERQ-predicted profiles shown in Fig. 4-87 exhibit satisfactory agreement. Note that the calculated temperatures have been 
normalized to an inner boundary concentration of $0.05 \mu \mathrm{g} / \mathrm{mg}$. This was necessary because the predicted concentrations were derived using an arbitrary source, a necessary prerequisite since the actual time-dependent cesium release from the fuel rods was not known. The similarity of the profiles indicates that the modeling assumptions and input temperatures used for this analysis are acceptably accurate and that the FIPERQ code provided a reasonable fit of the observed data."

Radionuclide profiles in the inner web were also analyzed. The Cs-134 and Cs-137 profiles are similar to those found in the outer web, a1though the loadings in the inner web are a factor of three lower than those in the outer web. In addition, Eu-154 was detected in the inner web. The steeper slope of the profile, compared with those of the cesium isotopes, reflects the much slower rate of diffusion of this metal in graphite (for HTGR design calculations, europium is assumed to behave like strontium; see Section 4.8). No europium was detected in the outer graphite web.

To summarize, the diffusion profiles of Cs-134, Cs-137, and Eu-154 in the inner and outer web adjacent to fuel hole 2 in fuel body 2 were determined by a sectioning-gamma counting technique. The similarity of $\mathrm{Cs}-134$ and Cs-137 profiles confirmed that release was by metallic diffusion, with contributions by gas phase precursors being negligible. The reference metallic release code, FIPERO, was used to model the observed Cs-137 concentration in the outer web. Using calculated FTE-6 temperatures lowered by $-100 \mathrm{~K}$ and reference input design data, good agreement between measured and predicted profiles were found. A reduction in temperature is justified because of axial heat conduction and fuel body and faces, presently not accounted for in the thermal analysis.

4.5.5.2. Graphite Gamma Scanning and Autoradiography. The graphite fuel bodies were gamma scanned using the same scanning geometry as was used

* A stronger confirmation of design methods is obtained when known values of the source and release of metal to the coolant stream are available (Ref. 4-18). Nevertheless, fitting of the metallic profile provides verification of one step in the overall metal release mechanisms. 
for the fuel rods. Quantitative isotope inventories were calculated using the fuel rod detector calibration to approximate quantitative results. The error in this method is that a fuel rod has higher gamma ray attenuation effects than the graphite fuel bodies. This tends to overestimate the quantitative amount of cesium found on the graphite fuel body.

Autoradiography of the graphite slice being analyzed showed the majority of the cesium plateout to be around fuel holes 1 and 2 (Fig. 4-85), which contained $\mathrm{UO}_{2}$ TRISO particles with high failure. It was therefore decided to scan holes 1 and 2 together and then rotate the body $180 \mathrm{deg}$ and scan holes 5 and 6 as a comparison. The area scanned in each case is shown in Fig. 4-91. Figure 4-92 gives a single-channel trace of the Cs-137 and Cs-134 inventories in the two axial scans. Only Cs-137 and Cs-134 were detected in these scans. The single-channel scans of holes 1 and 2 show a smooth profile of $\mathrm{Cs}-134$ and Cs-137 across the three fuel bodies. This profile is essentially the mirror image of the cesium loss from the fuel rods in stacks 1 and 2 shown in Fig. 4-84. The insignificant amount of cesium adjacent to the unfueled regions of the fuel bodies or near fuel rods which showed little fuel failure (i.e., body 1) suggests that the radial diffusion of cesium is more significant than the axial diffusion. The scans of holes 5 and 6 show only a small amount of cesium in the graphite. This corresponds to the low fuel failure in these fuel rods.

A quantitative analysis of the amount of cesium lost from the fuel rods and subsequent accumulation in fuel body 2 was determined. Body 2 was chosen because it had the greatest number of fuel rods scanned from holes 1 and 2 and the cesium profile was relatively flat across the body. The inventory of cesium of the fuel bodies was as follows:

\section{Holes 1 and 2}

Cs -137

Cs -134

$$
2.48 \pm 0.52 \mathrm{Ci}(2 \sigma)
$$$$
3.60 \pm 0.72 \mathrm{Ci}(2 \sigma)
$$

Holes 5 and 6

$$
\begin{aligned}
& 0.10 \pm 0.02 \mathrm{Ci}(2 \sigma) \\
& 0.22 \pm 0.04 \mathrm{Ci}(2 \sigma)
\end{aligned}
$$

Using the Cs-137/Zr-95 theoretical and measured ratios for the fuel rods in holes 1 and 2, a theoretical Cs-137 inventory was determined and compared with the measured $\mathrm{Cs}-137$ inventory. The results show an average 
Cs-137 loss of approximately $16 \%$ per fuel rod, which corresponds to a total cesium loss of $5.42 \pm 2.88(2 \sigma)$ curies from all 28 fuel rods in holes 1 and 2 of body 2 . The fact that on $1 y 45.8 \% \pm 26.2 \%(2 \sigma)$ of the lost Cs -137 accumulated on the fuel body suggests that some of the Cs-137 escaped from the fuel body and traveled to the sleeve or was removed by the purge gas flow. Unfortunately the sleeve section opposite holes 1 and 2 was destroyed during the unloading and could not be gamma scanned. The scan of the porous graphite trap in the bottom connector of the element showed a small amount of cesium, which indicates some cesium transport in the purge stream.

\subsubsection{Fue1 Performance Prediction}

With the large amount of in-pile and out-of-pile fuel performance data (Refs. 4-14 and 4-19) that has been collected on various types of candidate HTGR fuel types, the fuel performance in FTE-6 was estimated. Using the TREVER computer code, the fuel kernel migration was estimated and compared with the metallographically observed fuel kernel migration. Then, using the procedure outlined in Ref. 4-20, the failure by amoeba was approximated.

TREVER divides the rods into 10 radial segments which are divided by isotherms (Fig. 4-93). It then calculates the amount of fuel kernel migration of each fuel kernel type with Eq. 4-78 using the kernel migration coefficients shown in Table 4-45 and the calculated temperature and temperature gradient in each segment.

$$
\frac{\Delta x}{\Delta t}=(K M C) T^{-2} \frac{\Delta T}{\Delta x},
$$

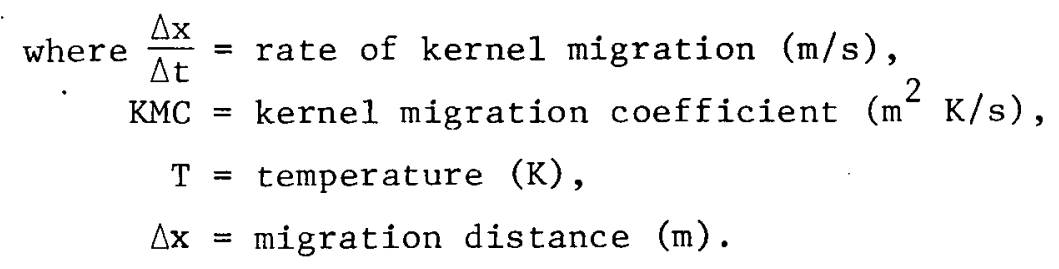

Present design standards consider fuel failure by amoeba to occur when the kernel migrates through the buffer layer. Because there is 
a distribution of layer thicknesses, a probabilistic approach must be used to determine when failure occurs. Using a normal distribution of layer thicknesses, the fraction of particles which have kernel migration greater than the buffer layer thickness is determined for each of the 10 TREVER radial segments and then assumed for the whole cross section using Eq. 4-79.

$$
F=\sum_{i=1}^{N} A i \Phi_{Z i},
$$

where $\Phi_{Z_{i}}=$ the fraction of particles with migration through the buffer in any one TREVER segment (cumulative normal distribution), $\mathrm{Z}=$ predicted migration distance from $\mathrm{Eq} \cdot 4-78$, $F=$ total fraction of particles with migration through the buffer in any one TREVER segment,

$A_{i}=$ area fraction of each TREVER segment, $N=$ number of TREVER segments (10).

Using the above analysis, it was calculated that all the fuel kernels in FTE-6 would migrate less than $10 \mu \mathrm{m}$ except for the $\mathrm{UO}_{2}$ type. This is consistent with the metallographic examination in Section 4.5. The $\mathrm{UO}_{2}$ kernels had predicted migration distances (Table 4-46) that extended through the buffer coating in the high temperature region of the element. The high measured fuel failure in these rods and the metallographically measured migration that extended through the buffer support the predicted behavior.

To compare the predicted fuel failure with the measured results, the cesium plateout on the graphite fuel bodies was used as a fuel failure monitor because of its smooth profile across the element (Fig. 4-92). The Cs activity profile in Fig. 4-92 was first normalized to the calculated burnup profile to take into account the axial differences in the cesium inventory. Using the fission gas release calculated failure from fuel rods $2-1-7$ and $2-2-7$, the cesium plateout curve was given a fuel 
failure scale which was assumed to be inear. This assumption neglects any effects of temperature or irradiation exposure on the release or the diffusion of cesium in the fuel and graphite bodies. Measurement of the failure at other axial locations is planned to determine the effect of these assumptions on the overall analysis of the Peach Bottom test element program. The results of the measured fuel failure is tabulated in Table 4-46.

Comparison of the measured and predicted fuel failure values in Table 4-46 shows that the current design criteria for fuel failure is conservative. The predicted failure in body 2 was in some cases greater by a factor of two than the measured fuel failure, which was accurately known in this region from the fuel rod fission gas release measurements. An alternate design criteria for fuel failure was also considered. In this case failure was assumed to occur when the kernel migrated through the buffer and inner pyrocarbon (IPyC) layer. An assumption was made that the rate of migration through the dense IPyC was the same as through the buffer. The results of this prediction are also shown in Table 4-45. Here the predicted values are approximately $20 \%$ lower than those measured, which suggests that failure occurs when the kernel migrates partially through the IPyC layer.

In summary, it can be stated that the current design criteria for fuel failure by amoeba migration is conservative. It appears that fuel failure occurs after the kernel has contacted the IPyC layer and the fission products in the kernel are allowed time for destructive attack of the SiC layer.

\subsection{THERMAL STABILITY SPINE SAMPLES}

The two thermal stability spine samples that were examined from FTE-6 were located in type II crucibles. A description of these crucibles is given in Fig. 4-94. This particular design guaranteed a relatively isothermal environment for each batch of particles in any particular hole. 
4.6.1. Visua1 Examination

The visual examination of samples TS18-3 and TS6-5 is summarized in Table 4-47 and a stereophotograph of each batch is shown in Fig. 4-95. Preirradiation data on batches 4000-302 (TS6-5) and 4000-242 (TS18-3) are given in Table 4-48. There was no observable fuel failure in any of the particles in batch 4000-302. The appearance of the 4000-242 batch was also good, with the exception of one doublet with a cracked $O P y C$ coating and four OPyC coating fragments.

\subsubsection{Fission Gas Release Measurements}

The $\mathrm{Kr}-85 \mathrm{~m}$ fission gas release measurements at $1100^{\circ} \mathrm{C}$ from the TRIGA reactor facility are shown in Table 4-47. Batch 4000-242 (ThC TRISO) and 4000-302 (UC 2 VSM TRISO) showed $0.6 \%$ and $0.3 \%$ deduced fuel failure assuming an $\mathrm{R} / \mathrm{B}$ per fuel failure of $2 \%$ in the unconstrained state.

\subsubsection{Meta1lography}

Metallographic examination results on spine samples TS18-3 and TS6-5 are shown in Table 4-47. Representative microphotographs of each of the fuel types are shown in Figs. 4-96 and 4-97. Batch 4000-242 (ThC 2 TRISO) showed very good fuel performance. There were no thermochemical effects and only one hairline SiC failure was observed. Hydrolysis of the kernels during the examination caused IPyC and buffer cracking. The $\mathrm{UC}_{2}$ (VSM) TRISO particles of batch 4000-302 showed no failure or kerne1 migration. All the particles observed had buffer densification and debonding and mixed fission products in the IPyC. In approximately $4 \%$ of the particles, the mixed fission products had attacked the SiC. In no case did this attack extend more than approximately $5 \mu \mathrm{m}$ into the surface of the particle.

\subsubsection{Spine Sample Gamma Scans}

Figures 4-98 through 4-100 show the relative activity of Ce-144, Cs-134, and Cs-137 in the spine samples from the three fuel bodies as 
determined by gamma spectra. The activity of some of the fueled spine samples indicates significant heat sources that could be used in a detailed thermal analysis (see Section 4.2.2).

\subsection{FUEL PERFORMANCE SUMMARY}

The fuel rods and loose particle spine samples in FTE- 6 behaved satisfactorily at peak exposures of $2.88 \times 10^{25} \mathrm{n} / \mathrm{m}^{2}(\mathrm{E}>29 \mathrm{fJ}){ }_{\mathrm{HTGR}}$ and $1250^{\circ} \mathrm{C}$. The current HTGR fuel performance codes were all substantiated at the peak irradiation conditions of FTE-6. No significant SiC attack, kerne1 migration, or matrix-coating interactions were observed except for the $\mathrm{UO}_{2}$ TRISO fuel. This particular fuel had kernel migration extending up to the SiC inner surface, which caused SiC attack and subsequent pressure vessel failure exceeding $20 \%$ in some of the fuel rods. The poor performance of the $\mathrm{UO}_{2}$ fuel has also been observed in the accelerated testing of $P 13 \mathrm{~N}$ and $\mathrm{P} 13 \mathrm{P}$ (Ref. 4-13) and $\mathrm{UO}_{2}$ fuel is now no longer a candidate HTGR fuel type. The $\mathrm{UO}_{2}$ migration was also successfully predicted using the TREVER computer code.

Specific fuel examination conclusions are as follows:

1. The appearance of the fuel rods in FTE-6 was satisfactory. Matrix end cap cracking and small surface cracks were found on the majority of the fuel rods.

2. Fuel rods from stacks 1 and 2 containing the $\mathrm{UO}_{2}-\mathrm{ThO}_{2}$ fuel blend were bowed up to $0.58 \mathrm{~mm}$ on their length $(48.3 \mathrm{~mm})$. This was apparently caused by inhomogeneity of the fuel particle loadings.

3. Fission gas release measurements of the $\mathrm{UO}_{2}-\mathrm{ThO}_{2}$ fuel rods show fuel failure fractions of approximately $23 \%$, which compares with metallographically measured fuel failure fractions of $8 \%$ to $17 \%$ for the $\mathrm{UO}_{2}$ TRISO particles. 
4. Using 0.0053 as the release fraction in a constrained geometry, the calculated fuel failure fractions of all the fuel blends except the $\mathrm{UO}_{2}-\mathrm{ThO}_{2}$ were between $0.5 \%$ and $5 \%$. In some cases this did not compare well with fuel failure measured during metallography. This discrepancy is believed to be caused by the hydrolysis of the thorium-bearing fuel, which would cause a higher $\mathrm{R} / \mathrm{B}_{\mathrm{f}}$.

5. Metallography showed little thermochemical effects of SiC attack, kernel migration, or matrix-coating interactions in the fuels tested except for the $\mathrm{UO}_{2}$ TRISO particles.

6. Kernel migration of the $\mathrm{UO}_{2}$ kernels caused $\mathrm{SiC}$ attack and up to $20 \%$ pressure vessel failure.

7. Gamma scanning indicates insignificant cesium release from any fuel rods except for the $\mathrm{UO}_{2}-\mathrm{ThO}_{2}$ fuel blend. Approximately $45.8 \% \pm 26.2 \%(2 \sigma)$ of the release cesium was found absorbed in the graphite fuel body. The other $54 \%$ of the released cesium could have gone into the adjacent sleeve material, which, however, was not available for counting.

8. Gamma-scan-measured total FIMA values for the fuel rods using Cs-137 as a burnup monitor indicate a systematic $10 \%$ to $20 \%$ higher burnup than that calculated by GAUGE.

9. Spine sample batches 4000-242 ( $\mathrm{ThC}_{2}$ TRISO) and 4000-320 (UC, TRISO) showed good irradiation behavior at $2.0 \times 10^{25} \mathrm{n} / \mathrm{m}^{2}$ ( $\mathrm{E}>$ $29 \mathrm{fJ})_{\mathrm{HTGR}}$ and approximately $1050^{\circ} \mathrm{C}$.

\subsection{METALLIC DIFFUSION SPINE SAMPLES}

\subsubsection{Test Description}

Nine fission metal diffusion samples were irradiated in the center spine position of FTE-6 (see Table 4-49). The spine samples were designed 
so as to provide data on the transport properties of fission product metals, including diffusion and sorption, in HTGR fuel material. They are particularly well suited for providing a measure of the metal behavior in graphite because (1) the temperature of crucibles is essentially uniform throughout, (2) the fission metal source in the matrix remains constant, and (3) the geometry of the crucibles is simple and well characterized. All of these factors are important when fitting the observed profiles with steady-state diffusion models. FTE-6 spine sample analyses were initially carried out to provide data on the following effects:

1. Transport properties of selected metal fission products in graphite under HTGR irradiation conditions as a function of metal concentration and temperature.

2. Distribution of metal fission products between matrix material and crucible graphite (partition factor).

3. Effects of other fission product metals on the migration rate of individual fission metals under study.

4. Effects of differing matrix materials.

5. Effect of fluence on measured diffusion coefficients.

Uncertainties in the calculated temperatures prevented the inclusion of these data in the reference data base at this time. Spine sample temperature uncertainties arose in part because of flux variations. This has been confirmed through the fuel gamma scan analysis, which revealed a flux depression originating from a boronated graphite sample located adjacent to some of the diffusion spine samples. This temperature microstructure information has not been quantified and is therefore not included in the thermal analysis presented in this report. The diffusion spine sample study is an extension of work begun under FTE-3 (Ref. 2-1) and FTE-4 (Ref. 2-2). 


\subsubsection{Experimental Procedure}

The nine diffusion samples in FTE- 6 were located in fuel body one. Following in part from a review of the data presented in Refs. $2 \frac{1}{1}$ and $2-2$, six samples were selected for analysis. The samples contained isotopes of strontium $(13,17,19)$, barium (33), or mixtures of cesium, strontium, barium, and samarium (europium) $(40,60)$. In addition, cesium released from the adjacent fuel rods was found to have passed through the crucible walls and matrix material of all samples into the crucible center posts. In some instances, values for the cesium diffusion coefficient were determined from the resultant cesium loading in the matrix.

The metallic source materials were initially loaded as carbides into previously outgassed H-327 graphite crucibles using inerted glovebox techniques. The carbides were formed by sorbing the metal isotopes of interest on matrix material and converting to the carbide at $1673 \mathrm{~K}$ in a vacuum furnace. The conversion was accomplished by heating the loaded matrix material in a tantalum crucible lined with graphite foil to minimize contamination of the matrix with tantalum. Two types of matrix materials were used: furfuryl alcohol resin coke (similar to Peach Bottom compact matrix material) and calcined petroleum coke containing approximately $0.5 \%$ sulfur. The latter material is of interest since it closely resembles the current HTGR reference matrix material. Following conversion to carbide, the matrix was loaded into the annulus of the $\mathrm{H}-327$ graphite crucible, shown in Fig. 4-101, and sealed with a screw-top lid. After loading, the crucibles were coated on the outside with polystyrene to protect the metals and carbides from exposure to moist air (thereby preventing hydrolysis or oxidation) prior to insertion in the reactor.

After irradiation to $645 \mathrm{EFPD}$ and a suitable cooling period, the crucibles were removed from the fuel test element, the matrix material was separated from the crucible and gamma-counted, and the samples sectioned by turning the crucible walls and center posts in a glove-box-enclosed lathe, uniformly removing a layer of graphite with each section. Samples 
of known weight were gamma-counted using a Ge(Li) crystal detector and a 4096-channel pulse height analyzer. The observed species were activation tracers of cesium, strontium (enriched in Sr-84), barium (tagged with $\mathrm{Ba}-133$ ), and samarium (enriched in $\mathrm{Sm}-152$ ) shown in Table 4-50. A 370 IBM mode1 165 computer was used in conjunction with the program CPROFIT (Concentration PROfile FIT) to assist in the analysis of some of the concentration profiles (Ref. 2-1).

The metallic diffusion spine sample temperatures presented in Table 2-6(b) have been weighted by the following activation energy factor (see Section 3.3.1):

$$
W_{i}=\frac{\Delta t_{i} \exp \left(-Q / R T_{i}\right)}{\sum_{i} \Delta t_{i} \exp \left(-Q / R_{i}\right)}
$$

where $\mathrm{T}_{i}=$ temperature $(\mathrm{K})$,

$$
\begin{aligned}
\Delta t_{i} & =\text { time at temperature } T_{i}, \\
Q & =\text { activation energy }(\mathrm{J} / \text { mole }), \\
R & =\text { gas constant }(8.3147 \mathrm{~J} / \mathrm{K} \text {-mole }) .
\end{aligned}
$$

This factor is used because changing temperatures bring about dissimilar changes in the rate of diffusion of metals having different activation energies. For strontium, barium, and europium analysis, $Q=$ $267 \mathrm{~kJ} / \mathrm{mole}$ was utilized; for cesium analysis, $\mathrm{Q}=144 \mathrm{~kJ} / \mathrm{mole}$ was used.

\subsubsection{Analysis and Results for Strontium, Europium, Barium, and Cesium}

4.8.3.1. Strontium. The concentration profiles for sr-85 in the center post and crucible wall of samples 13, 17, and 19 are presented in Figs. 4-102 through 4-107. No strontium was detectable in the center post or crucible wall graphite of samples 40 or 60 because of the low level of strontium remaining at the time of analysis (resulting from decay of $64.7 \mathrm{~d}$ half-life $\mathrm{Sr}-85$ ) and the high background of cesium present. No effort was made to chemically separate strontium from higher activity materials present in the graphite or matrix material. The error bars 
associated with each point in these figures (and those of other fission metals) represent the following:

1. The horizontal error bars represent the absolute root-meansquare $2 \sigma$ uncertainty associated with the radial position of each data point. The absolute uncertainty of each cut was estimated to be $50.4 \mu \mathrm{m}$.

2. The vertical error bars represent the relative $2 \sigma$ uncertainty in the fission metal concentration resulting from counting uncertainty (assuming a Poisson distribution). The uncertainty in the concentration introduced from weighing errors was found to be $<5 \%$ of the counting error in all cases and was neglected in the analysis. If the uncertainty was found to lie within the area of a datum symbol, no uncertainty was indicated.

Previous analyses of strontium profiles in graphite (Refs. 2-1 and 2-3, and summary in Ref. 4-21) have revealed that strontium profiles can usually be fit with a complementary error function equation. For the profiles in the crucible walls, the error function equation for diffusion in a plane sheet is utilized in the CPROFIT computer code:

$$
c=c_{0} \operatorname{erfc}\left(\frac{r-b}{2 \sqrt{D t}}\right) \text {, }
$$

where $\mathrm{C}=$ metal concentration in graphite at $\mathrm{r}(\mu \mathrm{g} / \mathrm{mg})$,

$\mathrm{C}_{\mathrm{o}}=$ metal concentration in the graphite region adjacent to the matrix material $(\mu \mathrm{g} / \mathrm{mg})$,

$\mathrm{r}-\mathrm{b}=$ depth of penetration (m) (see Fig. 4-101),

$\mathrm{D}=$ Fickian bulk diffusion coefficient $\left(\mathrm{m}^{2} / \mathrm{s}\right)$, and

$t$ = irradiation time $(s)$.

For diffusion in the cylindrical center post, the solution with $\mathrm{C}_{\mathrm{S}}^{\mathrm{O}}=$ concentration at $\mathrm{r}=\mathrm{a}$ (see Fig. 4-101) is: 


$$
\begin{aligned}
\frac{C_{s}}{C_{s}^{0}}= & \left(\frac{a}{r}\right)^{1 / 2} \text { erfc } \frac{a-r}{2 \sqrt{D t}}+\frac{(a-r)(D t a)^{1 / 2}}{4 a r^{3 / 2}} \operatorname{ierfc} \frac{a-r}{2 \sqrt{D t}} \\
& +\frac{\left(9 a^{2}-7 r^{2}-2 a r\right) D t}{32 a^{3 / 2} r^{5 / 2}} i^{2} \operatorname{erfc} \frac{a-r}{2 \sqrt{D t}}
\end{aligned}
$$

which is a good approximation provided $\mathrm{r} / \mathrm{a}$ is not too small (Ref. 4-22). Both equations are applicable when the period of the fuel test element operation is small relative to the characteristic slow diffusion time, $t_{D}\left(t_{D}=l^{2} / 4 D\right.$, where $l$ is the graphite sample radius or thickness).

The results of the CPROFIT analysis are shown in Table 4-51 and Figs. 4-102 through 4-107. All of the sample data can be fit satisfactory with the complementary error function, indicating Fickian control of diffusion. However, due to the low level of Sr-85 activity present, it is unlikely that any "tails" in the strontium profiles could have been detected had they been present. Such an indication of non-Fickian behavior, implying "fast path" diffusion of $\mathrm{Sr}$ along pores and grains in the graphite, has been reported by others (Refs. 4-18 and 4-23). Profiles for samples 17 and 19 show evidence of depletion of strontium near the matrix boundary. Since this effect appears in both the center post and crucible wall profiles, both of which involve separate sectioning operations, it is not likely this effect results from anomalous measurements. It is possible that the small decrease in tempratures that occurred during the final 30 days of irradiation brought a back-diffusion of strontium into the matrix.

An Arrhenius plot of strontium diffusion coefficients derived from FTEs $-3, *-4$, and -6 is presented in Fig. 4-108. Also presented is a least-squares fit of data from other sources as derived by Myers and Be11 (Ref. 4-21). These latter data exhibited a high degree of reproducibility; as such, the disparity between FTE data and the reference line may result from imprecise FTE temperatures. A review of the figure reveals

\footnotetext{
* The temperatures of the FTE-3 spine samples presented in Ref. 2-1 have been revised upward (Ref, 2-3). Figure 3-5 includes these updated temperatures.
} 
that the average calculated activation-energy-weighted temperatures for FTEs -4 and -6 may be too high by approximately $150 \mathrm{~K}$; temperatures for FTE-3 are in good accord with the reference time. The possibility also exists that the diffusivity of strontium in graphite is dependent upon neutron fluence. The FTE-6 spine samples, which experienced higher fluence $\left(\sim 3 \times 10^{25} \mathrm{n} / \mathrm{m}^{2}\right)$ than FTE-3 $\left(\sim 3 \times 10^{24} \mathrm{n} / \mathrm{m}^{2}\right)$ and FTE-4 $\left(\sim 2 \times 10^{25} \mathrm{n} / \mathrm{m}^{2}\right)$ samples, provided strontium diffusion coefficients that were significantly lower than those predicted using the reference data base or FTE- 3 and FTE-4 data. A similar comparison of FTE-3 and FTE-4 data shows that the latter provides somewhat smaller values of diffusivity than the former. These results indicate that increasing neutron fluence may result in lowered diffusion of strontium in graphite at a given temperature.

No effects of neutron fluence or isotope loading were noted. The lack of the latter effect is not surprising since the total metal loading at the end of irradiation was likely in a regime where the diffusion coefficient was expected to be independent of concentration (Ref. 4-21). In addition, the uncertainty in the sample temperatures tends to mask the presence of both these effects.

4.8.3.2. Europium. The profiles of Eu-154 in sample 40 are shown in Figs. 4-109 and 4-110 along with the CPROFIT fits to the data. No europium was detectable in sample 60 , although it was initially loaded into the source matrix along with cesium, strontium, and barium. This may have resulted from the lower loading of europium in this sample. The crucible wall profile exhibits apparent Fickian diffusion; the profile in the center post is more difficult to interpret. Although the profile adjacent to the matrix material exhibits the expected Fickian behavior, the accumulation of europium near the center post may be an indication of the presence of a non-Fickian "fast path" diffusion mechanism. * This phenomenon was also noted in FTE-3 and FTE-4 europium profiles where the existence of diffusion "tails" was noted for several samples. The diffusion coefficients derived from FTEs $-3,-4$, and -6 are presented in an Arrhenius plot in

* The lower sensitivity of the crucible wall gamma counting results may have precluded the detection of profile tails in this region. 
Fig. 4-111. Also shown is the least squares line for strontium data derived from other sources. This line is included since for reactor design purposes strontium and europium are assumed to diffuse at the same rate. The europium results confirmed the conclusions put forth for strontium, i.e., FTE-3 data show good agreement with the reference line, whereas FTE- 4 and -6 data provide diffusion coefficients that are lower than the reference line, the difference resulting from temperature uncertainties or a dependence of diffusivity on neutron fluence.

4.8.3.3. Barium. No barium was detected in the matrix or graphite of samples 33,40 , or 60 . This likely resulted from the high background activity of other isotopes present in the samples.

4.8.3.4. Cesium. The concentration profiles for $\mathrm{Cs}-134$ in the center post and crucible wall of all of the diffusion samples are shown in Figs. 4-112 through 4-123. The profiles observed in crucibles 13, 17, 19, and 33 resulted from cesium ingress from the adjacent fuel rods. In each case, a moderately steep profile was observed in the crucible wall and a much flatter profile in the center post. These profiles are felt to result from the diffusion of cesium along grain boundaries and within pores in the graphite, accompanied by a sorbtion/desorbtion process into the grain (Refs. 2-1, 4-18, and 4-24, and summary in Ref. 4-22). Each sample showed an increase in cesium loading near the matrix boundary, both for the center post and crucible wall. This effect has previously been ascribed to "slow path" Fickian diffusion of cesium within the grains of graphite (Ref. 2-1). However, since cesium was entering the crucible wall from the outside, a slow path component in the graphite adjacent to the matrix would not be expected. ${ }^{*}$ This effect may, instead, be associated with surface loading.

Since the external source of cesium for these crucibles was varying and uncertain, utilization of the constant source expressions developed

\footnotetext{
${ }^{*}$ If the crucibles had been subjected to markedly increased temperatures after the matrix had been loaded with cesium from the outside, a slow path profile might be produced by the diffusion cesium out of the matrix in the graphite.
} 
for the CPROFIT analysis of cesium diffusion (Ref. 2-1) was not appropriate. However, estimates of the diffusion coefficient were derived from the apparent time-integrated flux of cesium through the crucible wall into the matrix material. Since the matrix is highly sorptive of and initially free of cesium, it acts as a sink for cesium permeating the crucible wall. As such, the cesium loading in the matrix was assumed to be a measure of the integrated flux through the crucible wall (assuming insignificant cesium loss to the center post graphite). The diffusion coefficient of cesium in graphite was derived from the matrix loading using the following analysis. The linear matrix density was calculated using Eq. 4-83:

$$
\sigma_{m_{1}}=\left(b^{2}-a^{2}\right)(\pi)\left(\sigma_{m_{s}}\right)
$$

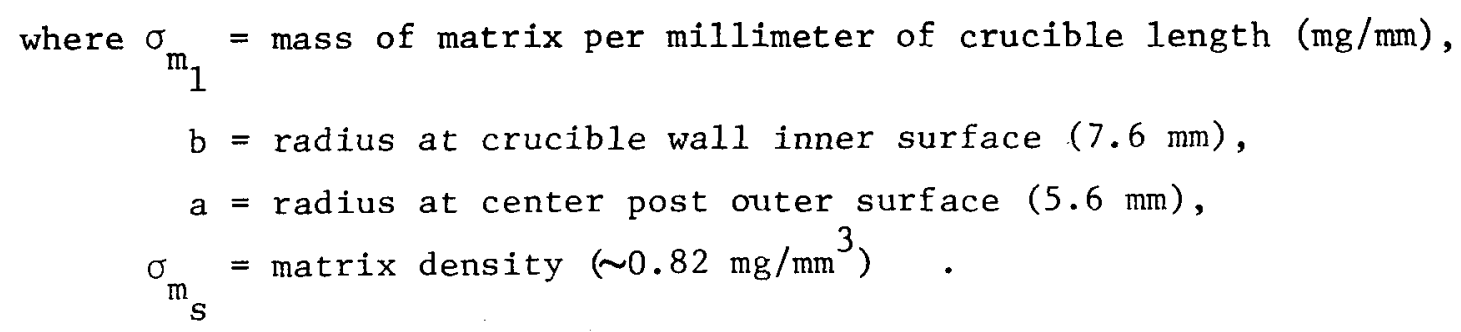

Using these values, a linear matrix density of $68.0 \mathrm{mg} / \mathrm{mm}$ was calculated. The flux of cesium into the matrix can be computed using Eq. 4-84:

$$
\bar{J}=\frac{\left(C_{m}\right)\left(\sigma_{m_{1}}\right)}{(t)(\pi)(2 b)},
$$

where $\bar{J}=$ mean flux $\left(\mu \mathrm{g} / \mathrm{mm}^{2}-\mathrm{s}\right)$,

$$
\begin{aligned}
\mathrm{C}_{\mathrm{m}} & =\mathrm{Cs}-134 \text { concentration in the matrix material }(\mu \mathrm{g} / \mathrm{mg}), \\
\mathrm{t} & =\text { real time }\left(6.84 \times 10^{7} \mathrm{~s}\right), \\
\mathrm{b} & =\text { inner radius of crucible wall }(7.62 \mathrm{~mm}) .
\end{aligned}
$$

The computed value of $\bar{J}$ is used in Eq. 4-85 to calculate $D_{\text {eff }}$ for the case of a hollow cylinder: 


$$
D_{\text {eff }}=\frac{(\bar{J})(2)(b) \ln (c / b)\left(10^{-6}\right)}{\left(C_{\ell}\right)\left(\sigma_{g}\right)},
$$

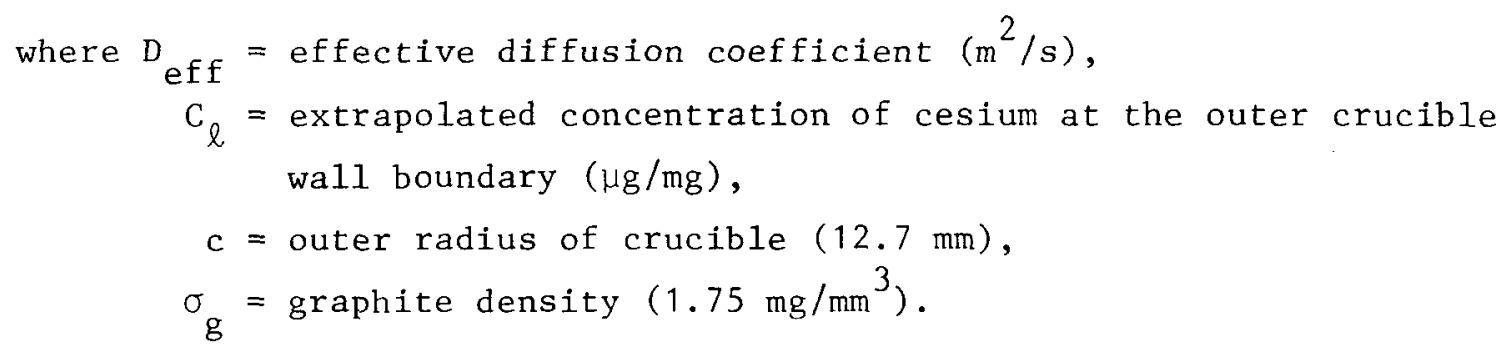

In the limit of an infinitely large cylinder, this expression reduces to:

$$
D_{\text {eff }}=\frac{(\bar{J})(2)(c-b)}{\left(C_{\ell}\right)\left(\sigma_{g}\right)} \text {, }
$$

since $\ln (c / b)=(c / b-1)-1 / 2(c / b-1)^{2}+\ldots$ or $b \ln (c / b) \simeq(c-b)$. The quantity $\left(C_{\ell}\right) / 2$ represents the time-average cesium loading in the crucible wa11.

The results of these calculations for FTE-6 are shown in Table 4-52. No values were derived by the flux method for samples 40 and 60 since cesium was initially present within the matrix material. It should be noted that the calculated cesium diffusion coefficient is dependent upon the concentration profile that exists across the crucible wall. If nonFickian behavior is present, as postulated in several sources, the shape and magnitude of the profile may be changing with time. However, since the rate of loading is likely to decrease with increasing time (Ref. 4-24), equilibrium concentrations of cesium in the graphite are likely to exist which would provide appropriate for determining $\mathrm{D}_{\text {eff }}$ (within the uncertainty of this type of measurement).

The profiles established in samples 40 and 60 (initially loaded with cesium) reveal that cesium ingress from adjacent fuel rods was significant. The crucible wall profile for sample 40 appears to be dominated by cesium from this source. The marked difference in the slope adjacent to the matrix and outer surface is difficult to reconcile, although the former may result in part from previously mentioned surface effects. 
Data from samples 40 and 60 have been analyzed with the CPROFIT code (Ref. 2-1). The model for cesium diffusion included in this code incorporates "fast path" diffusion along grain boundaries and in pores, with simultaneous first-order loading into the grain. The observed profile is postulated to result from cesium located on the grain and pore surface and within the bulk. Other models for cesium diffusion have been proposed but are not readily adaptable to computer modeling of the sample geometry. The results of the CPROFIT analysis of cesium profiles in samples 40 and 60 are presented in Table 4-51 and Figs. 4-120 through 4-123.

A summary of the effective diffusion coefficients for cesium derived from FTEs $-3,-4$, and -6 are presented in an Arrhenius plot in Fig. 4-124, along with data from the General Atomic Design Data Manual. If the temperatures of FTE- 6 are approximately $150 \mathrm{~K}$ too high, $\mathrm{D}_{\text {eff }}$ determined from cesium profile data $(40,60)$ would lie on or above the reference line, while values of $D_{\text {eff }}$ derived from flux measurements would lie closer to the mean reference line. Diffusion coefficients from FTE-3 show considerable scatter about the least squares fit of the reference data. The data from FTE-4, if temperature-corrected by $-150 \mathrm{~K}$, generally lie above the reference diffusion coefficient line. However, as suggested earlier, the lower diffusively shown in FTE-6 samples may result from the effect of increasing neutron fluence. This would agree with results found by Myers and $\mathrm{Be} 11$, wherein higher neutron fluences brought about a reduction in the measured diffusivity of cesium in graphite (Ref. 4-25).

The presence of apparent surface effects make it difficult to derive quantitative conclusions concerning the partition factors. However, a review of the data for sample 40 (Table 4-52), which had a furfury1 alcohol/resin coke matrix, and sample 60, which had a calcined petroleum coke matrix containing approximately $0.5 \%$ sulfur, reveals that the latter was significantly less sorbtive of cesium than the former. This result is similar to that noted in FTE-3 and is of interest since the calcined petroleum coke matrix is similar to the current reference matrix material. 


\subsubsection{Summary}

Analyses of diffusion spine samples, begun under FTE- 3 and -4 , have been continued with the higher fluence samples on FTE-6. Compilation of the data from the three elements has confirmed that strontium, barium, and europium can be treated using a Fickian model of diffusion (as is done in the reference and design codes FIPER and TRAFIC), and has aided in the assignment of temperature-dependent diffusion coefficients. Analysis of in-pile cesium behavior has confirmed that a "two-component" model may be required in order to accurately model diffusion in graphite. The model put forth by Zumwalt in FTE-3 has been found to provide reasonable fits of the observed data.

The diffusion profiles of $\mathrm{Cs}-134, \mathrm{Cs}-137$, and $\mathrm{Eu}-154$ in the inner and outer web adjacent to fuel hole 2 in fuel body 2 were determined by a sectioning-gamma counting technique. The similarity of Cs-134 and Cs-137 profiles confirmed that release was by metallic diffusion, with contributions by gas phase precursors being negligible. The reference metallic release code, FIPERQ, was used to model the observed Cs-137 concentration in the outer web. Using calculated FTE-6 temperatures (corrected by $100 \mathrm{~K}$ for end effects) and reference input design data, good agreement between measured and predicted profiles was found.

\section{REFERENCES}

4-1. Saurwein, J. J., and R. F. Korts, "STAT, A computer Code for Statistical Analysis of Fuel Test Element Metrology," General Atomic Company, unpublished data, May 1976.

4-2. Saurwein, J. J., "GAP, A Computer Code for Comparison of Calculated and Measured Heat Transfer Gaps in Fuel Test Elements," General Atomic Company, unpublished data, August 1976.

4-3. Saurwein, J.J., "STRAIN, A Computer Code for Comparison of Calculated and Measured Fuel Rod and Graphite Strain in Fuel Test Elements," General Atomic Company, unpublished data, August 1976. 
4-4. Mehner, A. S., "Test Plan for Gamma Scanning Peach Bottom Test and Drive Fuel Elements," General Atomic Company, unpublished data, November 15, 1974.

4-5. Smith, C. G., General Atomic Company, private communication, September 1976 .

4-6. Reese, H., and W. Whittemore, "HTGR Spent Fuel Shipping Casks - A Summary Report on Shielding Requirements," General Atomic Company, unpublished data, June 1976.

4-7. Mayweather, L. I., "Postirradiation Examination Procedure for Peach Bottom Test Element FTE-6," General Atomic Company, unpublished data, May 7, 1975.

4-8. Saurwein, J. J., and F. McCord, "FTE-4 Data Compilation of Nuclear, Thermal and Material Strain Evaluation," General Atomic Company, unpublished data, September 16, 1976.

4-9. Holzgraf, J. F., and C. M. Miller, Genera1 Atomic Company, unpublished data, May 19, 1976.

4-10. Scott, C. B., and D. P. Harmon, "Postirradiation Examination of Capsule F-30," General Atomic Report GA-A13208, Apri1 1, 1975.

4-11. Myers, B. F., N. L. Baldwin, W. E. Bell, and R. D. Burnett, "The Behavior of Fission Product Gases in HTGR fuel Materia1," General Atomic Company, to be published.

4-12. Myers, B. F., General Atomic Company, private communication.

4-13. Scott, C. B., D. P. Harmon and J. F. Holzgraf, "Postirradiation Examination of Capsules P13R and P13S," General Atomic Company, to be published.

4-14. Harmon, D. P., and C. B. Scott, "Development and Irradiation Performance of LHTGR Fue1," ERDA Report GA-A13173, General Atomic Company, October 31, 1975.

4-15. Forutanpour, B., and B. Roos, "FIPERX, a FORTRAN V Program for the Solution of One-Dimensional Linear and Non-Linear Diffusion Problems," USAEC Report GA-9904, Gulf General Atomic, September 1969.

4-16. Alberstein, D., P. D. Smith, and M. J. Haire, "Meta11ic Fission Product Release from the HTGR Core," GA-A13258, General Atomic Company, May 1975. 
4-17. "General Atomic Fuel Design Data Manual, Issue C," General Atomic Company, to be published.

4-18. "HTGR Fuels and Core Development Program Quarterly Progress Report for the Period Ending August 31, 1975," USERDA Report GA-A13592, Genera1 Atomic Company, September 30, 1975.

4-19. Smith, C. L., "Fuel Particle Behavior under Normal and Transient Conditions," USAEC Report GA-A12971, General Atomic Company, October 1, 1974.

4-20. Shamasundar, B. I., and A. Shenoy, General Atomic internal communication, August 6, 1975.

4-21. Crank, J., The Mathematics of Diffusion, Oxford University Press, London, 1956, p. 66.

4-22. "HTGR Base Program Quarterly Progress Report for the Period Ending February 29, 1968," USAEC Report GA-8530, Gulf Genera1 Atomic, March 29, 1968.

4-23. Besenbruch, G. E., J. H. Norman, C. L. Allen, and W. H. Weitzel, "Diffusion Behavior of Strontium in Graphite," Trans. Am. Nuc. Soc. 12, 81 (1969).

4-24. Chandra, D., and J. H. Norman, "Diffusion of Cesium Through Graphite," General Atomic Report GA-A13525, February 2, 1976.

4-25. Myers, B. F., and W. E. Be11, "Strontium Transport Data for HTGR Systems," USAEC Report GA-A13168, General Atomic Company, December 6,1974 . 


\section{DISCUSSION AND CONCLUSIONS}

The following is a discussion of the major conclusions drawn from the FTE-6 evaluation. Detailed results are presented at the end of the applicable sections within the report.

The evaluation of the irradiation performance of the fuel test element FTE- 6 has been directed toward the main objective of HTGR design verification. The attempted approach was to verify calculated irradiation parameters by measurements. In cases where more than one method had been available, measured results were tested for agreement. Calculated and measured parameters were compared with each other, and the established bias was tested for significance to discriminate the effect from possible measurement errors.

The temperature predictions were compared with measured in-pile thermocouple readings, which had been corrected for decalibration through a recalibration exercise of thermocouples retrieved from the FTE-5 and FTE-18 experiments. On a time average basis, very good agreement was found between measured and calculated temperatures. The actual time history, however, was significantly different for some time intervals.

Methods have been established to determine the thermal fluence profile from normalized cesium ( $\mathrm{Cs}-137$ and $\mathrm{Cs}-134 / \mathrm{Cs}-137$ ) distribution established from low failure TRISO fuel rods. The general indication is a slightly flatter (approximately $5 \%$ in peak) profile, with a shift towards the top end of the reactor, when compared with the predicted profile.

EOL power profiles were established through short-life isotopic (La-140) distributions. A comparison with longer-1ife isotopes revealed no significant change in the power profiles over the last one to two 
hundred days of reactor operations. It was therefore concluded that $\mathrm{Zr}-95$ could be used for EOL power distributions in cases where La-140 was already decayed away. Comparison of predicted with measured power profiles revealed good agreement for fuel rods and large axial perturbations coming from additional heat generation in spine samples.

The burnup calculations have been compared using several destructive and nondestructive methods of analysis. The methods of analysis were compared with each other and the combination of mass spectrometry and isotopic diluted mass spectrometry was selected as producing the most precise estimate. These methods are recommended for correcting calculated burnups as well as for calibrating nondestructive gamma scanning techniques for individual fuel rods. This combination was identified as the most cost effective method for burnup determination of fuel elements, and it has an additional advantage in providing power, fluence, and fuel failure information.

Stress calculations were carried out for all three graphite fuel bodies at midplane locations, and calculated stresses were compared with the ultimate tensile strength of the material as determined during manufacture of the graphite bodies and compensated for strength increase during irradiation. The peak stress situation was identified to have reached a stress leve1 of $153 \%$ during shutdowns within the period of irradiation. The residual stress level at end of life was still $128 \%$. A conservative failure criteria would expect structural failure when reaching a stress leve1 of $100 \%$. No defects were found however during destructive examination of the graphite.

Dimensional measurements of the graphite fuel bodies during PIE revealed significant residual bow, which exceeded more than four times the measured diametrical clearance within the sleeve. The stress level released through the deformation of the bow was assessed as up to $38 \%$ and the restraint stress within the sleeve was determined from the difference in bow and clearance to have reached a stress level of $45 \%$ at end of life shutdown. This stress level has to be superimposed onto the residual 
stresses quoted above, which will raise the predicted stress level within the fuel bodies even further above $100 \%$. This makes FTE- 6 an impressive stress experiment. Based on the evidence that no graphite damage was detected, it has been concluded that there was a certain conservatism of the stress calculation and failure criteria.

The deformation of the fuel bodies was partially traced toward azimuthal power variations between the four different fuel blends, each of which carried the same fissile but different fertile fuel loadings and therefore experienced a different amount of U-233 conversion. Systematical variation in the material properties cannot be excluded and will be measured during a destructive stress verification program outside the work scope of the results presented here.

Material strain measurements resulted in overprediction of the radial strain for the graphite fuel bodies, which was partially traced to the absence of modeling thermal and nuclear end effects for the unfueled ends of the graphite body with the one-dimensional heat transfer code (TREVER) used for the prediction. Inversion of the measured strain curvature was assessed for the low temperature range of the bottom body. This was consistent with FTE-4 results. Limited knowledge of the strain characteristic at low irradiation temperature could be the cause for not predicting the strain change correctly.

Fuel rod strain measurements were made on $80 \%$ of the 336 fuel rods irradiated in FTE- 6 . The axial versus radial strain ratio has been tested for isotopic behavior, which was the case for three out of the four fuel blends.* One blend clearly indicated an anisotropy factor of 0.8 , which meant larger shrinkage in the radial than in the axial direction. This may be attributed to some changes in the manufacturing process which evidently gave some preorientation to these fuel rods.

Comparison of predicted and measured strain values showed lack of agreement for a11 four blends in the positive and the negative sense. Fuel rod strain properties, derived from high exposure irradiation testing, * Within the accuracy of the measurements. 
apparently do not correlate with the fluence regime seen by the test elements. A stronger temperature correlation than that presently used for the strain data is also concluded from the strain measurements.

Dimensional changes of the sleeve dimensions are in reasonable agreement; sleeve-fuel body gaps, however, are overpredicted, although the number of measurements was very limited. Fuel rod/fuel body gaps did not deviate much from the predicted gaps because of the relative large BOL gap compared with the fuel rod dimensional change. The fuel rod strain, being an order of magnitude larger than for the graphite, primarily controls the gap change.

FTE-6 fuel performance was consistent with the results from previous companion test elements, FTE-3 and FTE-4 (Refs. 2-1 and 2-2). At peak exposures of $2.89 \times 10^{25} \mathrm{n} / \mathrm{m}^{2}(\mathrm{E} \geq 29 \mathrm{fJ})_{\mathrm{HTGR}}$ and $1430^{\circ} \mathrm{C}$ most of the fuel had low failure and little evidence of thermochemical effects (i.e., SiC attack, fuel kernel migration, or matrix coating interactions). The exception to this was the large amoeba migration and subsequent highpressure vessel failure of the $\mathrm{UO}_{2}$ TRISO fuel particles that were tested in one fuel rod variety. This behavior had also been observed in FTE-4 (Ref. 2-2) and $\mathrm{P} 13 \mathrm{~N}$ and $\mathrm{P} 13 \mathrm{P}$ (Ref. 4-12). The following results are highlighted.

- The visual appearance of most of the fuel rods was good. There were cases of surface fuel failure, unloading damage, matrix end cap cracking, and fuel rod bowing. Extensive fuel rod bowing of up to $0.58 \mathrm{~mm}$ over a fuel rod length of $49 \mathrm{~mm}$ was observed. This was traced to inhomogeneous fuel rod loadings and subsequent differences in the irradiation shrinkage along the cross section of the fuel rod. Part of this bow was restraint within the fuel hole geometry.

- Fuel failure measured by $\mathrm{Kr}-85 \mathrm{~m}$ fission gas release measurements showed up to $20 \%$ fuel failure in the $\mathrm{UO}_{2}-\mathrm{ThO}_{2}$ fuel rod 
blend and between $0.5 \%$ to $5 \%$ fuel failure in the remaining fuel blends and selected thermal stability spine samples.

- The amount of fuel failure observed for the $\mathrm{UO}_{2}$ variety was verified by prediction using the thermal analysis code TREVER by applying the kernel migration characteristics of this fuel.

- Gamma scan analysis of the fuel rods and graphite fuel bodies revealed a $20 \%$ cesium loss from the $\mathrm{UO}_{2}$ fuel variety for the center fuel body. Only $46 \%$ of the estimated cesium which escaped the fuel rods was found within the graphite fuel bodies. No cesium contamination was detected in the remainder of the sleeve left over from the disassembly operation. Unfortunately, a 120-deg section of the sleeve had to be destroyed during the unloading operation of the bend fuel bodies. This section happened to be opposite to the two releasing fuel holes 1 and 2. Therefore, escape of the unaccounted $54 \%$ of the cesium may have been possible through this section. A test for significance revealed that the amount of unaccounted cesium is outside the uncertainty of the measurements. A calibration of the gamma scanning geometry for the graphite bodies is required before final conclusions on the amount of cesium release can be made. The present assessment is that cesium left the element.

Analyses of diffusion spine samples began under FTE-3 and FTE-4 and have been continued with the higher fluence samples in FTE-6. The results derived have been added to the body of data assembled for the diffusion 'of the fission metals cesium, strontium, barium, and europium in graphite. Compilation of the data from the three elements has confirmed that strontium, barium, and europium can be treated using a Fickian model of diffusion (as is done in the reference and design codes FIPER and TRAFIC), and has aided in the assignment of temperature-dependent diffusion coefficients. Analysis of in-pile cesium behavior has confirmed that a twocomponent model is required in order to accurately model diffusion in 
graphite. The model put forth by Zumwalt in FTE-3 has been found to provide reasonable fits of the observed data.

The evaluation of the fuel test element FTE- 6 is regarded as a valuable source for fuel engineering data in support of HTGR fuel element designs. The presented statistical evaluation concept is proposed for use in the nuclear design verification of fission power fuel elements. 


\section{ACKNOWLEDGEMENT}

A comprehensive task such as that represented by this report cannot be carried out by a single person or group; it is, rather, the result of many contributions and contributors. The authors would particularly like to acknowledge the following personal contributions to this report.

- FTE-6 Test Element Design and Preparation

D. A. Nehrig, R. P. Morissette, W. J. Scheffel, and R. F. Turner

- FTE-6 Manufacturing and Assembly

Multiple group contributions

- FTE-6 Irradiation

Courtesy of Philadelphia Electric, represented by W. Birely

- Thermal Analysis and Design Verification

J. J. Saurwein

- Nuclear Analysis

F. McCord

- Hot Cell Data Acquisition

P. T. Mattson and staff, L. I. Mayweather, A. S. Mehner,

C. M. Miller, M. Scott, and W. Simpson

- Burnup Analysis

T. B. Crockett and D. M. Fleishman 
- Stress Analysis

D. D. Chiang and F. Tzung

- Peach Bottom Gamma Scanning

V. J. Orphen, V. C. Rodger, J. McKeney, all of Intelcom Rad Tech, and A. S. Mehner

- Fuel Gamma Scan Analysis

D. W. Hill, A. Bagierek, K. E. Buthe, R. De Nooy, and

S. Parikh

- Computer Program Development

T. L. Emrick and R. F. Korts

- Statistica1 Analysis Consultant

R. H. Leary

- Fission Gas Release Analysis

N. L. Baldwin, T. B. Crockett, J. H. Graves, and J. S. Greenwood

- Fission Product Analysis

D. Girotto, R. W. Tomlin, L. R. Zumwalt, and Glenda Hightower

- Editing

D. Novak, J. E. Baker, and J. E. Weaver and staff 
APPENDIX A

FIGURES 
FIGURE TITLE ERRATA

Figure titles as shown on Figs. 4-63 through 4-76 and 4-95 through 4-97 and 1isted in the List of Figures are incomplete. The full figure titles are as shown below:

Fig. 4-63. FTE-6 photomicrographs of fuel rod 2-1-7 irradiated to $2.88 \times 10^{25} \mathrm{n} / \mathrm{m}^{2}$ (E $\left.>29 \mathrm{fJ}\right)_{\mathrm{HTGR}}$ at $1128^{\circ} \mathrm{C}$ : (a) typical graphite matrix and (b) composite radial cross section

Fig. 4-64. FTE-6 photomicrographs of fuel rod 2-2-7 irradiated to $2.88 \times 10^{25} \mathrm{n} / \mathrm{cm}^{2}$ (E > $\left.29 \mathrm{fJ}\right)_{\mathrm{HTGR}}$ at $1128^{\circ} \mathrm{C}$ : (a) typical graphite matrix and (b) composite HTGR radial cross section

Fig. 4-65. FTE-6 photomicrographs of fuel rod 2-3-7 irradiated to $2.88 \times 10^{25} \mathrm{n} / \mathrm{m}^{2}$ (E $\left.>29 \mathrm{fJ}\right)_{\mathrm{HTGR}}$ at $1117^{\circ} \mathrm{C}$ : (a) typical graphite matrix and (b) composite radial cross section

Fig. 4-66. FTE-6 photomicrographs of fuel rod 2-6-7 irradiated to $2.88 \times 10^{25} \mathrm{n} / \mathrm{m}^{2}$ ( $\left.\mathrm{E}>29 \mathrm{fJ}\right)_{\mathrm{HTGR}}$ at $1121^{\circ} \mathrm{C}:$ (a) typical graphite matrix and (b) composite radial cross section

Fig. 4-67. FTE-6 photomicrographs of fuel rod 2-8-7 irradiated to $2.88 \times 10^{25} \mathrm{n} / \mathrm{m}^{2}$ (E $\left.>29 \mathrm{fJ}\right)_{\mathrm{HTGR}}$ at $1121^{\circ} \mathrm{C}$ : (a) typical graphite matrix and (b) composite radial cross section

Fig. 4-68. FTE-6 photomicrographs of representative fuel particles from fuel rod 2-1-7 irradiated to $2.88 \times 10^{25} \mathrm{n} / \mathrm{m}^{2}$ ( $\mathrm{E}>29 \mathrm{fJ}$ ) $\mathrm{HTGR}$ at $1128^{\circ} \mathrm{C}$ : (a) bright field of $\mathrm{UO}_{2}$ TRISO pressure vessel failure, (b) dark field of (a), (c) $\mathrm{ThO}_{2} \mathrm{BISO}-\mathrm{UO}_{2}$ TRISO pair 
Fig. 4-69. FTE-6 photomicrographs of UO 2 TRISO particle showing SiC attack from fuel rod 2-1-7 irradiated to $2.88 \times 10^{25} \mathrm{n} / \mathrm{m}^{2}$ $(\mathrm{E}>29 \mathrm{fJ})_{\mathrm{HTGR}}$ at $1128^{\circ} \mathrm{C}:$ (a) $\mathrm{UO}_{2}$ TRISO particle with migrating kernel, (b) dark field of particle in (a), (c) magnification of kernel-SiC interface showing SiC attack

Fig. 4-70. FTE-6 photomicrographs of representative fuel particles from fuel rod 2-2-7 irradiated to $2.88 \times 10^{25} \mathrm{n} / \mathrm{m}^{2}$ ( $\mathrm{E}>29 \mathrm{fJ}$ ) HTGR at $1128^{\circ} \mathrm{C}$ : (a) bright field of $\mathrm{UO}_{2}$ TRISO - ThO ${ }_{2}$ BISO pair (b) dark field of (a), (c) randomly migrating $\mathrm{UO}_{2}$ kernels

Fig. 4-71. FTE-6 photomicrographs of representative fuel particles from fuel rod 2-3-7 irradiated to $2.88 \times 10^{25} \mathrm{n} / \mathrm{m}^{2}$ ( $\mathrm{E}>29 \mathrm{fJ}$ ) $\mathrm{HTGR}$ at $1117^{\circ} \mathrm{C}$ : (a) bright field of (Th,U) $\mathrm{C}_{2}$ TRISO - $\mathrm{ThC}_{2}$ BISO pair, (b) dark field of (a)

Fig. 4-72. FTE-6 photomicrographs of (Th,U) $C_{2}$ TRISO particle from fuel rod 2-3-7 showing matrix-particle interaction: (a) light field, (b) dark field, (c) high-magnification interaction area

Fig. 4-73. FTE-6 photomicrographs of $\mathrm{ThC}_{2}$ TRISO particle in fuel rod 2-6-7 with OPyC failure: (a) bright field - kernel hydrolyzed during the examination, (b) dark field

Fig. 4-74. FTE-6 photomicrographs of representative fissile (Th,U) $\mathrm{C}_{2}$ particle from fuel rod 2-6-7 irradiated to $2.88 \times 10^{25} \mathrm{n} / \mathrm{m}^{2}$ (E > $29 \mathrm{fJ})_{\mathrm{HTGR}}$ at $1112^{\circ} \mathrm{C}$ : (a) bright field, (b) dark field

Fig. 4-75. FTE-6 photomicrographs of representative fuel particles from fuel rod 2-8-7 irradiated to $2.88 \times 10^{25} \mathrm{n} / \mathrm{m}^{2}$ (E $>29 \mathrm{fJ}$ ) $\mathrm{HTGR}$ at $1121^{\circ} \mathrm{C}$ : (a) bright field of $\mathrm{UC}_{2}$ TRISO - ThC 2 BISO pair, (b) dark field of (a) 
Fig. 4-76. FTE-6 photomicrographs of UC 2 TRISO particle from fuel rod 2-8-7 showing apparent contamination attack: (a) bright field, (b) dark field

Fig. 4-95. FTE-6 stereophotographs of thermal stability spine samples: (a) $\mathrm{UC}_{2}$ TRISO $(100 \mu \mathrm{m})$ VSM $(4000-302)$ particles from spine sample TS $6-5$ irradiated to $2.0 \times 10^{25} \mathrm{n} / \mathrm{m}^{2}$ (E $\left.>29 \mathrm{fJ}\right)_{\mathrm{HTGR}}$ at $1105^{\circ} \mathrm{C}$, (b) $\mathrm{ThC}_{2}$ TRISO particles (4000-247) from spine sample TS $18-3$ irradiated to $2.0 \times 10^{25} \mathrm{n} / \mathrm{m}^{2}$ ( $\left.\mathrm{E}>29 \mathrm{fJ}\right)_{\mathrm{HTGR}}$ at $1050^{\circ} \mathrm{C}$

Fig. 4-96. FTE-6 photomicrographs of typical microstructure of $\mathrm{ThC}_{2}$ TRISO particle (4000-247) from spine sample TS 18-3 irradiated to $2.0 \times 10^{25} \mathrm{n} / \mathrm{m}^{2}$ (E > $\left.29 \mathrm{fJ}\right)_{\mathrm{HTGR}}$ at $1139^{\circ} \mathrm{C}$; dark spots in kernel are due to hydrolysis during sample preparation: (a) bright field, (b) dark field

Fig. 4-97. FTE-6 photomicrographs of typical nilcrostructure of $\mathrm{UC}_{2} 120-\mu \mathrm{m}$ (VSM) particles (4000-302) from spine sample TS 6-5 irradiated to $2.0 \times 10^{25} \mathrm{n} / \mathrm{m}^{2}$ (E > $\left.29 \mathrm{fJ}\right)_{\mathrm{HTGR}}$ at $1105^{\circ} \mathrm{C}$ : (a) bright field, (b) dark field showing mixed fission products in IPyC, (c) high magnification of SiC attack in particle shown in (a) and (b) 


\section{LIST OF FIGURES}

2-1. Peach Bottom typical fuel test element assembly. . . . . . A-1

2-2. Peach Bottom graphite fuel body from fuel test elements . . A-2

2-3. Peach Bottom fuel test element eight-hole teledial cross section .................... $\mathrm{A}-3$

3-1. Peach Bottom Core 2 operating parameters, TREVER simulation. A-4

3-2. Peach Bottom Core 2 power history from thermal balance . . . A-5

3-3. Peach Bottom Core 2 thermal power, TREVER simulation . . . A-6

3-4. FTE-6 GAUGE-calculated power history . . . . . . . . . . . A-7

3-5. Peach Bottom Core 2 normalized axial fast flux, thermal flux, and power distribution .. . . . . . . . . . A-8

3-6a. FTE-6 normalized Cs-137 versus Cs-134/Cs-137 CPM profiles from fuel stack 3 and 5 gamma scans . . . . . . . . . A-9

3-6b. FTE-6 normalized, predicted, and measured fluence profiles determined from fuel stack 3 and 5 gamma scans . . . . . . A-10

3-7. FTE-6 radial temperature distribution (typica1). . . . . . A-11

3-8. FTE-6 time-averaged temperatures (fuel stacks 7 and 8) . . A-12

3-9. FTE-6 temperature envelope (fuel stacks 7 and 8) . . . . A-13

3-10. FTE-6 axial temperature profiles (BOL-298 EFPD). . . . . . A-14

3-11. FTE-6 axial temperature profiles (298-343 EFPD) . . . . . A-14

3-12. FTE-6 axial temperature profiles (343-385 EFPD) . . . . . A-14

3-13. FTE-6 axial temperature profiles (385-499 EFPD) . . . . . A-15

3-14. FTE-6 axial temperature profiles (499-564 EFPD) . . . . . A-15

3-15. FTE-6 axial temperature profiles (564-610 EFPD) . . . . . A-15

3-16. FTE-6 axial temperature profiles (610-701 EFPD) . . . . A-16

3-17. FTE-6 axial temperature profiles (701-748 EFPD) . . . . . A-16

3-18. FTE-6 axial temperature profiles (748-788 EFPD) . . . . . A-16

3-19. FTE-6 axial temperature profiles (788-818 EFPD) . . . . . A-17

3-20. FTE-6 axial temperature profiles (818-835 EFPD) . . . . . A-17

3-21. FTE-6 axial temperature profiles (835-858 EFPD) . . . . . A-17

3-22. FTE-6 axial temperature profiles (858-889 EFPD) . . . . . A-18

3-23. FTE-6 axial temperature profiles (889-EOL) . . . . . . A-18 


\section{LIST OF FIGURES (Continued)}

3-24. FTE-6 axial temperature profiles (EOL) . . . . . . . . A-18

3-25. Peach Bottom thermocouple recalibration plot of $\mathrm{Ti}$

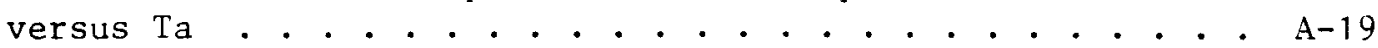

3-26. Peach Bottom thermocouple recalibration terms $K$ and $T_{0} \cdot . A-A 0$

3-27a. FTE-6 comparison of predicted temperatures and thermocouple readings . . . . . . . . . . . . . . . . $\mathrm{A}-21$

3-27b. FTE-6 TREVER simulation and thermocouple comparison . . . A-21

3-28. Peach Bottom Core 2 normalized axial distributions of fissile FIMA from FEVER calculations . . . . . . . . . A-22

3-29. Peach Bottom Core 2 normalized axial distribution of fertile FIMA from FEVER calculations . . . . . . . . . A-23

3-30. Peach Bottom Core 2 normalized axial distribution of mixed FIMA for (5.5 Th:U) particles from FEVER calculations . . A-24

3-31. Peach Bottom Core 2 normalized axial distributions for Th-232, U-233, U-237, and U-235 from. FEVER calculations. . A-25

3-32. Peach Bottom Core 2 normalized axial distribution of U-236, U-238, and Pu-239 from FEVER calculations. . . . . . . A-26

3-33. FTE-6 total FIMA measured by gamma spectroscopy . . . . . A-27

3-34. Finite element model . . . . . . . . . . . . A-28

3-35. FTE-6 fuel body 1 temperature and stress contour plots for startup at BOL................ . . A-29

3-36. FTE-6 fuel body 2 temperature and stress contour plots for startup at BOL . . . . . . . . . . . . . . . . A-30

3-37. FTE-6 fuel body 3 temperature and stress contour plots for startup at BOL . . . . . . . . . . . . . A $\mathrm{A}-31$

3-38. FTE- 6 fuel bodies 1,2 , and 3 stress directions for startup at BOL ................. 3-32

3-39. FTE-6 fuel body 1 temperature and stress contour plots for operation at EOL ................. . . A-33

3-40. FTE-6 fuel body 2 temperature and stress contour plots for operation at EOL ............ . . . . . . A-34

3-41. FTE-6 fuel body 3 temperature and stress contour plots for operation at EOL . . . . . . . . . . . . . A-35

3-42. FTE-6 fuel bodies 1, 2, and 3 stress directions for operation at EOL . . . . . . . . . . . . . A-36

3-43. FTE-6 fuel body 1 max-min temperatures and max-min stress histories for operation and shutdown . . . . . . . . . . A-37

3-44. FTE- 6 fuel body 2 max-min temperatures and max-min stress histories for operation and shutdown . . . . . . . . A-38 


\section{LIST OF FIGURES (Continued)}

3-45. FTE-6 fuel body 3 max-min temperatures and max-min stress histories for operation and shutdown . . . . . . . . . A-39

3-46. FTE-6 fuel body 1 temperature at EOL operation and stress contour plots for EOL shutdown . . . . . . . . . . A-40

3-47. FTE-6 fuel body 2 temperature at EOL operation and stress contour plots for EOL shutdown . . . . . . . . . . . A-41

3-48. FTE- 6 fuel body 3 temperature at EOL operation and stress contour plots for EOL shutdown . . . . . . . . . . . A-42

3-49. FTE-6 fuel bodies 1, 2, and 3 stress directions for EOL shutdown . . . . . . . . . . . . . . . . . . A-43

4-1. FTE-6 Peach Bottom single-channel gamma-scan charts . . . A-44

4-2. FTE-6 Peach Bottom EOL gamma scans: CPM profiles for $\mathrm{Cs}-134, \mathrm{Cs}-137$, and $\mathrm{Cs}-137 / \mathrm{Cs}-134$ ratios . . . . . . . . A-45

4-3. FTE-6 Peach Bottom EOL gamma scans: CPM profiles for $\mathrm{Ce}-141$, and $\mathrm{Ru}-103$. . . . . . . . . . . . . A $\mathrm{A}-45$

4-4. FTE-6 Peach Bottom EOL gamma scans: CPM profiles for $\mathrm{Pa}-233, \mathrm{La}-140$, and $\mathrm{Zr}-95$. . . . . . . . . . . A $\mathrm{A}-45$

4-5. FTE-6 Peach Bottom EOL gamma scans: profiles for $\mathrm{Zr}-95 /$ $\mathrm{Cs}-137, \mathrm{Ru}-103 / \mathrm{Zr}-95$, and $\mathrm{Ce}-141 / \mathrm{Zr}-95$ ratios . . . . A-45

4-6. FTE-6 Peach Bottom fuel gamma scan, azimuthal configuration A-46

4-7. FTE-6 comparison of La-140 and Zr-95 normalized CPM profiles as determined from Peach Bottom EOL gamma scan 1. . . . A-47

4-8. FTE-6 predicted and measured EOL axial power distribution determined from Peach Bottom EOL gamma scan $1 . . . . .9 A-47$

4-9. FTE-6 predicted and measured EOL axial power distribution determined from fuel stack 1 gamma scans. . . . . . . . A-48

4-10. FTE-6 Cs-137/2r-95 CPM profile versus core height for fuel stack 1.... . . . . . . . . . . . . . A A-49

4-11. FTE-6 fuel gamma scan comparison of Cs-137/2r-95 CPM profiles from two different methods . . . . . . . . A-49

4-12. FTE-6 fuel gamma scan comparison of normalized $\mathrm{Ce}-141 / \mathrm{Zr}-95$ and Ce-144/Zr-95 CPM profiles . . .. . . . . . A-50

4-13. FTE-6 fuel gamma scan comparison of normalized Ru-103/Zr-95 and $\mathrm{Ru}-106 / \mathrm{Zr}-95 \mathrm{CPM}$ profiles . . . . . . . . . . . A-50

4-14. FTE-6 neutron dose rate versus position along axis . . . . A-51

4-15. FTE-6 identification . . . . . . . . . . . . . . . A-52

4-16. FTE-6 composite photograph of total element . . . . . . A-53

4-17. Composite photographs of fuel bodies 1,2 , and $3 . . .$. A-55 


\section{LIST OF FIGURES (Continued)}

4-18. FTE-6 measured and calculated sleeve strain . . . . . . A-56

4-19. FTE-6 fue1 body bow measurement device . . . . . . . . . A-57

4-20. FTE-6 fuel body 1 bow . . . . . . . . . . . . . A-58

4-21. FTE-6 fuel body 2 bow . . . . . . . . . . . . A-59

4-22. FTE-6 fuel body 3 bow . . . . . . . . . . . . . A-60

4-23. FTE-6 stack power variations from radial gamma scanning of body 2. . . . . . . . . . . . . . . . . . . A-61

4-24. FTE-6 measured and calculated fuel body strain . . . . . A-62

4-25. FTE-6 measured and calculated sleeve fuel body radial gaps . A-63

4-26. FTE-6 measured and calculated fuel rod strain (holes 1 and 2)................... . . A-64

4-27. FTE-6 measured and calculated fuel rod strain (holes 3 and 4)................... . . A-64

4-28. FTE-6 measured and calculated fuel rod strain (holes 5 and 6)................... . A-64

4-29. FTE-6 measured and calculated fuel rod strain (holes 7 and 8)................... . . . . . . . . . . . . . .

4-30. FTE-6 fuel rod anisotropy (holes 1 and 2) ........ A-65

4-31. FTE-6 fuel rod anisotropy (holes 3 and 4) . . . . . . A-65

4-32. FTE-6 fuel rod anisotropy (holes 5 and 6) . . . . . . . A-65

4-33. FTE-6 fuel rod anisotropy (holes 7 and 8) . . . . . . A-65

4-34. FTE-6 measured and predicted fuel rod/fuel hole gap (holes 1 and 2)............................ A-66

4-35. FTE-6 measured and predicted fuel rod/fuel hole gap (holes 3 and 4)................... . . . . . . . . . . . . .

4-36. FTE-6 measured and predicted fuel rod/fuel hole gap (holes 5 and 6).................. . . A-66

4-37. FTE-6 measured and predicted fuel rod/fuel hole gap (holes 7 and 8)... . . . . . . . . . . . . A-66

4-38. FTE-6 composite photograph of stack 1 , body 1 . . . . . A-67

4-39. FTE-6 composite photograph of stack 2, body $1 . . . .$. . A-67

4-40. FTE-6 composite photograph of stack 6 , body 1 . . . . . A-67.

4-41. FTE-6 composite photograph of stack 8, body 1 . . . . . A-67

4-42. FTE-6 composite photograph of stack 1 , body 2 . . . . . A-68

4-43. FTE-6 composite photograph of stack 3, body 2 . . . . . A-68

4-44. FTE-6 composite photograph of stack 5, body 2 . . . . . A-68

4-45. FTE-6 composite photograph of stack 7, body 2 . . . . A-68 


\section{LIST OF FIGURES (Continued)}

4-46. FTE-6 composite photograph of stack 2, body 3 . . . . . . A-69

4-47. FTE-6 composite photograph of stack 4, body 3 . . . . . . A-69

4-48. FTE-6 composite photograph of stack 6 , body 3 . . . . . . . A-69

4-49. FTE-6 composite photograph of stack 8, body 3 . . . . . . A-69

4-50. FTE-6 typical bowed rod $(2-2-1)$ - center body . . . . . . A-70

4-51. FTE-6 typical bowed rod (3-1-3) - top body . . . . . . . A-70

4-52. FTE-6 typical bowed $\operatorname{rod}(3-1-12)$ - top body . . . . . . A-70

4-53. FTE-6 typical bowed $\operatorname{rod}(3-2-3)-$ top body . . . . . . . A-70

4-54. FTE-6 fue1 rod homogeneity of bowed fuel rods . . . . . . A-71

4-55. FTE-6 visual examination of fuel rod 2-1-7 . . . . . . . A-72

4-56. FTE-6 visual examination of fuel $\operatorname{rod} 2-2-7 . \ldots$. . . . . . A-73

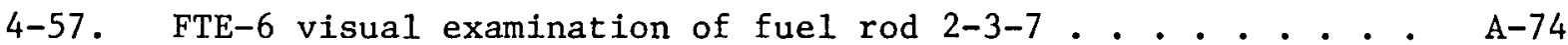

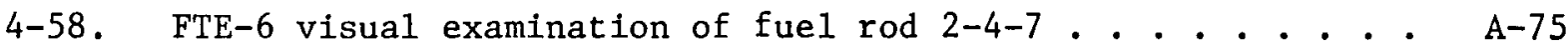

4-59. FTE-6 visual examination of fuel rod 2-5-7 . . . . . . . A-76

4-60. FTE-6 visual examination of fuel $\operatorname{rod} 2-6-7 . \ldots$. . . . . . A-77

4-61. FTE-6 visual examination of fuel rod 2-7-8 . . . . . . . A-78

4-62. FTE-6 visual examination of fuel rod 2-8-7 . . . . . . . . A-79

4-63. FTE-6 photomicrographs of fuel rod 2-1-7 . . . . . . . . . A-81

4-64. FTE-6 photomicrographs of fuel $\operatorname{rod} 2-2-7 . . . . . . . .4-83$

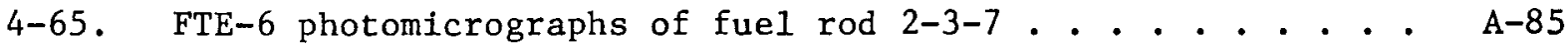

4-66. FTE-6 photomicrographs of fuel rod 2-6-7 . . . . . . . . . A-87

4-67. FTE-6 photomicrographs of fuel rod 2-8-7. . . . . . . . . A-89

4-68. FTE-6 photomicrography of representative fuel particles

from fuel rod $2-1-7$................ A-91

4-69. FTE-6 photomicrographs of $\mathrm{UO}_{2}$ TRISO particle showing SiC attack from fuel rod 2-1-7............ . . A-92

4-70. FTE-6 photomicrographs of representative fuel particles from fue1 $\operatorname{rod} 2-2-7$. . . . . . . . . . . . . . A-93

4-71. FTE-6 photomicrographs of representative fuel particles from fuel rod 2-3-7 . . . . . . . . . . . . . . A-94

4-72. FTE-6 photomicrographs of (Th,U) $C_{2}$ TRISO particle from fuel rod 2-3-7 showing matrix-particle interaction . . . . . A-95

4-73. FTE-6 photomicrographs of ThC2 TRISO particle in fuel

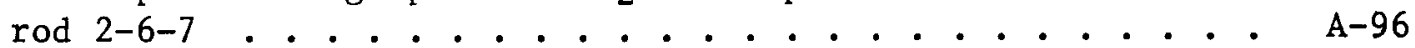

4-74. FTE-6 photomicrographs of representative fissile (Th,U) $\mathrm{C}_{2}$ particle from fuel rod 2-6-7. . . . . . . . . . . A-97 


\section{LIST OF FIGURES (Continued)}

4-75. FTE-6 photomicrographs of representative fuel particles from

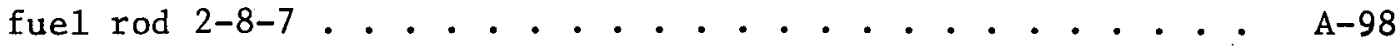

4-76. FTE-6 photomicrograph of $\mathrm{UC}_{2}$ TRISO particle from fuel rod 2-8-7 showing apparent contamination attach . . . . . . . A-99

4-77. FTE-6 single-channel gamma scans of fuel rods used in fuel examination .................. A-100

4-78. Schematic of gamma scanning apparatus at General Atomic hot cell....................... A-101

4-79. FTE-6 single-channel gamma scan comparison of fuel rods . . A-102 4-80. FTE-6 Cs-137/Zr-95 ratio for fuel rods in stacks 1 and 2 . . A-103 4-81. FTE-6 Cs-136/Zr-95 ratio for fuel rods in stacks 3 and 4.. A-103 4-82. FTE-6 Cs-136/2r-95 ratio for fuel rods in stacks 5 and 6 . . A-103 4-83. FTE-6 Cs-136/Zr-95 ratio for fuel rods in stacks 7 and 8 . . A-103 4-84. FTE-6 Cs-137 loss from fuel rods in stacks 1 and 2 . . . . A-104 4-85. FTE-6 autoradiography of graphite fuel body number 2 slice . A-105 4-86. FTE-6 Cs-134 (605 keV) profile in outer web of fuel body $2 . A-106$ 4-87. FTE-6 Cs-137 (662 keV) profile in outer web of fuel body 2 . A-107 4-88. FTE-6 Cs-134 (604 keV) profile in inner web of fuel body 2 . A-108 4-89. FTE-6 Cs-137 (662 keV) profile in inner web of fuel body 2 . A-109 4-90. FTE-6 Eu-154 (123 keV) profile in inner web of fuel body 2 . A-110 4-91. FTE-6 graphite fuel body gamma-scanned areas . . . . . . . A-111

4-92. FTE-6 fue1 body single-channel gamma scans of cesium . . . A-113

4-93. TREVER fuel rod cross section of fuel kernel migration segments ................... A-115

4-94. Design of type II thermal stability crucibles . . . . . . . A-116

4-95. FTE-6 stereophotographs of thermal stability spine samples . A-117

4-96. FTE-6 photomicrographs of typical microstructure of $\mathrm{ThC}_{2}$

TRISO particle from spine sample TS 18-3........ A-118

4-9.7. FTE-6 photomicrographs of typical microstructure of $\mathrm{UC}_{3}$ 120- $\mu \mathrm{m}$ (VSM) particles from spine sample TS 4-5 .... . A-119

4-98. FTE-6 GA hot cell gamma scan of body 1 spine samples . . . A-120

4-99. FTE-6 GA hot cell gamma scan of body 2 spine samples . . . A-121

4-100. FTE-6 GA hot cell gamma scan of body 3 spine samples . . . A-122

4-101. FTE-6 design of diffusion spine samples. . . . . . . . . . A-123

4-102. FTE-6 SR-85 profile in center post of diffusion sample 13. . A-124

4-103. FTE-6 Sr-85 profile in crucible wall of diffusion sample 13.................. . . A-124 


\section{LIST OF FIGURES (Continued)}

4-104. FTE-6 Sr-85 profile in center post of diffusion sample 17 . A-124

4-105. FTE-6 Sr-85 profile in crucible wall of diffusion

sample 17 ................... A-124

4-106. FTE-6 Sr-85 profile in the center post of diffusion

sample 19 . . . . . . . . . . . . . . . . . A-125

4-107. FTE-6 Sr-85 profile in crucible wall of diffusion

sample 19 . . . . . . . . . . . . . . . . . A-125

4-108. FTE-3, FTE-4, and FTE-6 strontium diffusion coefficients

plotted as a function of crucible irradiation

temperature. . . . . . . . . . . . . . . . A $\mathrm{A}-126$

4-109. FTE-6 Eu-154 profile in center post of diffusion sample 40 . A-127

4-110. FTE-6 Eu-154 profile in crucible wall of diffusion

sample 40................. . . A-127

4-111. FTE-3, FTE-4, and FTE-6 europium diffusion coefficients in graphite versus crucible irradiation temperature . . . . . A-128

4-112. FTE-6 Cs-134 profile in center post of diffusion sample 13 . A-129

4-113. FTE-6 Cs-134 profile in crucible wall of diffusion sample 13 . . . . . . . . . . . . . . . . . . A-129

4-114. FTE-6 Cs-134 profile in center post of diffusion sample 17 . A-129

4-115. FTE-6 Cs-134 profile in crucible wall of diffusion sample 17 .................. . . A-129

4-116. FTE-6 Cs-134 profile in center post of diffusion sample 19. A-130

4-117. FTE-6 Cs-134 profile in crucible wall of diffusion sample 19 .................. . . A-130

4-118. FTE-6 Cs-134 profile in center post of diffusion sample 33 . A-130

4-119. FTE-6 Cs-134 profile in crucible wall of diffusion sample 33 .................. A-130

4-120. FTE-6 Cs-134 profile in center post of diffusion sample 40 . A-131

4-121. FTE-6 Cs-134 profile in crucible wall of diffusion sample 40 . . . . . . . . . . . . . . . . . . A A-131

4-122. FTE-6 Cs-134 profile in center post of diffusion sample 60 . A-131

4-123. FTE-6 Cs-134 profile in crucible wall of diffusion sample 60 ................... . . . A-131

4-124. FTE-3, FTE-4, and FTE-6 diffusion coefficients of cesium (Deff) as a function of time-averaged irradiation temperature ............... . . A-132 


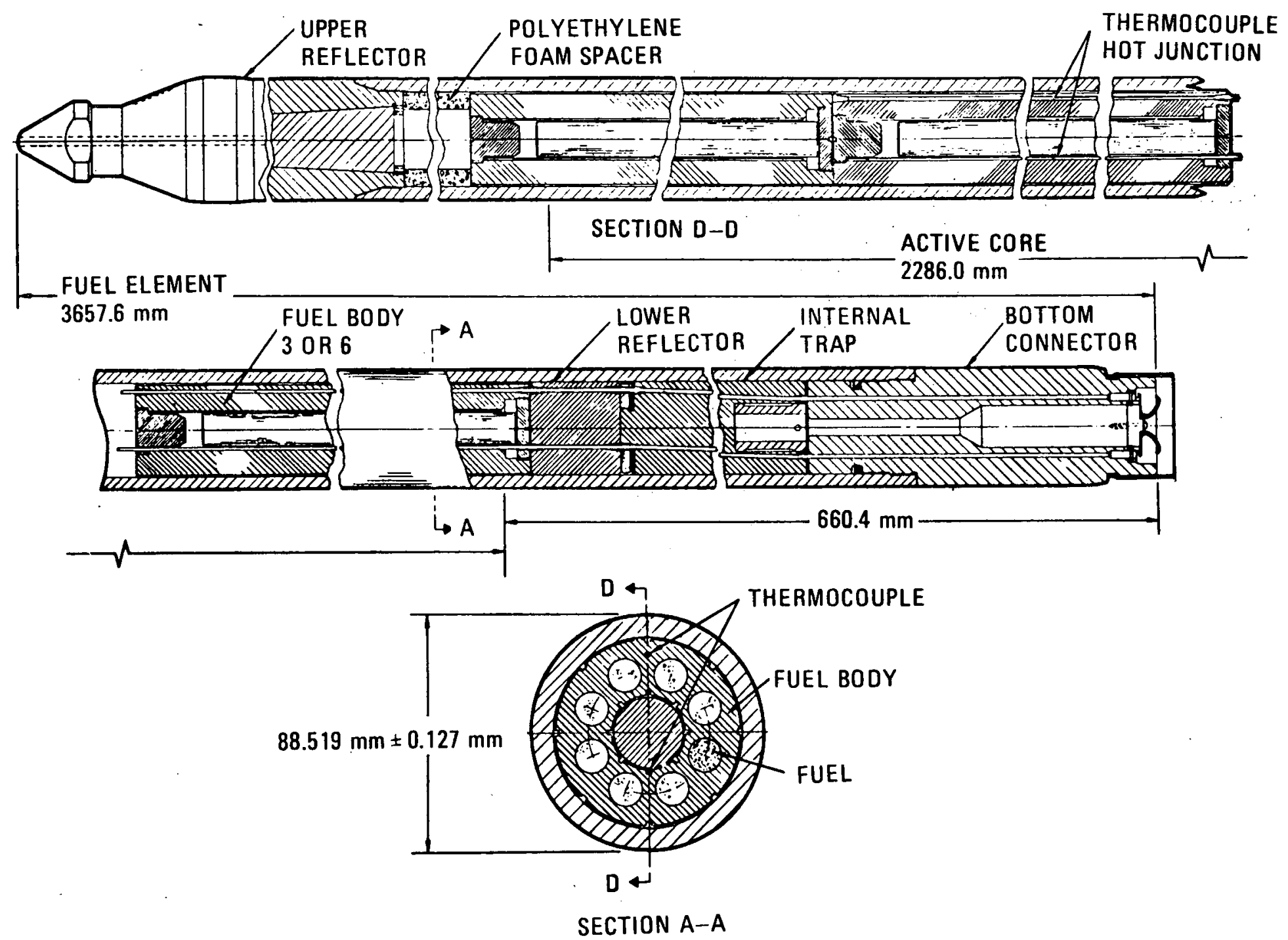

Fig. 2-1. Peach Bottom typical test element assembly 


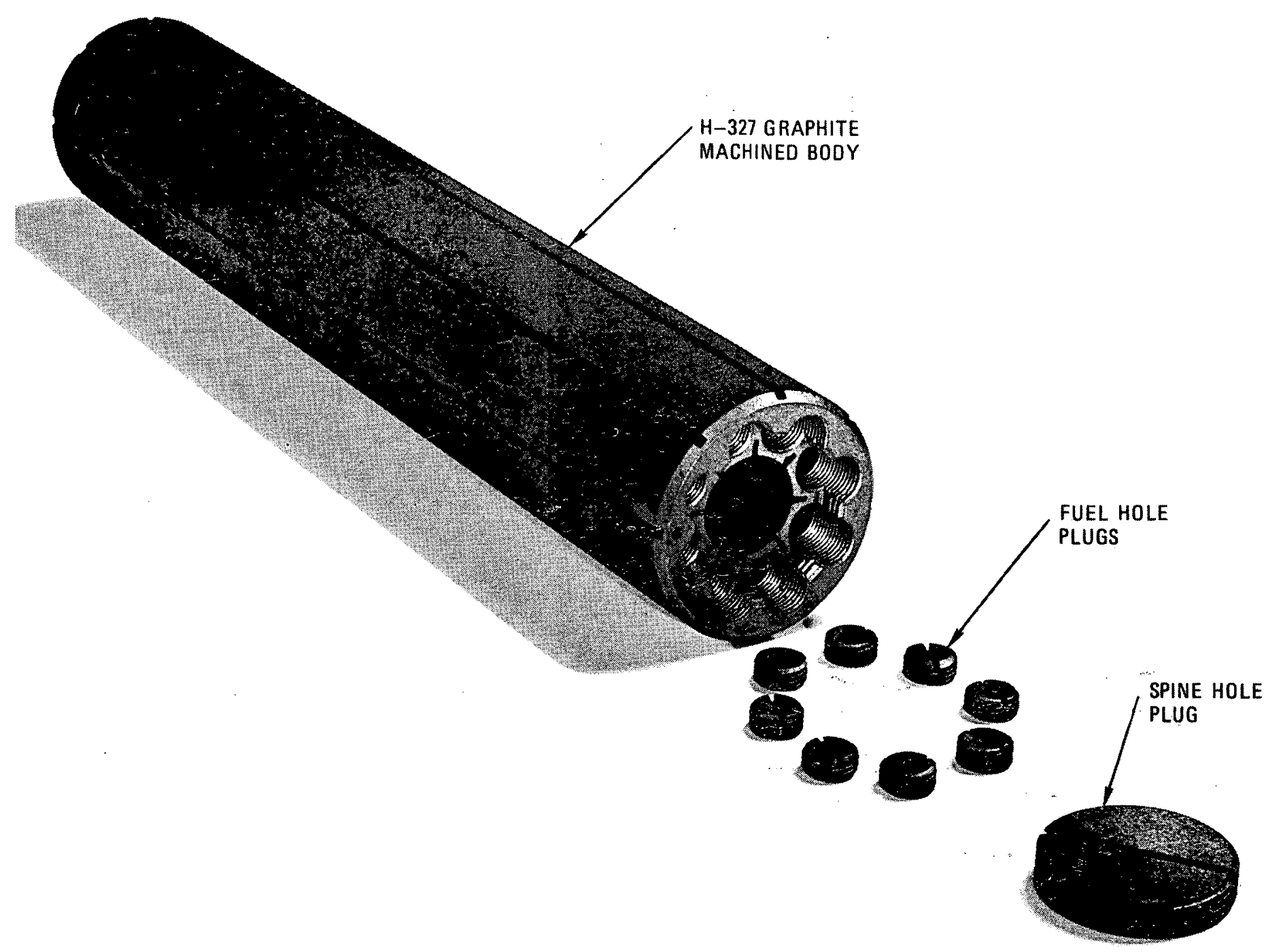

Fig. 2-2. Peach Bottom graphite fuel body from fuel test elements 


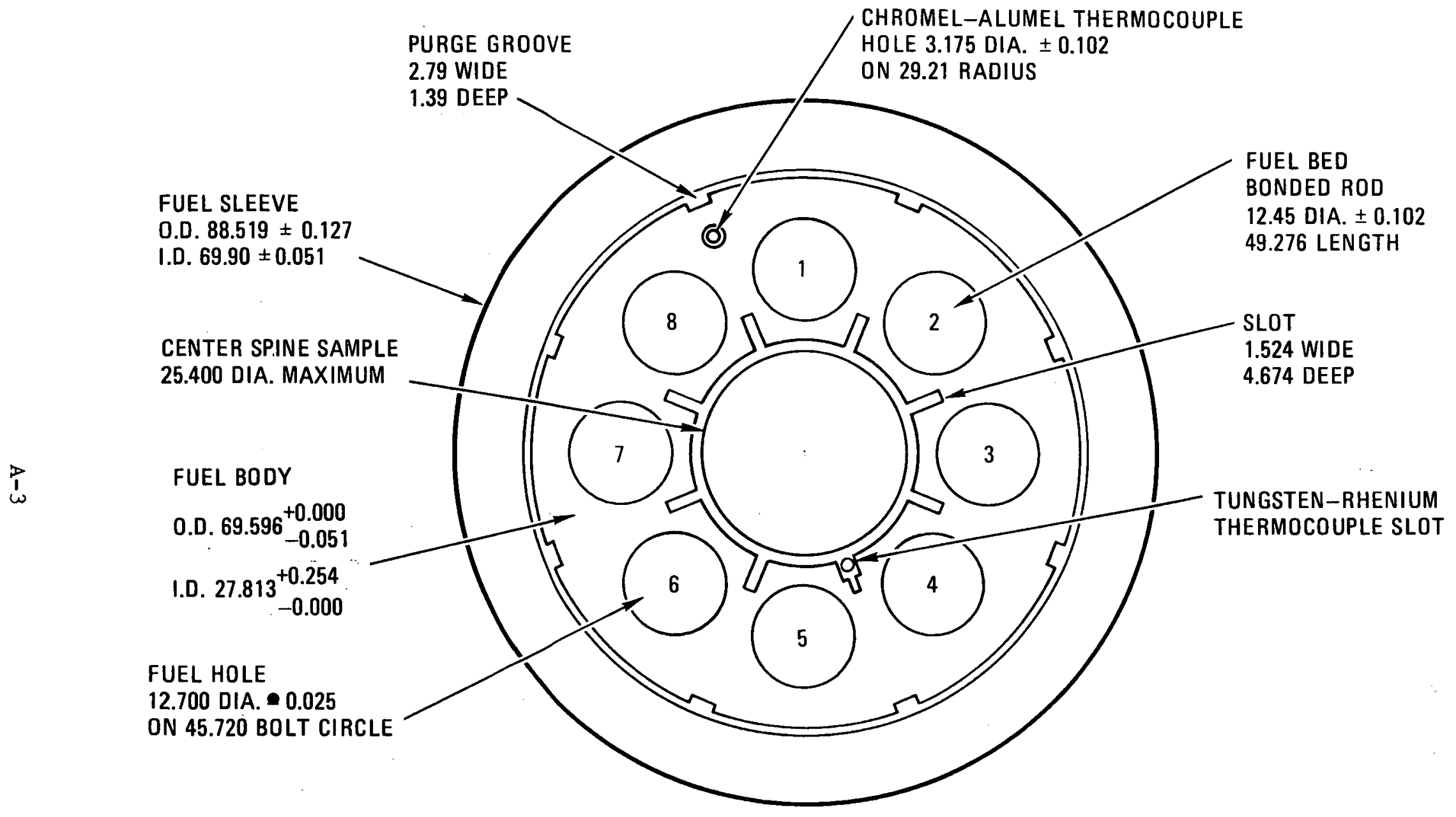

ALL DIMENSIONS

IN MILLIMETERS

Fig. 2-3. Peach Bottom fuel test element eight-hole teledial cross section 


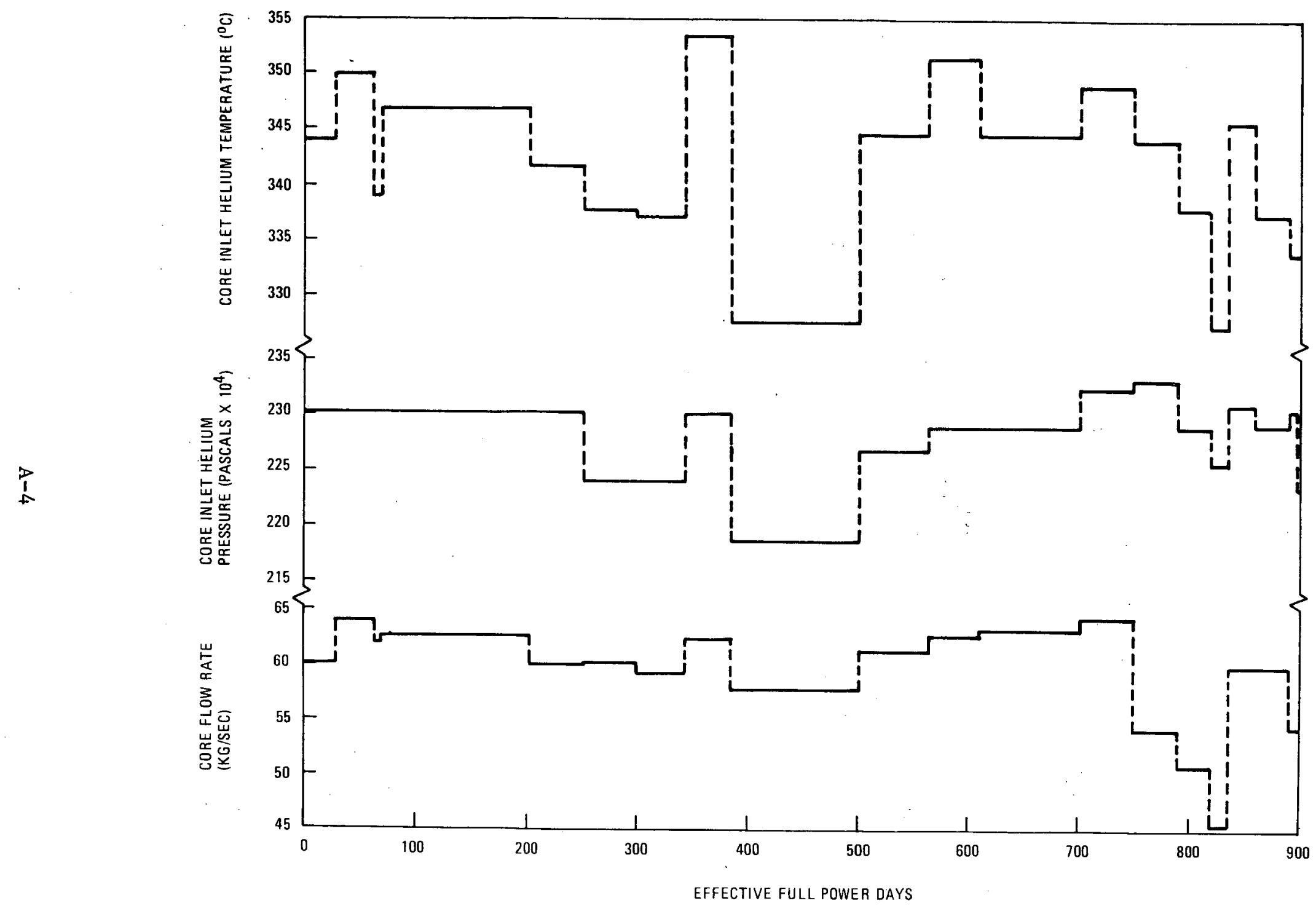

Fig. 3-1. Peach Bottom Core 2 operating parameters, TREVER simulation 


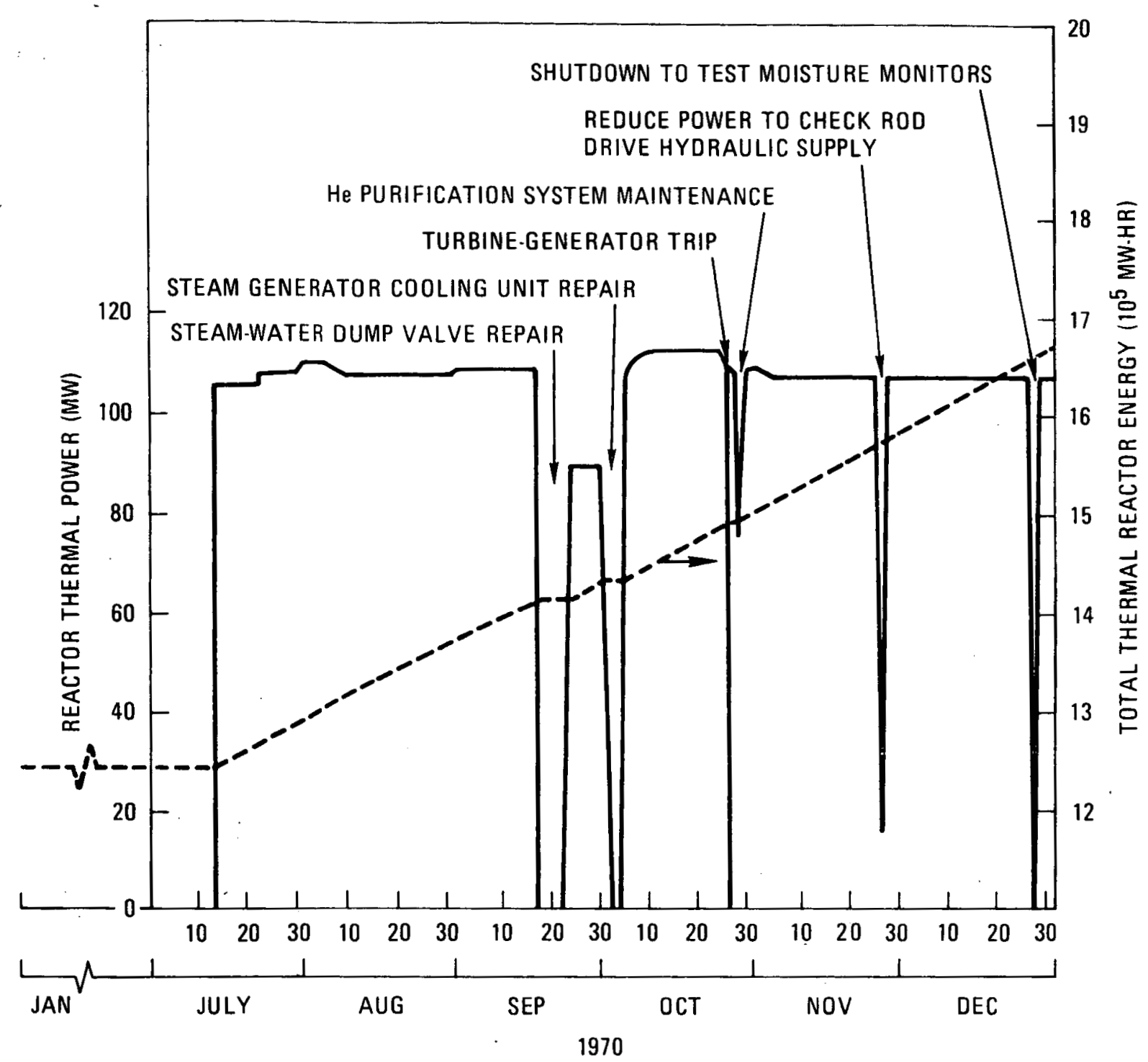

Fig. 3-2. Peach Bottom Core 2 power history from thermal balance 


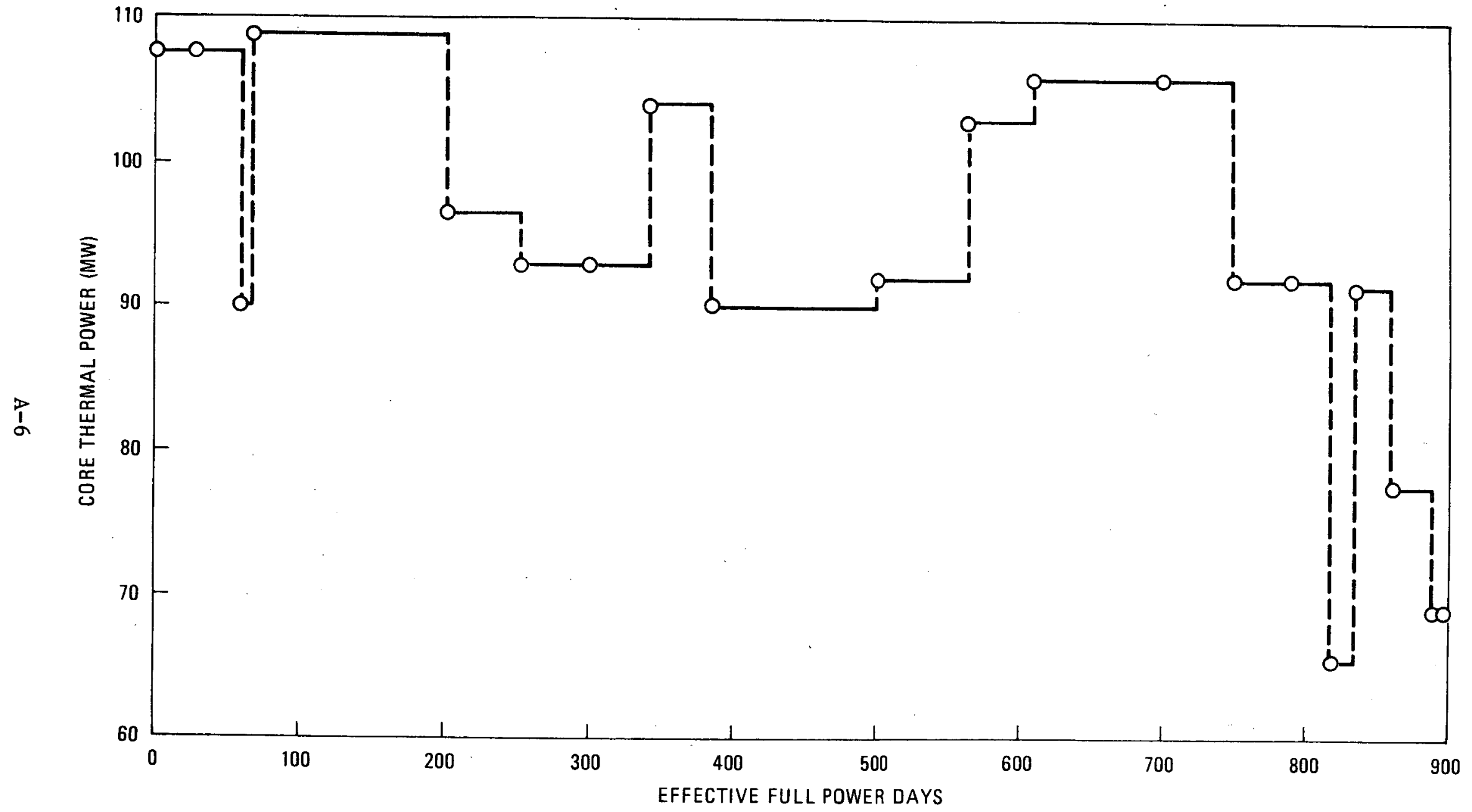

Fig. 3-3. Peach Bottom Core 2 thermal power, TREVER simulation 


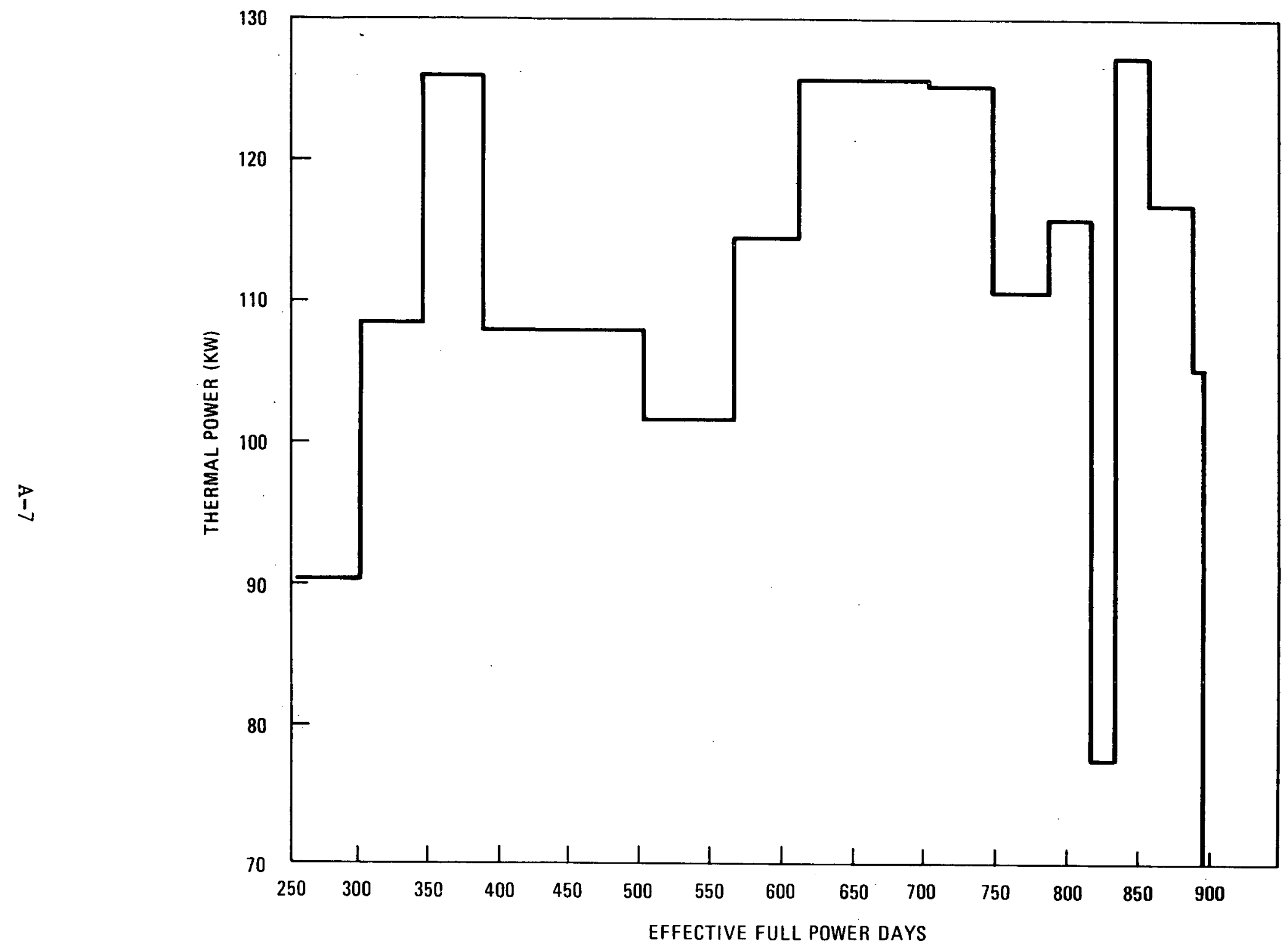

Fig. 3-4. FTE-6 GAUGE-calculated power history 

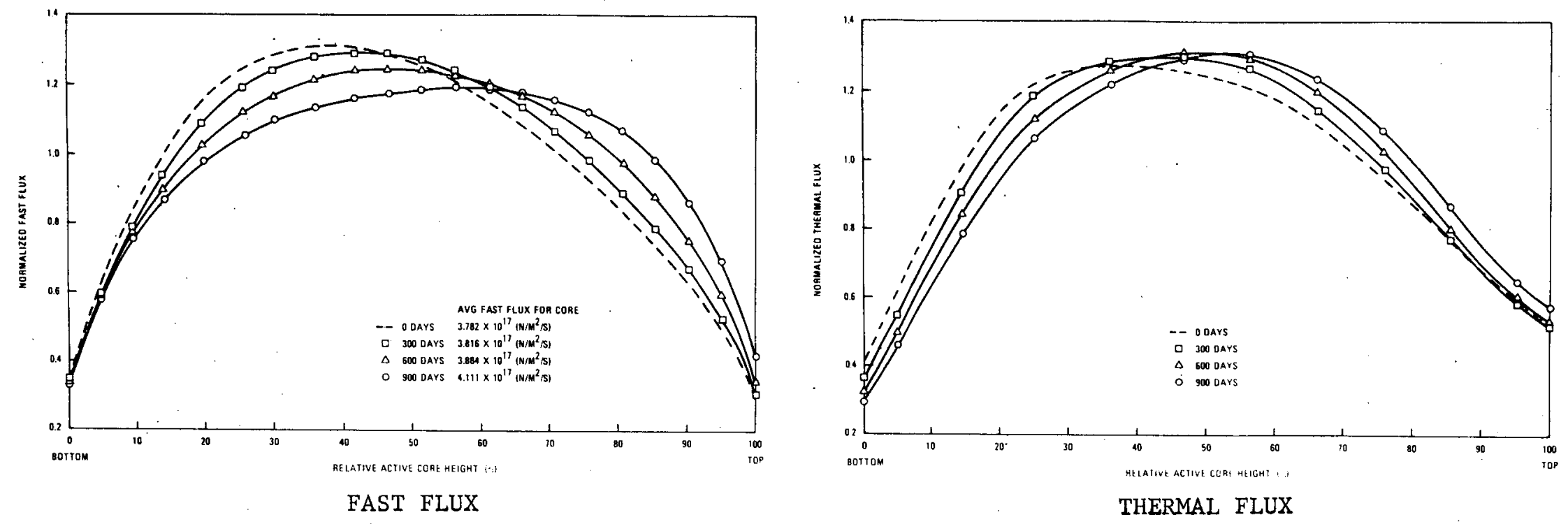

1
$\infty$

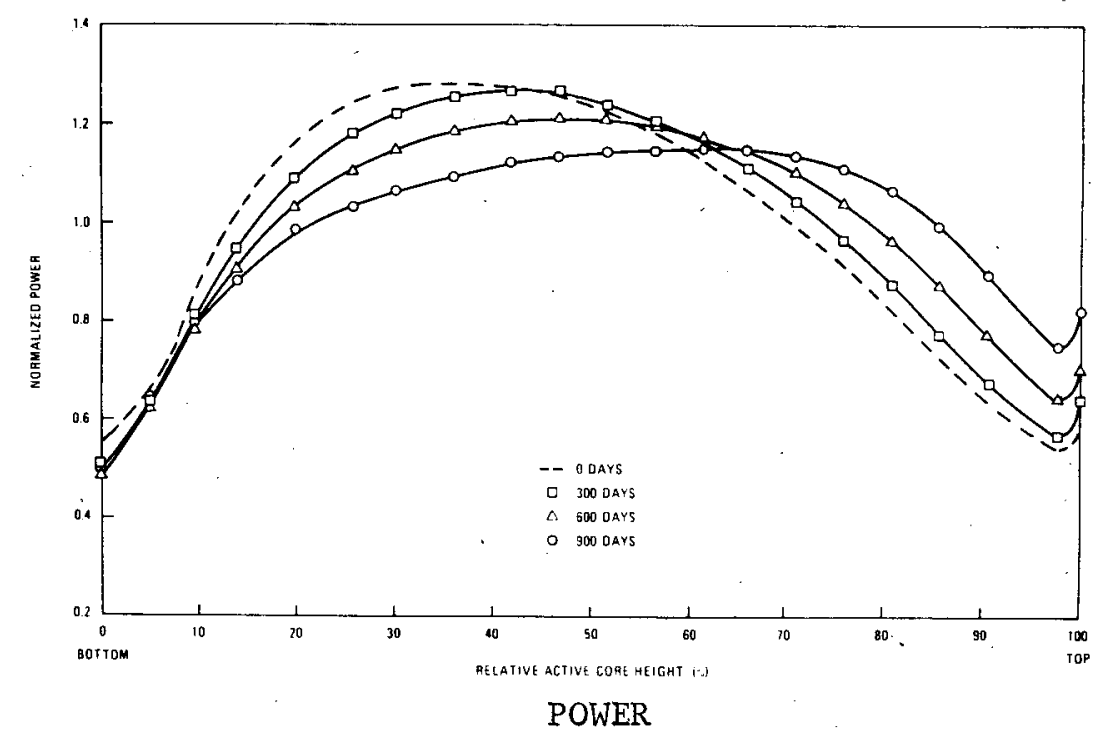

Fig. 3-5. Peach Bottom Core 2 normalized axial fast flux, thermal flux, and power distribution 


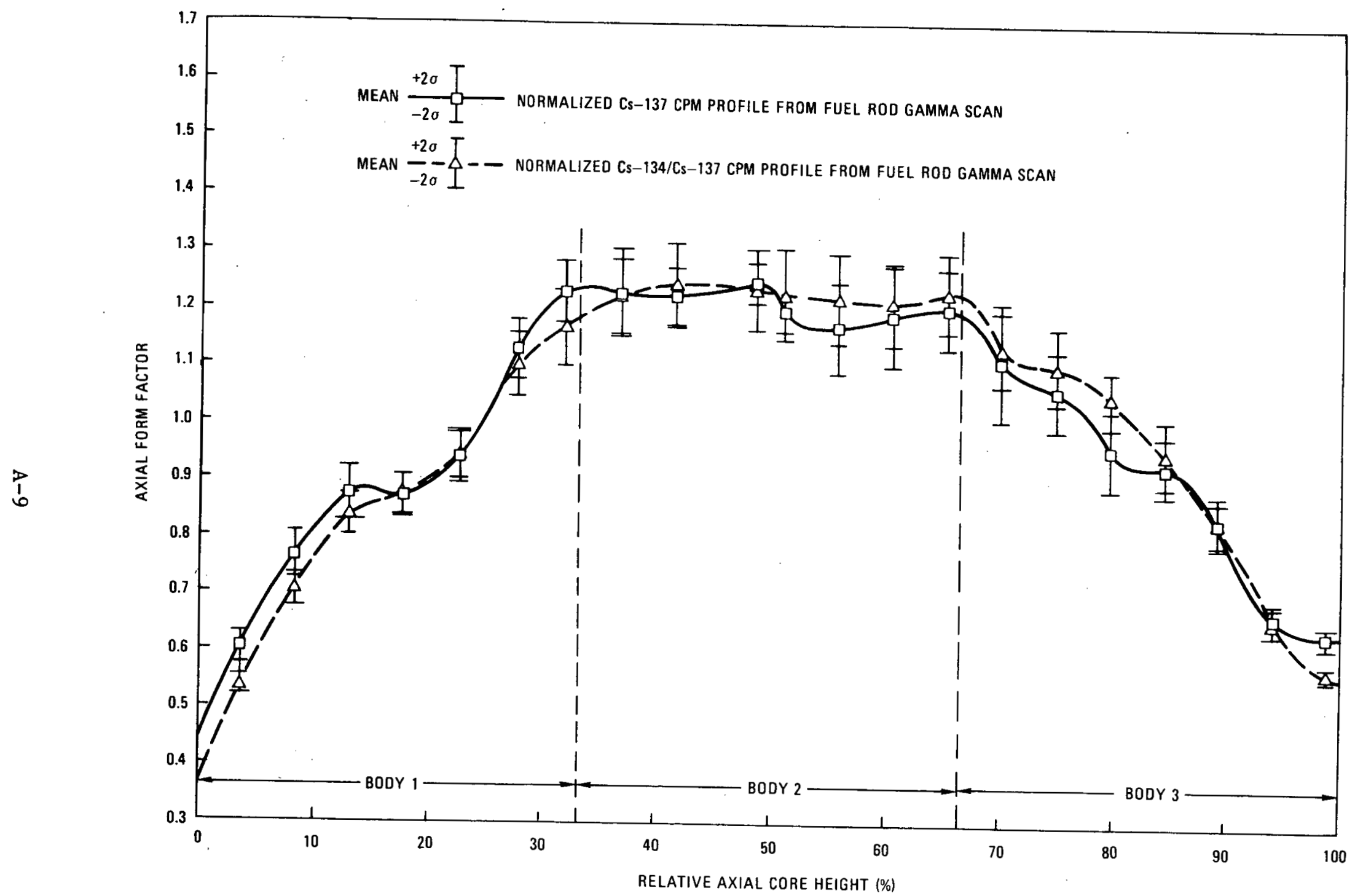

Fig. 3-6(a). FTE-6 normalized Cs-137 versus Cs-134/Cs-137 CPM profiles from fuel stack 3 and 5 gamma scans 


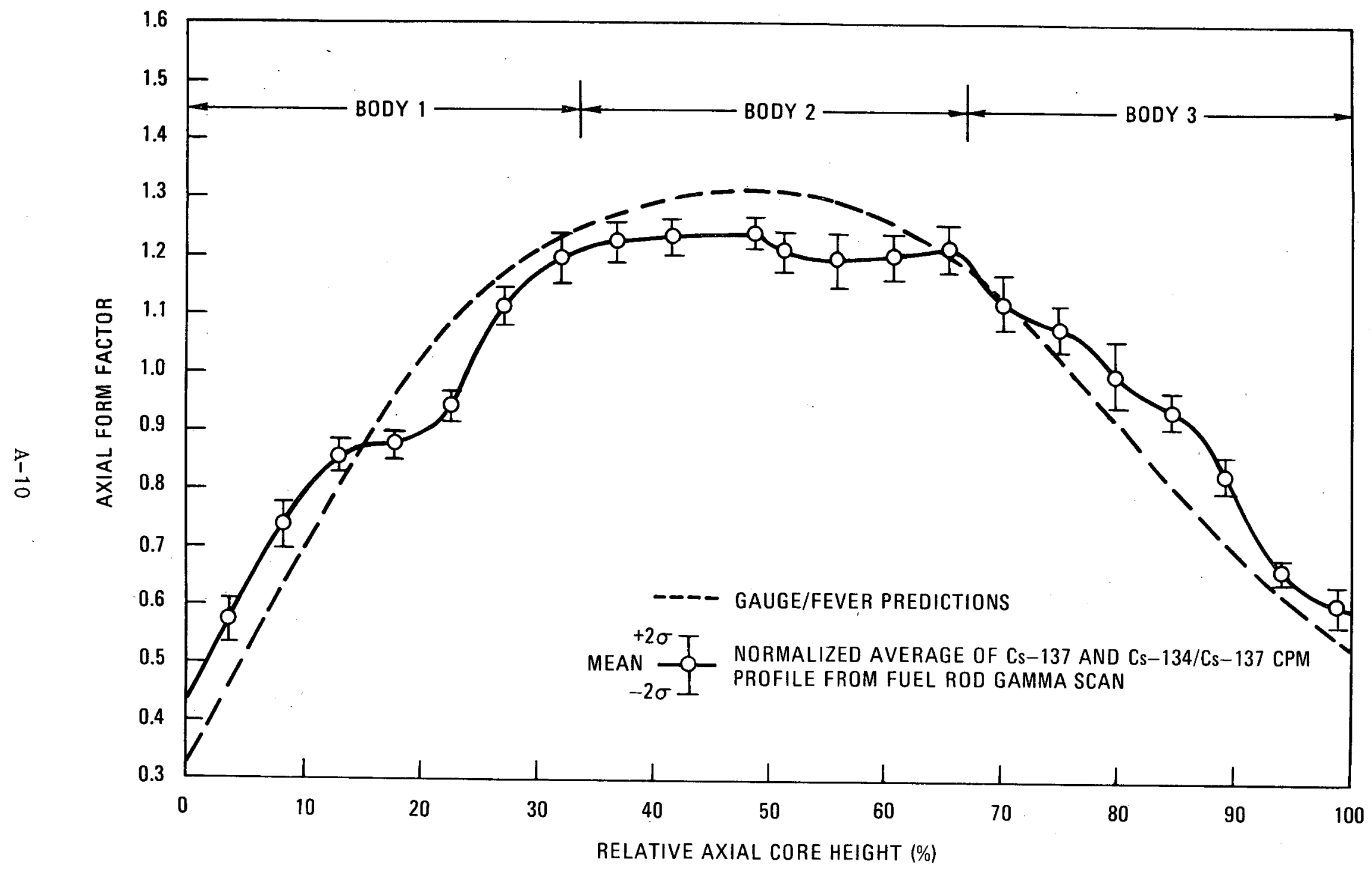
Fig. 3-6(b). FTE-6 normalized, predicted, and measured fluence profiles determined from fuel stack 3 and
5 gamma scans 


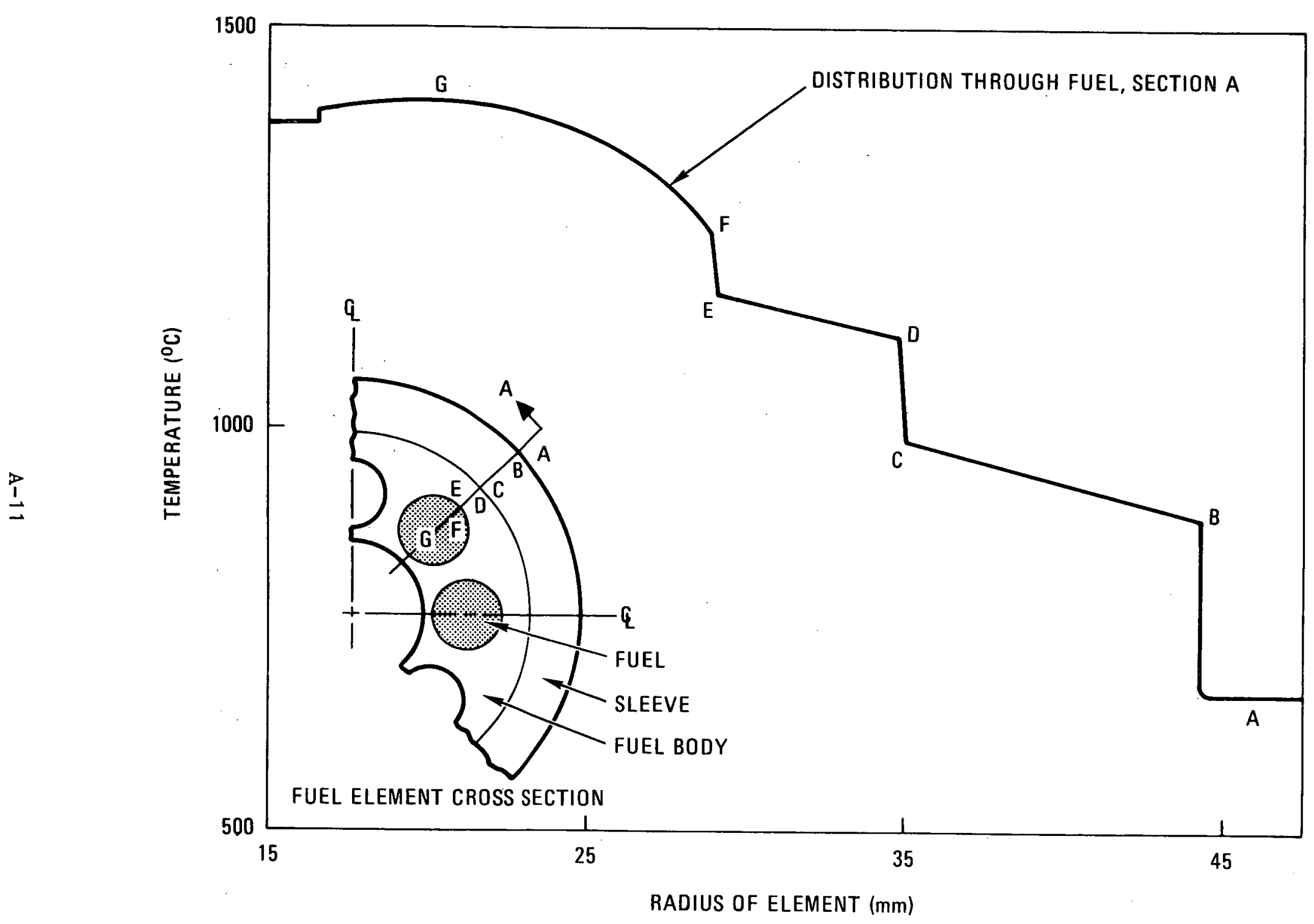

Fig. 3-7. FTE-6 radial temperature distribution (typical) 


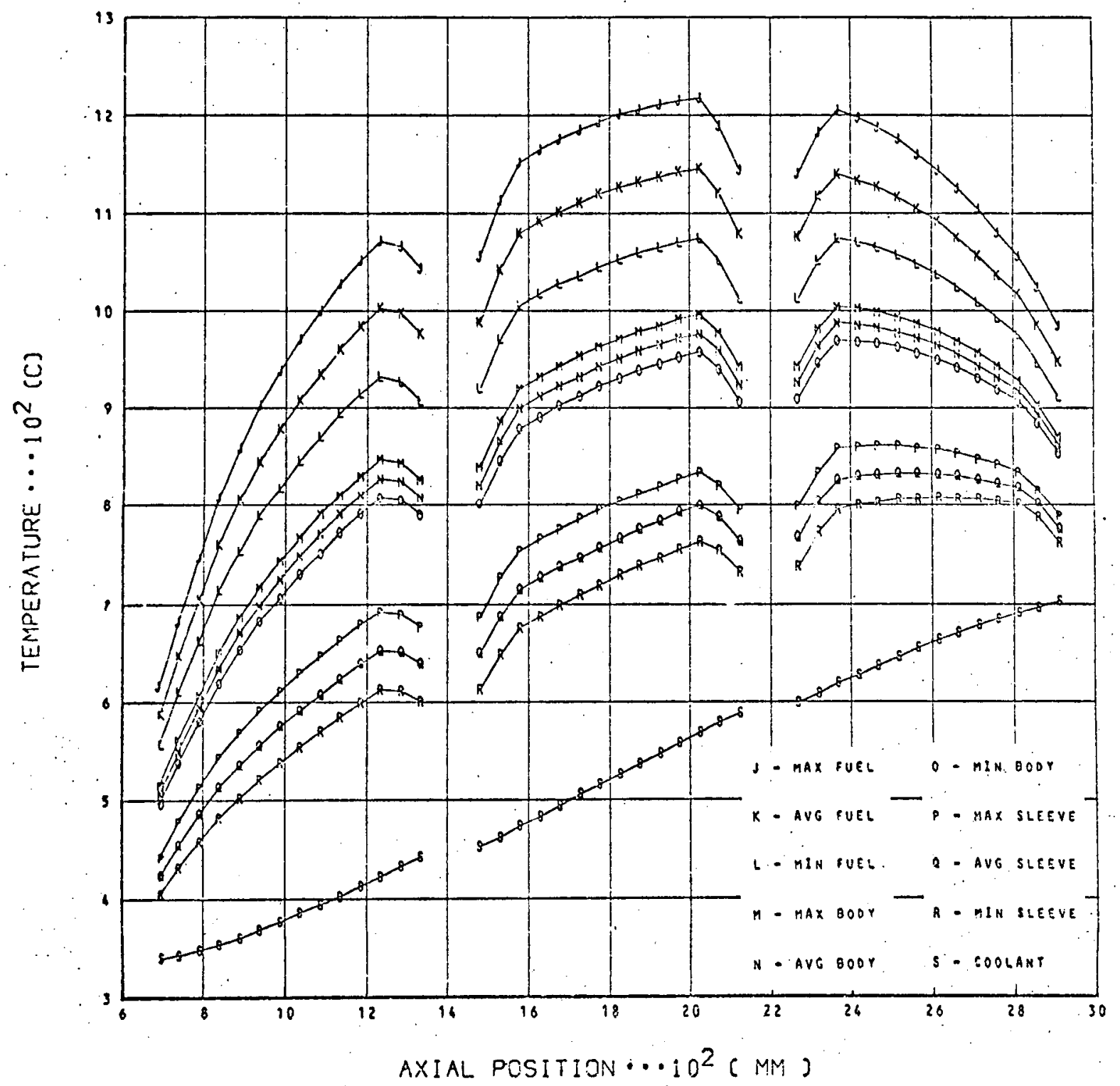

Fig. 3-8. FTE-6 time-averaged temperature (fuel stacks 7 and 8) 


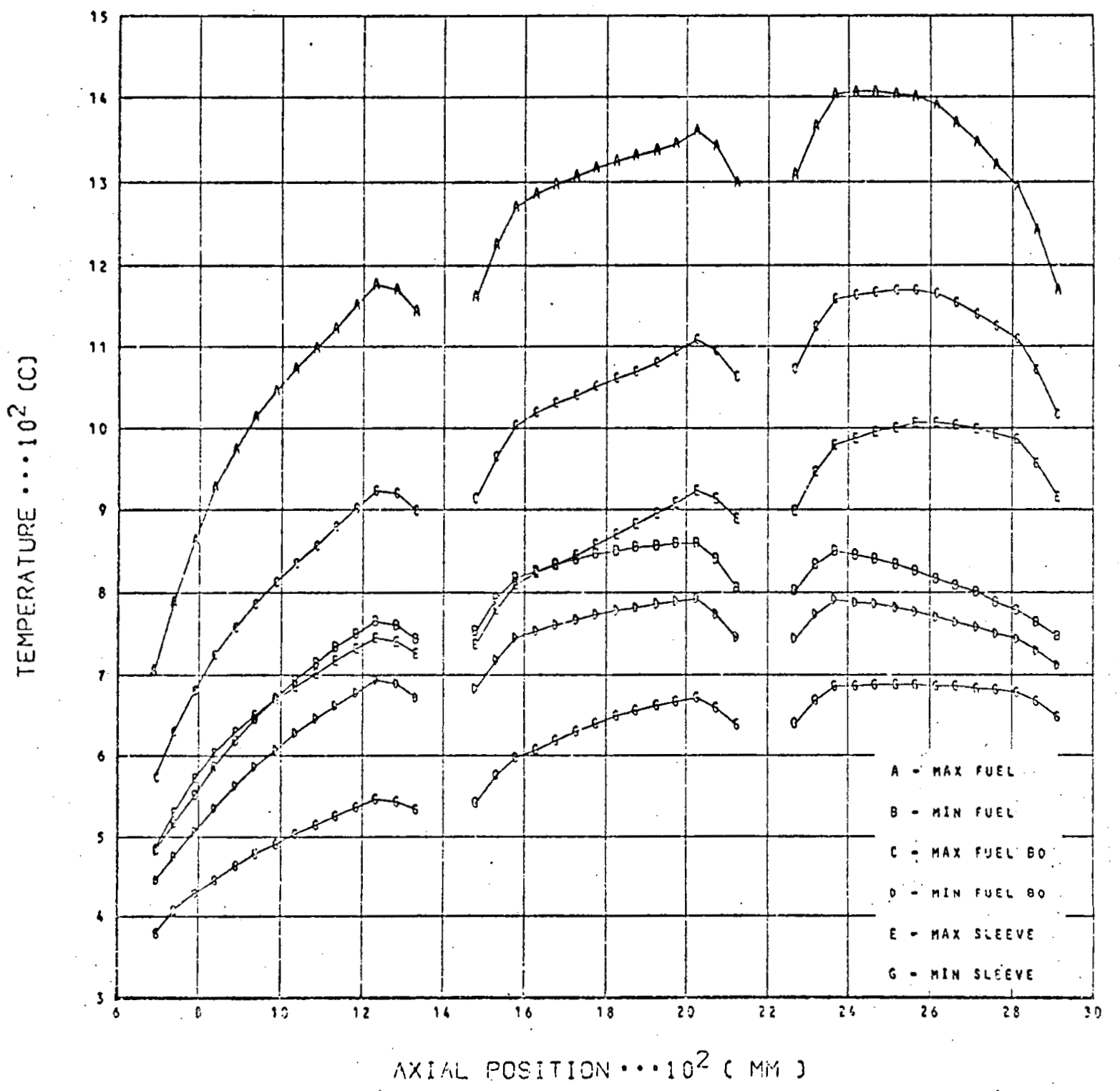

Fig. 3-9. FTE-6 temperature envelope (fuel stacks 7 and 8) 


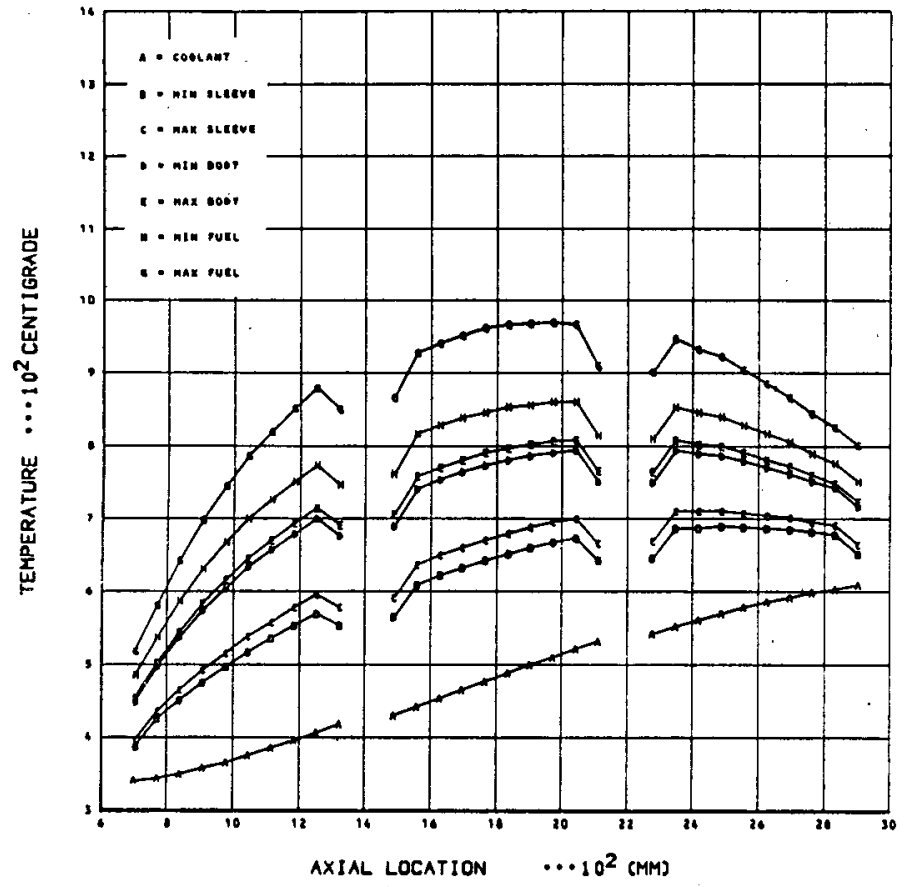

Fig. 3-10. FTE-6 axial temperature profiles (BOL-298 EFPD)

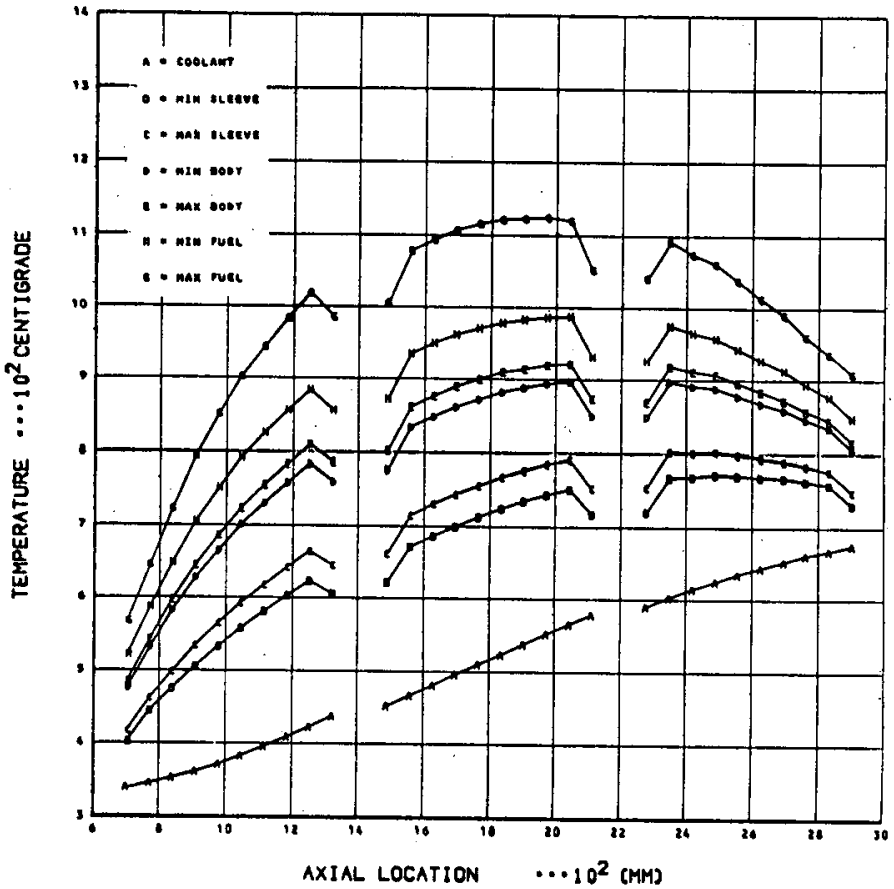

Fig. 3-11. FTE-6 axial temperature profiles (298-343 EFPD)

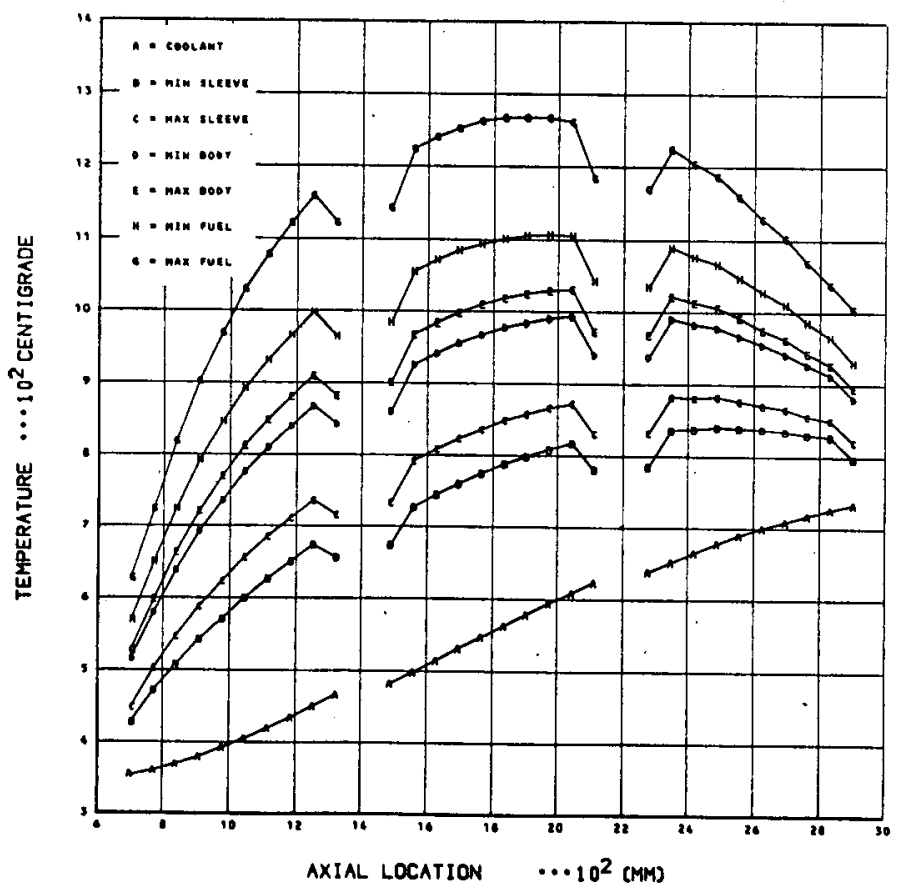

Fig. 3-12. FTE-6 axial temperature profiles (343-385 EFPD) 


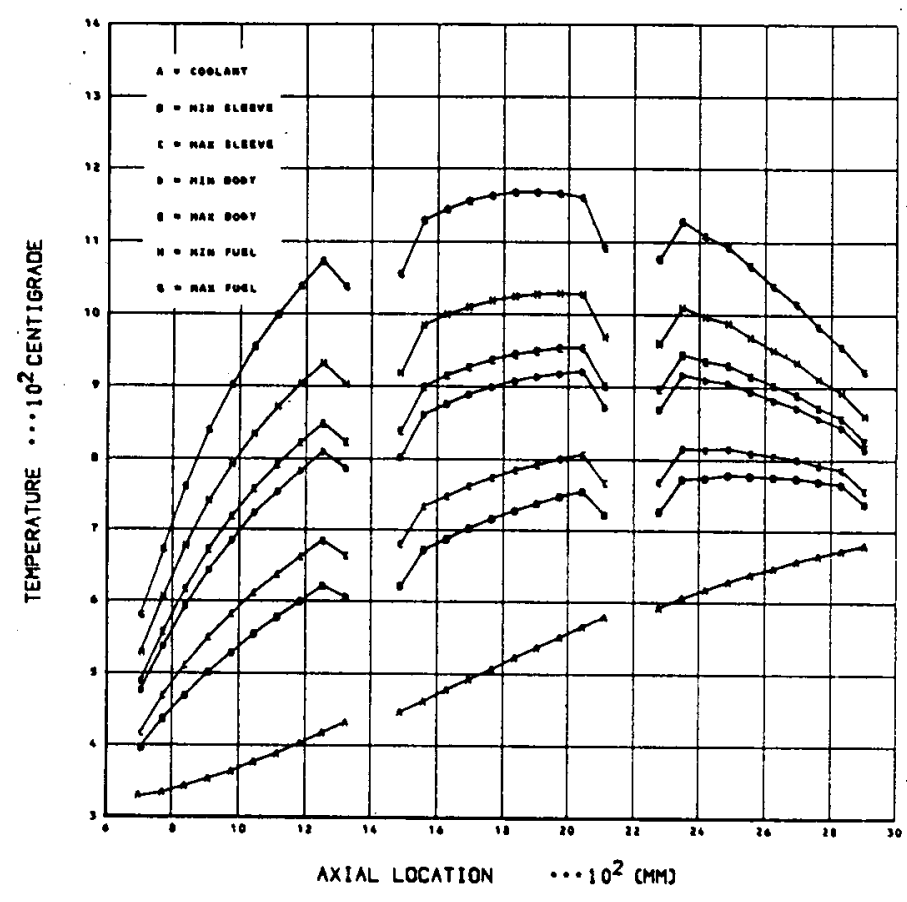

Fig. 3-13. FTE-6 axial temperature profiles (385-499 EFPD)

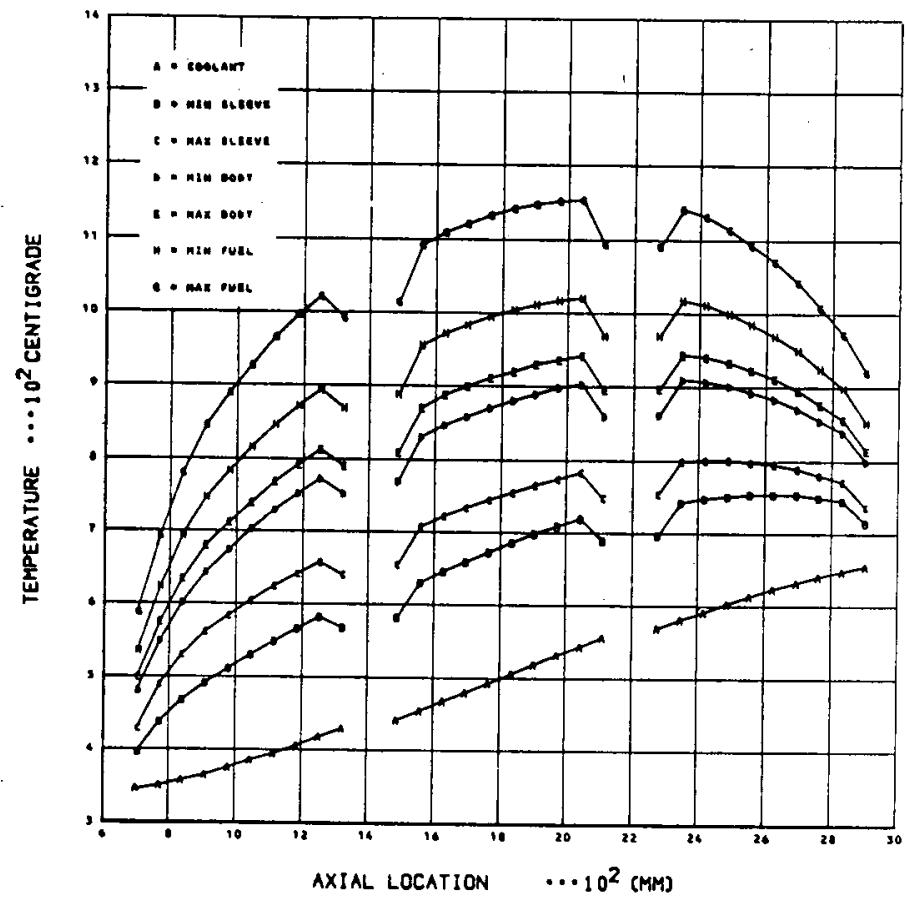

Fig. 3-14. FTE-6 axial temperature profiles (499-564 EFPD)

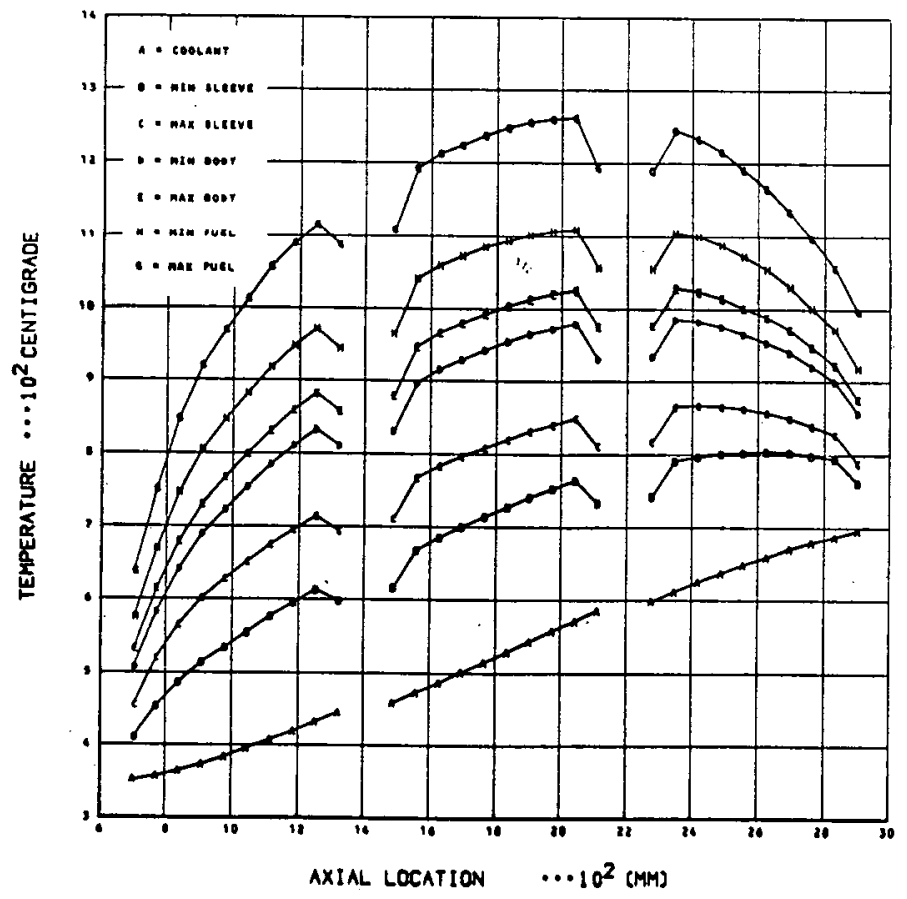

Fig. 3-15. FTE-6 axial temperature profiles (564-610 EFPD) 


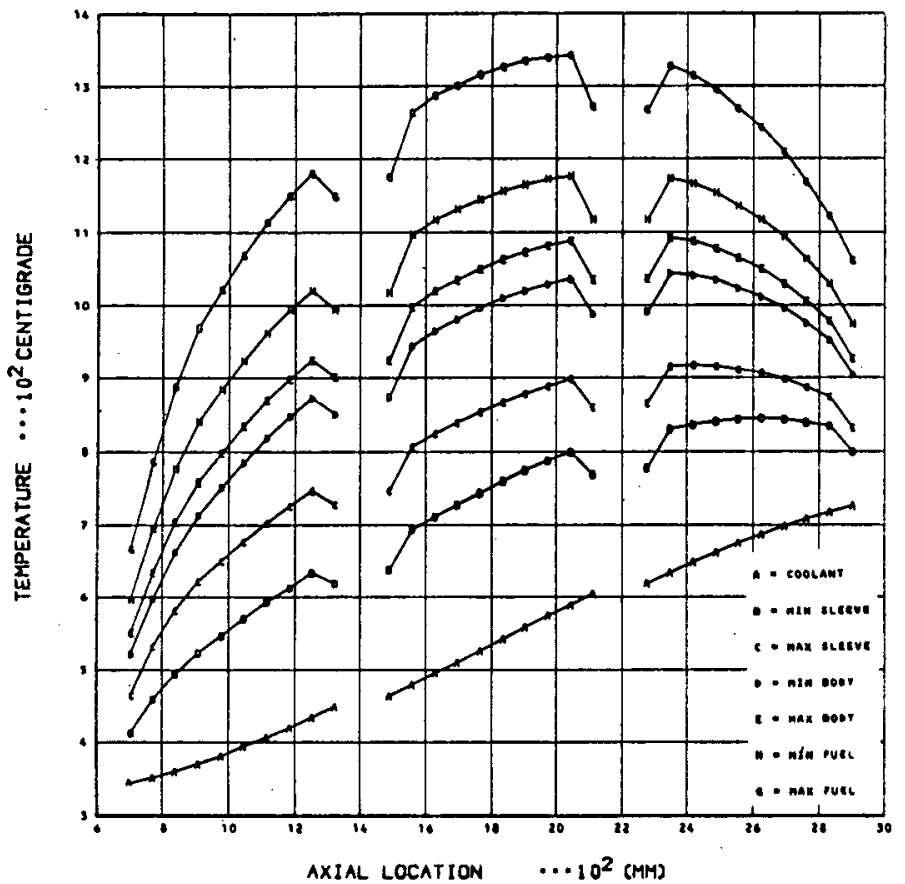

Fig. 3-16. FTE-6 axial temperature profiles (610-701 EFPD)

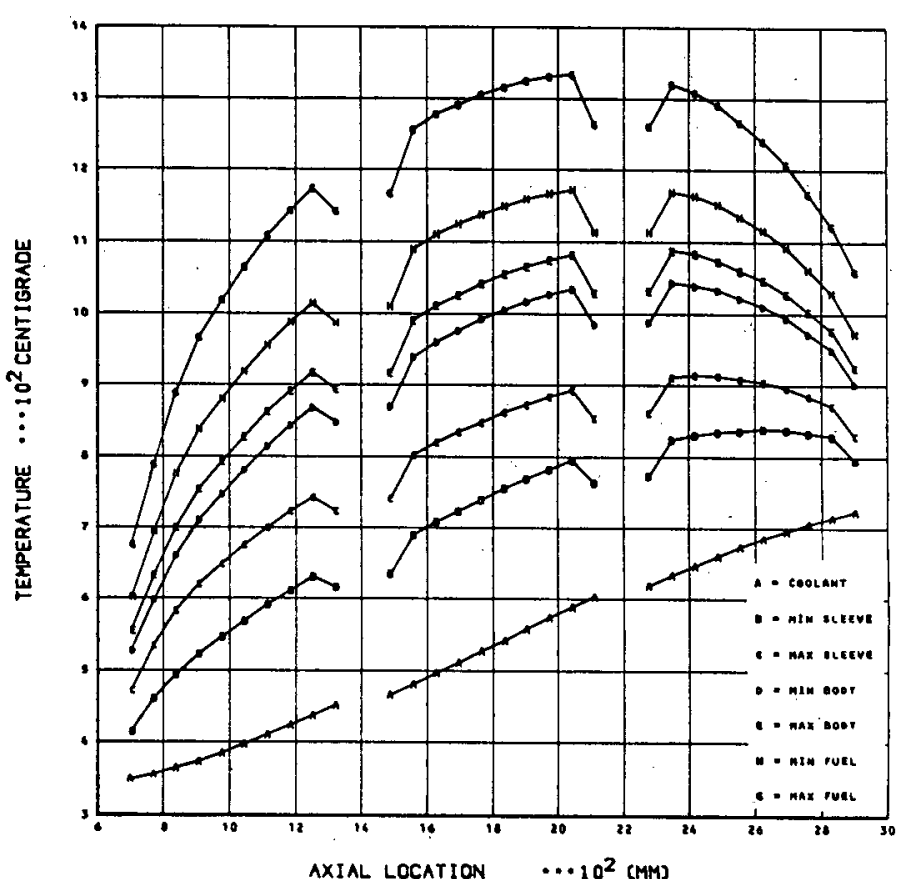

Fig. 3-17. FTE-6 axial temperature profiles (701-748 EFPD)

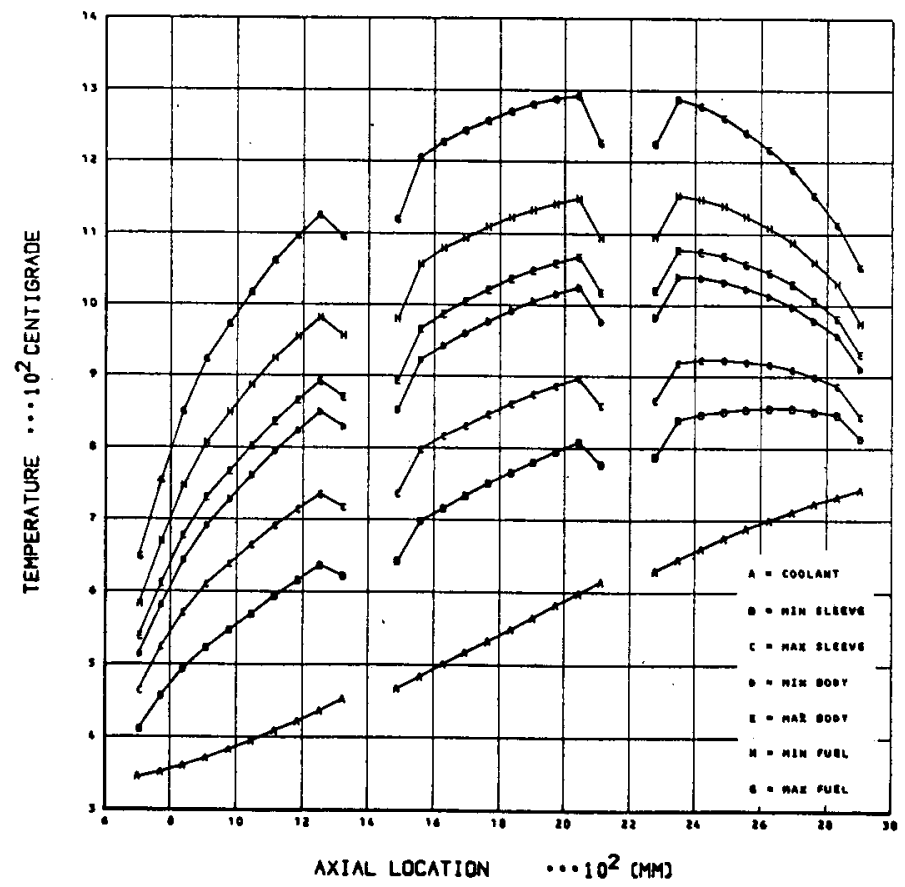

Fig. 3-18. FTE-6 axial temperature profiles (748-788 EFPD) 


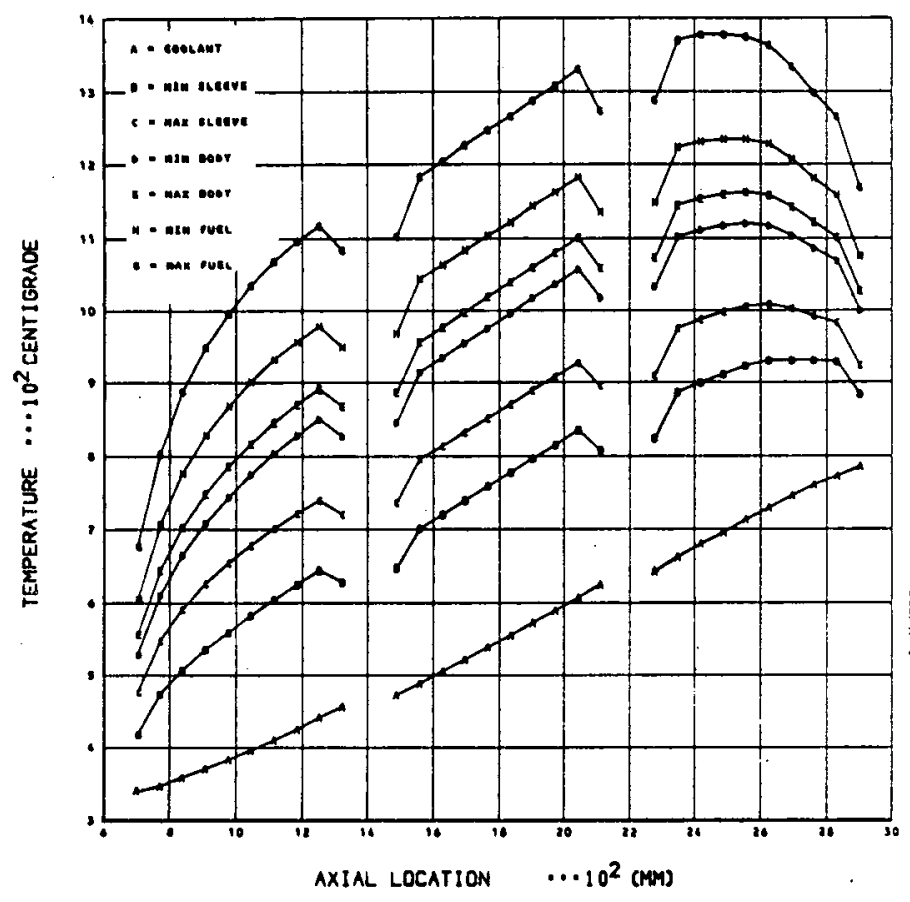

Fig. 3-19. FTE-6 axial temperature profiles (788-818 EFPD)

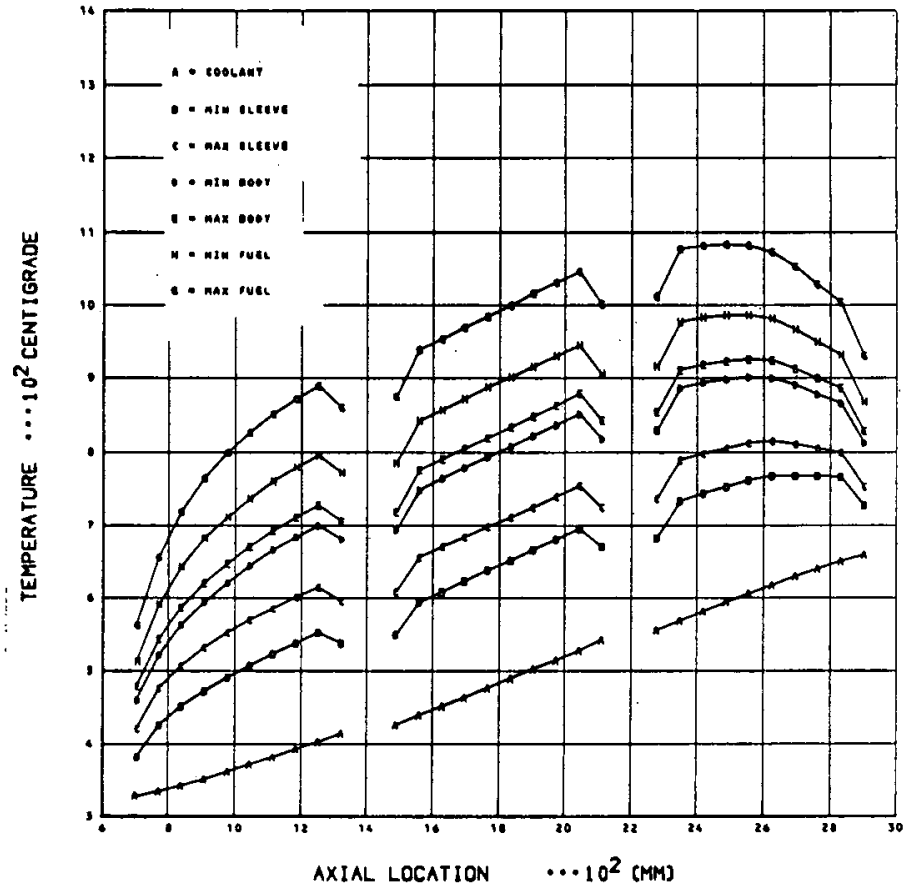

Fig. 3-20. FTE-6 axial temperature profiles (818-835 EFPD)

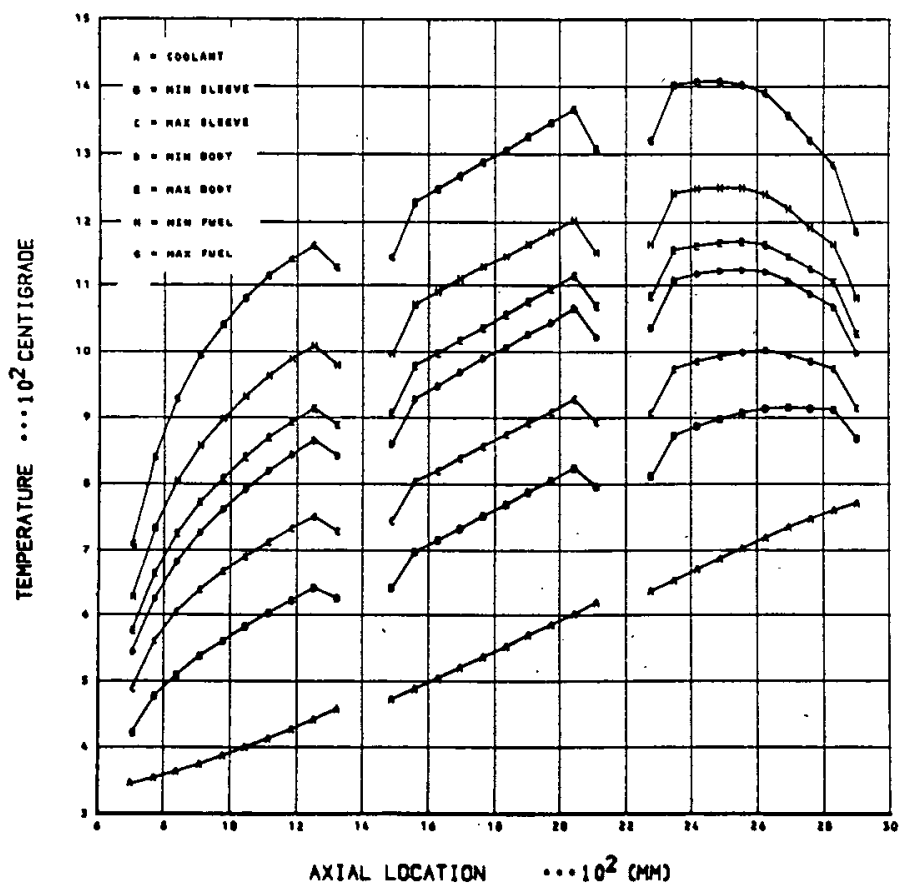

Fig. 3-21. FTE-6 axial temperature profiles (835-858 EFPD) 


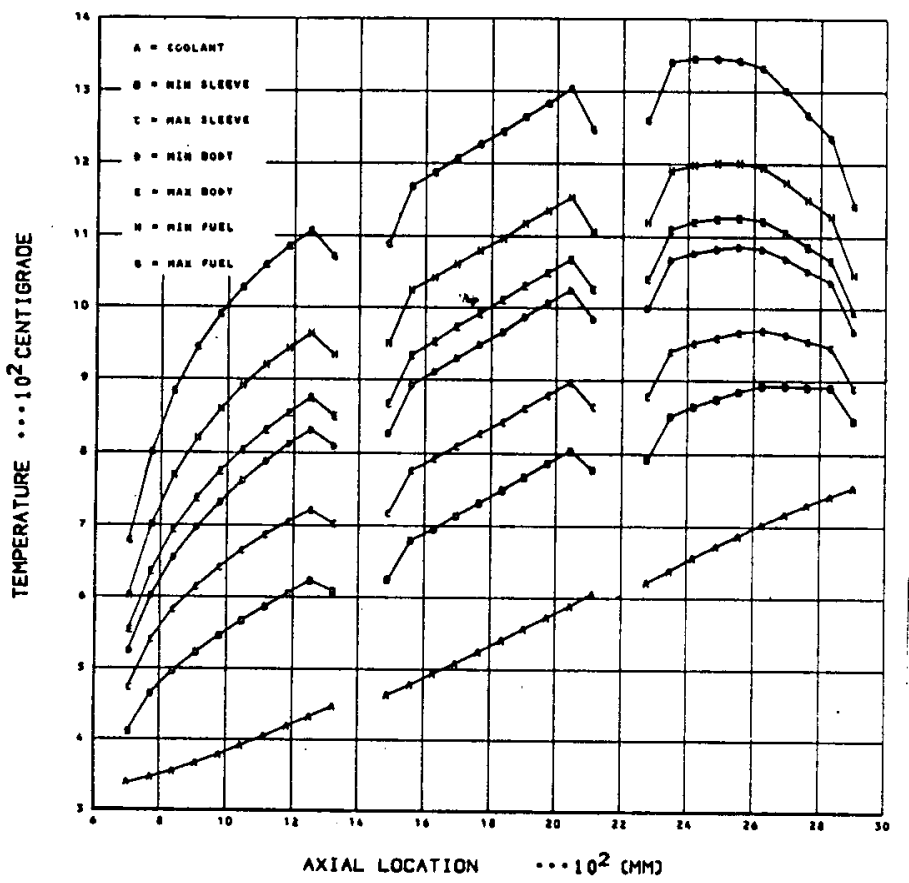

Fig. 3-22. FTE-6 axial temperature profiles (858-889 EFPD)

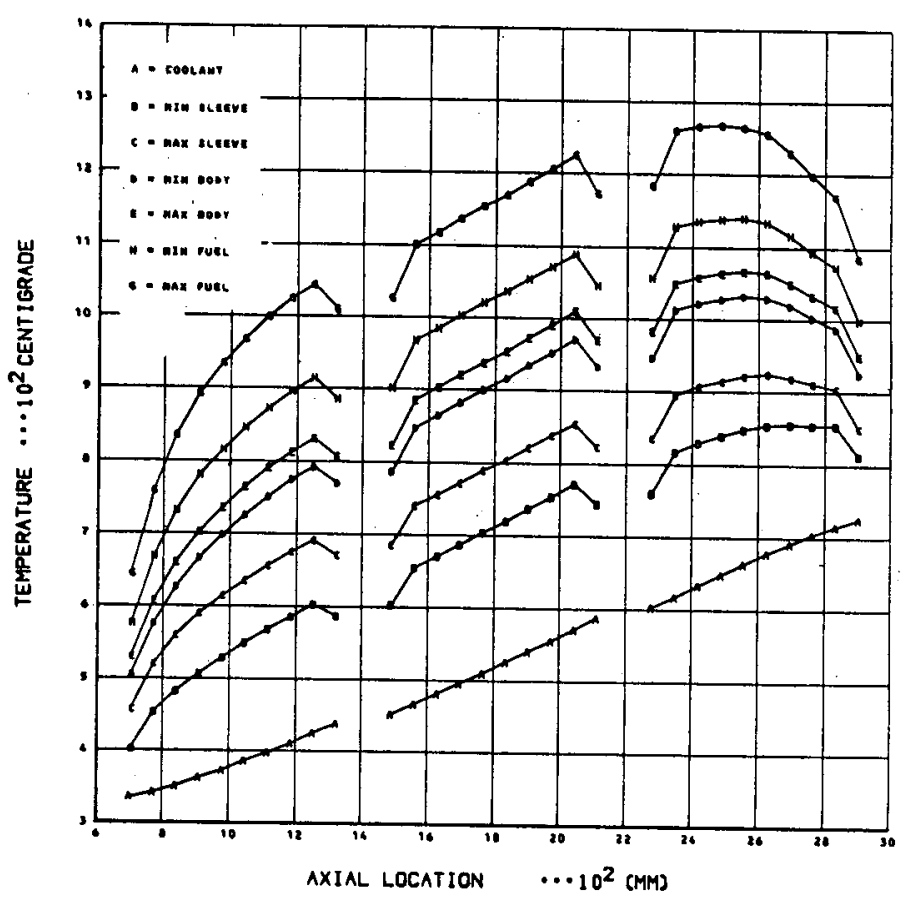

Fig. 3-23. FTE-6 axial temperature profiles (889-EOL)

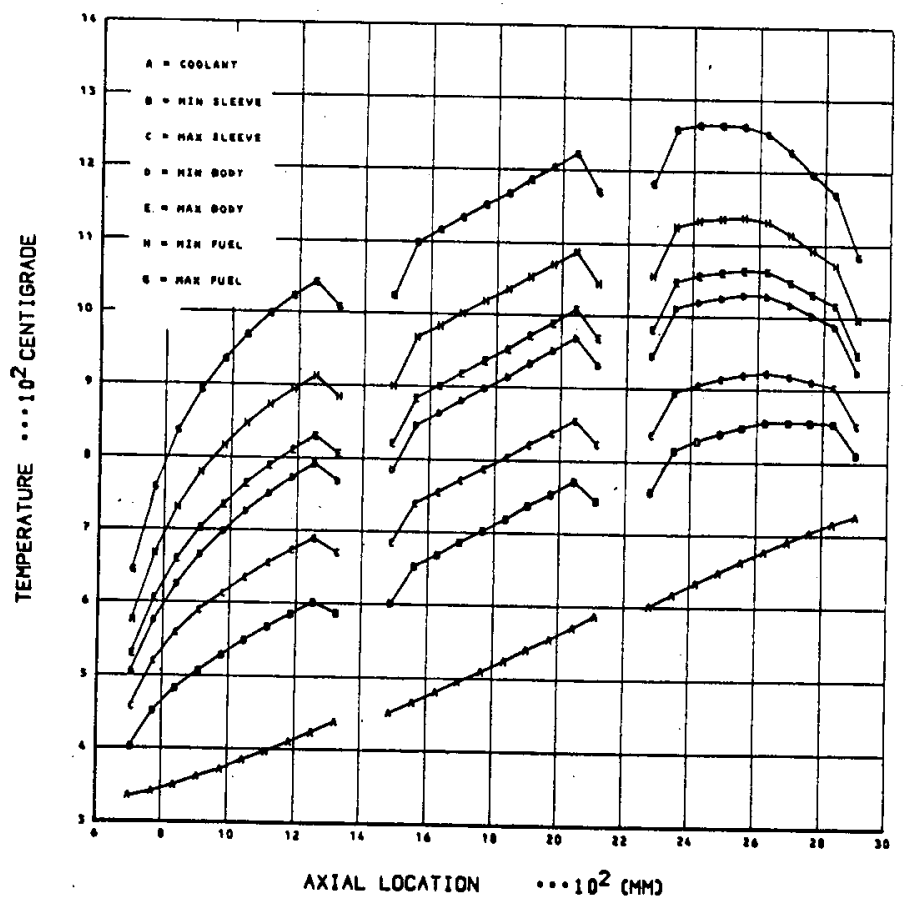

Fig. 3-24. FTE-6 axial temperature profiles (EOL) 


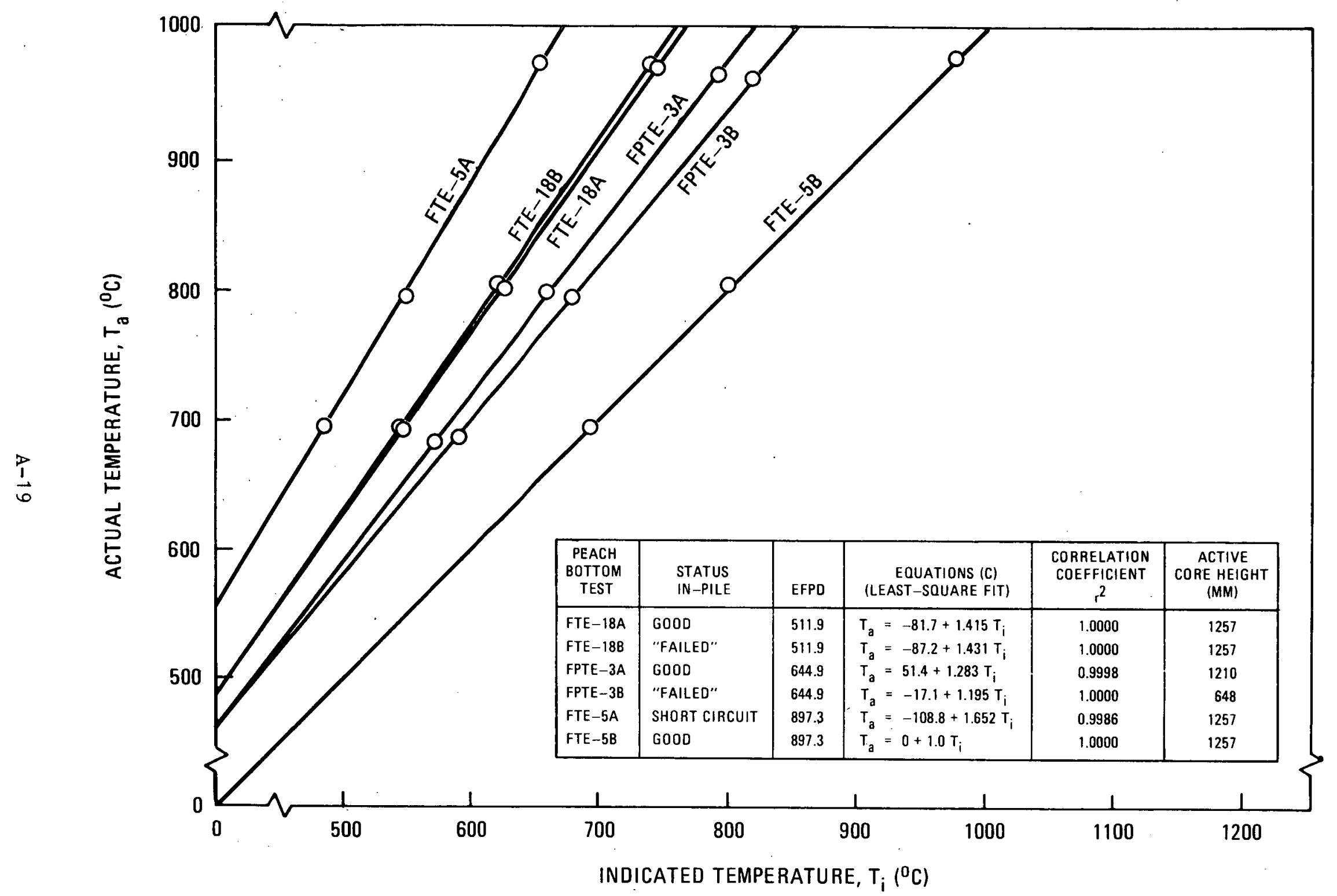

Fig. 3-25. Peach Bottom thermocouple recalibration plot of Ti versus Ta 


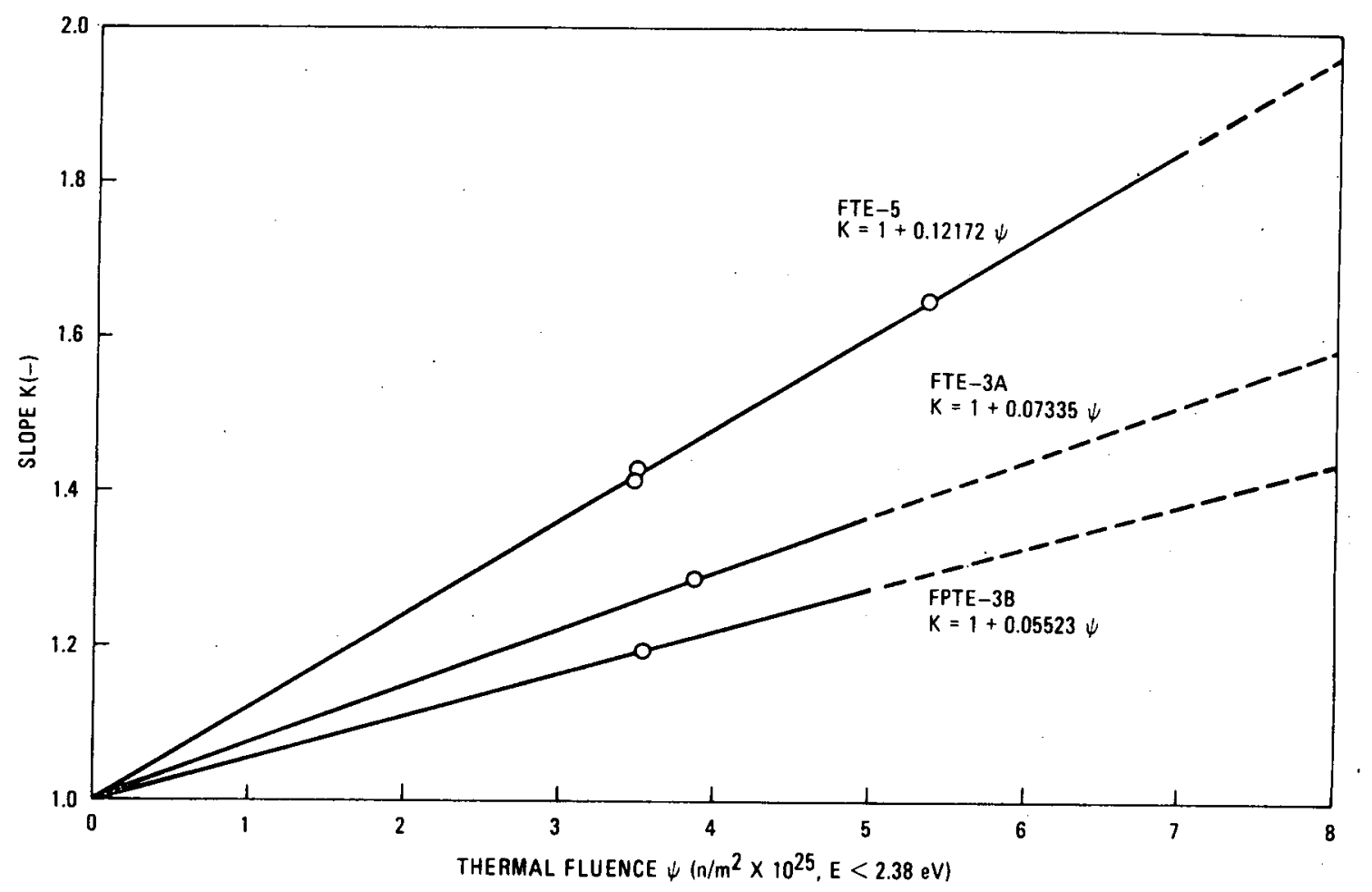

a. K(slope)

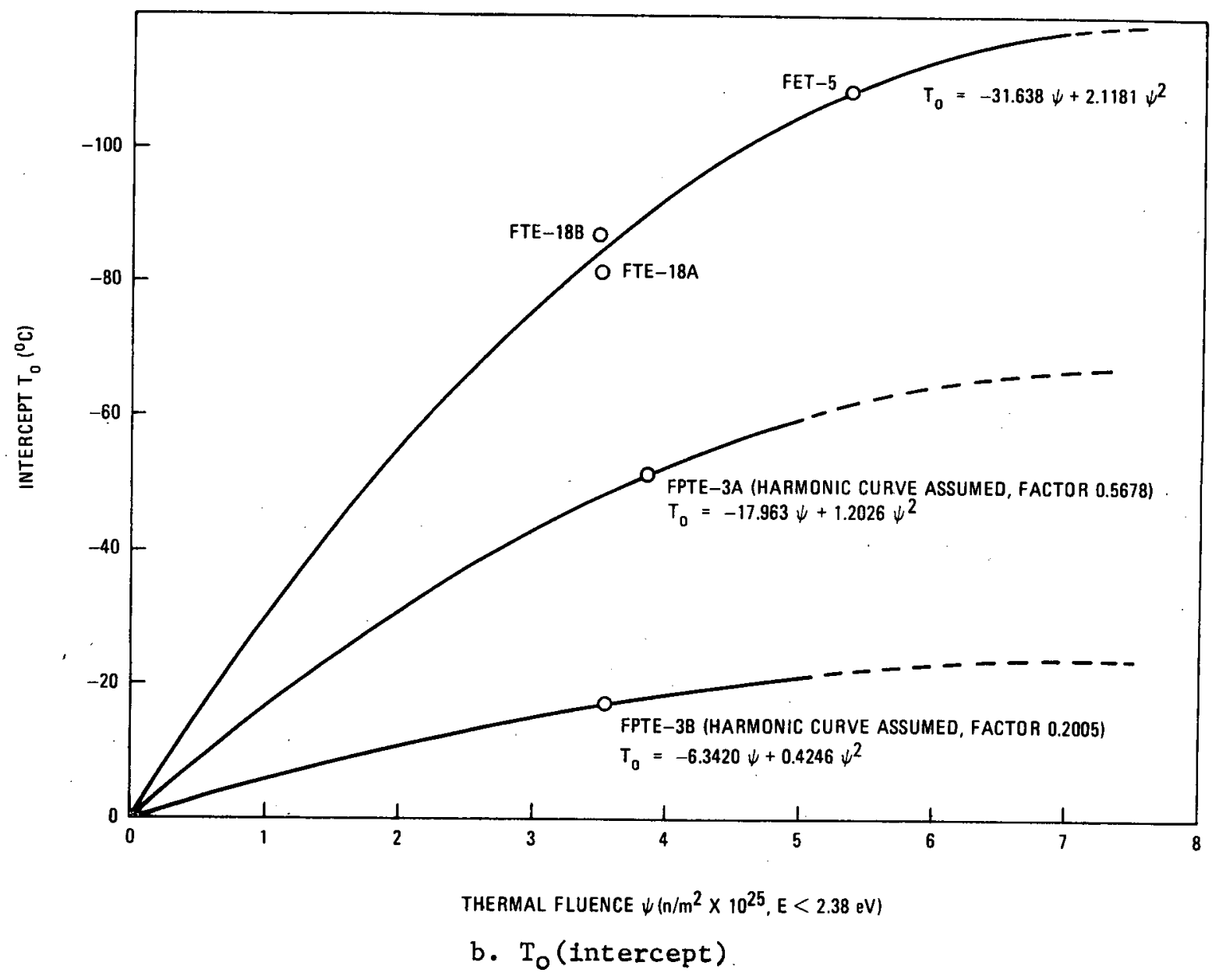

Fig. 3-26. Peach Bottom thermocouple recalibration terms $\mathrm{K}$ and $\mathrm{T}_{\mathrm{o}}$ 


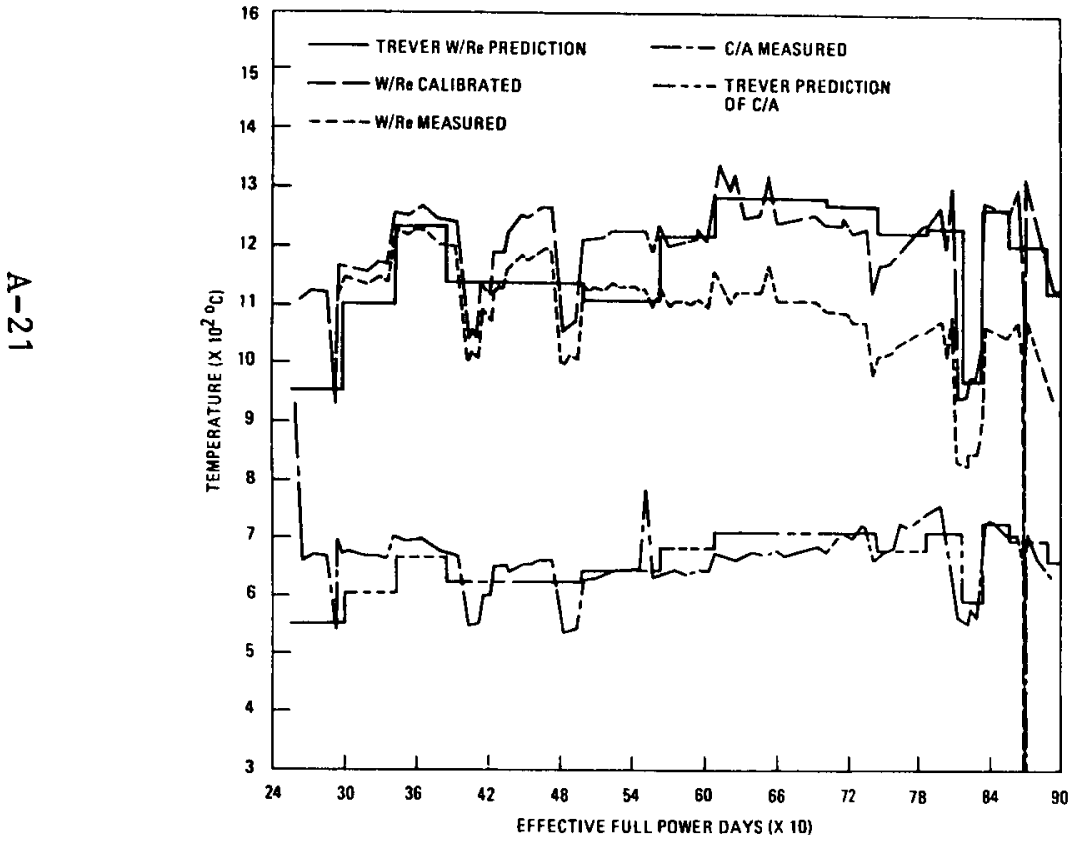

Fig. 3-27(a). FTE-6 comparison of predicted temperatures and thermocouple readings

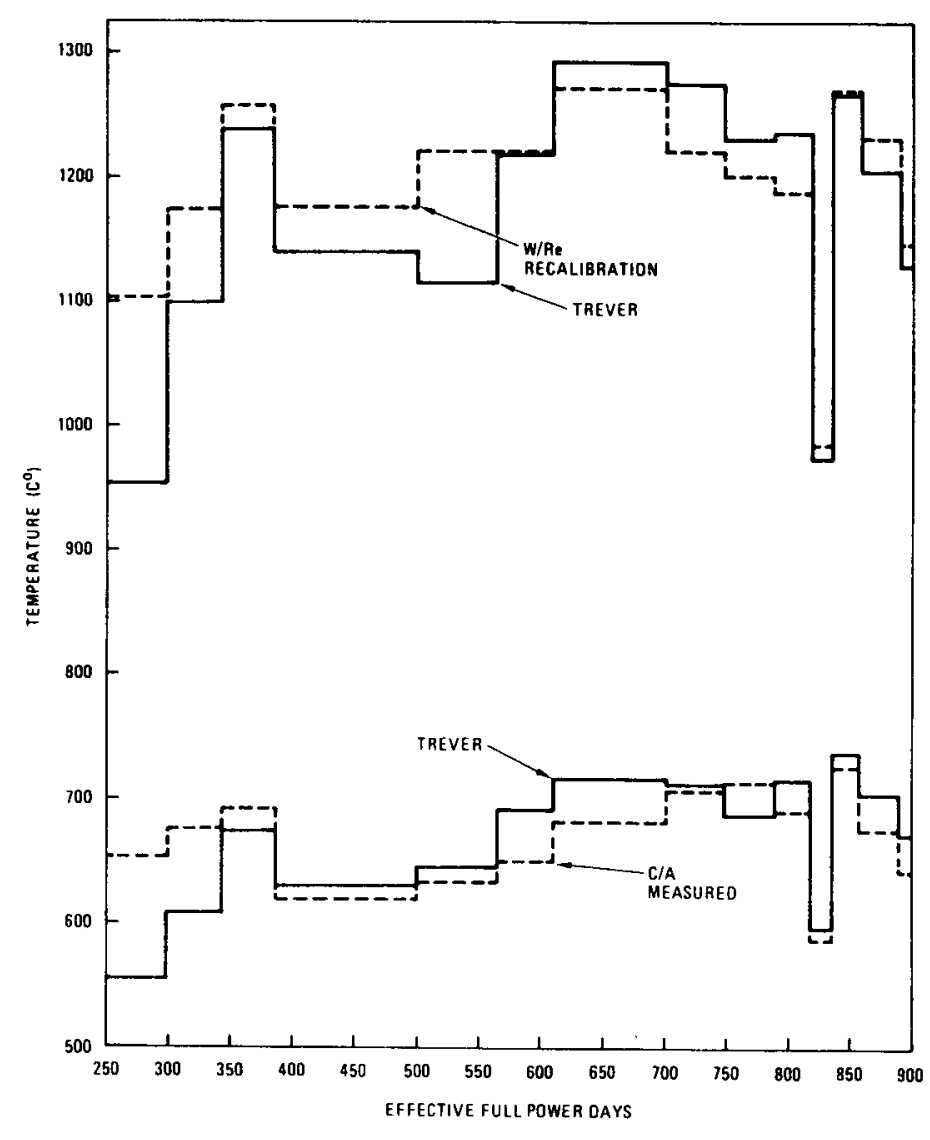

Fig. 3-27(b) FTE-6 TREVER simulation and thermocouple comparison 

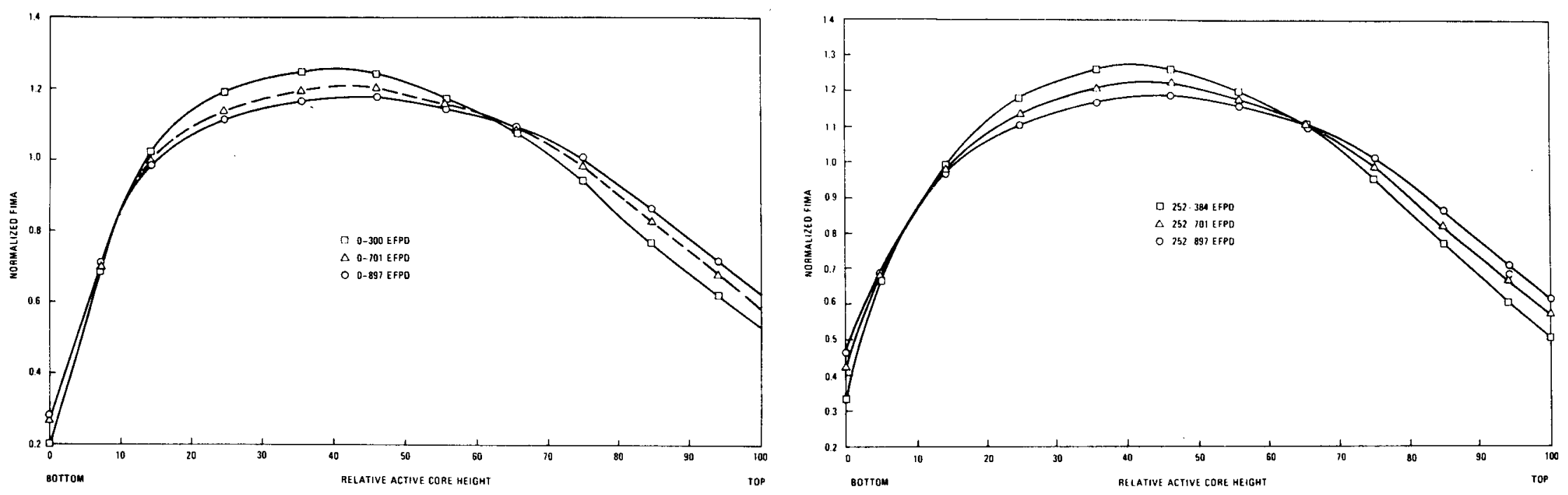

BOL - (0)EFPD

BOL - (252) EFPD

$\underset{n}{n}$

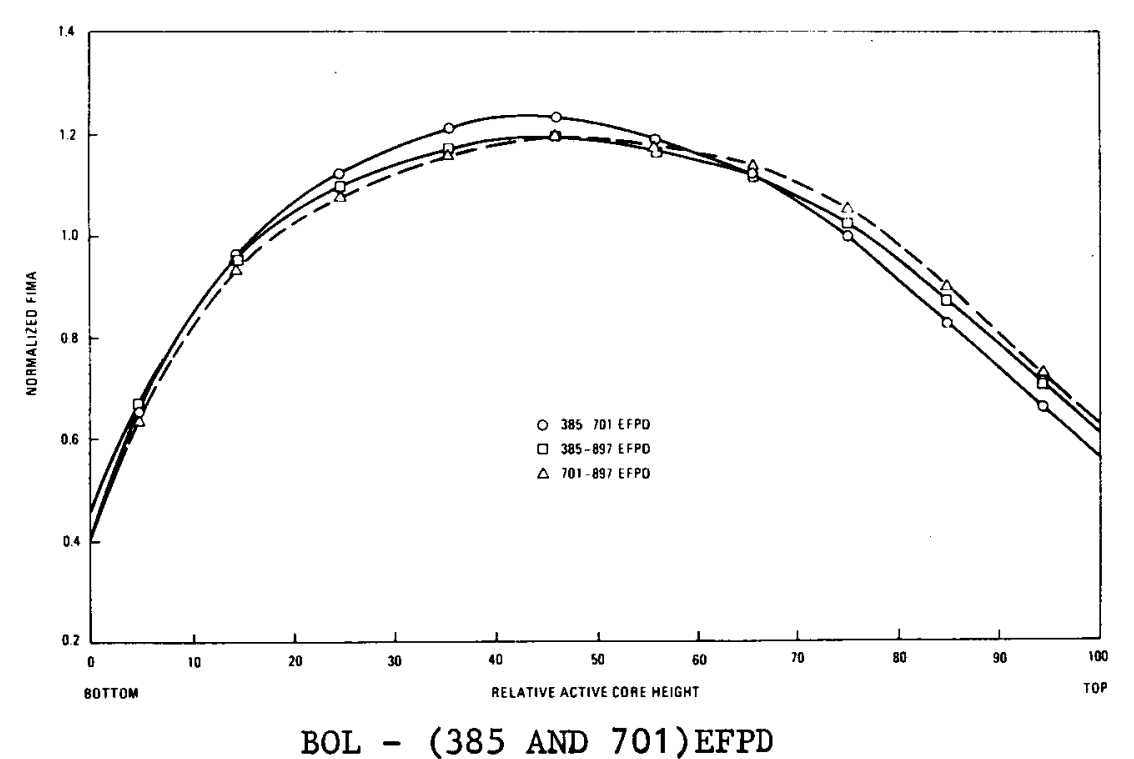

Fig. 3-28. Peach Bottom core 2 normalized axial distributions of fissile FIMA from FEVER calculations 


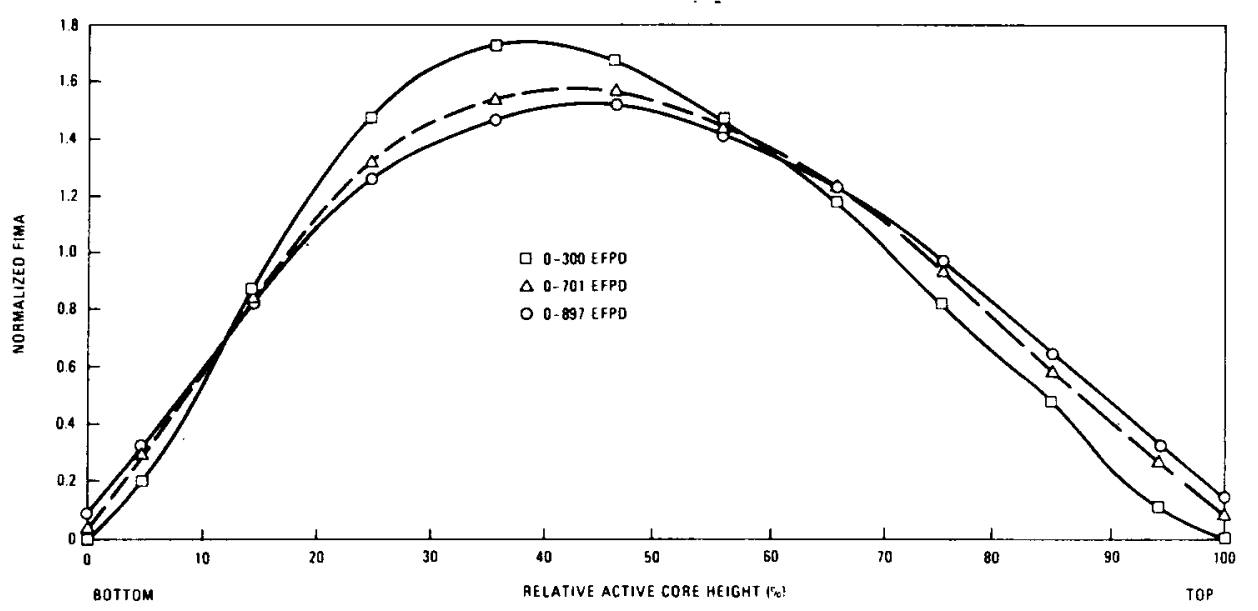

BOL - (0)EFPD

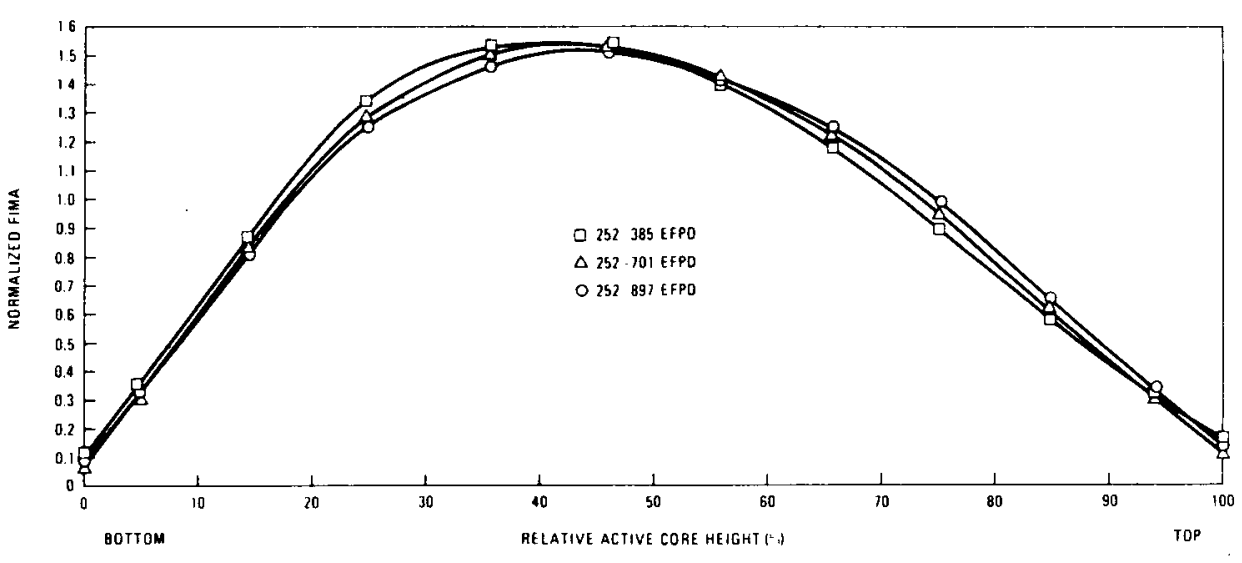

BOL - (252 EFPD)

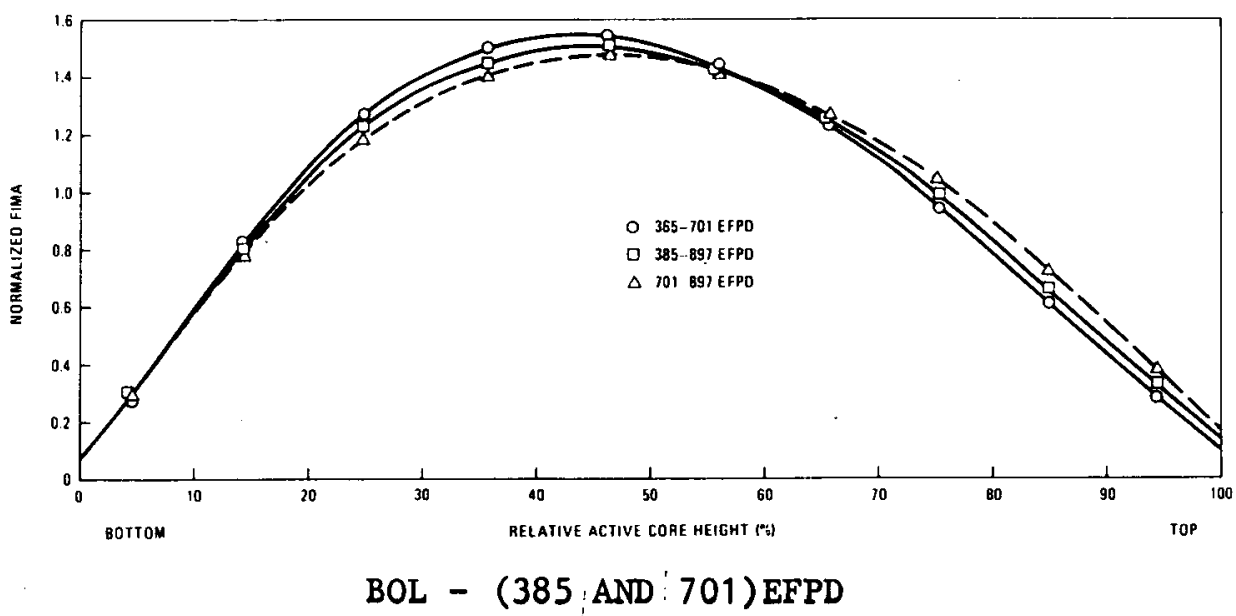

Fig. 3-29. Peach Bottom Core 2 normalized axial distribution of fertile FIMA from FEVER calculations 

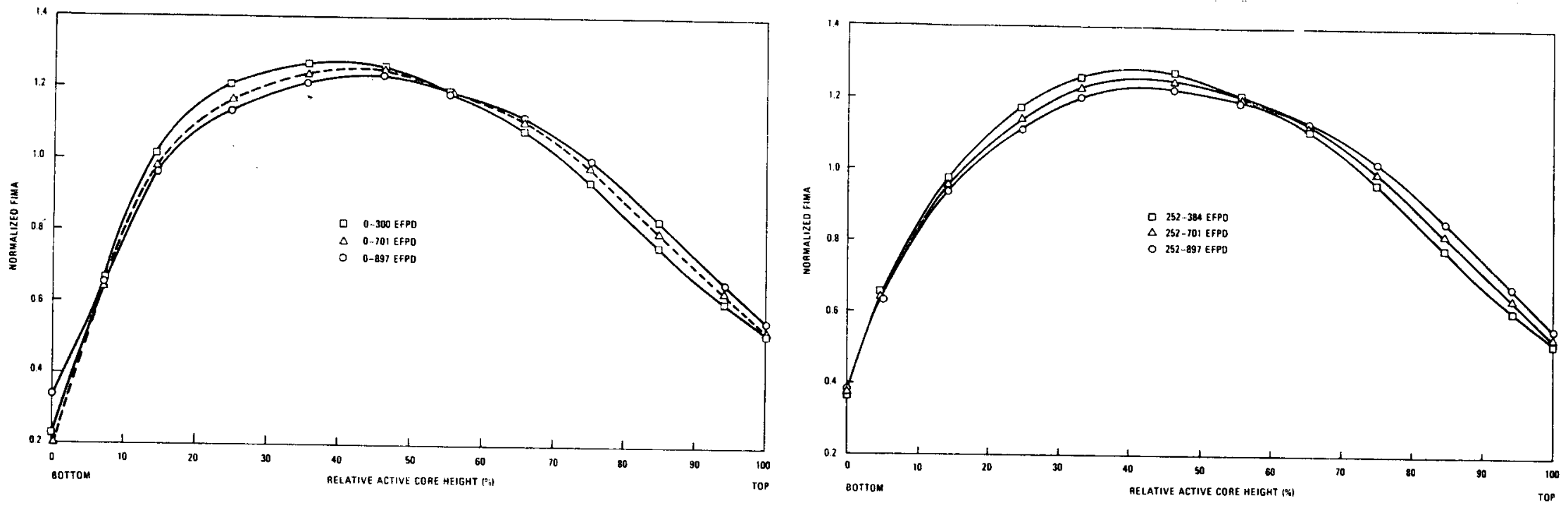

BOL - (0) EFPD

BOL - (252) EFPD

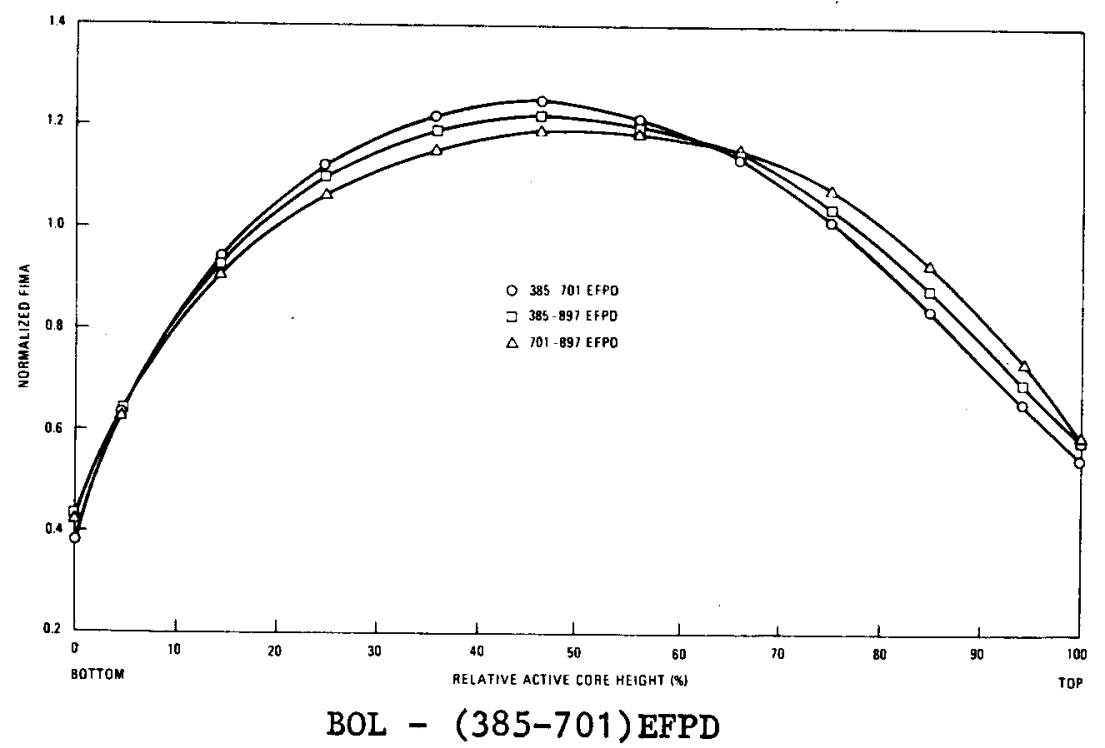

Fig. 3-30. Peach Bottom Core 2 normalized axial distribution of mixed FIMA for (5.5 Th:U) particles from FEVER calculations 


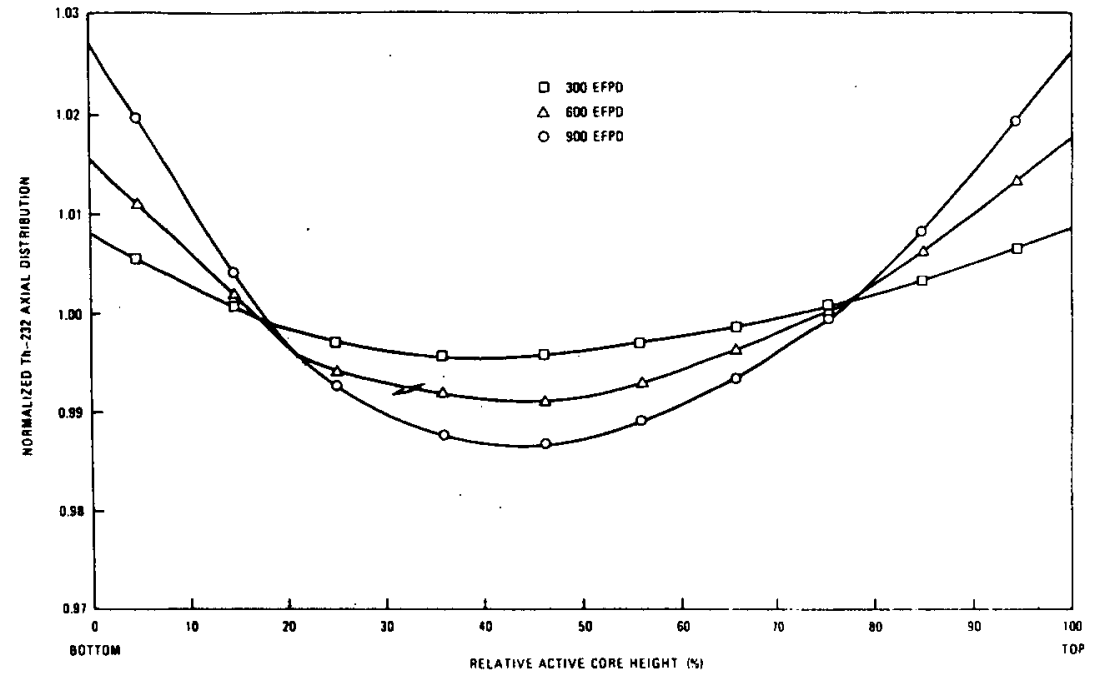

Th-232

3
$N$
$N$

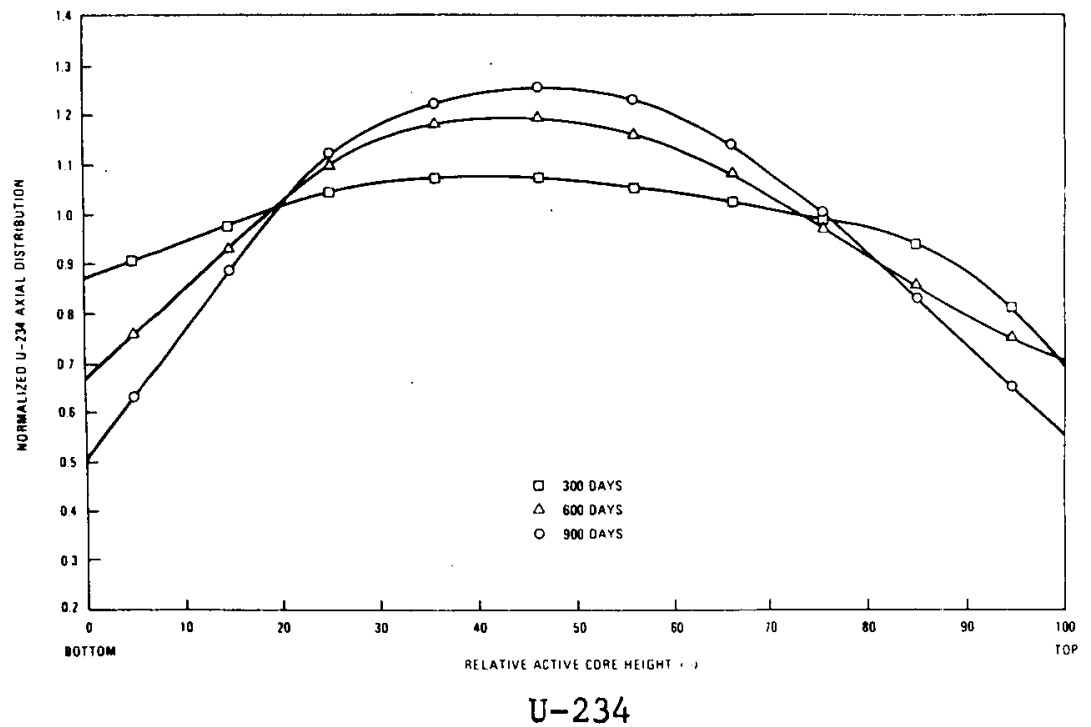

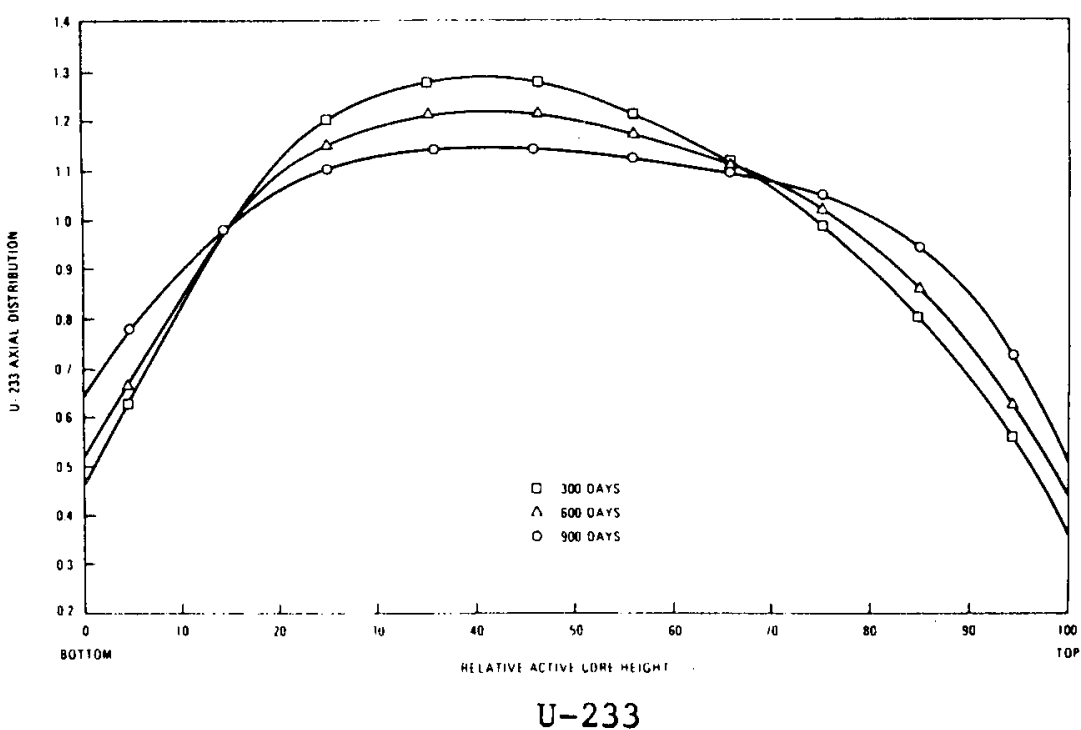

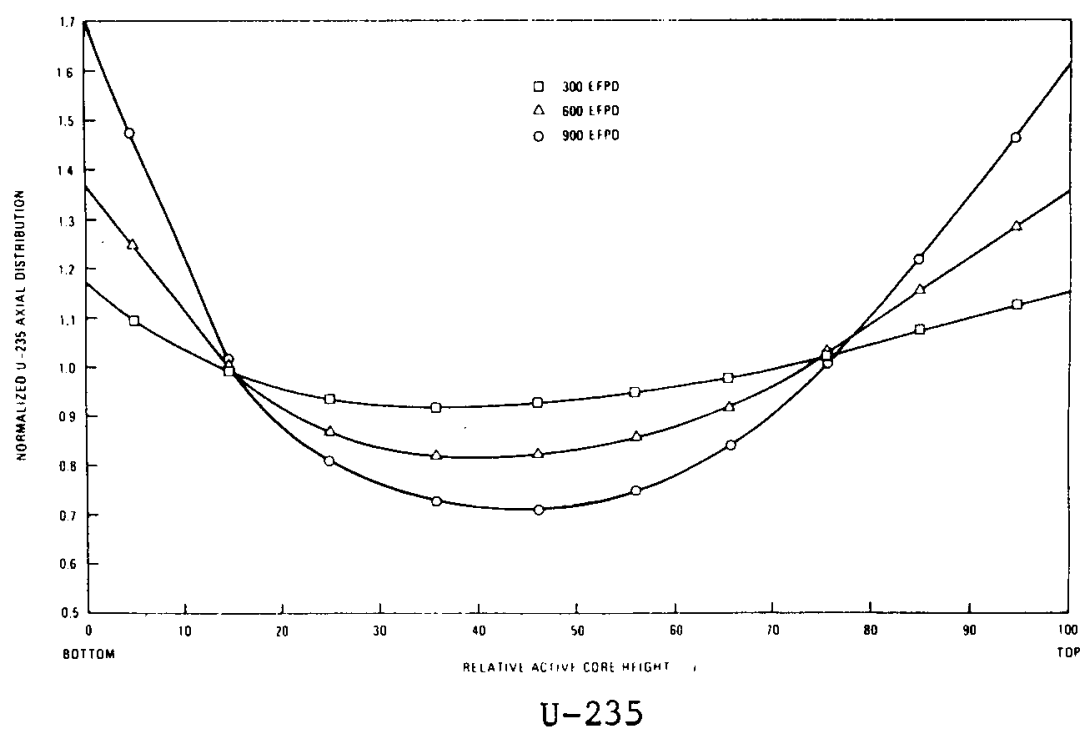

Fig. 3-31. Peach Bottom Core 2 normalized axial distributions for Th-232, U-233, U-237, and U-235 from FEVER calculations 

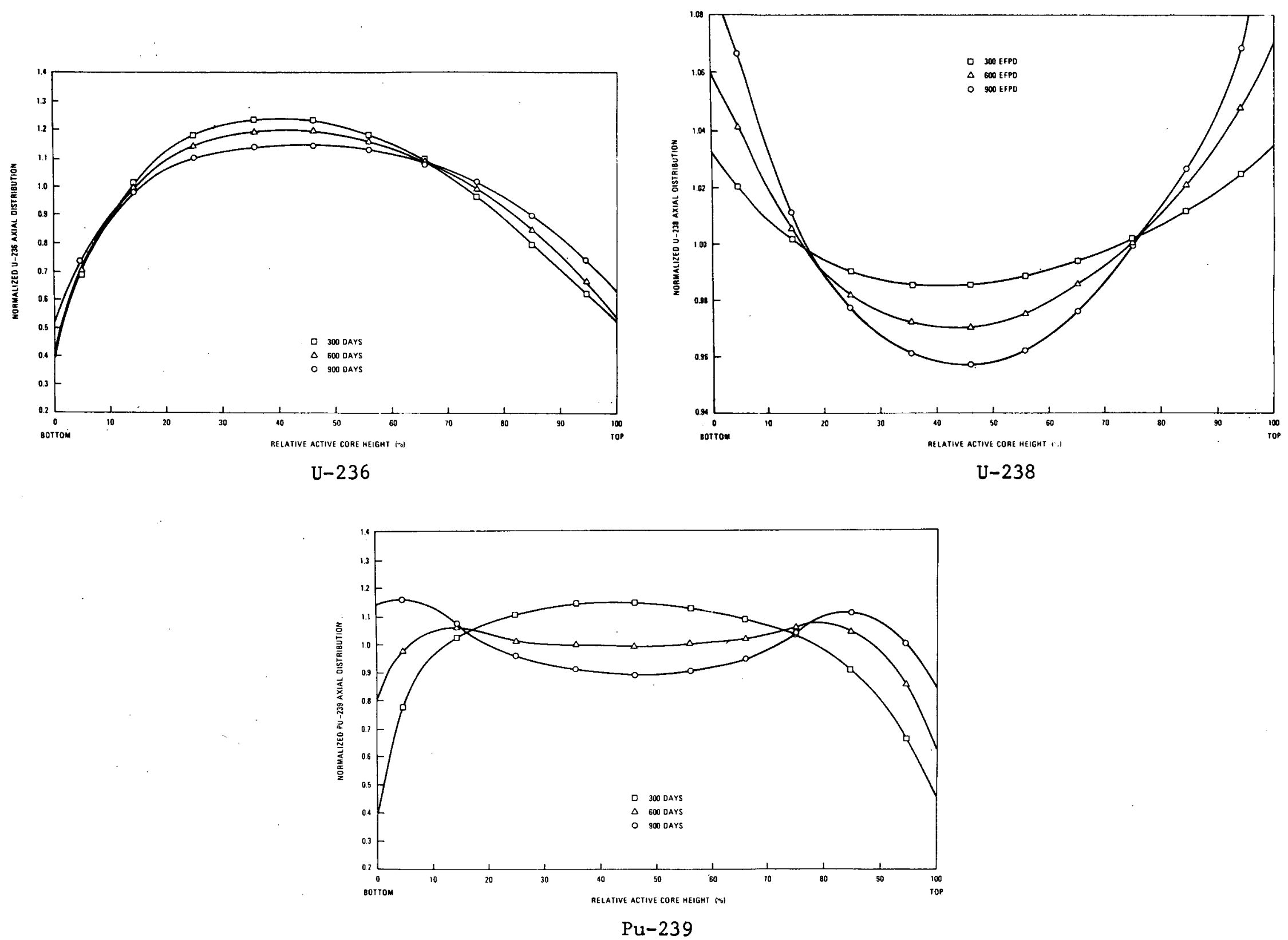

Fig. 3-32. Peach Bottom Core 2 normalized axial distribution of U-236, U-238, and Pu-239 from FEVER calculations 


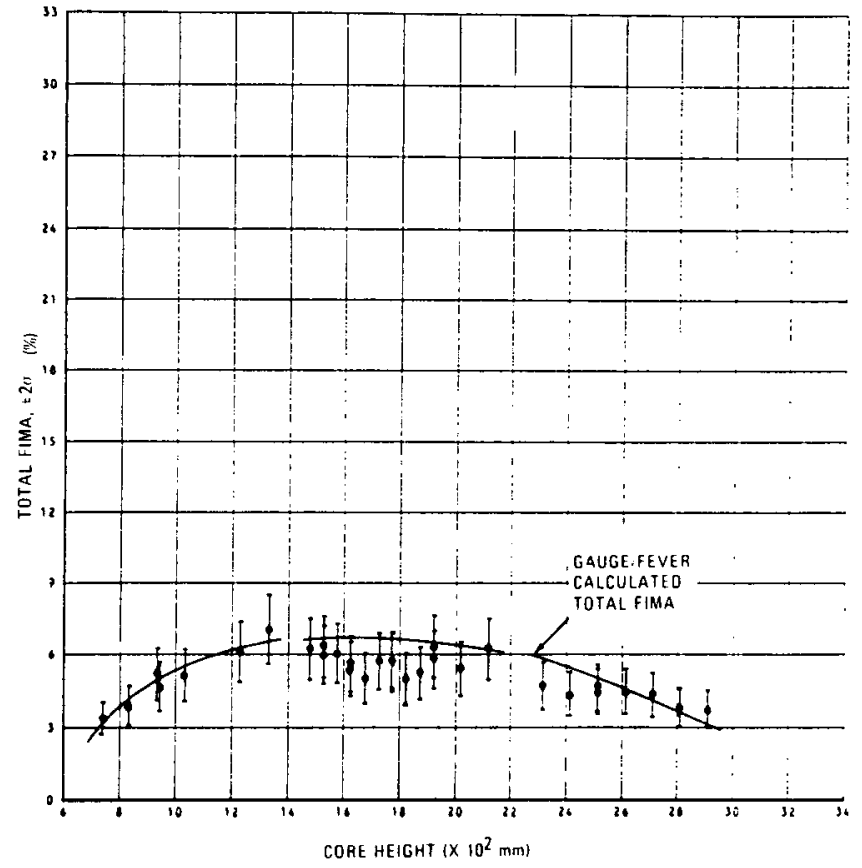

(a)

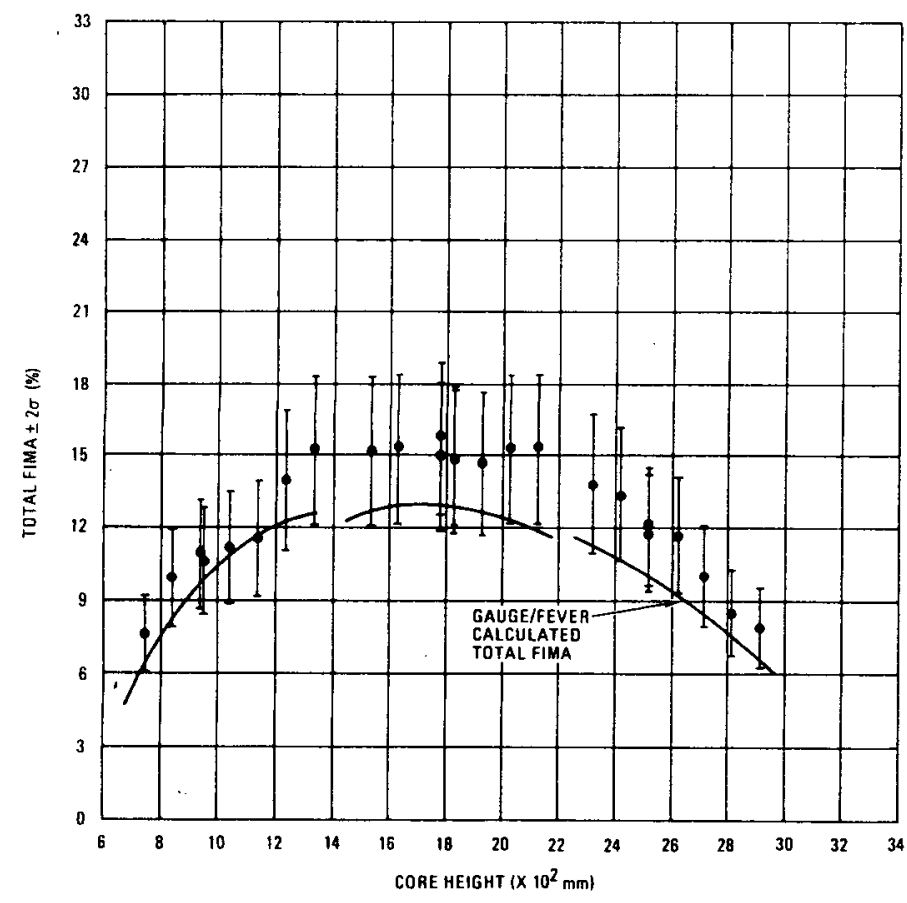

(c)

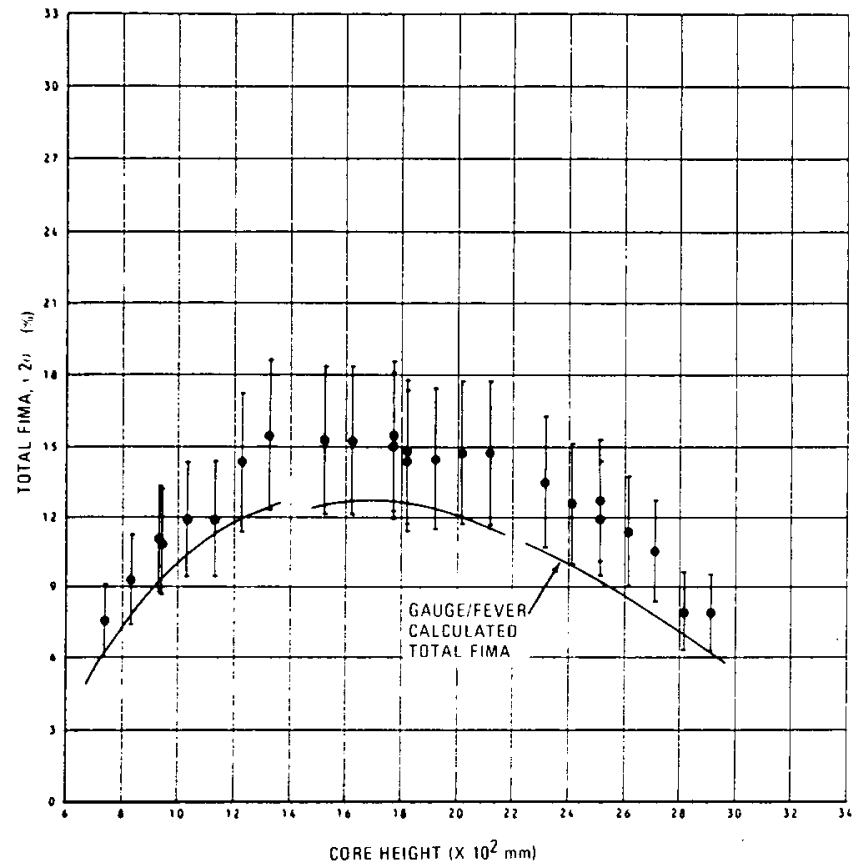

(b)

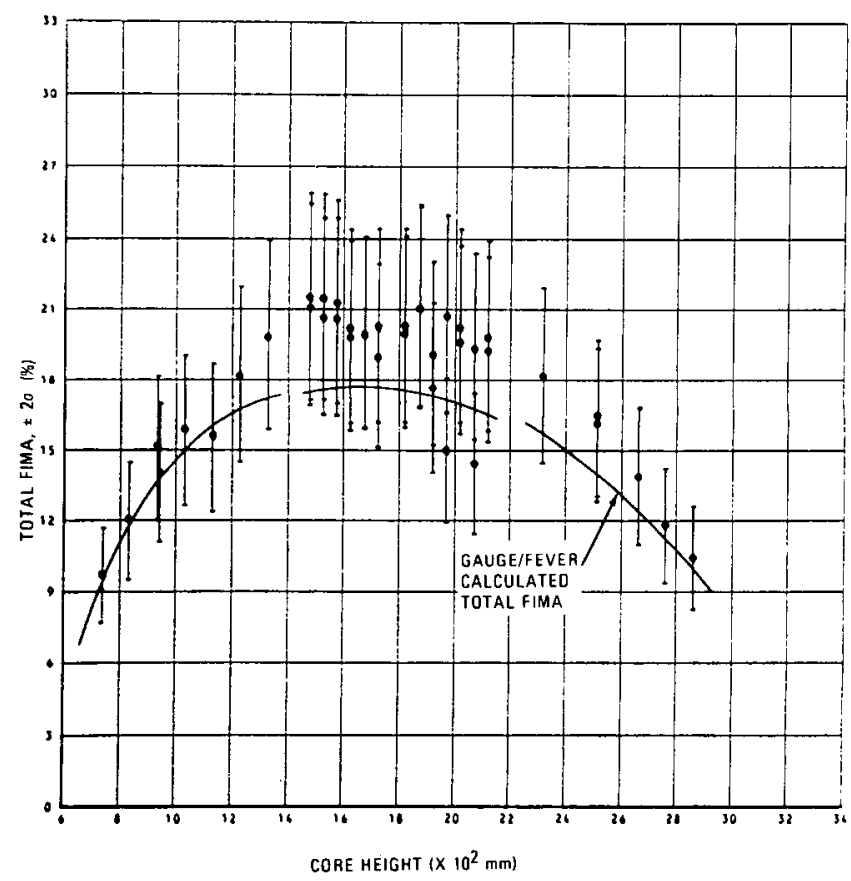

(d)

Fig. 3-33. FTE-6 total FIMA measured by gamma spectroscopy: (a) in fuel stacks 1 and 2; (b) in fuel stacks 3 and 4; (c) in fuel stacks 5 and $6 ;$ (d) in fuel stacks 7 and 8 

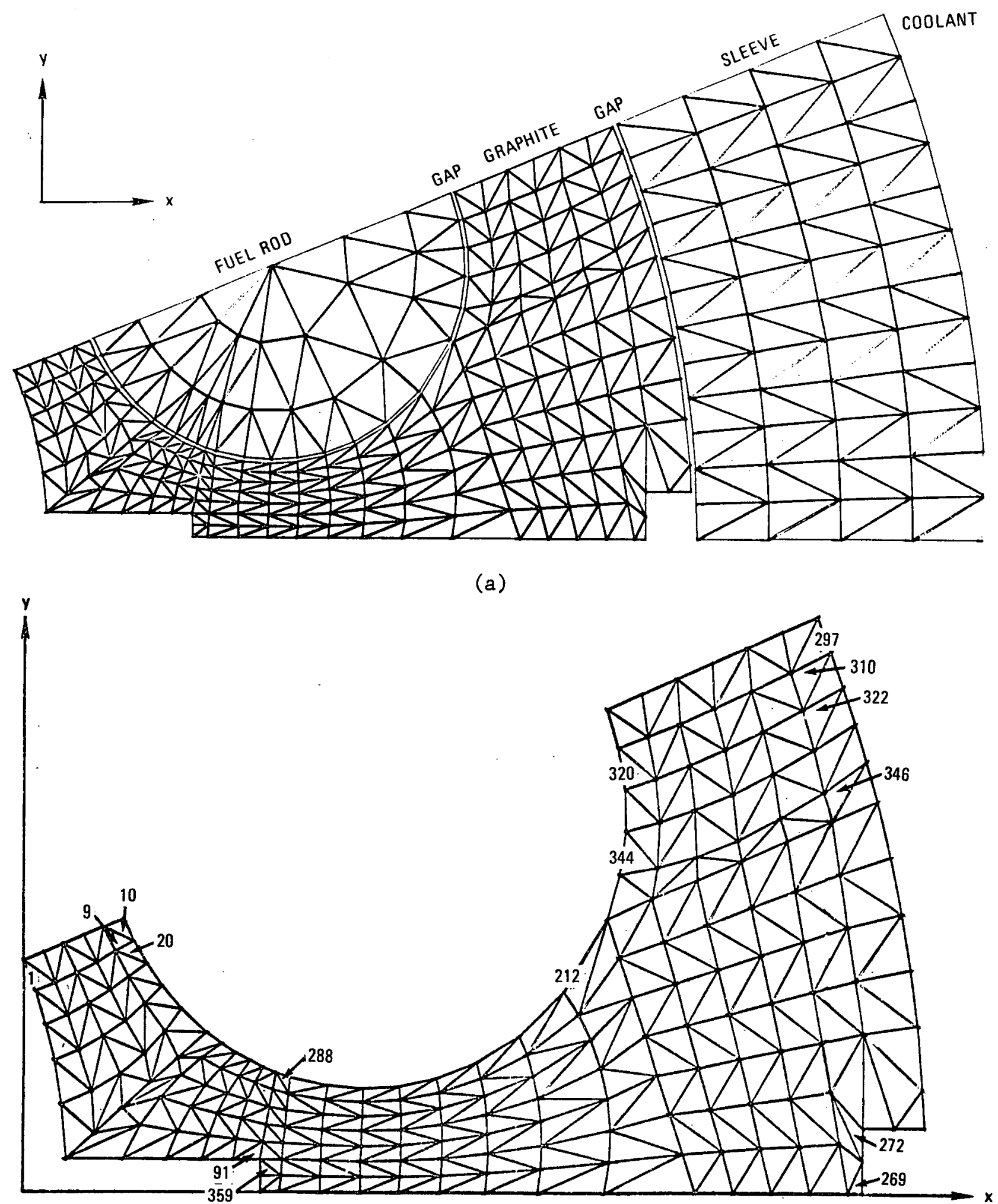

(b)

Fig. 3-34. Finite element model: (a) for thermal analysis; (b) for residual 

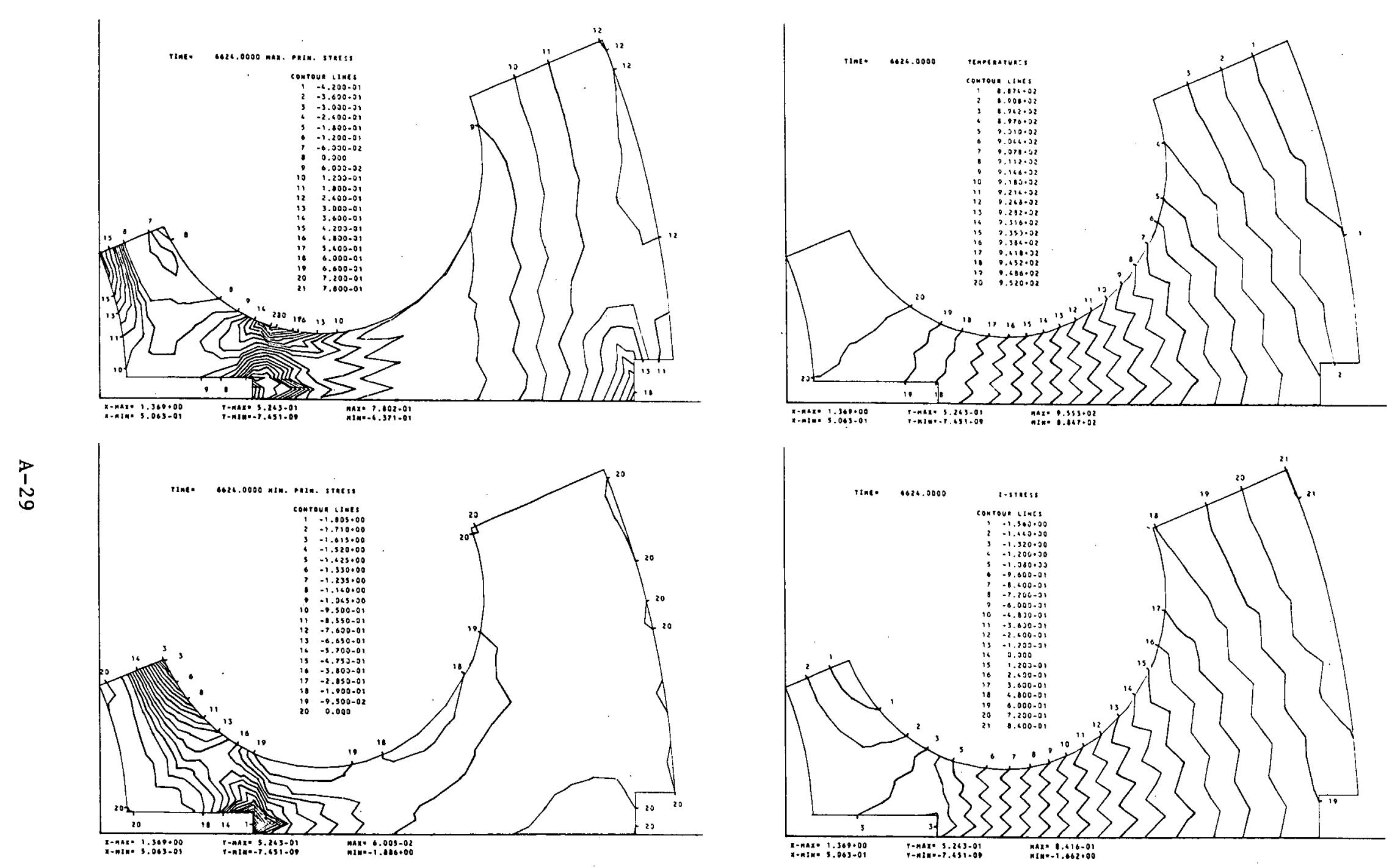

Fig. 3-35. FTE-6 fuel body 1 temperature and stress contour plots for startup at BOL 

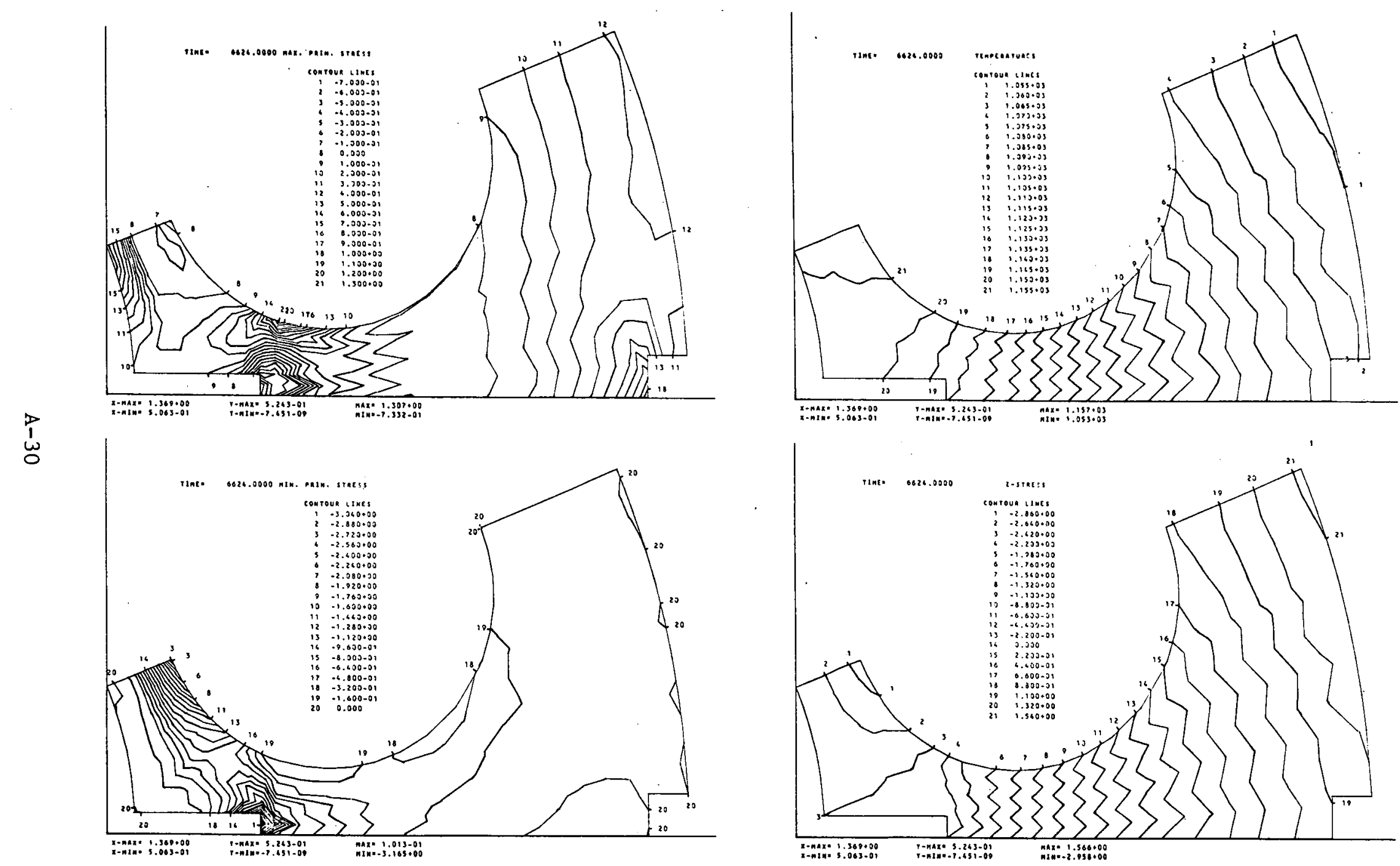

Fig. 3-36. FTE-6 fuel body 2 temperature and stress contour plots for startup at BOL 

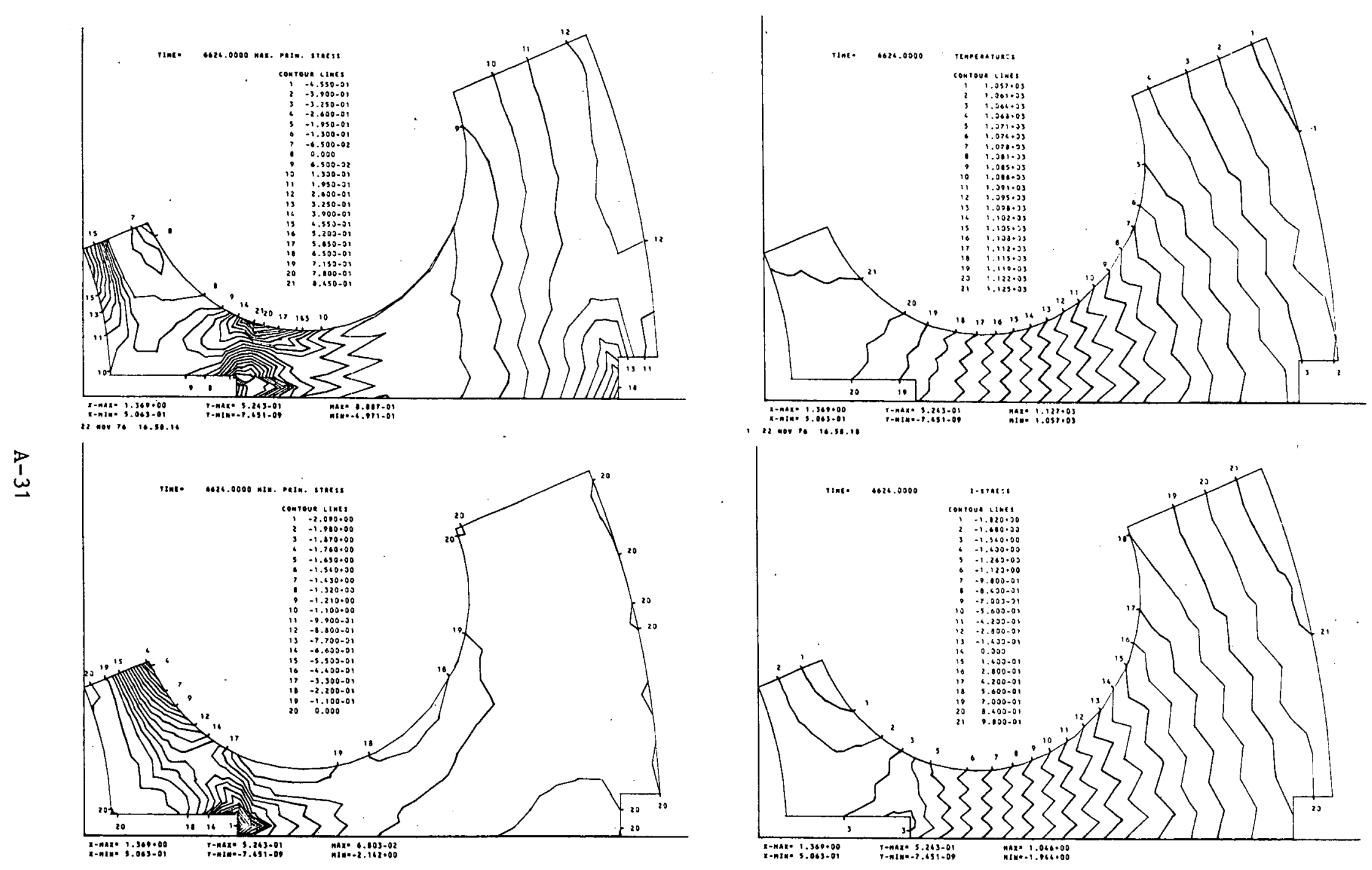

Fig. 3-37. FTE-6 fuel body 3 temperature and stress contour plots for startup at BOL 


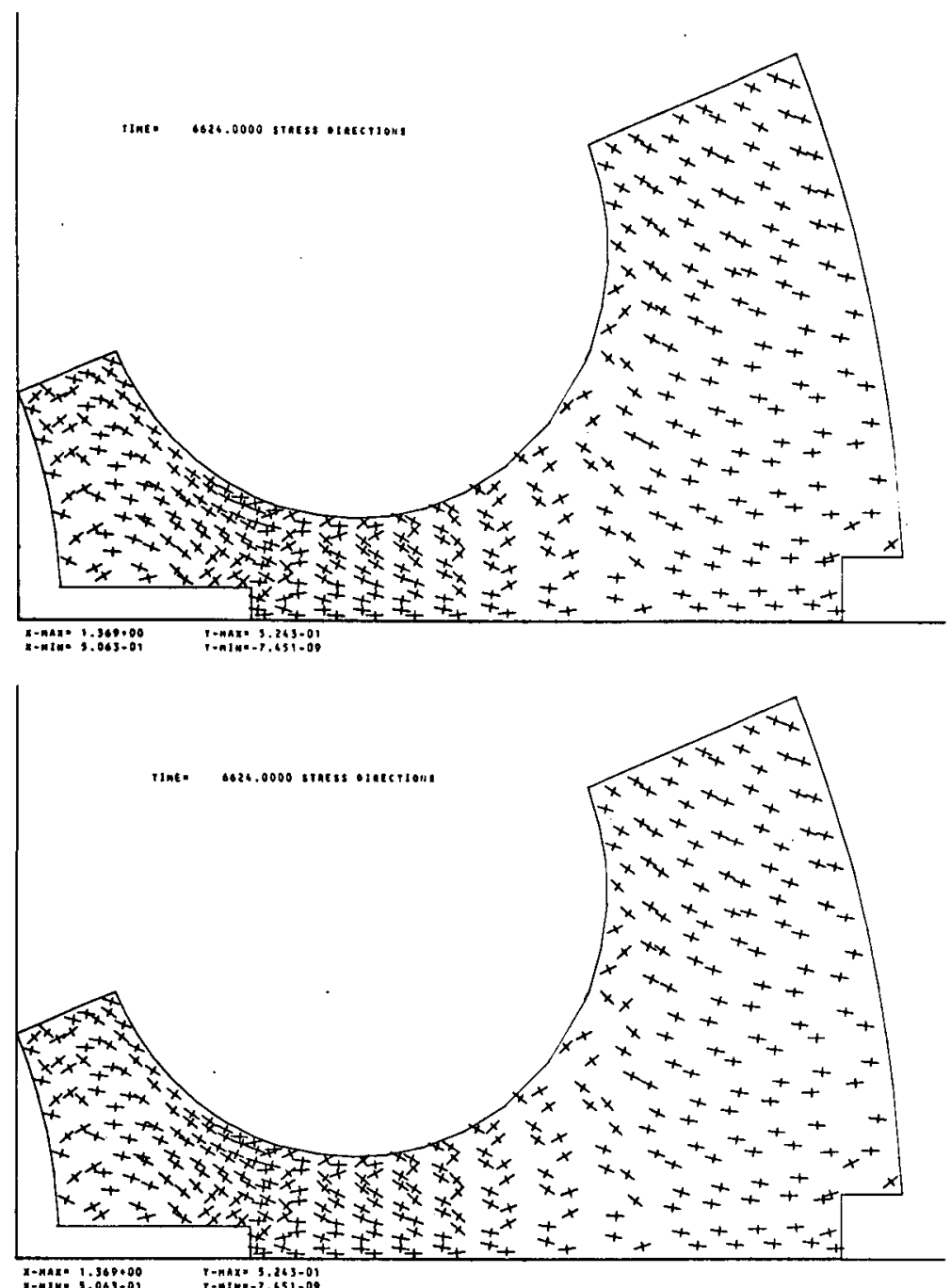

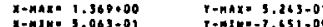

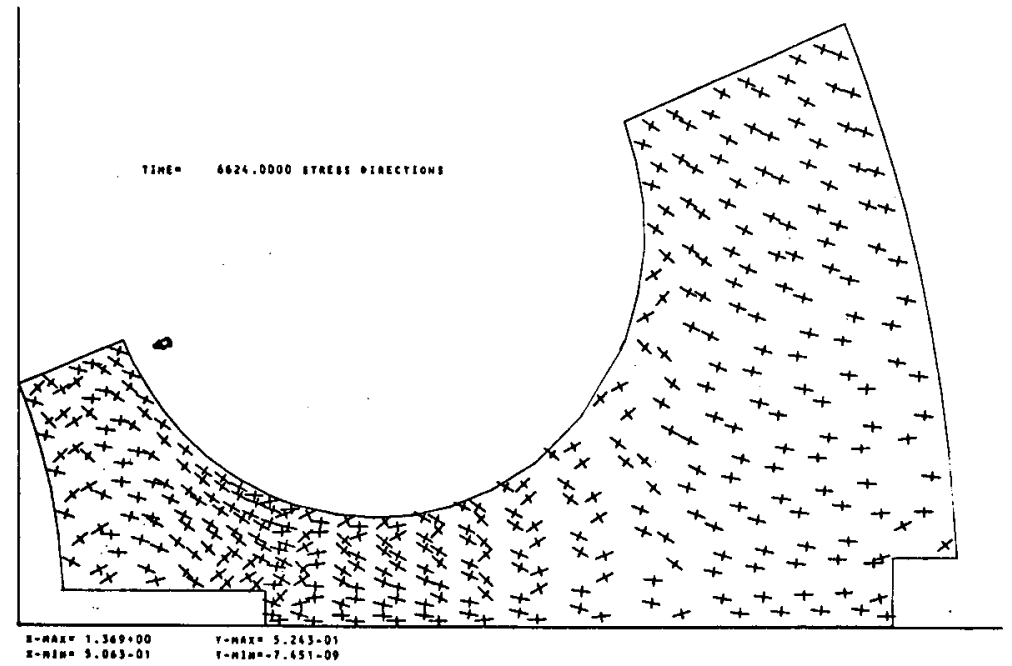

Fig. 3-38. FTE-6 fuel bodies 1, 2, and 3 stress directions for startup at BOL 

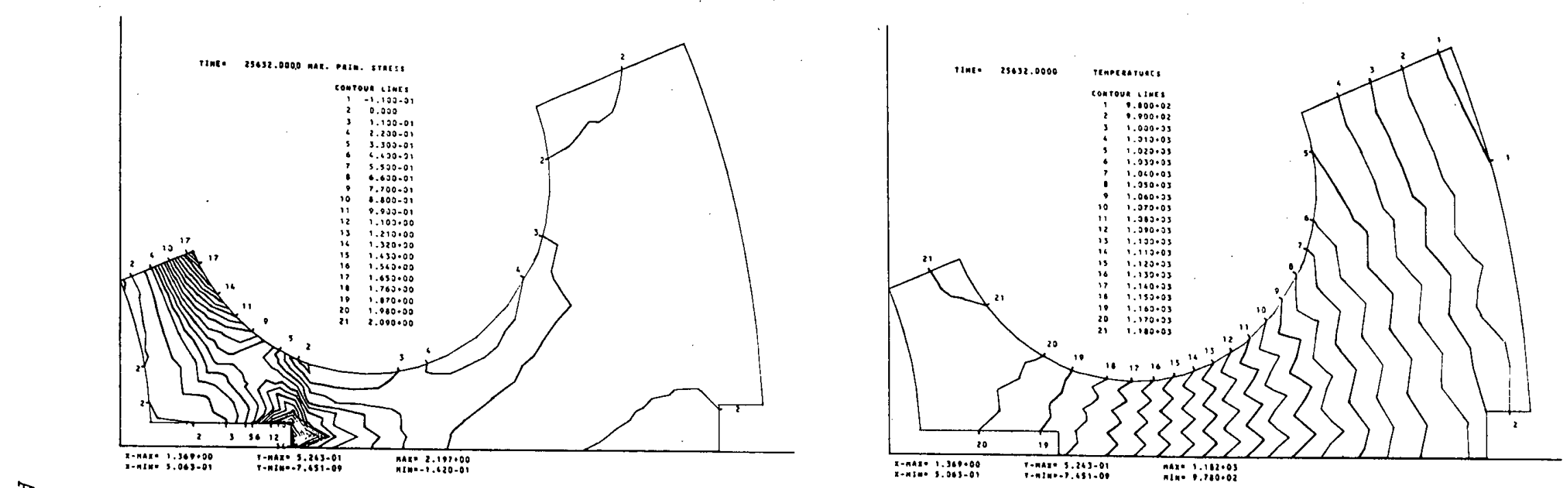

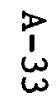
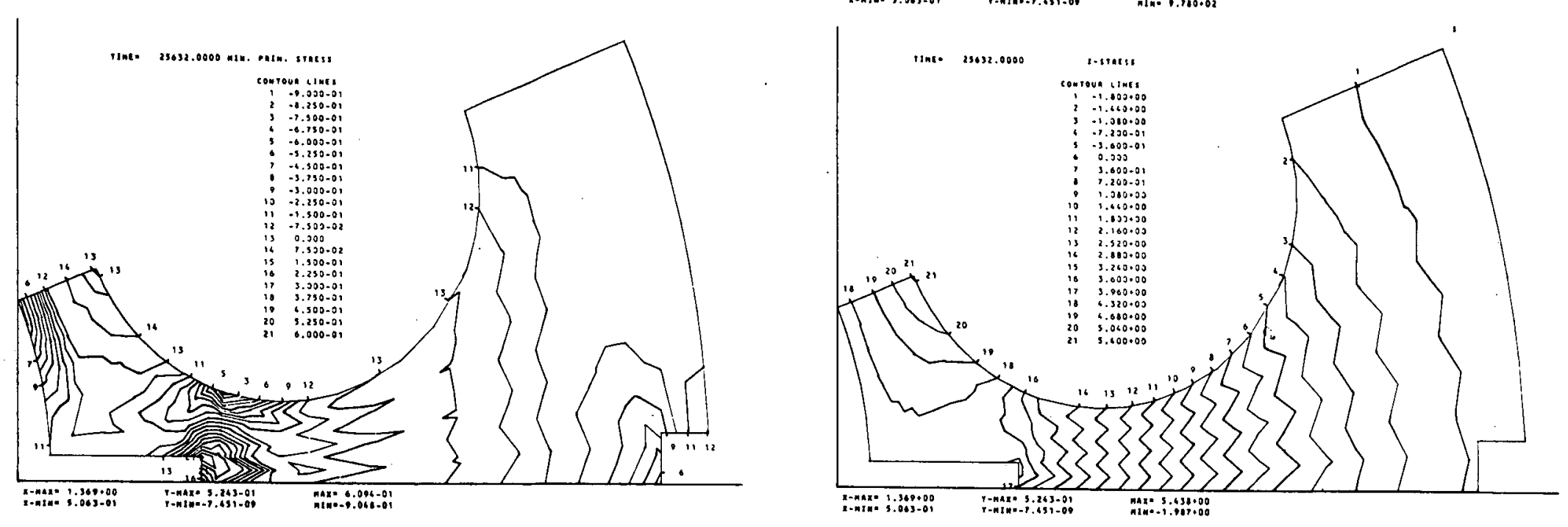

Fig. 3-39. FTE-6 fuel body 1 temperature and stress contour plots for operation at EOL 

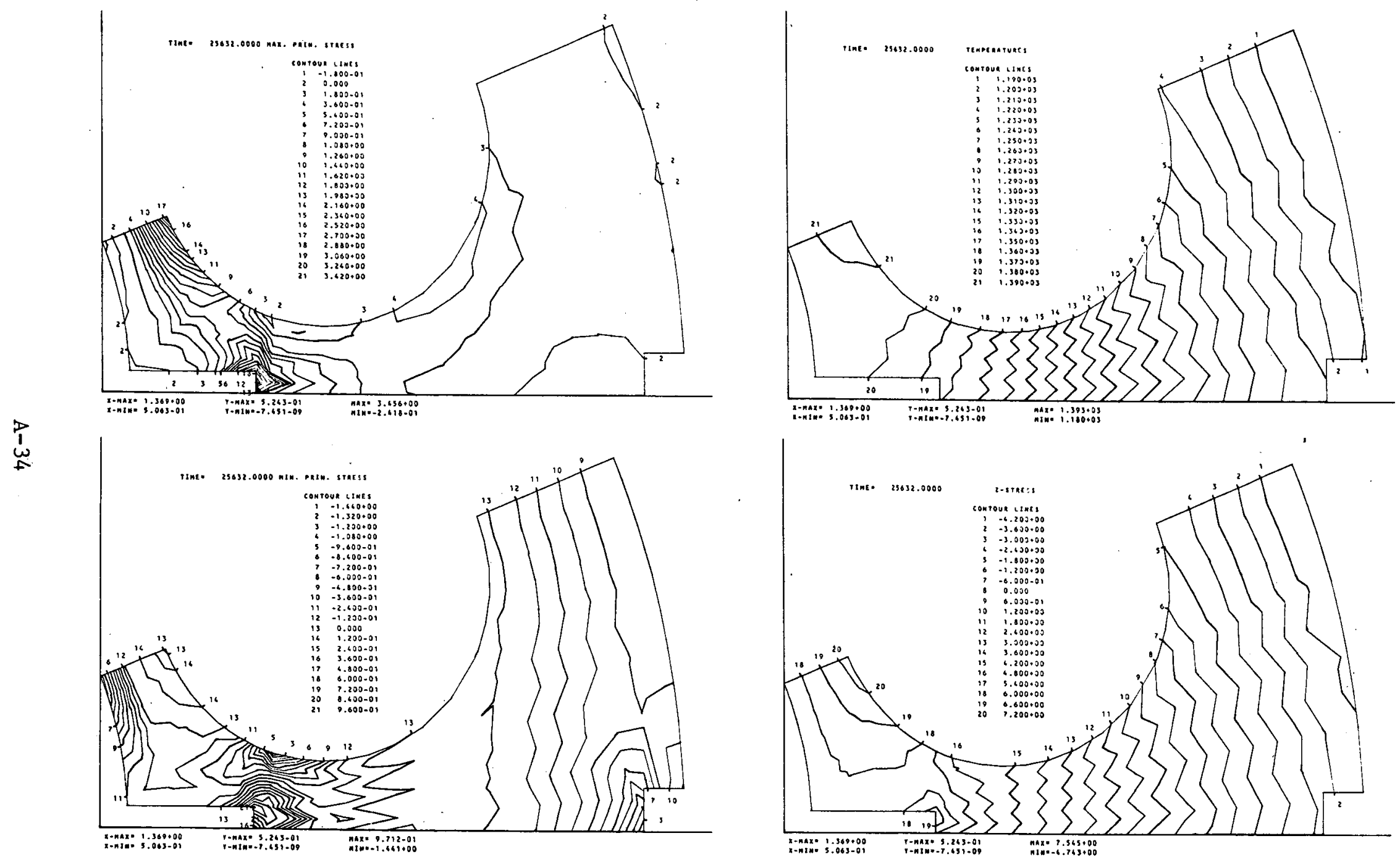

Fig. 3-40. FTE-6 fuel body 2 temperature and stress contour plots for operation at EOL 

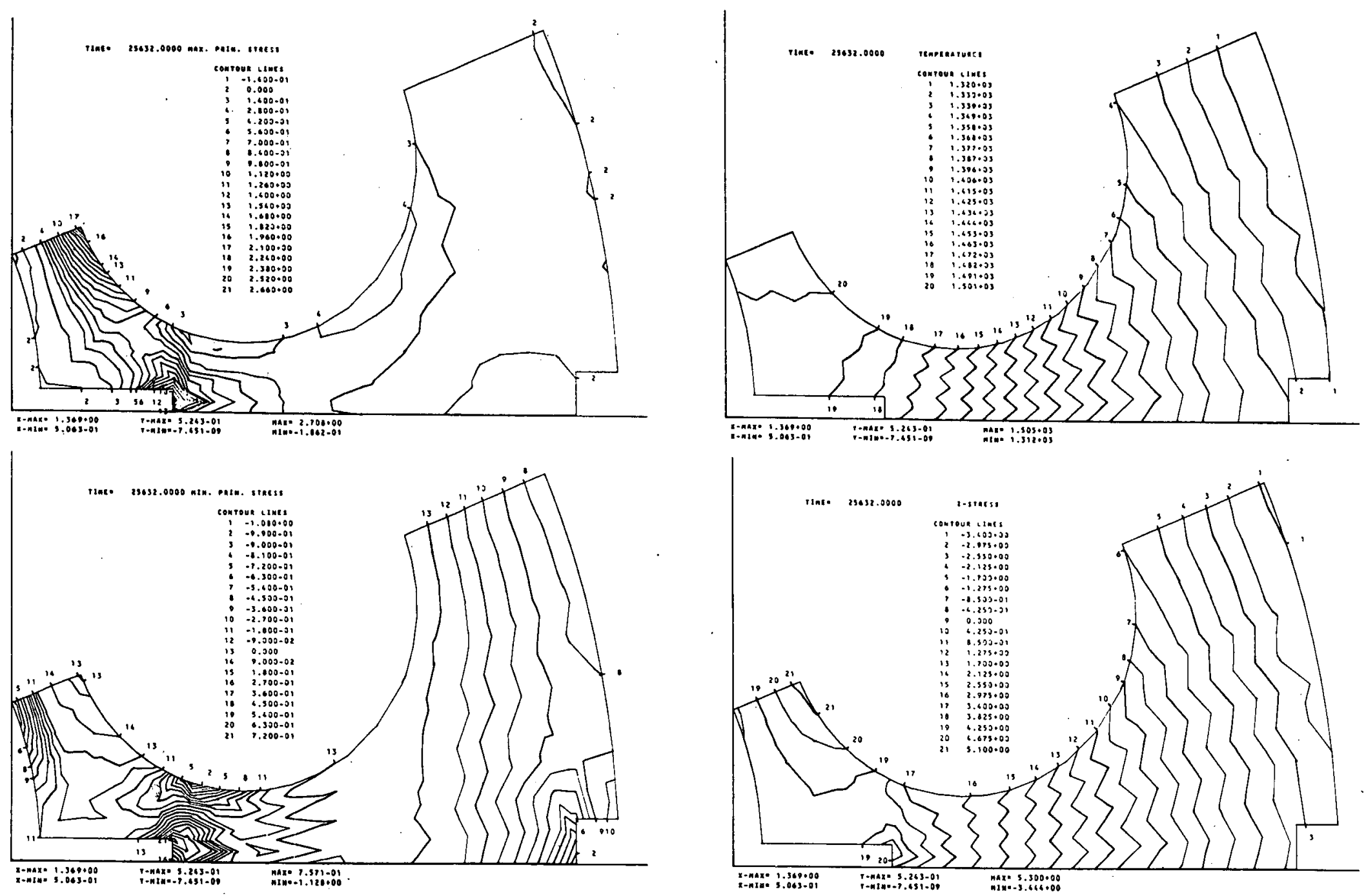

Fig. 3-41. FTE-6 fuel body 3 temperature and stress contour plots for operation at EOL 

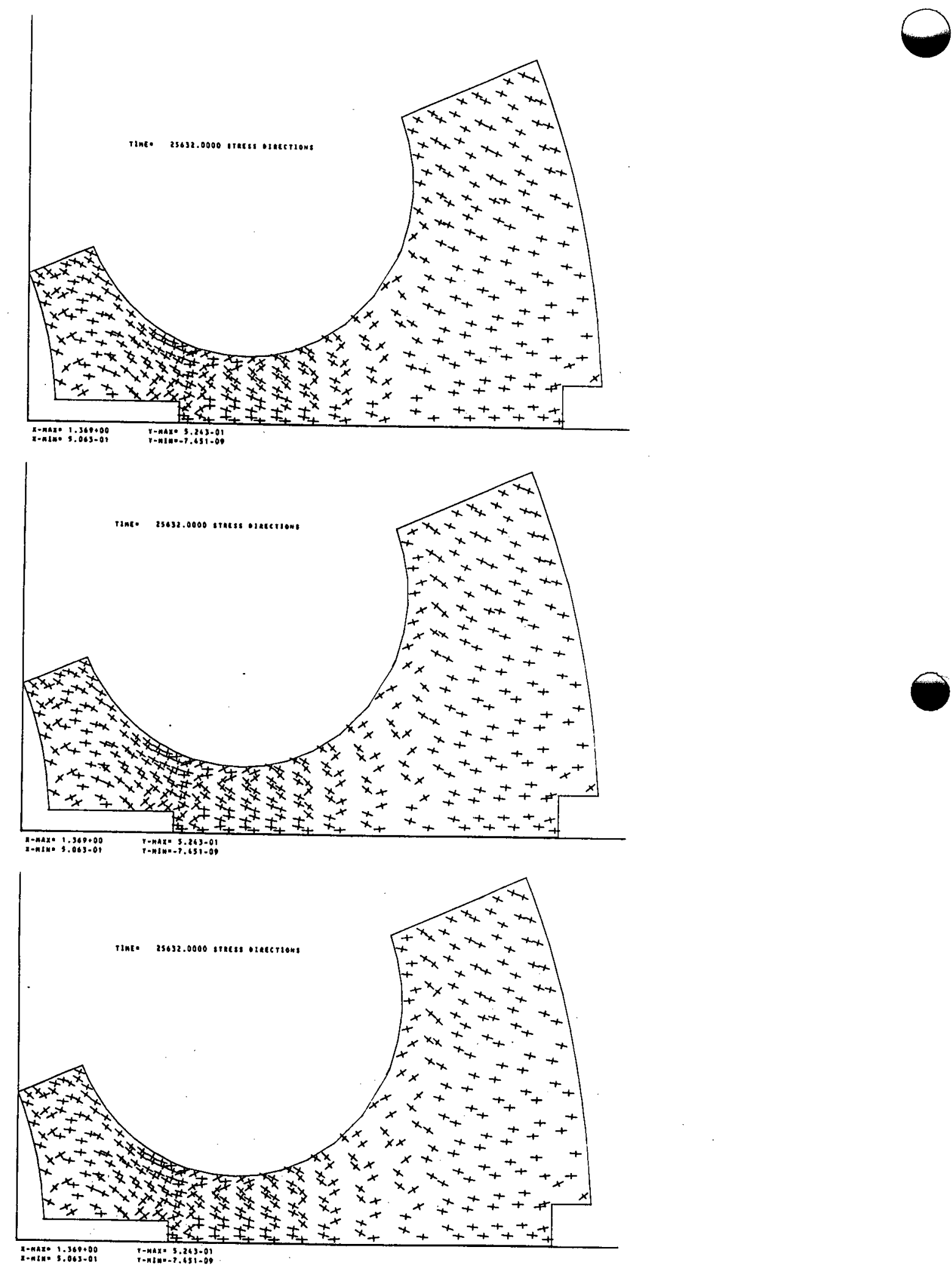

Fig. 3-42. FTE-6 fuel bodies 1, 2, and 3 stress directions for operation at EOL 

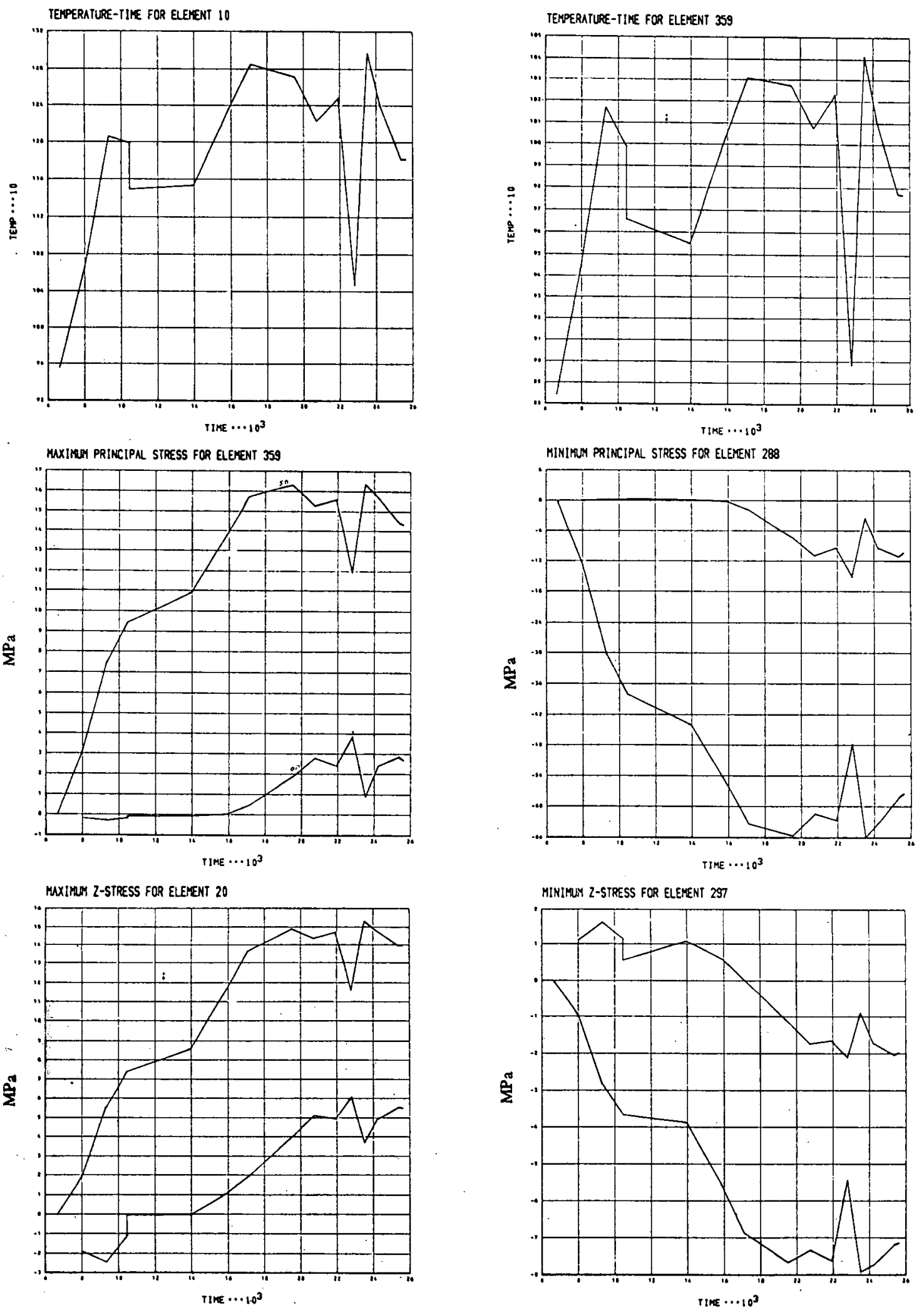

Fig. 3-43. FTE-6 fuel body 1 max-min temperatures and max-min stress histories for operation and shutdown 

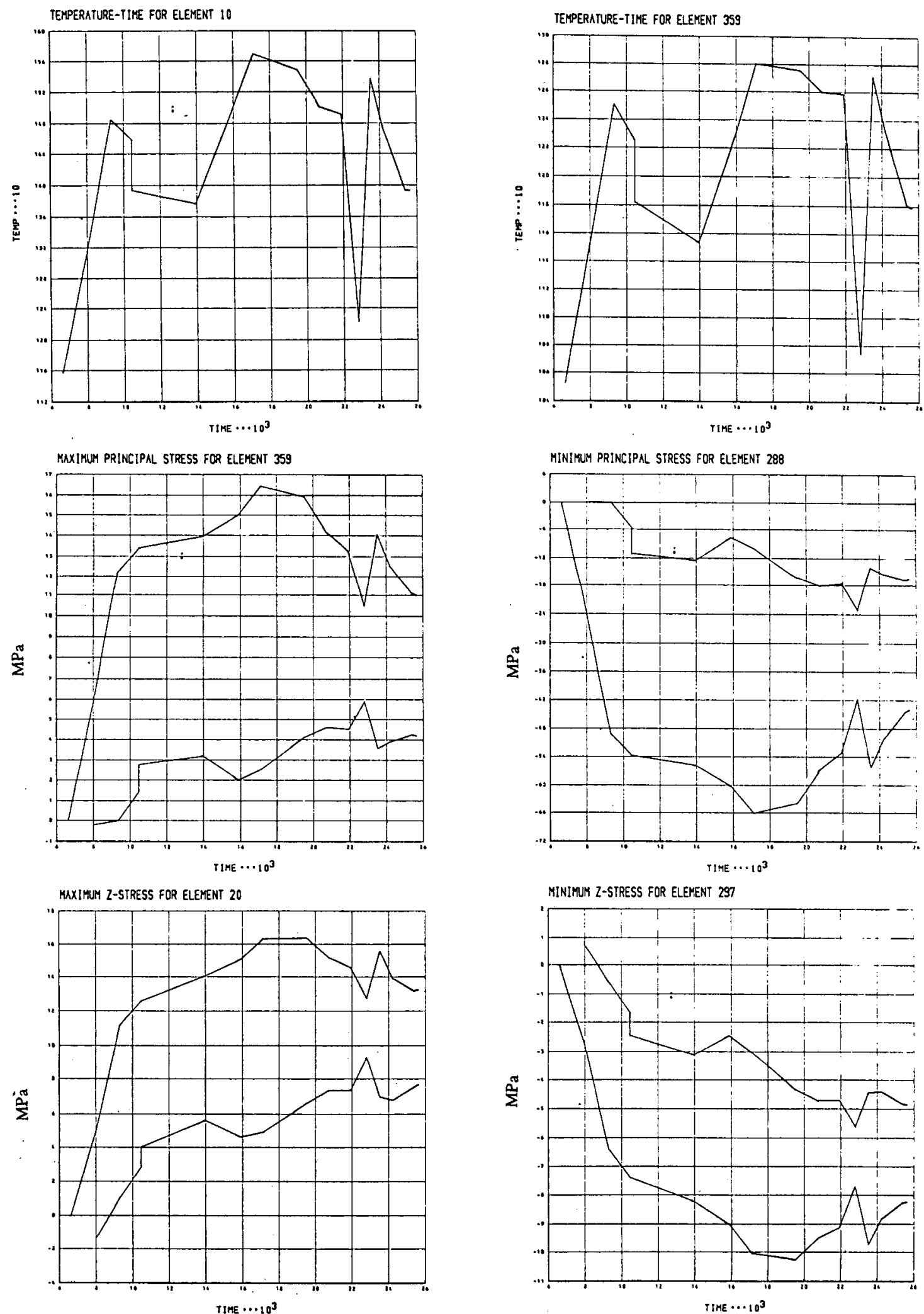

Fig. 3-44. FTE-6 fuel body 2 max-min temperatures and max-min stress histories for operation and shutdown 

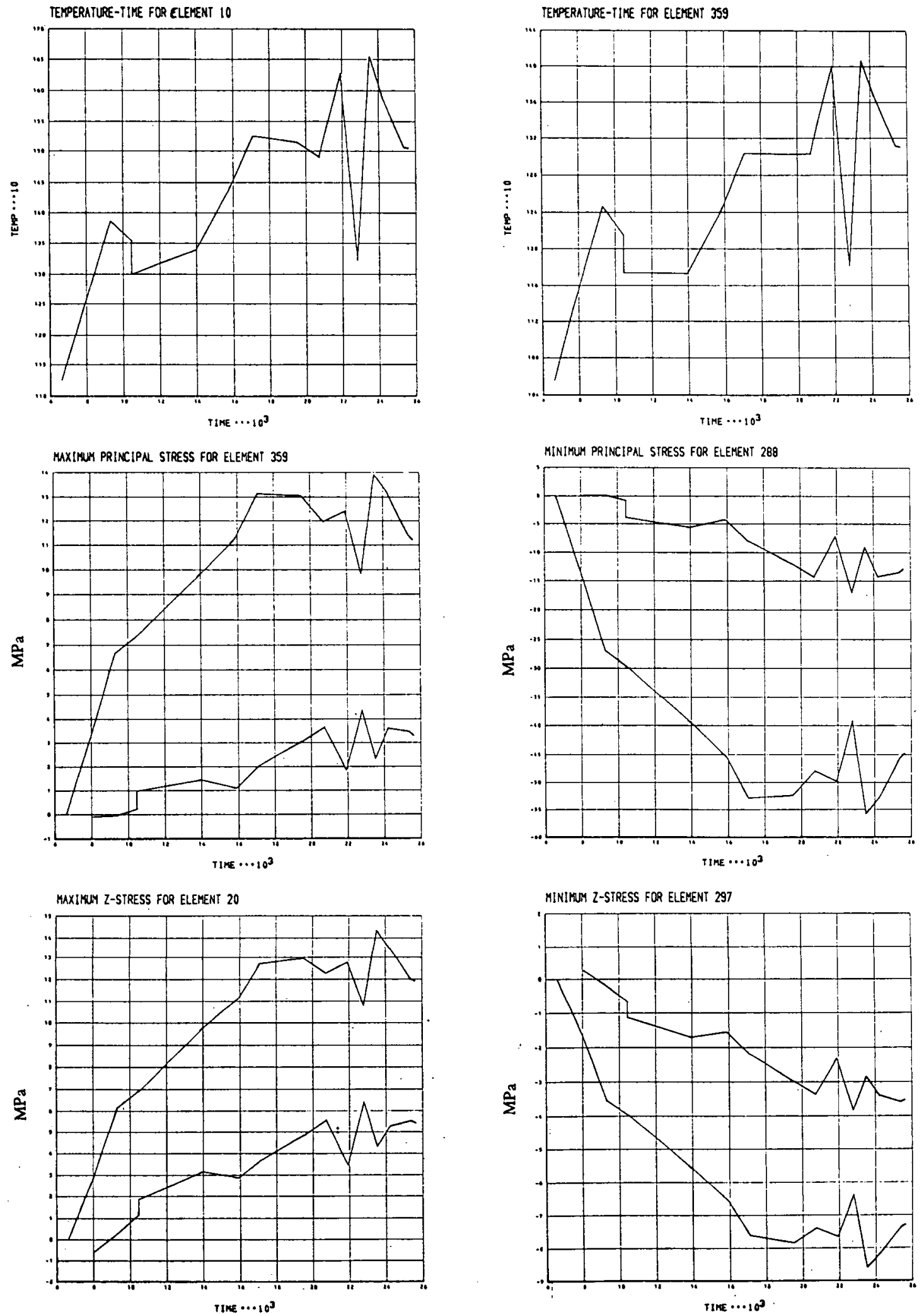

F1g. 3-45. FTE-6 fuel body 3 max-min temperatures and max-min stress histories for operation and shutdown 

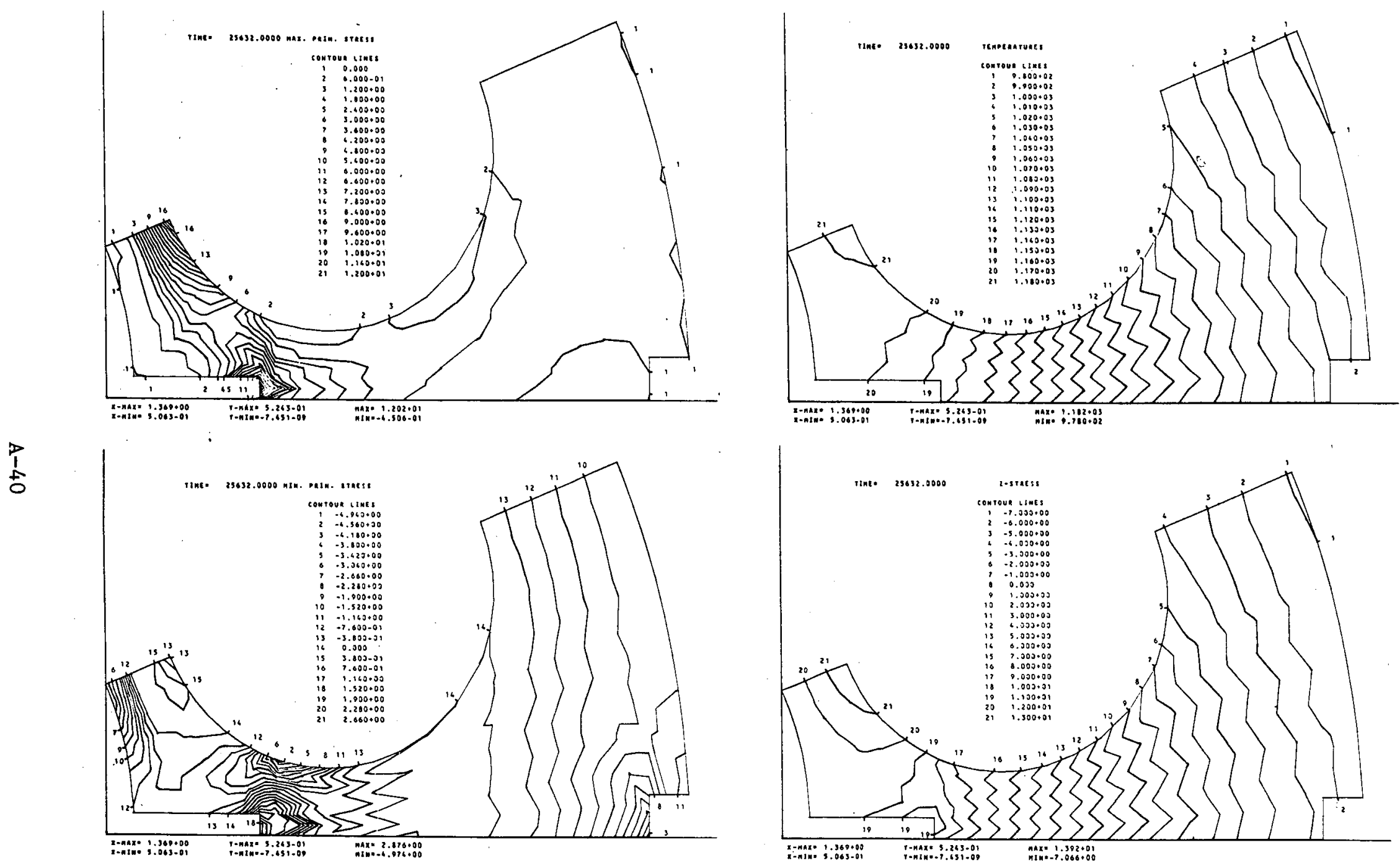

Fig. 3-46. FTE-6 fuel body 1 temperature at EOL operation and stress contour plots for EOL shutdown 

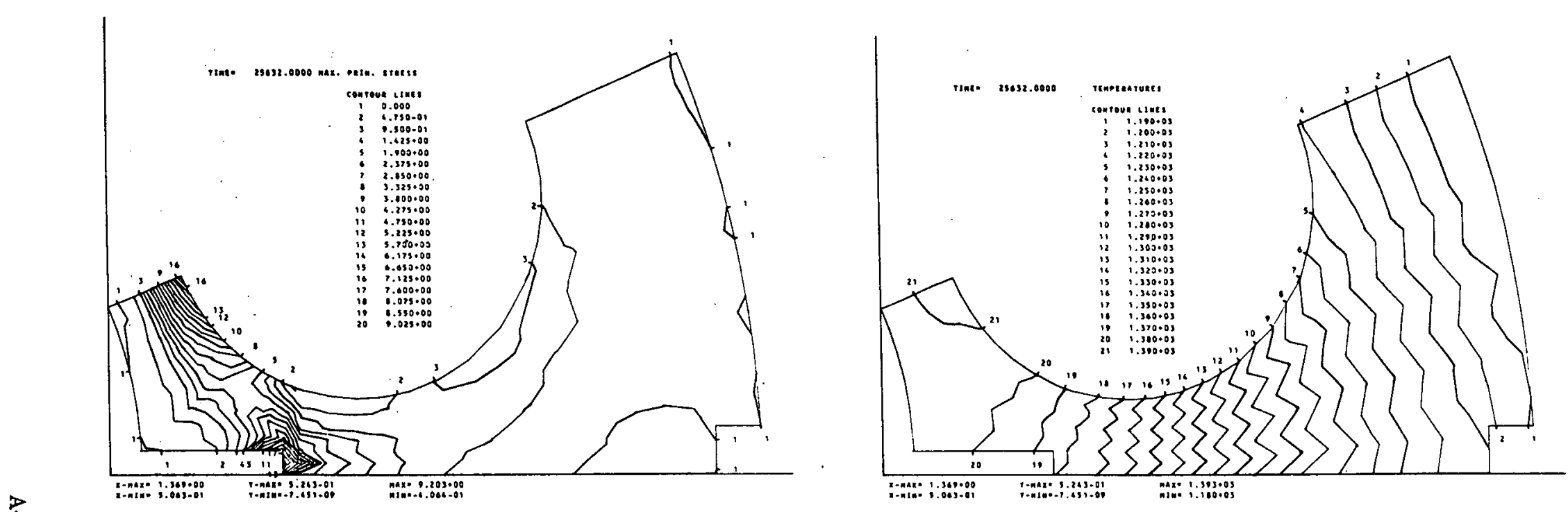

$\stackrel{p}{1}$
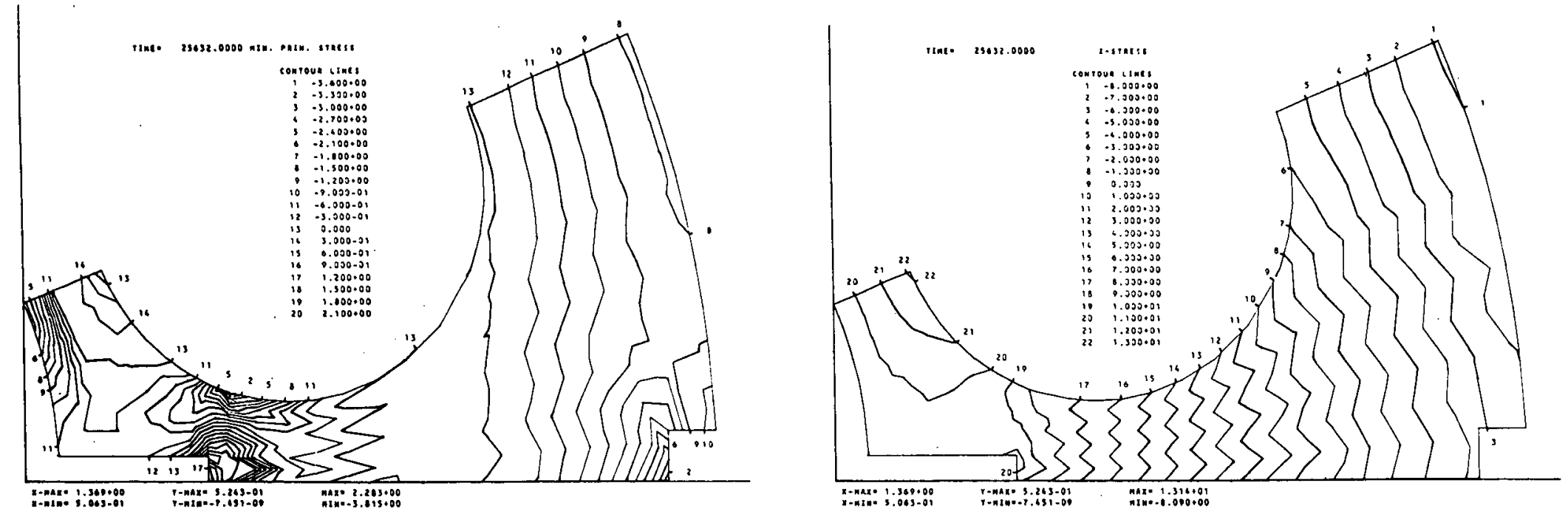

Fig. 3-47. FTE-6 fuel body 2 temperature at EOL operation and stress contour plots for EOL shutdown 

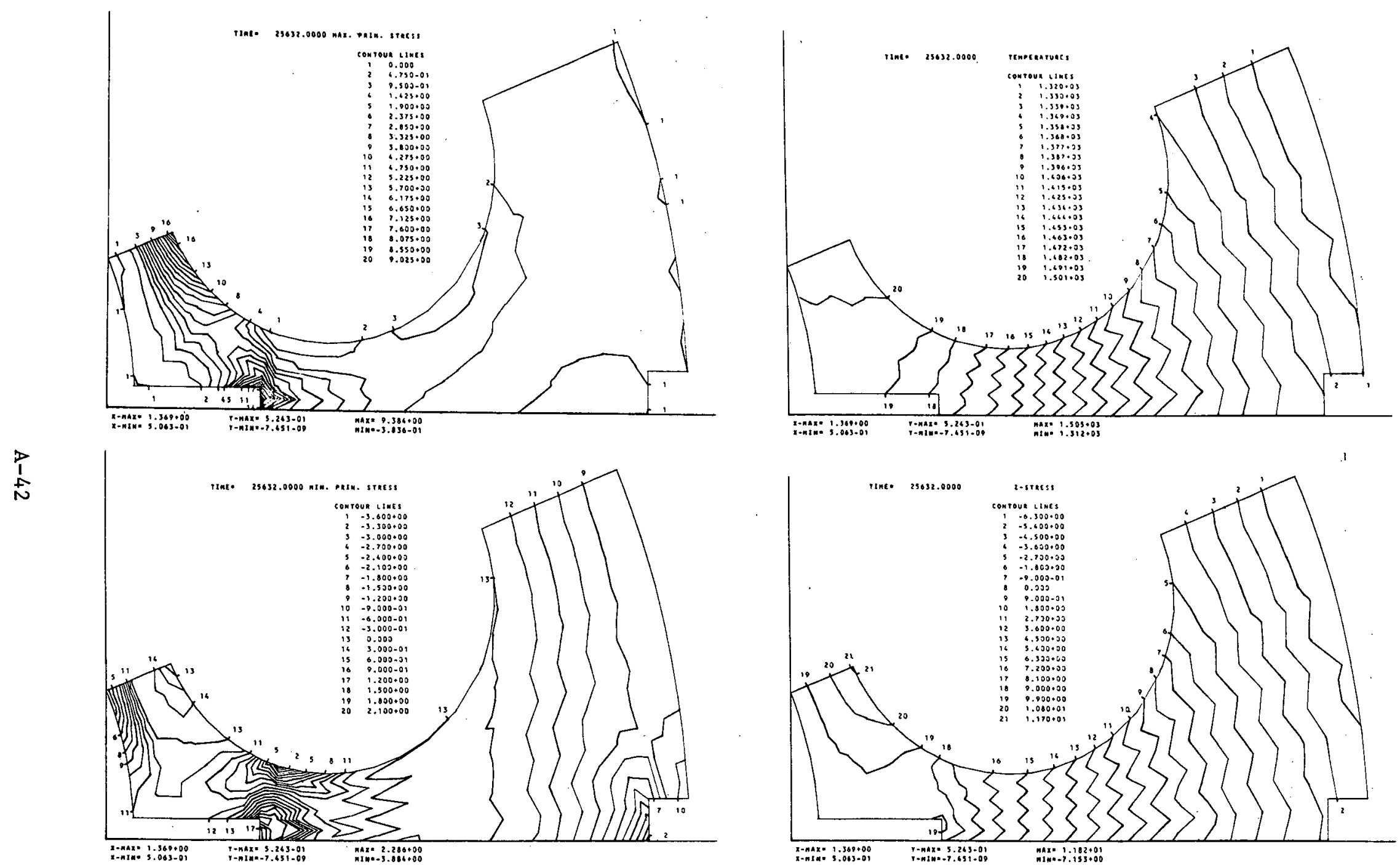

Fig. 3-48. FTE-6 fuel body 3 temperature at EOL operation and stress contour plots for EOL shutdown 

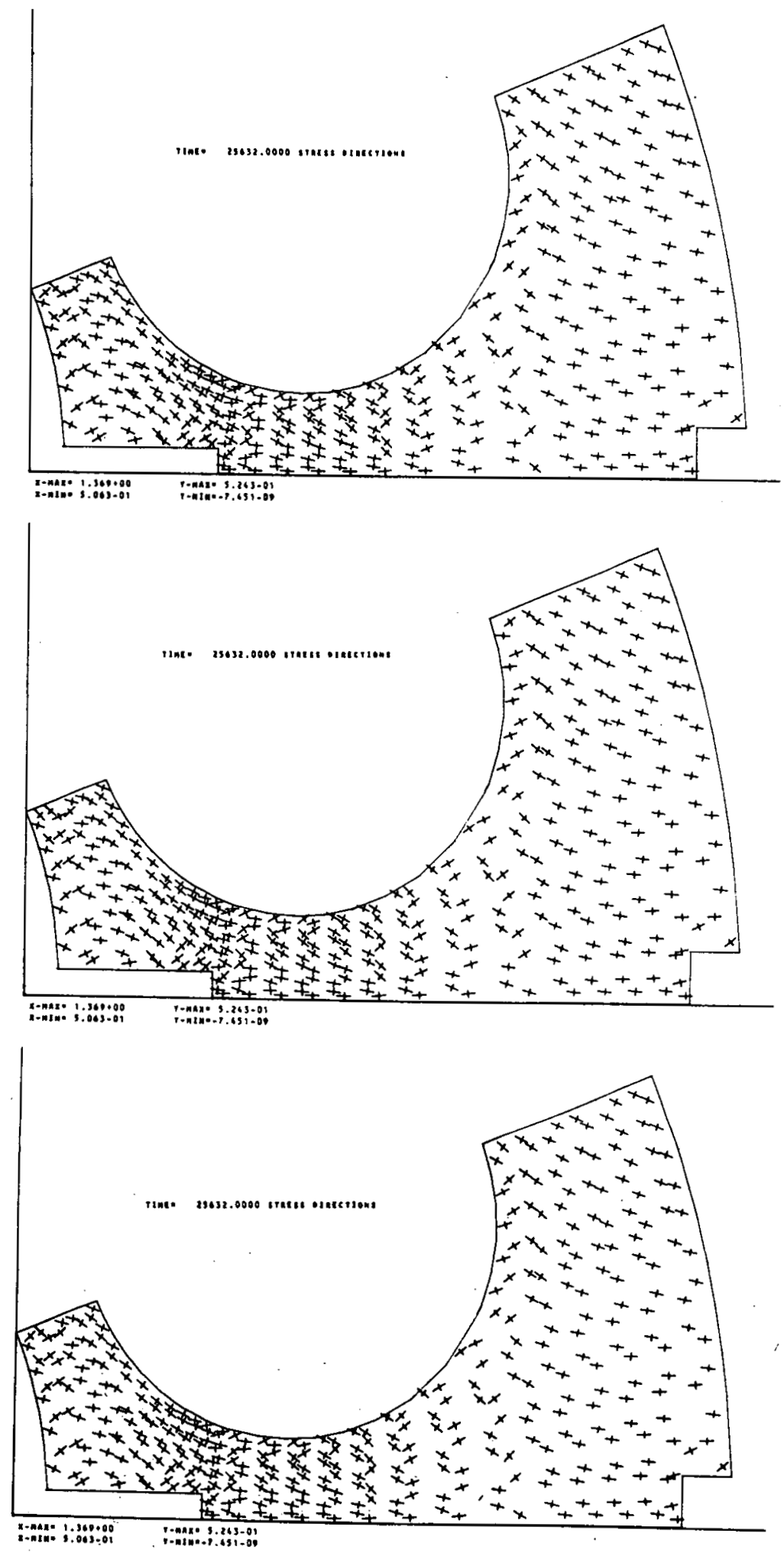

Fig. 3-49. FTE-6 fuel bodies 1, 2, and 3 stress directions for EOL shutdown 


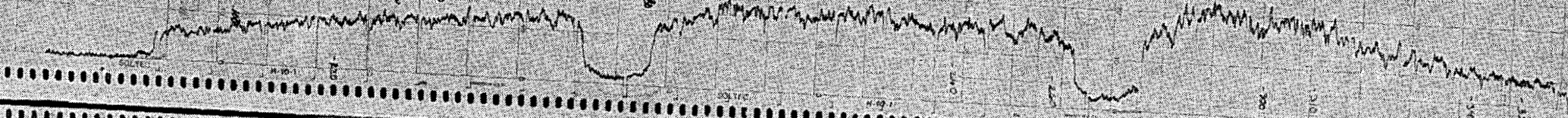

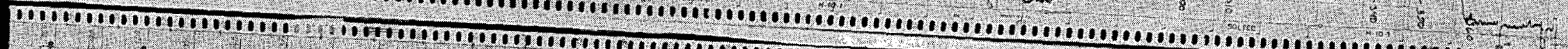

(2)

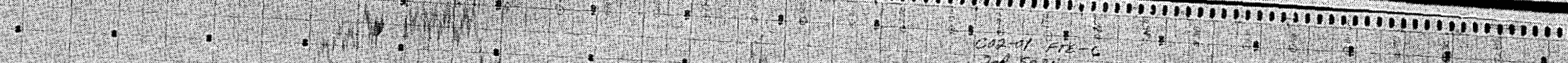

(2)

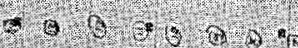

3) 6.6 .6

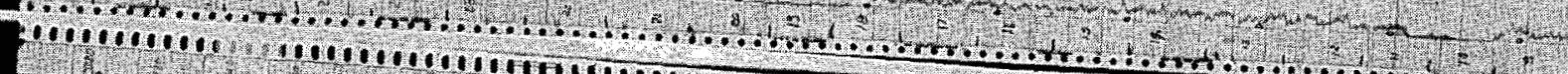

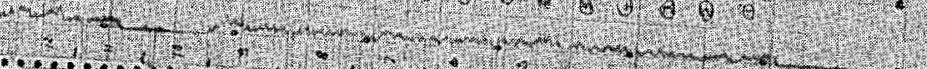

NWM

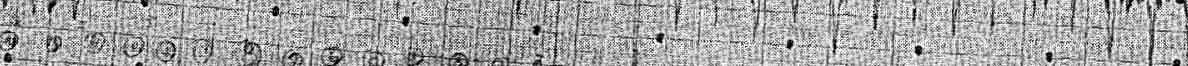

-3 e $^{2}$

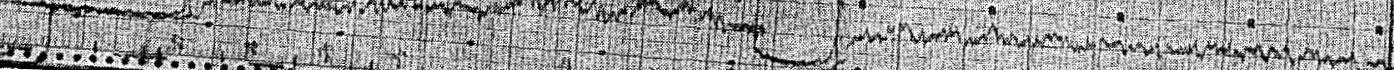

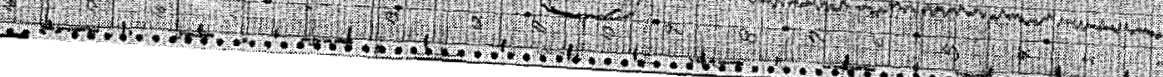

Fig. 4-1. FTE-6 Peach Bottom single-channel gamma-scan charts 


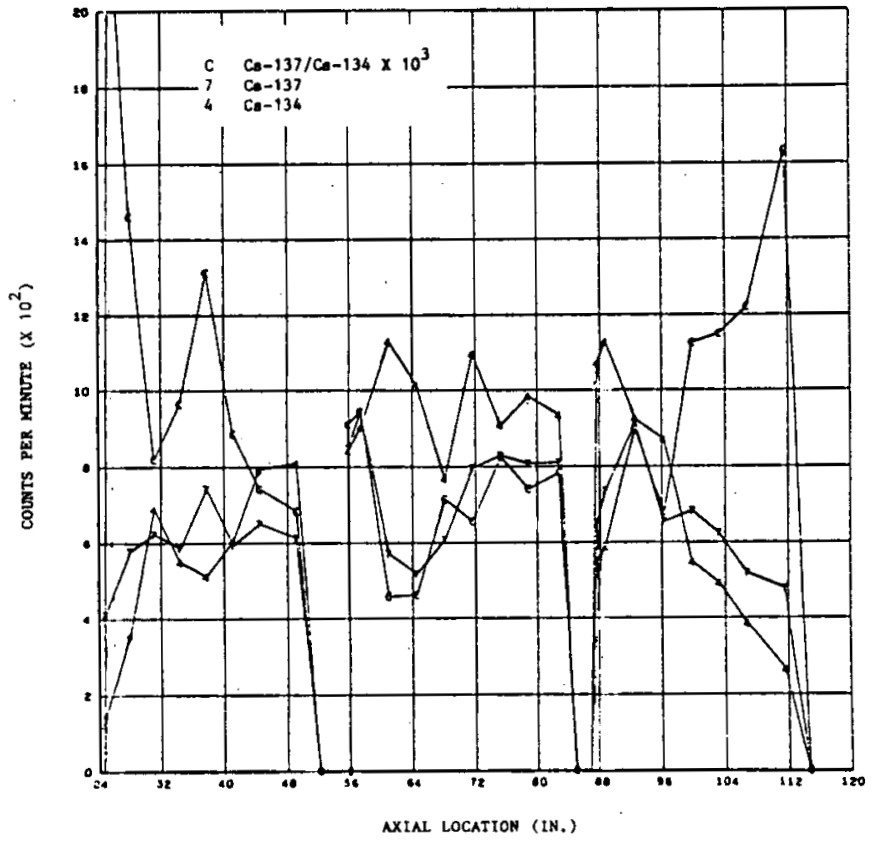

Fig. 4-2. FTE-6 Peach Bottom EOL gamma scans: CPM profiles for $\mathrm{Cs}-134, \mathrm{Cs}-137$, and $\mathrm{Cs}-137 /$ Cs-134 ratios

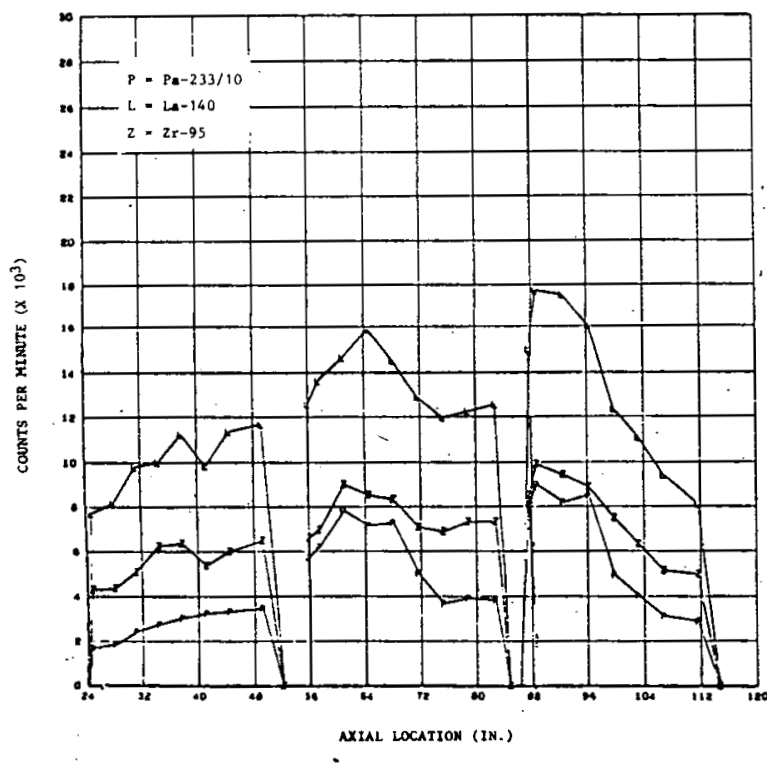

Fig. 4-4. FTE-6 Peach Bottom EOL gamma scans: CPM profiles for $\mathrm{Pa}-233, \mathrm{La}-140$, and $\mathrm{Zr}-95$

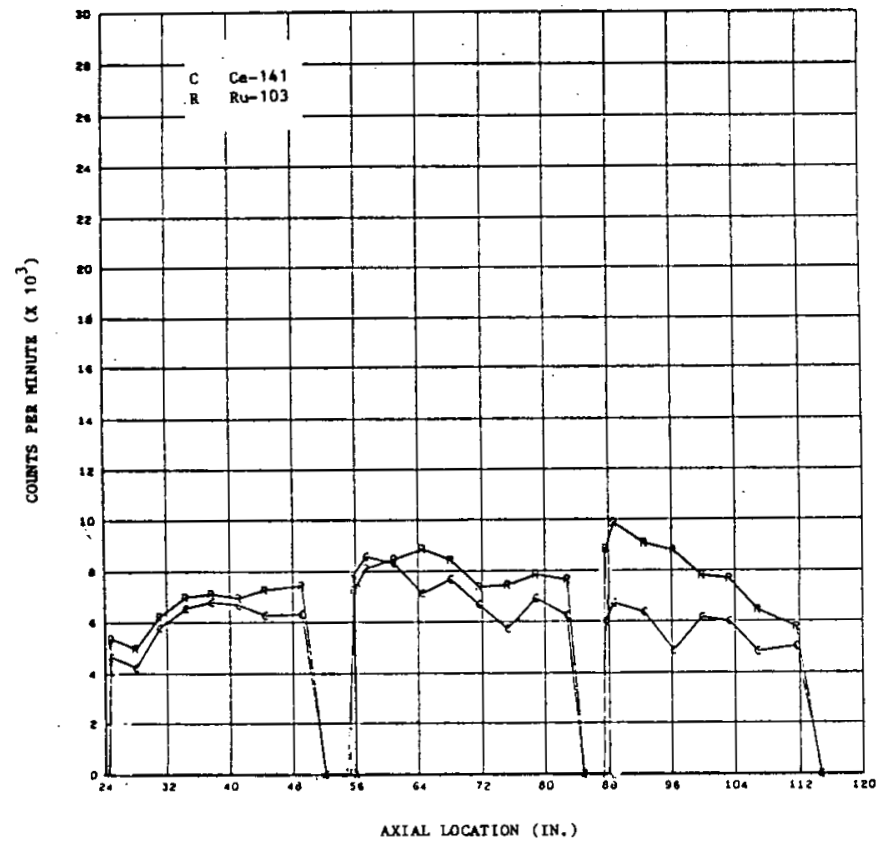

Fig. 4-3. FTE-6 Peach Bottom EOL gamma scans: CPM profiles for $\mathrm{Ce}-141$, and $\mathrm{Ru}-103$

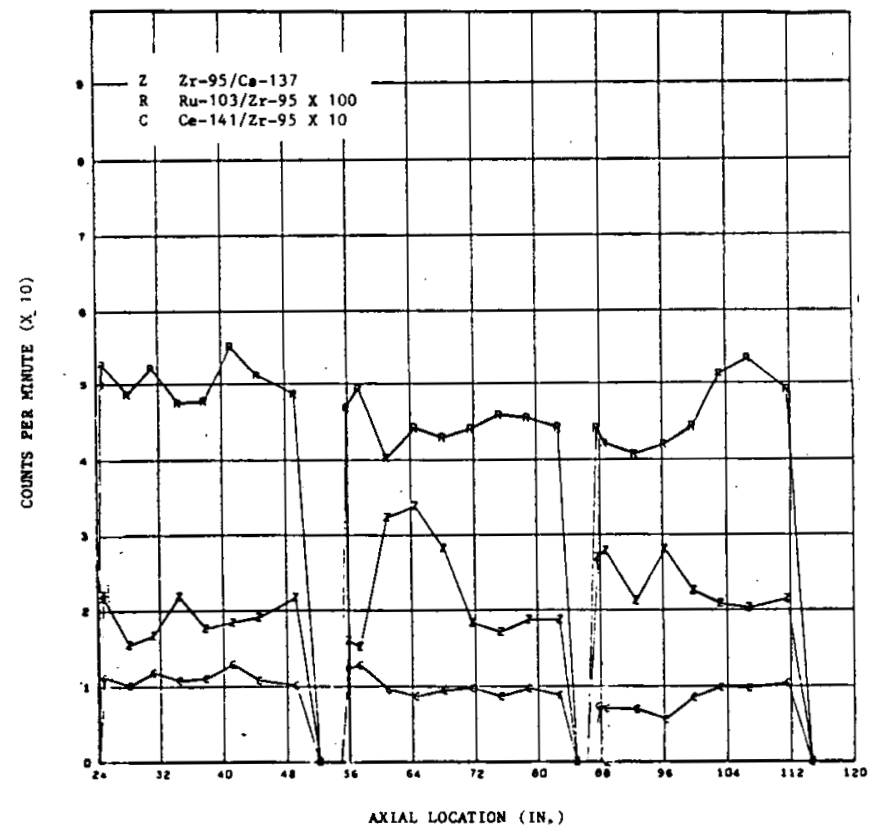

FIg. 4-5. FTE-6 Peach Bottom EOL gamma scans: profiles for $\mathrm{Zr}-95 /$ $\mathrm{Cs}-137, \mathrm{Ru}-103 / 2 \mathrm{r}-95$, and $\mathrm{Ce}-141 / \mathrm{Zr}-95$ ratios 


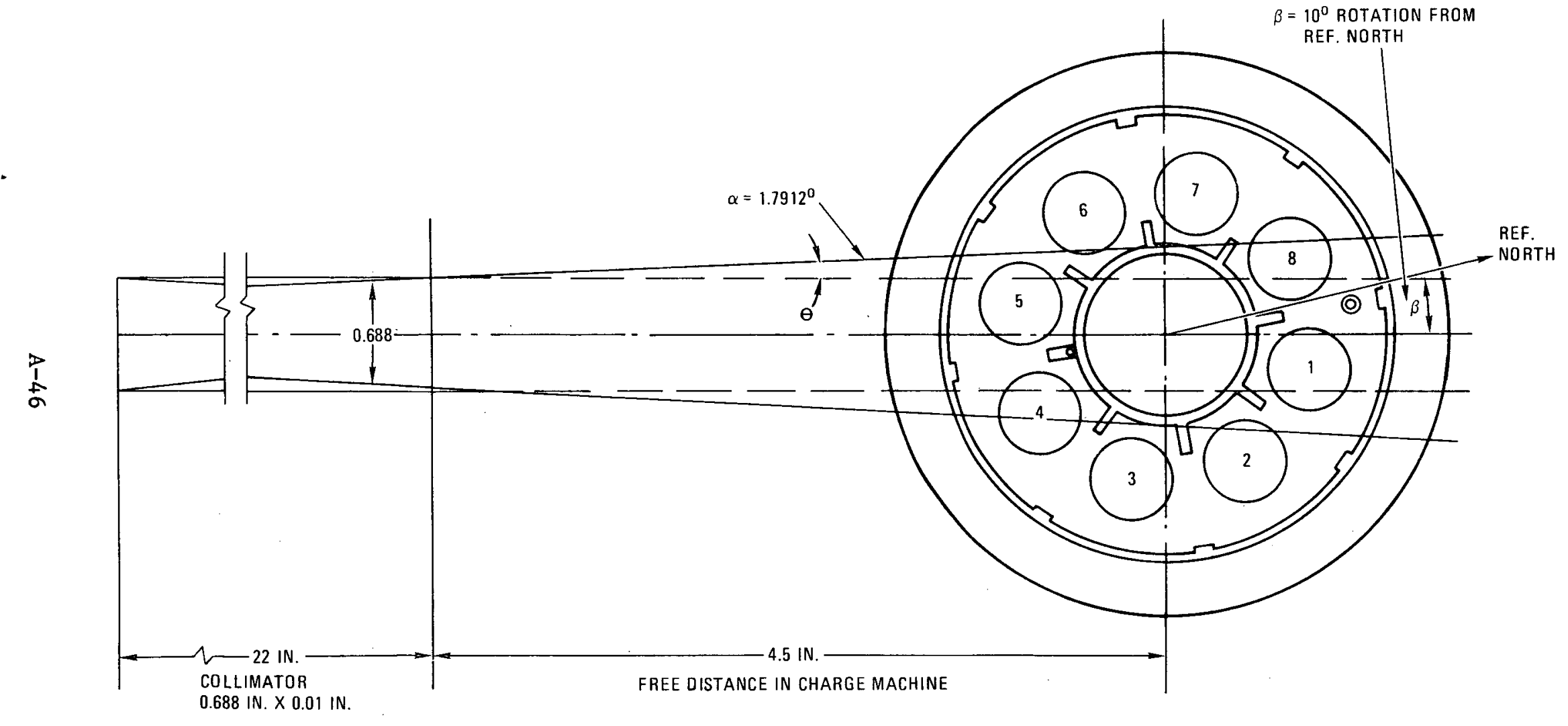

Fig. 4-6. FTE-6 Peach Bottom fuel gamma scan, azimuthal configuration 


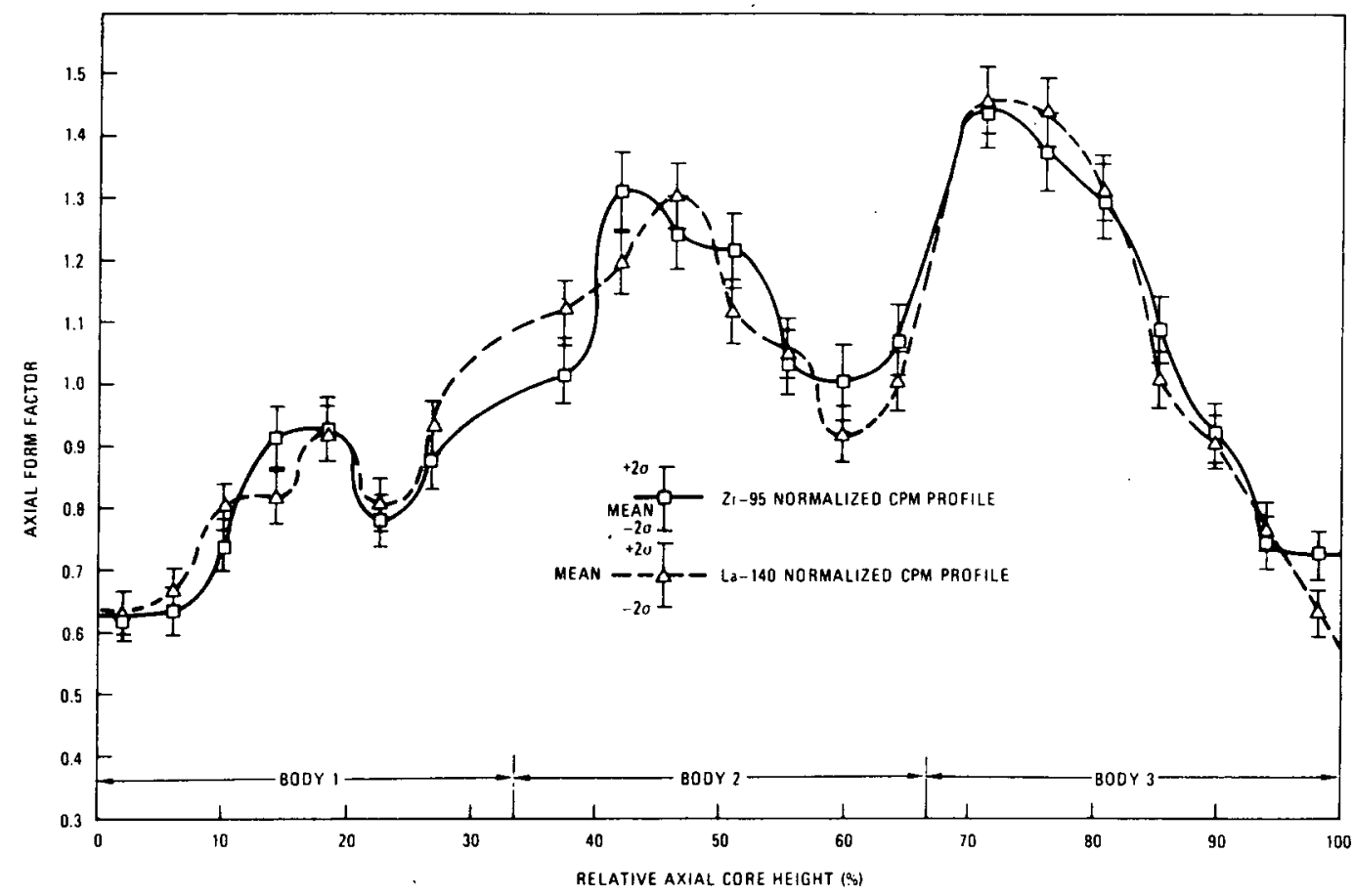

Fig. 4-7. FTE-6 comparison of La-140 and Zr-95 normalized CPM profiles as determined from Peach Bottom EOL gamma scan 1.

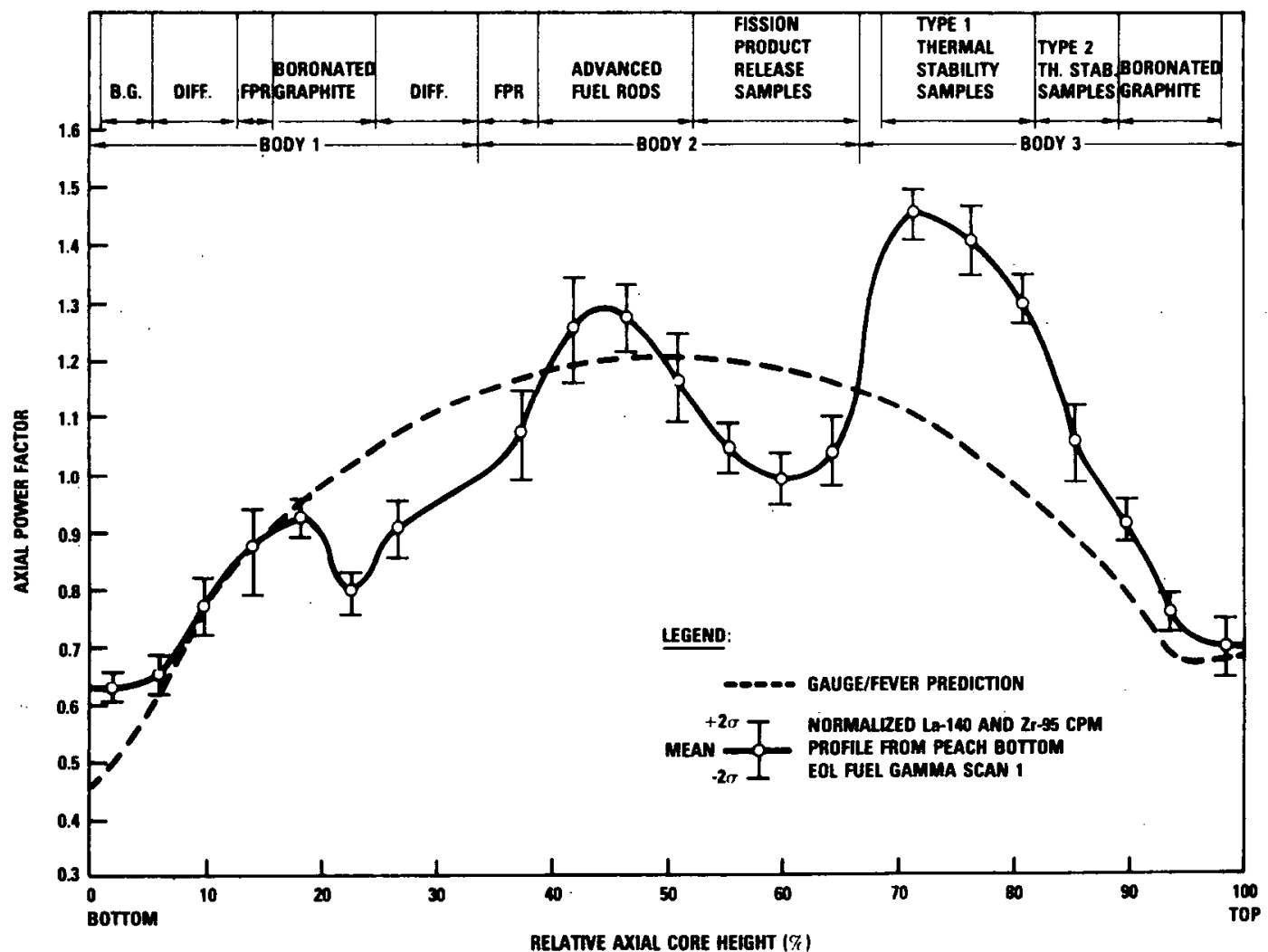

F1g. 4-8. FTE-6 predicted and measured EOL axial power distribution determined from Peach Bottom EOL gamma scan 1 


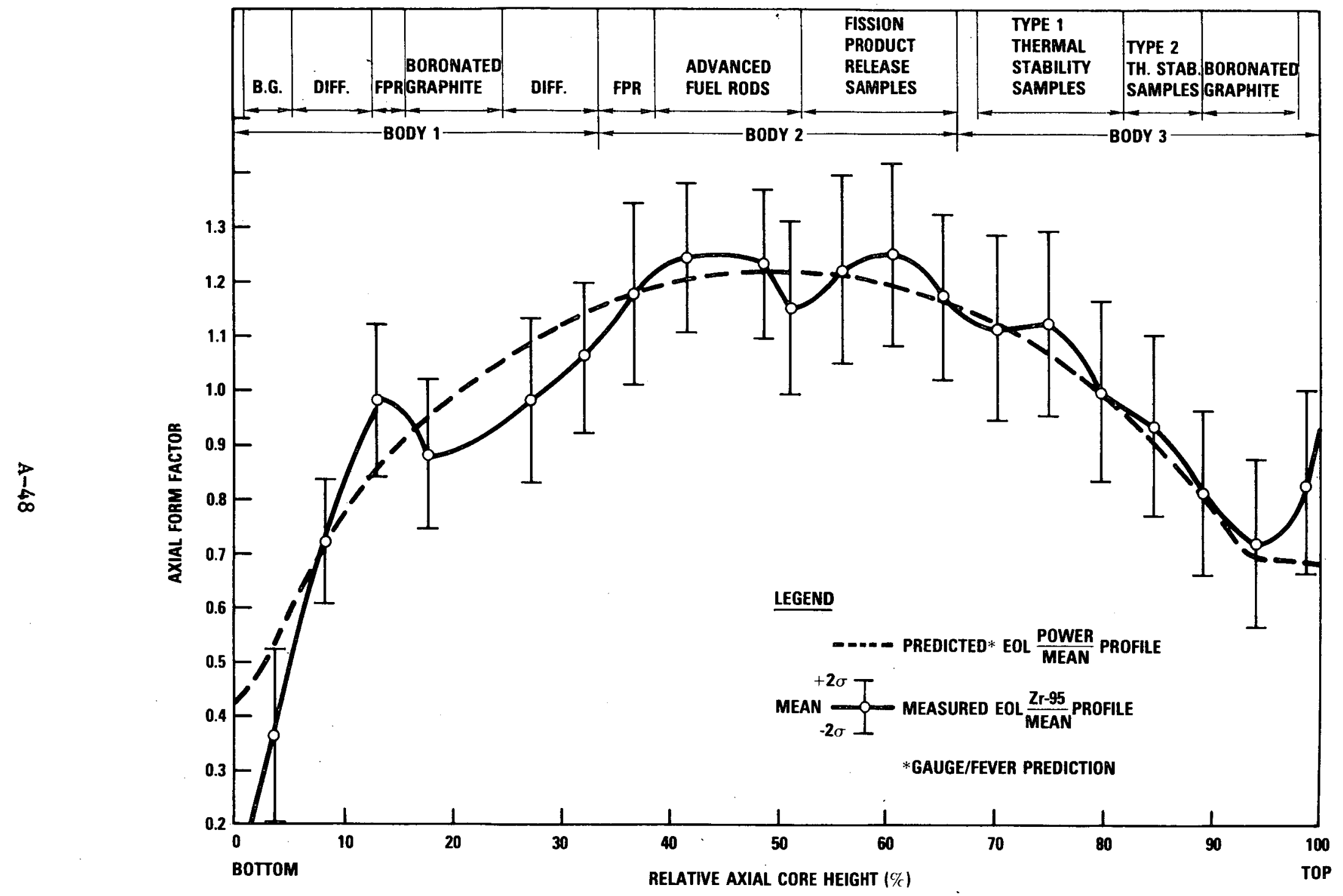

Fig. 4-9. FTE-6 predicted and measured EOL axial power distribution determined from fuel stack 1 gamma scans 


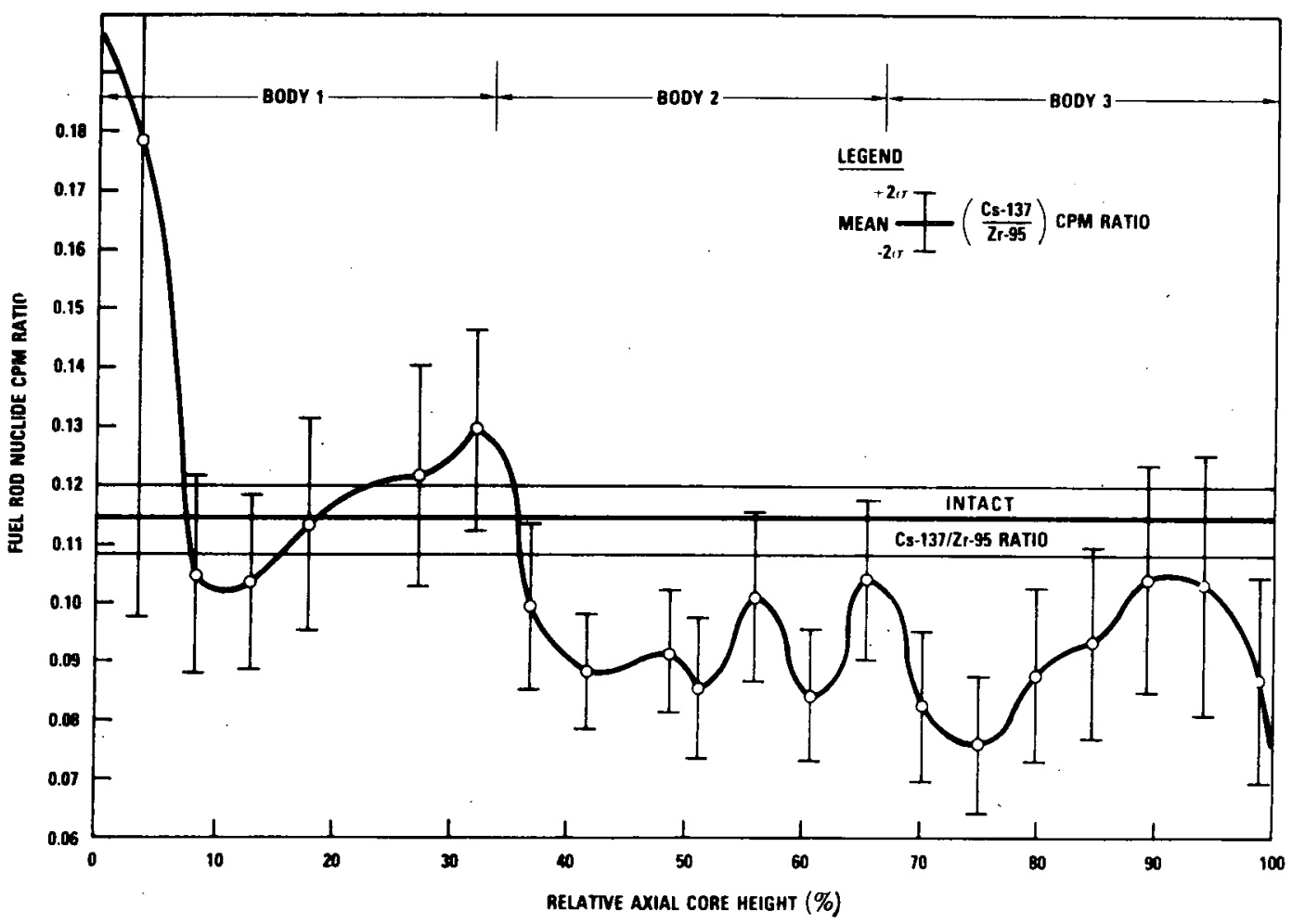

Fig. 4-10. FTE-6 Cs-137/Zr-95 CPM profile versus core height for fuel stack 1

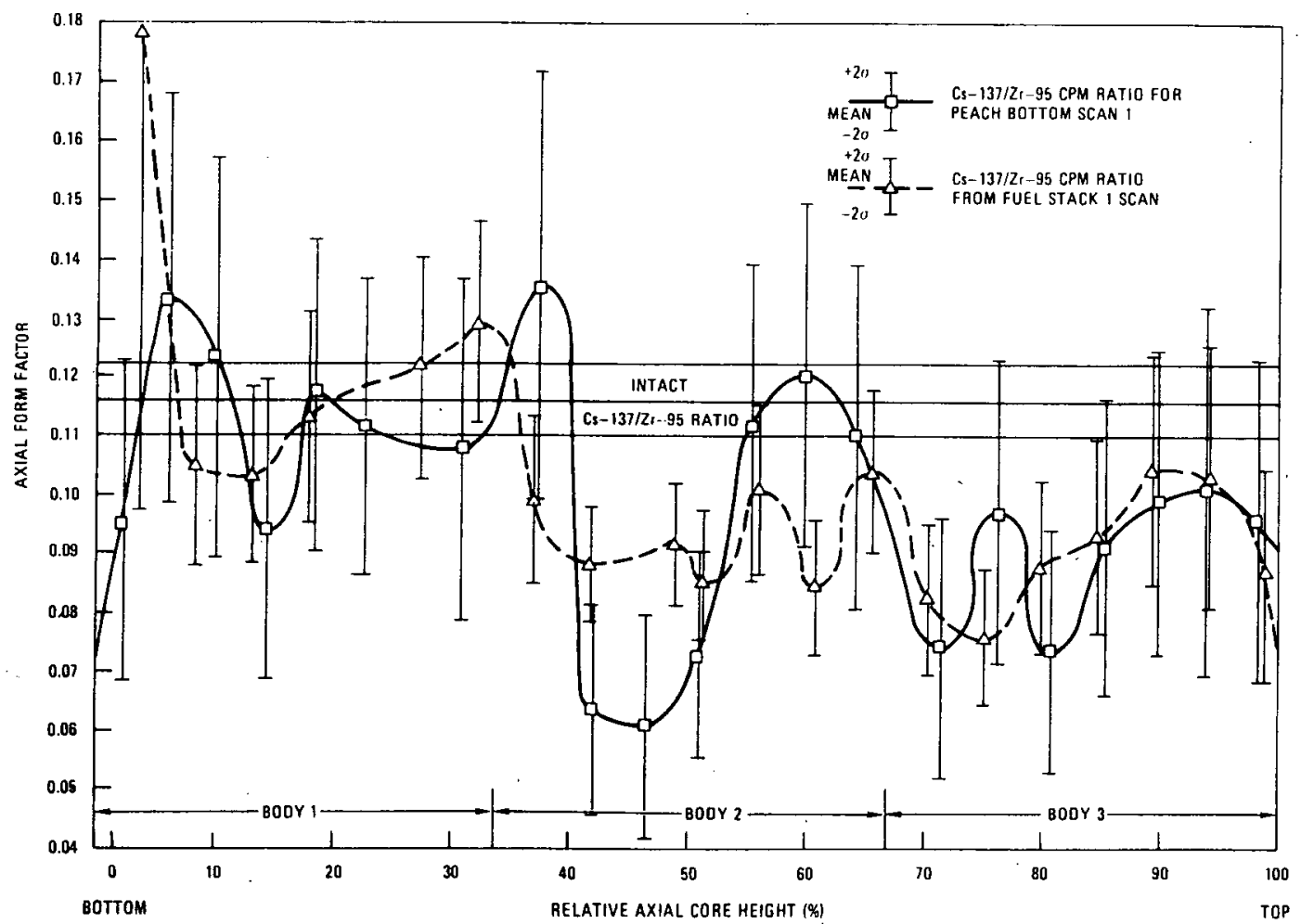

Fig. 4-11. FTE-6 fuel gamma scan comparison of Cs-137/Zr-95 CPM profiles from two different methods 


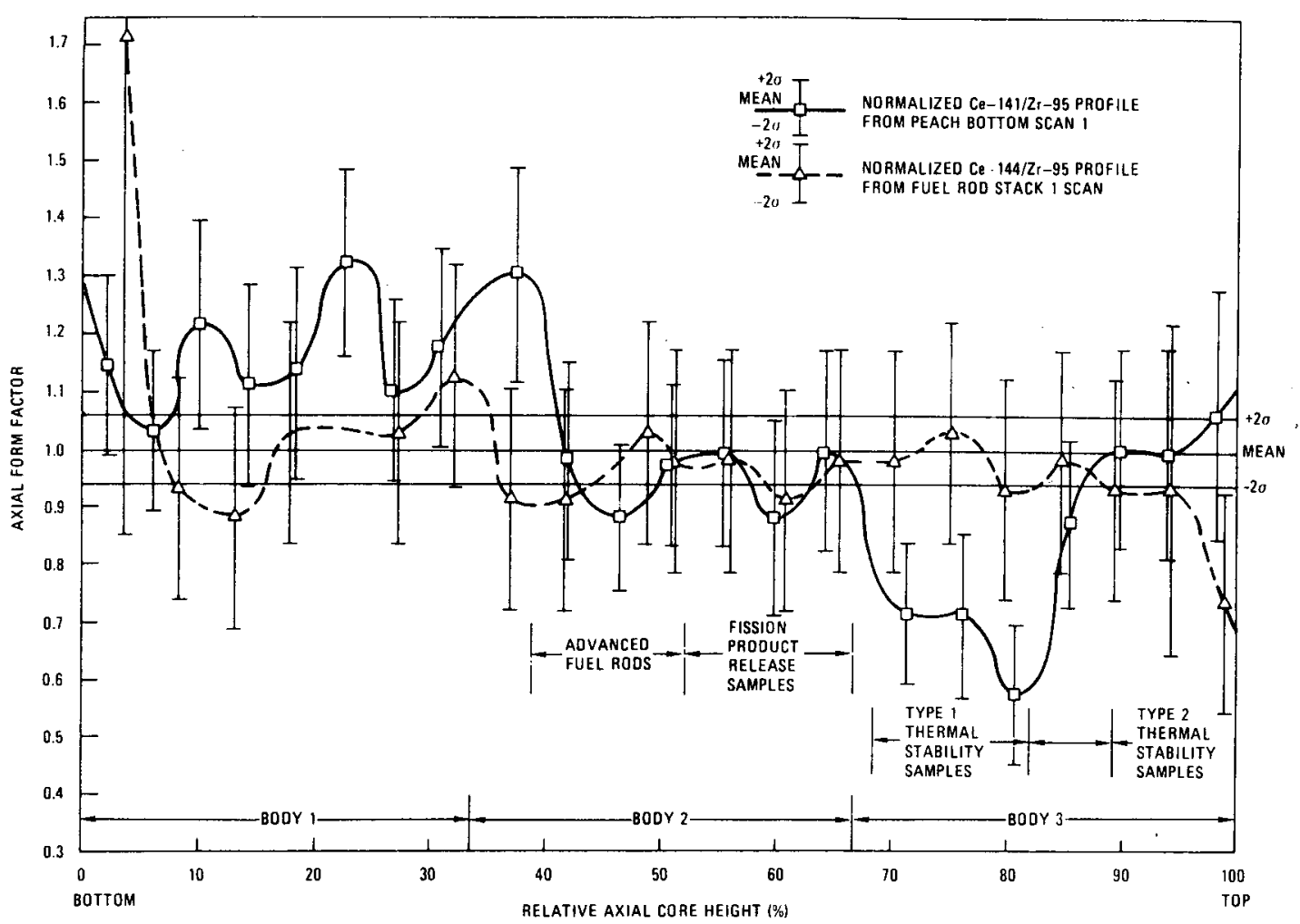

Fig. 4-12. FTE-6 fuel gamma scan comparison of normalized Ce-141/Zr-95 and Ce-144/Zr-95 CPM profiles

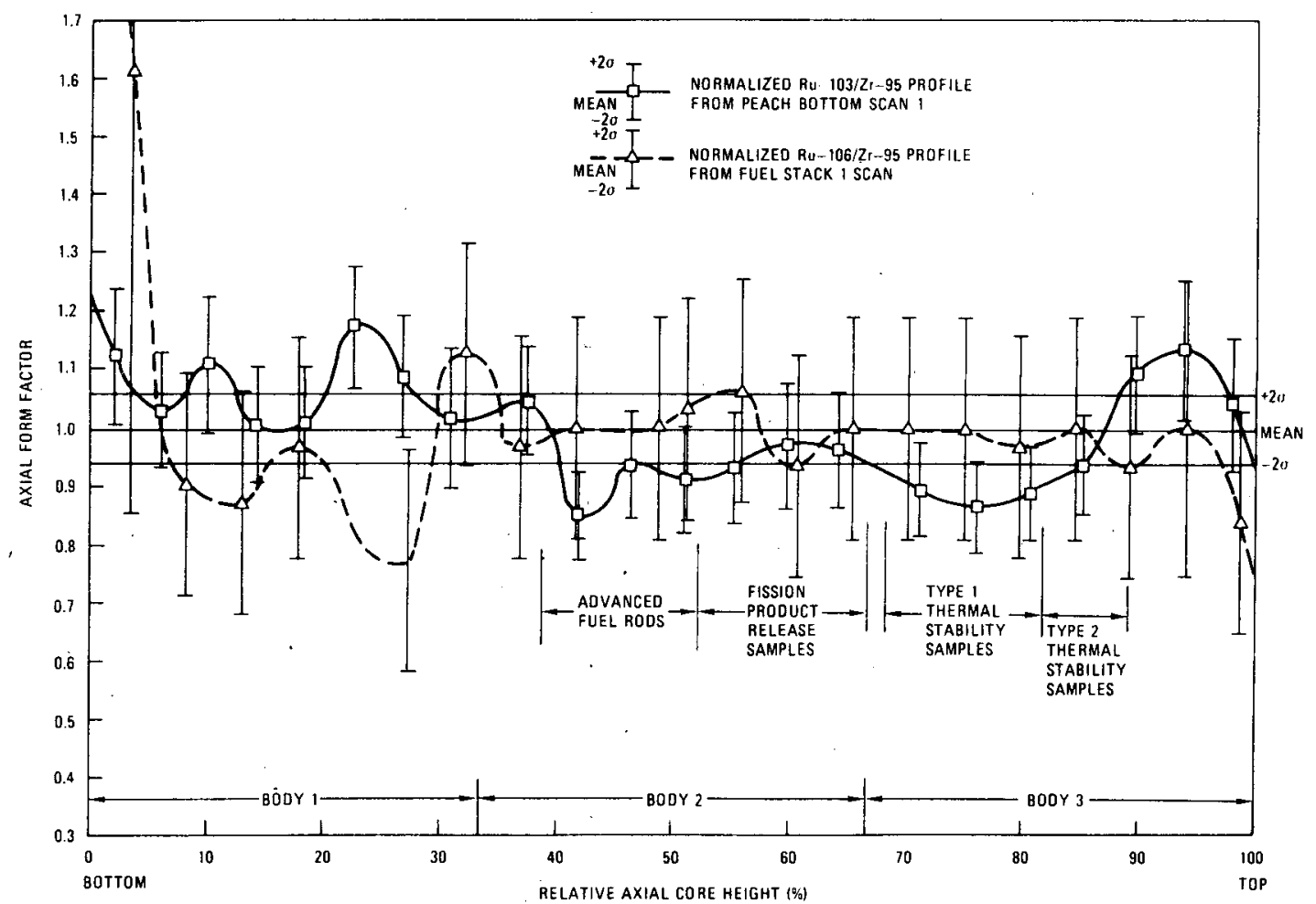

Fig. 4-13. FTE-6 fuel gamma scan comparison of normalized Ru-103/Zr-95 and $\mathrm{Ru}-106 / 2 r-95$ CPM profiles 


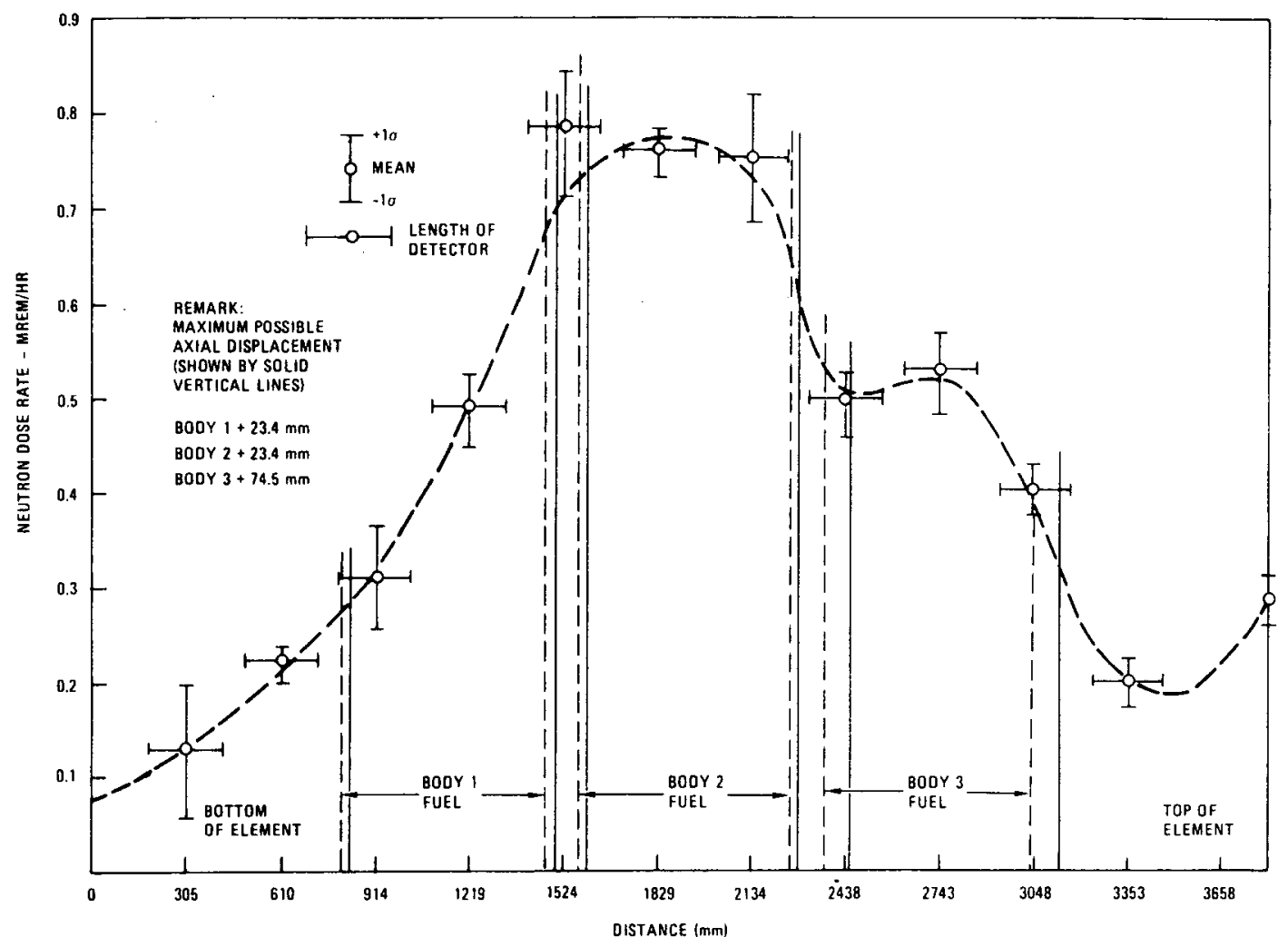

a. FTE-6 fuel element in Hallam cask

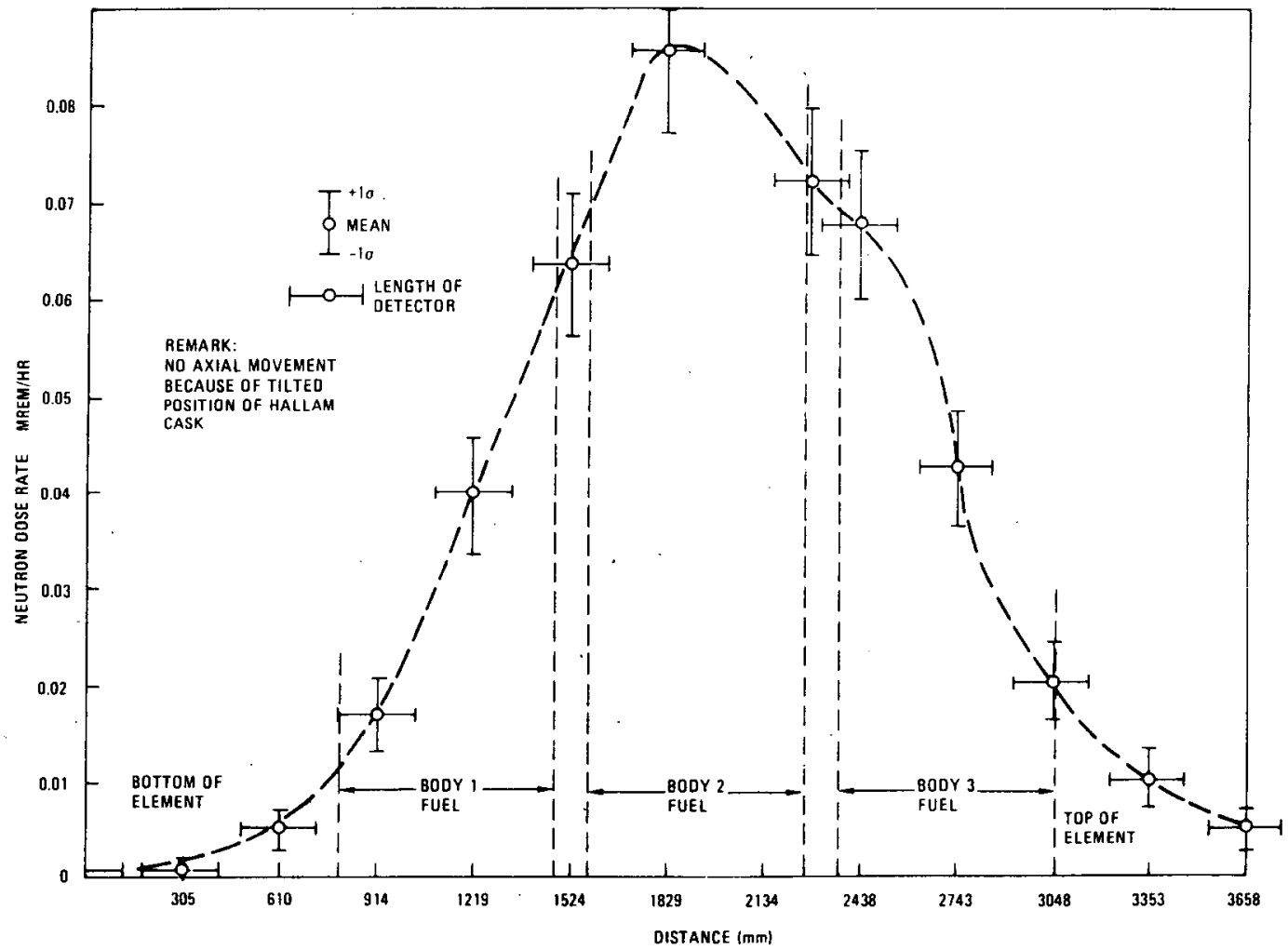

b. FTE-6 bare fuel element in inner shipping container

Fig. 4-14. FTE-6 neutron dose rate versus position along axis 


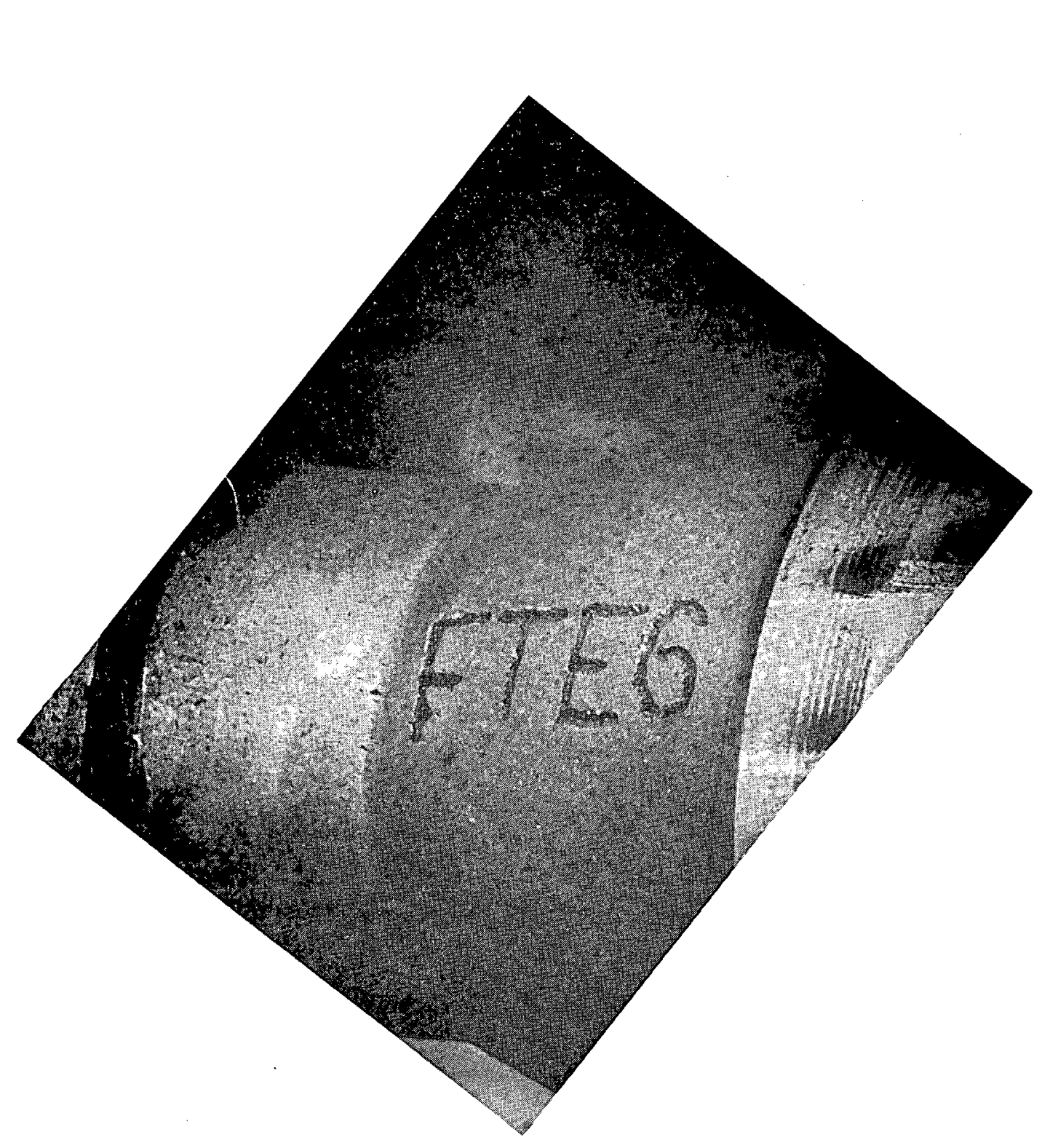

$\ominus$

Fig. 4-15. FTE-6 identification

$\theta$ 


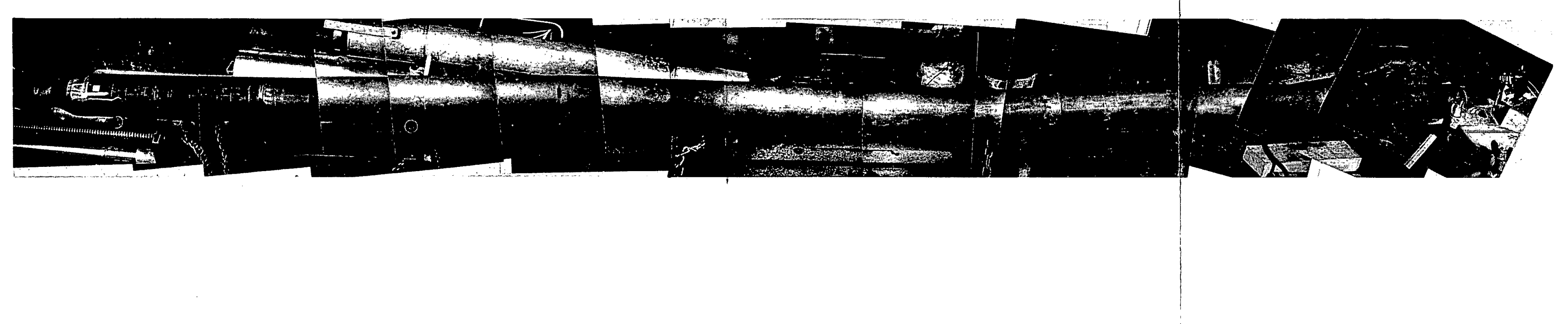




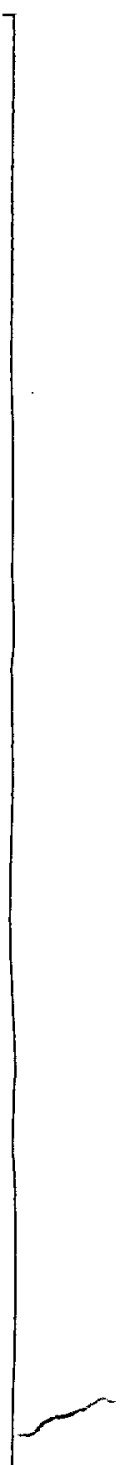

$-$

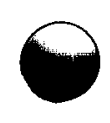


Reactor Bottom

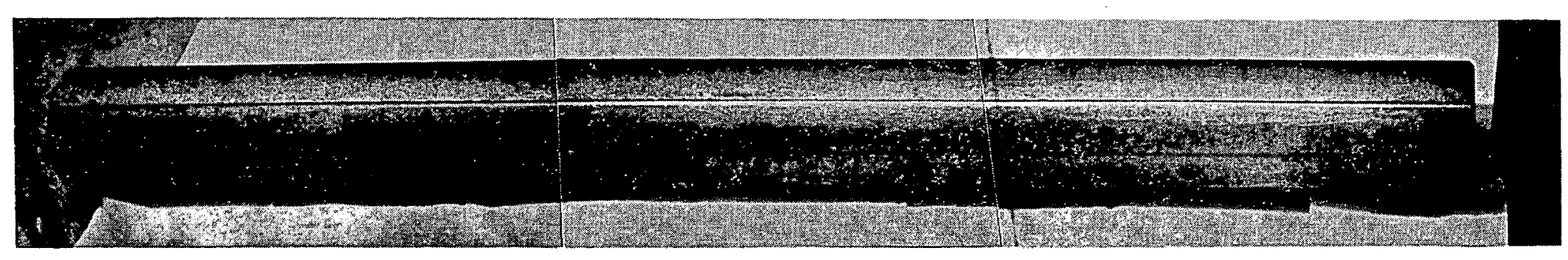

Body 1

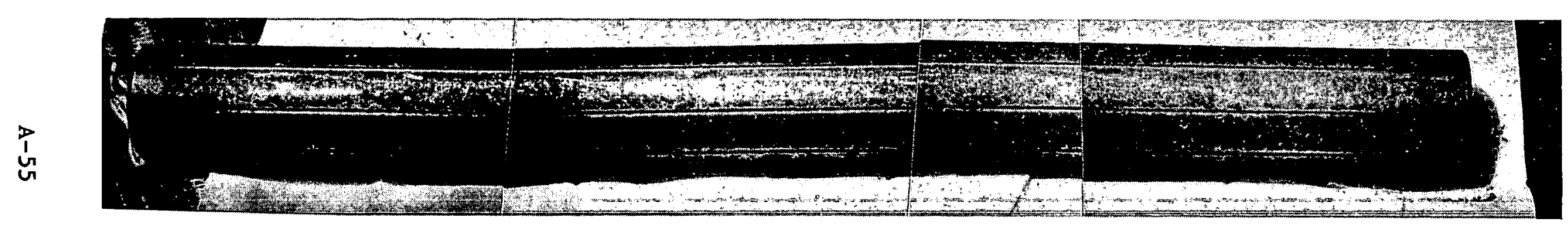

Body 2

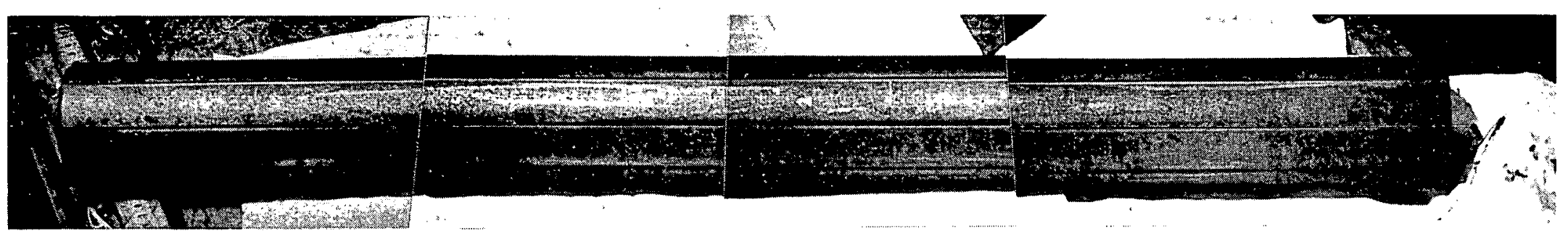

Body 3

Reactor Top

Fig. 4-17. Composite photographs of fuel bodies 1, 2, and 3 


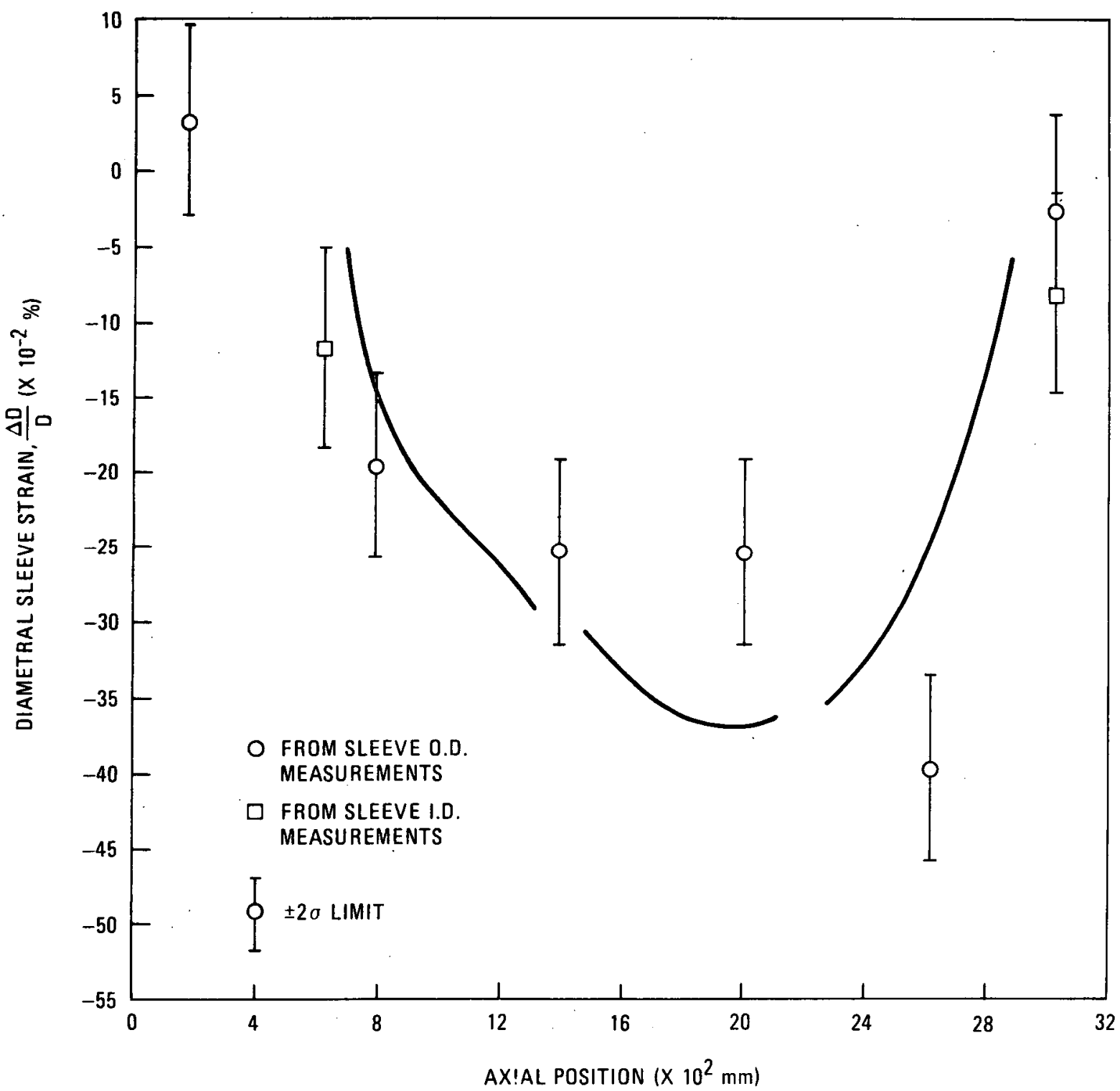

Fig. 4-18. FTE-6 measured and calculated sleeve strain 


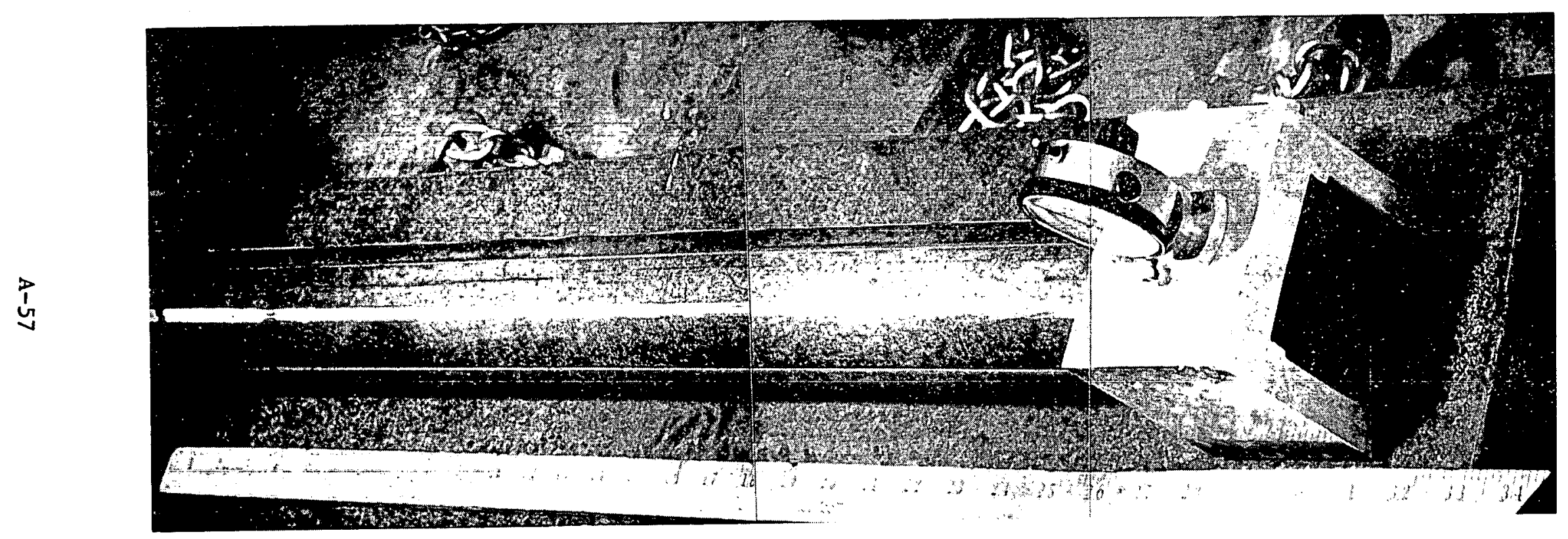

Fig. 4-19. FTE-6 fuel body bow measurement device 


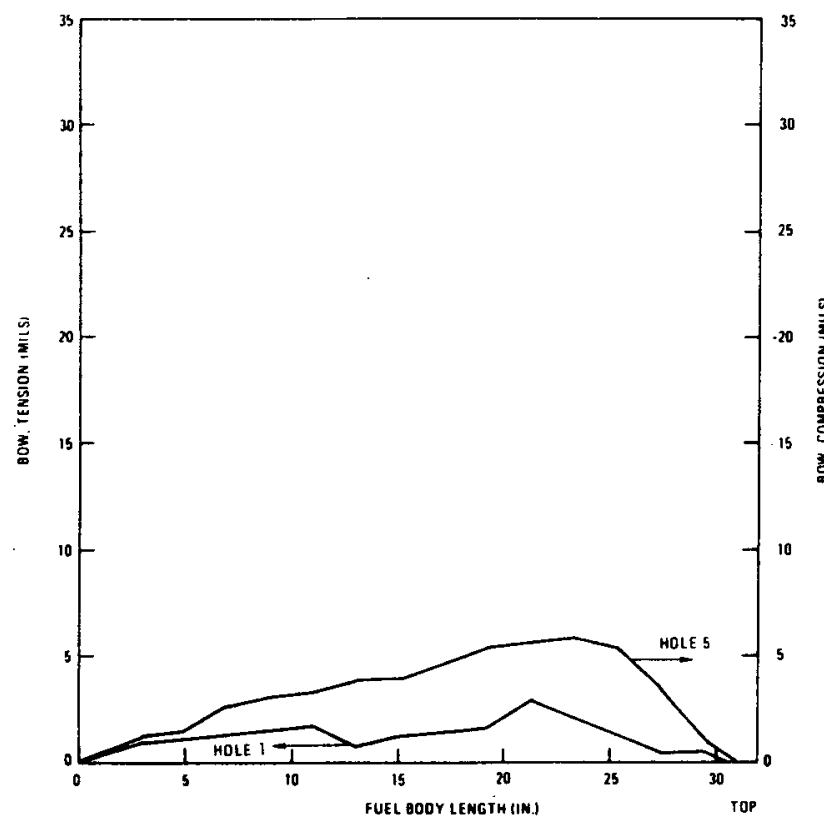

a. Holes 1 and 5

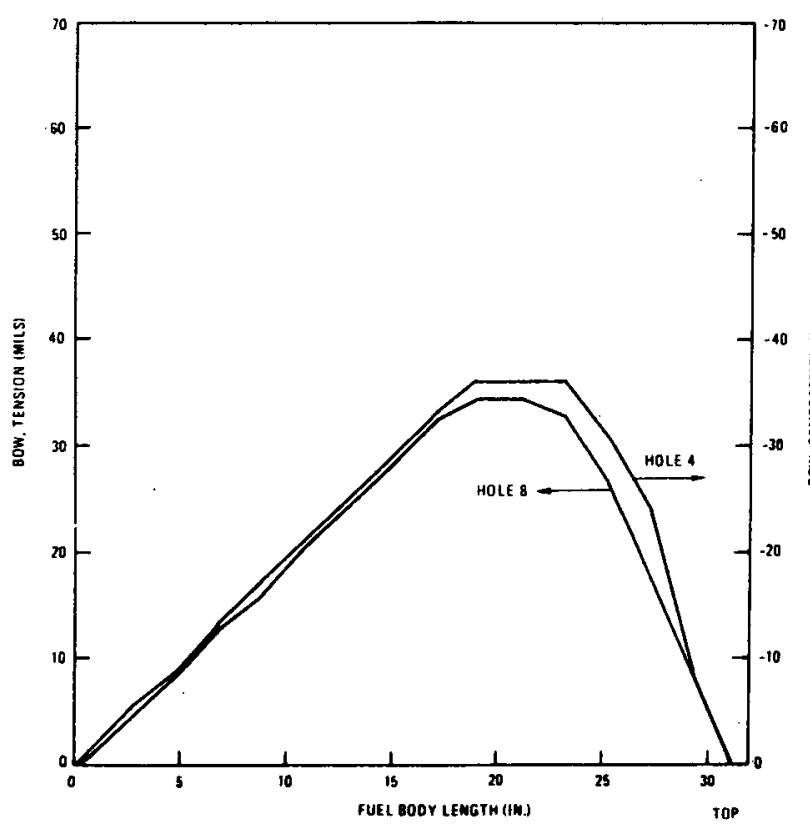

c. Holes 4 and 8

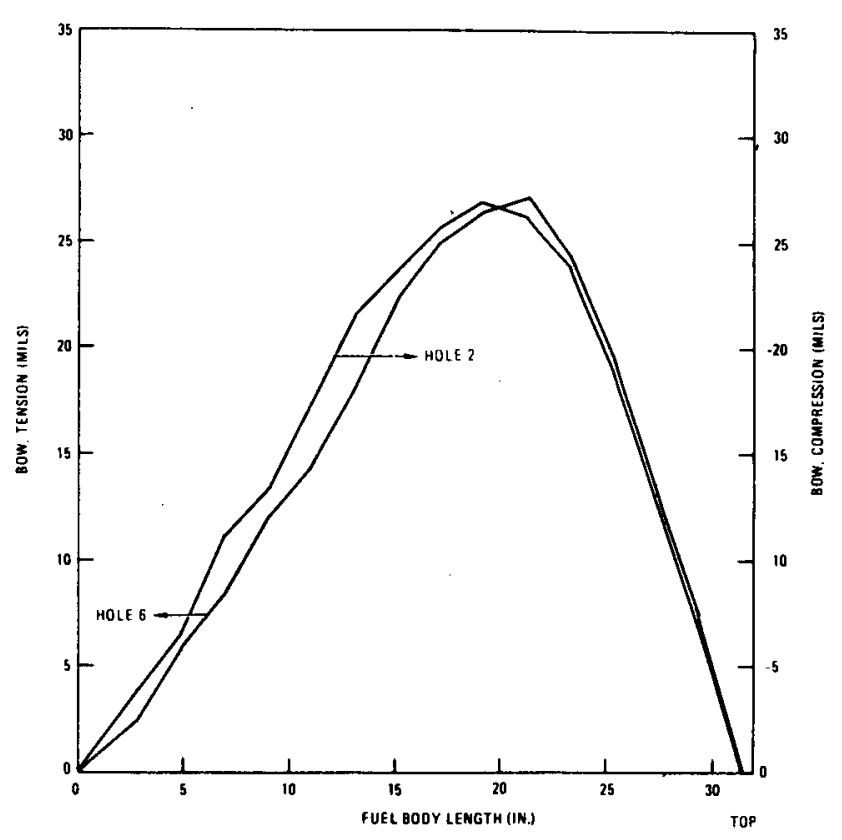

b. Holes 2 and 6

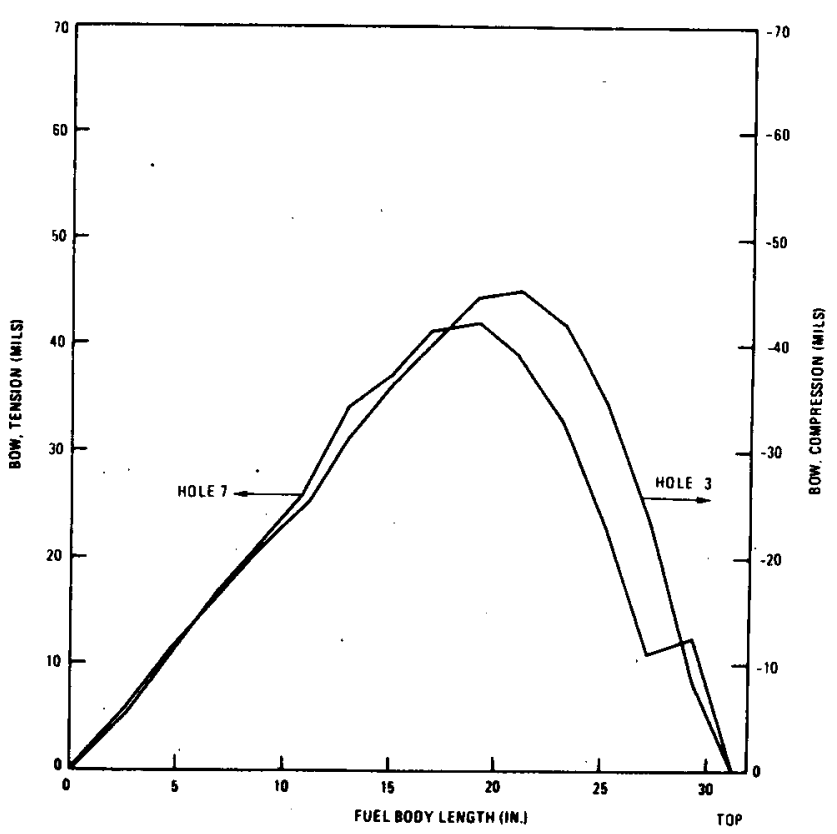

d. Holes 3 and 7

Fig. 4-20. FTE-6 fuel body 1 bow 


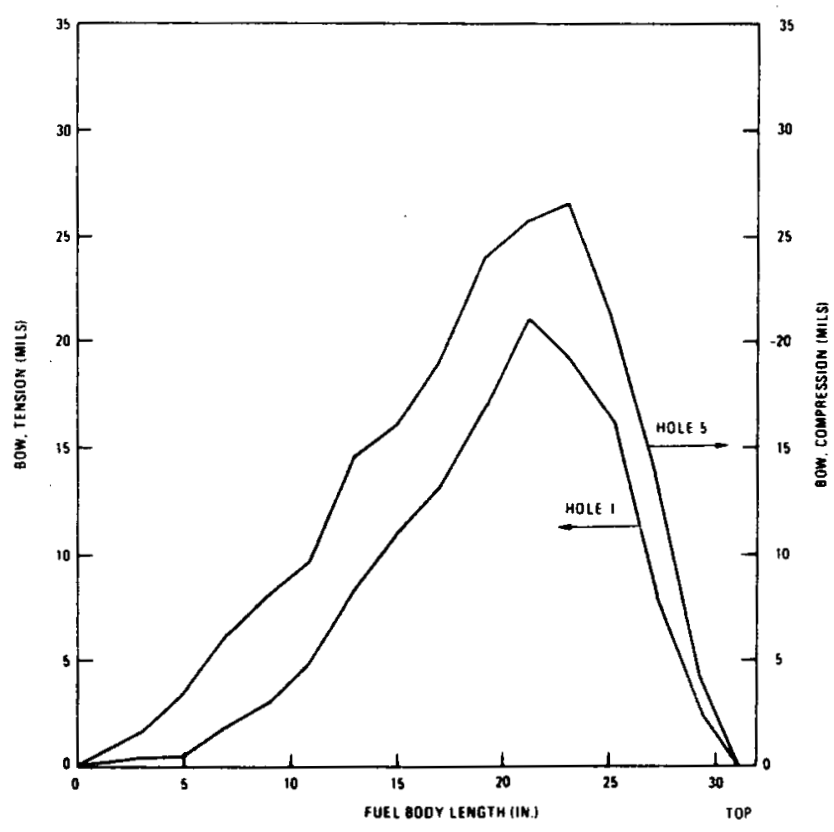

a. Holes 1 and 5

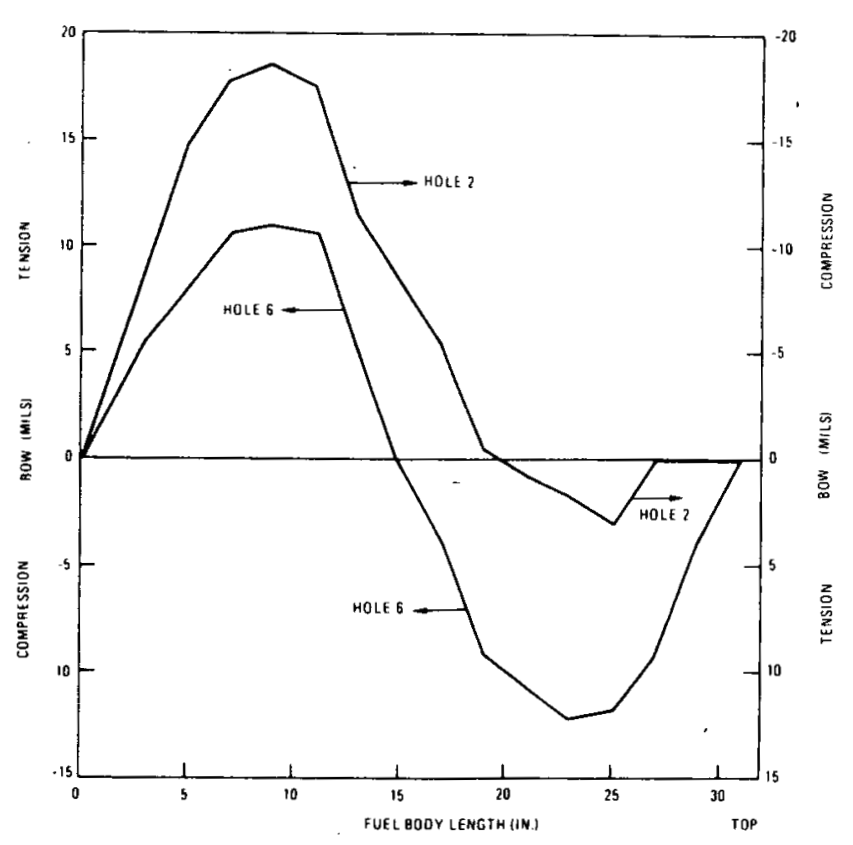

b. Holes 2 and 6

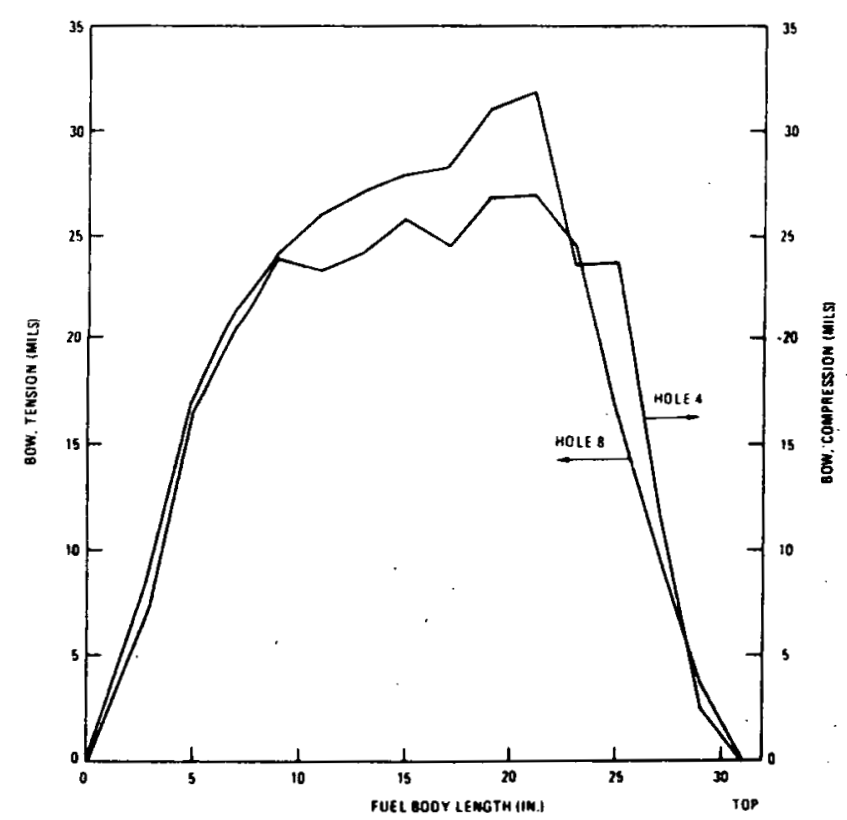

c. Holes 4 and 8

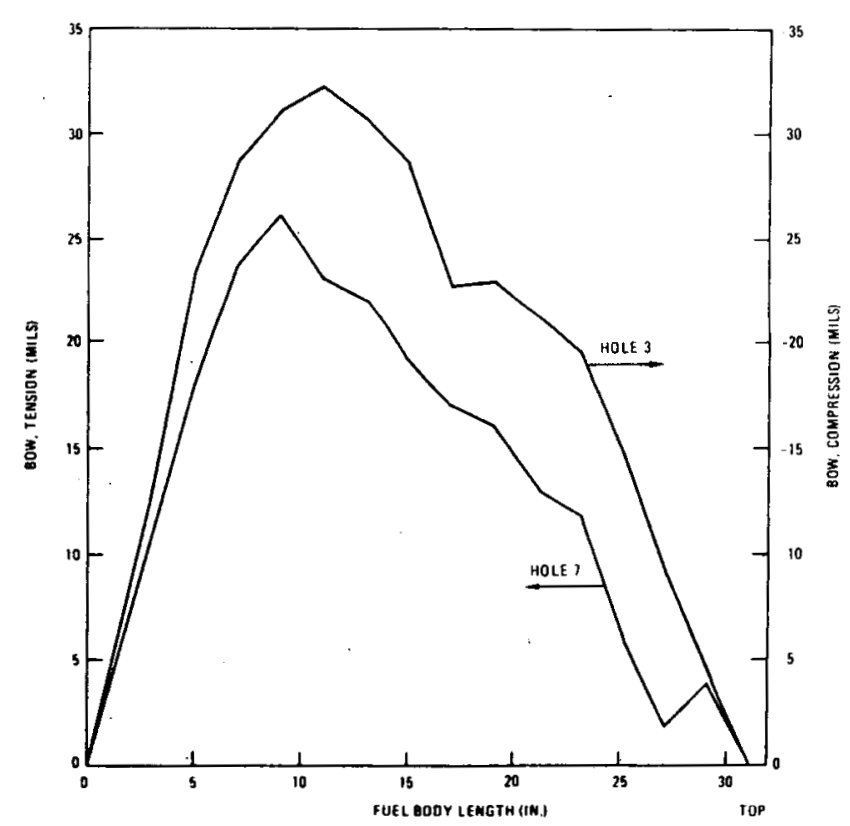

d. Holes 3 and 7

Fig. 4-21. FTE-6 fuel body 2 bow 


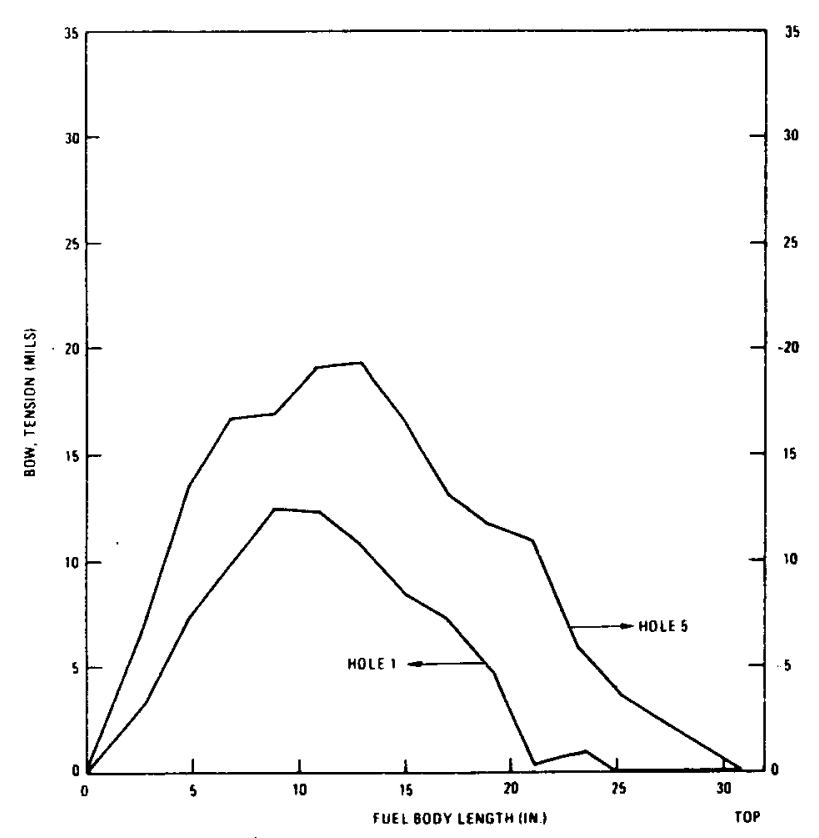

a. Holes 1 and 5

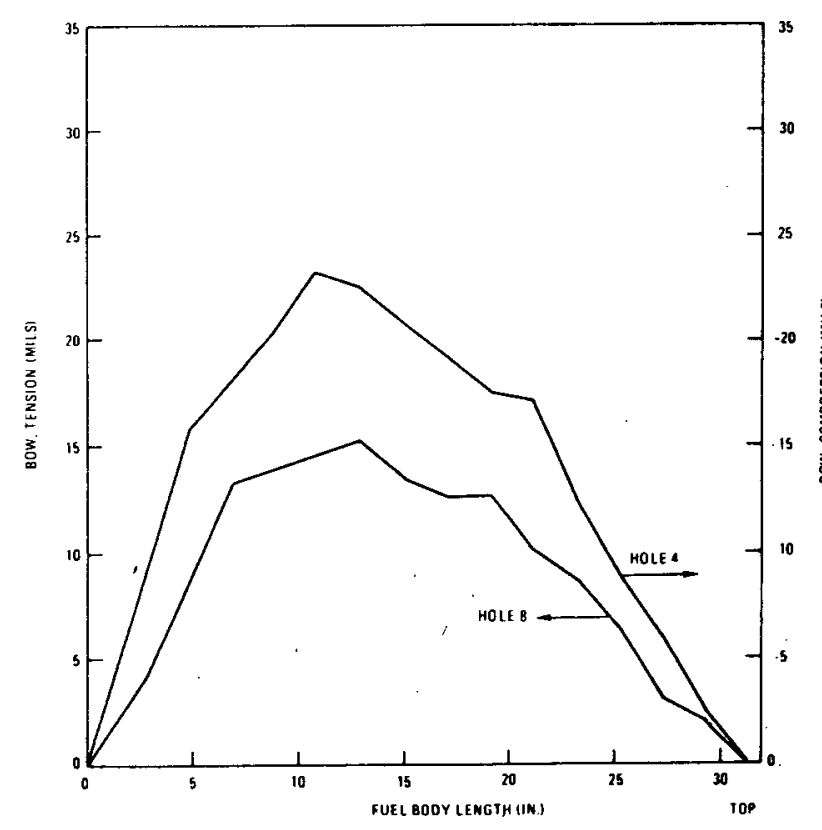

c. Holes 4 and 8

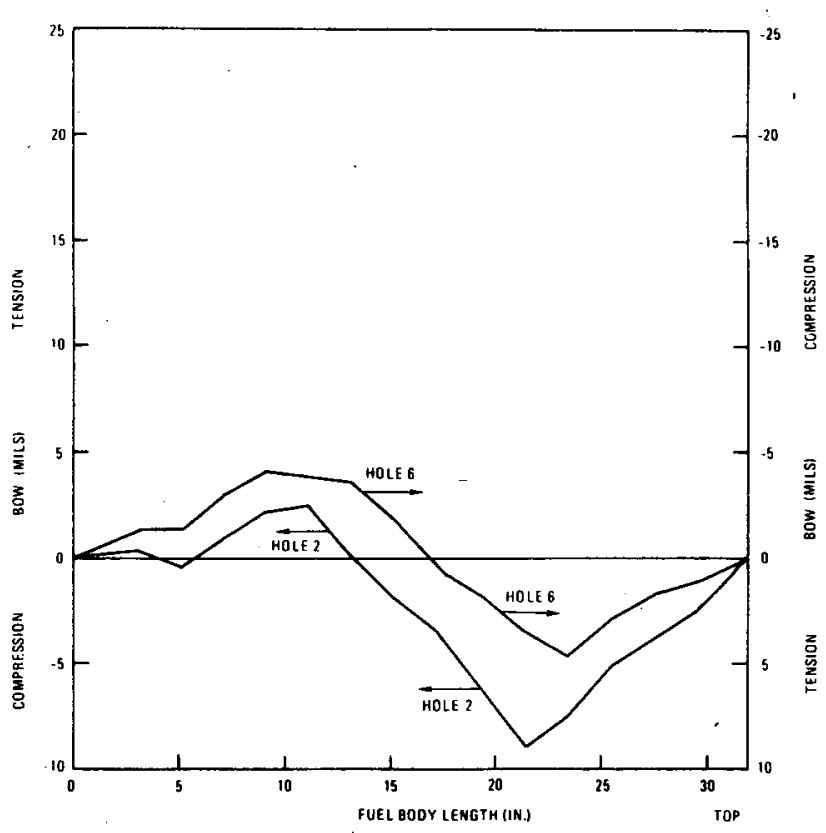

b. Holes 2 and 6

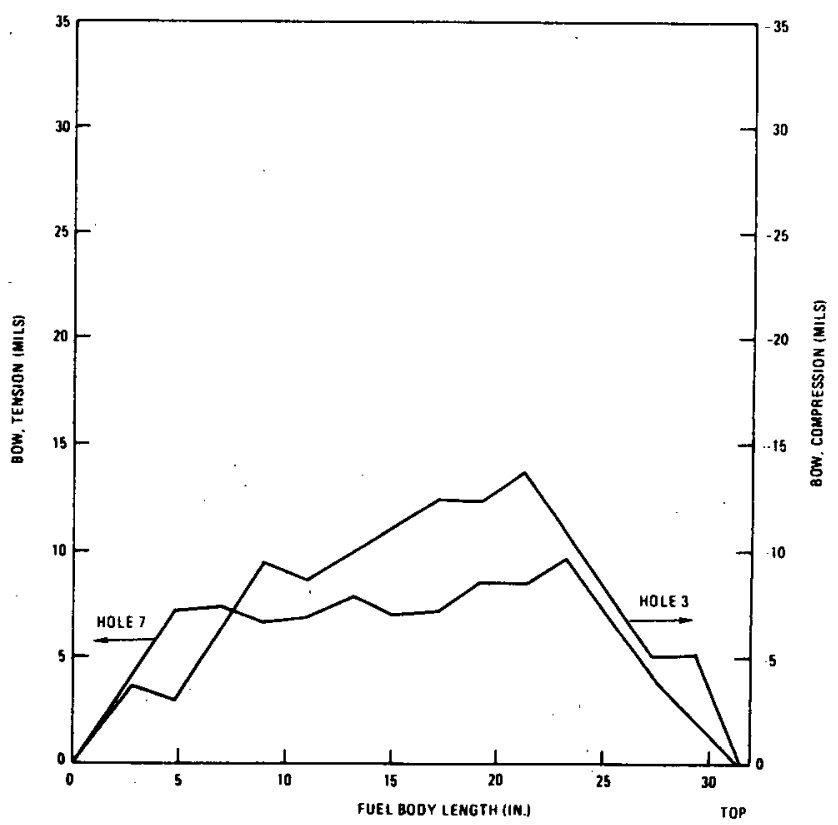

d. Holes 3 and 7

Fig. 4-22. FTE-6 fuel body 3 bow 


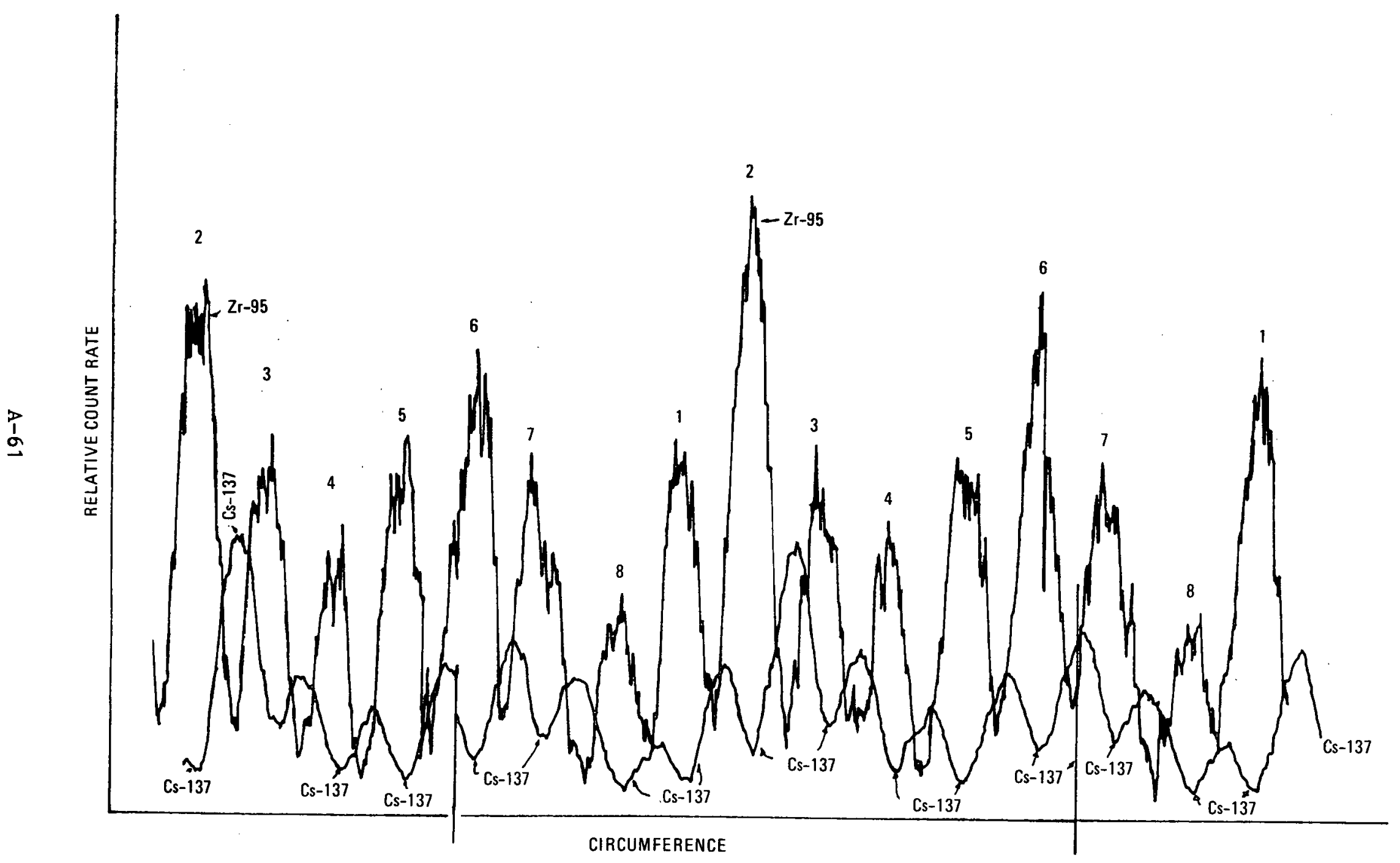

Fig. 4-23. FTE-6 stack power variations from radial gamma scanning of body 2 


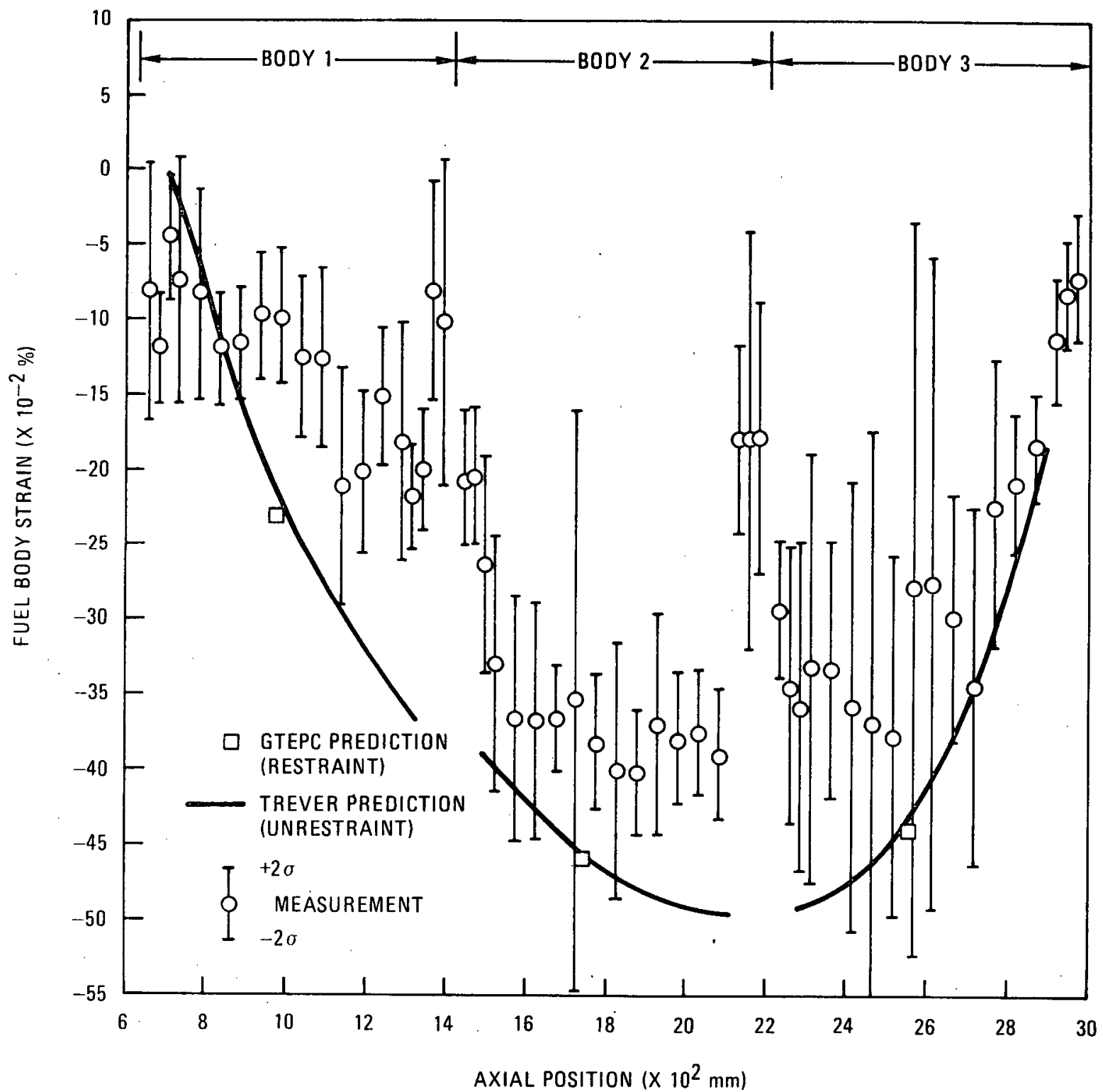

Fig. 4-24. FTE-6 measured and calculated fuel body strain 


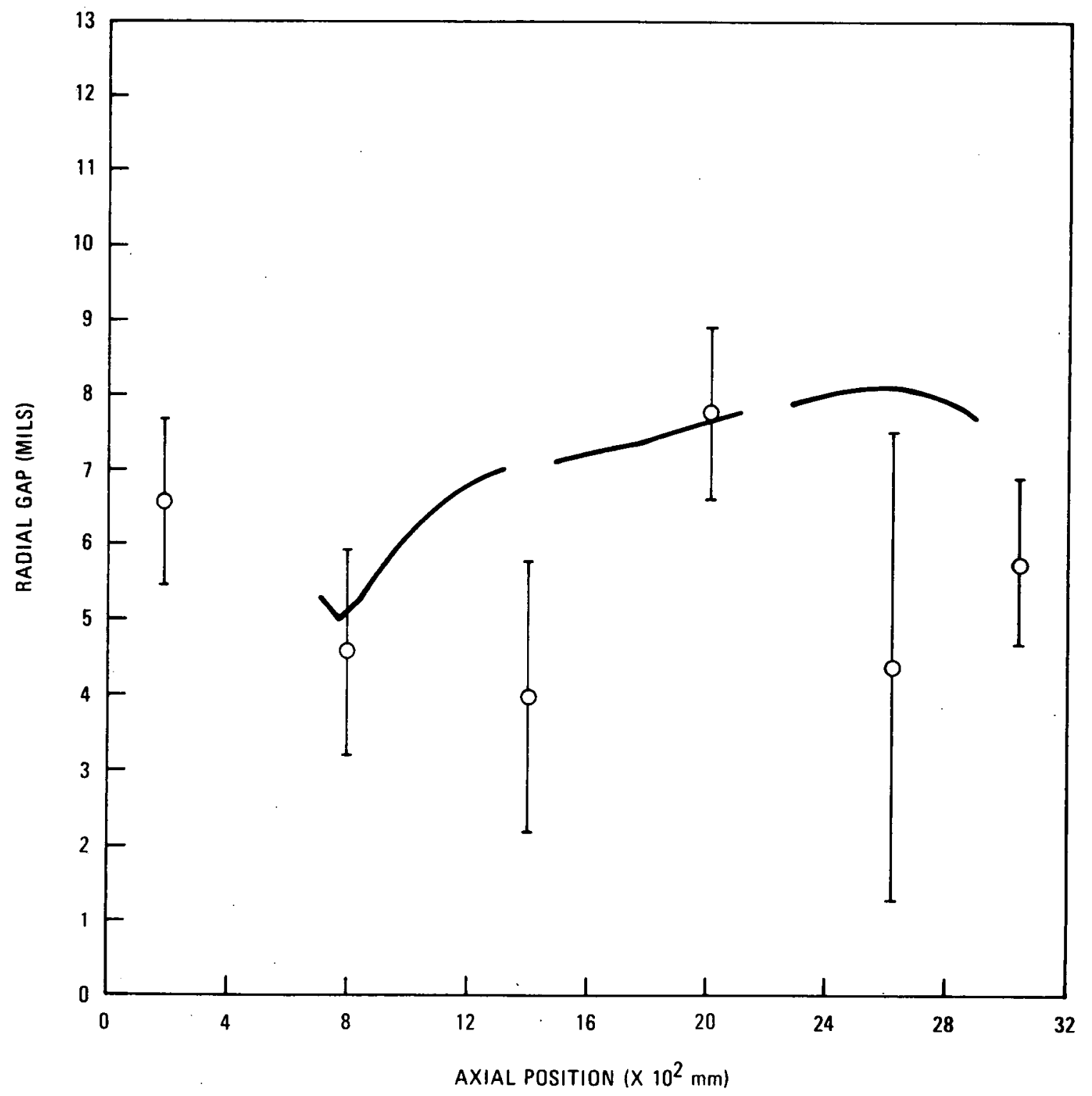

Fig. 4-25. FTE-6 measured and calculated sleeve fuel body radial gaps 


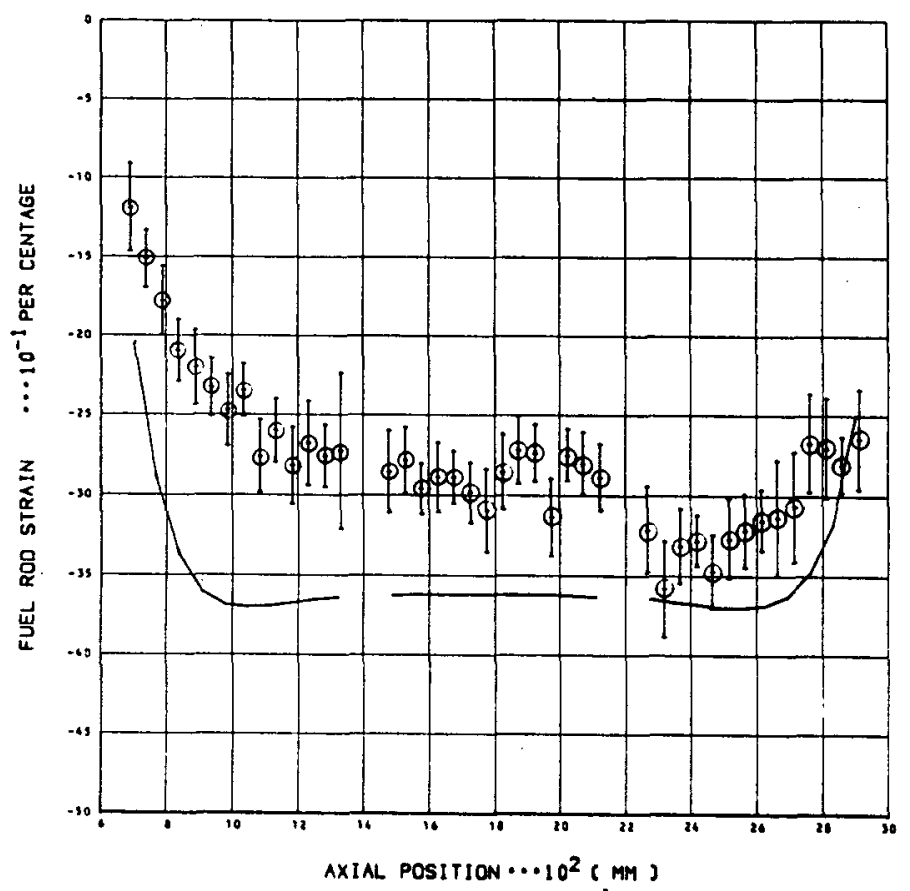

Fig. 4-26. FTE-6 measured and calculated fuel rod strain (holes 1 and 2)

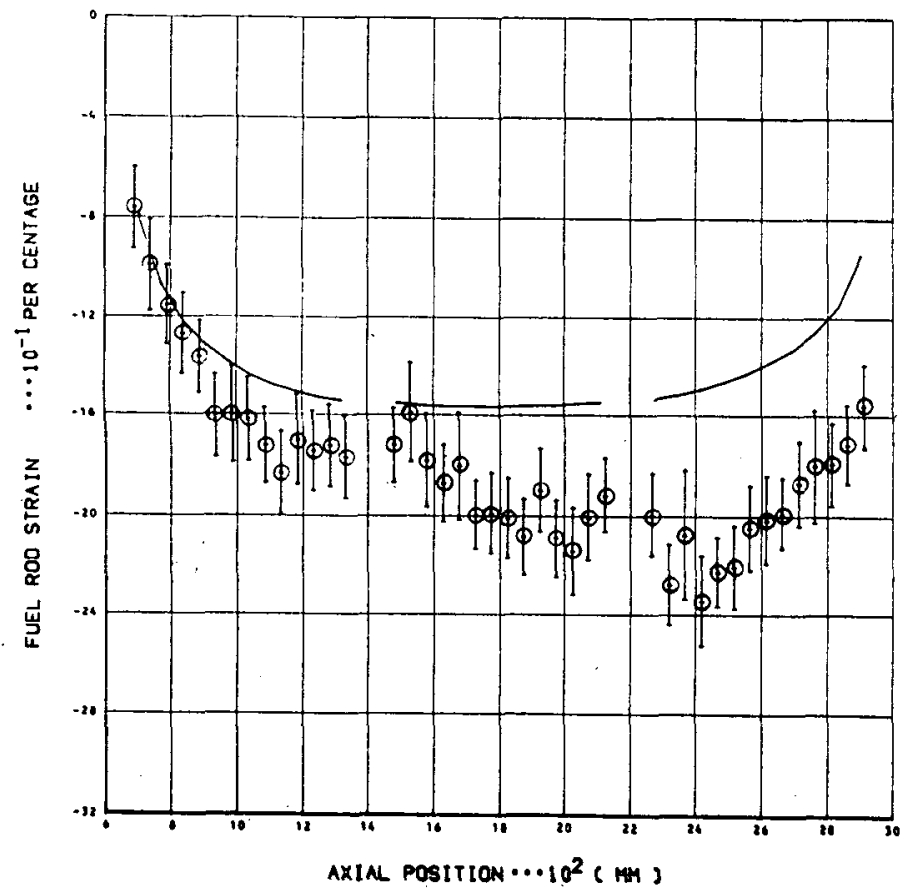

Fig. 4-28. FTE-6 measured and calculated fuel rod strain (holes 5 and 6)

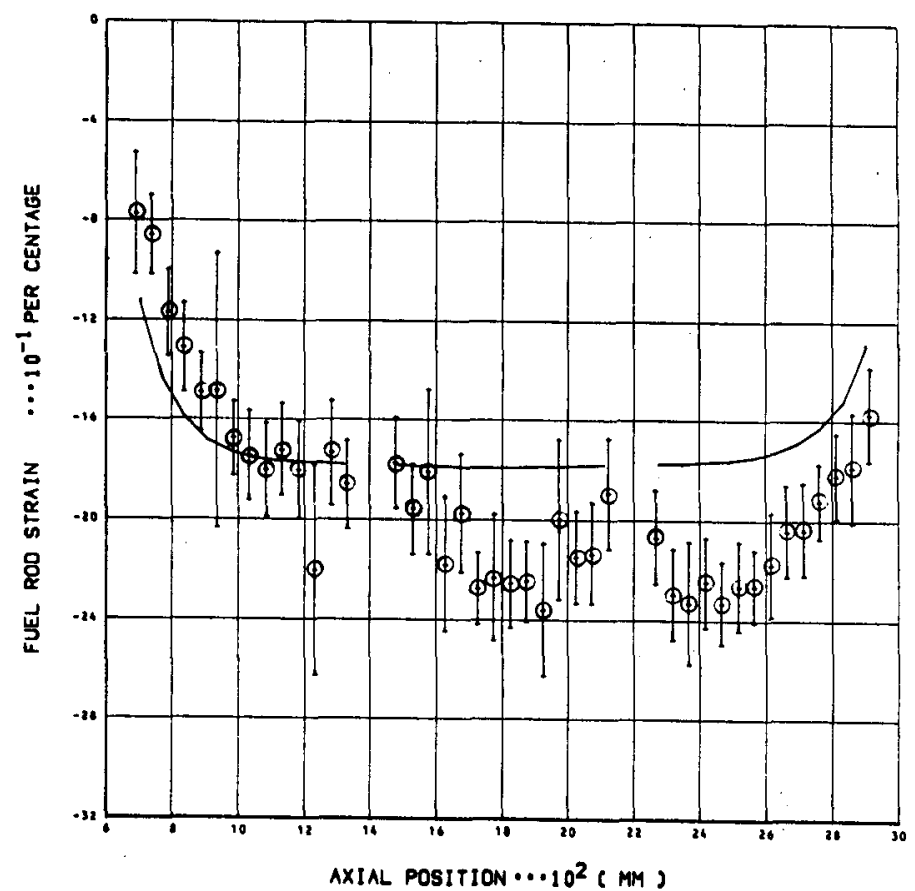

Fig. 4-27. FTE-6 measured and calculated fuel rod strain (holes 3 and 4)

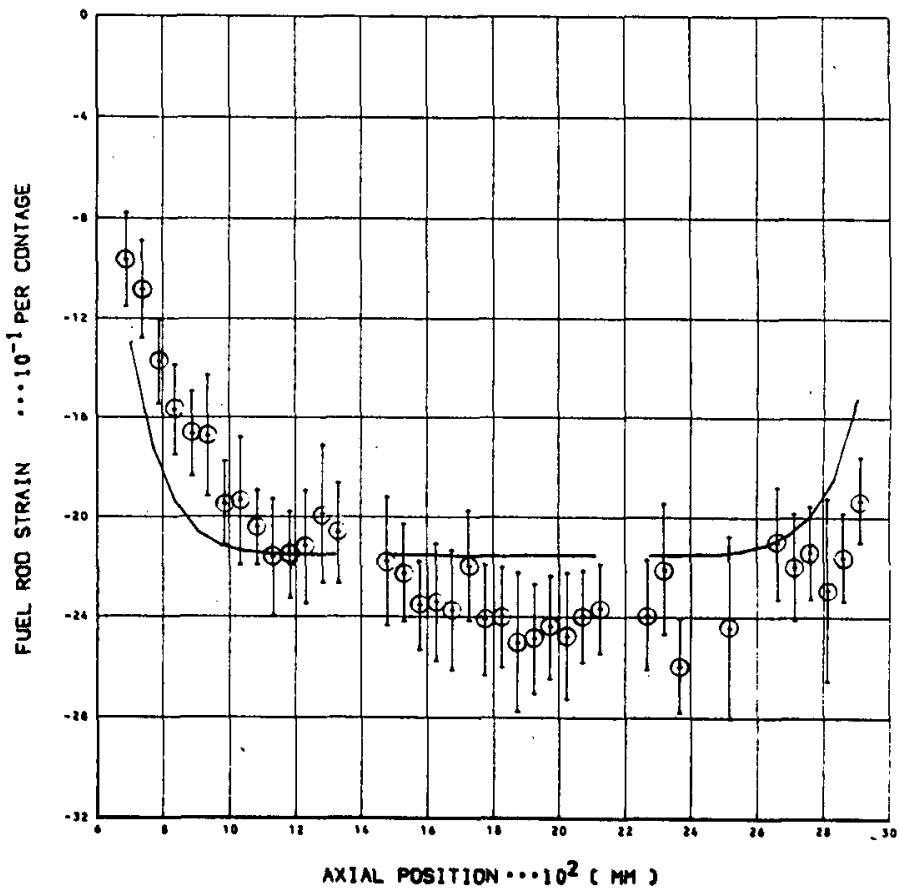

Fig. 4-29. FTE-6 measured and calculated fuel rod strain (holes 7 and 8 ) 


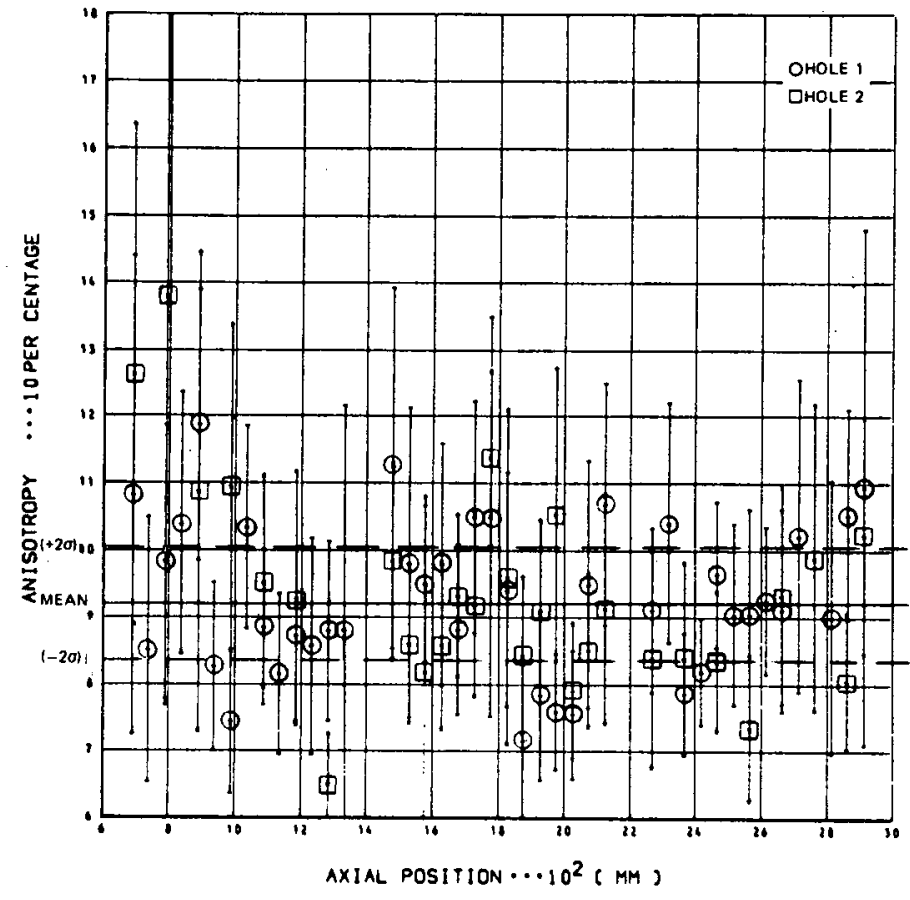

Fig. 4-30. FTE-6 fuel rod anisotropy (holes 1 and 2)

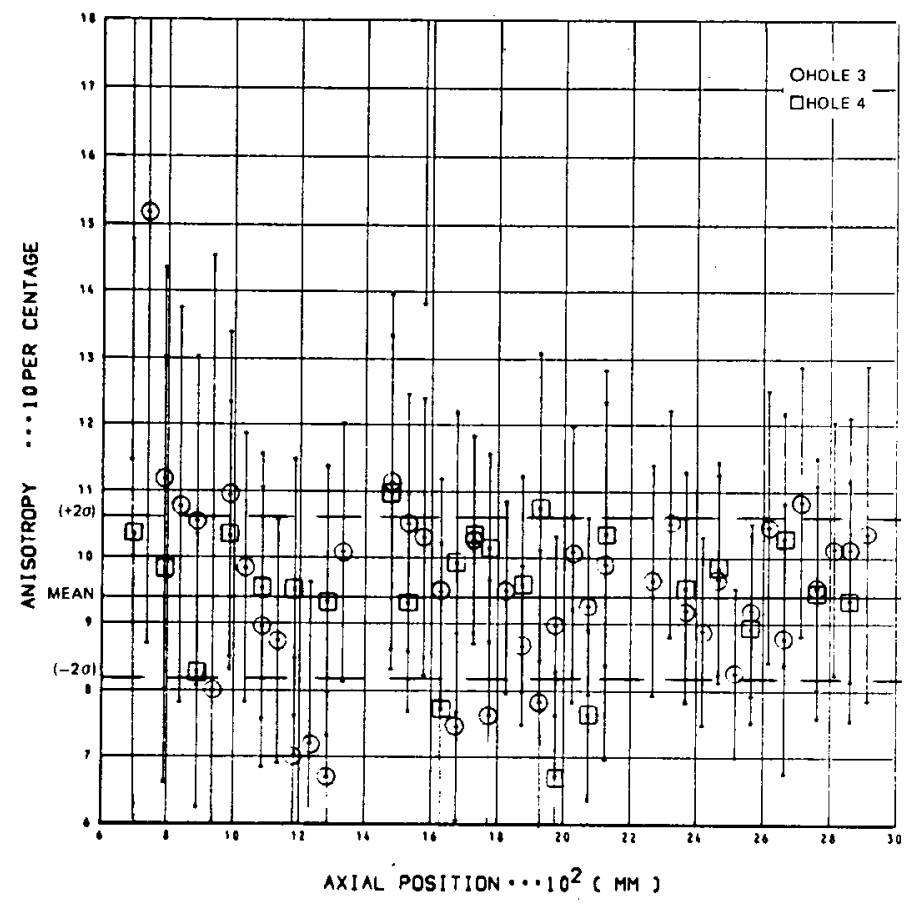

Fig. 4-31. FTE-6 fuel rod anisotropy (holes 3 and 4)

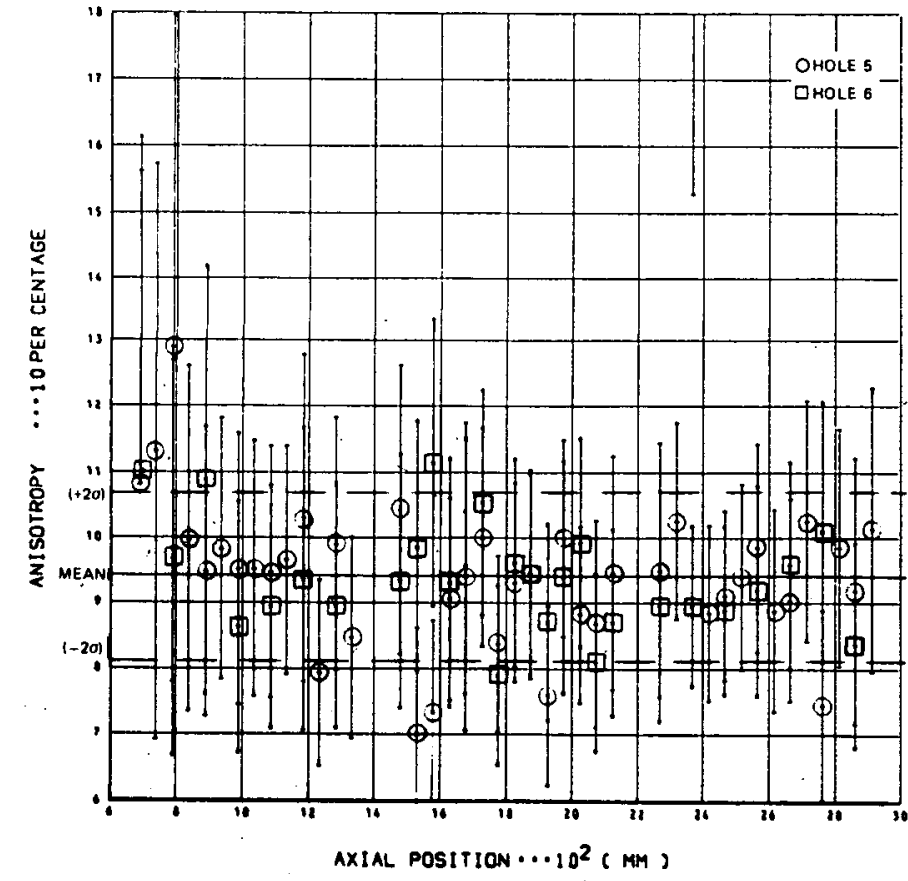

Fig. 4-32. FTE-6 fuel rod anisotropy (holes 5 and 6)

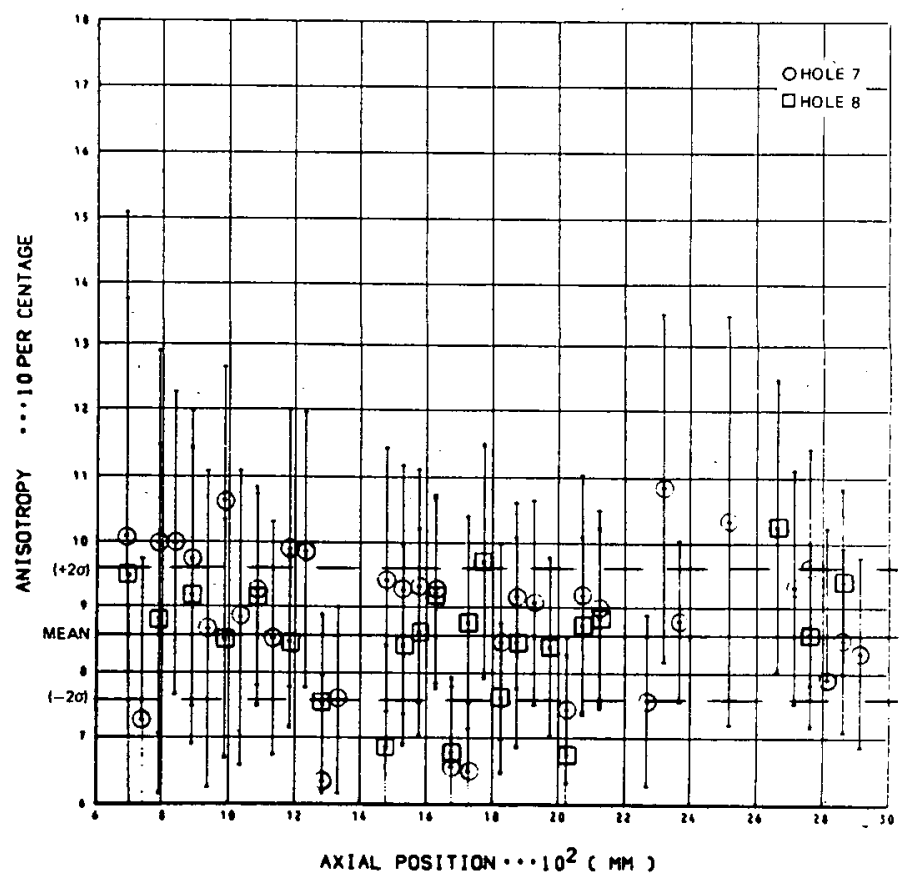

Fig. 4-33. FTE-6 fuel rod anisotropy (holes 7 and 8) 


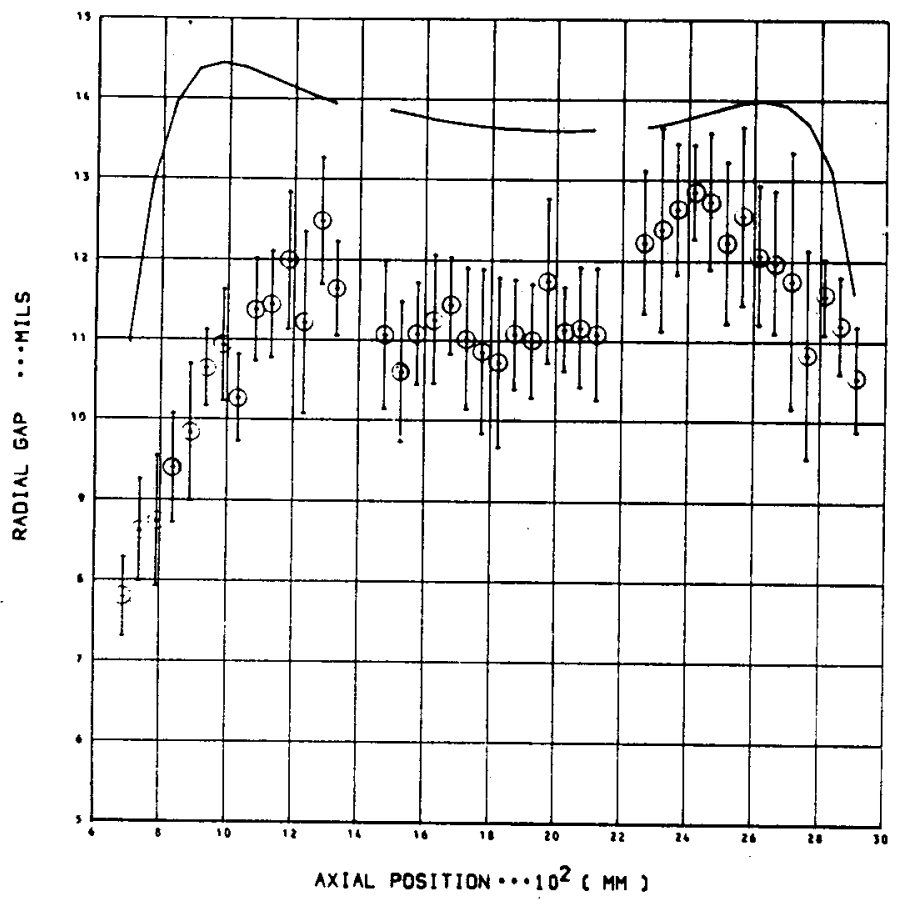

Fig. 4-34. FTE-6 measured and predicted fuel rod/fuel hole gap (holes 1 and 2)

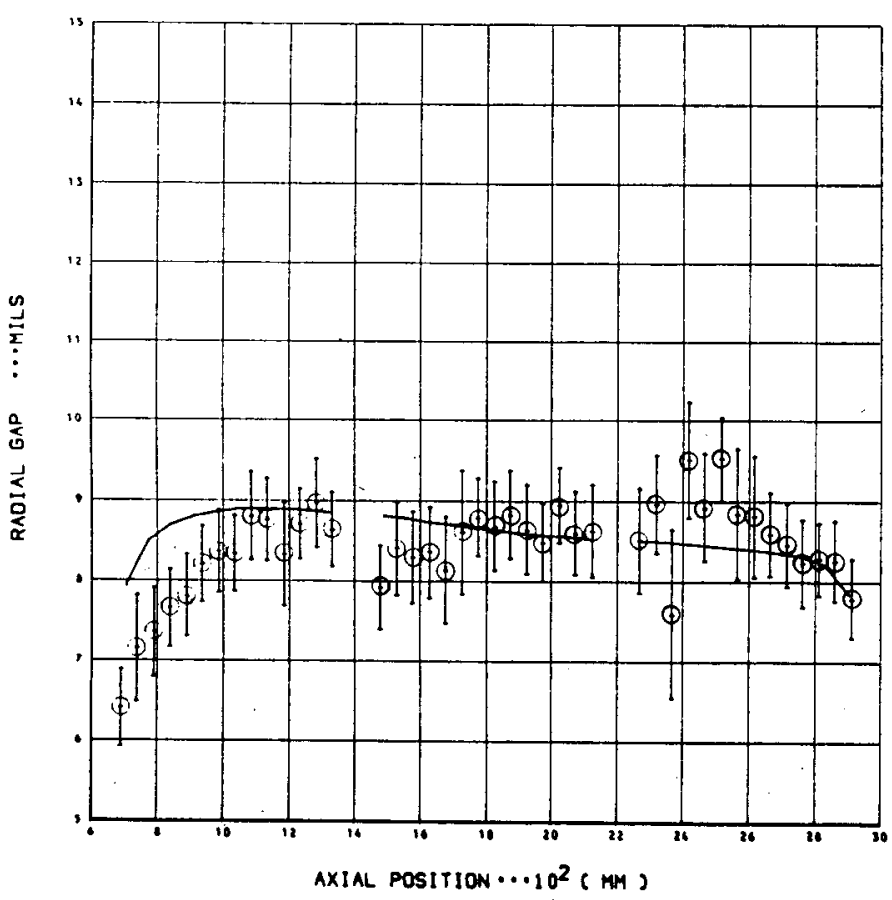

Fig. 4-36. FTE-6 measured and predicted fuel rod/fuel hole gap (holes 5 and 6)

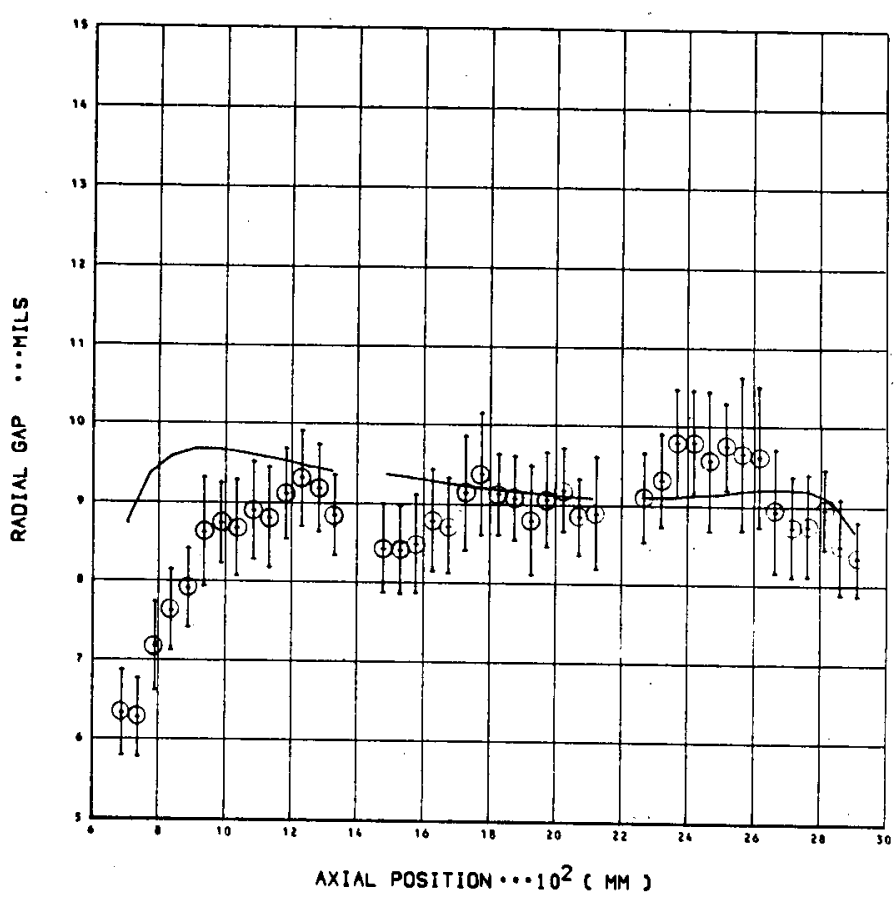

Fig. 4-35. FTE-6 measured and predicted fuel rod/fuel hole gap (holes 3 and 4)

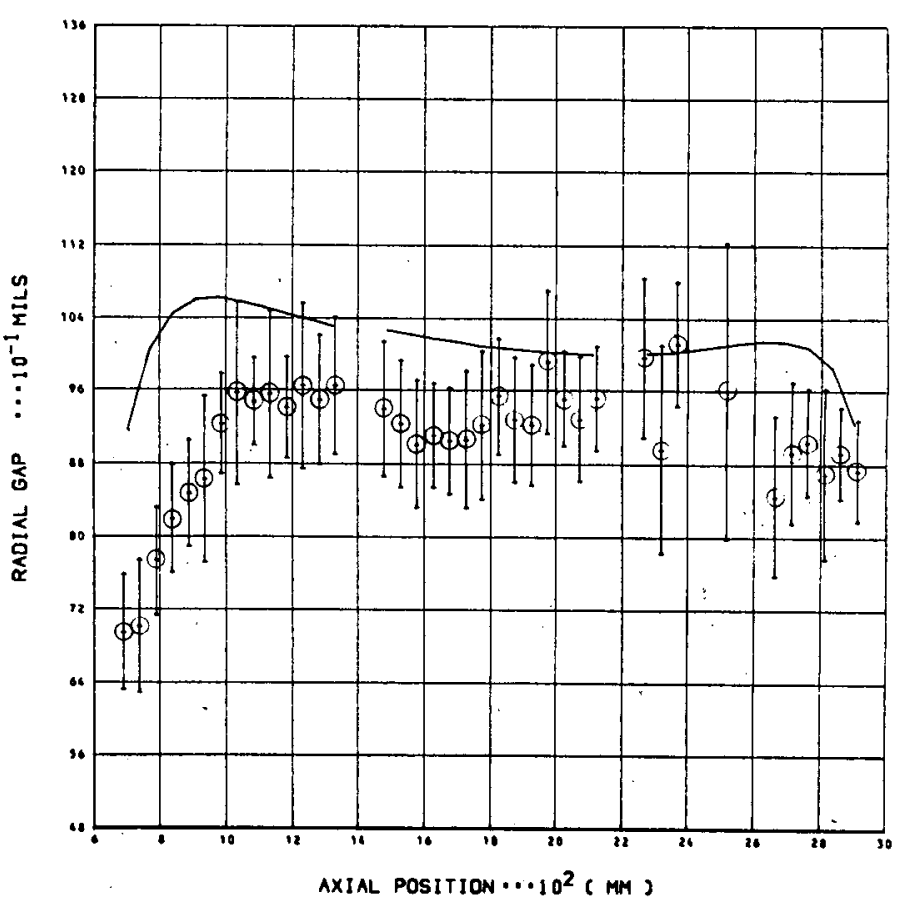

Fig. 4-37. FTE-6 measured and predicted fuel rod/fuel hole gap (holes 7 and 8 ) 


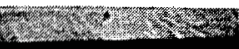

(1)

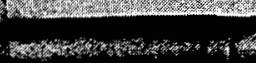

$-2$

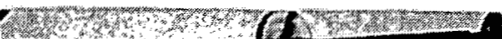

(r)

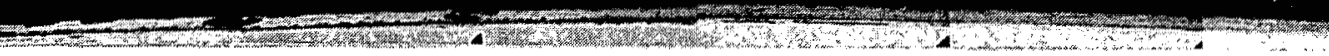

Fig. 4-38. FTE-6 composite photograph of stack 1 , body 1

6.

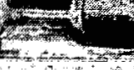

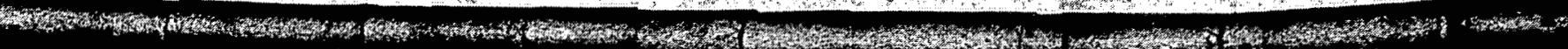

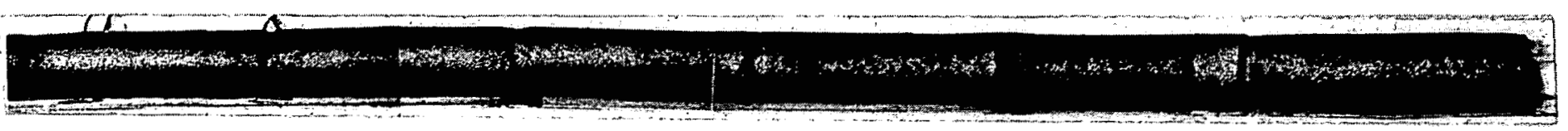

Fig. 4-39. FTE-6 composite photograph of stack 2, body 1

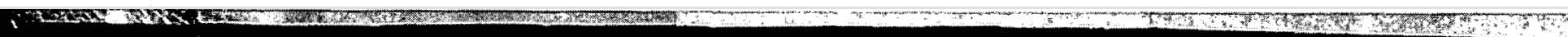

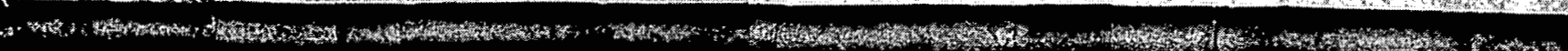

(2)

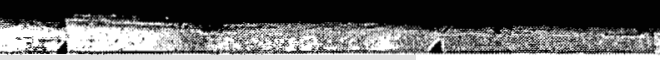

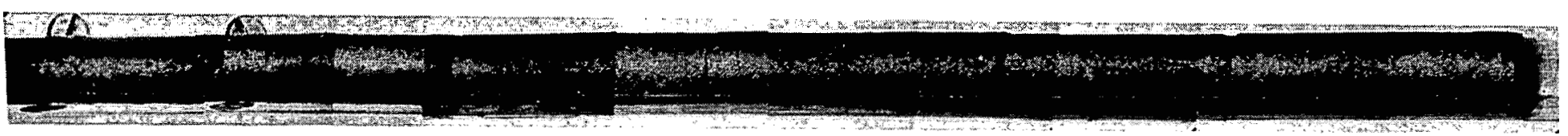

Fig. 4-40. FTE-6 composite photograph of stack 6 , body 1

(2. 4. 4.

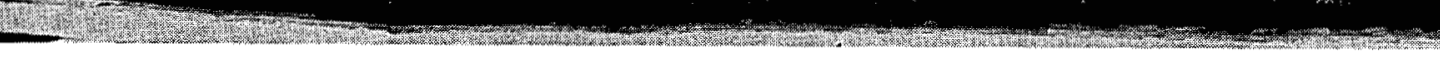

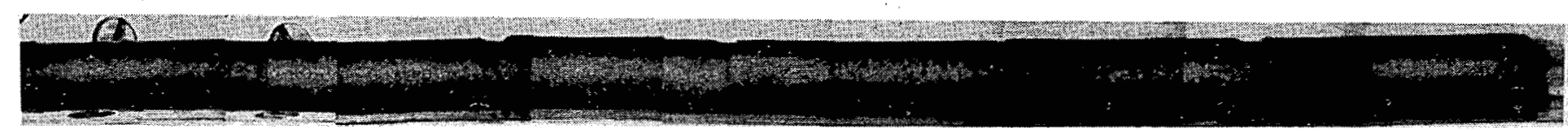

Fig. 4-41. FTE-6 composite photograph of stack 8 , body 1 


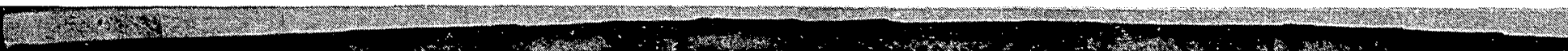
s.

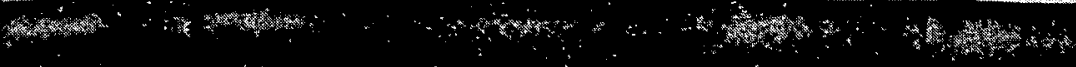

1.

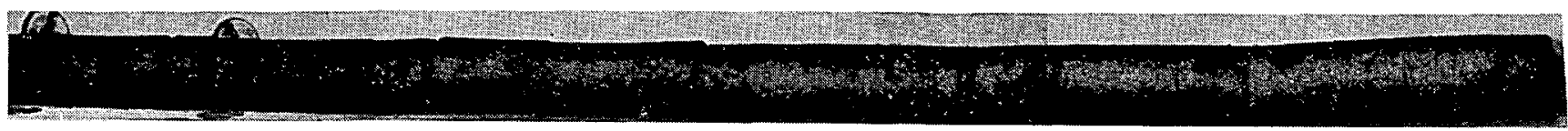

Fig. 4-42. FTE-6 composite photograph of stack 1 , body 2

Fig. 4-43. FTE-6 composite photograph of stack 3, body 2

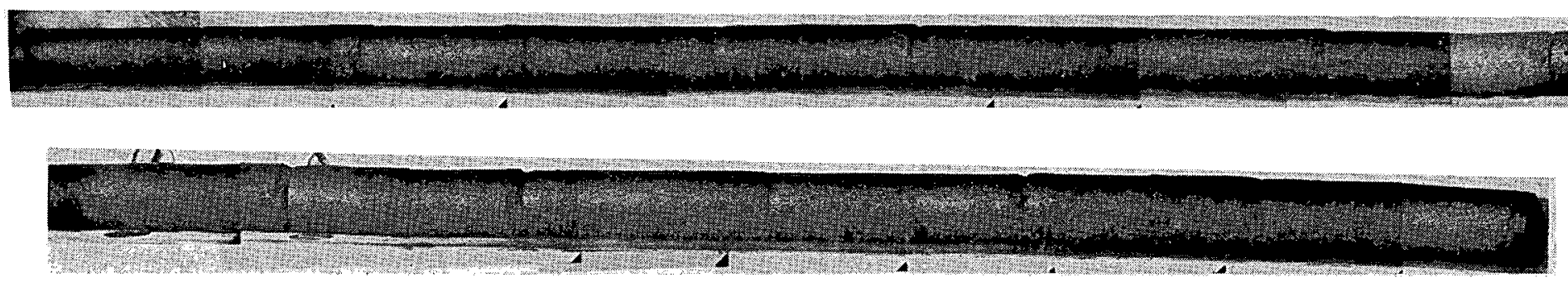

Fig. 4-44. FTE-6 composite photograph of stack 5, body 2

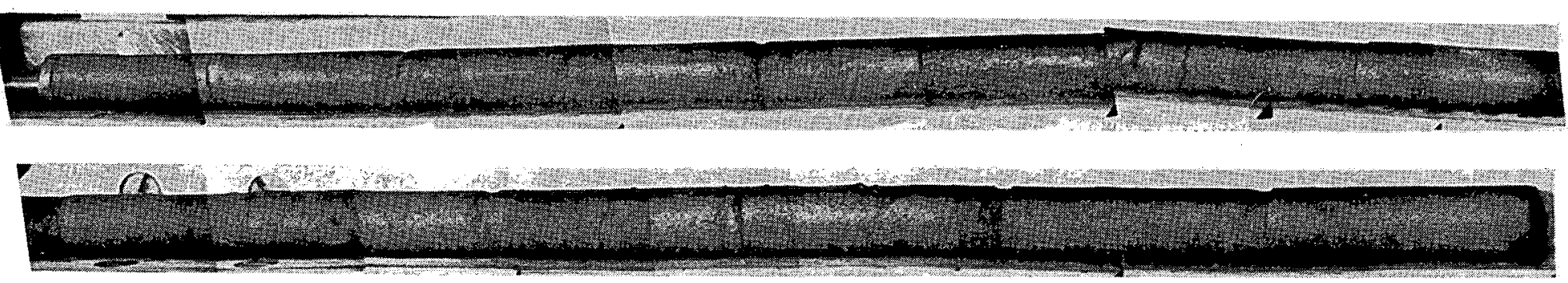

Fig. 4-45. FTE-6 composite photograph of stack 7, body 2 


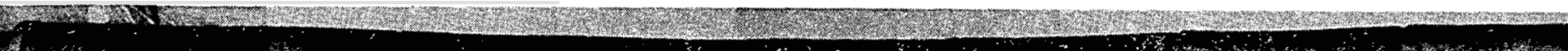

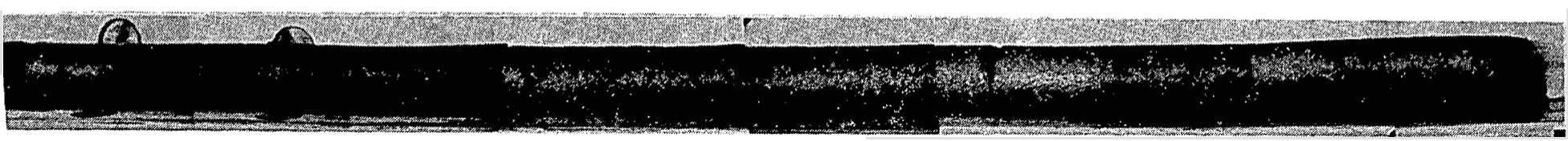

Fig. 4-46. FTE-6 composite photograph of stack 2, body 3

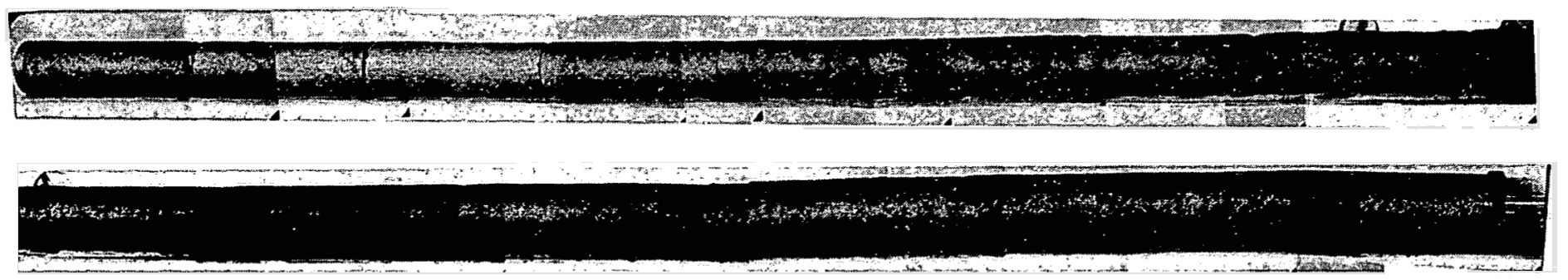

Fig. 4-47. FTE-6 composite photograph of stack 4 , body 3

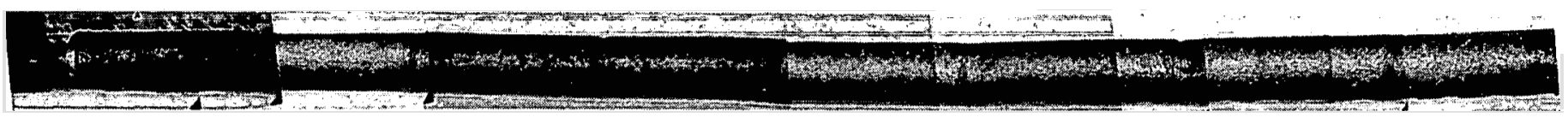

$\rightarrow$ a

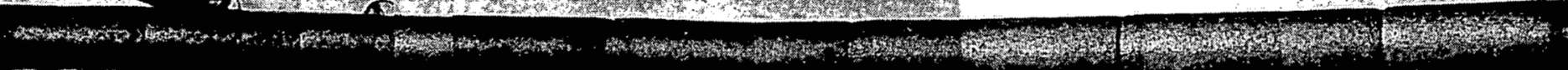

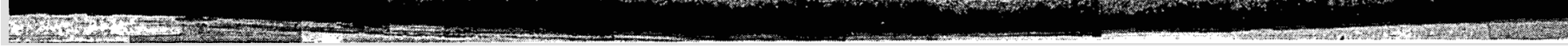

Fig. 4-48. FTE-6 composite photograph of stack 6 , body 3

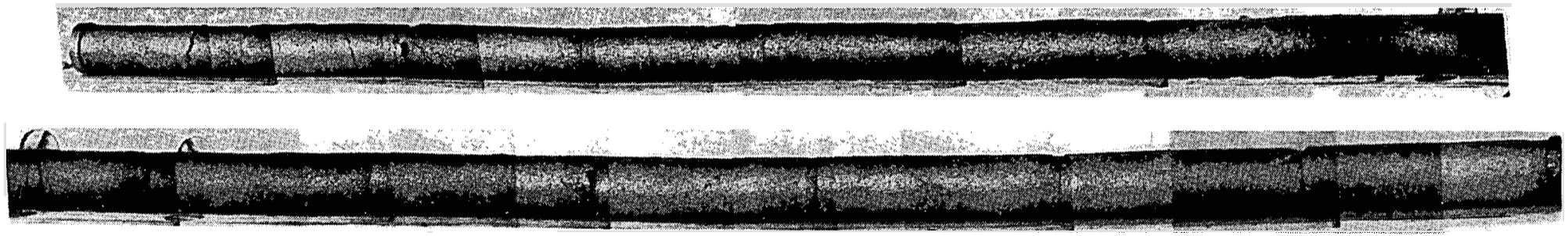

Fig. 4-49. FTE-6 composite photograph of stack 8 , body 3 


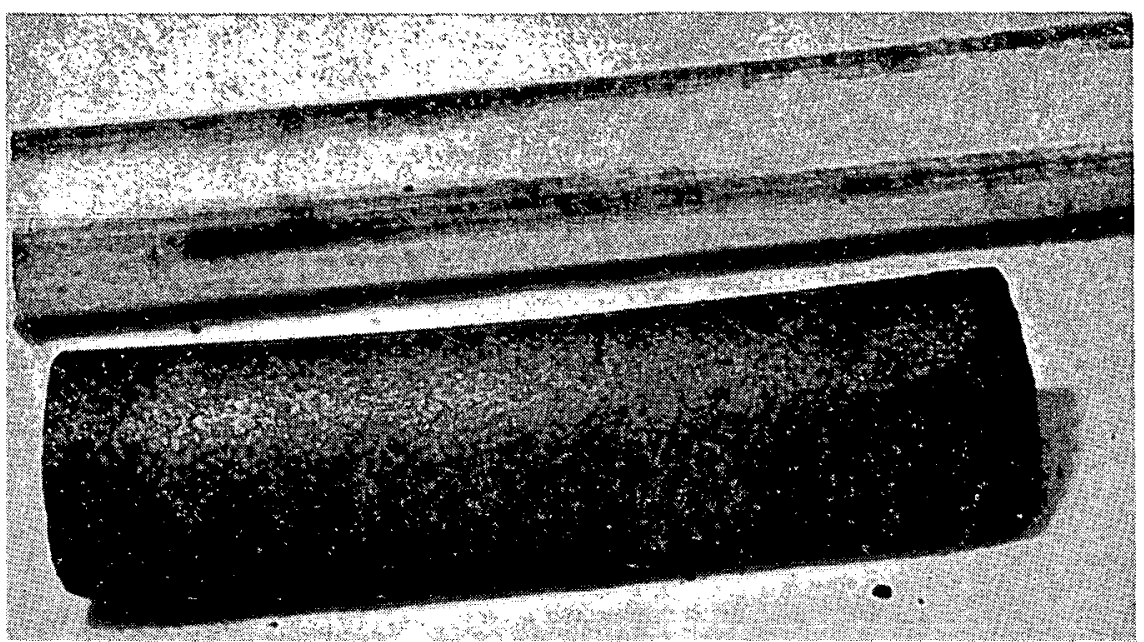

Fig. 4-50. FTE-6 typical bowed rod (2-2-1) - center $\frac{\text { t }}{0}$

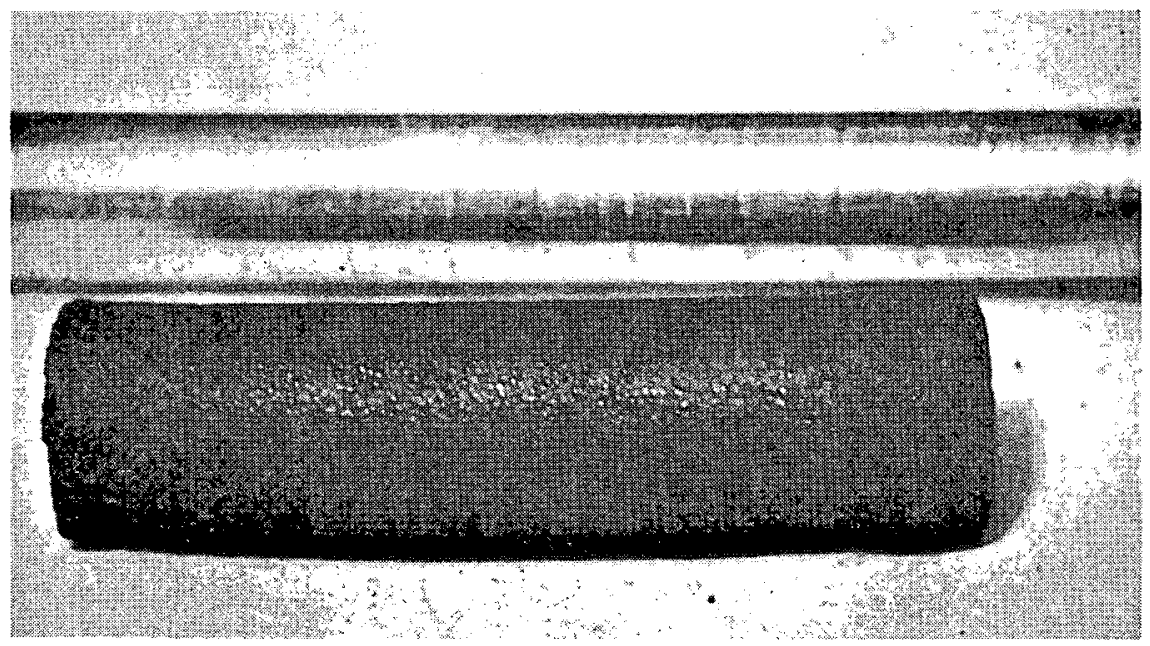

Fig. 4-52. FTE-6 typical bowed rod (3-1-12) - top body

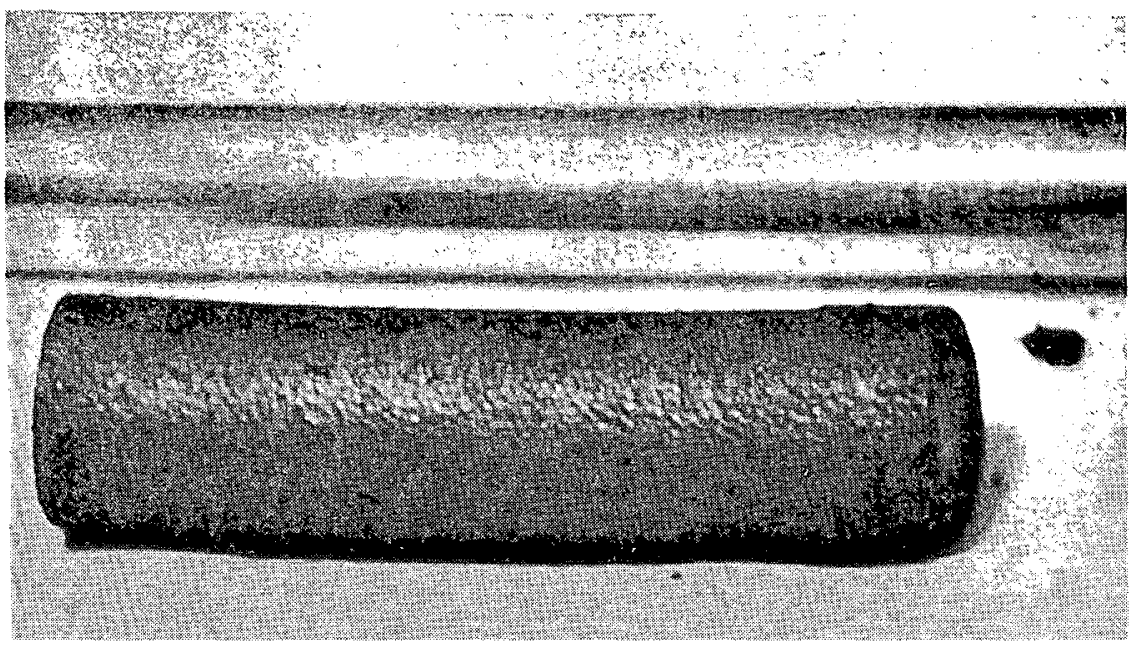

Fig. 4-51. FTE-6 typical bowed rod (3-1-3) - top body

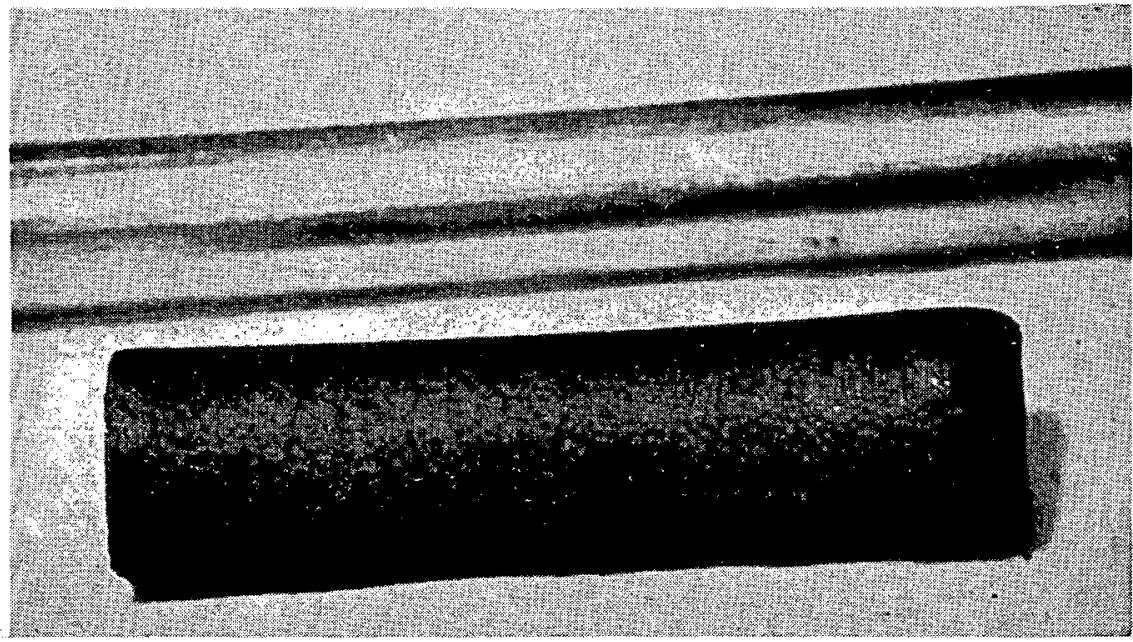

Fig. 4-53. FTE-6 typical bowed rod $(3-2-3)$ - top body 


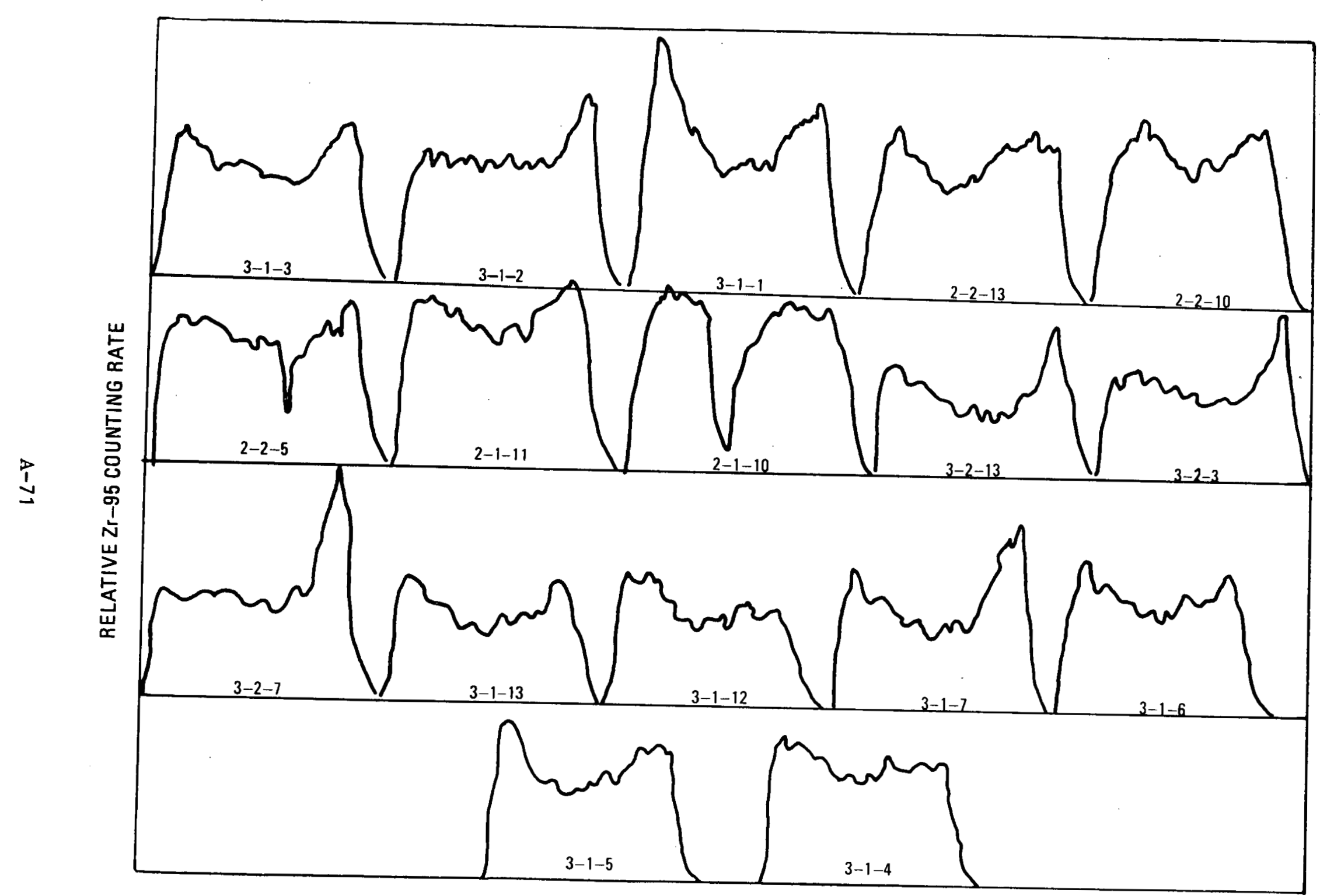

Fig. 4-54. FTE-6 fuel rod homogeneity of bowed fuel rods 


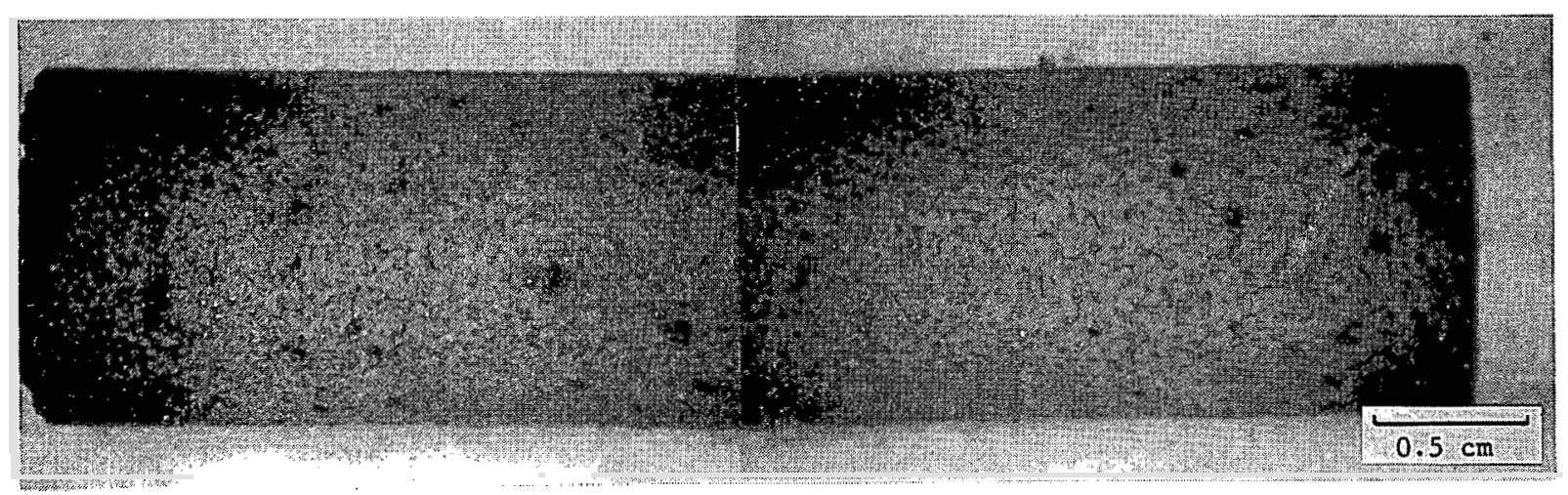

S7519 (3-4)

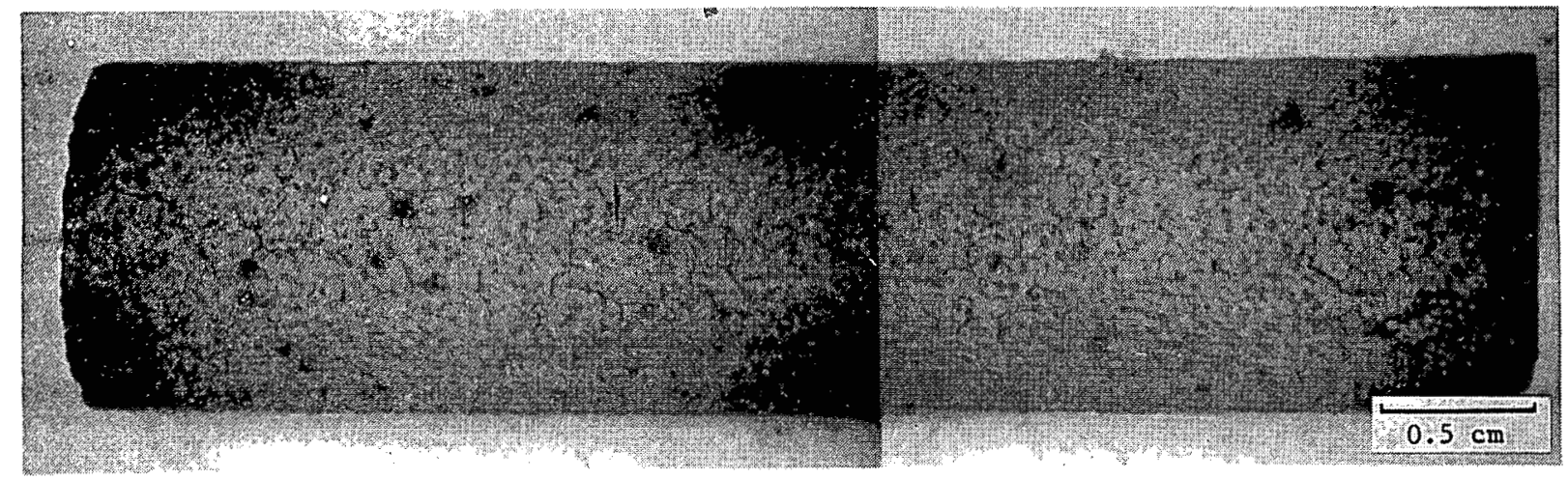

S7519 (5-6)

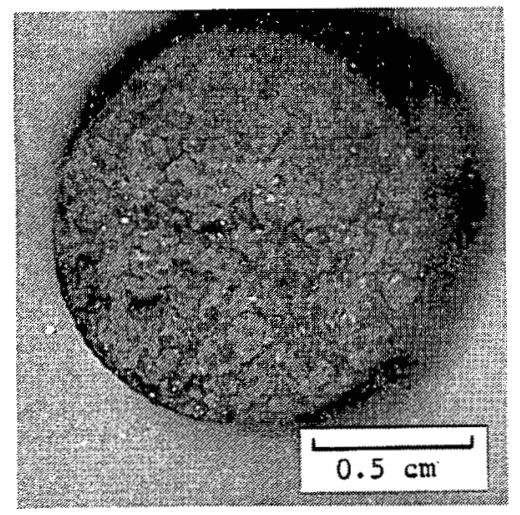

S7519-1

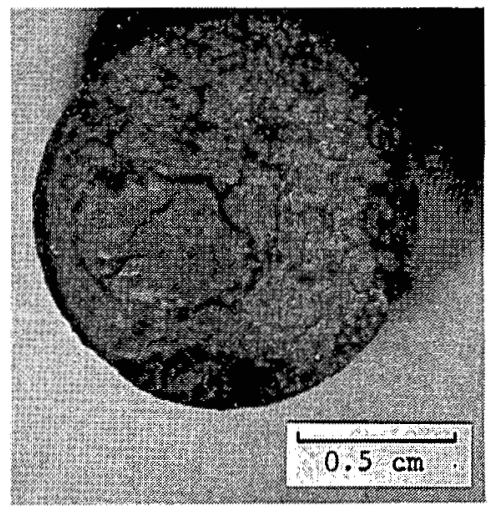

S7519-2

Fig. 4-55. FTE-6 visual examination of fuel rod 2-1-7 


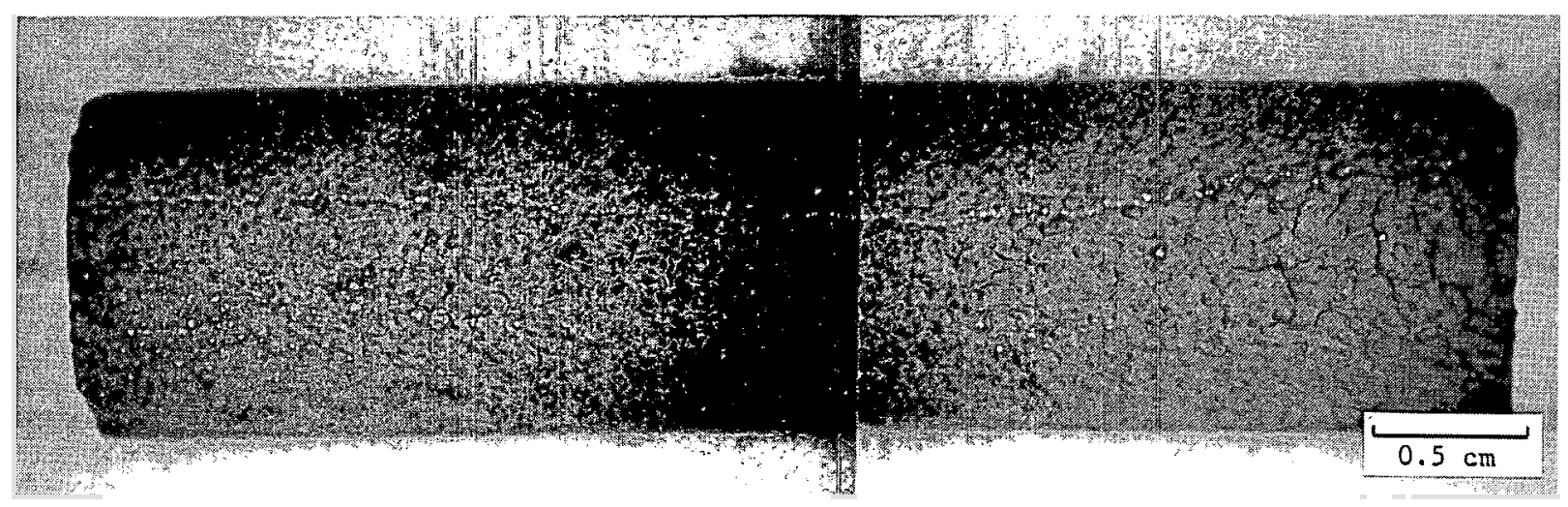

S7519 (9-10)

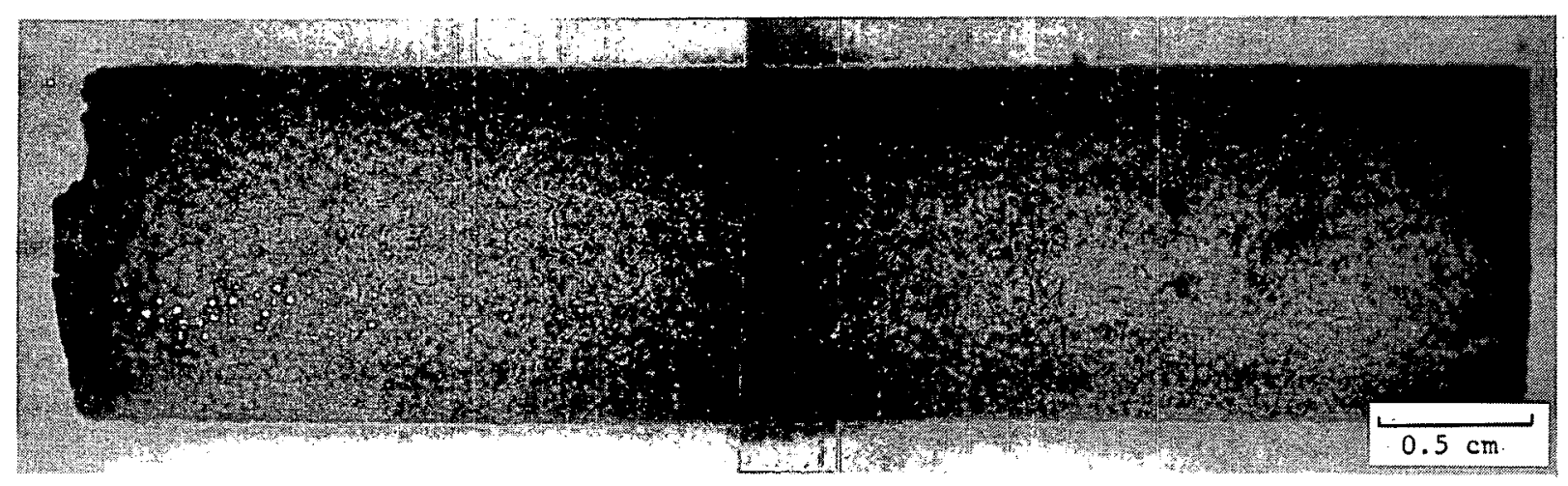

S7519 (11-12)

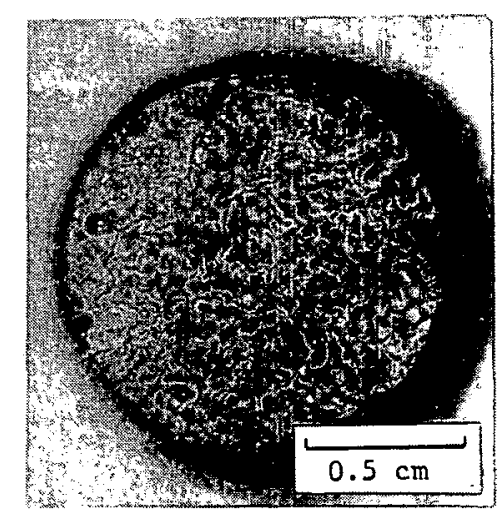

S7519-8

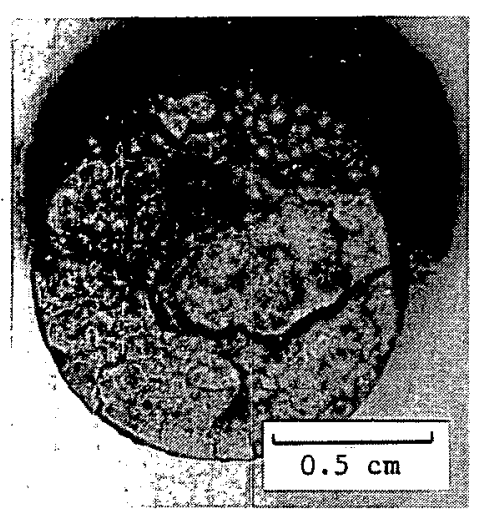

S7519-7

Fig. 4-56. FTE-6 visual examination of fuel rod 2-2-7 


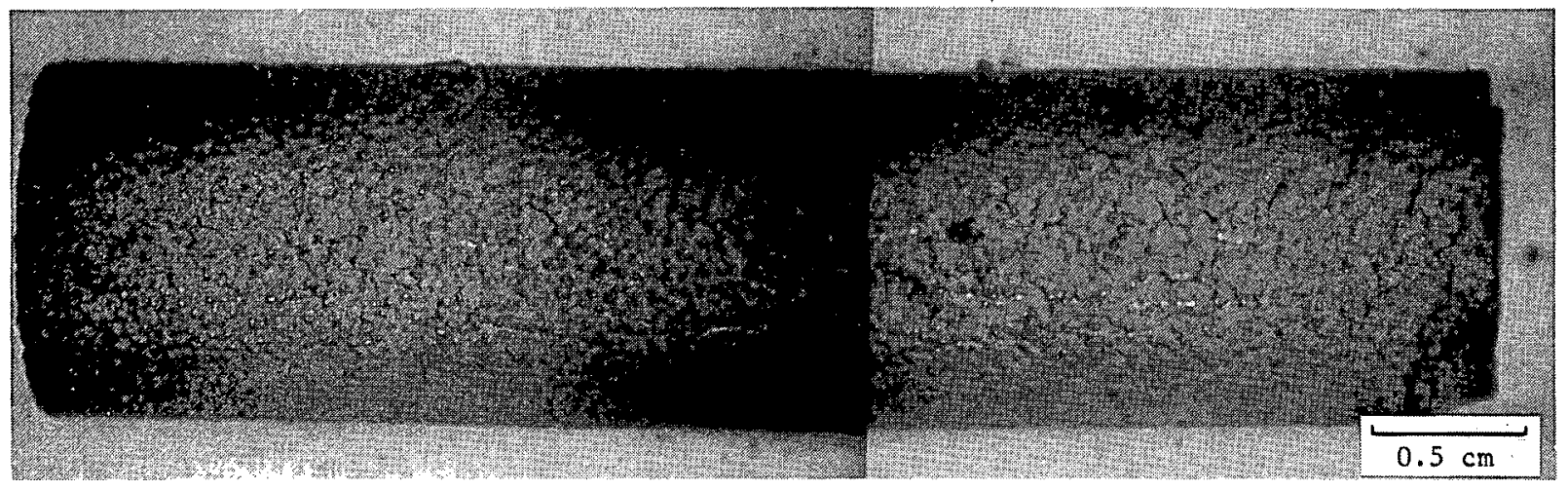

$57519(15-16)$

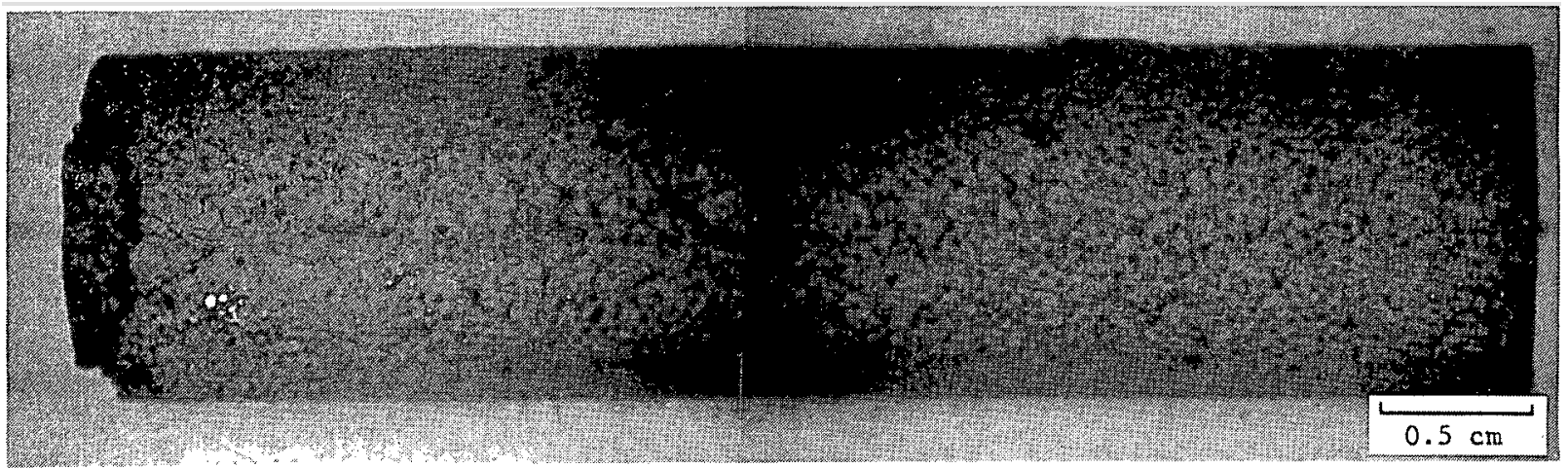

S7519 (17-18)

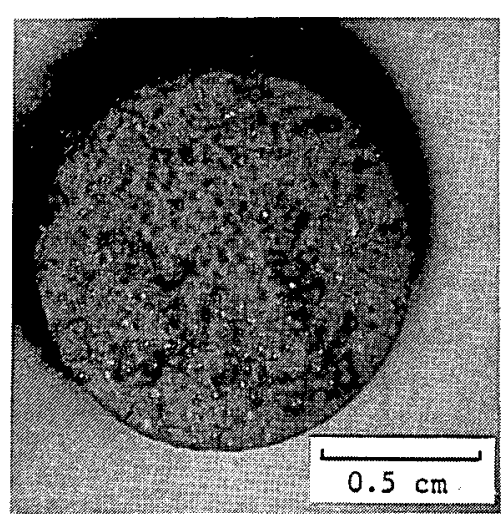

S7519-14

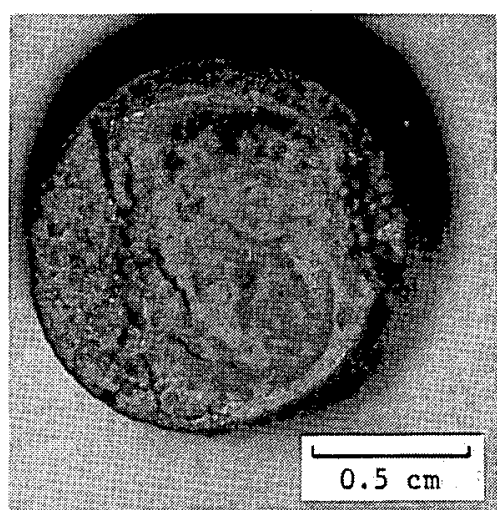

S7519-13

Fig. 4-57. FTE-6 visual examination of fuel rod 2-3-7 


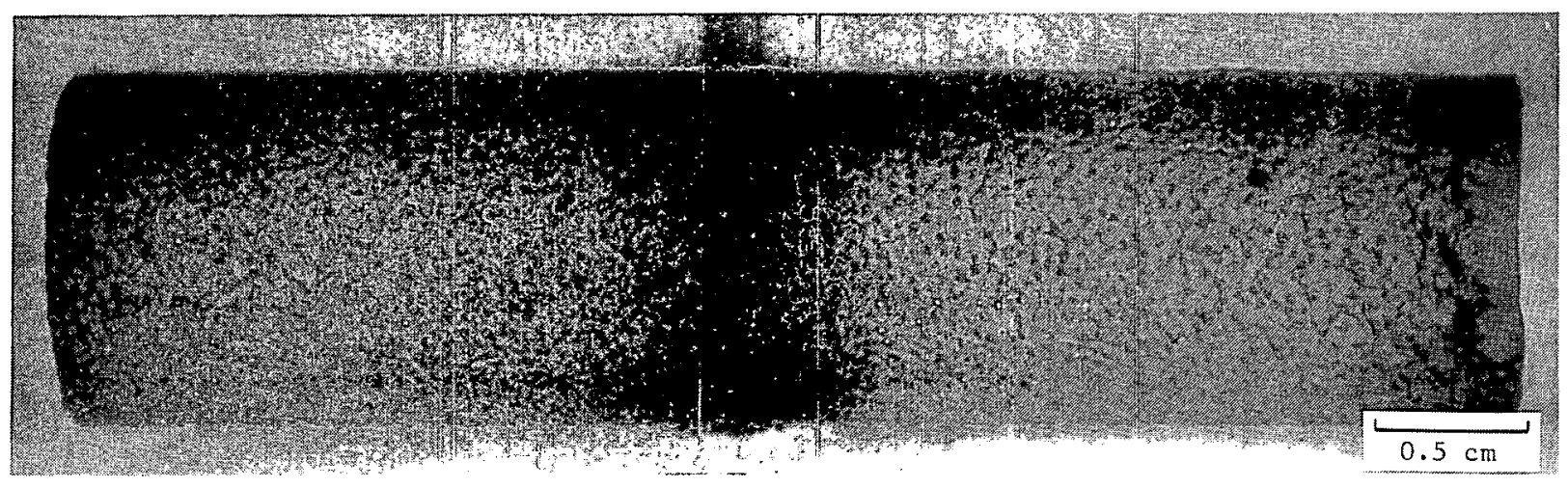

$57519(21-22)$

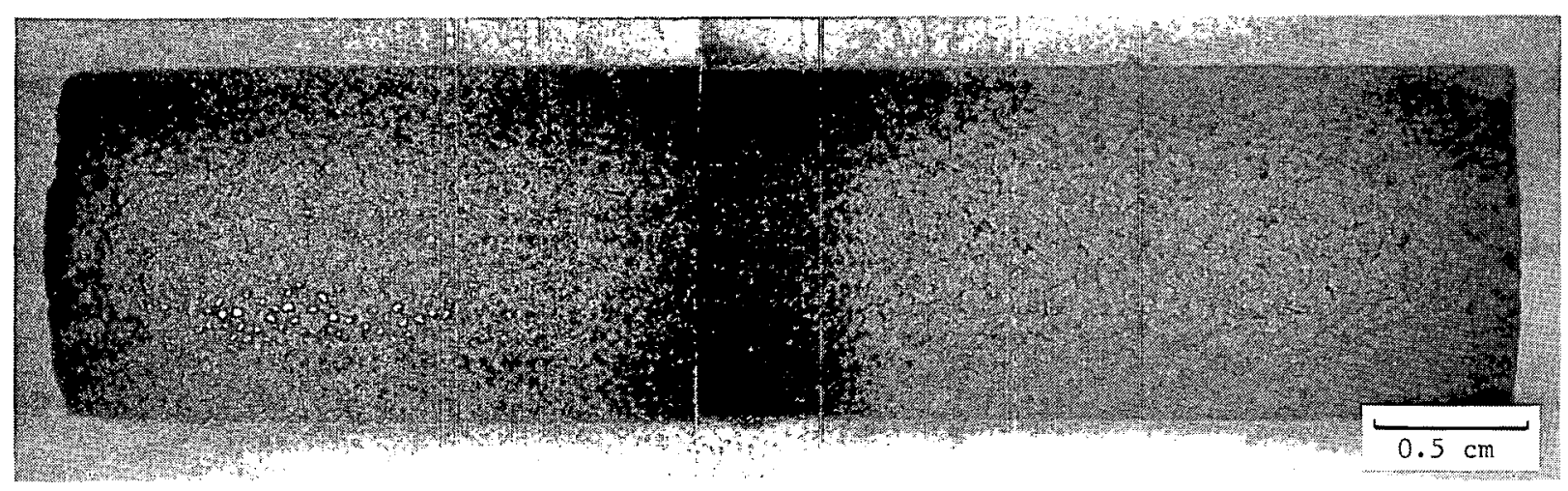

S7519 (23-24)

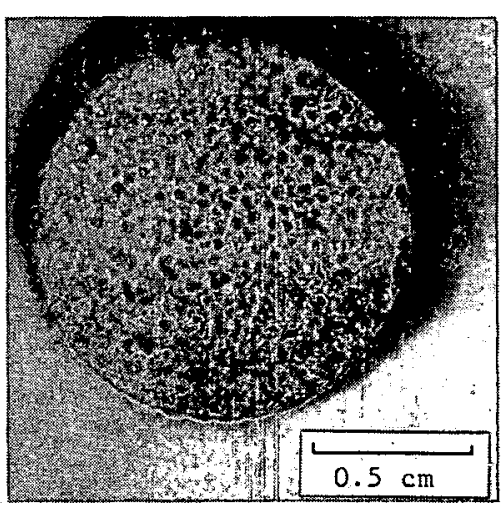

S7519-19

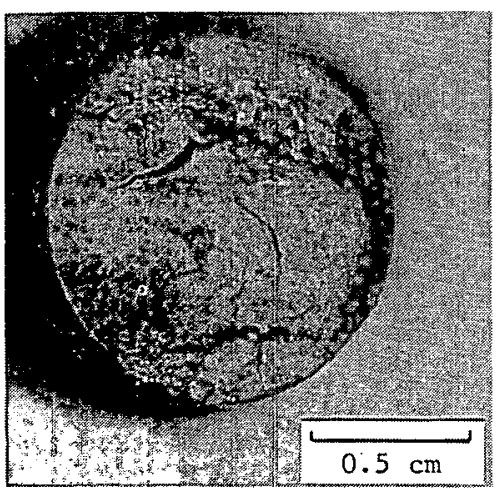

S7519-20B

Fig. 4-58. FTE-6 visual examination of fuel rod 2-4-7 


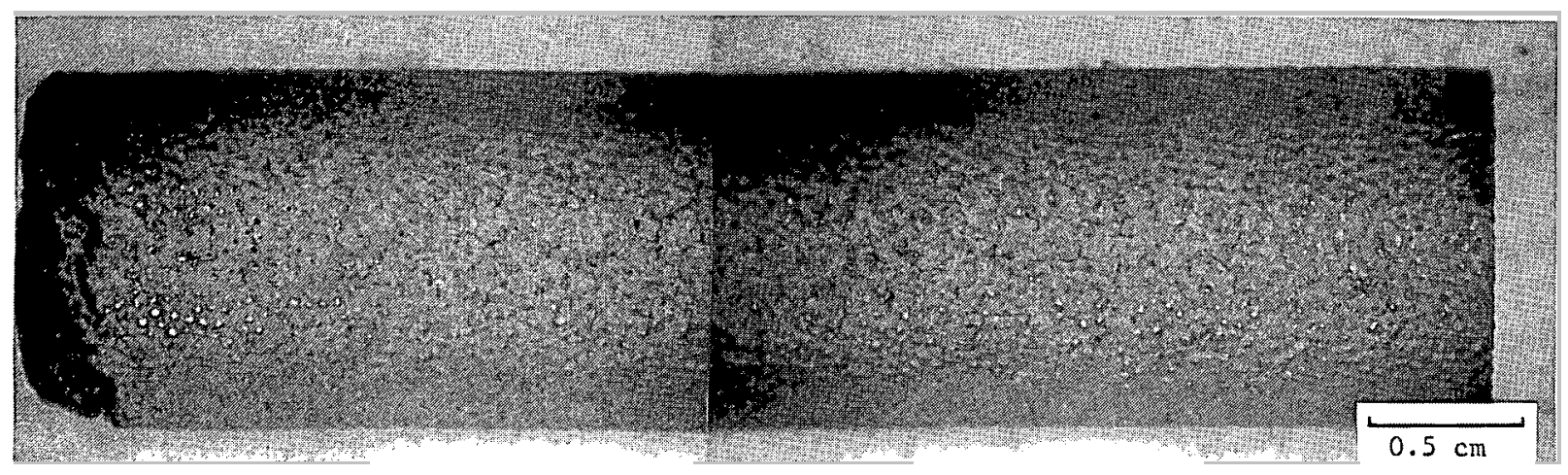

S7519 (27-28)

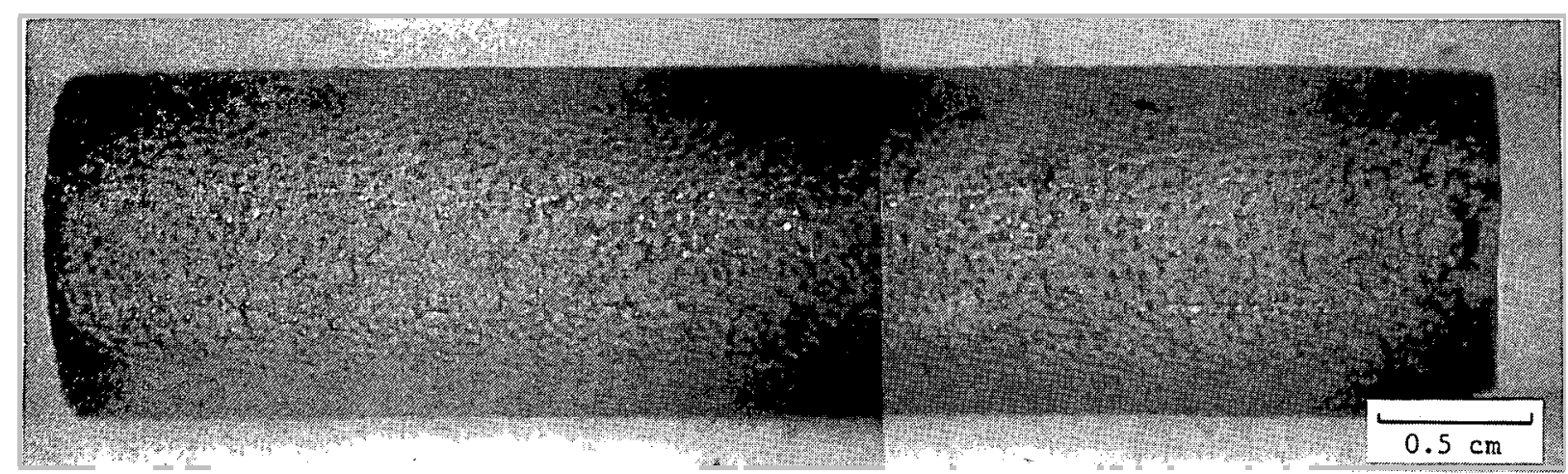

S7519 (29-30)

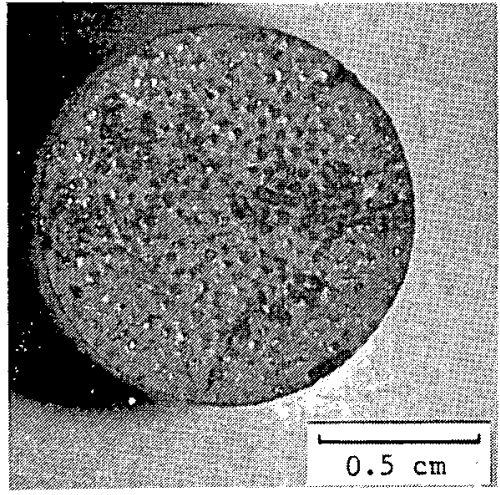

S7519-26

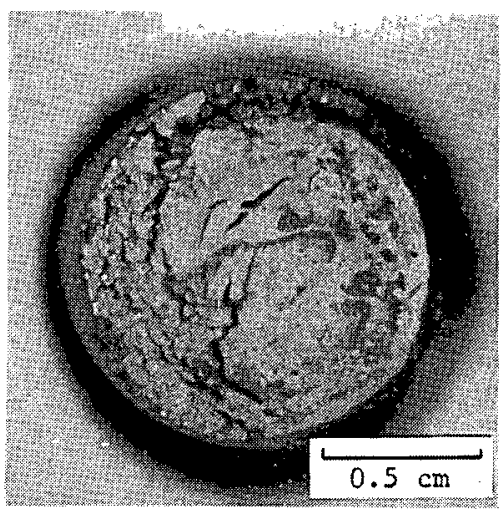

S7519-25

Fig. 4-59. FTE-6 visual examination of fuel rod 2-5-7 

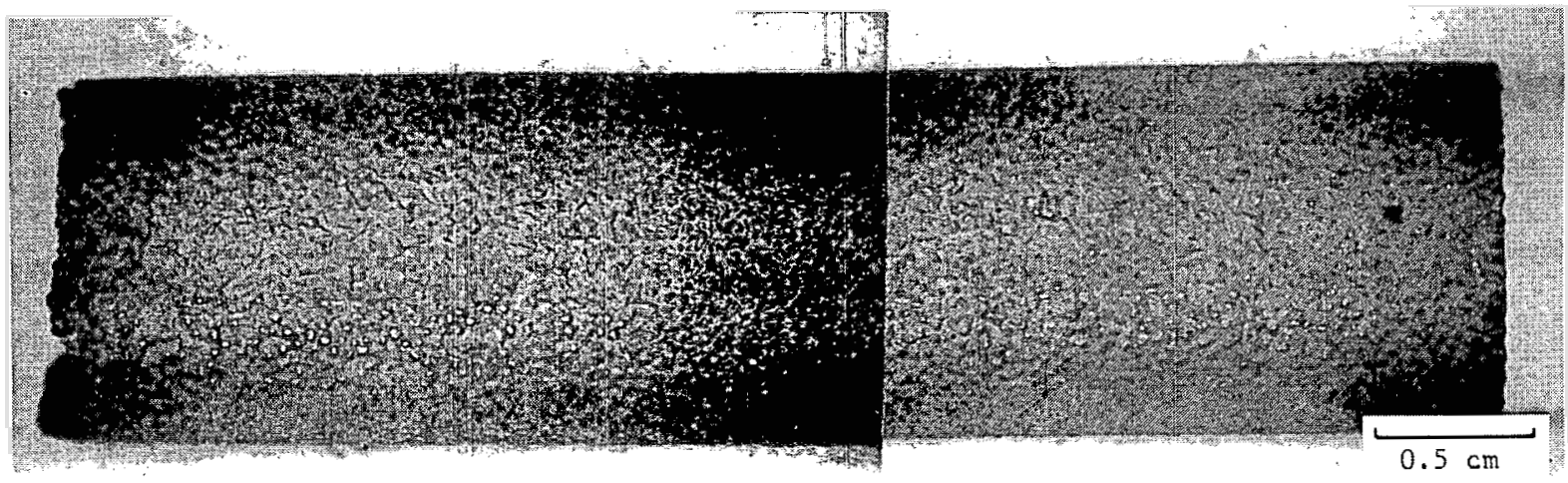

S7519 (33-34)

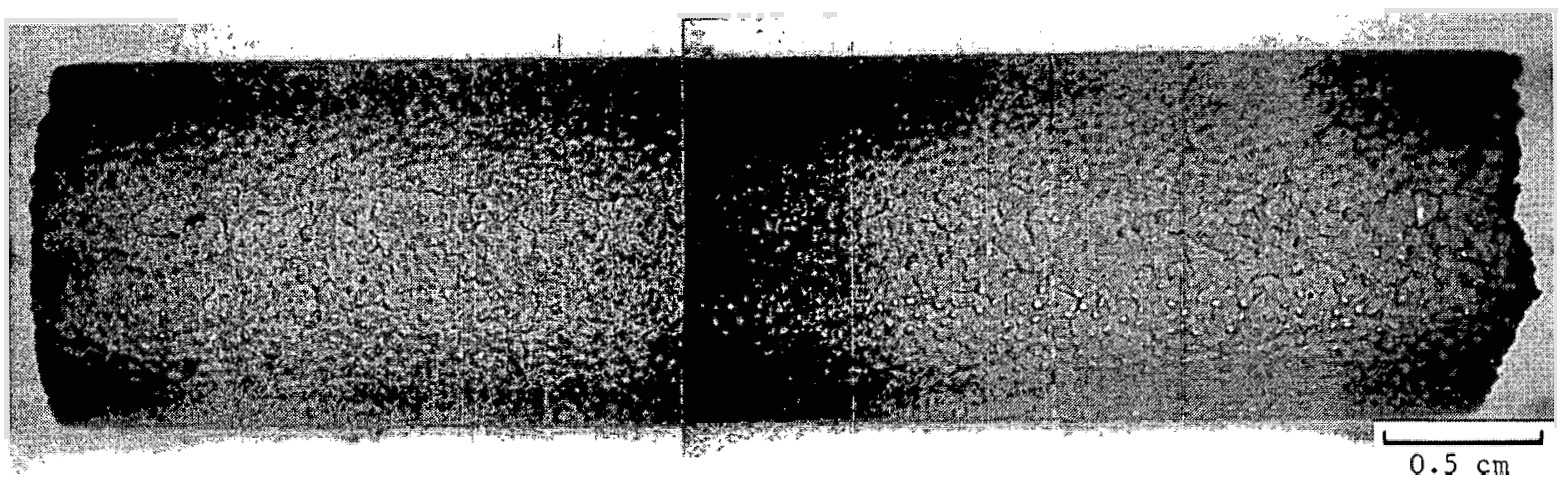

S7519 (35-36)

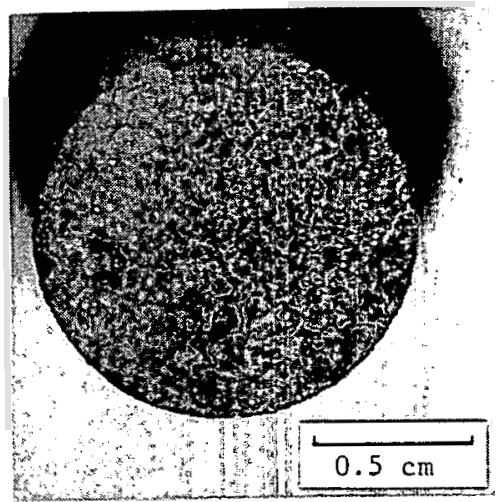

S7519-32

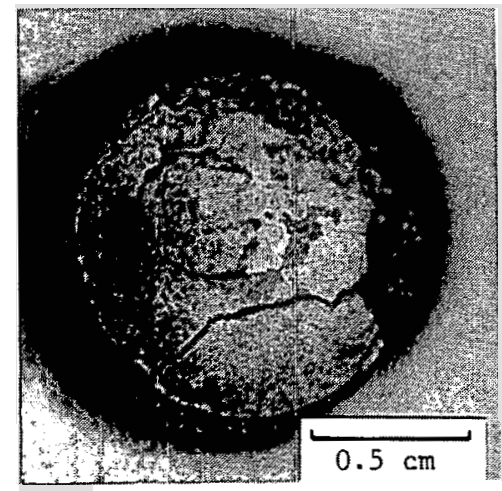

S7519-31

Fig. 4-60. FTE-6 visual examination of fuel rod 2-6-7 


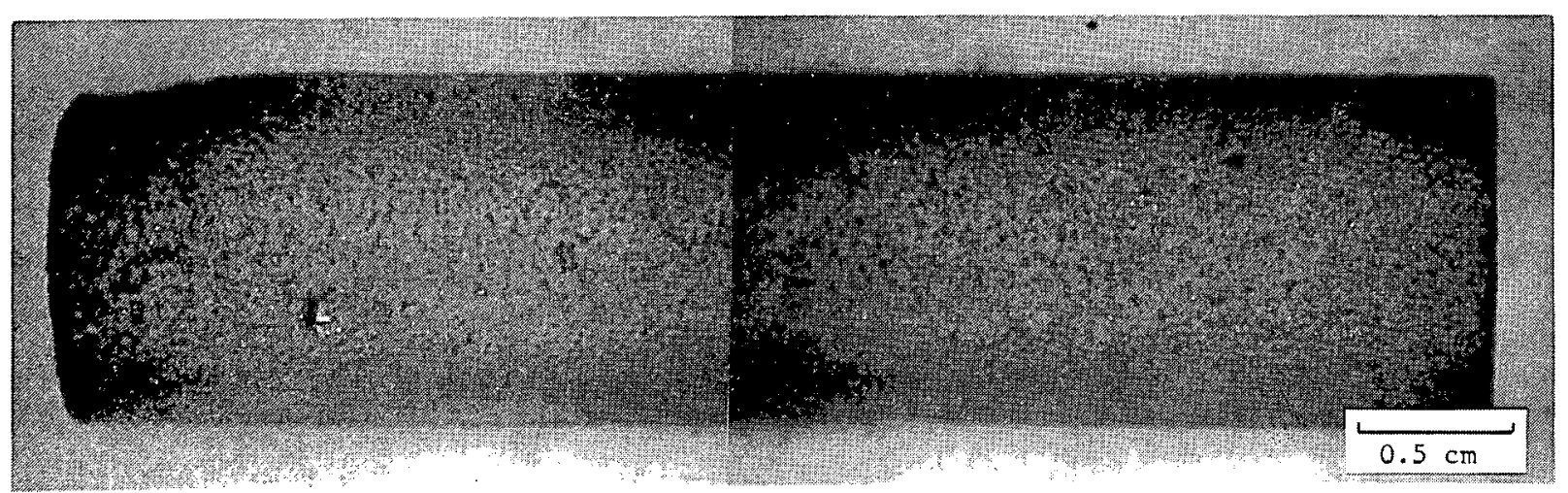

S7519 (45-46)

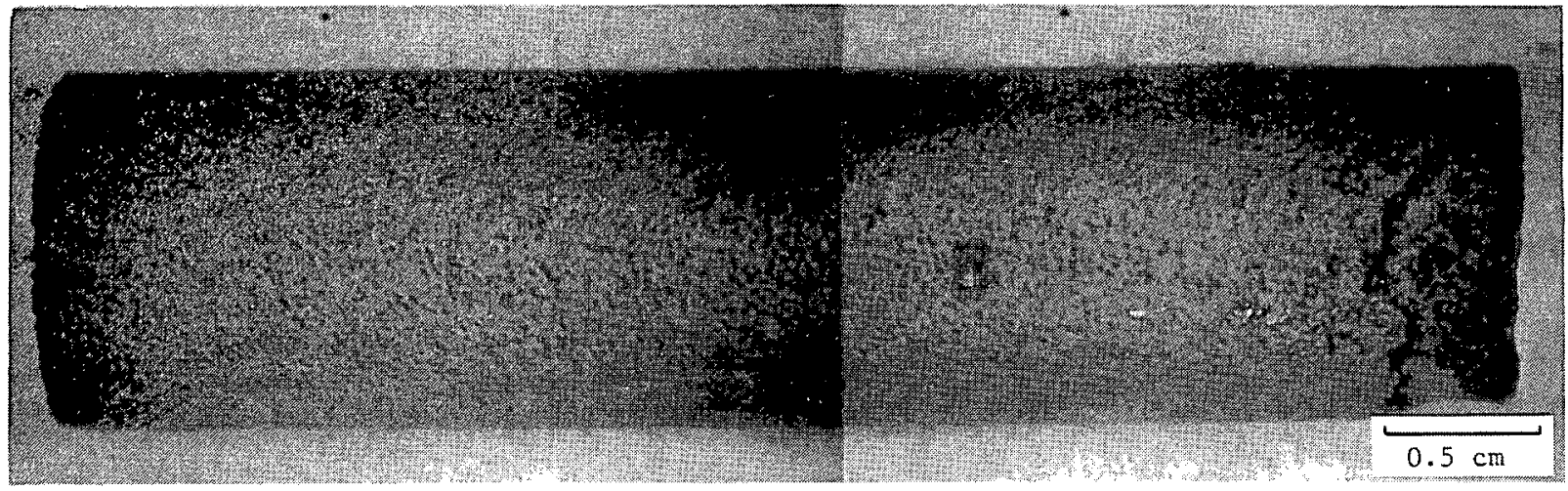

S7519 (47-48)

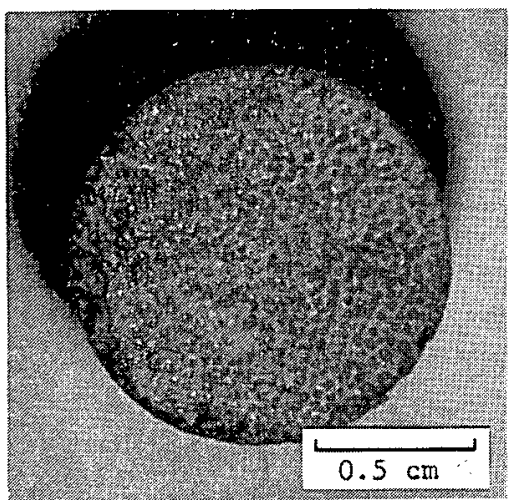

$57519-44$

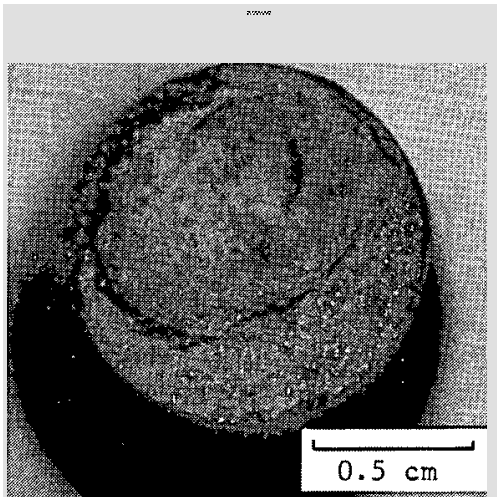

S7519-43

Fig. 4-61. FTE-6 visual examination: of fuel rod 2-7-8 


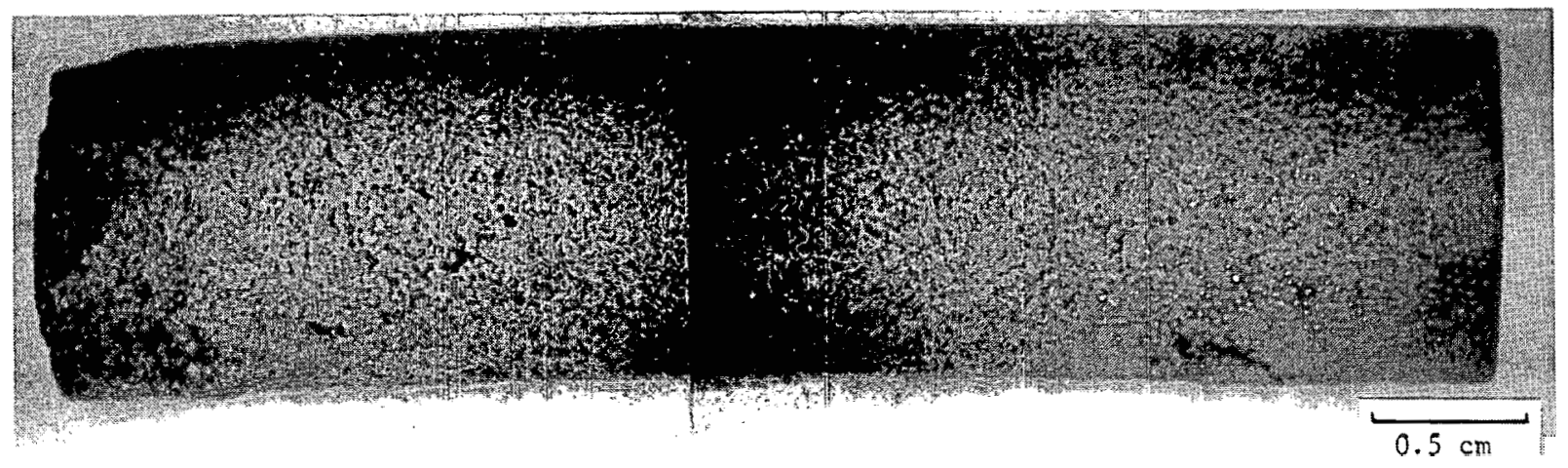

S7519 (39-40)

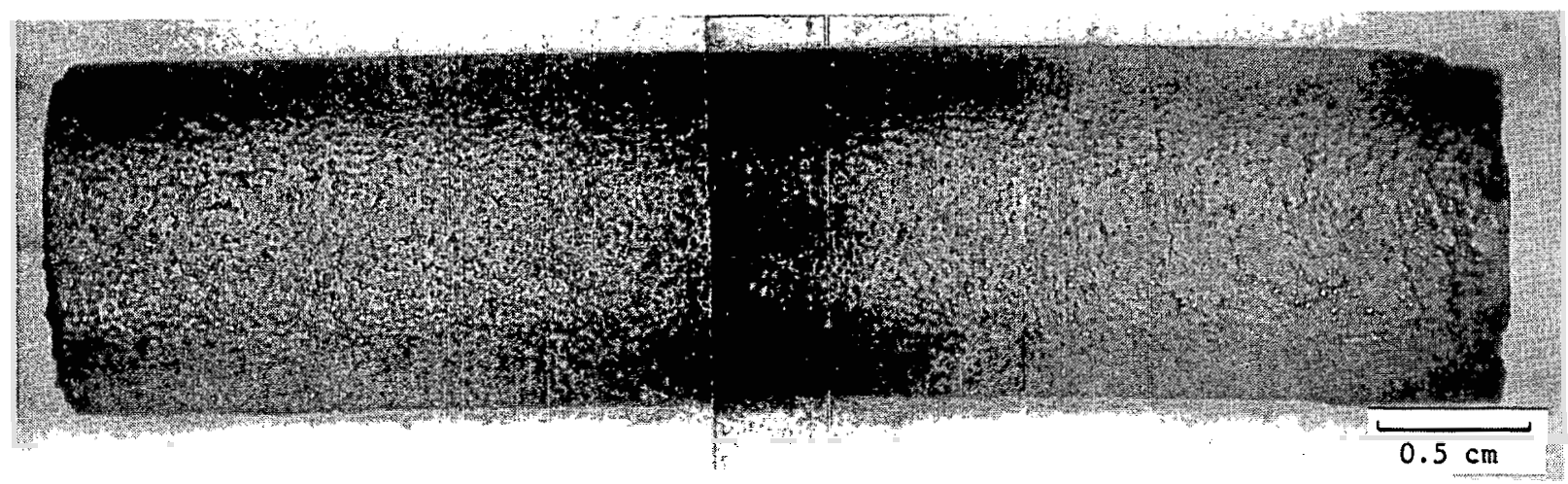

S7519 (41-42)

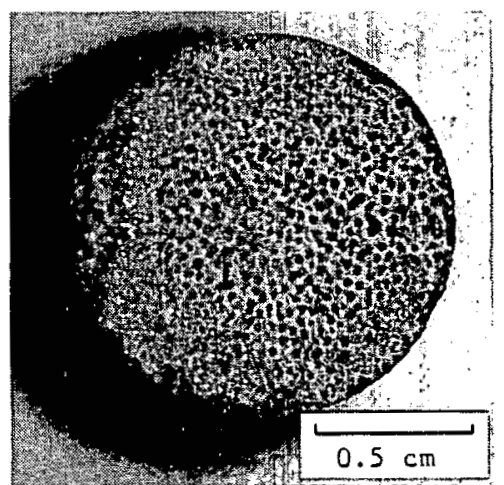

S7519-38

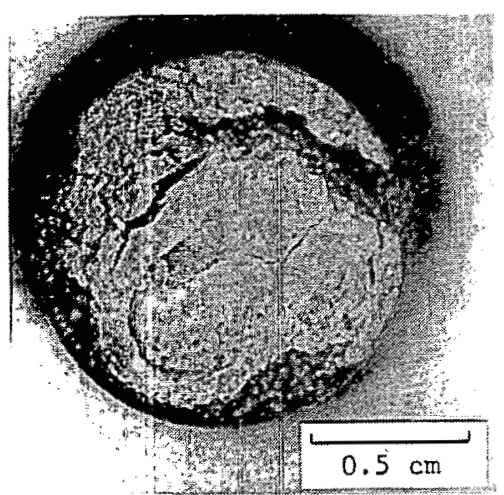

S7519-37

Fig. 4-62. FTE-6 visual examination of fuel rod 2-8-7 
○ • 

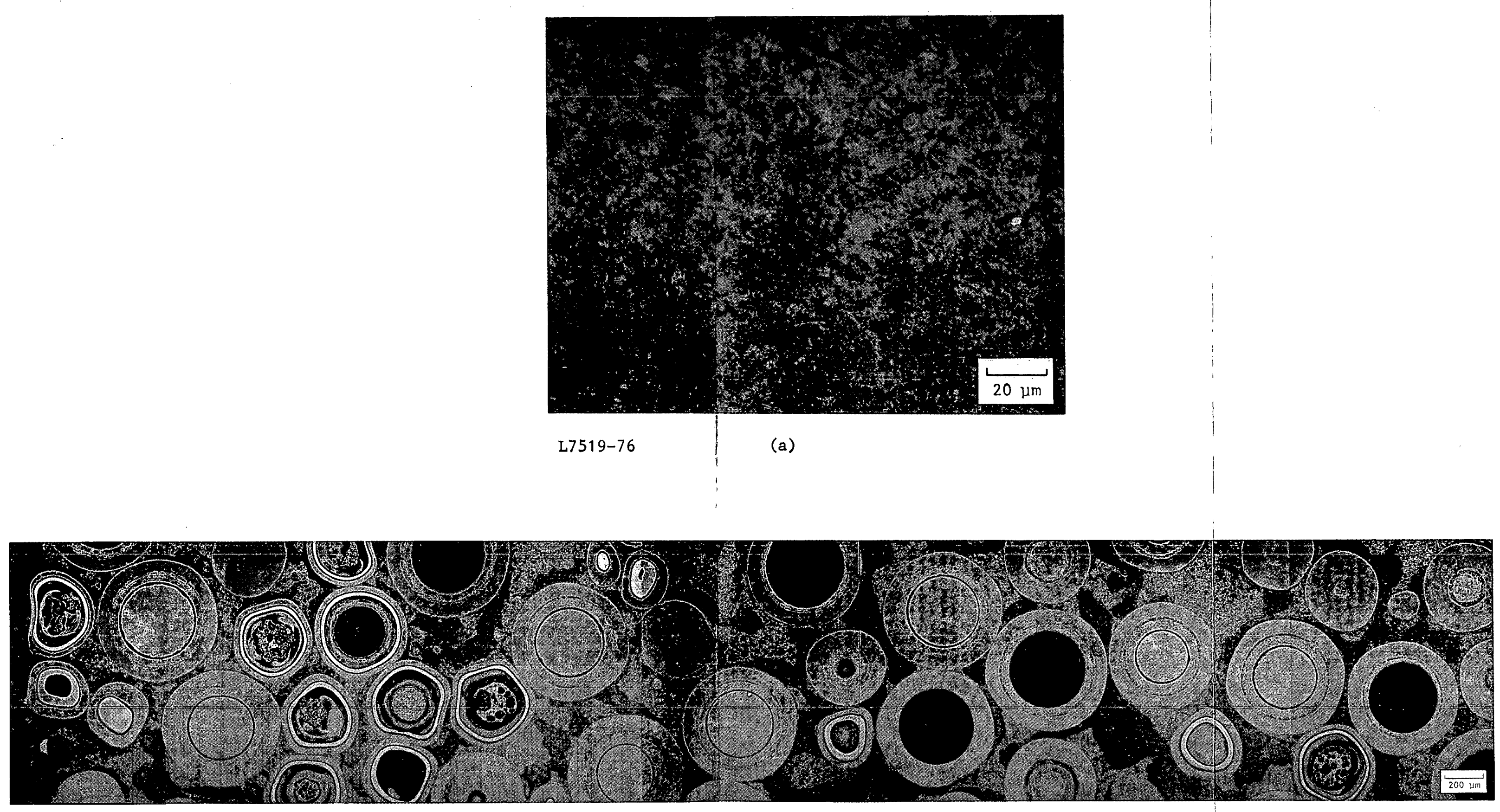

L7519 (68-74)

(b)

Fig. 4-63. FTE-6 photomicrographs of fuel $\operatorname{rod} 2-1-7$ 


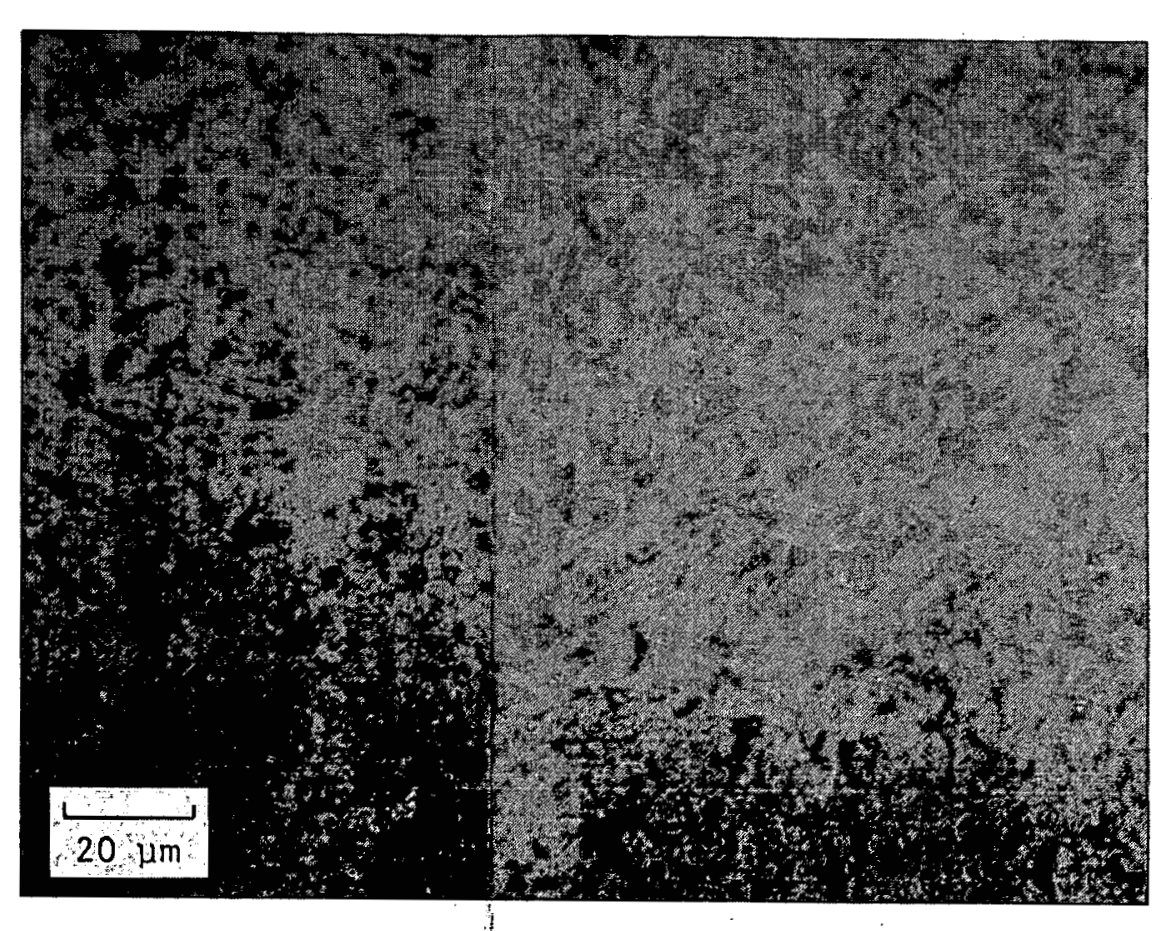

L7519-124

(a)

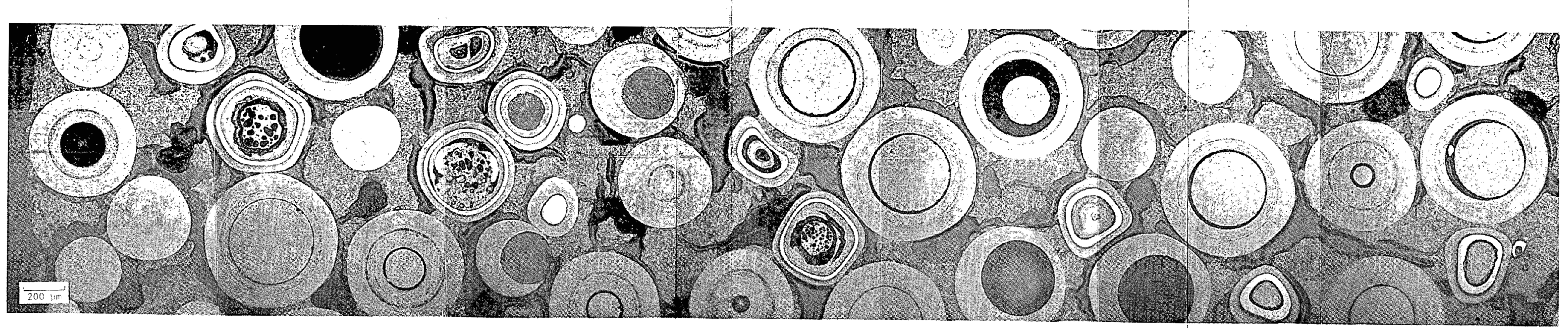




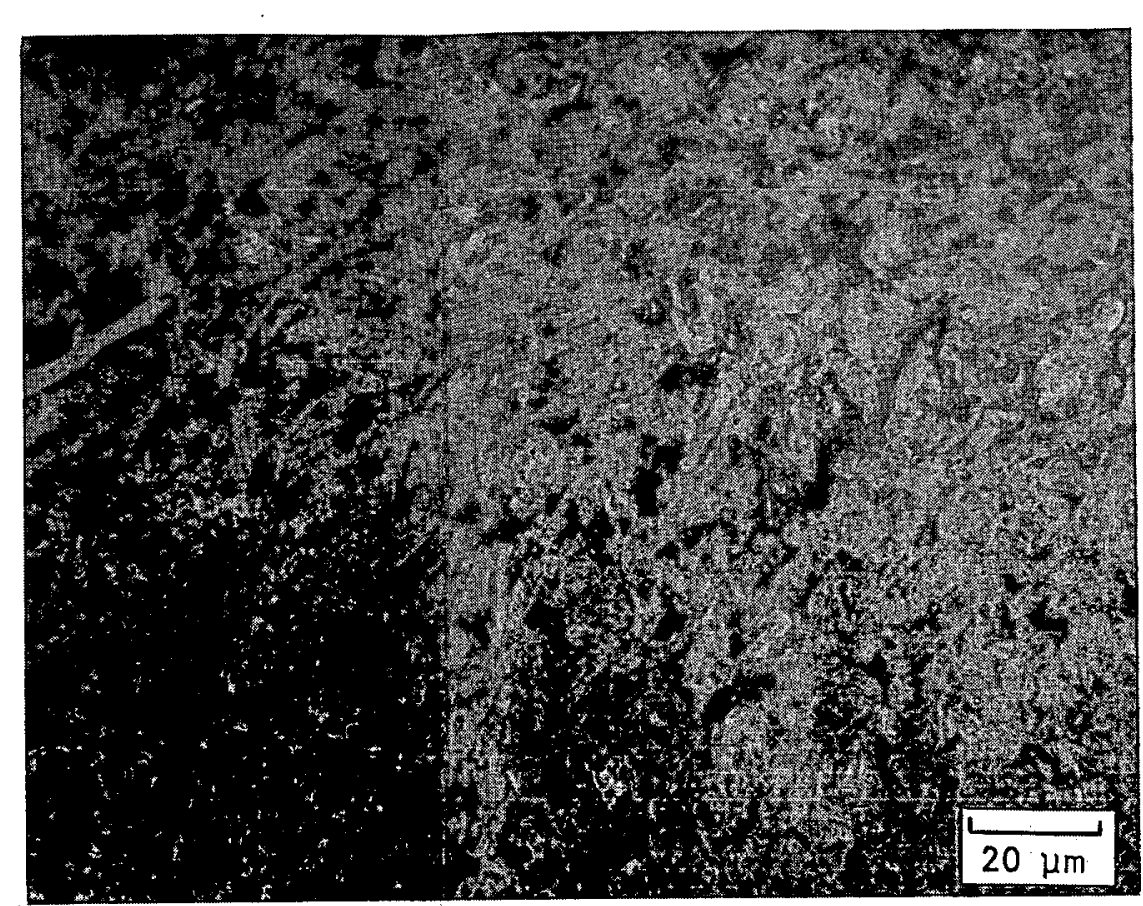

L7519-48

(a)

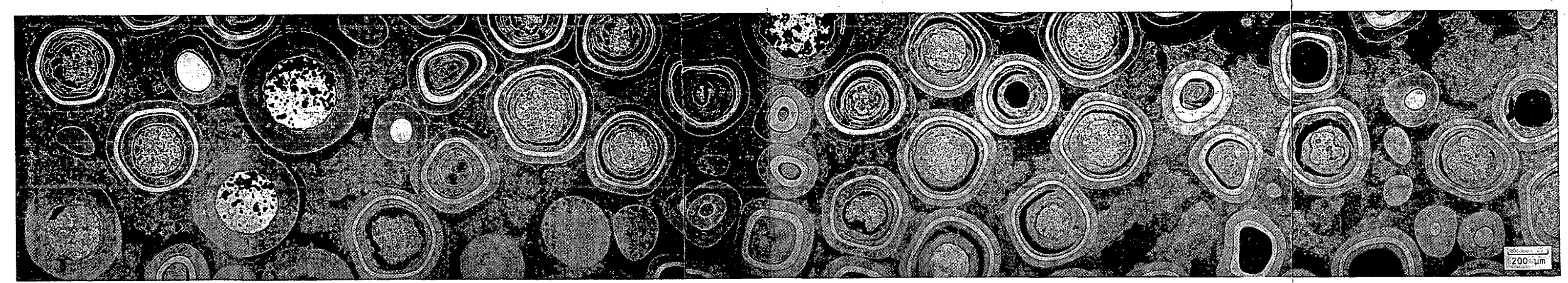

L7519 (40-46)

(b)

Fig. 4-65. FTE-6 photomicrographs of fuel $\operatorname{rod} 2-3-7$ 


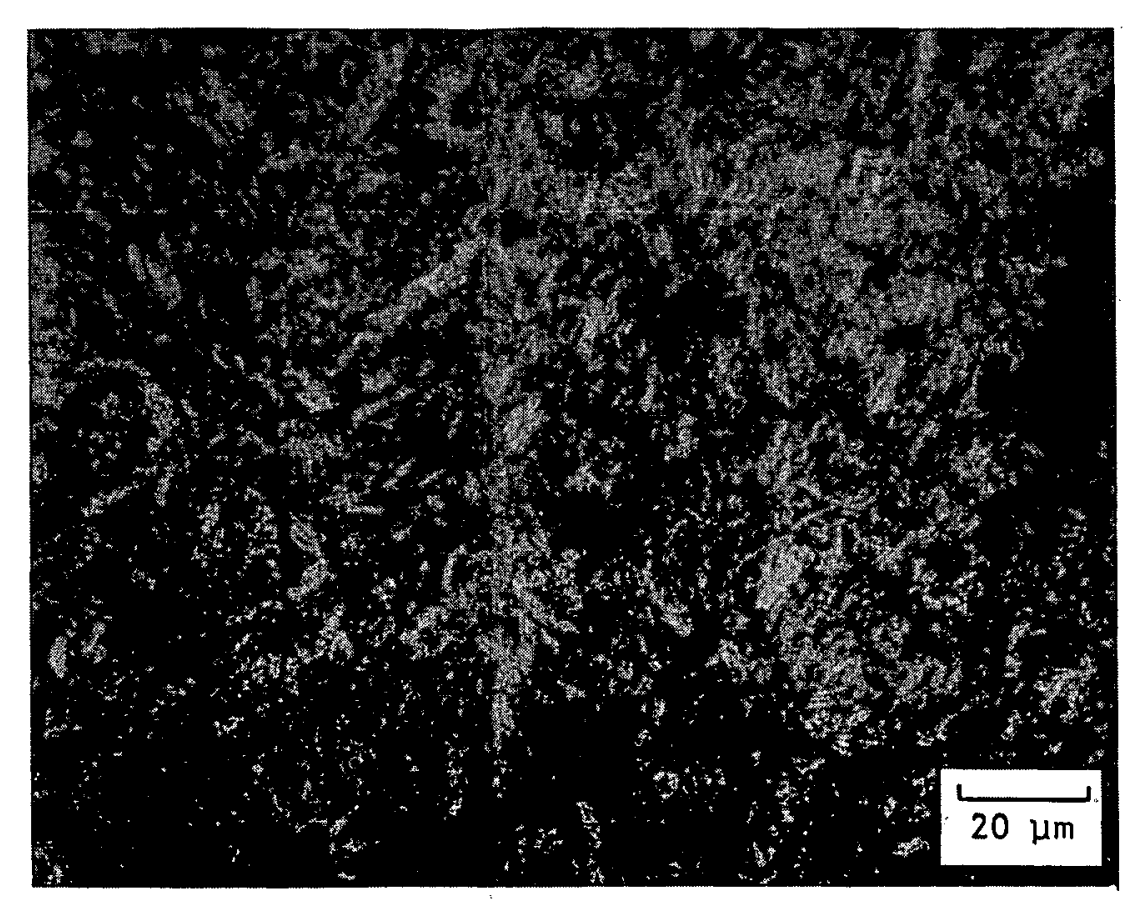

L7519-105

i(a)

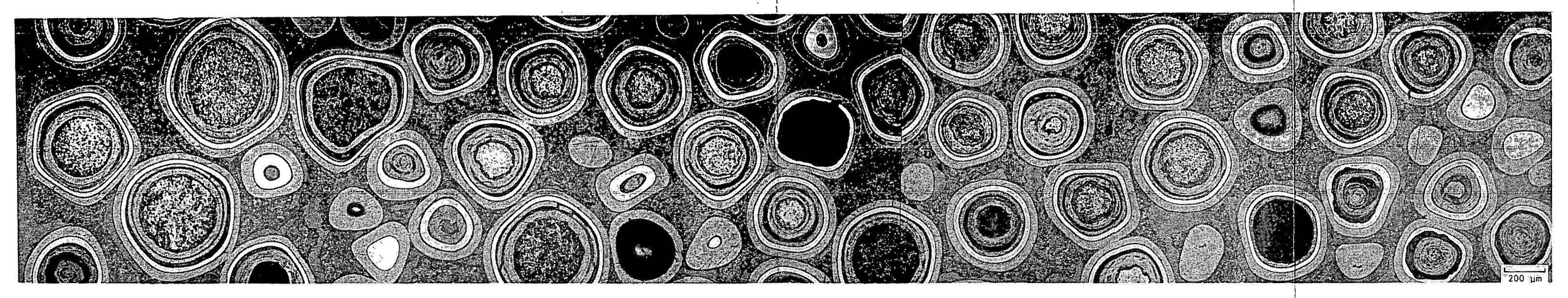




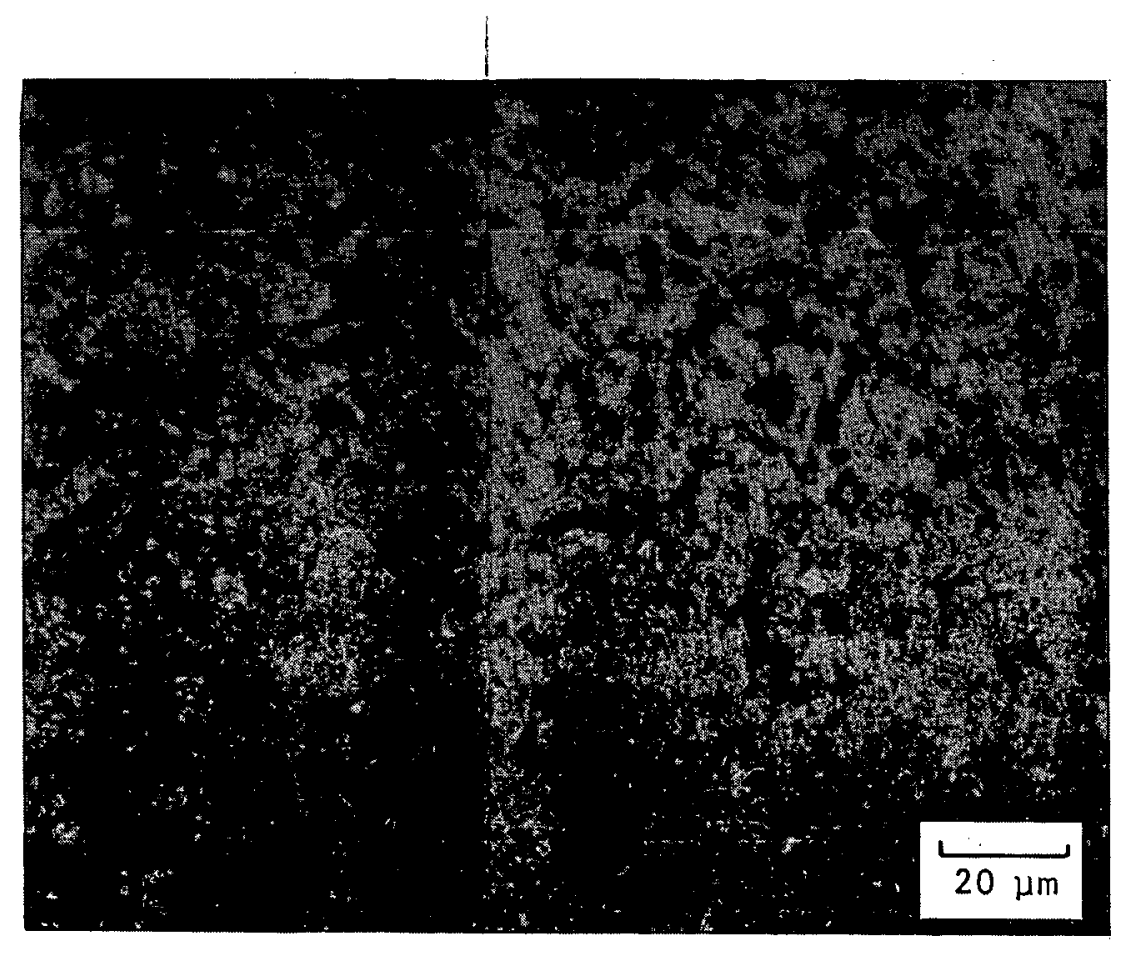

L7519-28

(a)

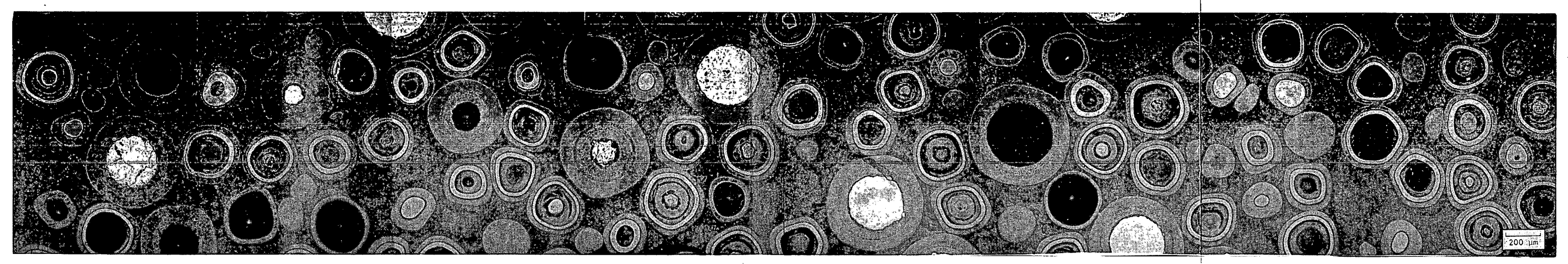

(b)

Fig. 4-67. FTE-6 photomicrographs of fuel rod 2-8-7 



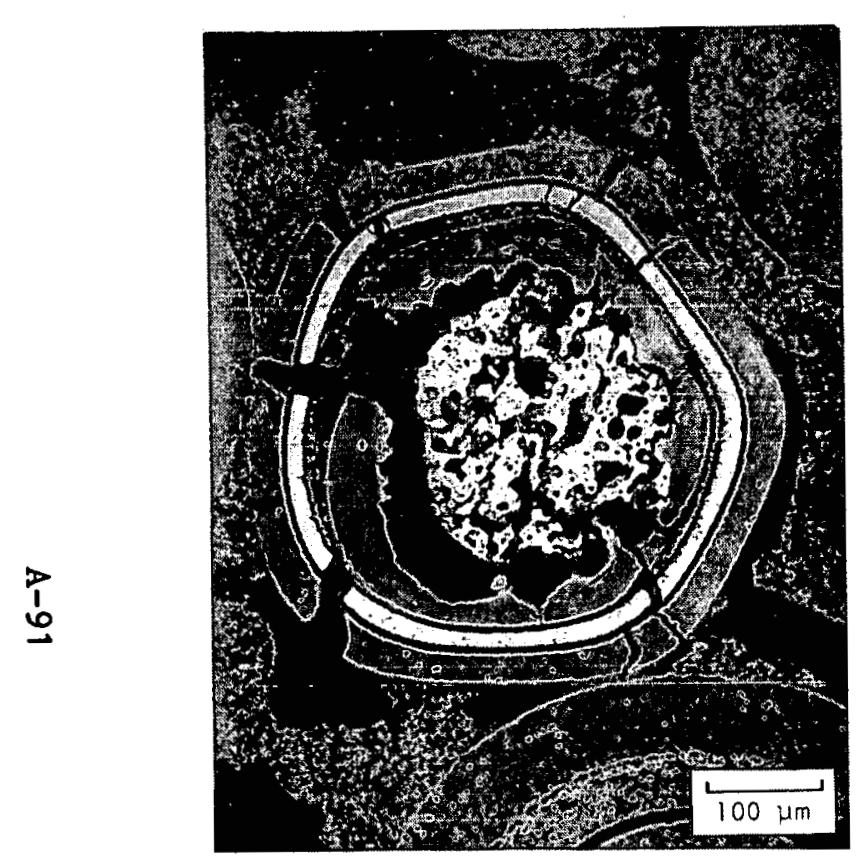

L7519-66

(a)

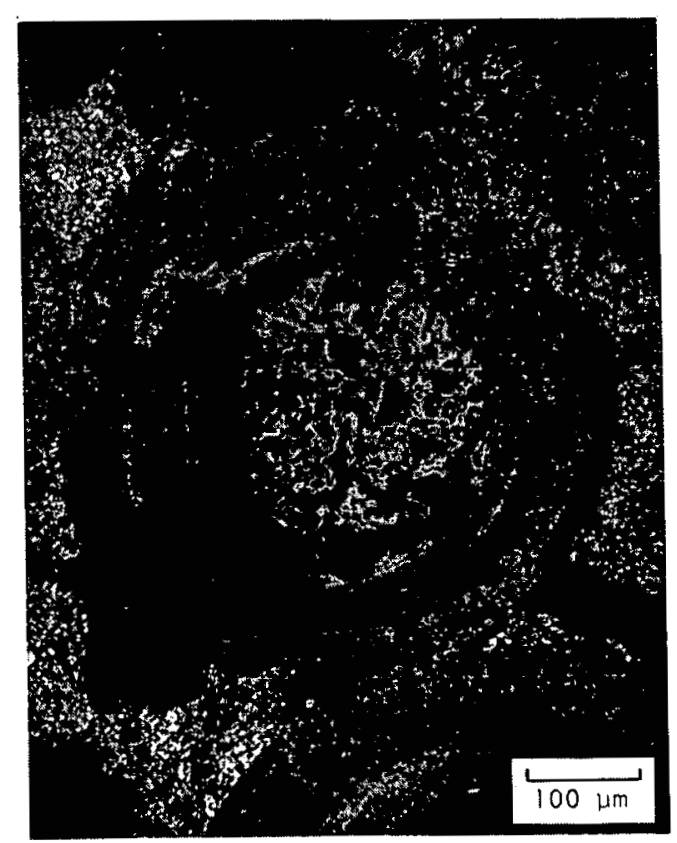

(b)

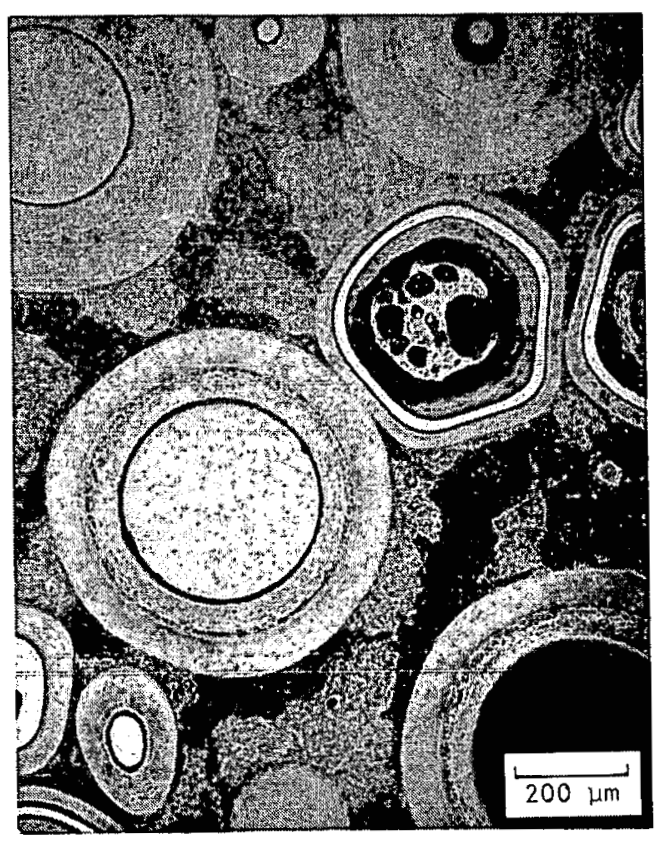

L7519-70

(c) 


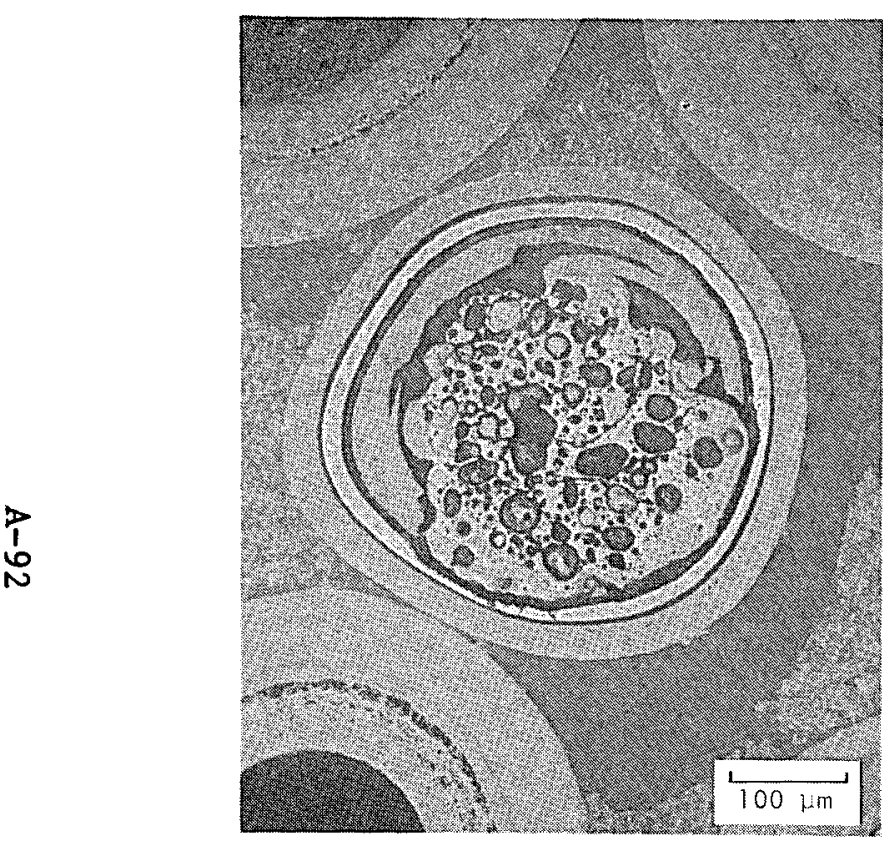

L7519-58

(a)

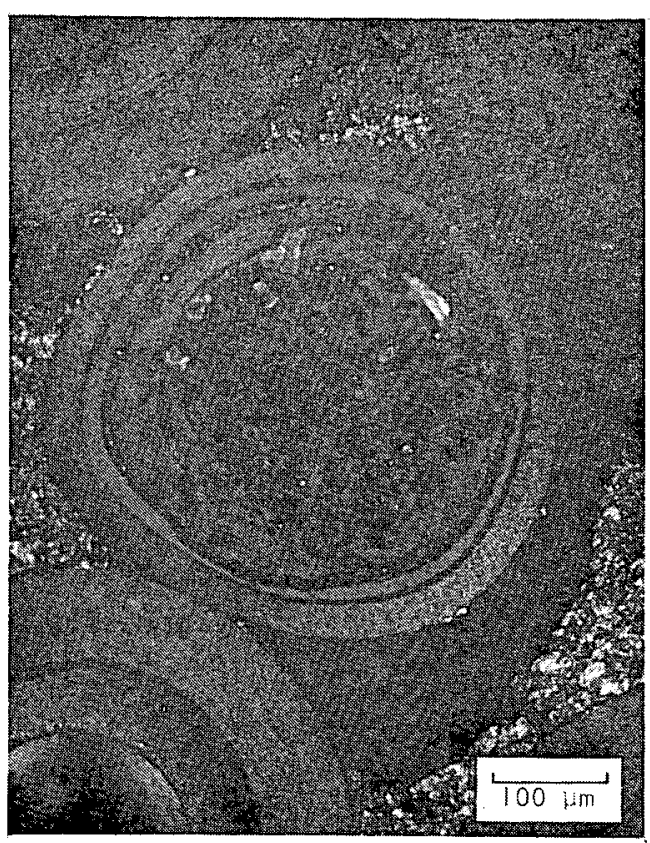

L7519-59

(b)

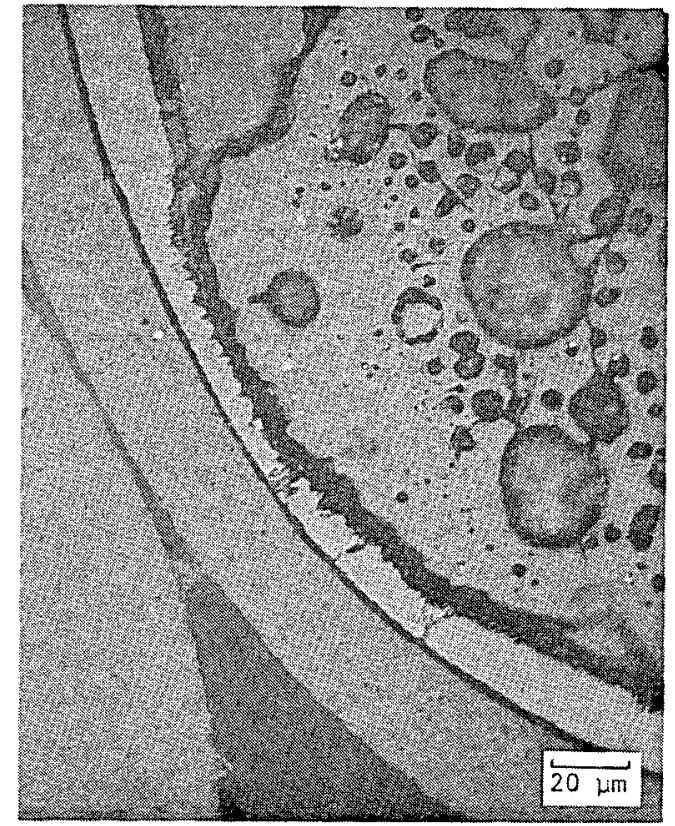

L7519-60

(c)

Fig. 4-69. FTE-6 photomicrographs of $\mathrm{UO}_{2}$ TRISO particle showing SiC attack from fuel rod 2-1-7 


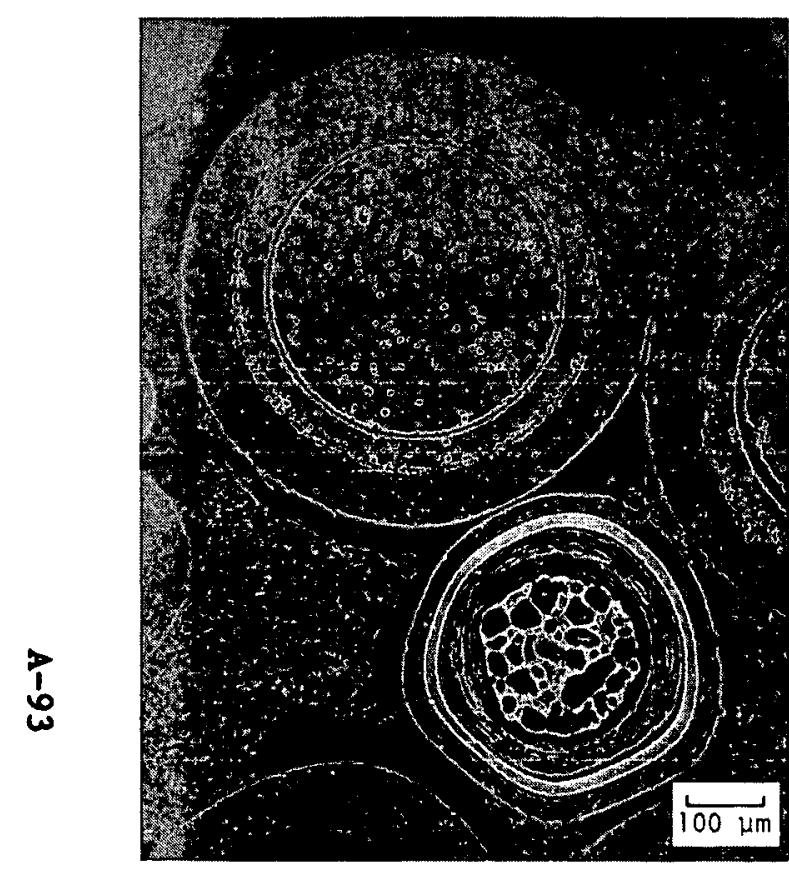

L7519-112 (a)

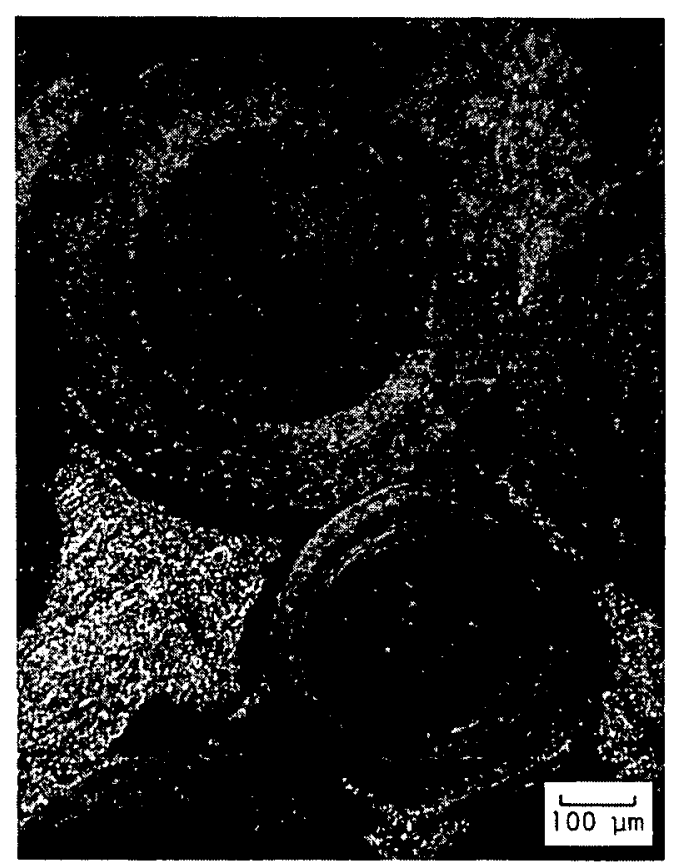

(b)

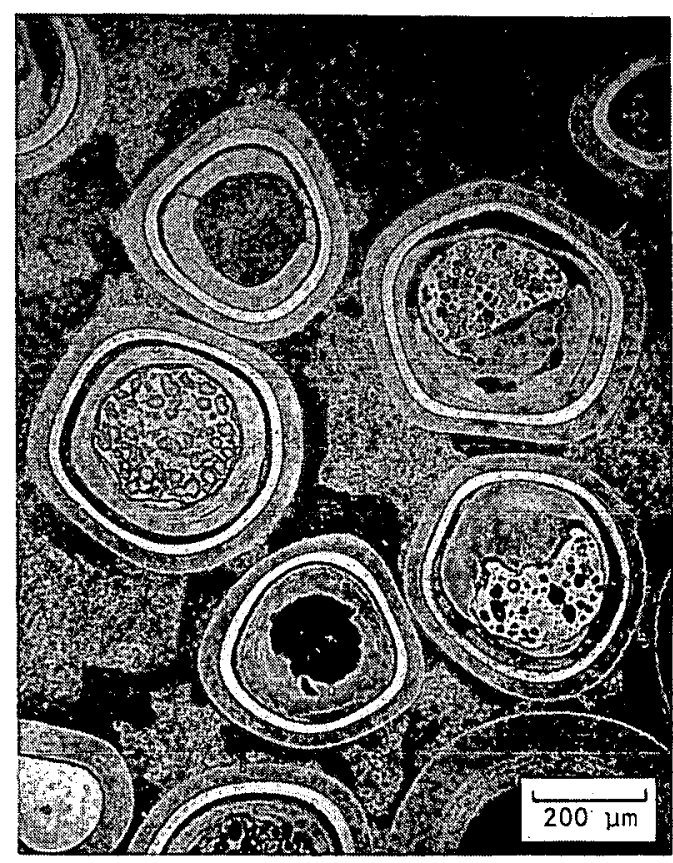

L7519-125

(c)

Fig. 4-70. FTE-6 photomicrographs of representative fuel particles from fuel rod 2-2-7 


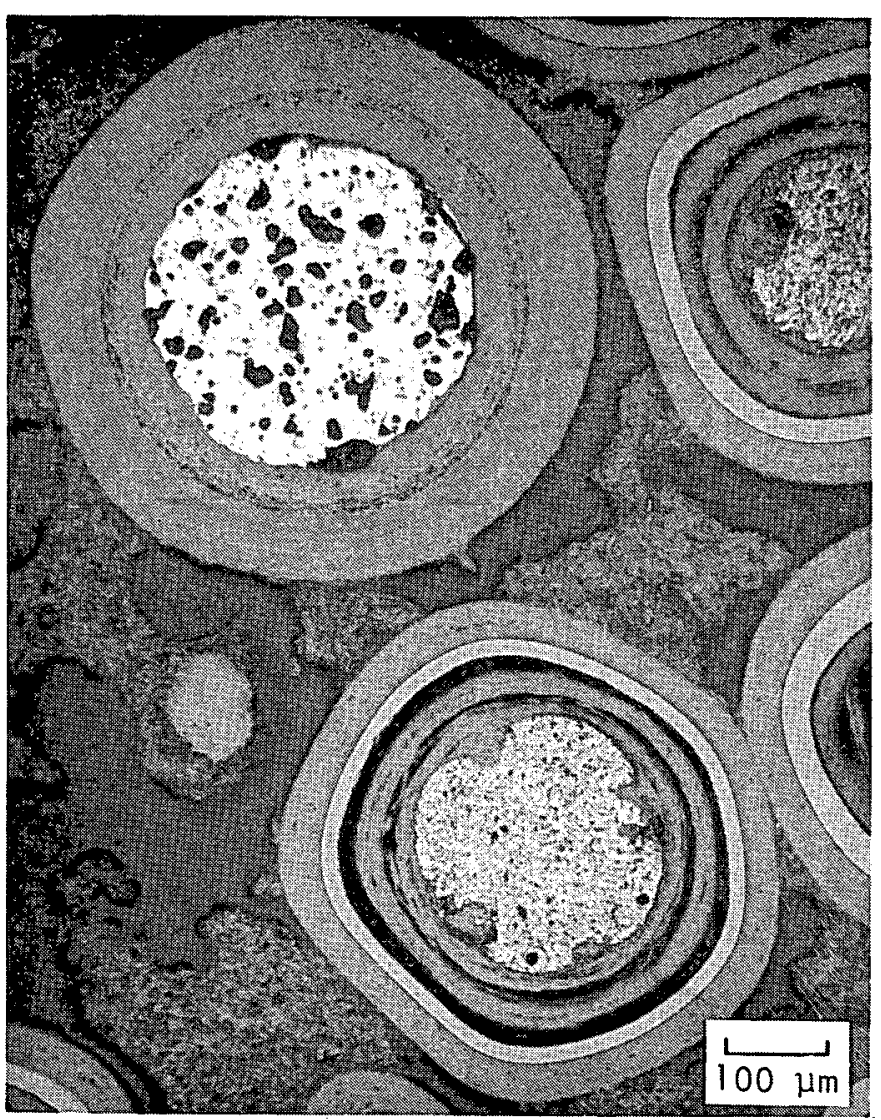

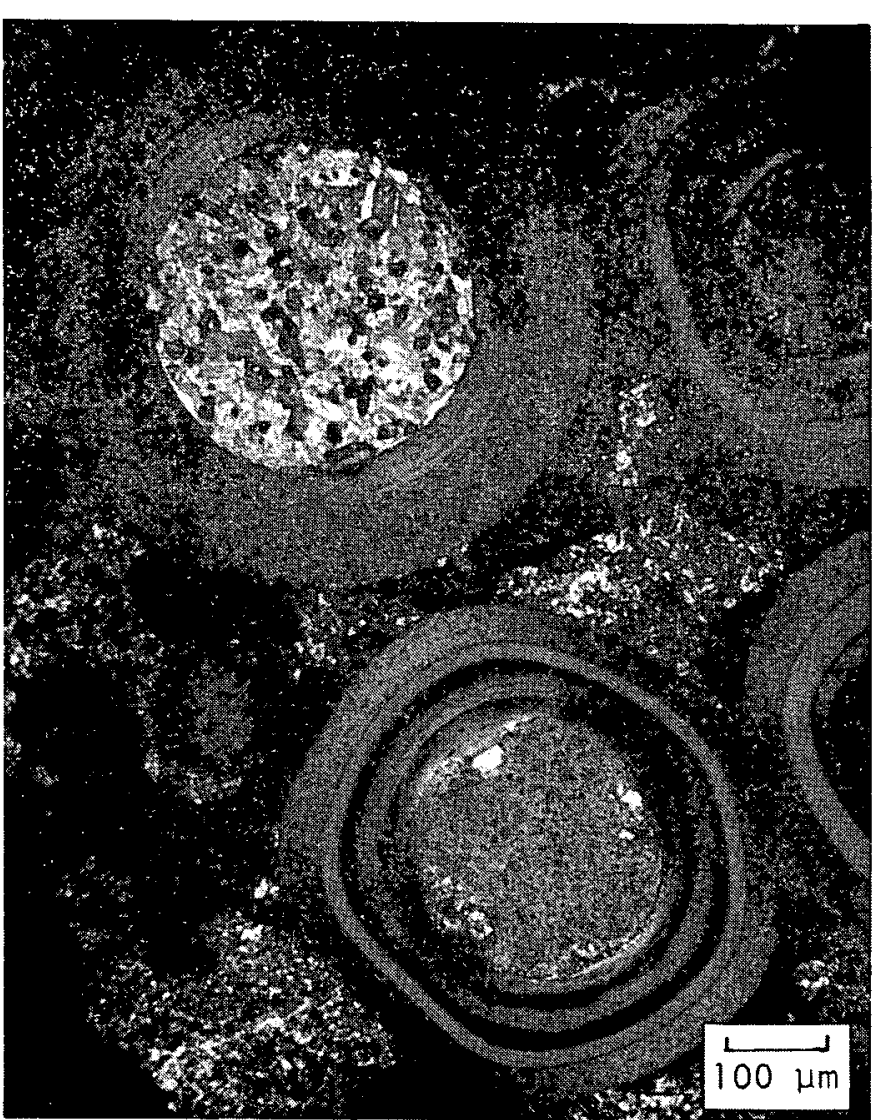

(b)

Fig. 4-71. FTE-6 photomicrographs of representative fuel particles from fuel rod 2-3-7

(a)

L7519-29

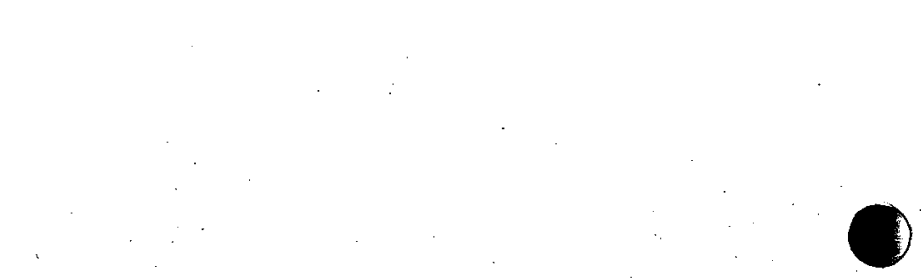




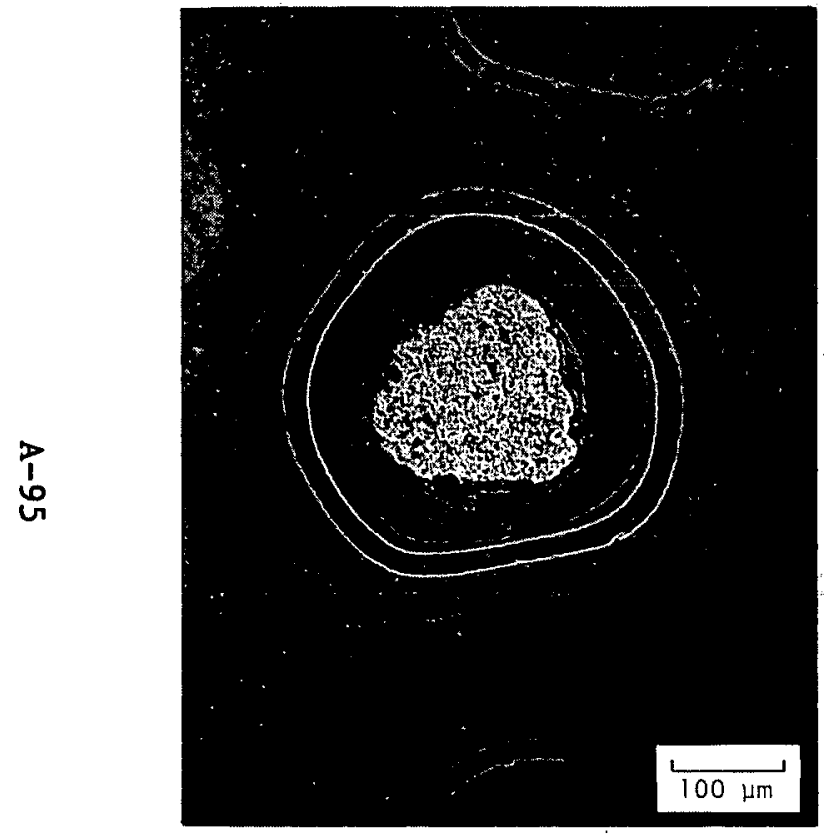

L7519-33 (a)

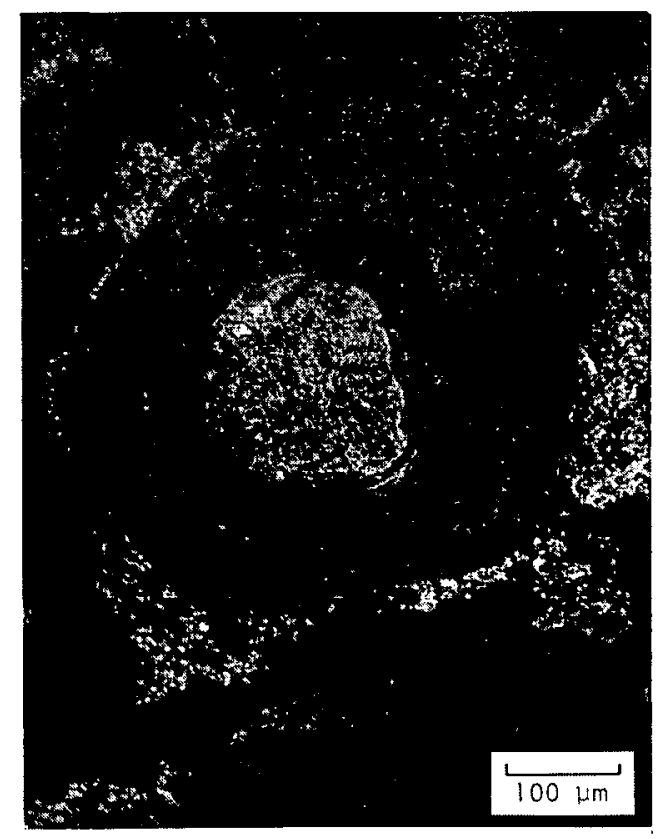

L7519-34

(b)

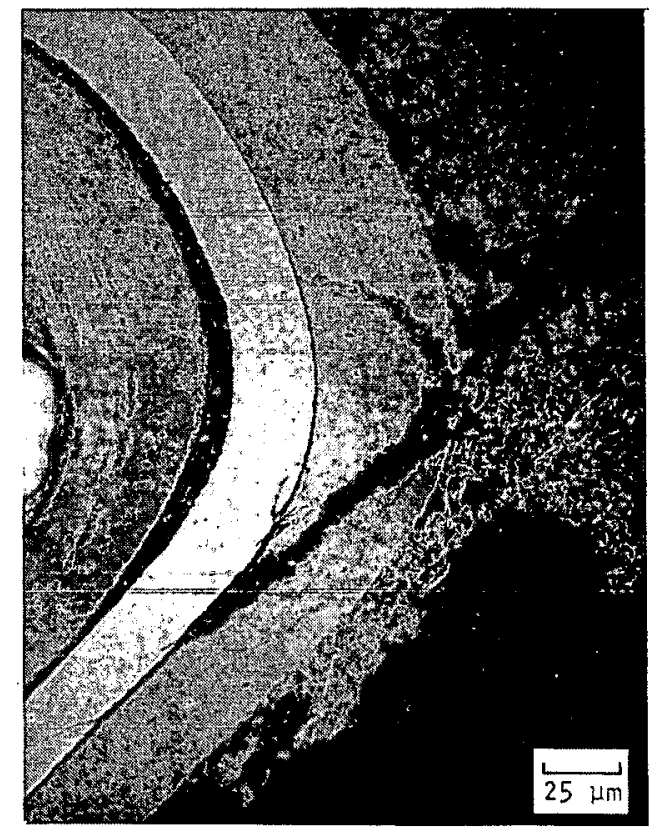

L7519-35

(c)

Fig. 4-72. FTE-6 photomicrographs of (Th,U) $C_{2}$ TRISO particle from fuel rod 2-3-7 showing matrix-particle interaction 


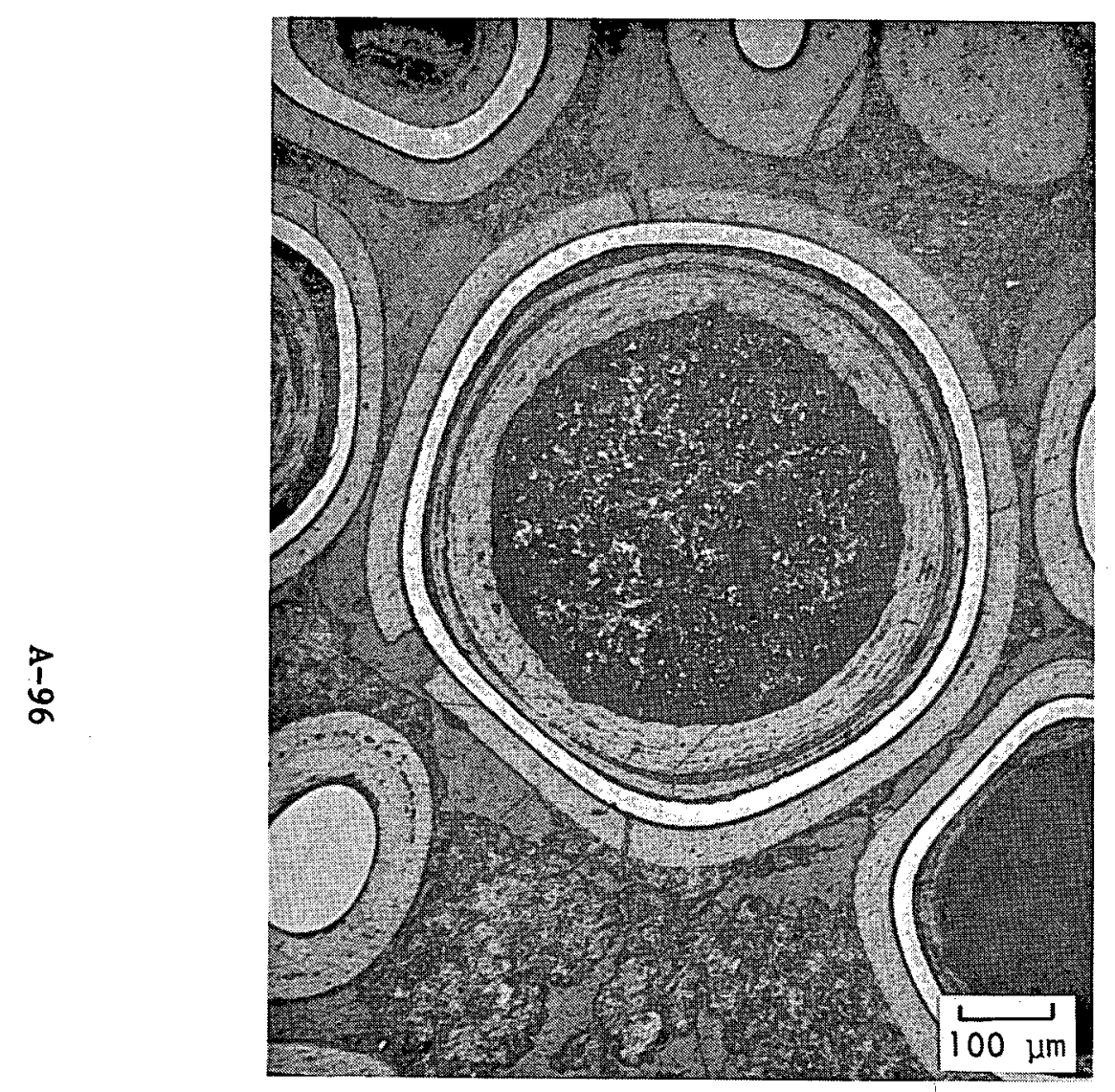

L7519-95

(a)

Fig. 4-73. FTE photomicrographs of $\mathrm{ThC}_{2}$ TRISO particle in fuel rod 2-6-7

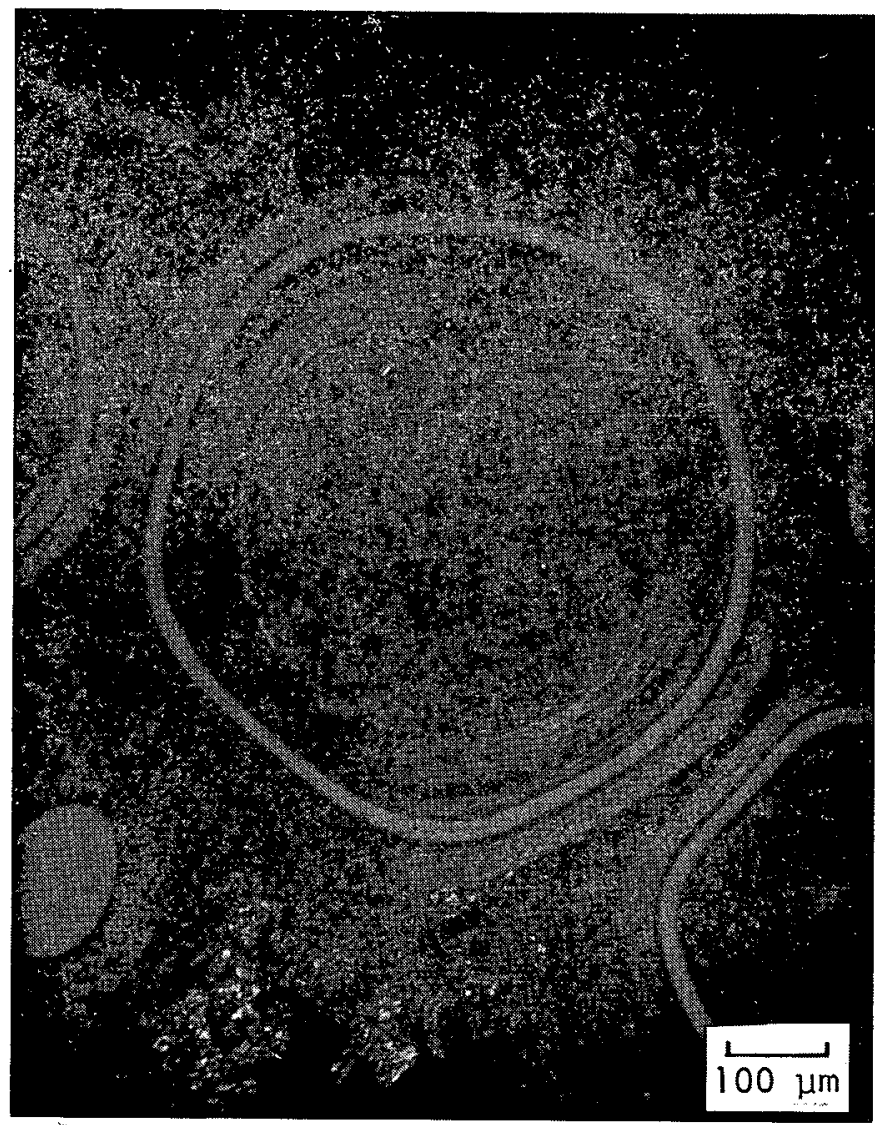

L7519-96

(b) 


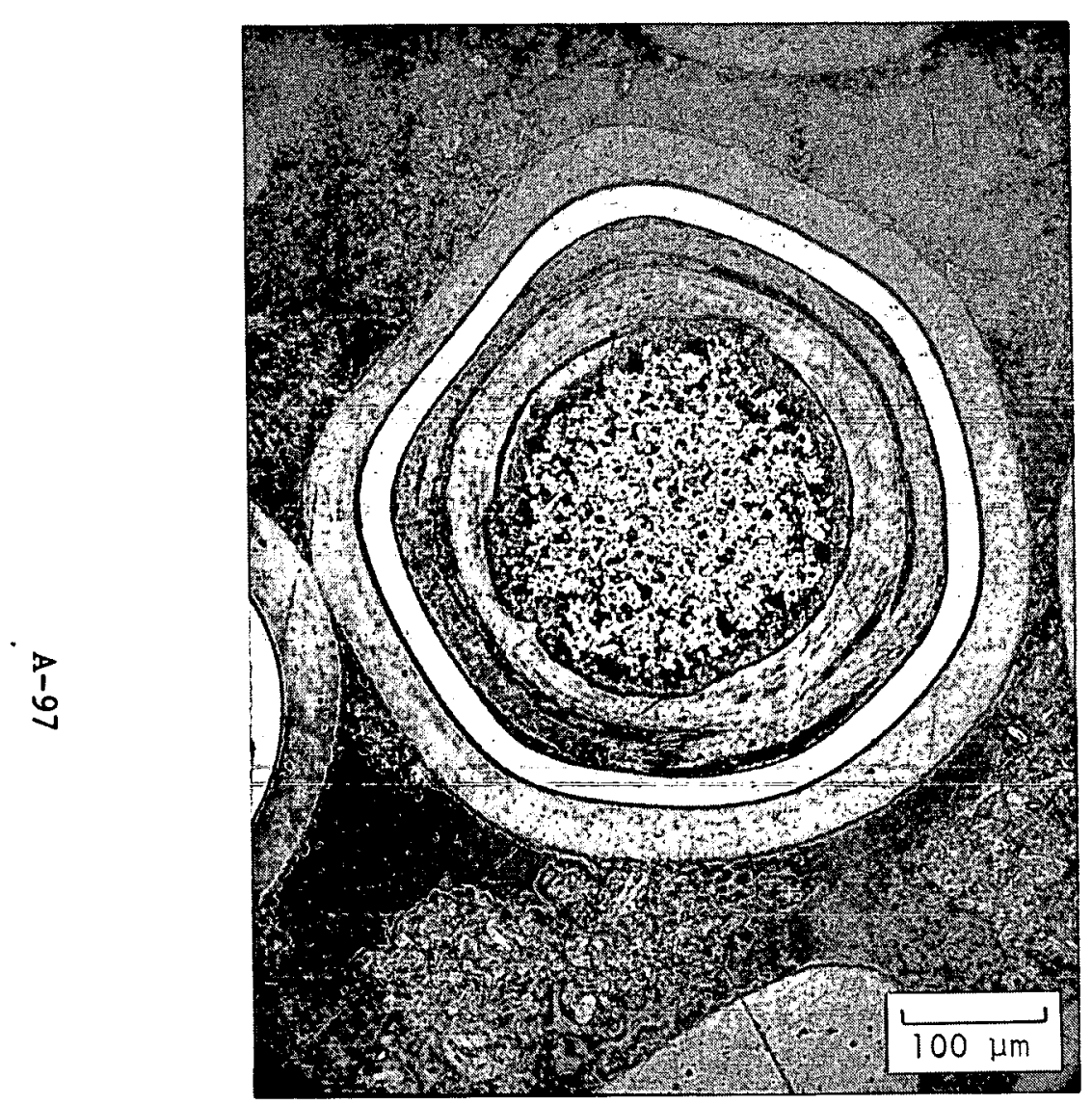

L7519-92

(a)

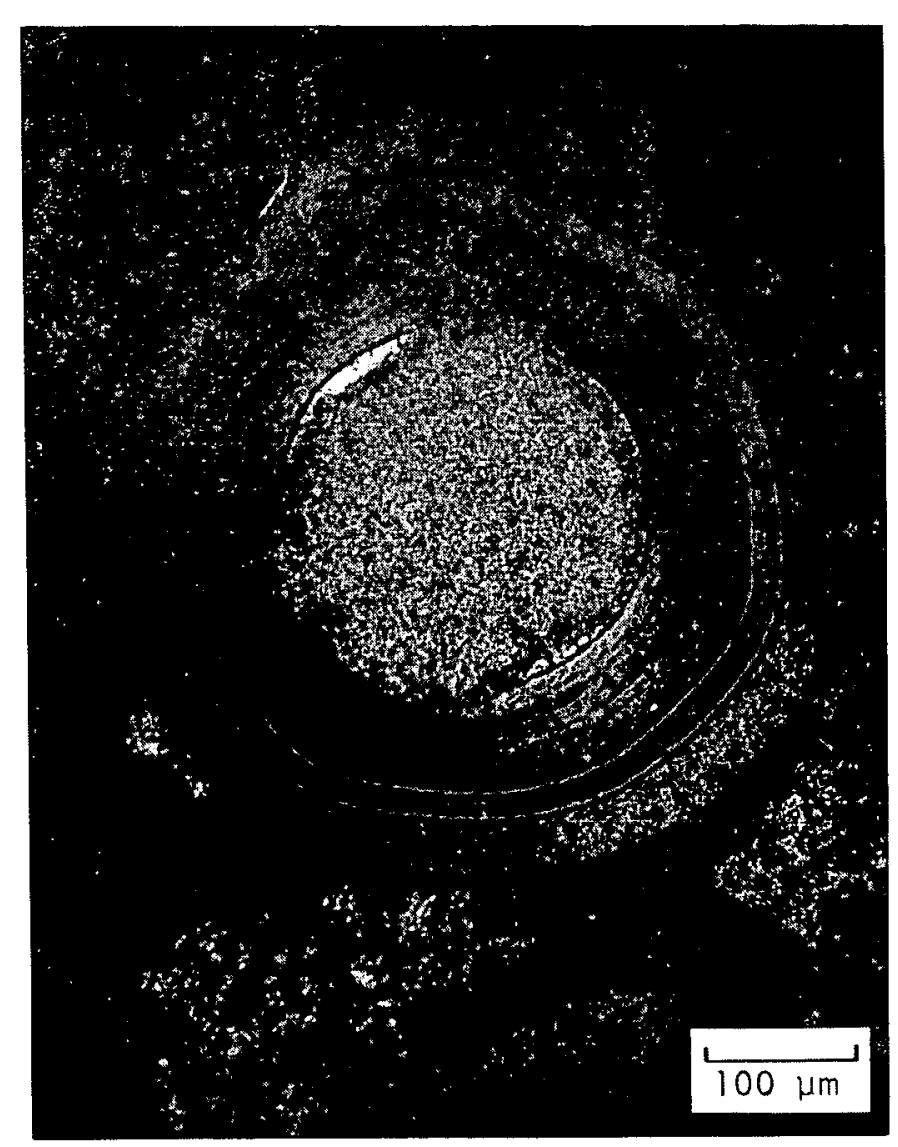

L7519-92A

(b)

Fig. 4-74. FTE-6 photomicrographs of representative fissile (Th,U) ${ }_{2}$ particle from fuel rod 2-6-7 


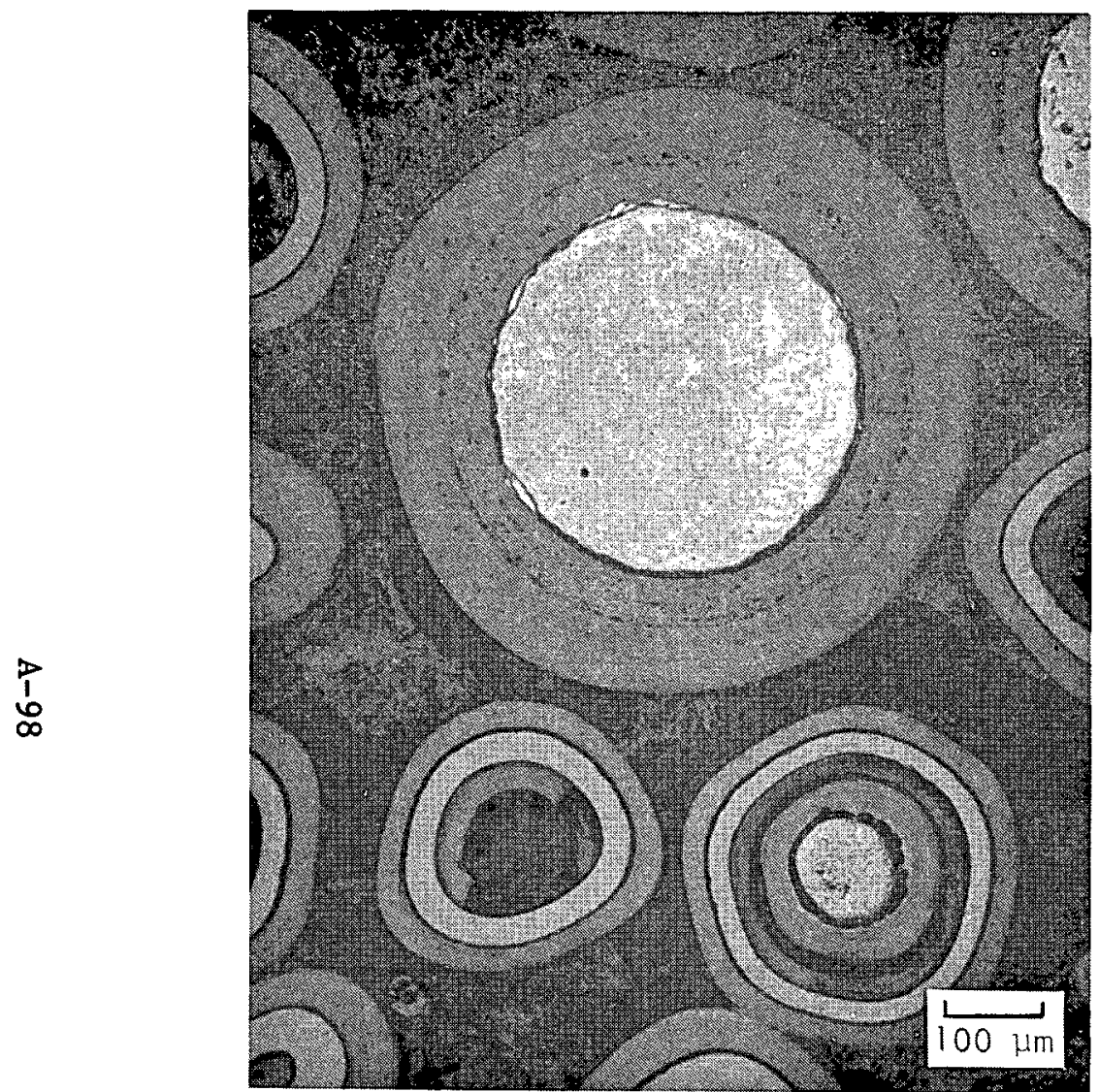

L7519-17

(a)

Fig. 4-75. FTE-6 photomicrographs of representative fuel particles from fuel rod 2-8-7

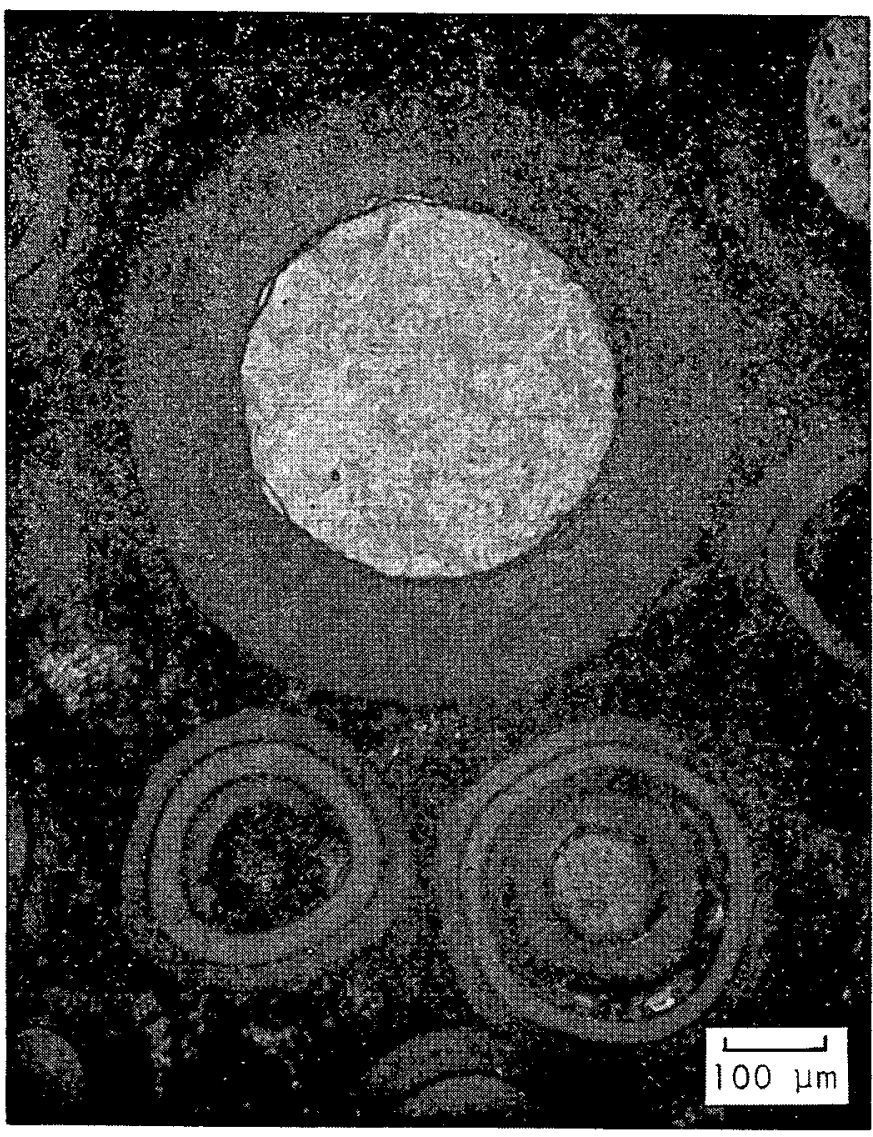

L7519-18

(b) 


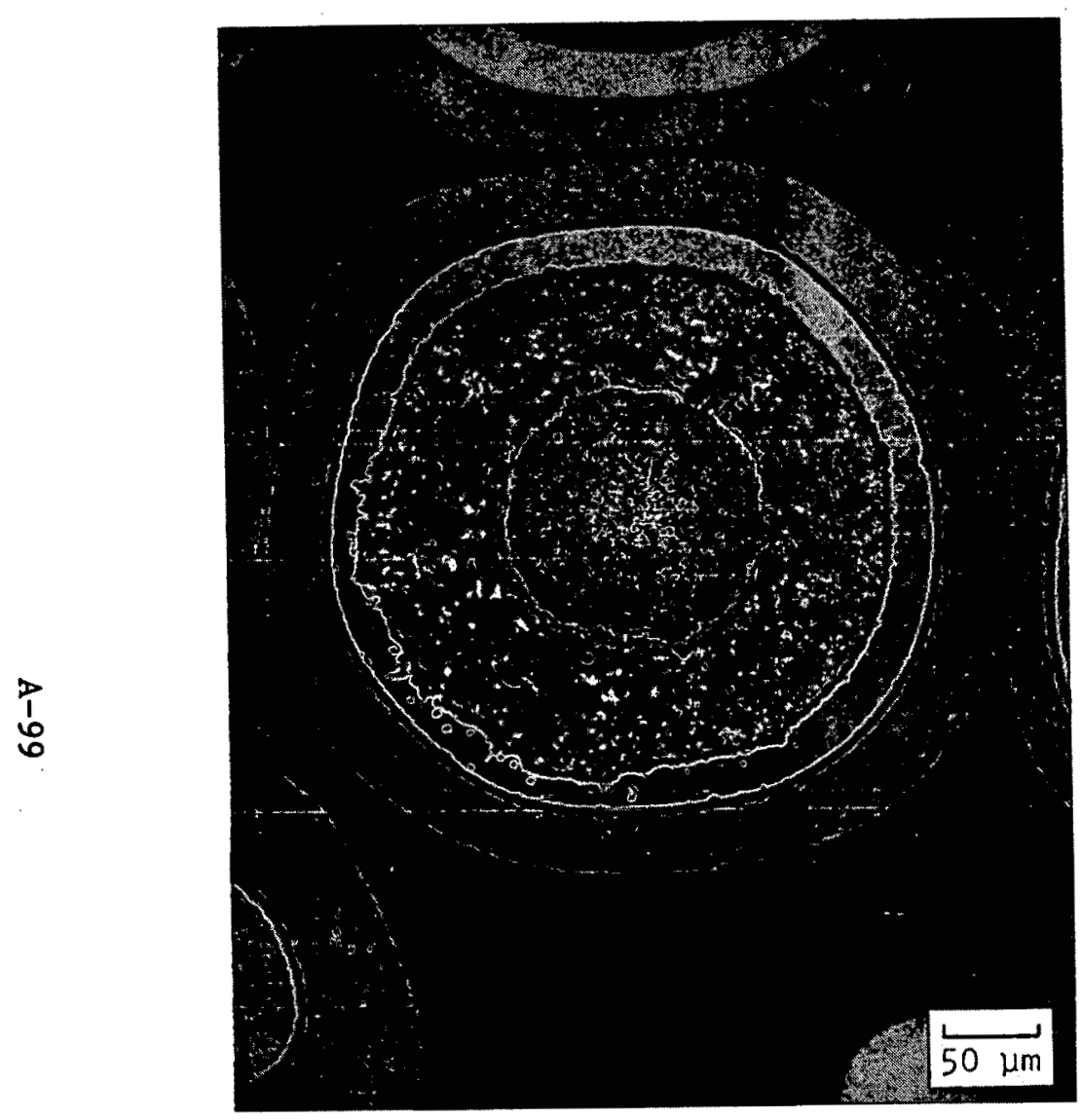

L7519-5

(a)

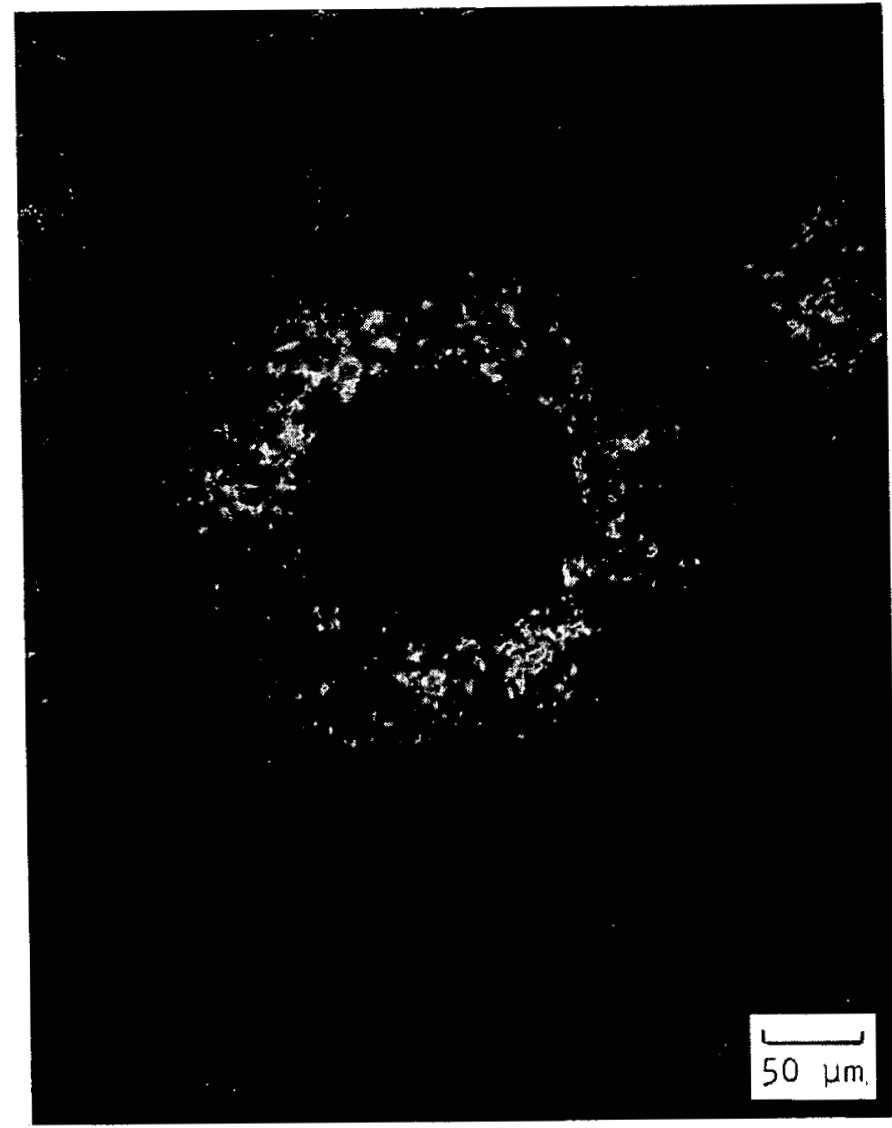

L7519-6

(b)

Fig. 4-76. FTE-6 photomicrograph of UC TRISO particle from fuel rod 2-8-7 showing apparent contamination attack 


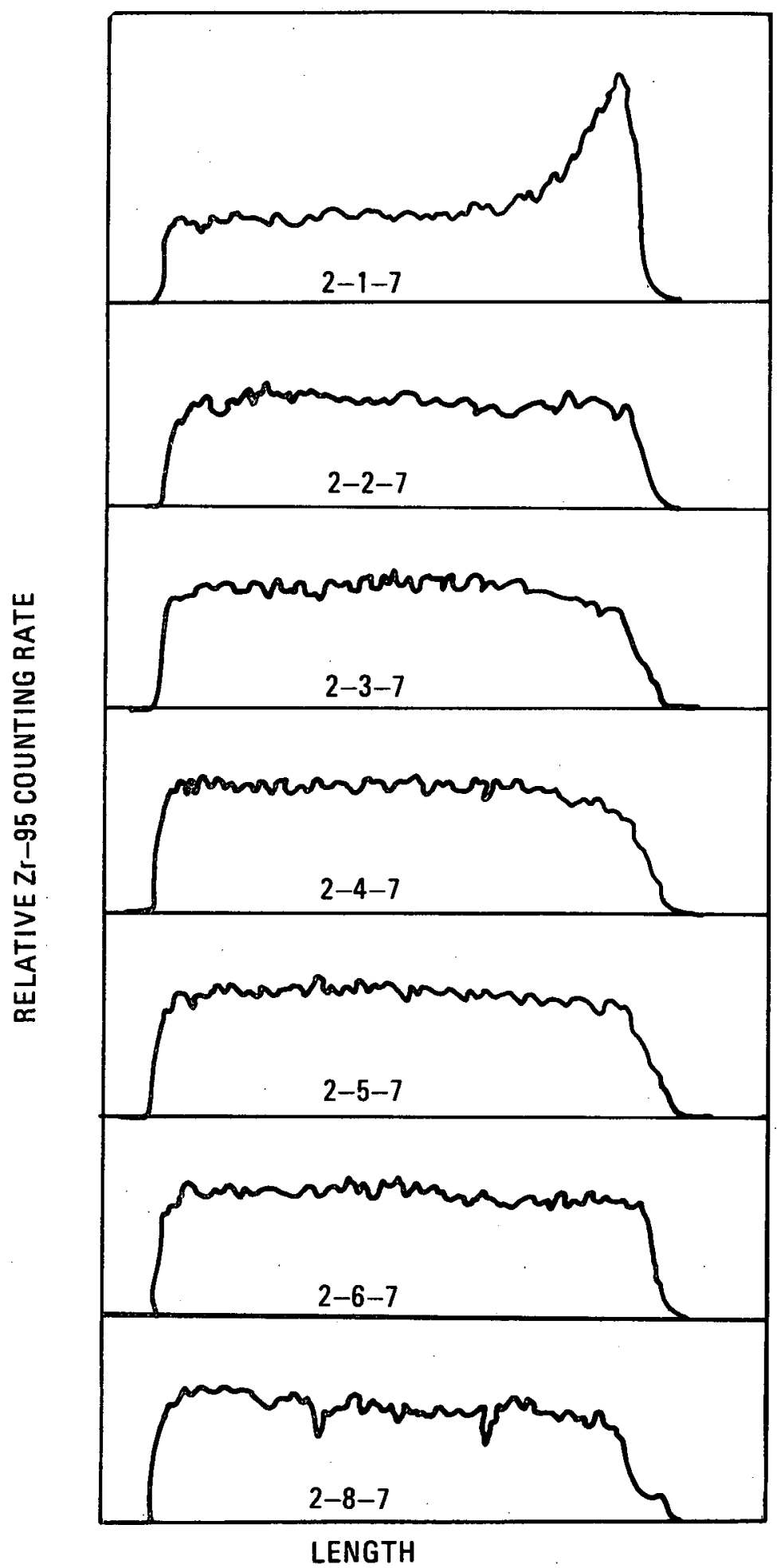

Fig. 4-77. FTE-6 single-channel gamma scans of fuel rods used in fuel examination 


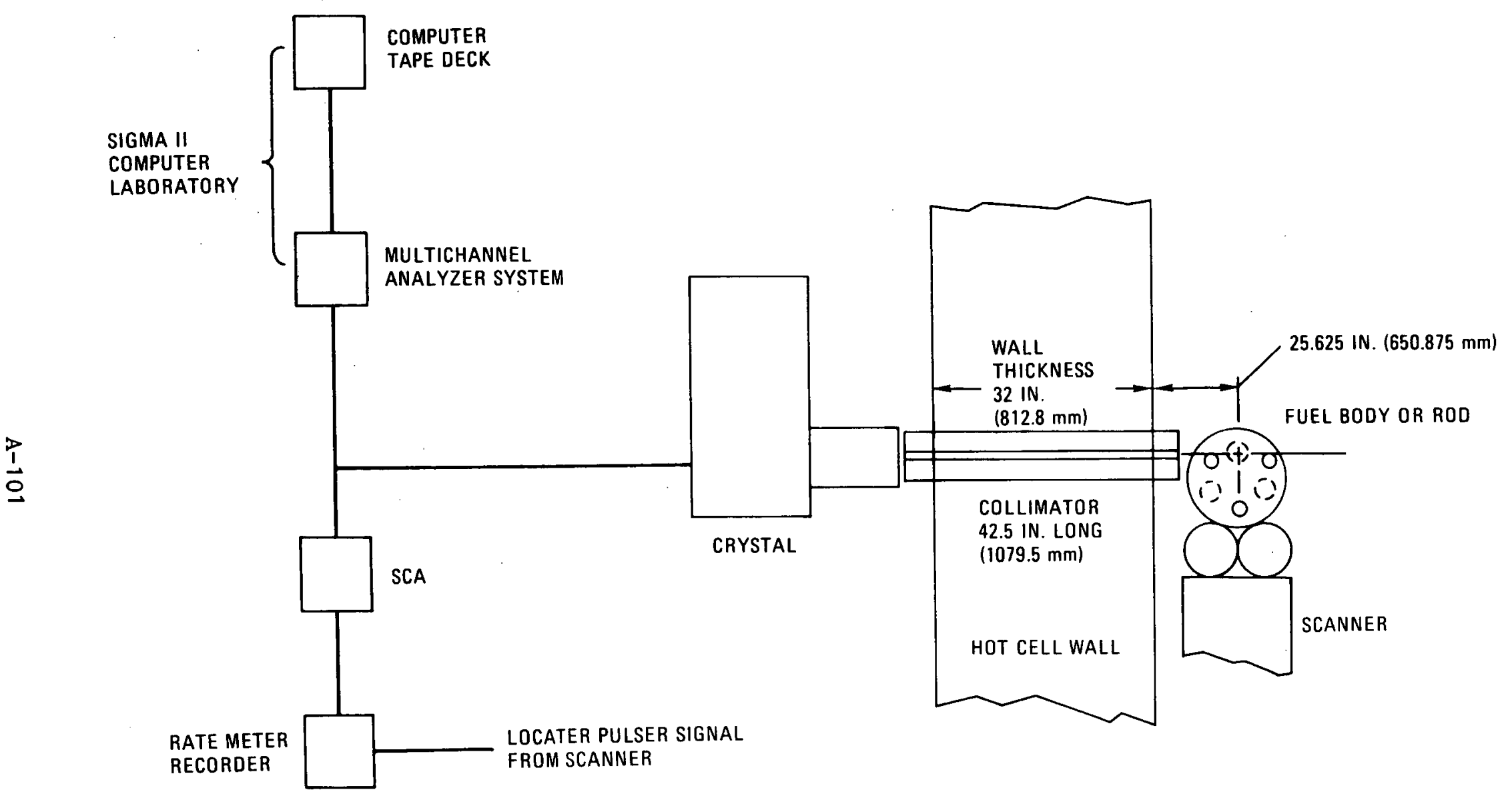

Fig. 4-78. Schematic of gamma scanning apparatus at General Atomic hot cell 

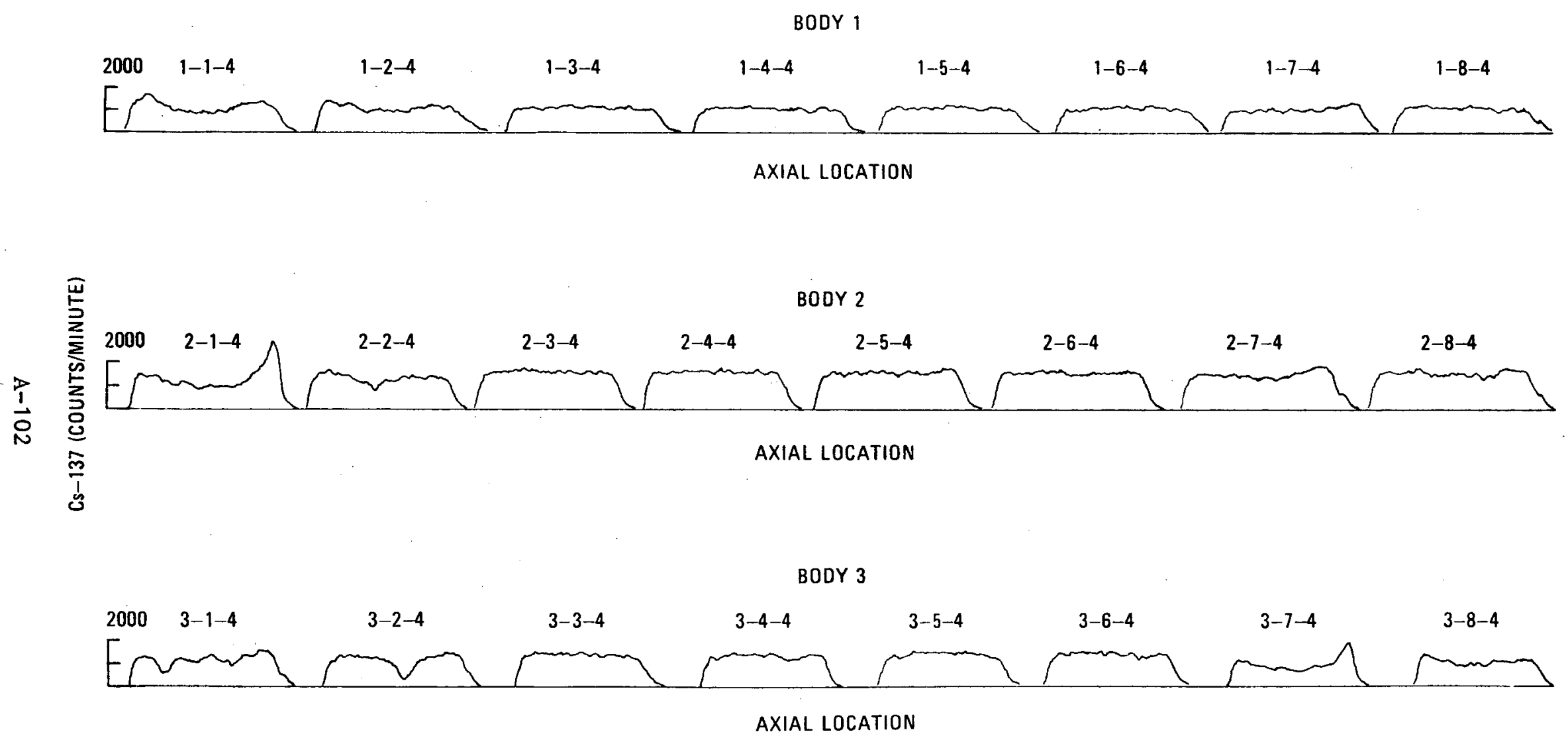

Fig. 4-79. FTE-6 single-channel gamma scan comparison of fuel rods 


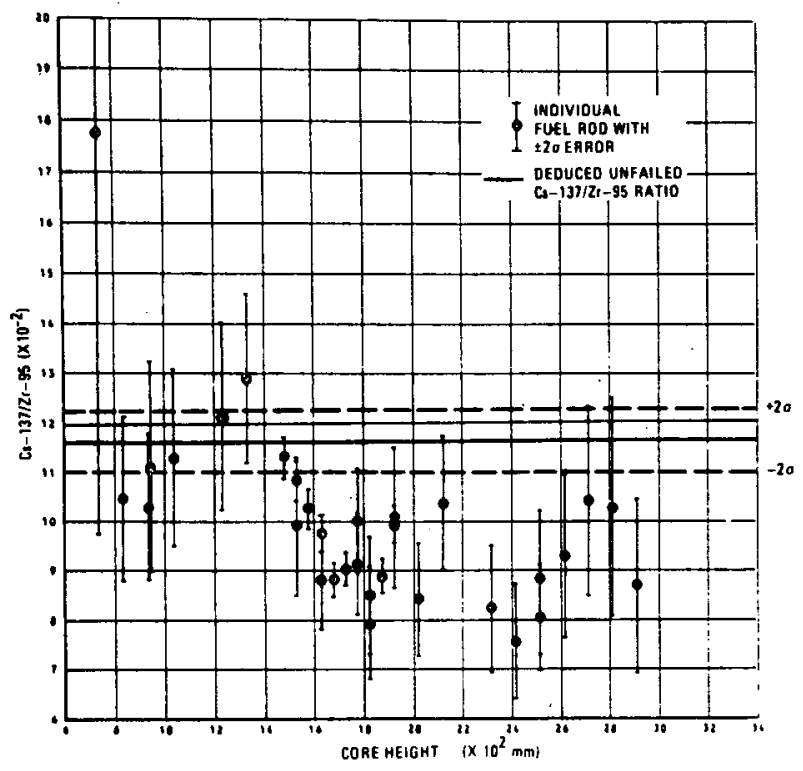

Fig. 4-80. FTE-6 Cs-137/Zr-95 ratio for fuel rods in stacks 1 and 2

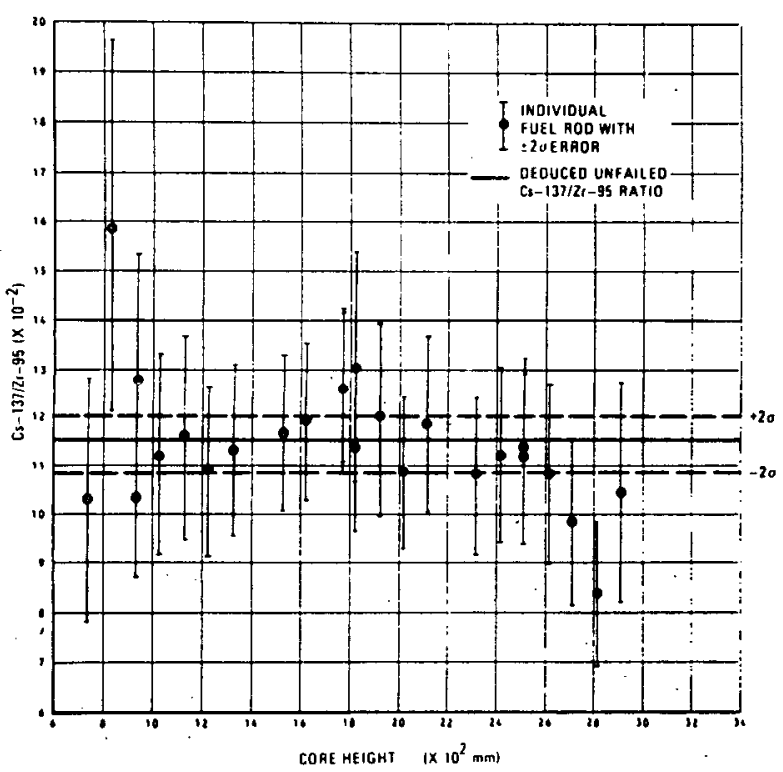

Fig. 4-82. FTE-6 Cs-136/Zr-95 ratio for fuel rods in stacks 5 and 6

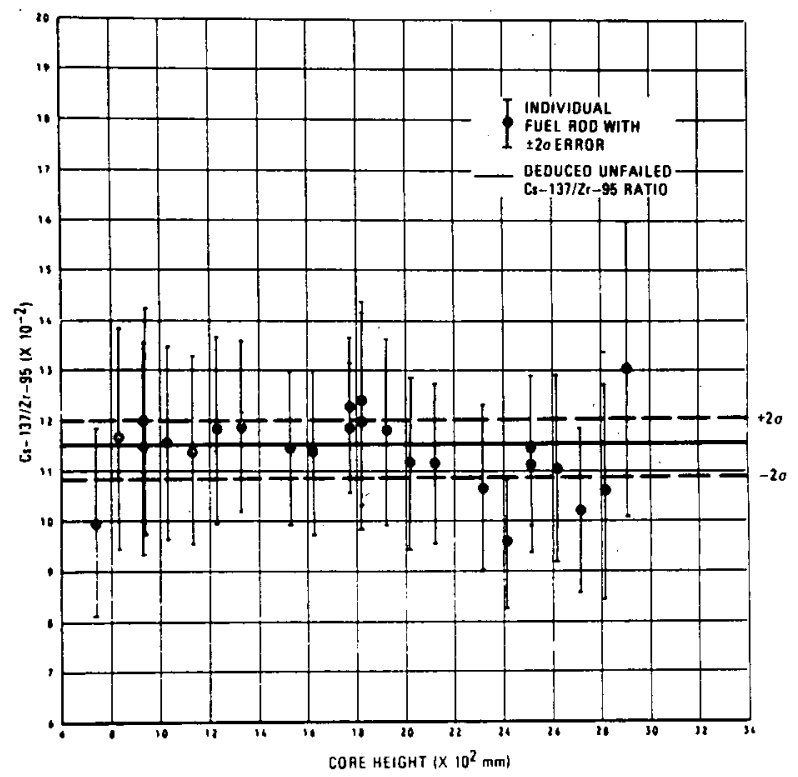

Fig. 4-81. FTE-6 Cs-136/Zr-95 ratio for fuel rods in stacks 3 and 4

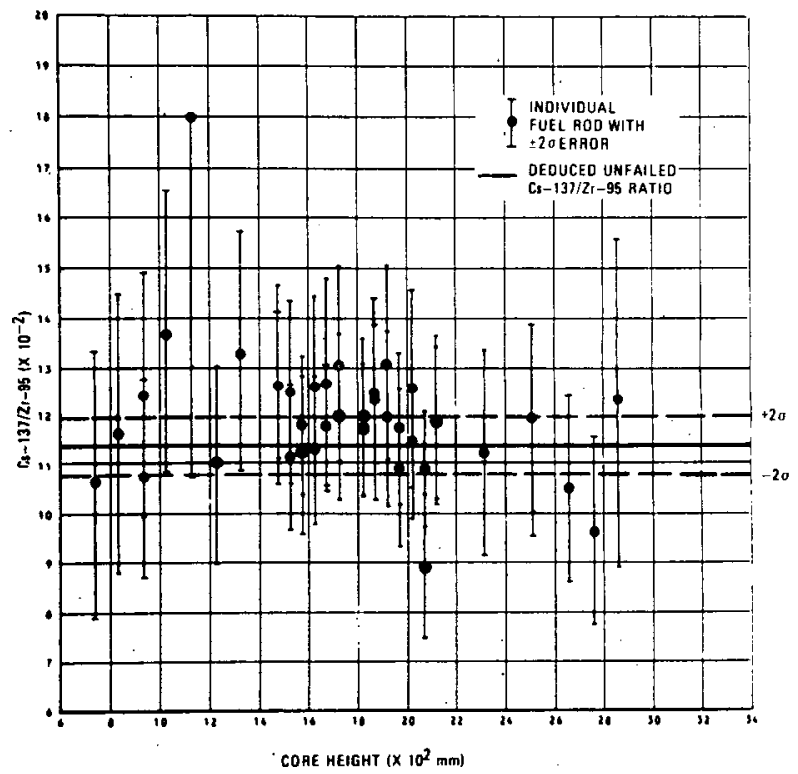

Fig. 4-83. FTE-6 Cs-136/2r-95 ratio for fuel rods in stacks 7 and 8 


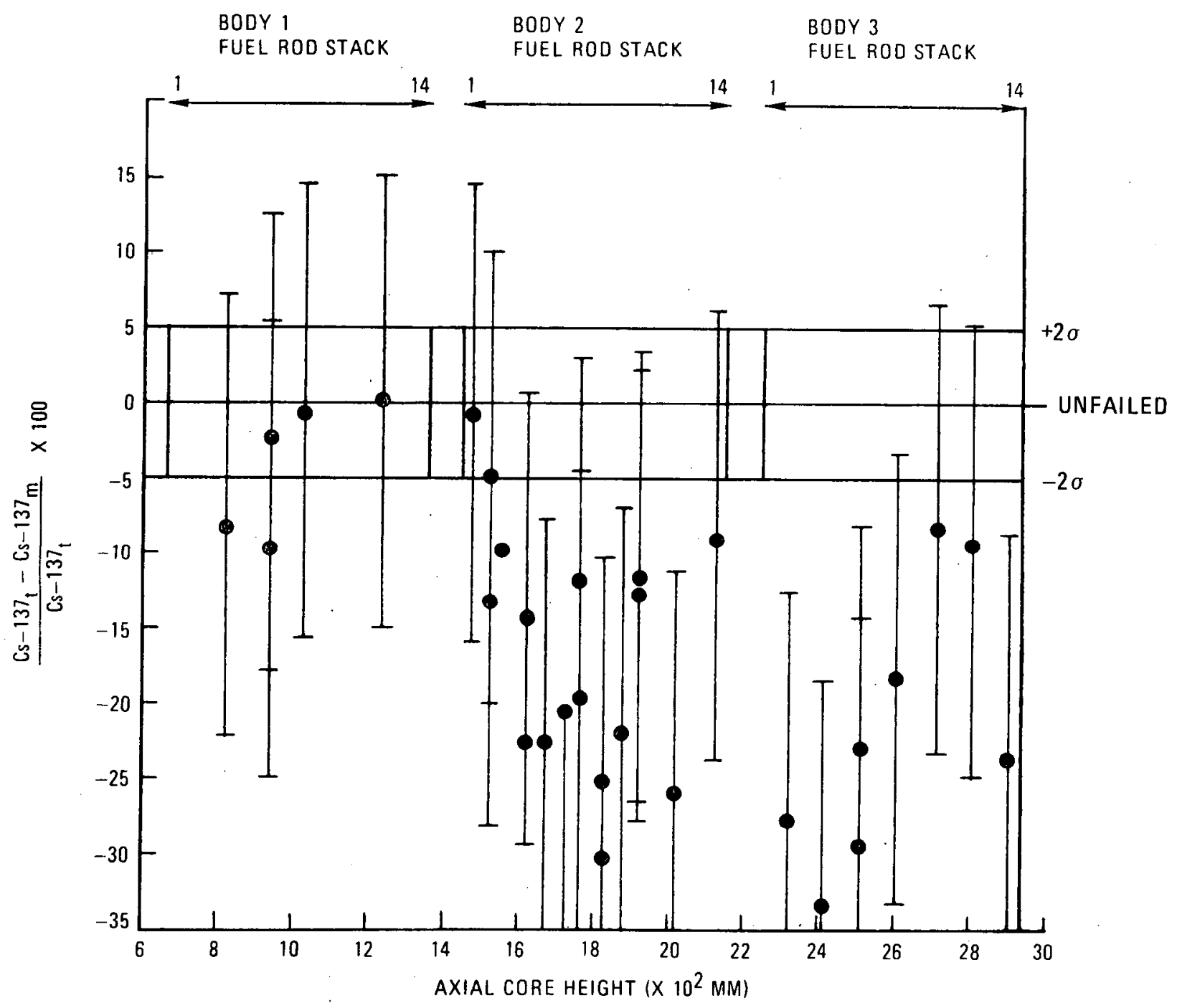

Fig. 4-84. FTE-6 Cs-137 loss from fuel rods in stacks 1 and 2 


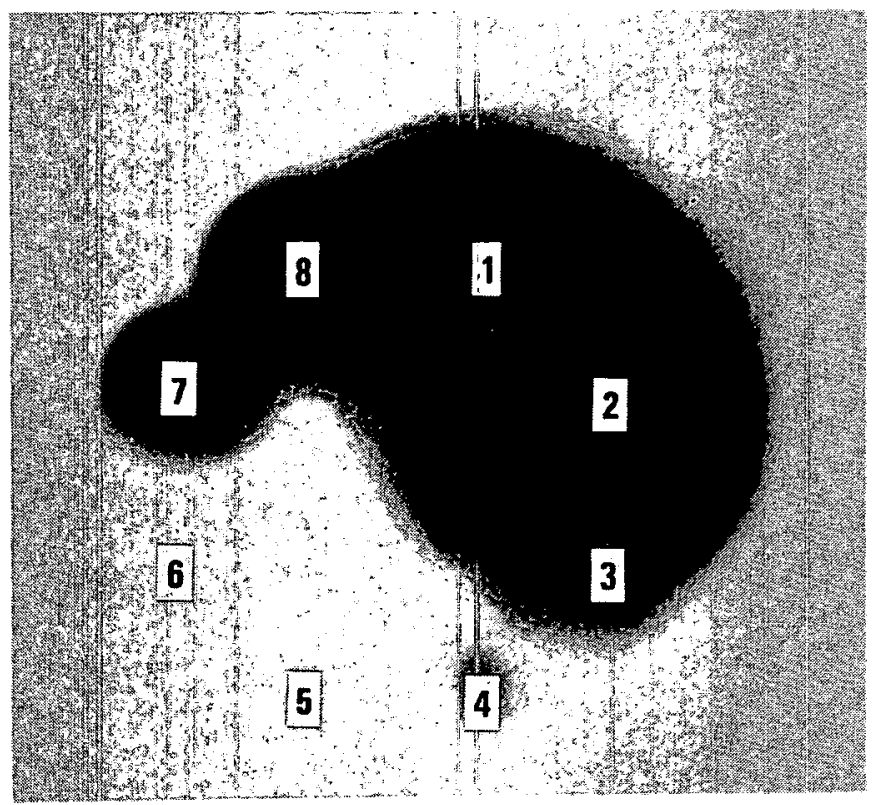

Fig. 4-85. FTE-6 autoradiography of graphite fuel body number 2 slice 


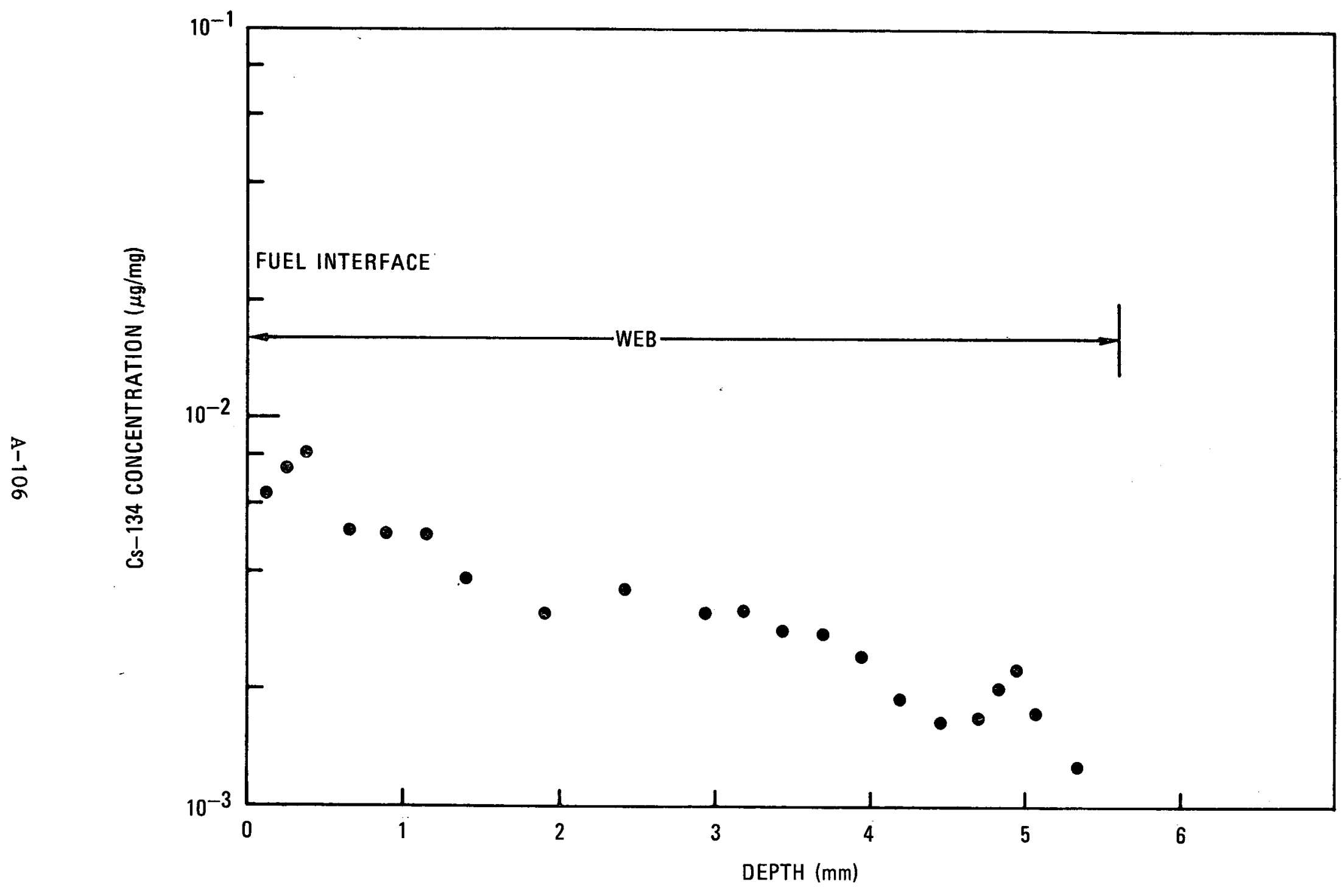

Fig. 4-86. FTE-6 Cs-134 (605 keV) profile in outer web of fuel body 2 


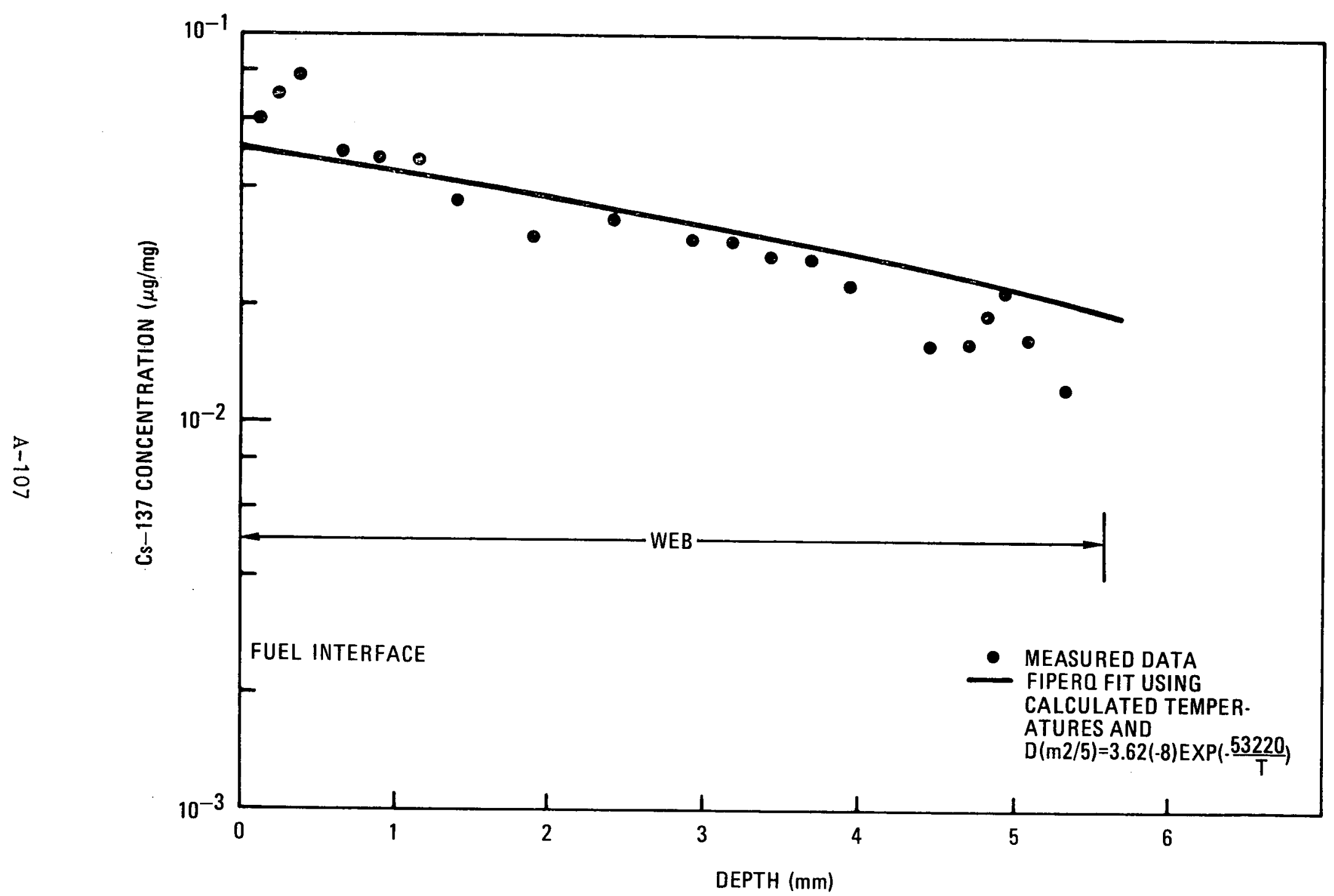

Fig. 4-87. FTE-6 Cs-137 (662 keV) profile in outer web of fuel body 2 


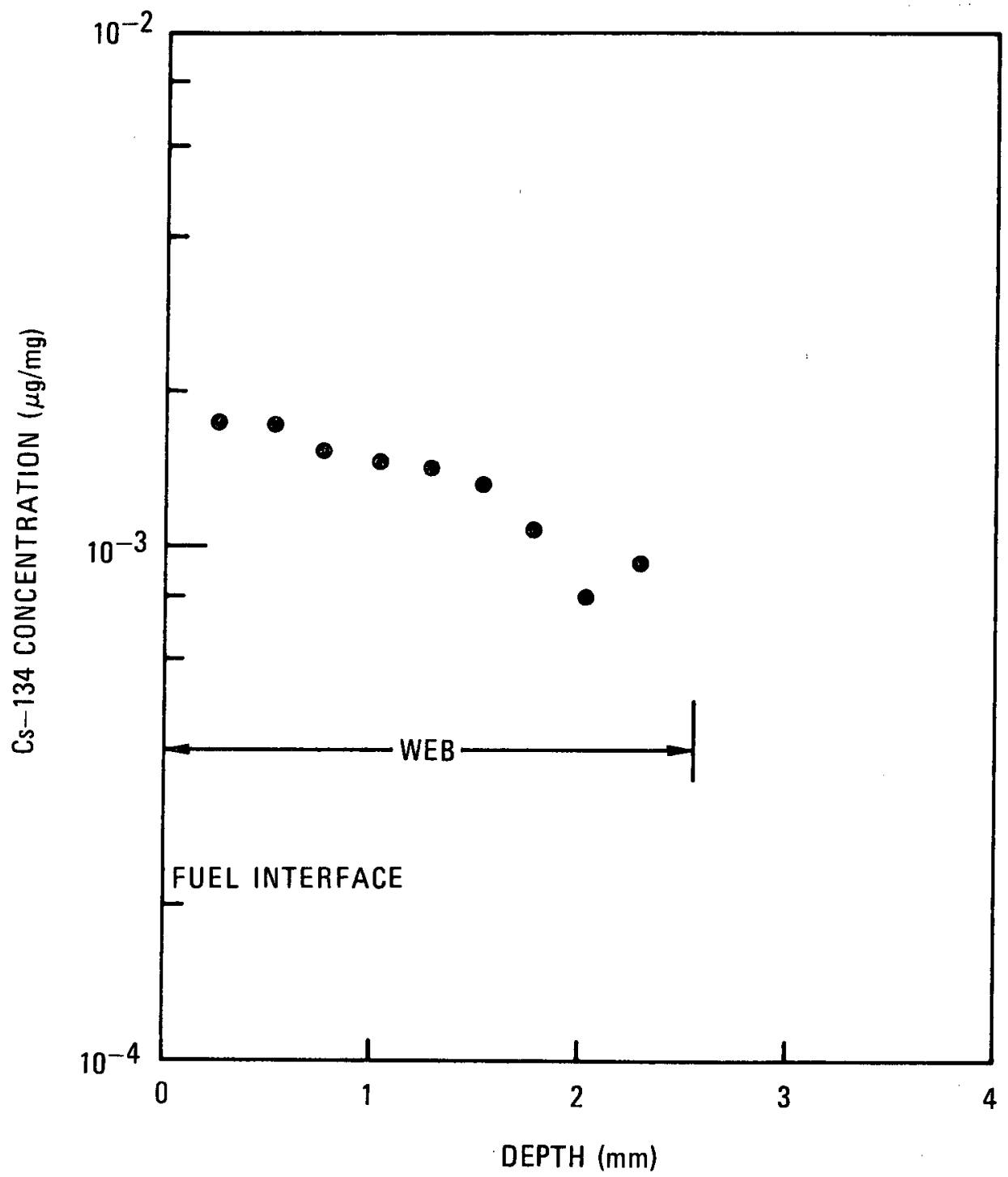

Fig. 4-88. FTE-6 Cs-134 (604 keV) profile in inner web of fuel body 2 


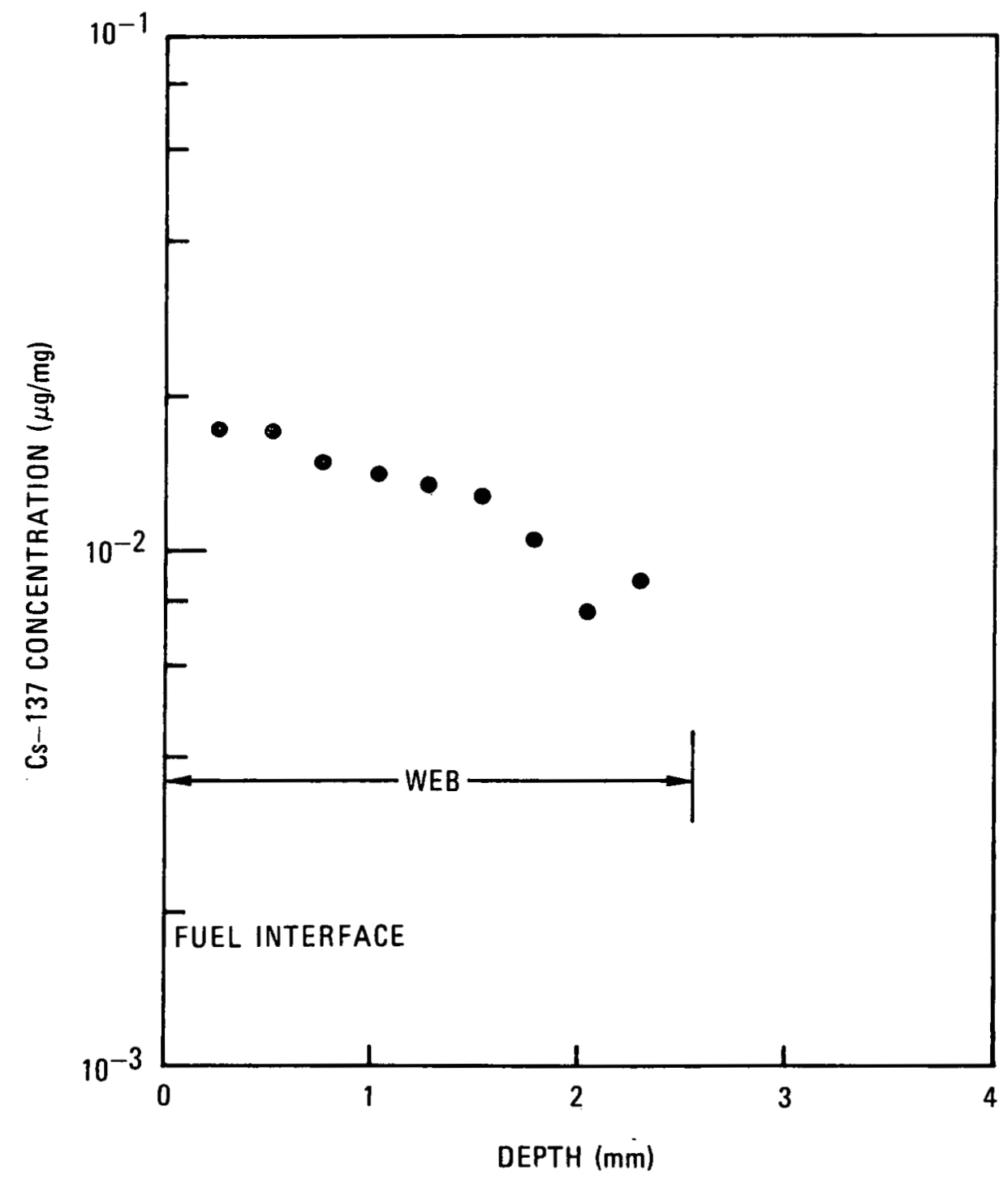

Fig. 4-89. FTE-6 Cs-137 (662 keV) profile in inner web of fuel body 2 


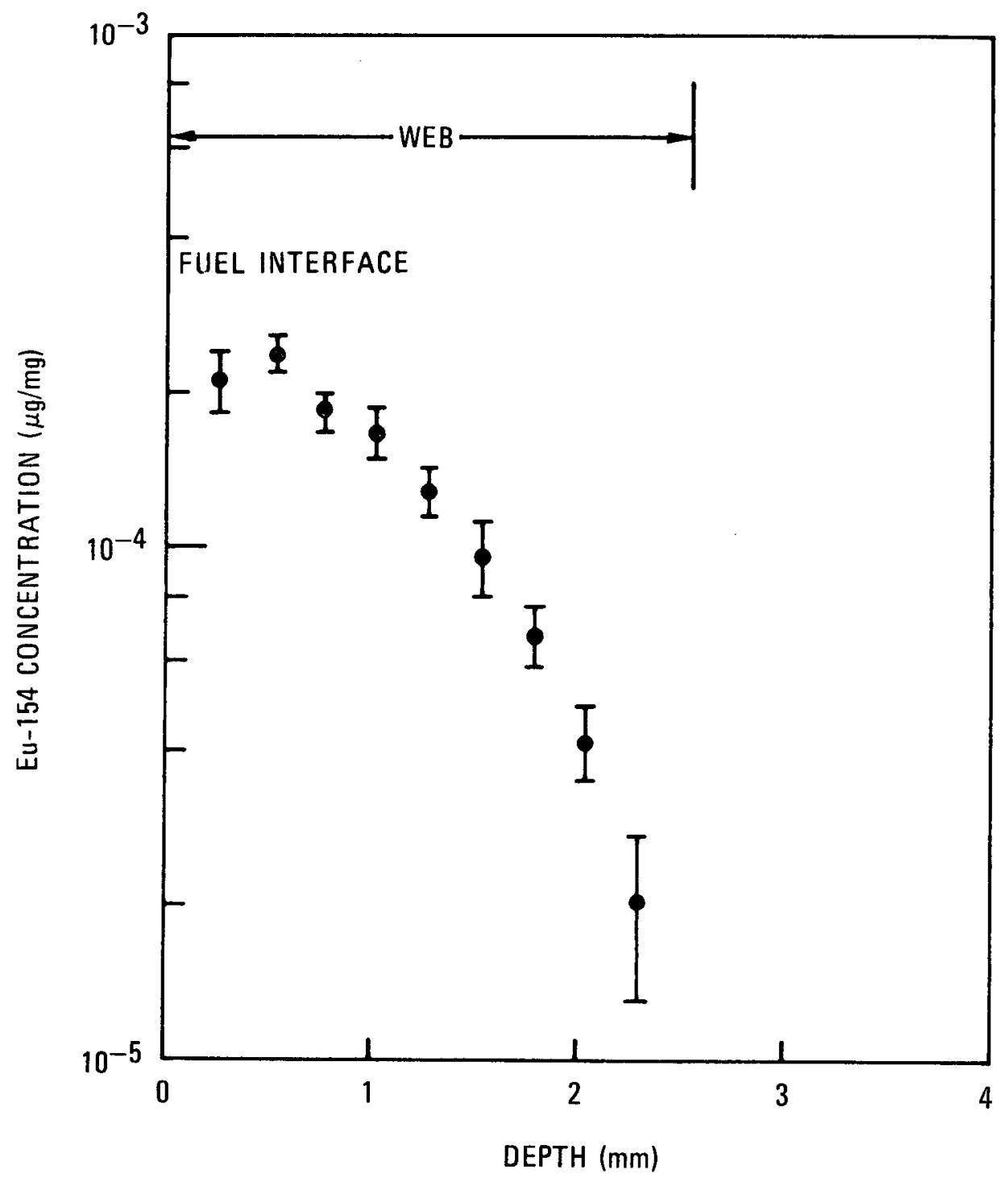

Fig. 4-90. FTE-6 Eu-154 (123 keV) profile in inner web of fuel body 2 


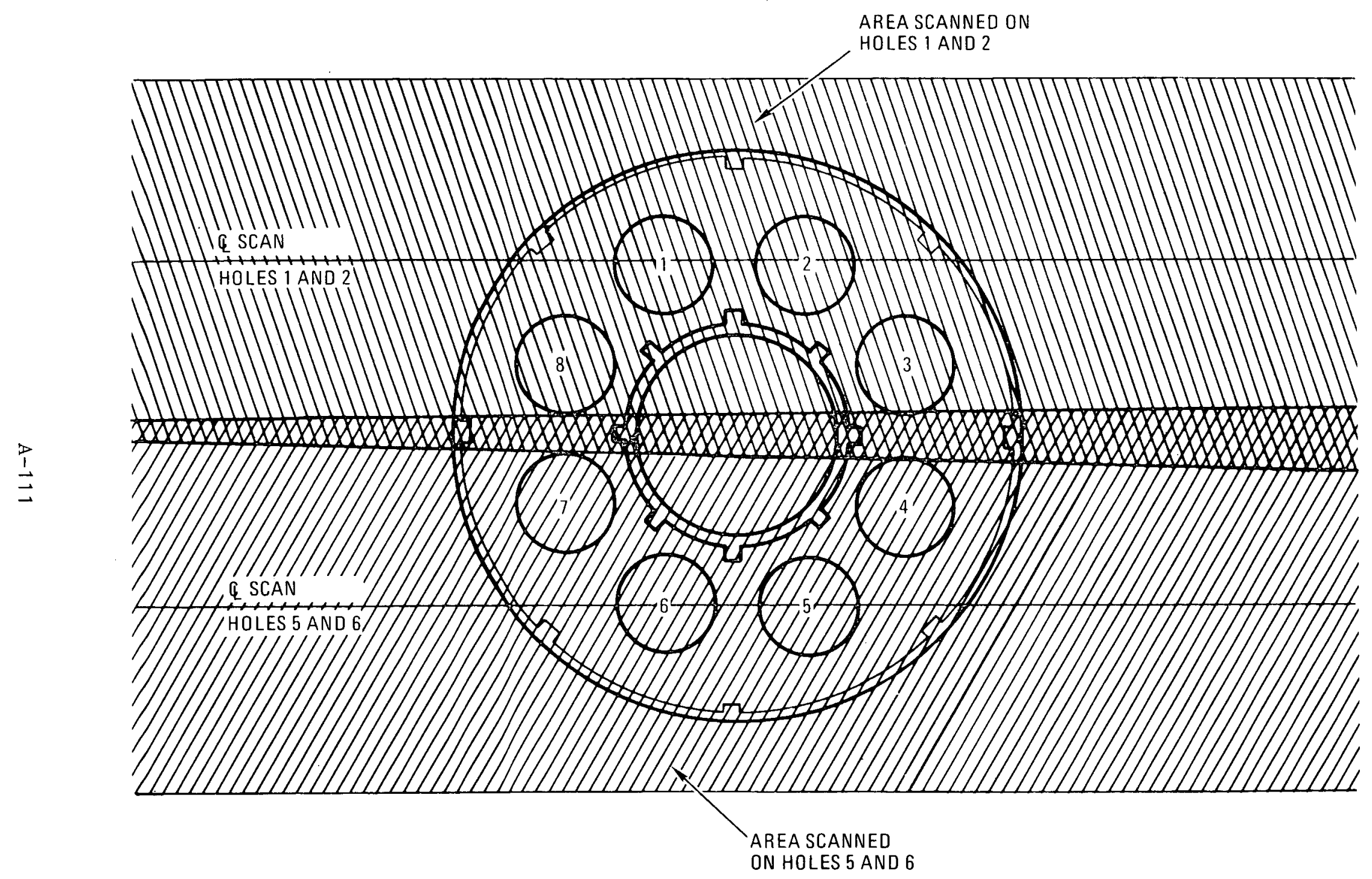

Fig. 4-91. FTE-6 graphite fuel body gamma-scanned areas 


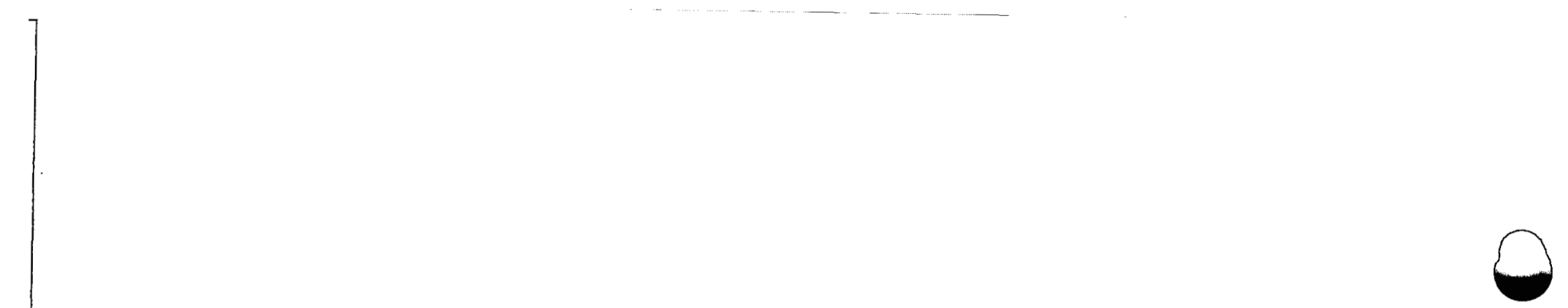

- 


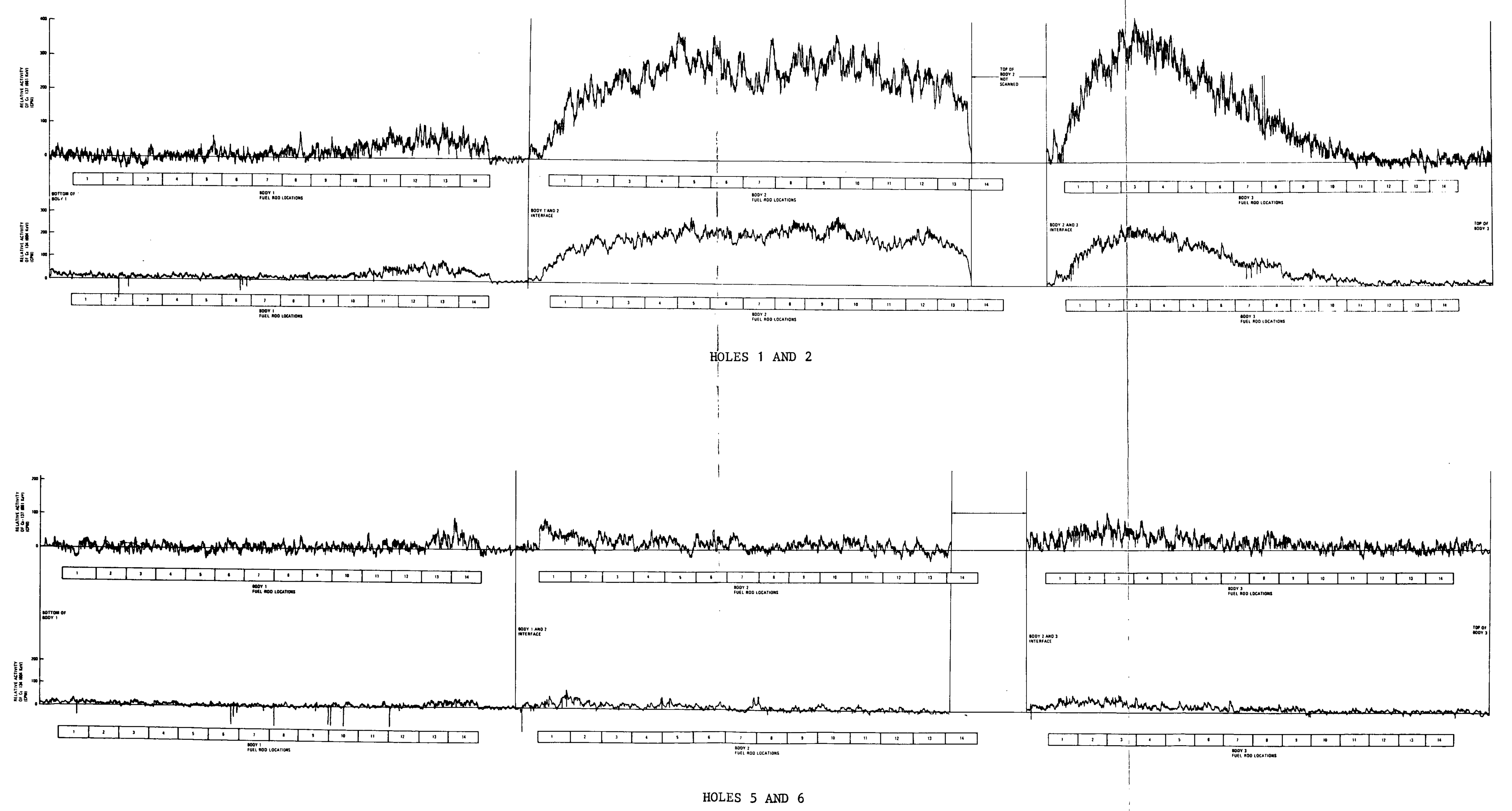

Fig. 4-92. FTE-6 fuel body singlechannel gamma scans of 


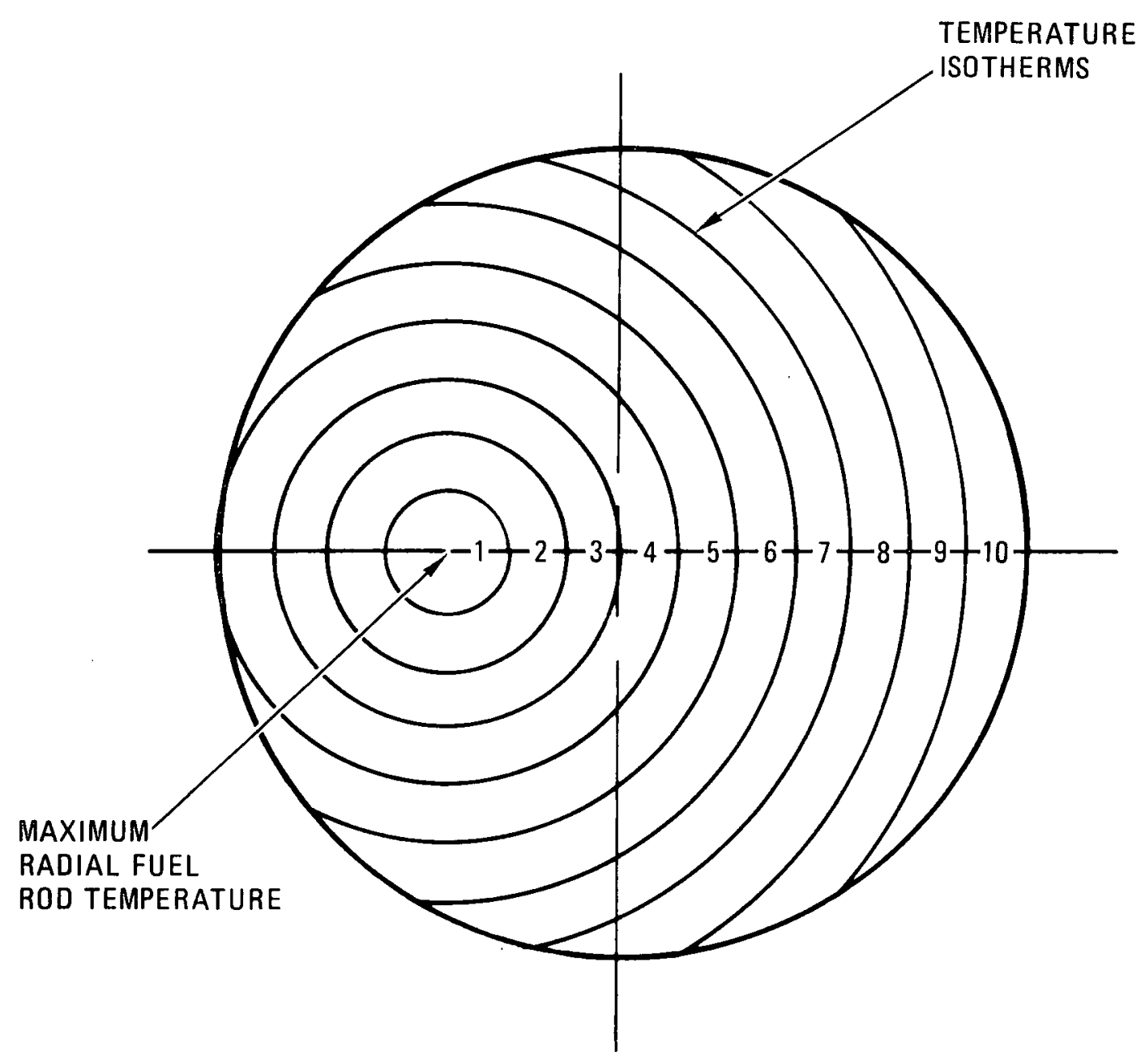

Fig. 4-93. TREVER fuel rod cross section of fuel kernel migration segments 

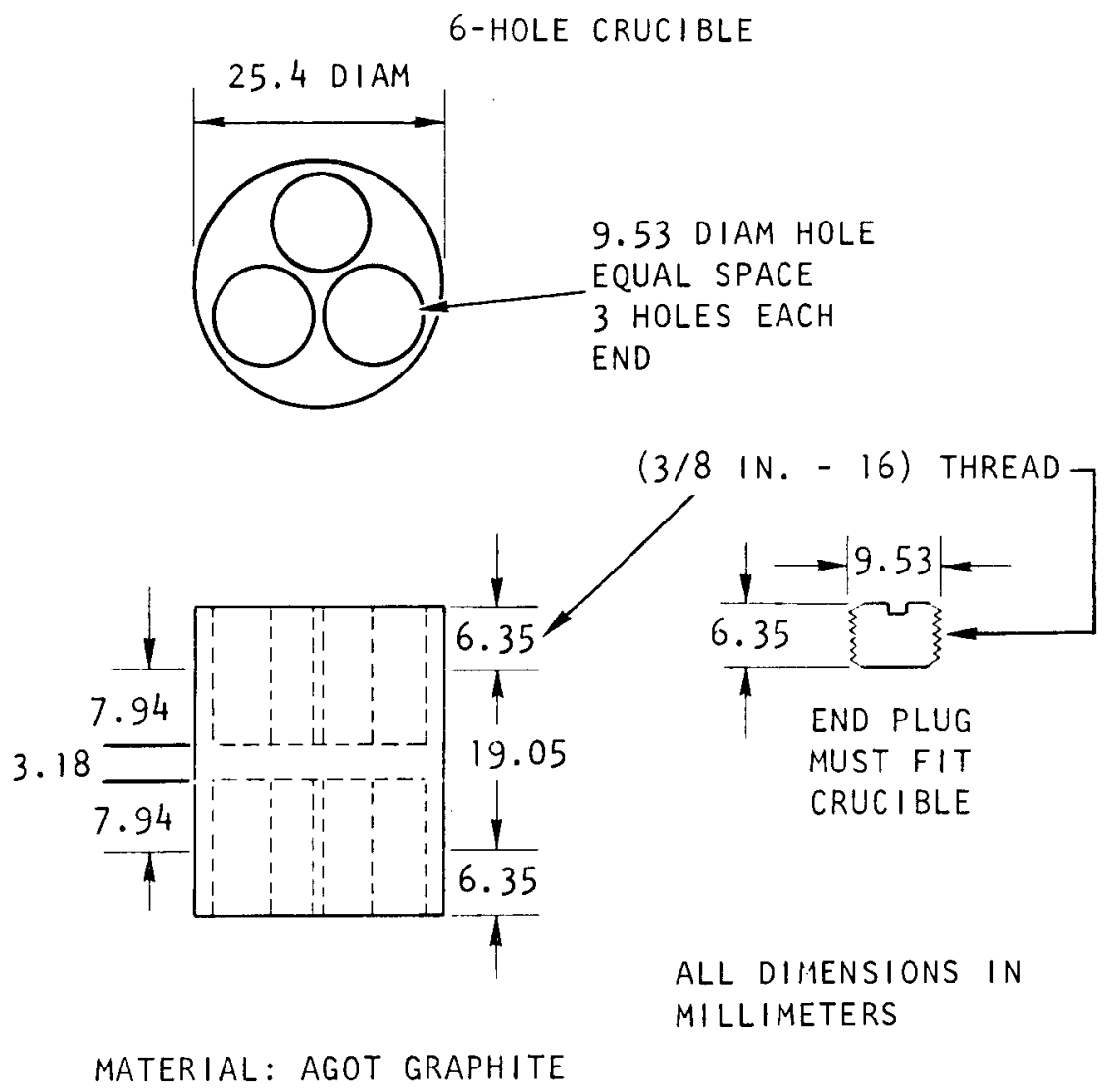

Fig. 4-94. Design of type II thermal stability crucibles 


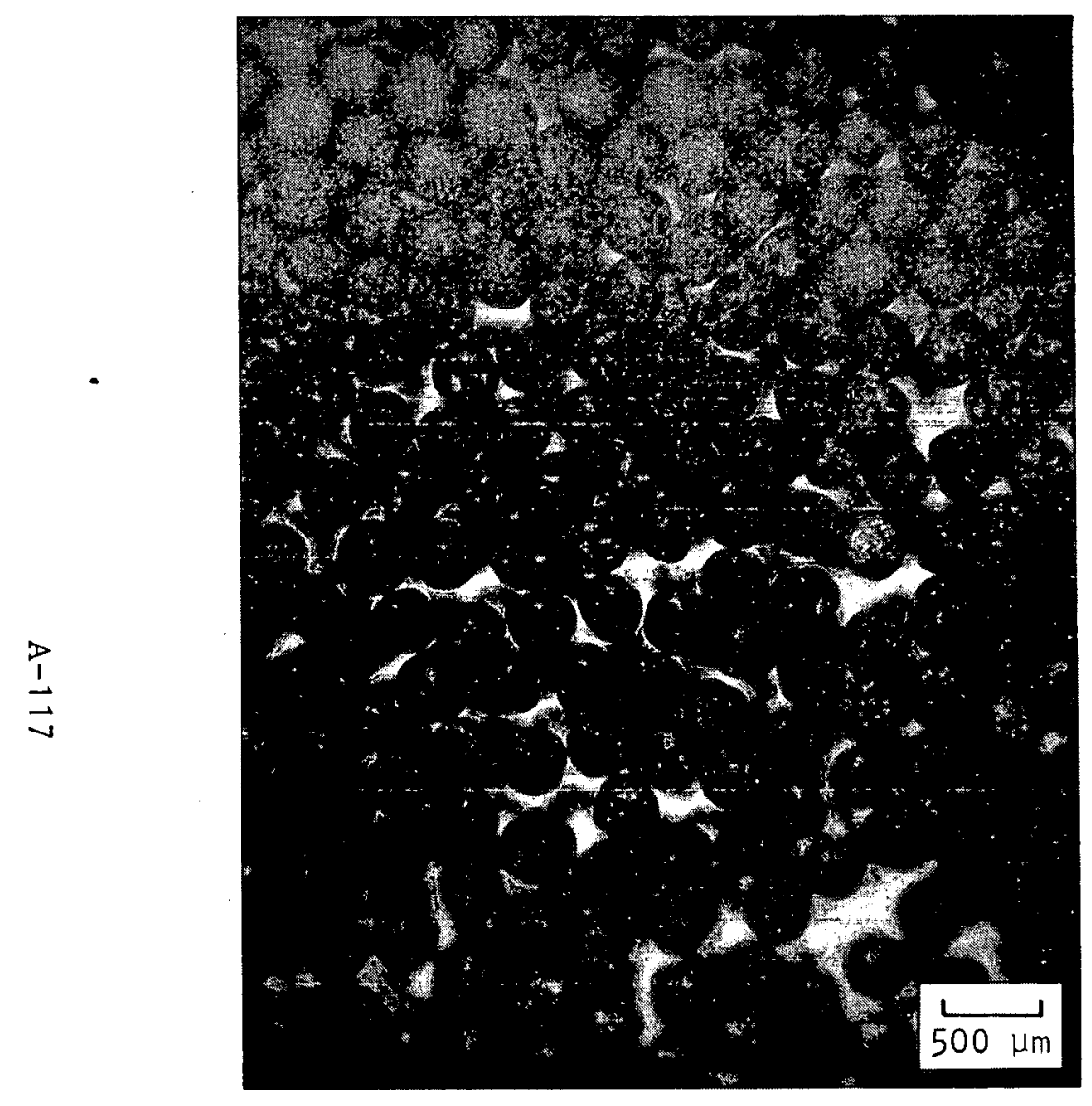

S7519-54

(a)

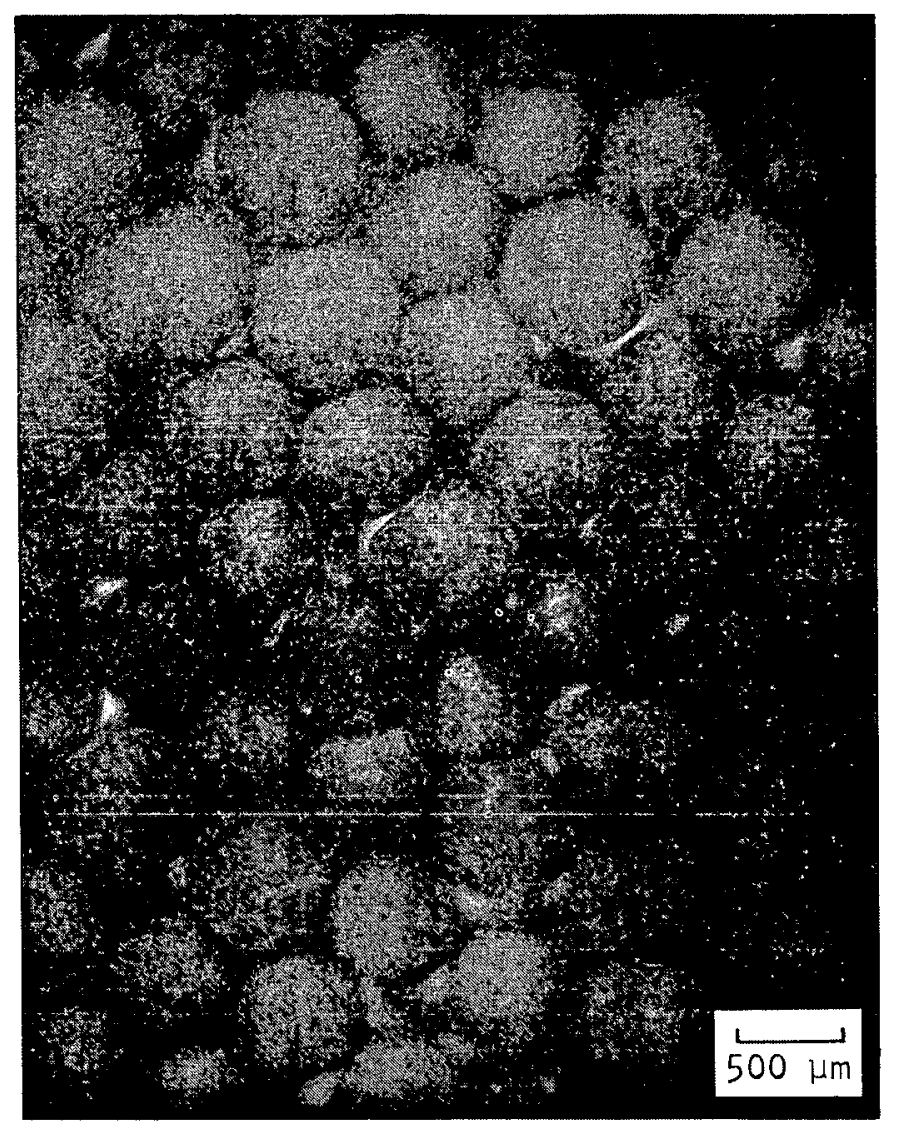

S7519-52

(b)

Fig. 4-95. FTE-6 stereophotographs of thermal stability spine samples 


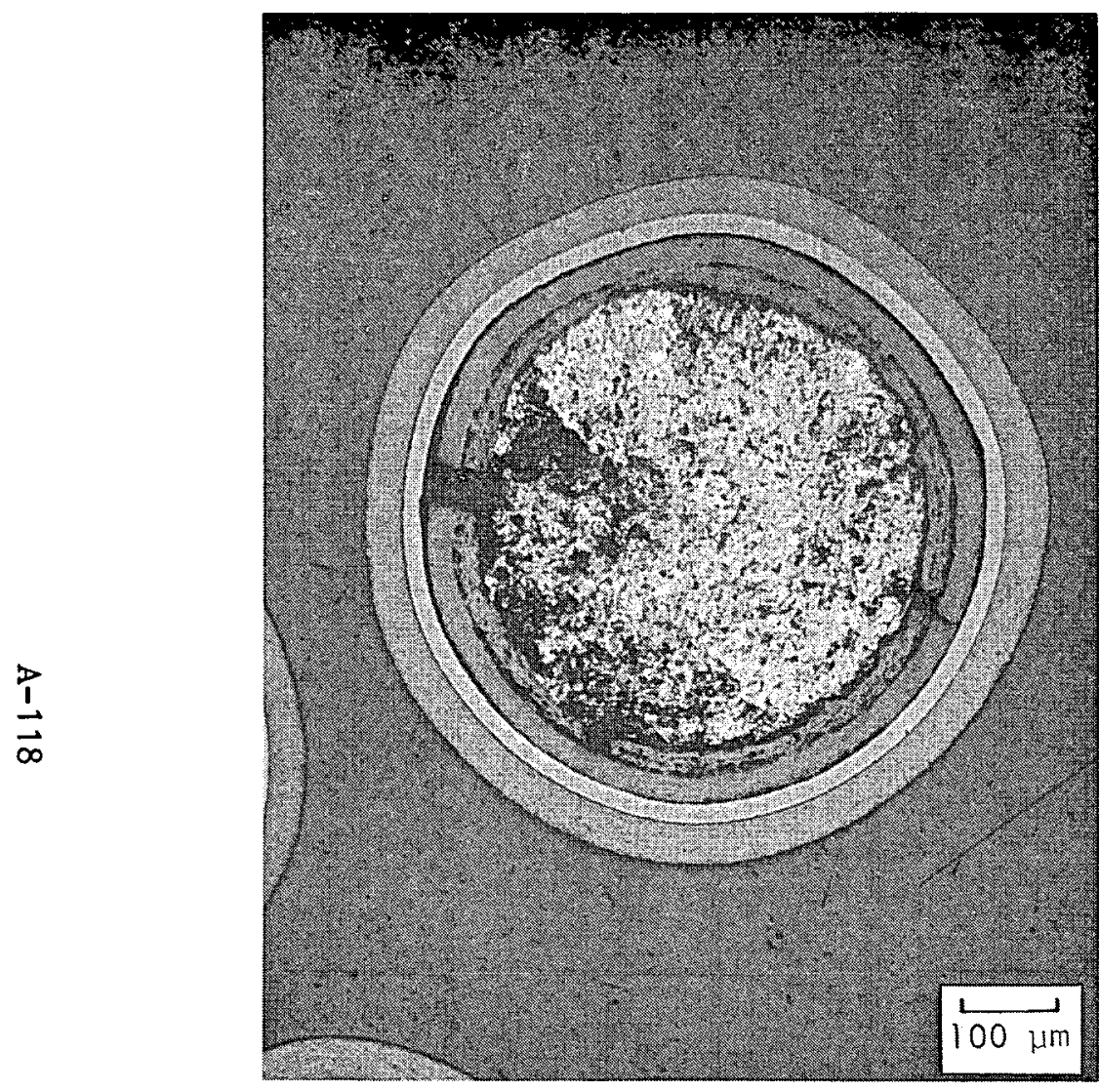

L7519-78

(a)

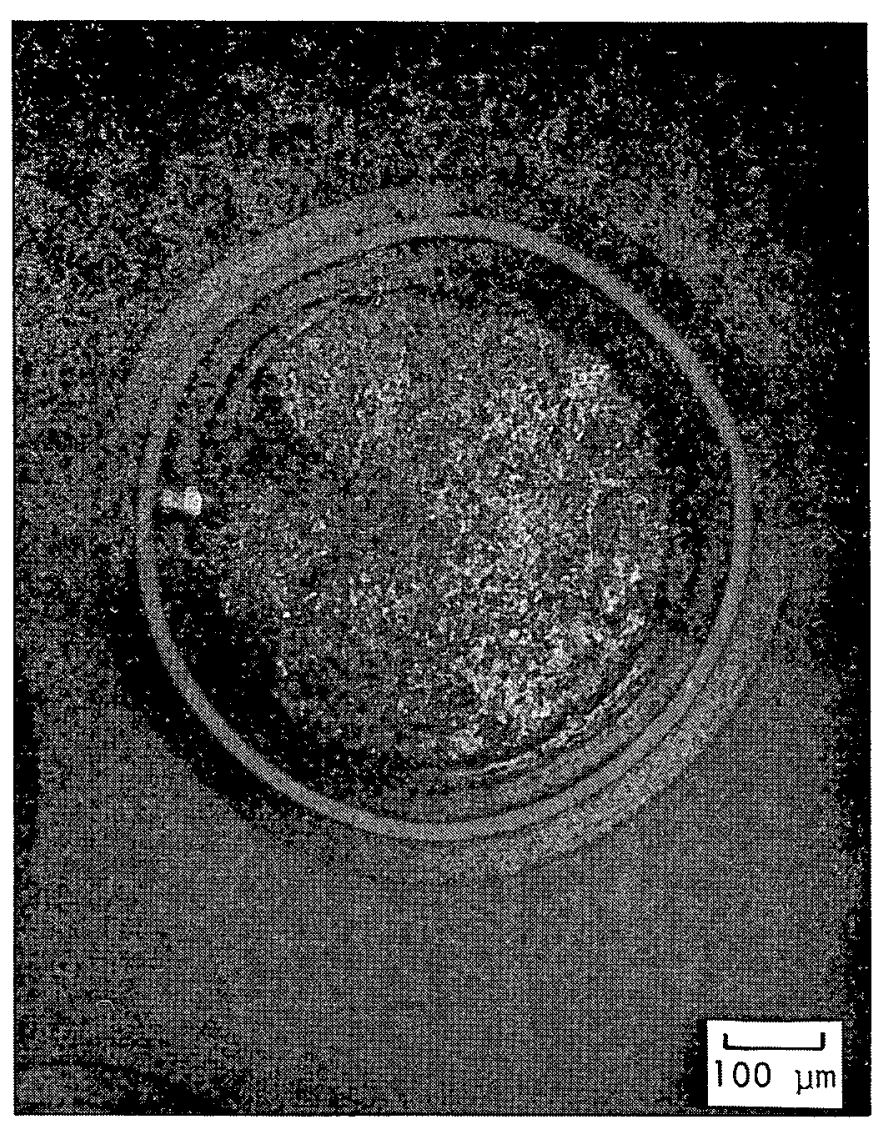

L7519-79

(b)

Fig. 4-96. FTE-6 photomicrographs of typical microstructure of $\mathrm{ThC}_{2}$ TRISO particle from spine sample TS 18-3 


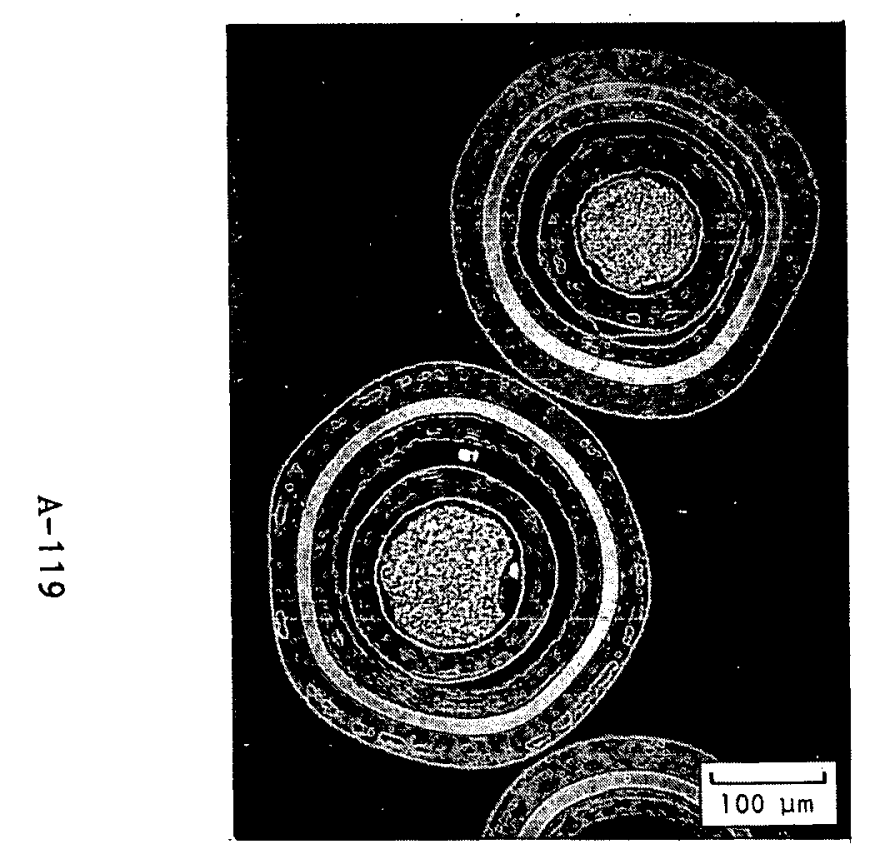

L7519-90

(a)

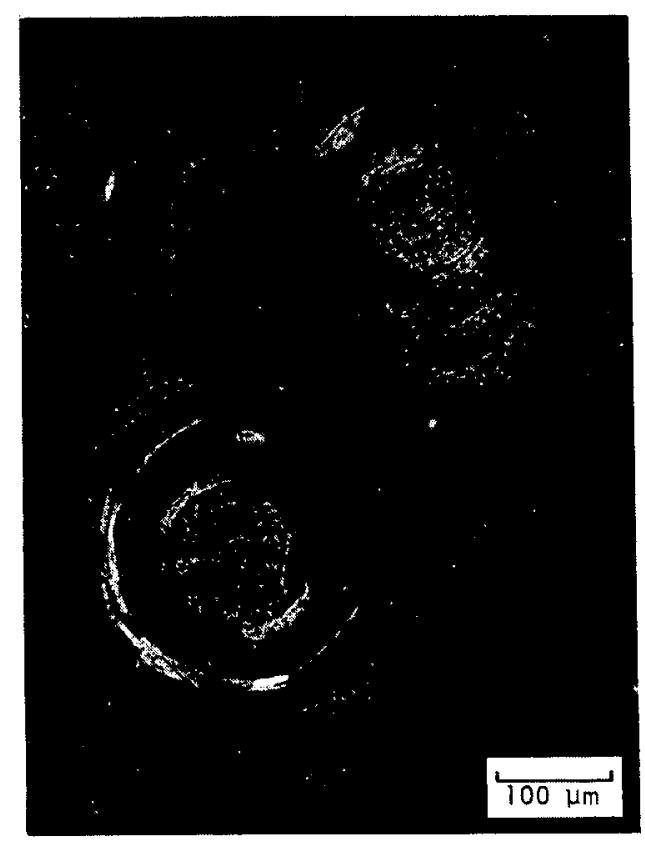

(b)

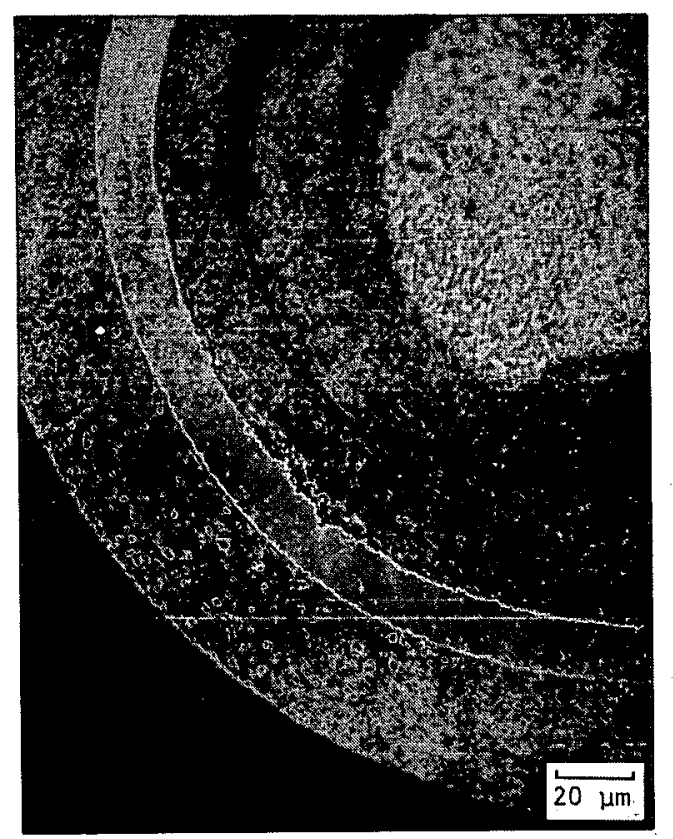

L7519-91A

(c)

Fig. 4-97. FTE-6 photomicrographs of typical microstructure of $\mathrm{UC}_{3}$ 120- $\mu \mathrm{m}$ (VSM) particles from spine sample TS 4-5 


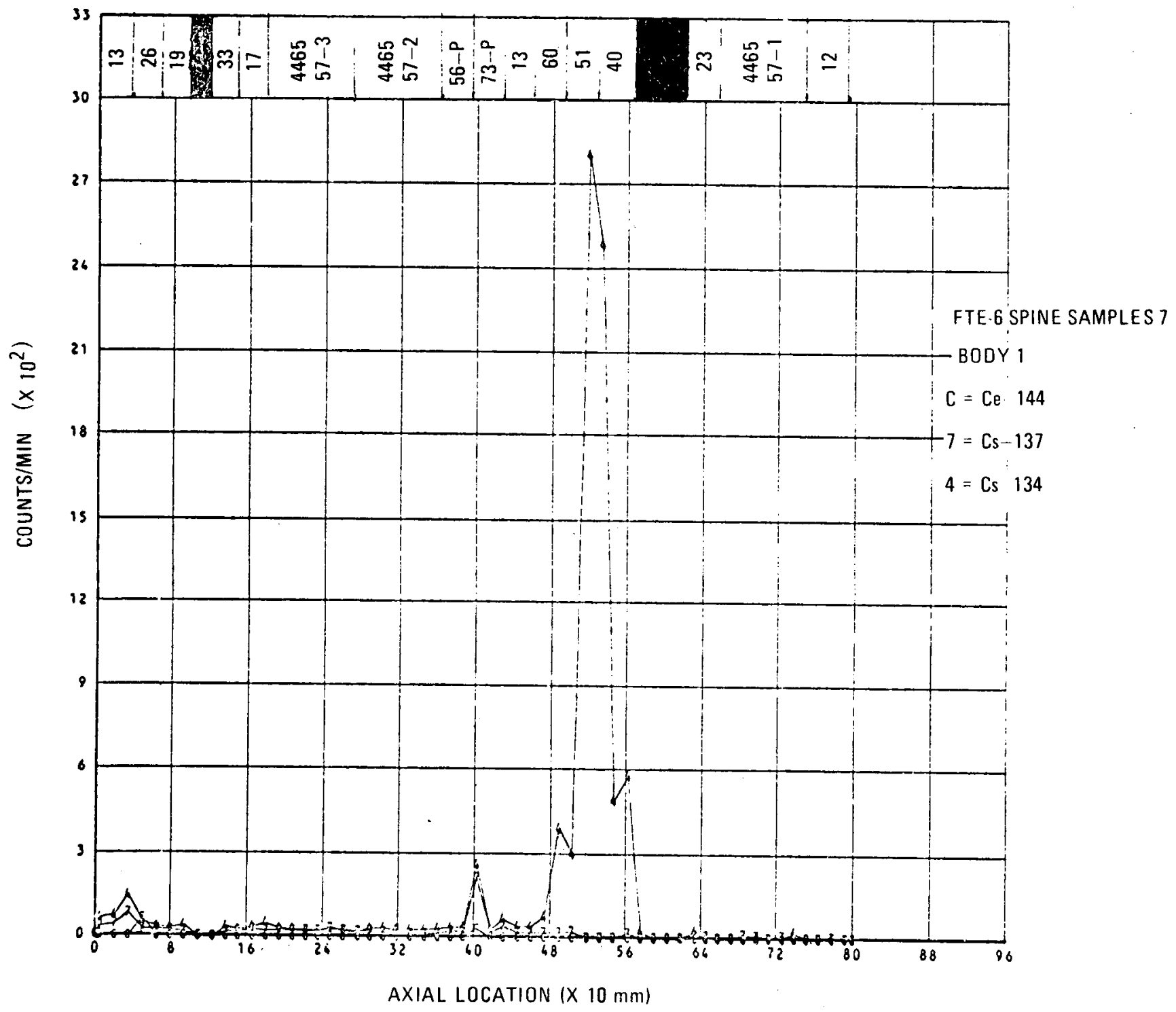

Fig. 4-98. FTE-6 GA hot cell gamma scan of body 1 spine samples 


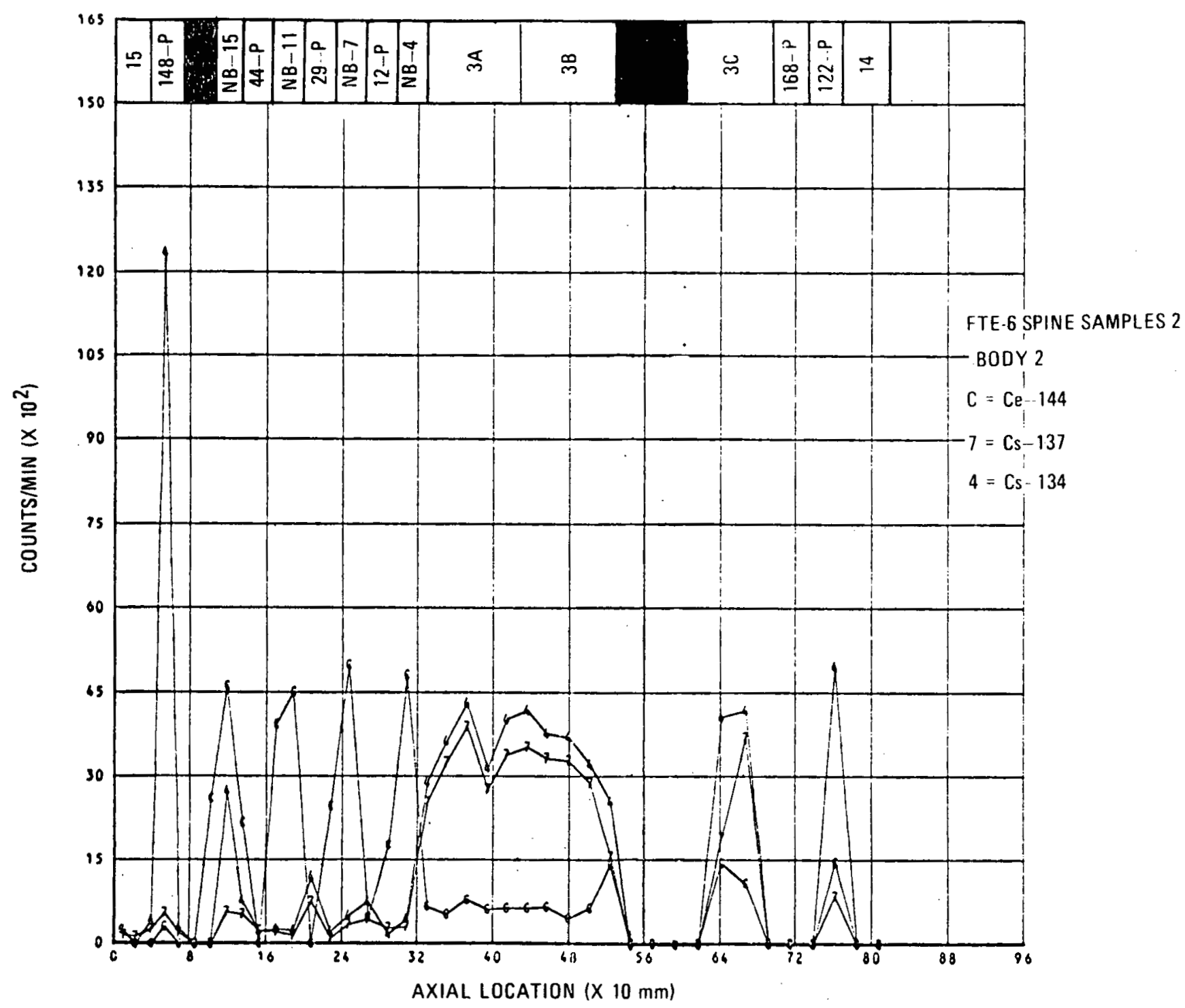

Fig. 4-99. FTE-6 GA hot cell gamma scan of body 2 spine samples 


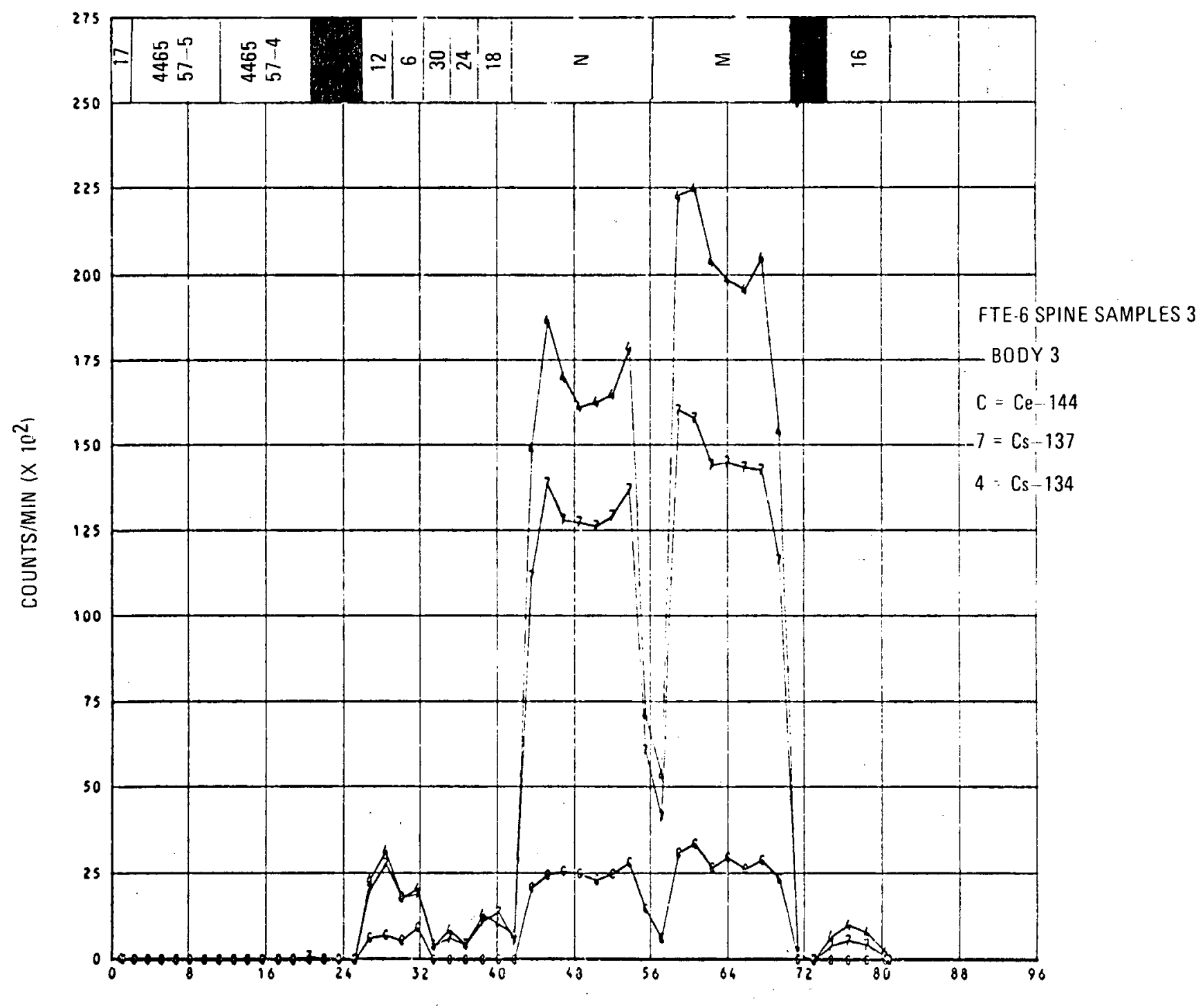

AXIAL LOCATION (X $10 \mathrm{~mm})$

Fig. 4-100. FTE-6 GA hot cell gamma scan of body 3 spine samples 


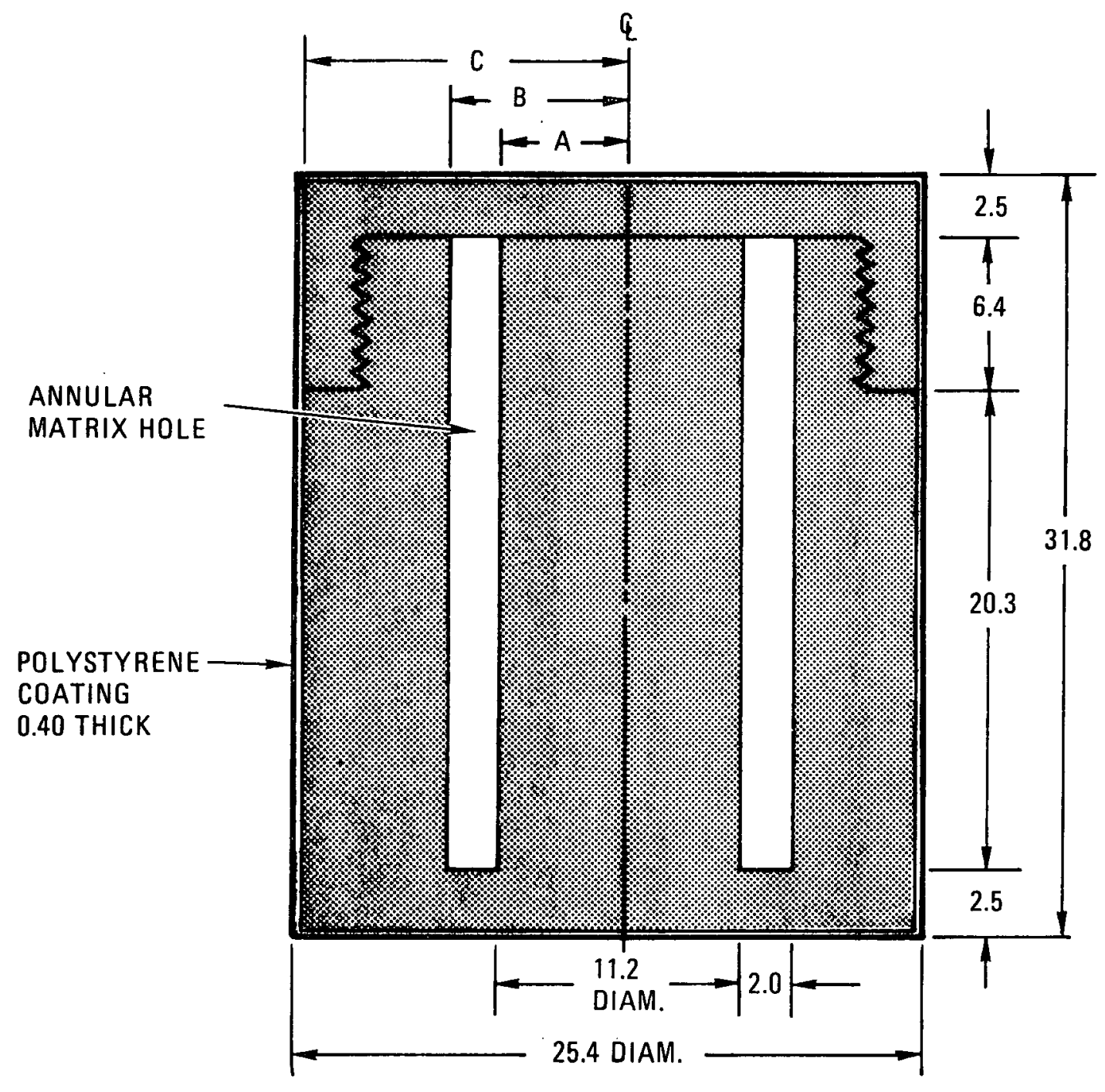

ALL DIMENSIONS IN mm

Fig. 4-101. Design of FTE-6 diffusion spine samples 


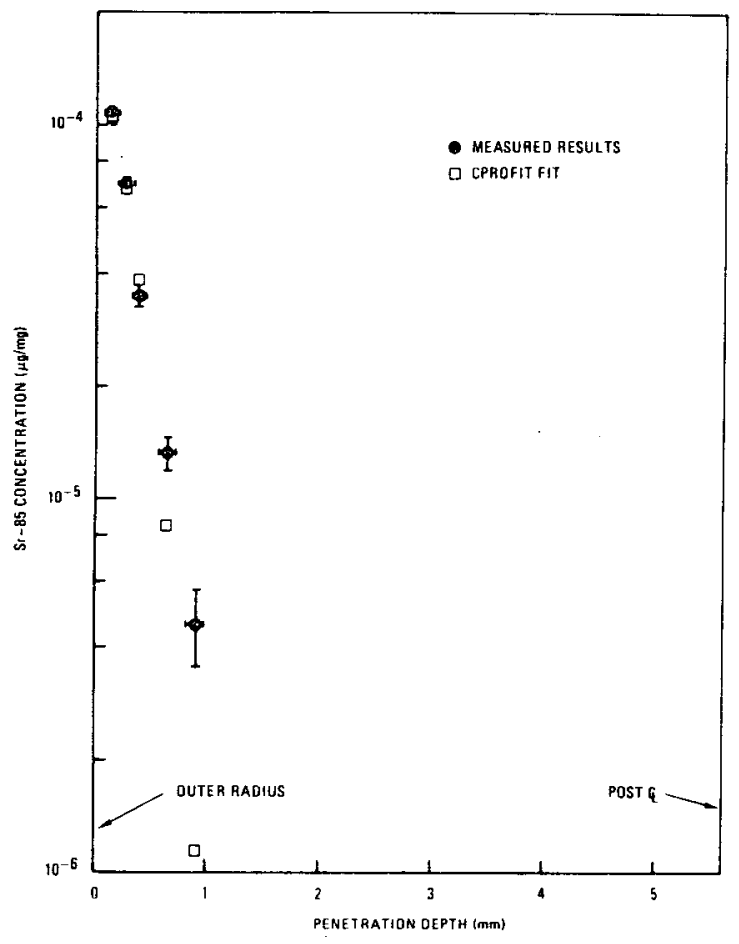

Fig. 4-102. FTE-6 Sr-85 profile in center post of diffusion sample 13

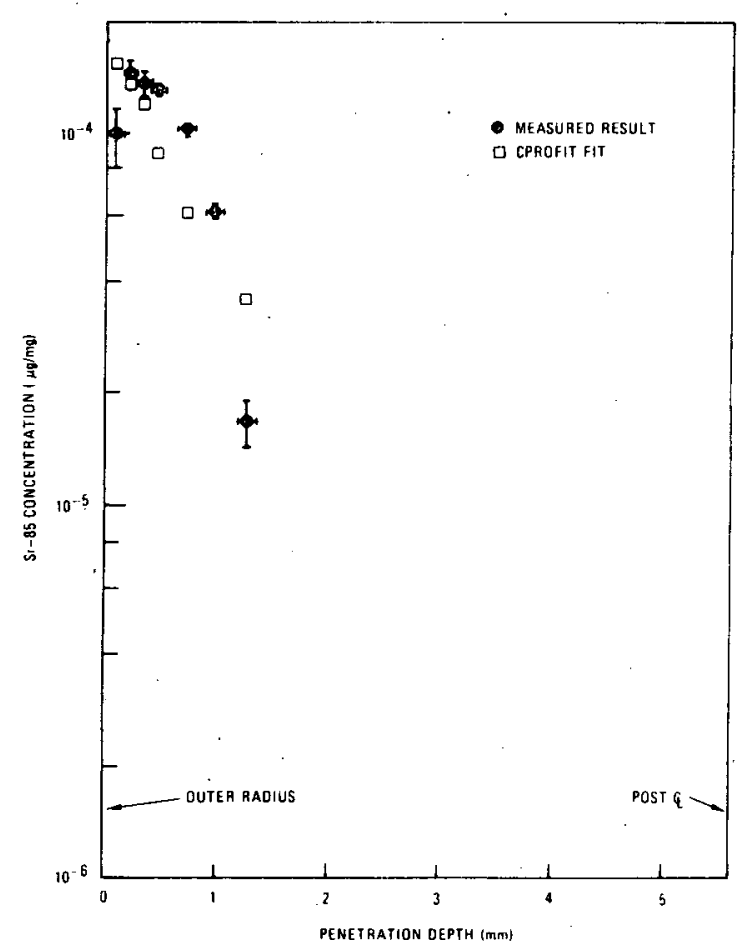

Fig. 4-104. FTE-6 Sr-85 profile in center post of diffusion sampel 17

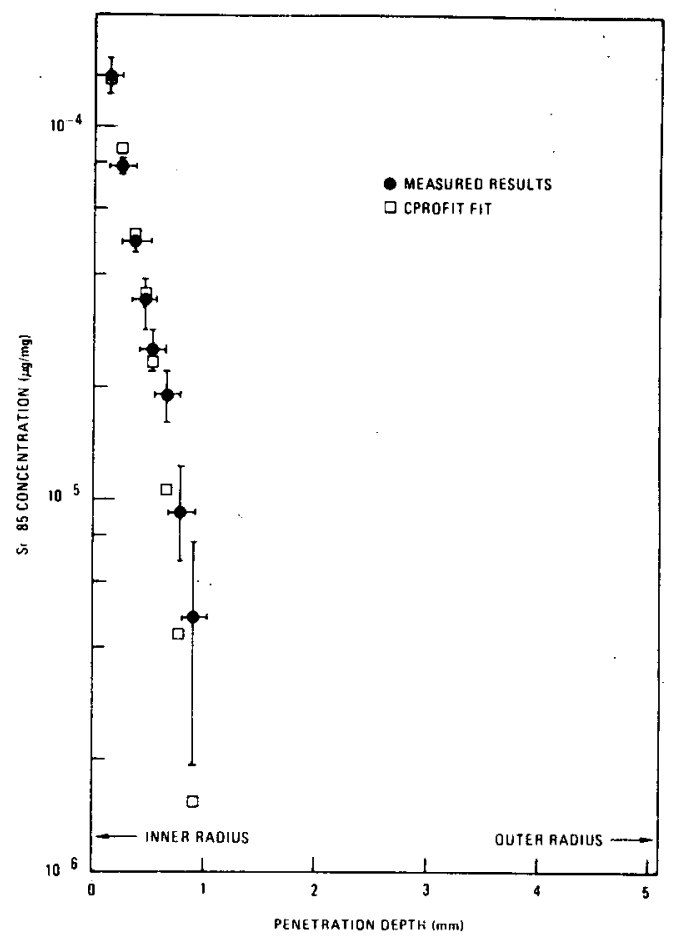

Fig. 4-103. FTE-6 Sr-85 profile in crucible wall of diffusion sample 13

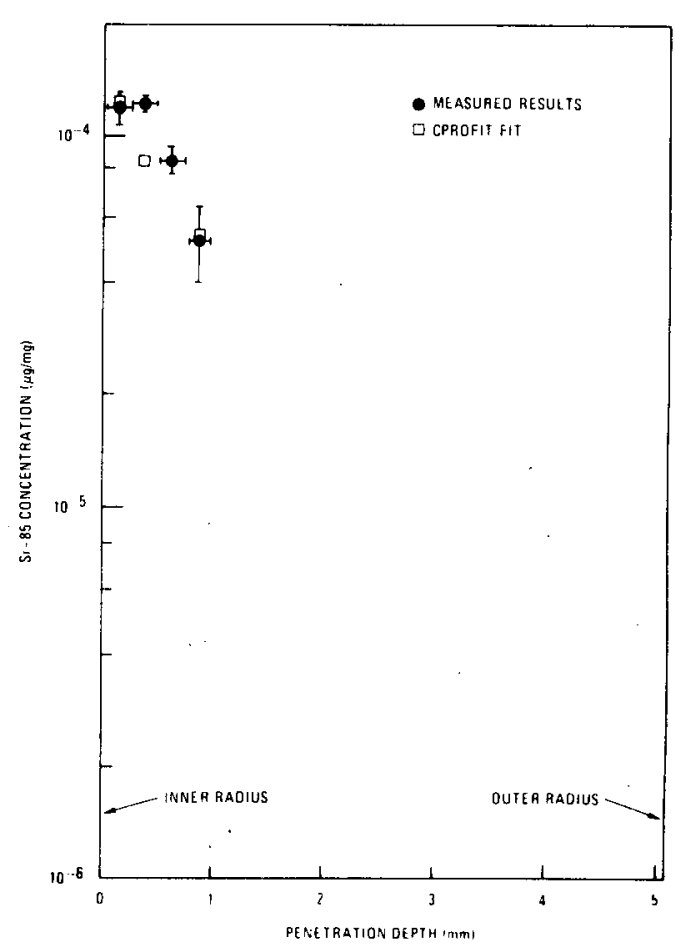

Fig. 4-105. FTE-6 Sr-85 profile in crucible wall of diffusion sample 17 


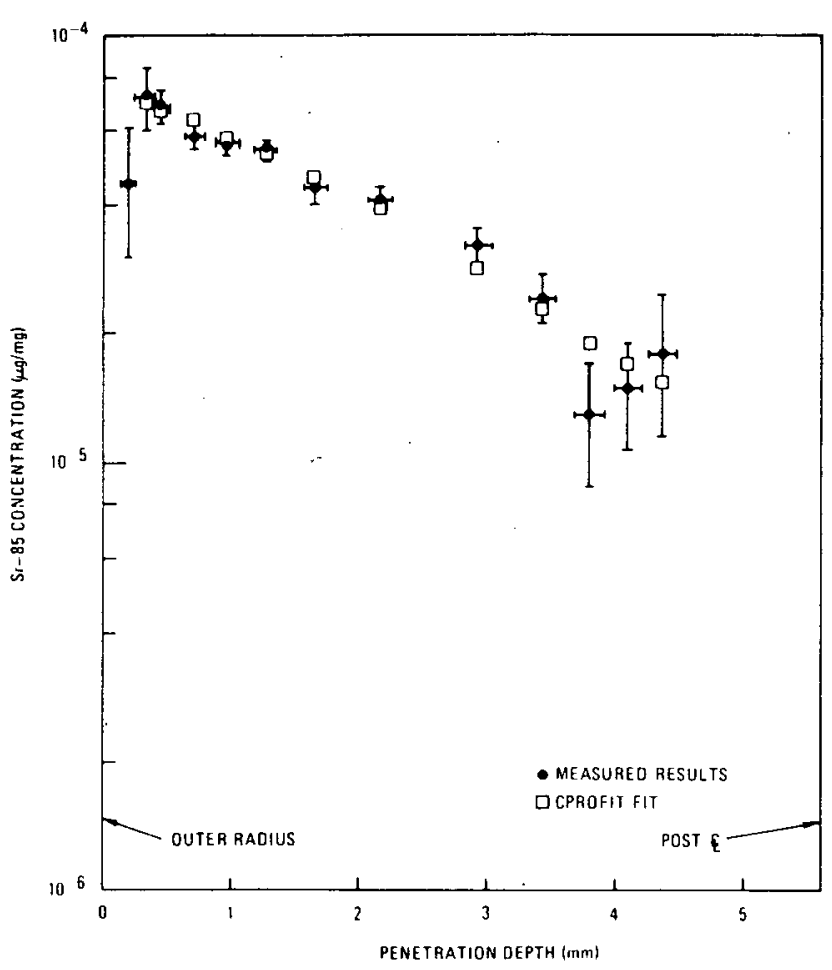

Fig. 4-106. FTE-6 Sr-85 profile in the center post of diffusion sample 19

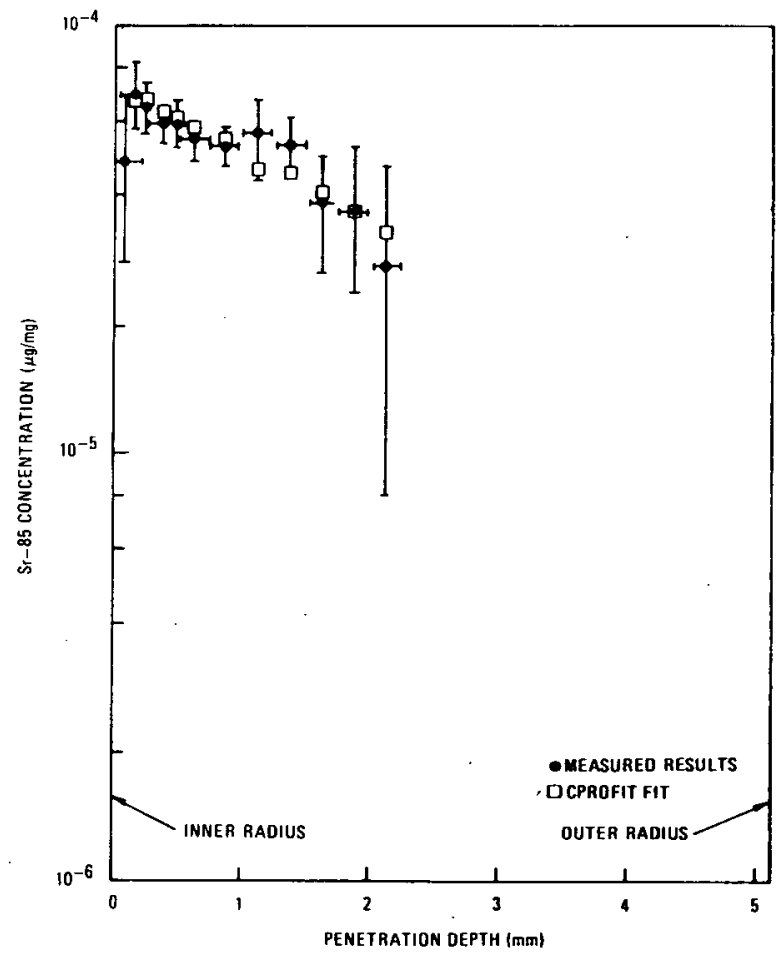

Fig. 4-107. FTE-6 Sr-85 profile in crucible wall of diffusion sample 19 


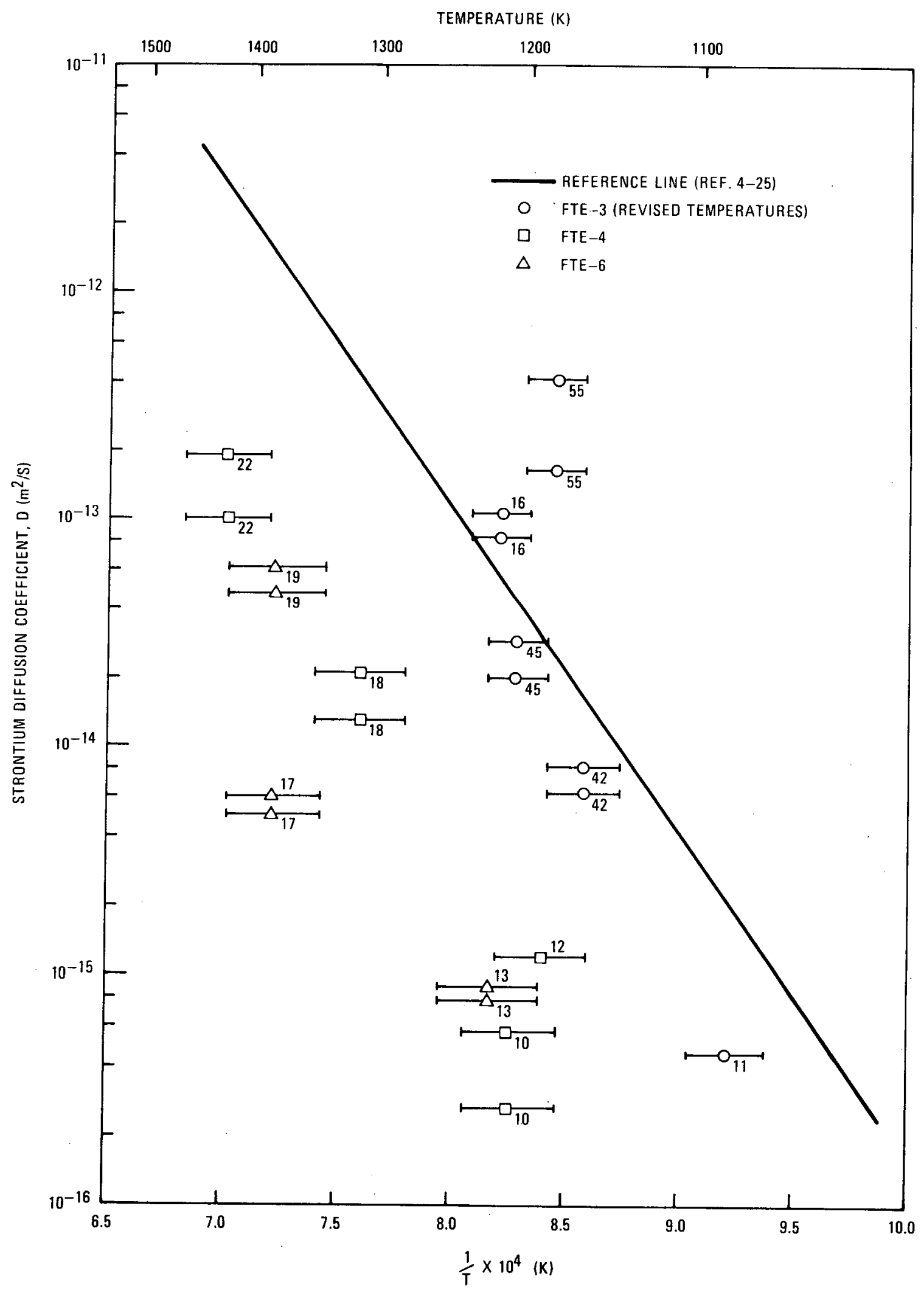

Fig. 4-108. FTE-3, FTE-4, and FTE-6 strontium diffusion coefficients plotted as a function of crucible irradiation temperature 
0
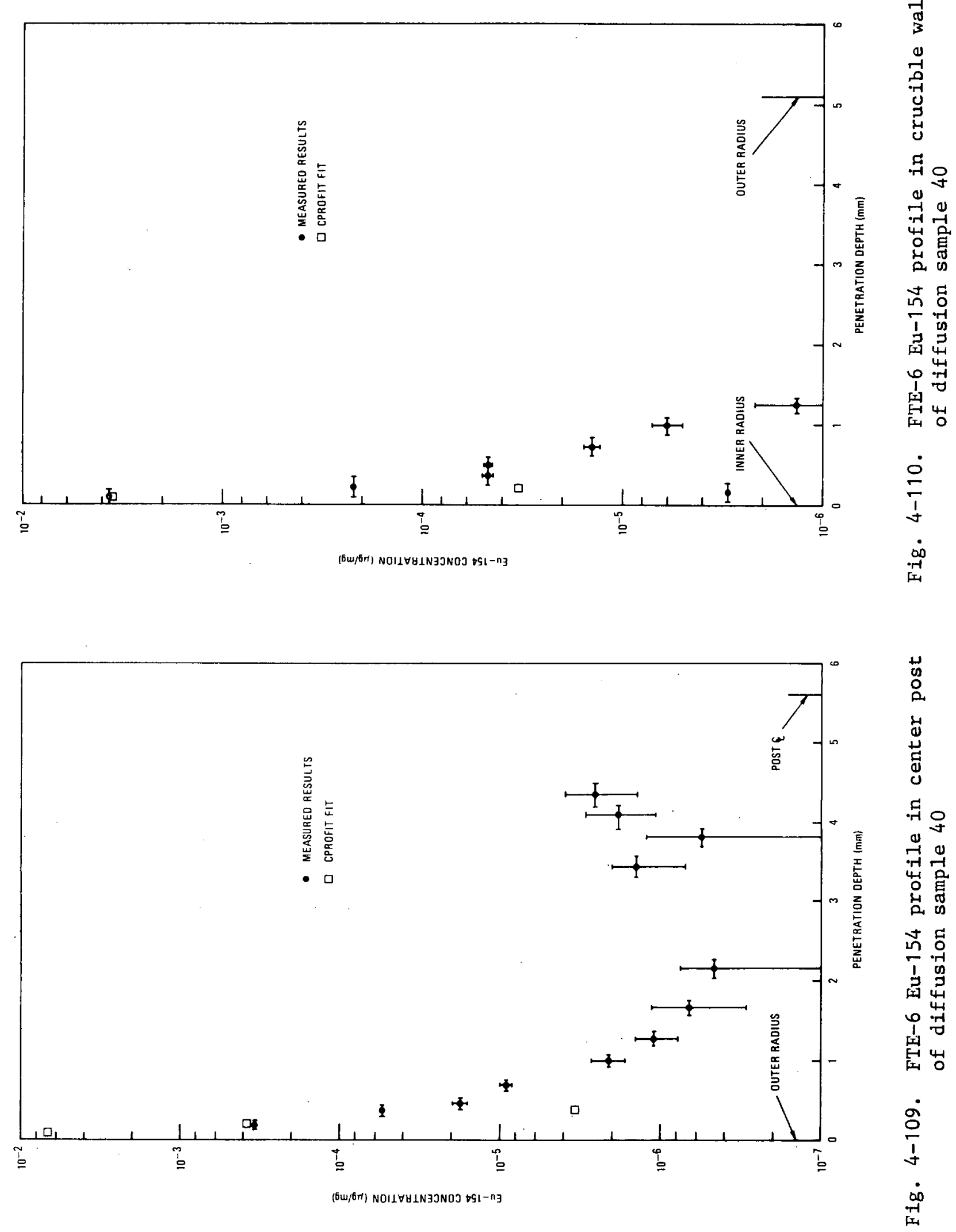


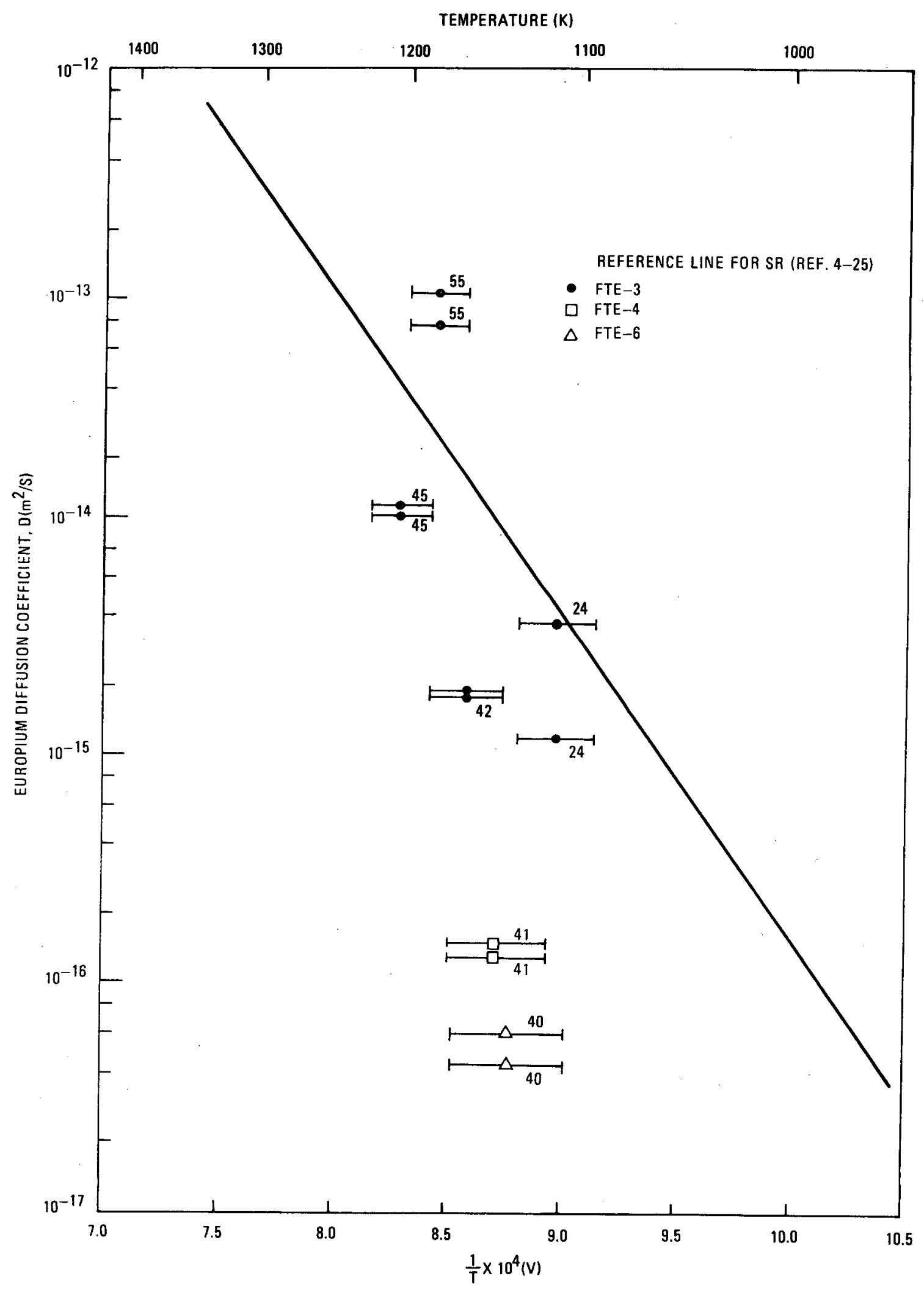

Fig. 4-111. FTE-3, FTE-4, and FTE-6 europium diffusion coefficients in graphite versus crucible irradiation temperature 


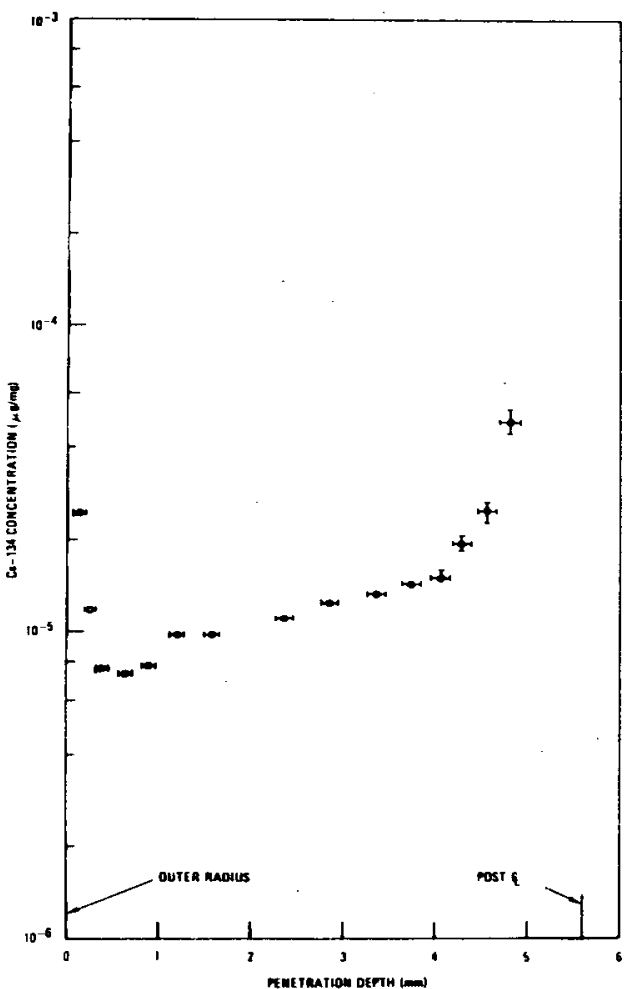

Fig. 4-112. FTE-6 Cs-134 profile in center post of diffusion sample 13

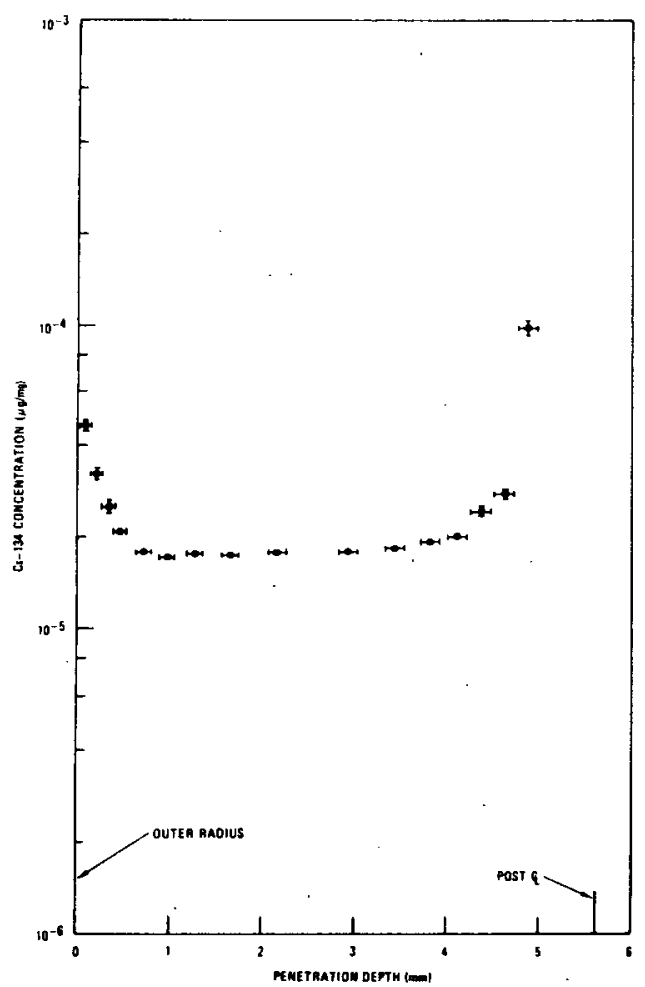

Fig. 4-114. FTE-6 cs-134 profile in center post of diffusion sample 17

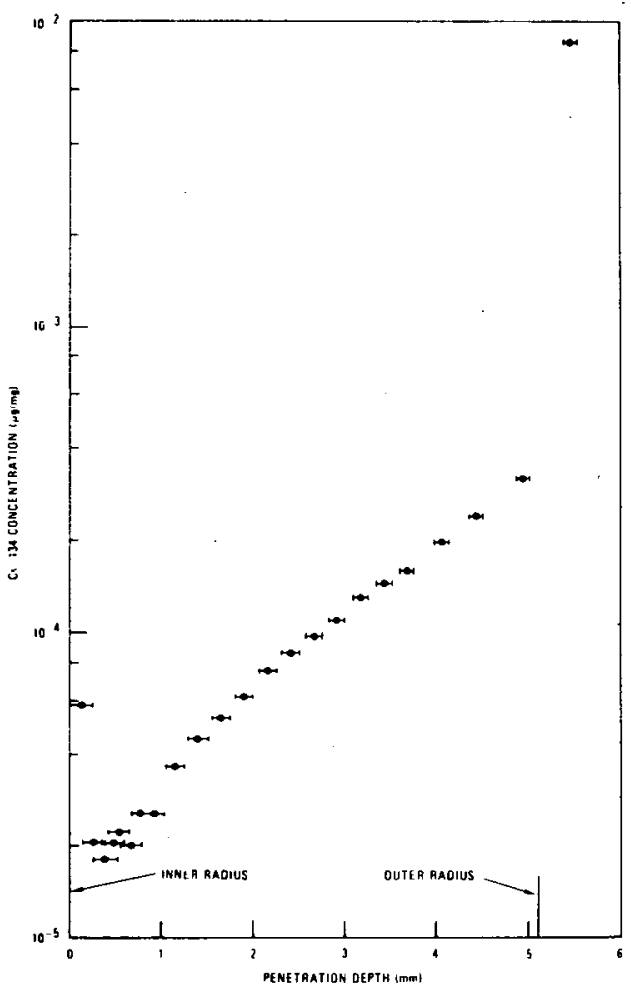

Fig. 4-113. FTE-6 Cs-134 profile in crucible wall of diffusion sample 13

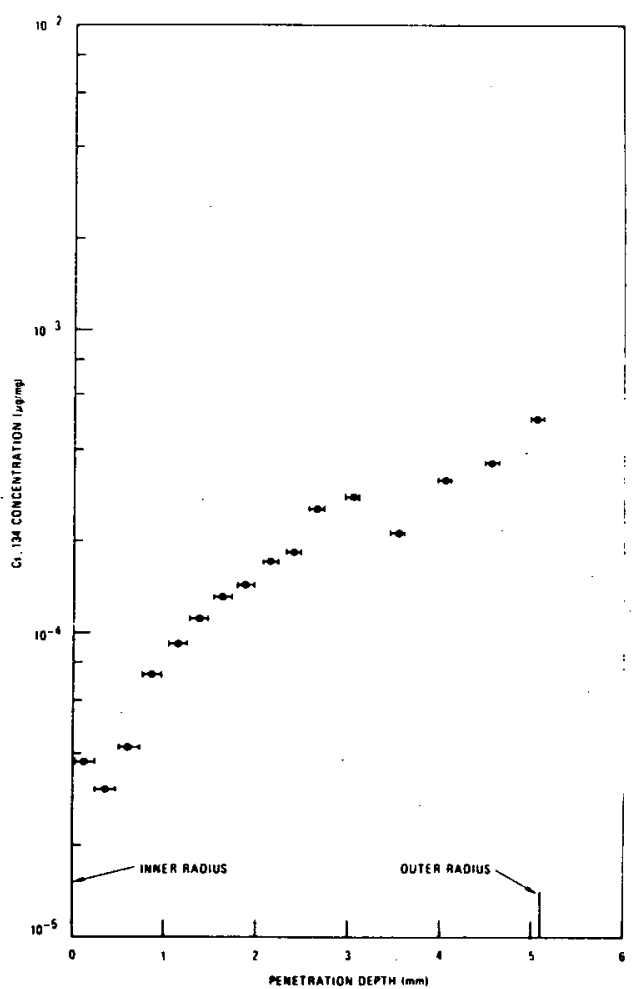

Fig. 4-115. FTE-6 Cs-134 profile in crucible wall of diffusion sample 17 


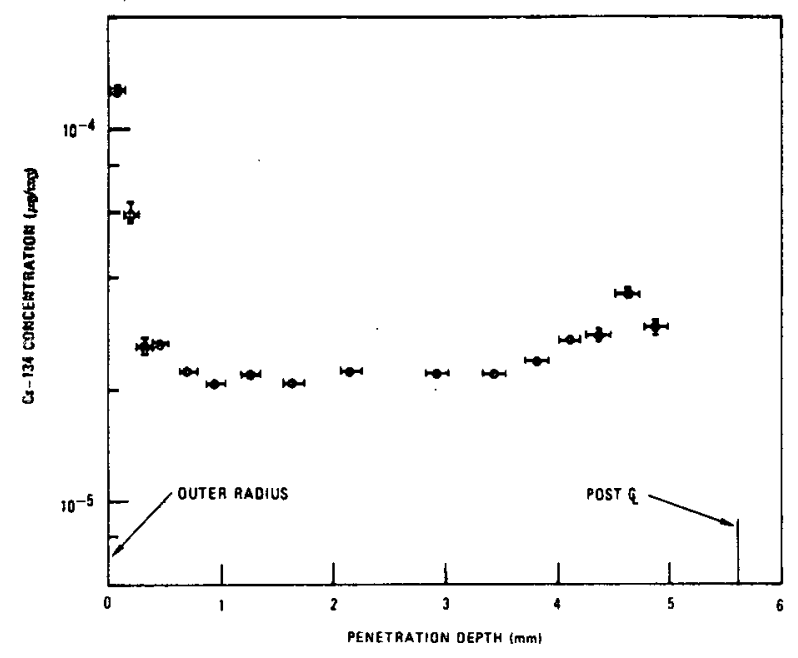

Fig. 4-116. FTE-6 Cs-134 profile in center post of diffusion sample 19

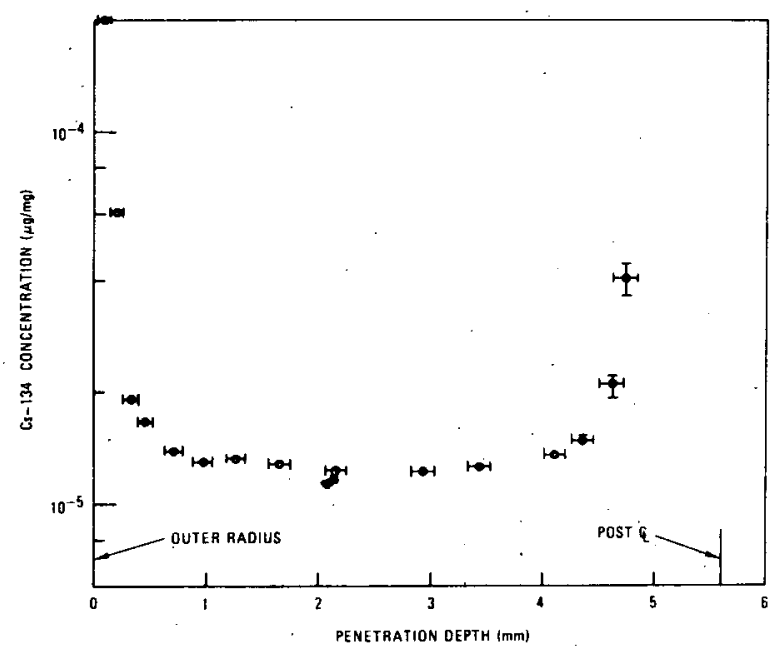

Fig. 4-118. FTE-6 Cs-134 profile in center post of diffusion sample 33

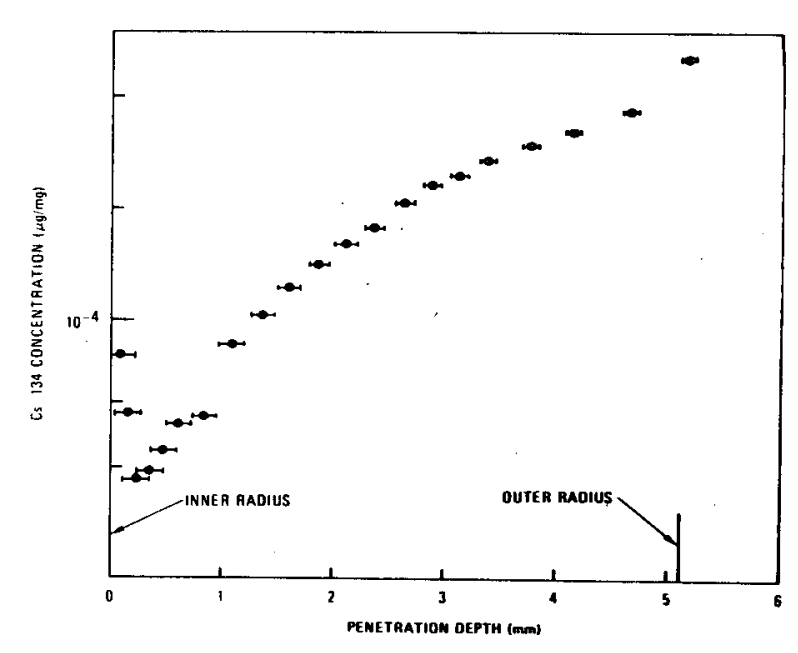

Fig. 4-117. FTE-6 Cs-134 profile in crucible wall of diffusion sample 19

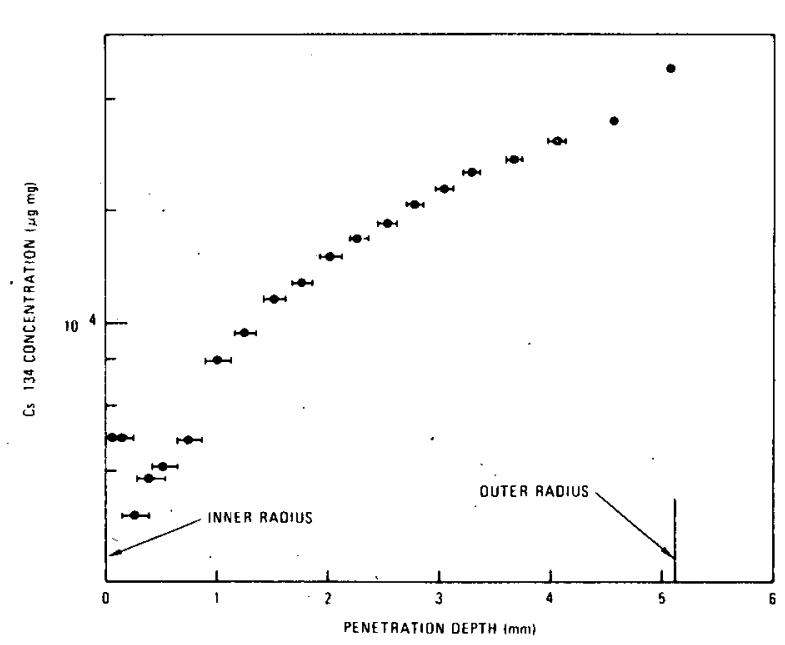

Fig. 4-119. FTE-6 Cs-134 profile in crucible wall of diffusion sample 33 


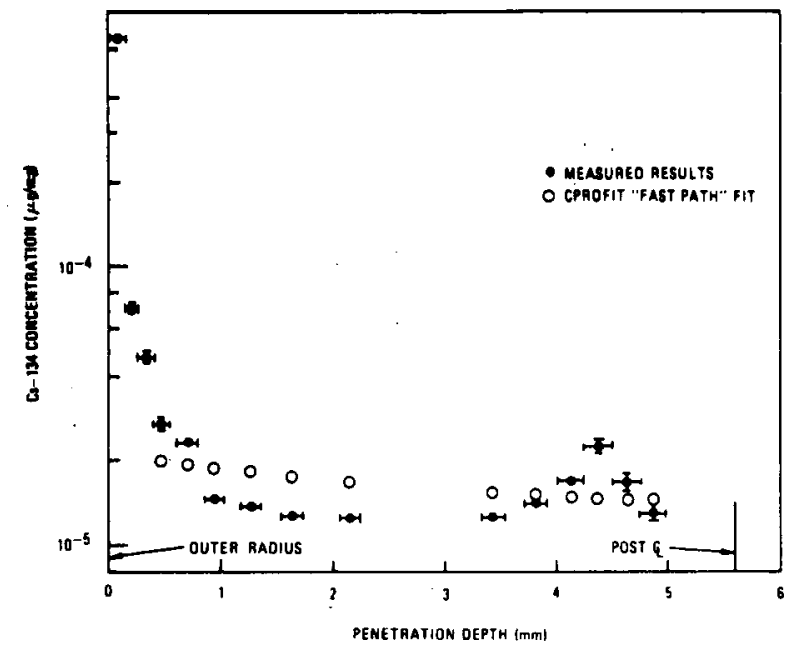

Fig. 4-120. FTE-6 Cs-134 profile in center post of diffusion sample 40

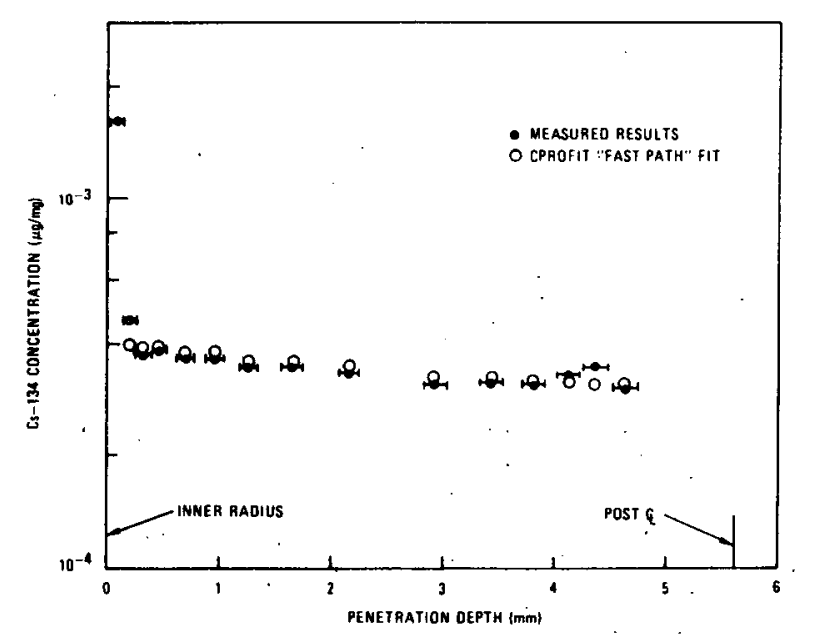

Fig. 4-122. FTE-6 Cs-134 profile in center post of diffusion sample 60

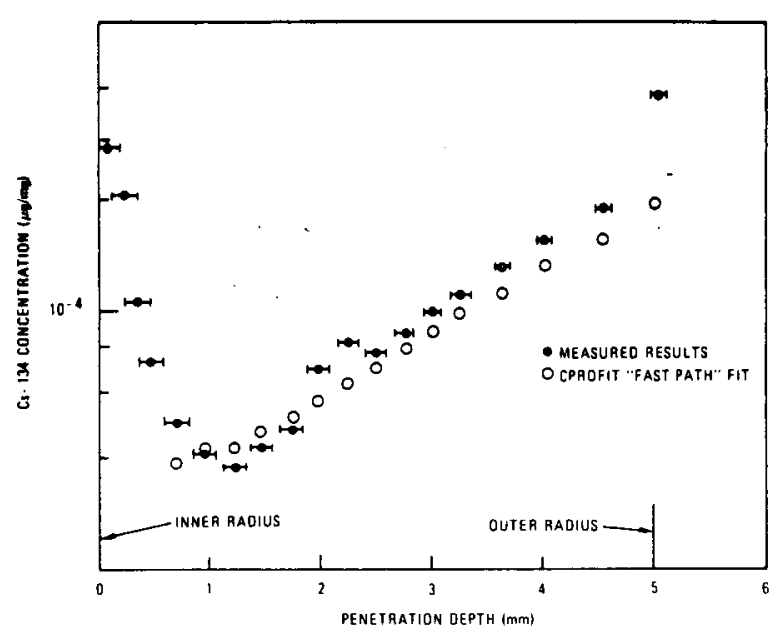

Fig. 4-121. FTE-6 Cs-134 profile in crucible wall of diffusion sample 40

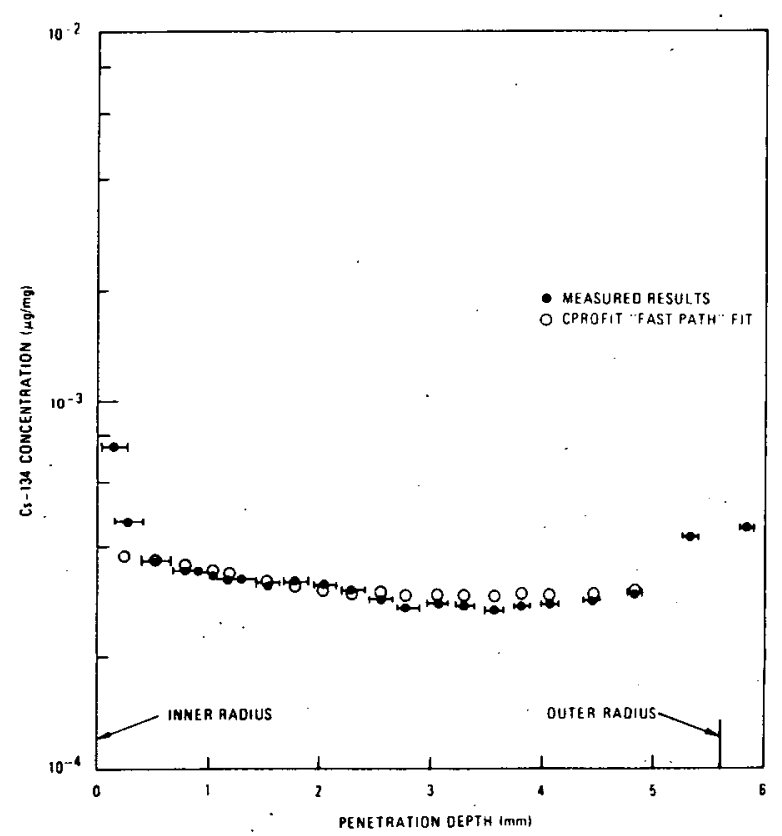

Fig. 4-123. FTE-6 Cs-134 profile in crucible wall of diffusion sample 60 


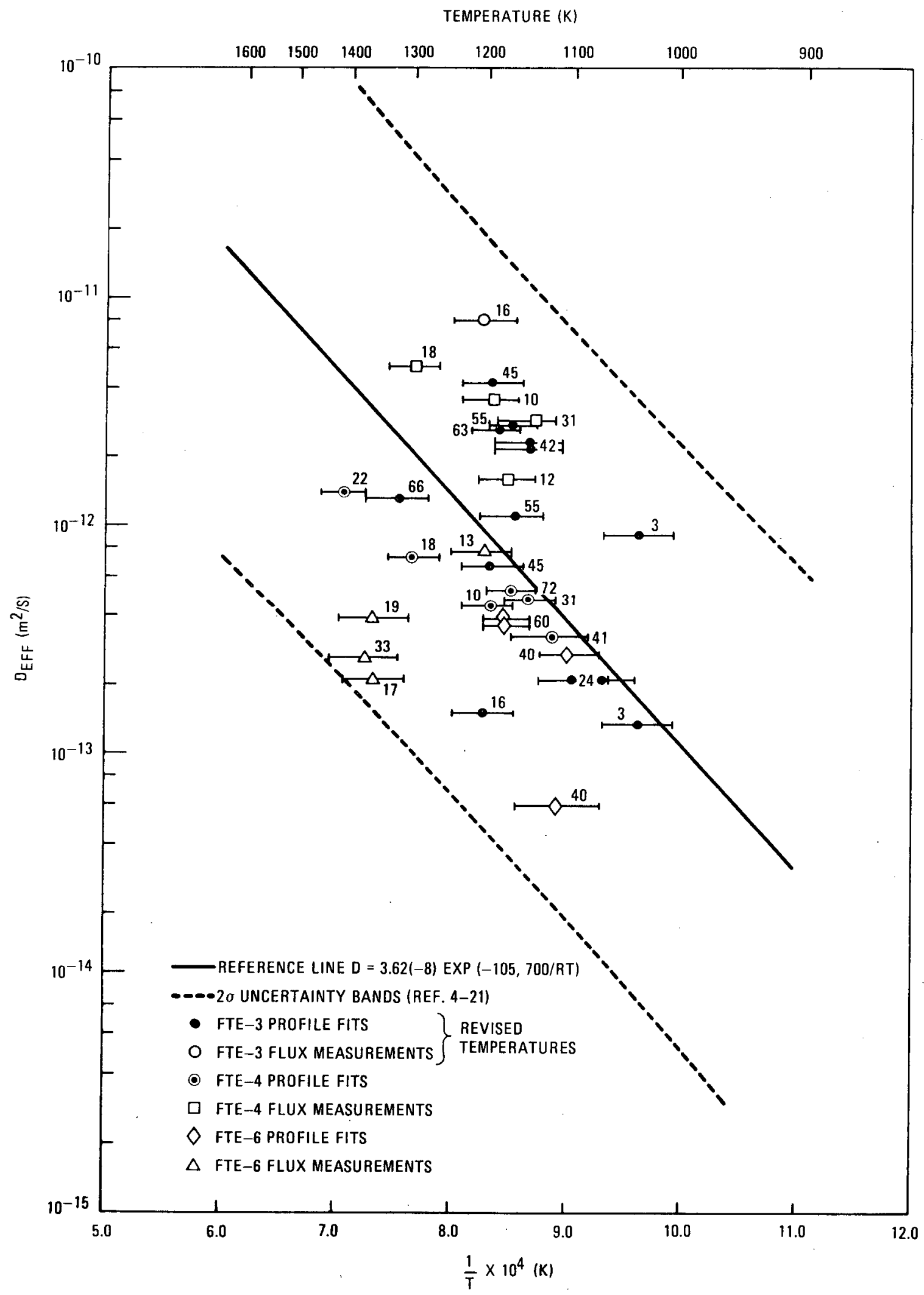

Fig. 4-124. FTE-3, FTE-4, and FTE-6 diffusion coefficients of cesium $\left(D_{\text {eff }}\right)$ as a function of time-averaged irradiation temperature 
APPENDIX B

TABLES

$\ominus$

$\ominus$ 
0

•

$\theta$ 


\section{LIST OF TABLES}

2-1. FTE-6 drawing and parts 1ist . . . . . . . . . . . . B-1

2-2. Peach Bottom graphite materials used in fuel elements . . . B-3

2-3. FTE-6 fuel particle attributes . . . . . . . . . . B-4

2-4. FTE-6 fuel rod attributes . . . . . . . . . . . . B-5

2-5. Preirradiation data for FTE-6 type II thermal stability

2-6a. FTE-6 spine sample, burnup and fluence tabulation (calculated values) ................ B-7

2-6b. FTE-6 spine sample temperature (K) tabulation . . . . . B-8

2-7. FTE-6 purge flow description . . . . . . . . . . . . B-9

3-1. FTE-6 power, flow, and four group flux history . . . . . B-10

3-2. FTE-6 fast and thermal fluence calculations . . . . . . . B-11

3-3a. FTE-6 tabulation of irradiation temperatures for holes 1 and $2\left(\mathrm{UO}_{2}\right.$ TRISO, ThO2 2 BISO fuel rods) . . . . . . . . . B-12

3-3b. FTE-6 tabulation of irradiation temperatures for holes 3

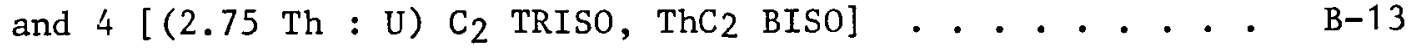

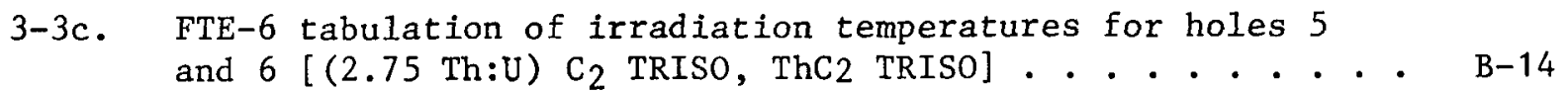

3-3d. FTE-6 tabulation of irradiation temperatures for holes 7 and 8 (UC, 2 TRISO, ThC2 BISO) . . . . . . . . . . . B-15

3-4a. FTE-6 comparison of predicted and time-averaged chromelalumel thermocouple temperatures ... . . . . . . B-16

3-4b. FTE- 6 comparison of predicted and time-averaged tungstenrhenium thermocouple temperatures . . . . . . . . B-17

3-5. FTE-6 average nuclide weights for fuel rods, spine samples, and total element ............... B-18

3-6. FTE-6 peak and mean burnup values and gradients . . . . B-19

3-7. Nuclear constants and uncertainty limits used in burnup determinations ............. . . B-21

3-8. FTE-6 mass spectrometry and IDMS results and comparison with GAUGE/FEVER predictions . . . . . . . . . . . B-22

3-9. FTE-6 isotopic dilution mass spectrometry results . . . . B-23

3-10. FTE-6 comparison of IDMS and radiochemistry results . . . B-24

3-11. FTE-6 fissile and fertile burnup measurements . . . . . . B-25 


\section{LIST OF TABLES (Continued)}

3-12. FTE-6 comparison between gamma spectroscopy and GAUGE/

FEVER calculated fuel rod burnups . . . . . . . . B-26

3-13. FTE-6 composite burnup measurements and comparison with

GAUGE/FEVER calculations ........... . . B-27

3-14a. FTE-6 stress summary for midplane of body 1 (Axial position 5) ............... . B-28

3-14b. FTE-6 stress summary for midplane of body 2 (Axial position 15)... . . . . . . . . . . . B-30

3-14c. FTE-6 stress summary for midplane of body 3 (Axial position 25)... . . . . . . . . . . . . B-32

3-15. H-327 graphite ultimate tensile and compression strength and elastic modulus . . . . . . . . . . . . . B-34

3-16. FTE-6 irradiation summary . . . . . . . . . . . B-35

4-1. Formula summary on error analysis . . . . . . . . . B-39

4-2. Error analysis default table . . . . . . . . . . . B-40

4-3. FTE-6 Peach Bottom EOL fuel gamma scan 1, nuclide CPM ratios and normalized profiles ... . . . . . . . . . B-41

4-4. FTE-6 Peach Bottom EOL fuel gamma scan 1, nuclide CPM tabulation . . . . . . . . . . . . . . B-42

4-5. FTE-6 fuel stack 1 gamma scans, nuclide CPM tabulation . . B-43

4-6. FTE-6 fuel stack 1 gamma scans, nuclide CPM ratios, and normalized profiles . . . . . . . . . . . . . B-44

4-7. FTE-6 fuel gamma scan comparison of calculated and measured EOL power profiles for Peach Bottom scan $1 \mathrm{Zr}-95+\mathrm{La}-140$. B-45

4-8. Measured EOL power profiles for stack 1 fuel $\operatorname{rods}$. . . . B-46

4-9. Fuel gamma scan comparison of Cs-137/Zr-95 profiles for cesium mobility ............. . B-47

4-10. FTE-6 fuel gamma scan comparison of Cs-137/Zr-95 profiles from two different scanning methods . . . . . . . . B-48

4-11. FTE-6 fuel gamma scan comparison of normalized Cs/Zr-95 and $\mathrm{Ru} / \mathrm{Zr}-95$ profiles from two different scanning methods . B-49

4-12. FTE-6 fuel gamma scan comparison of calculated and measured thermal fluence profiles for stack 3 and 5 fuel rods . . . B-50

4-13. FTE-6 comparison of accumulative and composite fuel stack lengths derived from metrology and gamma scanning . . . . B-51

4-14. FTE-6 thermocouple resistance data . . . . . . . . . B-52

4-15. FTE-6 distance d - top of spine sample to edge of hole . . B-53

4-16. FTE-6 fuel stack length . . . . . . . . . . . B-54 


\section{LIST OF TABLES (Continued)}

4-17. FTE-6 fuel rod removal summary . . . . . . . . . . B-55

4-18. FTE-6 total element length and fuel body weight . . . . . B-56

4-19. FTE-6 sleeve diameter . . . . . . . . . . . B-57

4-20. FTE-6 fuel body bow . . . . . . . . . . . . B-58

4-21. FTE-6 fuel body bow analysis . . . . . . . . . . B-59

4-22. FTE-6 fuel body 1 dimensional changes . . . . . . . . B-60

4-23. FTE-6 fuel body 2 dimensional changes . . . . . . . . B-61

4-24. FTE-6 fuel body 3 dimensional changes . . . . . . . . B-62

4-25. FTE-6 fuel body strain summary . . . . . . . . . . . B-63

4-26. FTE-6 comparison of measured and calculated fuel body strain B-64

4-27. FTE-6 sleeve fuel body gaps . . . . . . . . . . . B-66

4-28. FTE-6 comparison of measured and calculated fuel rod strain Holes 1 and $2 \mathrm{UO}_{2}$ TRISO and $\mathrm{ThO}_{2}$ BISO fuel . . . . . . B-67

4-29. FTE-6 comparison of measured and calculated fuel rod strain Holes 3 and 4 (Th,U) $C_{2}$ TRISO and $\mathrm{ThC}_{2}$ BISO fuel ..... B B-68

4-30. FTE-6 comparison of measured and calculated fuel rod strain Holes 5 and $6(\mathrm{Th}, \mathrm{U}) \mathrm{C}_{2}$ TRISO and $\mathrm{ThC}_{2}$ TRISO fuel .. . . . B-69

4-31. FTE-6 comparison of measured and calculated fuel rod strain Holes 7 and $8 \mathrm{UC}_{2}$ TRISO and $\mathrm{ThC}_{2}$ BISO fuel........ B-70

4-32. FTE-6 hole 1 and 2 fuel rod strain summary . . . . . . B-71

4-33. FTE-6 hole 3 and 4 fuel rod strain summary . . . . . . B-72

4-34. FTE-6 hole 5 and 6 fuel rod strain summary . . . . . . B-73

4-35. FTE-6 hole 7 and 8 and total element strain summary . . . B-74

4-36. FTE-6 comparison of measured and calculated fuel rod -

4-37. FTE-6 comparison of measured and calculated fuel rod fuel hole gaps, holes 3 and 4 .......... B-76 4-38. FTE-6 comparison of measured and calculated fuel rod -
fuel hole gaps, holes 5 and 6 . . . . . . . . B-77

4-39. FTE-6 comparison of measured and calculated fuel rod -
fuel hole gaps, holes 7 and 8 . . . . . . . B-78

4-40. FTE-6 fuel rod bow . . . . . . . . . . . . . B-79

4-41. FTE-6 summary of postirradiation examination of fuel rods $. \quad B-80$

4-42. FTE-6 fission gas release summary . . . . . . . . . . B-81

4-43. FTE-6 fuel rod gamma scan summary . . . . . . . . . . B-82 


\section{LIST OF TABLES (Continued)}

4-44. FTE-6 TREVER-calculated temperatures used as input to the FIPERQ fits of the Cs-137 profile in fuel body 2

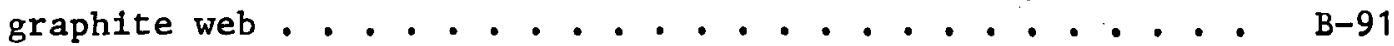

4-45. KMC data for kernel types used in Peach Bottom test elements B-92 4-46. FTE-6 UO ${ }_{2}$ fuel performance predictions . . . . . . . . B-93

4-47. FTE- 6 postirradiation data for the type II thermal stability spine samples ............... B-94

4-48. FTE-6 preirradiation data for the type II thermal stability spine samples ............... . . B-94

4-49. Summary data on FTE-6 diffusion spine samples . . . . . . B-95

4-50. Nuclear transformations of metal diffusants . . . . . . B-96

4-51. FTE-6 diffusion coefficients for strontium and europium derived from diffusion sample analysis . . . . . . . . B-97

4-52. FTE-6 cesium diffusion coefficients and partition factors derived from diffusion sample analysis . . . . . . . . B-98 
TABLE 2-1

FTE-6 DRAWING AND PARTS LIST

\begin{tabular}{|c|c|c|}
\hline Drawing/Part No. & Issue & Title \\
\hline $11497-1,2$ & B & Assembly \\
\hline $11666-1$ & B & Sleeve \\
\hline $11668-1$ & B & Brazing Ring \\
\hline $11669-6$ & B & Upper Reflector \\
\hline $11670-2$ & B & Bottom Connector \\
\hline $11671-1$ & B & Screen \\
\hline $11672-1,2$ & B & W/Re, C/A Thermocouples \\
\hline $11673-1$ & B & Upper Contact \\
\hline $11541-1$ & B & Spacer \\
\hline $11542-1$ & $B$ & Internal Trap Assemb1y \\
\hline $11543-1$ & B & Lower Reflector \\
\hline $11510-1$ & B & Fuel Body Assembly, 6-1 \\
\hline $11503-3$ & B & Fuel Body \\
\hline $11503-4$ & B & Fuel Hole Plug \\
\hline $11503-5$ & $\mathrm{~B}$ & Compact Pusher \\
\hline $11503-6$ & B & Sample Hole Plug \\
\hline $11503-7$ & B & Sample Hole Plug \\
\hline $11400-1$ & A & Fue1 Stack Assemb1y \\
\hline $11704-1$ & A & Fuel Stack Assembly \\
\hline $11410-1$ & A & Fuel Stack Assembly \\
\hline $11411-1$ & A & Fuel Stack Assembly \\
\hline $11510-15$ & B & Graphite Cement (P-511) \\
\hline $11510-16$ & B & Polystyrene Solution \\
\hline $11510-17$ & B & Graphite Spacer \\
\hline $11510-18$ & B & Boronated Graphite Sample \\
\hline $11510-19,20,21,22,23$ & B & Diffusion Sample \\
\hline $11510-24,25$ & B & Fission Product Release Sample \\
\hline $11510-26,27$ & B & Boronated Graphite Sample \\
\hline $11510-28,29,30,31$ & B & Diffusion Sample \\
\hline
\end{tabular}


TABLE 2-1 (Continued)

\begin{tabular}{|c|c|c|}
\hline Drawing/Part No. & Issue & Title \\
\hline $11510-32$ & B & Graphite Spacer \\
\hline $11511-1$ & B & Fuel Body Assembly 6-2 \\
\hline $11511-2$ & B & Fuel Body \\
\hline $11511-3$ & B & Fuel Hole Plug \\
\hline $11511-4$ & B & Compact Pusher \\
\hline $11511-5$ & B & Sample Hole Plug \\
\hline $11511-6$ & B & Sample Hole Plug \\
\hline $11511-7,8,9,10$ & B & Fuel Stack Assembly \\
\hline $11511-15$ & B & Graphite Cement (P-511) \\
\hline $11511-16$ & B & Polystyrene Solution \\
\hline $11511-17$ & B & Graphite Spacer \\
\hline $11511-18,19$ & B & Fission Product Release Sample \\
\hline $11511-20,21,22$ & B & Advanced Fuel Rod Samples \\
\hline $\begin{array}{l}11511-24,25,26,27 \\
28,29,30\end{array}$ & B & Fission Product Release Sample \\
\hline $11511-31$ & B & Graphite Spacer \\
\hline $11512-1$ & B & Fuel Body Assembly 6-3 \\
\hline $11512-2$ & B & Fue1 Body \\
\hline $11512-3$ & B & Fuel Hole Plug \\
\hline $11512-4$ & B & Compact Pusher \\
\hline $11512-5$ & B & Sample Hole Plug \\
\hline $11512-6$ & B & Sample Hole Plug \\
\hline $11512-7,8,9,10$ & B & Fuel Stack Assembly \\
\hline $11512-14$ & B & Polystyrene Solution \\
\hline $11512-15$ & B & Graphite Cement (P-511) \\
\hline $11512-16$ & B & Graphite Spacer \\
\hline $11512-17,18$ & B & Therma1 Stability Sample, Type 1 \\
\hline $11512-19,20,21,22,23$ & B & Thermal Stability Sample, Type 2 \\
\hline $11512-24,25$ & $\mathrm{~B}$ & Boronated Graphite Sample \\
\hline $11512-26$ & B & Graphite Spacer \\
\hline
\end{tabular}


TABLE 2-2

PEACH BOTTOM GRAPHITE MATERIALS USED IN FUEL ELEMENTS

\begin{tabular}{|c|c|c|c|c|c|c|}
\hline \multirow[b]{2}{*}{ Fuel Element Component } & \multicolumn{2}{|c|}{$\begin{array}{l}\text { Core } 1 \text { Driver } \\
\text { Graphite Type }\end{array}$} & \multicolumn{2}{|c|}{$\begin{array}{l}\text { Core } 2 \text { Driver } \\
\text { Graphite Type }\end{array}$} & \multicolumn{2}{|c|}{$\begin{array}{c}\text { FTE-3, }-4,-5,-6 \\
\text { Graphite Type }\end{array}$} \\
\hline & Base Stock & Manufacturer & Base Stock & Manufacturer & Base Stock & Manufacturer \\
\hline Top reflector & $\mathrm{H}-253^{(\mathrm{a})}$ & GLCC & $\mathrm{H}-381^{(\mathrm{b})}$ & GLCC & $\mathrm{H}-381^{(b)}$ & GLCC \\
\hline Top spine & $711-G S X Y$ & Speer & $711-T$ & Speer & $\mathrm{H}-327$ & GLCC \\
\hline $\begin{array}{l}\text { Lower two spines (with } \mathrm{T} / \mathrm{C} \\
\text { slots) }\end{array}$ & $711-G S X Y$ & Speer & $711-G S X Y$ & Speer & $\mathrm{H}-327$ & GLCC \\
\hline Fuel compact or rod & & & & & & \\
\hline Filler & GP-38 flour & UCC & $\mathrm{GP}-38$ flour & UCC & 6353 & Ashbury \\
\hline Binder & Barrett No. 30 & $\begin{array}{l}\text { Allied } \\
\text { Chemical }\end{array}$ & Barrett No. 30 & $\begin{array}{l}\text { Allied } \\
\text { Chemical }\end{array}$ & $15 \mathrm{~V}$ & $\begin{array}{l}\text { Allied } \\
\text { Chemical }\end{array}$ \\
\hline Graphite fuel body & $\mathrm{NA}(\mathrm{c})$ & & NA & & $\mathrm{H}-327$ & GLCC \\
\hline Sleeve & $\mathrm{H}-253^{(\mathrm{a})}$ & GLCC & $\mathrm{H}-382^{(b)}$ & GLCC & $H-382^{(b)}$ & GLCC \\
\hline Internal trap & $806 \mathrm{RL}$ & Speer & 580 & Speer & 580 & Speer \\
\hline Lower reflector & $806 \mathrm{RL}$ & Speer & 580 & Speer & $\mathrm{H}-327$ & GLCC \\
\hline Bottom connector & $\mathrm{H}-253^{(\mathrm{a})}$ & GLCC & $\mathrm{H}-381$ & GLCC & $\mathrm{H}-253^{(\mathrm{a})}$ & GLCC \\
\hline
\end{tabular}

(a) Also referred to as HLM-85-10, made with Texas Lockport coke.

(b) Also referred to as HLM-85-10, made with "Y" coke. Discrimination into H-381 and H-382 1acks explanation. It should be noted that most of the Core 1 and all Core 2 HLM-85-10 material was graphitized in a standard Acheson-type furnace for about 28 days with temperatures up to $2800^{\circ} \mathrm{C}$. However, some of the Core 1 material went through a rapid graphitization process in a tube furnace for about $1 \mathrm{hr}$ at $2800^{\circ} \mathrm{C}$ (which also has been used for impregnation purposes of Core 1 and 2 sleeves after machining). About 40 elements in Core 2 were made out of leftover Core 1 rapid graphitized material. This material happened to shrink more than the Core 2 standard material, as realized during the Core 2 unloading exercise.

(c) $\mathrm{NA}=$ not applicable. 
TABLE $2-3$
FTE-6 FUEL PARTICLE ATTRIBUTES
(a)

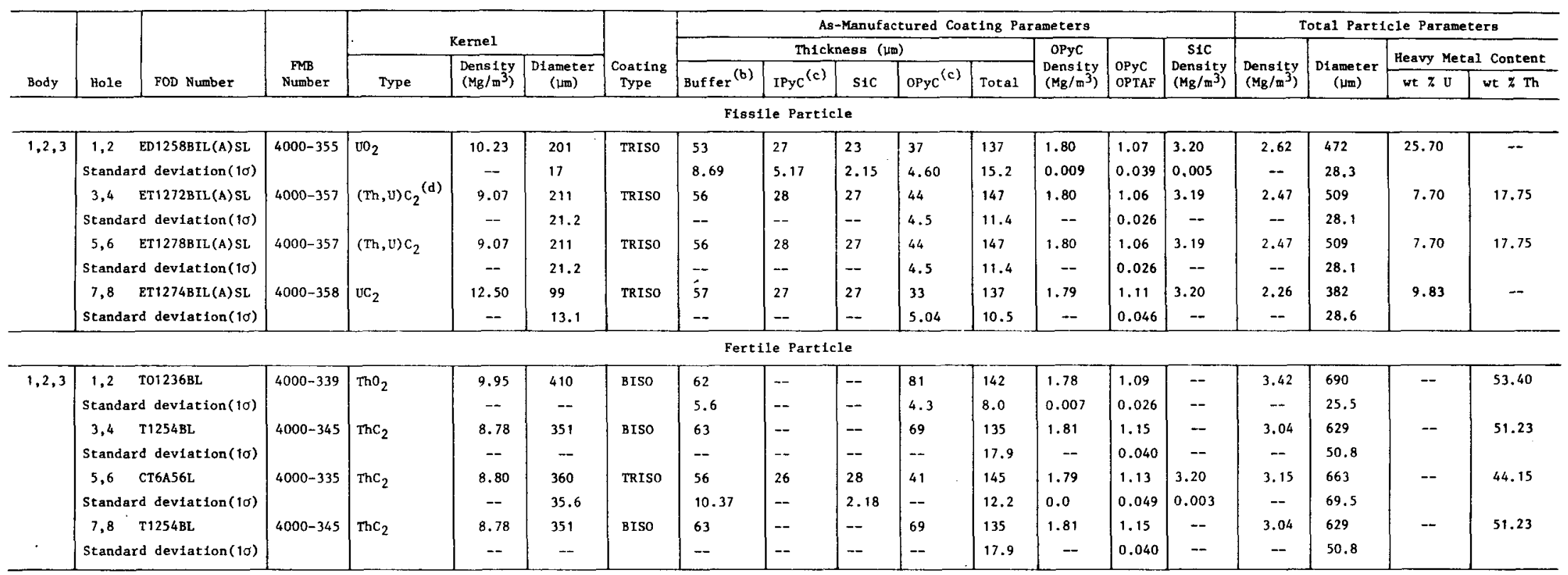

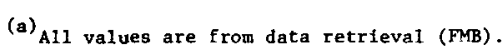

(b) $\mathrm{C}_{2} \mathrm{H}_{2}$ coating gas.

(c) $\mathrm{C}_{3} \mathrm{H}_{6}$ coating gas.

(d) $\mathrm{Th} / \mathrm{U}$ ratio $=2.75$. 
TABLE 2-4
FTE-6 FUEL ROD ATTRIBUTES
$($ a)

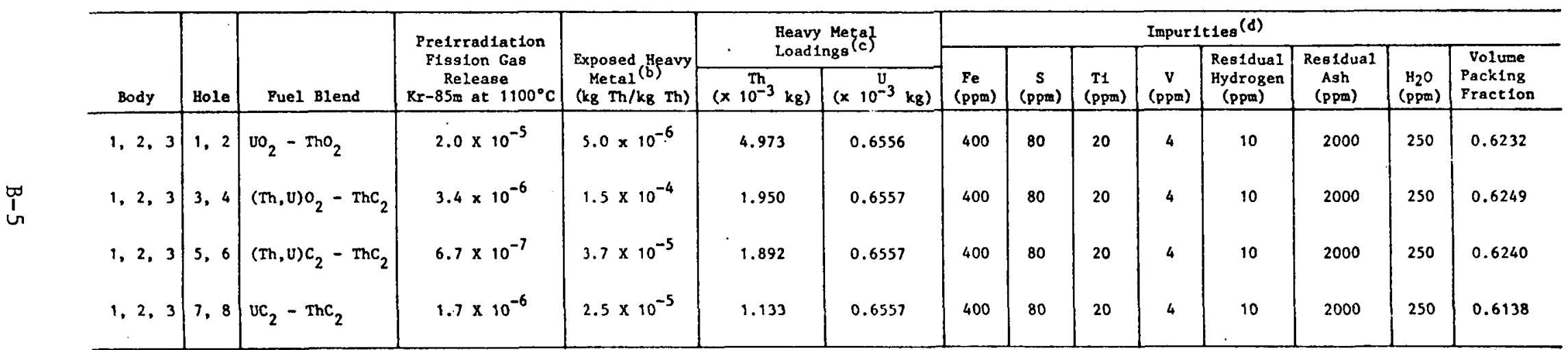

(a) Representative rod attributes, all values retrleved from Quality Control records.

(b) Determined by hydrolysis technique.

(c) Design loadings (nominal values).

(d) Nitrogen <1000 ppm determined on similar production runs. 
TABLE 2-5

PREIRRADIATION DATA FOR FTE-6 TYPE II THERMAL STABILITY SPINE SAMPLES

\begin{tabular}{|c|c|c|c|c|c|c|c|c|c|c|c|c|c|c|c|c|}
\hline \multirow{5}{*}{$\begin{array}{c}\text { FMB } \\
\text { No:(a) }\end{array}$} & \multirow{2}{*}{\multicolumn{3}{|c|}{ Kernel }} & \multirow{5}{*}{$\begin{array}{l}\text { Coating } \\
\text { Type }\end{array}$} & \multirow{2}{*}{\multicolumn{8}{|c|}{ As-Manufactured Coating Parameters }} & \multirow{5}{*}{$\begin{array}{c}\text { Density } \\
\left(\mathrm{Mg} / \mathrm{m}^{3}\right)\end{array}$} & \multirow{5}{*}{$\begin{array}{c}\text { Diameter } \\
(\mu \mathrm{m})\end{array}$} & \multirow{3}{*}{\multicolumn{2}{|c|}{$\begin{array}{c}\text { Hameters } \\
\text { Heavy Metal } \\
\text { Content }\end{array}$}} \\
\hline & & & & & & & & & & & & & & & & \\
\hline & \multirow[b]{3}{*}{ Type } & \multirow{3}{*}{$\begin{array}{l}\text { Density } \\
\left(\mathrm{Mg} / \mathrm{m}^{3}\right)\end{array}$} & \multirow{3}{*}{$\begin{array}{c}\text { Nominal } \\
\text { Diameter } \\
(\mu \mathrm{m})\end{array}$} & & \multirow{2}{*}{\multicolumn{5}{|c|}{ Mean Thickness $(\mu \mathrm{m})$}} & \multirow{3}{*}{$\begin{array}{l}\text { OPyC } \\
\text { Density } \\
\left(\mathrm{Mg} / \mathrm{m}^{3}\right)\end{array}$} & \multirow{3}{*}{$\begin{array}{l}\text { OPyC } \\
\text { OPTAF (d) }\end{array}$} & \multirow{3}{*}{$\begin{array}{c}\text { SiC } \\
\text { Dens1ty } \\
\left(\mathrm{Mg}_{\mathrm{g}} / \mathrm{m}^{3}\right)\end{array}$} & & & & \\
\hline & & & & & & & & & & & & & & & $\mathrm{U}$ & \\
\hline & & & & & $\begin{array}{l}\text { Buffer } \\
\text { (b) }\end{array}$ & $\begin{array}{l}\text { IPyC } \\
\text { (c) }\end{array}$ & S1C & $\begin{array}{l}\text { OPyC } \\
\text { (c) }\end{array}$ & Total & & & & & & (wt $z$ ) & (wt X) \\
\hline $4000-242$ & $\mathrm{ThC}_{2}$ & 8.86 & 384 & TRISO & 46 & 28 & 23 & 42 & 133 & 1.71 & 1.04 & 3.19 & 3.30 & 650 & - & 48.71 \\
\hline $4000-302$ & $\mathrm{UC}_{2}$ & 10.43 & 100 & TRISO & 50 & 18 & 20 & 36 & 122 & 1.71 & 1.05 & 3.19 & 2.05 & 344 & 11.57 & -- \\
\hline
\end{tabular}

(a) Fuel Materials Branch data retrleval number.

(b) $\mathrm{C}_{2} \mathrm{H}_{2}$ coating gas.

(c) $\mathrm{C}_{3} \mathrm{H}_{6}$ coating gas.

(d) BAF disc OPTAF.

(e) Density in air. 
TABLE 2-6(a)

FTE-6 SPINE SAMPLE, BURNUP AND FLLUENCE TABULATION (CALCULATED VALUUE)

\begin{tabular}{|c|c|c|c|c|c|c|c|c|c|c|c|c|}
\hline \multirow[b]{3}{*}{ Postition } & \multirow{2}{*}{\multicolumn{2}{|c|}{ Pre-Irrad. }} & \multirow{3}{*}{$\begin{array}{c}\text { Mean } \\
\text { Core } \\
\text { He1ght (a) } \\
\text { (mm) }\end{array}$} & \multirow[b]{3}{*}{ Sample Type } & \multirow[b]{3}{*}{$\begin{array}{l}\text { Ident. } \\
\text { Number }\end{array}$} & \multirow{2}{*}{\multicolumn{2}{|c|}{$\begin{array}{c}\text { Composite } \\
\text { Spine Length } \\
\text { (nm) }\end{array}$}} & \multirow{2}{*}{\multicolumn{3}{|c|}{ FIMA }} & \multicolumn{2}{|c|}{ Fluence } \\
\hline & & & & & & & & & & & \multirow{2}{*}{$\begin{array}{c}\text { Fast } \\
\left(\begin{array}{c}x \quad 10^{25} \mathrm{n} / \mathrm{m}^{2} \dot{d} \\
\mathbf{E}>0.18 \mathrm{MeV}\end{array}\right)\end{array}$} & \multirow{2}{*}{ 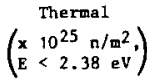 } \\
\hline & \begin{tabular}{|c|} 
Length \\
(mm)
\end{tabular} & $\begin{array}{c}\text { Welght } \\
\text { (kg) }\end{array}$ & & & & $\begin{array}{c}\text { Pre- } \\
\text { Irrad. }\end{array}$ & $\begin{array}{l}\text { Post- } \\
\text { Irrad. }\end{array}$ & $\begin{array}{c}\text { Fissile } \\
(z)\end{array}$ & $\begin{array}{c}\text { Fertile } \\
(z)\end{array}$ & $\begin{array}{l}\text { Mixed } \\
(z)\end{array}$ & & \\
\hline \multicolumn{13}{|l|}{ Body 1} \\
\hline$\Delta$ & 9.652 & & & & & & & & & & & \\
\hline 1 & 45.720 & 0.04090 & 668 & Graphtce spacer & 12 & & & 19.80 & 0.14 & 4.16 & 0.90 & 1.11 \\
\hline 2 & 96.520 & 0.07721 & 739 & Boronated graphite & $4665-57-1$ & & & 25.44 & 0.31 & 5.45 & 1.31 & 1.45 \\
\hline 3 & 32.004 & 0.02453 & 803 & DIffusion & 23 & & & 29.57 & 0.46 & 6.42 & 1.59 & 1.77 \\
\hline 4 & 32.004 & 0.02469 & 835 & Diffuston & 40 & & & 31.49 & 0.54 & & 1.73 & 1.93 \\
\hline 5 & 32.004 & 0.02471 & 867 & Diffusion & 51 & & & 33.32 & 0.62 & 7.31 & 1.84 & 2.09 \\
\hline 6 & 32.004 & 0.02513 & 899 & Diffusion & 60 & & & 34.67 & 0.70 & 7.64 & 1.95 & 2.24 \\
\hline 7 & 32.004 & 0.02448 & 931 & Deffusion & 13 & & & 36.02 & 0.77 & 7.98 & 2.06 & 2.40 \\
\hline 8 & 32.004 & 0.02475 & 963 & Fisaion product release & $73-8$ & 724.66 & 719.40 & 37.37 & 0.85 & 8.32 & 2.14 & 2.55 \\
\hline 9 & 32.004 & 0.02501 & 995 & Fisaion product release & $56-P$ & The & & 38.34 & 0.93 & 8.58 & 2.23 & 2.70 \\
\hline 10 & 96.520 & 0.07853 & 1059 & Boronated graphite & $4465-57-2$ & $\frac{\Delta L}{I}=-0$ & $.7255 \%$ & 40.04 & 1.08 & 9.04 & 2.37 & 2.97 \\
\hline 11 & 96.520 & 0.07899 & 1156 & Boronated graphite & $4465-57-3$ & & & 41.78 & 1.24 & 9.53 & 2.55 & 3.31 \\
\hline 12 & 32.004 & 0.02472 & 1220 & Diffuston & 17 & & & 42.69 & 1.32 & 9.78 & 2.65 & 3.49 \\
\hline 13 & 32.004 & 0.02455 & 1252 & Diffusion & 33 & & & 43.08 & 1.36 & 9.89 & 2.68 & 3.55 \\
\hline 14 & 32.004 & 0.02452 & 1284 & Diffusion & 19 & & & 43,48 & 1.40 & 10.00 & 2.71 & 3.60 \\
\hline 15 & 32.004 & 0.02463 & 1316 & D1ffusion & 26 & & & 43.82 & 1.43 & 10.10 & 2.74 & 3.65 \\
\hline 16 & 37.338 & 0.03358 & 1351 & Graphtte spacer & 13 & & & 44.16 & 1.45 & 10.19 & 2.77 & 3.70 \\
\hline$\Delta$ & 52.857 & & & & & & & & & & & \\
\hline$\Sigma$ & 787.174 & & & & & & & & & & & \\
\hline \multicolumn{13}{|l|}{ Body 2} \\
\hline$\Delta$ & 9.652 & & & & & & & & & & & \\
\hline 1 & 63.500 & 0.05738 & 1464 & Graphite spacer & 14 & & & 44.66 & 1.49 & 10.32 & 2.81 & 3.76 \\
\hline 2 & 31.496 & 0.02494 & 1511 & Fission product release & $122-\mathrm{P}$ & & & 44.98 & 1.52 & 10.41 & 2.83 & 3.81 \\
\hline 3 & 31.750 & 0.02500 & 1543 & F1ssion product release & $168-P$ & & & 45.18 & 1.54 & 10.46 & 2.85 & 3.83 \\
\hline 4 & 101.854 & 0.09358 & 1609 & Advanced fuel rods & $3 c$ & & & 45.44 & 1.56 & 10.53 & 2.87 & 3.88 \\
\hline 5 & 101.600 & 0.09108 & 1711 & Advanced fuel rods & 36 & & & 45.47 & 1.55 & 10.54 & 2.88 & 3.92 \\
\hline 6 & 101.600 & 0.09257 & 1813 & Advanced fuel rods & $3 a$ & & & 45.10 & 1.52 & 10.43 & 2.87 & 3.92 \\
\hline 7 & 31.750 & 0.10158 & 1880 & Fission product release & NB-4 & & & 44.63 & 1.48 & 10.31 & 2.85 & 3.90 \\
\hline 8 & 31.750 & 0.02455 & 1912 & Fisston product release & $12-p$ & $>723.39$ & 720.98 & 44.40 & 1.47 & 10.25 & 2.84 & 3.88 \\
\hline 9 & 31.750 & 0.10144 & 1943 & Fission product release & NB-7 & & & 44.16 & 1.44 & 10.18 & 2.83 & 3.85 \\
\hline 10 & 31.750 & 0.02506 & 1975 & Fission product release & 29-p & $\frac{\Delta L}{I}=-0$ & $.3329 \%$ & 43.91 & 1.42 & 10.11 & 2.81 & 3.82 \\
\hline 11 & 31.750 & 0.10158 & 2007 & Fission product release & NB-11 & & & 43.67 & 1.39 & 10.04 & 2.79 & 3.78 \\
\hline 12 & 31.750 & 0.02467 & 2039 & Fission product release & $44-P$ & & & 43.34 & 1.37 & 9.95 & 2.77 & 3.74 \\
\hline 13 & 31.750 & 0.10167 & 2070 & Fission product release & NB-15 & & & 43.01 & 1.34 & 9.86 & 2.75 & 3.67 \\
\hline 14 & 31.750 & 0.02460 & 2102 & Fission product release & $148-P$ & & & 42.67 & 1.31 & 9.77 & 2.73 & 3.64 \\
\hline 15 & 37.338 & 0.03367 & 2137 & Graphite spacer & 15 & & & 42.28 & 1.28 & 9.66 & 2.70 & 3.57 \\
\hline$\Delta$ & 52.400 & & & & & & & & & & & \\
\hline$\Sigma$ & 785.444 & & & & & & & & & & & \\
\hline \multicolumn{13}{|l|}{ Body 3} \\
\hline$\Delta$ & 9.652 & & & & & & & & & & & \\
\hline 1 & 63.500 & 0.05725 & 2249 & Graphite spacer & 16 & & & 41.66 & 1.22 & 9.49 & 2.65 & 3.46 \\
\hline 2 & 146.050 & 0.13749 & 2354 & Thermal stability - 1 & $M$ & & & 39.81 & 1.08 & 9.00 & 2.51 & 3.20 \\
\hline 3 & 146.050 & 0.13741 & 2500 & Theraal stability - 1 & $N$ & & & 36.44 & 0.86 & 8.13 & 2.27 & 2.78 \\
\hline 4 & 31.750 & 0.02603 & 2589 & Thermal stablifty - 2 & 18 & & & 33.96 & 0.71 & 7.51 & 2.09 & 2.50 \\
\hline 5 & 31.750 & 0.02383 & 2620 & Theraal stab111ty - 2 & 24 & 725.17 & 721.61 & 33.06 & 0.66 & 7.29 & 2.01 & 2.39 \\
\hline 6 & 31.750 & 0.02390 & 2652 & Thermal stability - 2 & 30 & & & 32.12 & 0.61 & 7.06 & 1.93 & 2.28 \\
\hline 7 & 37.750 & 0.02463 & 2684 & Thermal stability - 2 & 6 & $\frac{\Delta \mathrm{L}}{\Delta}=-0$. & $.4904 \pi$ & 31.18 & 0.56 & 6.59 & 1.84 & 2.18 \\
\hline 8 & 31.750 & 0.02479 & 2716 & Thermal stability - 2 & 12 & & & 30.25 & 0.51 & 6.59 & 1.75 & 2.09 \\
\hline 9 & 96.520 & 0.07988 & 2780 & Boronated graphite & $4465-57-4$ & & & 28.41 & 0.40 & 6.13 & 1.53 & 1.92 \\
\hline 10 & 96.520 & 0.07855 & 2876 & Boronated graphite & $4465-57-5$ & & & 25.64 & 0.25 & 5.14 & 1.10 & 1.71 \\
\hline 11 & 17.780 & 0.01598 & 2934 & GraphIte apacer & & & & 23.99 & 0.16 & 5.03 & 0.76 & 1.61 \\
\hline$\Delta$ & 52.654 & & & & & & & & & & & \\
\hline$\Sigma$ & 787,476 & & & & & & & & & & & \\
\hline
\end{tabular}

(a) Counted from core bed plate. 
TABLE 2-6(b)

FTE-6 SPINE SAMPLE TEMPERATURE (K) TABULATION

CASE A (LINEAR)

CASE B CARRMENIUS?

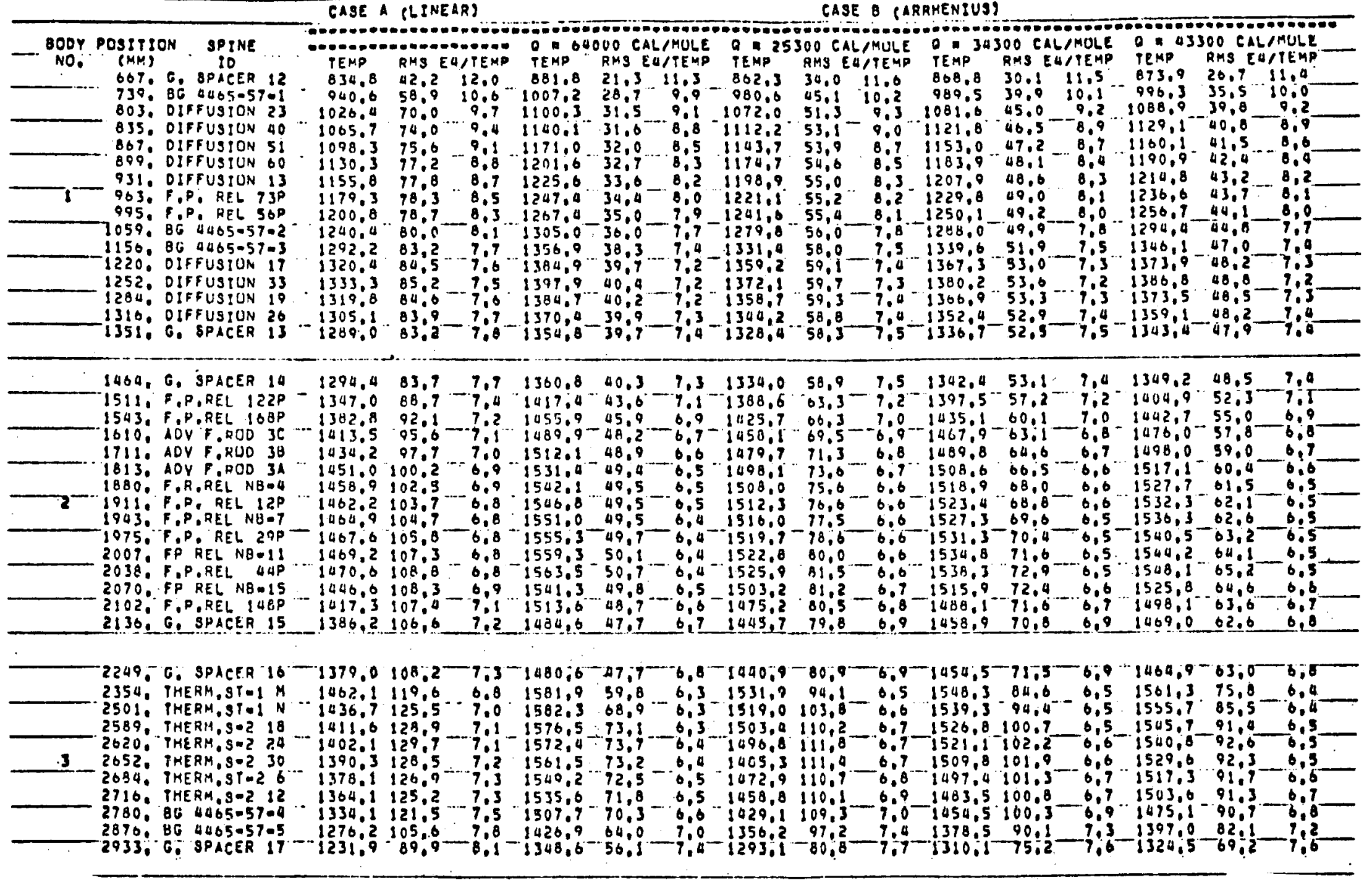


TABLE 2-7

FTE-6 PURGE FLOW DESCRIPTION

\begin{tabular}{|c|c|c|c|c|c|c|c|c|c|c|c|}
\hline \multirow[b]{3}{*}{$\begin{array}{l}\text { Puel } \\
\text { Body }\end{array}$} & \multirow{2}{*}{\multicolumn{3}{|c|}{ Beginning of Life }} & \multicolumn{8}{|c|}{ End of Life } \\
\hline & & & & \multicolumn{4}{|c|}{ Measured (a) } & \multicolumn{4}{|c|}{ Calculated (b) } \\
\hline & $\begin{array}{l}\text { Inner } \\
\text { Sleeve Radius } \\
\text { (in.) }\end{array}$ & $\begin{array}{l}\text { Outer Fuel } \\
\text { Body Rad1us } \\
\text { (In.). }\end{array}$ & $\begin{array}{l}\text { Flow } \\
\text { Area } \\
\text { (in. 2) }\end{array}$ & $\begin{array}{l}\text { Inner } \\
\text { Sleeve Radius } \\
\text { (In.) }\end{array}$ & $\begin{array}{l}\text { Outer Fuel } \\
\text { Body Radlus } \\
\text { (1n.) }\end{array}$ & $\begin{array}{l}\text { Plow } \\
\text { Area } \\
\left(1 n .{ }^{2}\right)\end{array}$ & $\begin{array}{c}\text { Relative Change } \\
\mathrm{A}_{2} / \mathrm{A}_{1}-1 \\
(\mathrm{Z})\end{array}$ & $\begin{array}{l}\text { Inner } \\
\text { Sleeve Radtus } \\
\text { (In.) }\end{array}$ & $\begin{array}{l}\text { Outer Puel } \\
\text { Body Radilus } \\
\text { (In.) }\end{array}$ & $\begin{array}{c}\text { Flow } \\
\text { Area } \\
(\text { In. 2) }\end{array}$ & $\begin{array}{l}\text { Relative Change } \\
\mathrm{A}_{2} / \mathrm{A}_{1}-1 \\
(\overline{(z)})\end{array}$ \\
\hline 1 & 1.3761 & 1.370 & .053 & 1.3730 & 1.3682 & .041 & -22.6 & 1.3732 & 1.3682 & .043 & -18.9 \\
\hline 2 & 1.3761 & 1.370 & .053 & 1.3726 & 1.3657 & .060 & +13.2 & 1.3711 & 1.3657 & .047 & -11.3 \\
\hline 3 & 1.3761 & 1.370 & .053 & 1.3706 & 1.3664 & .036 & -32.1 & 1.3727 & 1.3664 & .054 & +1.9 \\
\hline
\end{tabular}

(a) The inner sleeve rad11 are obtained from the BOL inner sleeve radius and the BOL and BOL outer sleeve dlameter measurements. outer fuel body rad11 are obtalned from fuel body diameter measurements.

(b) The inner sleeve radil are obtalned from the BOL Inner sleeve radius and the sleeve strains calculated by the TREVER code. outer fuel body radil are obtained from fuel body diameter measurements. 
TABLE 3-2

FTE-6 FAST AND THERMAL FLUENCE CALCULATIONS

\begin{tabular}{|c|c|c|c|c|c|c|}
\hline $\begin{array}{l}\text { Fuel } \\
\text { Body }\end{array}$ & $\begin{array}{l}\text { Fue 1 } \\
\text { Rod }\end{array}$ & $\begin{array}{l}\text { Core Height } \\
\text { (MM) } \\
\text { (BOL) }\end{array}$ & $\begin{array}{l}\text { Calculated } \\
\text { Fast Fluence } \\
\left(10^{25} \mathrm{n} / \mathrm{m}^{2}\right) \\
(\mathrm{E}>29 \mathrm{fJ})\end{array}$ & $\begin{array}{l}\text { Calculated } \\
\text { Thermal Fluence } \\
\left(10^{25} \mathrm{n} / \mathrm{m}^{2}\right) \\
(E<0.38 \mathrm{aJ})\end{array}$ & $\begin{array}{l}\text { Measured Thermal } \\
\text { Fluence Profile } \\
\left(10^{25} \mathrm{n} / \mathrm{m}^{2}\right)\end{array}$ & $\frac{\text { Calculated }}{\text { Measured }}-1$ \\
\hline 1 & $\begin{array}{l}1 \\
2 \\
3 \\
4 \\
5 \\
6 \\
7 \\
8 \\
9 \\
10 \\
11 \\
12 \\
13 \\
14\end{array}$ & $\begin{array}{r}689 . \\
738 \\
788 \\
837 \\
887 \\
936 . \\
986 . \\
1036 . \\
1085 \\
1134 \\
1184 \\
1233 \\
1283 . \\
1332 .\end{array}$ & $\begin{array}{l}.92 \\
1.22 \\
1.47 \\
1.69 \\
1.88 \\
2.05 \\
2.19 \\
2.32 \\
2.42 \\
2.52 \\
2.60 \\
2.67 \\
2.72 \\
2.76\end{array}$ & $\begin{array}{l}1.12 \\
1.37 \\
1.63 \\
1.88 \\
2.14 \\
2.39 \\
2.66 \\
2.87 \\
3.07 \\
3.25 \\
3.41 \\
3.52 \\
3.61 \\
3.69\end{array}$ & $\begin{array}{l}1.42 \\
1.70 \\
1.96 \\
2.20 \\
2.40 \\
2.55 \\
2.60 \\
2.60 \\
2.67 \\
2.81 \\
3.09 \\
3.33 \\
3.48 \\
3.58\end{array}$ & $\begin{array}{r}-21.89 \\
-19.28 \\
-16.67 \\
-14.33 \\
-10.22 \\
-6.27 \\
1.15 \\
10.23 \\
15.02 \\
15.28 \\
9.28 \\
5.91 \\
3.78 \\
3.14\end{array}$ \\
\hline \multicolumn{2}{|c|}{$\begin{array}{l}\text { Mean } \\
\text { Range/RMS }\end{array}$} & $\begin{array}{r}1011 \\
693 .\end{array}$ & $\begin{array}{l}2.10 \\
\pm .57\end{array}$ & $\begin{array}{r}2.61 \\
\pm .84\end{array}$ & $\begin{array}{r}2.60 \\
\pm 0.62\end{array}$ & $\begin{array}{r}-1.776 \\
\pm 12.375\end{array}$ \\
\hline 2 & $\begin{array}{l}1 \\
2 \\
3 \\
4 \\
5 \\
6 \\
7 \\
8 \\
9 \\
10 \\
11 \\
12 \\
13 \\
14\end{array}$ & $\begin{array}{l}1479 . \\
1529 . \\
1578 . \\
1628 \\
1677 \\
1727 . \\
1776 . \\
1826 . \\
1875 \\
1925 \\
1974 \\
2024 \\
2073 . \\
2123 .\end{array}$ & $\begin{array}{l}2.80 \\
2.83 \\
2.86 \\
2.87 \\
2.88 \\
2.88 \\
2.88 \\
2.87 \\
2.86 \\
2.84 \\
2.81 \\
2.78 \\
2.74 \\
2.71\end{array}$ & $\begin{array}{l}3.75 \\
3.80 \\
3.85 \\
3.88 \\
3.90 \\
3.92 \\
3.93 \\
3.92 \\
3.90 \\
3.87 \\
3.82 \\
3.76 \\
3.68 \\
3.59\end{array}$ & $\begin{array}{l}3.62 \\
3.66 \\
3.67 \\
3.68 \\
3.69 \\
3.70 \\
3.70 \\
3.61 \\
3.59 \\
3.56 \\
3.57 \\
3.58 \\
3.60 \\
3.63\end{array}$ & $\begin{array}{r}3.47 \\
3.97 \\
4.56 \\
5.40 \\
5.67 \\
5.82 \\
6.04 \\
8.61 \\
8.75 \\
8.52 \\
7.03 \\
4.96 \\
2.16 \\
-1.06\end{array}$ \\
\hline \multicolumn{2}{|c|}{$\begin{array}{l}\text { Mean } \\
\text { Range/RMS }\end{array}$} & $\begin{array}{r}1801 . \\
693 .\end{array}$ & $\begin{array}{l}2.828 \\
\pm .052\end{array}$ & $\begin{array}{l}3.83 \\
\pm .10\end{array}$ & $\begin{array}{r}3.63 \\
\pm 0.05\end{array}$ & $\begin{array}{r}5.279 \\
\pm 2.581\end{array}$ \\
\hline 3 & $\begin{array}{l}1 \\
2 \\
3 \\
4 \\
5 \\
6 \\
7 \\
8 \\
9 \\
10 \\
11 \\
12 \\
13 \\
14\end{array}$ & $\begin{array}{l}2269 . \\
2318 . \\
2368 \\
2418 \\
2467 \\
2517 \\
2566 \\
2616 \\
2665 \\
2714 \\
2764 \\
2814 \\
2863 \\
2913\end{array}$ & $\begin{array}{l}2.65 \\
2.59 \\
2.52 \\
2.45 \\
2.36 \\
2.27 \\
2.16 \\
2.04 \\
1.91 \\
1.76 \\
1.61 \\
1.40 \\
1.17 \\
.86\end{array}$ & $\begin{array}{l}3.47 \\
3.35 \\
3.22 \\
3.08 \\
2.93 \\
2.78 \\
2.61 \\
2.44 \\
2.26 \\
2.11 \\
1.96 \\
1.84 \\
1.73 \\
1.64\end{array}$ & $\begin{array}{l}3.51 \\
3.34 \\
3.27 \\
3.22 \\
3.14 \\
2.98 \\
2.88 \\
2.80 \\
2.68 \\
2.47 \\
2.17 \\
1.97 \\
1.86 \\
1.79\end{array}$ & $\begin{array}{r}-0.68 \\
0.39 \\
-1.46 \\
-4.40 \\
-6.58 \\
-6.92 \\
-9.87 \\
-12.87 \\
-15.37 \\
-14.70 \\
-8.97 \\
-6.78 \\
-6.60 \\
-8.70\end{array}$ \\
\hline \multicolumn{2}{|c|}{$\begin{array}{l}\text { Mean } \\
\text { Range/RMS }\end{array}$} & $\begin{array}{r}2591 . \\
693 .\end{array}$ & $\begin{array}{r}1.98 \\
\pm .54\end{array}$ & $\begin{array}{l}2.53 \\
\pm .60\end{array}$ & $\begin{array}{r}2.72 \\
\pm 0.56\end{array}$ & $\begin{array}{l}-7.394 \\
\pm 4.698\end{array}$ \\
\hline $\begin{array}{r}\text { Total } \\
\text { Total } \\
\text { /RMS }\end{array}$ & $\begin{array}{l}\text { Mean } \\
\text { Range }\end{array}$ & $\begin{array}{l}1801 \\
2273\end{array}$ & $\begin{array}{l}2.30 \\
\pm .59\end{array}$ & $\begin{array}{l}2.99 \\
\pm .84\end{array}$ & $\begin{array}{l}2.98 \\
\pm .67\end{array}$ & $\begin{array}{l}-1.297 \\
\pm 9.354\end{array}$ \\
\hline
\end{tabular}


TABLE 3-3(a)

FTE-6 TABULATION OF IRRADIATION TEMPERATURES FOR HOLES 1 AND 2 (UO ${ }_{2}$ TRISO, ThO ${ }_{2}$ BISO FUEL RODS)

\begin{tabular}{|c|c|c|c|c|c|c|c|c|c|c|c|c|c|c|c|c|c|}
\hline \multirow[b]{2}{*}{ Fuel Body } & \multirow{2}{*}{$\begin{array}{l}\text { Mean } \mathrm{Pqs} \\
\text { Core } \mathrm{Ht}(\mathrm{a}) \\
\text { BOL (MM) }\end{array}$} & \multicolumn{6}{|c|}{ Temperature Envelope (C) } & \multicolumn{10}{|c|}{ Time Weighted Averages (C) } \\
\hline & & $\begin{array}{l}\text { Fue 1 } \\
\text { Max }\end{array}$ & $\begin{array}{l}\text { Fuel } \\
\text { Min }\end{array}$ & $\begin{array}{l}\text { Body } \\
\text { Max }\end{array}$ & $\begin{array}{l}\text { Body } \\
\text { Min }\end{array}$ & $\begin{array}{l}\operatorname{Siv} \\
\operatorname{Max}\end{array}$ & $\begin{array}{l}\text { Slv } \\
\text { Min }\end{array}$ & $\begin{array}{l}\text { Fuel } \\
\text { Max }\end{array}$ & $\begin{array}{c}\text { Fue } \\
\text { Avg }\end{array}$ & $\begin{array}{l}\text { Fuel } \\
\text { Min }\end{array}$ & $\begin{array}{l}\text { Body } \\
\text { Max }\end{array}$ & $\begin{array}{c}\text { Body } \\
\text { Avg }^{(b)}\end{array}$ & $\begin{array}{l}\text { Body } \\
\text { Min }\end{array}$ & $\begin{array}{l}\text { S1v } \\
\operatorname{Max}\end{array}$ & $\begin{array}{c}S 1 y \\
A v_{g}(a)\end{array}$ & $\begin{array}{l}\text { S1v } \\
\text { Min }\end{array}$ & Coolant \\
\hline $\begin{array}{c}\text { Body } \\
1\end{array}$ & $\begin{array}{r}689 . \\
738 . \\
788 . \\
837 . \\
887 . \\
936 . \\
986 . \\
1036 . \\
1085 . \\
1134 . \\
1184 . \\
1233 . \\
1283 . \\
1332 .\end{array}$ & $\begin{array}{r}713 . \\
802 . \\
882 . \\
948 . \\
996 . \\
1037 . \\
1070 . \\
1098 . \\
1123 . \\
1148 . \\
1174 . \\
1196 . \\
1191 . \\
1167 .\end{array}$ & $\begin{array}{l}482 . \\
517 . \\
552 . \\
587 . \\
619 . \\
647 . \\
672 . \\
695 . \\
715 . \\
734 . \\
751 . \\
767 . \\
762 . \\
744 .\end{array}$ & $\begin{array}{l}573 . \\
630 . \\
682 . \\
725 . \\
759 . \\
788 . \\
814 . \\
837 . \\
858 . \\
881 . \\
904 . \\
925 . \\
921 . \\
901 .\end{array}$ & $\begin{array}{l}447 . \\
478 . \\
508 . \\
537 . \\
564 . \\
588 \\
609 . \\
629 . \\
647 . \\
663 . \\
679 . \\
694 . \\
690 . \\
673 .\end{array}$ & $\begin{array}{l}486 . \\
532 . \\
573 . \\
605 . \\
630 . \\
651 . \\
671 . \\
687 . \\
703 . \\
718 . \\
733 . \\
745 . \\
741 . \\
726 .\end{array}$ & $\begin{array}{l}380 . \\
408 . \\
431 . \\
449 . \\
466 . \\
480 . \\
492 . \\
504 . \\
516 . \\
527 . \\
538 . \\
548 . \\
545 . \\
535 .\end{array}$ & \begin{tabular}{|r|}
619. \\
688. \\
754. \\
819. \\
872. \\
916. \\
953. \\
985. \\
1016. \\
1043. \\
1068. \\
1088. \\
1083. \\
1059.
\end{tabular} & $\begin{array}{r}590 . \\
652 . \\
713 . \\
772 . \\
820 . \\
860 . \\
894 . \\
924 . \\
952 . \\
977 . \\
1000 . \\
1019 . \\
1014 . \\
992 .\end{array}$ & $\begin{array}{l}560 . \\
617 . \\
672 . \\
725 . \\
768 . \\
804 . \\
835 . \\
862 . \\
888 . \\
911 . \\
932 . \\
950 . \\
945 . \\
925 .\end{array}$ & $\begin{array}{l}515 . \\
563 . \\
608 . \\
651 . \\
687 . \\
718 . \\
745 . \\
769 . \\
792 . \\
813 . \\
833 . \\
850 . \\
846 . \\
829 .\end{array}$ & $\begin{array}{l}505 . \\
591 . \\
595 . \\
636 . \\
671 . \\
700 . \\
726 . \\
750 . \\
773 . \\
793 . \\
813 . \\
830 . \\
827 . \\
810 .\end{array}$ & $\begin{array}{l}496 . \\
539 . \\
581 . \\
620 . \\
654 . \\
683 . \\
708 . \\
732 . \\
763 . \\
774 . \\
793 . \\
810 . \\
807 . \\
791 .\end{array}$ & $\begin{array}{l}441 . \\
478 . \\
513 . \\
544 . \\
569 . \\
592 . \\
611 . \\
630 . \\
647 . \\
664 . \\
679 . \\
693 . \\
690 . \\
677 .\end{array}$ & $\begin{array}{l}422 . \\
455 . \\
486 . \\
513 . \\
536 . \\
556 . \\
575 . \\
592 . \\
608 . \\
624 . \\
639 . \\
653 . \\
651 . \\
639 .\end{array}$ & $\begin{array}{l}404 . \\
432 . \\
459 . \\
482 . \\
502 . \\
521 . \\
538 . \\
554 . \\
570 . \\
585 . \\
600 . \\
613 . \\
612 . \\
601 .\end{array}$ & $\begin{array}{l}340 . \\
344 . \\
349 . \\
355 . \\
362 . \\
369 \\
377 \\
386 . \\
394 . \\
403 . \\
413 . \\
423 . \\
433 . \\
443 .\end{array}$ \\
\hline $\begin{array}{l}\text { Mean } \\
\text { Range/RMS }(c)\end{array}$ & $\begin{array}{l}1011 . \\
\pm 693 .\end{array}$ & $\begin{array}{r}1039 . \\
147 .\end{array}$ & $\begin{array}{r}660 . \\
92 .\end{array}$ & $\begin{array}{l}800 . \\
108 .\end{array}$ & $\begin{array}{c}600 . \\
79 .\end{array}$ & $\begin{array}{l}657 . \\
\pm 79 .\end{array}$ & $\begin{array}{l}487 \\
\pm 52\end{array}$ & $\begin{array}{l}926 . \\
149 .\end{array}$ & $\begin{array}{l}870 . \\
136 .\end{array}$ & $\begin{array}{l}814 . \\
123 .\end{array}$ & $\begin{array}{l}730 . \\
106 .\end{array}$ & $\begin{array}{l}713 . \\
103 .\end{array}$ & $\begin{array}{l}696 . \\
100 .\end{array}$ & $\begin{array}{l}602 . \\
\pm 79 .\end{array}$ & $\begin{array}{l}568 . \\
\pm 73 .\end{array}$ & $\begin{array}{l}534 . \\
\pm 67 .\end{array}$ & $\begin{array}{l}385 \\
\pm 33\end{array}$ \\
\hline $\begin{array}{l}\text { Body } \\
2\end{array}$ & $\begin{array}{l}1479 . \\
1529 . \\
1578 . \\
1628 . \\
1677 . \\
1727 . \\
1776 . \\
1826 . \\
1875 . \\
1925 . \\
1974 . \\
2024 . \\
2073 . \\
2123 .\end{array}$ & $\begin{array}{l}1185 \\
1249 . \\
1294 . \\
1311 . \\
1320 . \\
1331 . \\
1340 . \\
1348 . \\
1355 . \\
1360 . \\
1368 . \\
1382 . \\
1362 . \\
1320 .\end{array}$ & $\begin{array}{l}753 . \\
793 . \\
820 . \\
828 . \\
839 . \\
842 . \\
848 . \\
852 \text {. } \\
856 . \\
858 \text {. } \\
861 \text {. } \\
861 . \\
841 \text {. } \\
807 .\end{array}$ & $\begin{array}{c}9.15 . \\
967 . \\
1005 . \\
1020 . \\
1031 . \\
1041 . \\
1052 . \\
1062 . \\
1070 . \\
1082 . \\
1096 . \\
1110 . \\
1096 . \\
1064 .\end{array}$ & $\begin{array}{l}683 . \\
719 . \\
745 . \\
753 . \\
761 . \\
768 . \\
774 . \\
779 . \\
783 . \\
787 . \\
791 . \\
793 . \\
775 . \\
746 .\end{array}$ & $\begin{array}{l}738 . \\
781 . \\
812 . \\
826 . \\
836 . \\
847 . \\
860 . \\
872 . \\
885 . \\
898 . \\
911 . \\
924 . \\
915 . \\
891 .\end{array}$ & $\begin{array}{l}543 . \\
576 . \\
599 . \\
609 . \\
619 . \\
630 . \\
640 . \\
650 . \\
657 . \\
662 . \\
668 . \\
672 . \\
660 . \\
638 .\end{array}$ & $\begin{array}{l}1073 . \\
1130 . \\
1169 . \\
1182 . \\
1192 . \\
1201 . \\
1210 . \\
1216 . \\
1221 . \\
1226 . \\
1230 . \\
1232 . \\
1204 . \\
1157 .\end{array}$ & $\begin{array}{l}1005 . \\
1059 . \\
1096 . \\
1109 . \\
1118 . \\
1127 . \\
1136 . \\
1143 . \\
1149 . \\
1154 . \\
1158 . \\
1161 . \\
1136 . \\
1092 .\end{array}$ & $\begin{array}{c}937 . \\
988 . \\
1023 . \\
1035 . \\
1045 . \\
1054 . \\
1063 . \\
1070 . \\
1076 . \\
1082 . \\
1087 . \\
1090 . \\
1067 . \\
1028 .\end{array}$ & $\begin{array}{l}842 . \\
889 . \\
922 . \\
934 . \\
945 . \\
955 . \\
964 . \\
972 . \\
980 . \\
986 . \\
993 . \\
997 . \\
979 . \\
944 .\end{array}$ & $\begin{array}{l}823 . \\
869 . \\
902 . \\
914 . \\
924 . \\
934 . \\
944 . \\
952 . \\
960 . \\
967 . \\
973 . \\
978 . \\
960 . \\
926 .\end{array}$ & $\begin{array}{l}803 . \\
849 . \\
881 . \\
893 . \\
904 . \\
914 . \\
924 . \\
932 . \\
940 . \\
947 . \\
954 . \\
959 . \\
942 . \\
909 .\end{array}$ & $\begin{array}{l}688 . \\
727 . \\
756 . \\
767 . \\
777 . \\
787 . \\
797 . \\
806 . \\
814 . \\
822 . \\
830 . \\
837 . \\
823 . \\
797 .\end{array}$ & $\begin{array}{l}650 . \\
688 . \\
716 . \\
728 . \\
738 . \\
748 . \\
758 . \\
768 . \\
777 . \\
785 . \\
794 . \\
801 . \\
789 . \\
765 .\end{array}$ & $\begin{array}{l}613 . \\
649 . \\
676 . \\
688 . \\
699 . \\
710 . \\
720 . \\
730 . \\
739 . \\
748 . \\
757 . \\
765 . \\
755 . \\
733 .\end{array}$ & $\begin{array}{l}453 . \\
463 . \\
474 . \\
484 . \\
495 . \\
506 . \\
516 . \\
527 . \\
537 . \\
548 . \\
558 . \\
569 . \\
579 . \\
589 .\end{array}$ \\
\hline $\begin{array}{l}\text { Mean } \\
\text { Range/RMS }\end{array}$ & $\begin{array}{r}1801 . \\
693 .\end{array}$ & $\begin{array}{r}1323 . \\
51 .\end{array}$ & $\begin{array}{r}832 . \\
30 .\end{array}$ & $\begin{array}{r}1044 . \\
52 .\end{array}$ & $\begin{array}{l}761 . \\
30 .\end{array}$ & $\begin{array}{l}857 \\
\pm 52\end{array}$ & $\begin{array}{l}630 \\
\pm 36\end{array}$ & $\begin{array}{r}1189 . \\
43 .\end{array}$ & $\begin{array}{r}1117 . \\
42 .\end{array}$ & $\begin{array}{r}1046 . \\
41 .\end{array}$ & $\begin{array}{r}950 . \\
42 .\end{array}$ & $\begin{array}{r}930 . \\
42 .\end{array}$ & $\begin{array}{r}911 . \\
42 .\end{array}$ & $\begin{array}{l}788 \\
\pm 41\end{array}$ & $\begin{array}{l}750 . \\
\pm 42 .\end{array}$ & $\begin{array}{l}713 \\
\pm 43\end{array}$ & $\begin{array}{l}521 \\
\pm 43 .\end{array}$ \\
\hline $\begin{array}{c}\text { Body } \\
3\end{array}$ & $\begin{array}{l}2269 . \\
2318 . \\
2368 . \\
2418 . \\
2467 . \\
2517 . \\
2566 . \\
2616 . \\
2665 . \\
2714 . \\
2764 . \\
2814 . \\
2863 . \\
2913 .\end{array}$ & $\begin{array}{l}1328 . \\
1388 . \\
1426 . \\
1429 . \\
1428 . \\
1425 . \\
1420 . \\
1410 . \\
1389 . \\
1363 . \\
1336 . \\
1308 . \\
1253 . \\
1179 .\end{array}$ & $\begin{array}{l}804 . \\
835 . \\
852 . \\
846 . \\
842 . \\
836 . \\
827 . \\
818 . \\
810 . \\
801 . \\
789 . \\
780 . \\
765 . \\
747 .\end{array}$ & $\begin{array}{l}1074 . \\
1126 . \\
1159 . \\
1165 . \\
1168 . \\
1170 . \\
1170 . \\
1166 . \\
1155 . \\
1141 . \\
1126 . \\
1110 . \\
1072 . \\
1017 .\end{array}$ & $\begin{array}{l}744 . \\
775 . \\
792 . \\
789 . \\
787 . \\
783 . \\
777 . \\
771 . \\
765 . \\
759 . \\
751 . \\
744 . \\
730 . \\
712 .\end{array}$ & $\begin{array}{c}901 . \\
948 . \\
980 . \\
989 . \\
996 . \\
1002 . \\
1007 . \\
1008 . \\
1005 . \\
1000 . \\
993 . \\
987 . \\
958 . \\
916 .\end{array}$ & $\begin{array}{l}640 . \\
669 . \\
686 . \\
687 . \\
689 . \\
689 . \\
688 . \\
687 . \\
686 . \\
684 . \\
682 . \\
679 . \\
667 . \\
648 .\end{array}$ & $\begin{array}{r}1154 . \\
1196 . \\
1218 . \\
1210 . \\
1200 . \\
1188 . \\
1172 . \\
1156 . \\
1136 . \\
1114 . \\
1089 . \\
1064 . \\
1030 . \\
989 .\end{array}$ & $\begin{array}{c}1090 . \\
1132 . \\
1154 . \\
1147 . \\
1139 . \\
1129 . \\
1116 . \\
1102 . \\
1085 . \\
1066 . \\
1045 . \\
1023 . \\
901 . \\
952 .\end{array}$ & \begin{tabular}{|c|}
1027. \\
1067. \\
1089. \\
1084. \\
1078. \\
1070. \\
1060. \\
1049. \\
1035. \\
1019. \\
1000. \\
982. \\
952. \\
915.
\end{tabular} & $\begin{array}{c}945 . \\
983 . \\
1006 . \\
1004 . \\
1000 . \\
995 . \\
988 . \\
980 . \\
970 . \\
958 . \\
944 . \\
930 . \\
905 . \\
872 .\end{array}$ & $\begin{array}{l}928 . \\
966 . \\
989 . \\
987 . \\
984 . \\
979 . \\
973 . \\
966 . \\
956 . \\
945 . \\
932 . \\
919 . \\
895 . \\
864 .\end{array}$ & $\begin{array}{l}911 . \\
949 . \\
971 . \\
970 . \\
968 . \\
964 . \\
958 . \\
951 . \\
943 . \\
933 . \\
921 . \\
909 . \\
686 . \\
855 .\end{array}$ & $\begin{array}{l}801 . \\
837 . \\
860 . \\
862 . \\
863 . \\
863 . \\
861 . \\
859 . \\
855 . \\
850 . \\
844 . \\
837 . \\
818 . \\
790 .\end{array}$ & $\begin{array}{l}769 . \\
806 . \\
829 . \\
832 . \\
834 . \\
835 . \\
835 . \\
834 . \\
832 . \\
829 . \\
825 . \\
820 . \\
803 . \\
777 .\end{array}$ & $\begin{array}{l}738 . \\
774 . \\
797 . \\
802 . \\
805 . \\
808 . \\
809 . \\
810 . \\
809 . \\
808 . \\
806 . \\
803 . \\
788 . \\
764 .\end{array}$ & $\begin{array}{l}599 . \\
609 . \\
619 . \\
628 . \\
637 . \\
646 . \\
655 . \\
663 . \\
670 . \\
678 . \\
685 . \\
691 . \\
697 . \\
703 .\end{array}$ \\
\hline $\begin{array}{l}\text { Mean } \\
\text { Range/RMS }\end{array}$ & $\begin{array}{r}2591 . \\
693 .\end{array}$ & $\begin{array}{r}1363 . \\
73 .\end{array}$ & $\begin{array}{l}811 . \\
31 .\end{array}$ & $\begin{array}{r}1130 \\
45 .\end{array}$ & $\begin{array}{l}763 \\
\pm 23\end{array}$ & $\begin{array}{l}978 \\
\pm 33\end{array}$ & $\begin{array}{l}677 . \\
\pm 15 .\end{array}$ & $\begin{array}{r}1137 \\
68 .\end{array}$ & $\begin{array}{r}1084 . \\
59 .\end{array}$ & $\begin{array}{r}1031 . \\
50 .\end{array}$ & $\begin{array}{c}963 . \\
39 .\end{array}$ & $\begin{array}{c}949 . \\
36 .\end{array}$ & $\begin{array}{r}935 . \\
34 .\end{array}$ & $\begin{array}{l}843 \\
\pm 23\end{array}$ & $\begin{array}{l}819 . \\
\pm 21 .\end{array}$ & $\begin{array}{l}794 \\
\pm 21\end{array}$ & $\begin{array}{l}656 \\
\pm 32\end{array}$ \\
\hline $\begin{array}{l}\text { Grand Mean } \\
\text { Range/RMS, }\end{array}$ & $\begin{array}{l}1801 . \\
2273 .\end{array}$ & $\begin{array}{r}1242 . \\
175 .\end{array}$ & $\begin{array}{r}768 . \\
96 .\end{array}$ & $\begin{array}{l}991 . \\
158 .\end{array}$ & $\begin{array}{r}708 . \\
92 .\end{array}$ & $\begin{array}{r}831 \\
\pm 141\end{array}$ & $\begin{array}{l}598 \\
\pm 89\end{array}$ & $\begin{array}{r}1080 . \\
150 .\end{array}$ & $\begin{array}{c}1080 . \\
150 .\end{array}$ & $\begin{array}{l}963 . \\
133 .\end{array}$ & $\begin{array}{l}881 . \\
127 .\end{array}$ & $\begin{array}{l}864 . \\
127 .\end{array}$ & $\begin{array}{l}867 . \\
126 .\end{array}$ & $\begin{array}{r}744 \\
\pm 116\end{array}$ & $\begin{array}{r}712 . \\
\pm 117 .\end{array}$ & $\begin{array}{r}680 \\
\pm 119\end{array}$ & $\begin{aligned} & 521 \\
& \pm 116\end{aligned}$ \\
\hline
\end{tabular}

(a) Core helght above core bed plate based on Bol metrology.

(b) Time and volume averaged.

(c) Root mean square deviation for gradient information. 
TABLE 3-3(b)

FTE-6 TABULATION OF IRRADIATION TEMPERATURES FOR

HOLES 3 AND $4\left[(2.75 \mathrm{Th}: U) \mathrm{C}_{2}\right.$ TRISO, $\mathrm{ThC}_{2}$ BISO $]$

\begin{tabular}{|c|c|c|c|c|c|c|c|c|c|c|c|}
\hline \multirow[b]{2}{*}{ Fuel Body } & \multirow{2}{*}{$\begin{array}{l}\text { Mean Pos } \\
\text { Core Ht (a) } \\
\text { BOL (MM) }\end{array}$} & \multicolumn{4}{|c|}{$\begin{array}{c}\text { Temperature } \\
\text { Envelope (C) (d) }\end{array}$} & \multicolumn{6}{|c|}{ Time Weighted Averages (c) ${ }^{\text {(d) }}$} \\
\hline & & $\begin{array}{l}\text { Fuel } \\
\text { Max }\end{array}$ & $\begin{array}{l}\text { Fuel } \\
\text { M1n }\end{array}$ & $\begin{array}{l}\text { Body } \\
\text { Max }\end{array}$ & $\begin{array}{l}\text { Body } \\
\text { Min }\end{array}$ & $\begin{array}{l}\text { Fuel } \\
\text { Max }\end{array}$ & $\begin{array}{c}\text { Fuel } \\
\operatorname{Avg}(\mathrm{b})\end{array}$ & $\begin{array}{l}\text { Fuel } \\
\text { Min }\end{array}$ & $\begin{array}{l}\text { Body } \\
\text { Max }\end{array}$ & $\begin{array}{l}\text { Body } \\
\text { Avg(b) }\end{array}$ & $\begin{array}{l}\text { Body } \\
\text { Min }\end{array}$ \\
\hline $\begin{array}{c}\text { Body } \\
1\end{array}$ & $\begin{array}{r}689 . \\
738 . \\
788 . \\
837 . \\
887 . \\
936 . \\
986 . \\
1036 . \\
1085 . \\
1134 . \\
1184 . \\
1233 . \\
1283 . \\
1332 .\end{array}$ & $\begin{array}{c}704 . \\
787 . \\
862 . \\
925 . \\
971 . \\
1009 . \\
1042 . \\
1069 . \\
1094 . \\
1120 . \\
1150 . \\
1177 . \\
1171 . \\
1143 .\end{array}$ & $\begin{array}{l}482 . \\
517 . \\
552 . \\
587 . \\
619 . \\
647 . \\
672 . \\
695 . \\
715 . \\
734 . \\
751 . \\
767 . \\
762 . \\
744 .\end{array}$ & $\begin{array}{l}573 . \\
630 . \\
682 . \\
725 . \\
759 . \\
788 . \\
814 . \\
837 . \\
858 . \\
881 . \\
904 . \\
925 . \\
921 . \\
901 .\end{array}$ & $\begin{array}{l}447 . \\
478 . \\
508 . \\
537 . \\
564 . \\
588 . \\
609 . \\
629 . \\
647 . \\
663 . \\
679 . \\
694 . \\
690 . \\
673 .\end{array}$ & $\begin{array}{r}615 . \\
681 . \\
745 . \\
807 . \\
858 . \\
900 . \\
936 . \\
963 . \\
998 . \\
1025 . \\
1049 . \\
1069 . \\
1064 . \\
1041 .\end{array}$ & $\begin{array}{c}585 . \\
645 . \\
704 . \\
760 . \\
806 . \\
844 . \\
877 . \\
906 . \\
933 . \\
958 . \\
981 . \\
1000 . \\
99.5 \\
973 .\end{array}$ & $\begin{array}{l}556 . \\
610 . \\
662 . \\
712 . \\
753 . \\
788 . \\
817 . \\
844 . \\
869 . \\
892 . \\
912 . \\
930 . \\
925 . \\
905 .\end{array}$ & $\begin{array}{l}515 . \\
563 . \\
608 . \\
651 . \\
687 . \\
718 . \\
745 . \\
769 . \\
792 . \\
813 . \\
833 . \\
850 . \\
846 . \\
829 .\end{array}$ & $\begin{array}{l}505 . \\
551 . \\
595 . \\
636 . \\
671 . \\
700 . \\
726 . \\
750 . \\
773 . \\
793 . \\
813 . \\
830 . \\
827 . \\
810 .\end{array}$ & $\begin{array}{l}496 . \\
539 . \\
581 . \\
620 . \\
654 . \\
683 . \\
708 . \\
732 . \\
753 . \\
774 . \\
793 . \\
810 . \\
807 . \\
791 .\end{array}$ \\
\hline $\begin{array}{l}\text { Mean } \\
\text { Range/RMS }\end{array}$ & $\begin{array}{l}1011 . \\
693 .\end{array}$ & $\begin{array}{l}1016 \\
\pm 143\end{array}$ & $\begin{array}{l}660 \text {. } \\
\pm 92\end{array}$ & $\begin{array}{r}800 \\
\pm 108\end{array}$ & $\begin{array}{l}600 \\
\pm 79\end{array}$ & $\begin{array}{r}911 . \\
\pm 144 .\end{array}$ & $\begin{array}{r}855 \\
\pm 131\end{array}$ & $\begin{array}{r}798 \\
\pm 118 \text {. }\end{array}$ & $\begin{array}{r}730 \\
\pm 106\end{array}$ & $\begin{aligned} & 713 \\
\pm & 103\end{aligned}$ & $\begin{array}{r}696 \\
\pm 100\end{array}$ \\
\hline $\begin{array}{c}\text { Body } \\
2\end{array}$ & $\begin{array}{l}1479 . \\
1529 . \\
1578 . \\
1628 . \\
1677 \\
1727 \\
1776 \\
1826 . \\
1875 \\
1925 . \\
1974 . \\
2024 . \\
2073 . \\
2123 .\end{array}$ & $\begin{array}{l}1158 . \\
1221 . \\
1266 . \\
1282 . \\
1292 . \\
1302 . \\
1312 . \\
1320 . \\
1326 . \\
1332 . \\
1342 . \\
1356 . \\
1337 . \\
1295 .\end{array}$ & $\begin{array}{l}753 . \\
793 . \\
820 . \\
828 . \\
835 . \\
842 . \\
848 . \\
852 . \\
856 . \\
858 . \\
861 . \\
861 . \\
841 . \\
807 .\end{array}$ & $\begin{array}{r}915 . \\
967 . \\
1005 . \\
1020 . \\
1031 . \\
1041 . \\
1052 . \\
1062 . \\
1070 . \\
1082 . \\
1096 . \\
1110 . \\
1096 . \\
1064 .\end{array}$ & $\begin{array}{l}683 . \\
719 . \\
745 . \\
753 . \\
761 . \\
768 . \\
774 . \\
779 . \\
783 . \\
787 . \\
791 . \\
793 . \\
775 . \\
746 .\end{array}$ & $\begin{array}{l}1055 . \\
1111 . \\
1150 . \\
1163 . \\
1173 . \\
1182 . \\
1191 . \\
1198 . \\
1203 . \\
1208 . \\
1212 . \\
1214 . \\
1188 . \\
1141 .\end{array}$ & $\begin{array}{c}986 . \\
1039 . \\
1076 . \\
1089 . \\
1099 . \\
1108 . \\
1117 . \\
1124 . \\
1130 . \\
1135 . \\
1140 . \\
1143 . \\
1119 . \\
1076 .\end{array}$ & $\begin{array}{r}918 . \\
968 . \\
1003 . \\
1015 . \\
1025 . \\
1034 . \\
1043 . \\
1050 . \\
1057 . \\
1063 . \\
1068 . \\
1072 . \\
1050 . \\
1011 .\end{array}$ & $\begin{array}{l}842 . \\
889 . \\
922 . \\
934 . \\
945 . \\
955 . \\
964 . \\
972 . \\
980 . \\
986 . \\
993 . \\
997 . \\
979 . \\
944 .\end{array}$ & $\begin{array}{l}823 . \\
869 . \\
902 . \\
914 . \\
924 . \\
934 . \\
944 . \\
952 . \\
960 . \\
967 . \\
973 . \\
978 . \\
960 . \\
926 .\end{array}$ & $\begin{array}{l}803 . \\
849 . \\
881 . \\
893 . \\
904 . \\
914 . \\
924 . \\
932 . \\
940 . \\
947 . \\
954 . \\
959 . \\
942 . \\
909 .\end{array}$ \\
\hline $\begin{array}{l}\text { Mean } \\
\text { Range/RMS }\end{array}$ & $\begin{array}{r}1801 . \\
693 .\end{array}$ & $\begin{aligned} 1296 . \\
\pm 51\end{aligned}$ & $\begin{array}{l}832 \\
\pm 30\end{array}$ & $\begin{array}{r}1044 \\
\pm 52\end{array}$ & $\begin{array}{l}761 \\
\pm 30\end{array}$ & $\begin{array}{l}1171 \text {. } \\
\pm 43 .\end{array}$ & $\begin{array}{l}1099 \text {. } \\
\pm 42 \text {. }\end{array}$ & $\begin{array}{r}1027 \\
\pm 41\end{array}$ & $\begin{array}{l}950 . \\
\pm 42 .\end{array}$ & $\begin{array}{l}930 . \\
\pm 42 .\end{array}$ & $\begin{array}{l}911 . \\
\pm 42 .\end{array}$ \\
\hline $\begin{array}{l}\text { Body } \\
3\end{array}$ & $\begin{array}{l}2269 \text {. } \\
2318 \text {. } \\
2368 . \\
2418 \text {. } \\
2467 . \\
2517 . \\
2566 \text {. } \\
2616 \text {. } \\
2665 . \\
2714 . \\
2764 . \\
2814 . \\
2863 \text {. } \\
2913 \text {. }\end{array}$ & $\begin{array}{l}1304 . \\
1363 . \\
1400 . \\
1404 . \\
1403 . \\
1401 . \\
1397 . \\
1387 . \\
1367 . \\
1343 . \\
1317 . \\
1291 . \\
1239 . \\
1168 .\end{array}$ & $\begin{array}{l}804 . \\
835 . \\
852 . \\
846 . \\
842 . \\
836 . \\
827 . \\
818 . \\
810 . \\
801 . \\
789 . \\
780 . \\
765 . \\
747 .\end{array}$ & $\begin{array}{l}1074 . \\
1126 . \\
1159 . \\
1165 . \\
1168 . \\
1170 . \\
1170 . \\
1166 . \\
1155 . \\
1141 . \\
1126 . \\
1110 . \\
1072 . \\
1017 .\end{array}$ & $\begin{array}{l}744 . \\
775 . \\
792 . \\
789 . \\
787 . \\
783 . \\
777 . \\
771 . \\
765 . \\
759 . \\
751 . \\
744 . \\
730 . \\
712 .\end{array}$ & $\begin{array}{r}1139 . \\
1181 . \\
1203 . \\
1195 . \\
1186 . \\
1174 . \\
1160 . \\
1144 . \\
1125 . \\
1104 . \\
1080 . \\
1056 . \\
1023 . \\
985 .\end{array}$ & $\begin{array}{c}1075 . \\
1116 . \\
1138 . \\
1132 . \\
1125 . \\
1115 . \\
1103 . \\
1090 . \\
1074 . \\
1056 . \\
1036 . \\
1015 . \\
985 . \\
948 .\end{array}$ & $\begin{array}{c}1011 . \\
1051 . \\
1073 . \\
1069 . \\
1064 . \\
1056 . \\
1047 . \\
1036 . \\
1023 . \\
1008 . \\
991 . \\
974 . \\
946 . \\
911 .\end{array}$ & $\begin{array}{r}945 . \\
983 . \\
1006 . \\
1004 . \\
1000 . \\
995 . \\
988 . \\
980 . \\
970 . \\
958 . \\
944 . \\
930 . \\
905 . \\
872 .\end{array}$ & $\begin{array}{l}928 . \\
966 . \\
989 . \\
987 . \\
984 . \\
979 . \\
973 . \\
966 . \\
956 . \\
945 . \\
932 . \\
919 . \\
895 . \\
864 .\end{array}$ & $\begin{array}{l}911 . \\
949 . \\
971 . \\
970 . \\
968 . \\
964 . \\
958 . \\
951 . \\
943 . \\
933 . \\
921 . \\
909 . \\
886 . \\
855 .\end{array}$ \\
\hline $\begin{array}{l}\text { Mean } \\
\text { Range/RMS }\end{array}$ & $\begin{array}{r}2591 . \\
693 .\end{array}$ & $\begin{array}{l}1342 \\
\pm 69\end{array}$ & $\begin{array}{l}811 \\
\pm 31\end{array}$ & $\begin{array}{l}1130 \\
\pm 45\end{array}$ & $\begin{array}{l}763 \\
\pm 23\end{array}$ & $\begin{array}{l}1125 \text {. } \\
\pm 65\end{array}$ & $\begin{array}{l}1072 \\
\pm 56\end{array}$ & $\begin{array}{l}1019 \\
\pm 47\end{array}$ & $\begin{array}{l}963 \text {. } \\
\pm 39 \text {. }\end{array}$ & $\begin{array}{l}949 . \\
\pm 36 .\end{array}$ & $\begin{array}{l}935 \\
\pm 34\end{array}$ \\
\hline $\begin{array}{l}\text { Mean } \\
\text { Range/RMS }\end{array}$ & $\begin{array}{l}1801 \\
2273\end{array}$ & $\begin{array}{l}1218 \\
\pm 173\end{array}$ & $\begin{array}{l}768 \\
\pm 96\end{array}$ & $\begin{aligned} 991 \\
\pm 158\end{aligned}$ & $\begin{array}{l}708 \\
\pm 92\end{array}$ & $\begin{array}{l}1069 \\
\pm 147\end{array}$ & $\begin{array}{l}1008 \\
\pm 139\end{array}$ & $\begin{array}{r}948 \\
\pm 131\end{array}$ & $\begin{array}{r}881 \\
\pm 127\end{array}$ & $\begin{array}{r}864 \\
\pm 127\end{array}$ & $\begin{array}{r}847 \\
\pm 126\end{array}$ \\
\hline
\end{tabular}

(a) Core helght above core bed plate based on BOL metrology.

(b) Time and volume averaged.

(c) Root mean square deviation for gradient information.

(d) For sleeve temperatures see Table 3-3(a). 
TABLE 3-3(c)

FTE-6 TABULATION OF IRRADIATION TEMPERATURES FOR HOLES 5 AND $6\left[(2.75 \mathrm{Th}: \mathrm{U}) \mathrm{C}_{2}\right.$ TRISO, ThC $\mathrm{T}_{2}$ TRISO $]$

\begin{tabular}{|c|c|c|c|c|c|c|c|c|c|c|c|}
\hline \multirow[b]{2}{*}{ Fuel Body } & \multirow{2}{*}{$\begin{array}{c}\text { Mean Pos } \\
\text { Core Ht (a) } \\
\text { BOL (MM) }\end{array}$} & \multicolumn{4}{|c|}{$\begin{array}{l}\text { Temperature } \\
\text { Envelope (C) (d) }\end{array}$} & \multicolumn{6}{|c|}{ Time-Welghted Averages (c) (d) } \\
\hline & & $\begin{array}{l}\text { Fuel } \\
\text { Max }\end{array}$ & $\begin{array}{l}\text { Fue1 } \\
\text { MIn }\end{array}$ & $\begin{array}{l}\text { Body } \\
\text { Max }\end{array}$ & $\begin{array}{l}\text { Body } \\
\text { Min }\end{array}$ & $\begin{array}{l}\text { Fuel } \\
\text { Max }\end{array}$ & $\begin{array}{c}\text { Fue } 1 \\
\text { Avg }(b)\end{array}$ & $\begin{array}{l}\text { Fuel } \\
\text { Min }\end{array}$ & $\begin{array}{l}\text { Body } \\
\text { Max }\end{array}$ & $\begin{array}{l}\text { Bòdy } \\
\operatorname{Avg}(b)\end{array}$ & $\begin{array}{l}\text { Body } \\
\text { Min }\end{array}$ \\
\hline $\begin{array}{c}\text { Body } \\
1\end{array}$ & $\begin{array}{c}689 . \\
738 . \\
788 . \\
837 \\
887 \\
936 . \\
986 . \\
1036 \\
1085 \\
1134 \\
1184 \\
1233 \\
1283 . \\
1332\end{array}$ & $\begin{array}{r}700 . \\
783 . \\
857 . \\
919 . \\
965 . \\
1004 . \\
1036 . \\
1063 . \\
1088 . \\
1114 . \\
1144 . \\
1171 . \\
1165 . \\
1137 .\end{array}$ & $\begin{array}{l}482 . \\
517 . \\
552 . \\
587 . \\
619 . \\
647 \\
672 . \\
695 . \\
715 . \\
734 . \\
751 \\
767 . \\
762 . \\
744 .\end{array}$ & $\begin{array}{l}573 . \\
630 . \\
682 . \\
725 . \\
759 . \\
788 . \\
814 . \\
837 . \\
858 . \\
881 . \\
904 . \\
925 . \\
921 . \\
901 .\end{array}$ & $\begin{array}{l}447 . \\
478 . \\
508 . \\
537 \\
564 . \\
588 \\
609 \\
629 \\
647 \\
663 . \\
679 \\
694 . \\
690 \\
673 .\end{array}$ & $\begin{array}{r}613 . \\
678 . \\
742 . \\
803 . \\
854 . \\
896 . \\
932 . \\
963 . \\
993 . \\
1020 . \\
1044 . \\
1065 . \\
1059 . \\
1036 .\end{array}$ & $\begin{array}{l}583 . \\
642 . \\
700 . \\
756 . \\
801 . \\
839 . \\
872 . \\
901 . \\
928 . \\
953 . \\
975 . \\
995 . \\
990 . \\
968 .\end{array}$ & $\begin{array}{l}553 . \\
607 . \\
659 . \\
708 . \\
749 . \\
783 . \\
812 . \\
839 . \\
863 . \\
886 . \\
907 . \\
925 . \\
920 . \\
900 .\end{array}$ & $\begin{array}{l}515 . \\
563 . \\
608 . \\
651 . \\
687 . \\
718 . \\
745 . \\
769 . \\
792 . \\
813 . \\
833 . \\
850 . \\
846 . \\
829 .\end{array}$ & $\begin{array}{l}505 . \\
551 . \\
595 . \\
636 . \\
671 . \\
700 . \\
726 . \\
750 . \\
773 . \\
793 . \\
813 . \\
830 . \\
827 . \\
810 .\end{array}$ & $\begin{array}{l}496 . \\
539 . \\
581 . \\
620 . \\
654 . \\
683 . \\
708 . \\
732 . \\
753 . \\
774 . \\
793 . \\
810 . \\
807 . \\
791 .\end{array}$ \\
\hline $\begin{array}{l}\text { Mean } \\
\text { Range/RMS }\end{array}$ & $\begin{array}{l}1011 \\
\pm 693\end{array}$ & $\begin{array}{l}1011 \\
\pm 143\end{array}$ & $\begin{array}{l}660 \\
\pm 92\end{array}$ & $\begin{array}{r}800 \\
\pm 108\end{array}$ & $\begin{array}{l}600 \\
\pm 79\end{array}$ & $\begin{array}{r}907 \\
\pm 143\end{array}$ & $\begin{array}{r}850 \\
\pm 130\end{array}$ & $\begin{array}{r}794 \\
\pm 118\end{array}$ & $\begin{array}{r}730 \\
\pm 106\end{array}$ & $\begin{array}{r}713 \\
\pm 103\end{array}$ & $\begin{array}{r}696 \\
\pm 100\end{array}$ \\
\hline $\begin{array}{c}\text { Body } \\
2\end{array}$ & $\begin{array}{l}1479 . \\
1529 \\
1578 . \\
1628 \\
1677 \\
1727 \\
1776 . \\
1826 . \\
1875 \\
1925 \\
1974 \\
2024 \\
2073 . \\
2123\end{array}$ & $\begin{array}{l}1151 . \\
1215 . \\
1259 . \\
1276 . \\
1285 . \\
1296 . \\
1305 . \\
1313 . \\
1320 . \\
1326 . \\
1337 . \\
1351 . \\
1332 . \\
1291 .\end{array}$ & $\begin{array}{l}753 . \\
793 . \\
820 \\
828 . \\
835 . \\
842 . \\
848 . \\
852 . \\
856 . \\
858 . \\
861 . \\
861 . \\
841 . \\
807 .\end{array}$ & $\begin{array}{r}915 . \\
967 . \\
1005 . \\
1020 \\
1031 . \\
1041 . \\
1052 . \\
1062 . \\
1070 . \\
1082 . \\
1096 . \\
1110 . \\
1096 . \\
1064 .\end{array}$ & $\begin{array}{l}683 . \\
719 . \\
745 . \\
753 . \\
761 . \\
768 . \\
774 . \\
779 . \\
783 . \\
787 . \\
791 . \\
793 . \\
775 . \\
746 .\end{array}$ & $\begin{array}{l}1050 . \\
1106 . \\
1145 . \\
1158 . \\
1168 . \\
1177 . \\
1186 . \\
1193 . \\
1198 . \\
1203 . \\
1208 . \\
1210 . \\
1183 . \\
1137 .\end{array}$ & $\begin{array}{r}981 . \\
1034 . \\
1071 . \\
1084 . \\
1094 . \\
1103 . \\
1112 . \\
1119 . \\
1125 . \\
1130 . \\
1135 . \\
1138 . \\
1114 . \\
1072 .\end{array}$ & $\begin{array}{r}913 . \\
963 . \\
997 . \\
1010 . \\
1019 . \\
1029 . \\
1038 . \\
1045 . \\
1052 . \\
1058 . \\
1063 . \\
1067 . \\
1045 . \\
1006 .\end{array}$ & $\begin{array}{l}842 . \\
889 . \\
922 . \\
934 . \\
945 . \\
955 . \\
964 . \\
972 . \\
980 . \\
986 . \\
993 . \\
997 . \\
979 . \\
944 .\end{array}$ & $\begin{array}{l}823 . \\
869 . \\
902 . \\
914 . \\
924 . \\
934 . \\
944 . \\
952 . \\
960 . \\
967 . \\
973 . \\
978 . \\
960 . \\
926 .\end{array}$ & $\begin{array}{l}803 . \\
849 . \\
881 . \\
893 . \\
904 . \\
914 . \\
924 . \\
932 . \\
940 . \\
947 . \\
954 . \\
959 . \\
942 . \\
909 .\end{array}$ \\
\hline $\begin{array}{l}\text { Mean } \\
\text { Range/RMS }\end{array}$ & $\begin{array}{l}1801 \\
\pm 693\end{array}$ & $\begin{array}{r}1290 \\
\pm 51\end{array}$ & $\begin{array}{l}832 \\
\pm 30\end{array}$ & $\begin{array}{r}1044 \\
\pm 52\end{array}$ & $\begin{array}{l}761 \\
\pm 30\end{array}$ & $\begin{array}{r}1166 \\
\pm 43\end{array}$ & $\begin{array}{r}1094 \\
\pm 42\end{array}$ & $\begin{array}{r}1022 \\
\pm 41\end{array}$ & $\begin{array}{l}950 \\
\pm 42\end{array}$ & $\begin{array}{l}930 \\
\pm 42\end{array}$ & $\begin{array}{l}911 \\
\pm 42\end{array}$ \\
\hline $\begin{array}{c}\text { Body } \\
3\end{array}$ & $\begin{array}{l}2269 . \\
2318 \\
2368 \\
2418 \\
2467 \\
2517 \\
2566 \\
2616 \\
2665 \\
2714 \\
2764 \\
2814 \\
2863 \\
2913 .\end{array}$ & $\begin{array}{l}1300 . \\
1359 . \\
1396 . \\
1399 . \\
1398 . \\
1396 . \\
1392 . \\
1382 . \\
1362 . \\
1338 . \\
1312 . \\
1287 . \\
1235 . \\
1165 .\end{array}$ & $\begin{array}{l}804 . \\
835 . \\
852 . \\
846 . \\
842 . \\
836 . \\
827 . \\
818 . \\
810 . \\
801 . \\
789 . \\
780 . \\
765 . \\
747 .\end{array}$ & $\begin{array}{l}1074 . \\
1126 . \\
1159 . \\
1165 . \\
1168 . \\
1170 . \\
1170 . \\
1166 . \\
1155 . \\
1141 . \\
1126 . \\
1110 . \\
1072 . \\
1017 .\end{array}$ & $\begin{array}{l}744 . \\
775 . \\
792 . \\
789 . \\
787 . \\
783 . \\
777 . \\
771 . \\
765 . \\
759 . \\
751 . \\
744 . \\
730 . \\
712 .\end{array}$ & $\begin{array}{c}1135 . \\
1177 . \\
1199 . \\
1191 . \\
1182 . \\
1170 . \\
1156 . \\
1140 . \\
1122 . \\
1101 . \\
1077 . \\
1053 . \\
1021 . \\
983 .\end{array}$ & $\begin{array}{c}1070 . \\
1111 . \\
1134 . \\
1128 . \\
1121 . \\
1111 . \\
1099 . \\
1086 . \\
1071 . \\
1053 . \\
1032 . \\
1012 . \\
982 . \\
946 .\end{array}$ & $\begin{array}{c}1006 . \\
1046 . \\
1068 \\
1065 \\
1059 \\
1052 \\
1043 . \\
1032 \\
1020 \\
1005 \\
988 \\
971 . \\
944 . \\
909 .\end{array}$ & $\begin{array}{r}945 . \\
983 . \\
1006 . \\
1004 . \\
1000 . \\
995 . \\
988 . \\
980 . \\
970 . \\
958 . \\
944 . \\
930 . \\
905 . \\
872 .\end{array}$ & $\begin{array}{l}928 . \\
966 . \\
989 . \\
987 . \\
984 . \\
979 . \\
973 . \\
966 . \\
956 . \\
945 . \\
932 . \\
919 . \\
895 . \\
864 .\end{array}$ & $\begin{array}{l}911 . \\
949 . \\
971 . \\
970 . \\
968 . \\
964 . \\
958 . \\
951 . \\
943 . \\
933 . \\
921 . \\
909 . \\
886 . \\
855 .\end{array}$ \\
\hline $\begin{array}{l}\text { Mean } \\
\text { Range/RMS }\end{array}$ & $\begin{array}{l}2591 . \\
\pm 693\end{array}$ & $\begin{array}{r}1337 \\
\pm 68\end{array}$ & $\begin{array}{l}811 \\
\pm 31\end{array}$ & $\begin{array}{r}1130 \\
\pm 45\end{array}$ & $\begin{array}{l}763 \\
\pm 23\end{array}$ & $\begin{array}{r}1122 \\
\pm 64\end{array}$ & $\begin{array}{r}1068 \\
\pm 55\end{array}$ & $\begin{array}{r}1015 \\
\pm 46\end{array}$ & $\begin{array}{l}963 \text {. } \\
\pm 39\end{array}$ & $\begin{array}{l}949 \\
\pm 36\end{array}$ & $\begin{array}{l}935 . \\
\pm 34 .\end{array}$ \\
\hline $\begin{array}{l}\text { Mean } \\
\text { Range/RMS }\end{array}$ & $\begin{array}{l}1801 . \\
2273 .\end{array}$ & $\begin{array}{r}1213 \\
173\end{array}$ & $\begin{array}{r}768 . \\
96\end{array}$ & $\begin{array}{l}991 . \\
158 .\end{array}$ & $\begin{array}{r}708 . \\
92 .\end{array}$ & $\begin{array}{r}1065 \\
147\end{array}$ & $\begin{array}{r}1004 \\
139\end{array}$ & $\begin{array}{l}943 . \\
131 .\end{array}$ & $\begin{array}{l}881 . \\
127 .\end{array}$ & $\begin{array}{l}864 . \\
127 .\end{array}$ & $\begin{array}{l}847 . \\
126 .\end{array}$ \\
\hline
\end{tabular}

(a) Core height above core bed plate based on BOL metrology.

(b) Time and volume averaged.

(c) Root mean square devlation for gradient information.

(d) For sleeve temperatures see Table 3-3(a). 
TABLE 3-3(d)

FTE-6 TABULATION OF IRRADIATION TEMPERATURES FOR HOLES 7 AND $8\left(\mathrm{UC}_{2}\right.$ TRISO, $\mathrm{ThC}_{2}$ BISO)

\begin{tabular}{|c|c|c|c|c|c|c|c|c|c|c|c|}
\hline \multirow[b]{2}{*}{ Fuel Body } & \multirow{2}{*}{$\begin{array}{l}\text { Mean Pos } \\
\text { Core Ht (a) } \\
\text { BOL (MM) }\end{array}$} & \multicolumn{4}{|c|}{$\begin{array}{c}\text { Temperature } \\
\text { Envelope (C) (d) }\end{array}$} & \multicolumn{6}{|c|}{ Time Weighted Averages (C) (d) } \\
\hline & & $\begin{array}{l}\text { Fue1 } \\
\text { Max }\end{array}$ & $\begin{array}{l}\text { Fuel } \\
\text { Min }\end{array}$ & $\begin{array}{l}\text { Body } \\
\text { Max }\end{array}$ & $\begin{array}{l}\text { Body } \\
\text { Min }\end{array}$ & $\begin{array}{l}\text { Fuel } \\
\text { Max }\end{array}$ & $\begin{array}{c}\text { FueI } \\
\operatorname{Avg}(b)\end{array}$ & $\begin{array}{l}\text { Fuel } \\
\text { Min }\end{array}$ & $\begin{array}{l}\text { Body } \\
\text { Max }\end{array}$ & $\begin{array}{l}\operatorname{Body} \\
\operatorname{Avg}(b)\end{array}$ & $\begin{array}{l}\text { Body } \\
\text { Min }\end{array}$ \\
\hline $\begin{array}{c}\text { Body } \\
1\end{array}$ & $\begin{array}{r}689 . \\
738 . \\
788 . \\
837 \\
887 \\
936 \\
986 . \\
1036 . \\
1085 \\
1134 . \\
1184 . \\
1233 . \\
1283 . \\
1332\end{array}$ & $\begin{array}{r}706 . \\
790 . \\
866 . \\
930 . \\
976 . \\
1015 . \\
1048 . \\
1075 . \\
1100 . \\
1125 . \\
1153 . \\
1179 . \\
1173 . \\
1145 .\end{array}$ & $\begin{array}{l}482 . \\
517 . \\
552 . \\
587 . \\
619 . \\
647 . \\
672 . \\
695 . \\
715 . \\
734 . \\
751 . \\
767 . \\
762 . \\
744 .\end{array}$ & $\begin{array}{l}573 . \\
630 . \\
682 . \\
725 . \\
759 . \\
788 . \\
814 . \\
837 . \\
858 . \\
881 . \\
904 . \\
925 . \\
921 . \\
901 .\end{array}$ & $\begin{array}{l}447 . \\
478 . \\
508 . \\
537 . \\
564 . \\
588 . \\
609 . \\
629 . \\
647 . \\
663 . \\
679 . \\
694 . \\
690 . \\
673 .\end{array}$ & $\begin{array}{c}616 . \\
682 . \\
747 \\
810 \\
861 . \\
904 . \\
940 . \\
972 . \\
1002 . \\
1029 . \\
1053 . \\
1074 . \\
1068 . \\
1045 .\end{array}$ & $\begin{array}{r}586 . \\
647 . \\
706 . \\
762 . \\
809 . \\
847 . \\
880 . \\
910 . \\
937 . \\
962 . \\
985 . \\
1004 . \\
999 . \\
977 .\end{array}$ & $\begin{array}{l}556 . \\
611 . \\
664 . \\
715 . \\
756 . \\
791 . \\
821 . \\
848 . \\
873 . \\
896 . \\
916 . \\
934 . \\
929 . \\
909 .\end{array}$ & $\begin{array}{l}515 . \\
563 . \\
608 . \\
651 . \\
687 . \\
718 . \\
745 . \\
769 . \\
792 . \\
813 . \\
833 . \\
850 . \\
846 . \\
829 .\end{array}$ & $\begin{array}{l}505 . \\
551 . \\
595 . \\
636 . \\
671 . \\
700 . \\
726 . \\
750 . \\
773 . \\
793 . \\
813 . \\
830 . \\
827 . \\
810 .\end{array}$ & $\begin{array}{l}496 . \\
539 . \\
581 . \\
620 . \\
654 . \\
683 . \\
708 . \\
732 . \\
753 . \\
774 . \\
793 . \\
810 . \\
807 . \\
791 .\end{array}$ \\
\hline $\begin{array}{l}\text { Mean } \\
\text { Range/RMS }\end{array}$ & $\begin{array}{r}1011 . \\
693 .\end{array}$ & $\begin{array}{l}1020 \\
\pm 144\end{array}$ & $\begin{array}{l}660 \\
\pm 92\end{array}$ & $\begin{array}{r}800 \\
\pm 108\end{array}$ & $\begin{array}{l}600 \\
\pm 79\end{array}$ & $\begin{array}{r}914 \\
\pm 145\end{array}$ & $\begin{array}{r}858 \\
\pm 132\end{array}$ & $\begin{array}{r}802 \\
\pm 120\end{array}$ & $\begin{array}{r}730 \\
\pm 106\end{array}$ & $\begin{array}{r}713 \\
\pm 103\end{array}$ & $\begin{array}{r}696 \\
\pm 100\end{array}$ \\
\hline $\begin{array}{l}\text { Body } \\
2\end{array}$ & $\begin{array}{l}1479 . \\
1529 . \\
1578 . \\
1628 . \\
1677 . \\
1727 . \\
1776 . \\
1826 . \\
1875 \\
1925 . \\
1974 . \\
2024 . \\
2073 . \\
2123 .\end{array}$ & $\begin{array}{l}1163 . \\
1227 . \\
1272 . \\
1288 . \\
1298 . \\
1308 . \\
1318 . \\
1326 . \\
1332 . \\
1338 . \\
1347 \\
1362 . \\
1343 . \\
1300 .\end{array}$ & $\begin{array}{l}753 . \\
793 . \\
820 . \\
828 . \\
835 . \\
842 . \\
848 . \\
852 . \\
856 . \\
858 . \\
861 . \\
861 . \\
841 . \\
807 .\end{array}$ & $\begin{array}{r}915 . \\
967 . \\
1005 . \\
1020 . \\
1031 . \\
1041 . \\
1052 . \\
1062 . \\
1070 . \\
1082 . \\
1096 . \\
1110 . \\
1096 \\
1064 .\end{array}$ & $\begin{array}{l}683 . \\
719 . \\
745 . \\
753 . \\
761 . \\
768 . \\
774 . \\
779 . \\
783 . \\
787 . \\
791 . \\
793 . \\
775 . \\
746 .\end{array}$ & $\begin{array}{l}1058 . \\
1115 . \\
1154 . \\
1167 . \\
1177 . \\
1186 . \\
1195 . \\
1202 . \\
1207 . \\
1212 . \\
1216 . \\
1218 . \\
1191 . \\
1145 .\end{array}$ & $\begin{array}{r}990 . \\
1044 . \\
1081 . \\
1093 . \\
1103 . \\
1112 . \\
1121 . \\
1128 . \\
1134 . \\
1139 . \\
1144 . \\
1147 . \\
1122 . \\
1080 .\end{array}$ & $\begin{array}{r}922 . \\
972 . \\
1007 . \\
1019 . \\
1029 . \\
1038 . \\
1047 . \\
1055 . \\
1061 . \\
1067 . \\
1072 . \\
1076 . \\
1054 . \\
1014 .\end{array}$ & $\begin{array}{l}842 . \\
889 . \\
922 . \\
934 . \\
945 . \\
955 . \\
964 . \\
972 . \\
980 . \\
986 . \\
993 . \\
997 . \\
979 . \\
944 .\end{array}$ & $\begin{array}{l}823 . \\
869 . \\
902 . \\
914 . \\
924 . \\
934 . \\
944 . \\
952 . \\
960 . \\
967 . \\
973 . \\
978 . \\
960 . \\
926 .\end{array}$ & $\begin{array}{l}803 . \\
849 . \\
881 . \\
893 . \\
904 . \\
914 . \\
924 . \\
932 . \\
940 . \\
947 . \\
954 . \\
959 . \\
942 . \\
909 .\end{array}$ \\
\hline $\begin{array}{l}\text { Mean } \\
\text { Range/RMS }\end{array}$ & $\begin{array}{r}1801 . \\
693 .\end{array}$ & $\begin{array}{r}1301 \\
\pm 51\end{array}$ & $\begin{array}{l}832 \\
\pm 30\end{array}$ & $\begin{array}{r}1044 \\
\pm 52\end{array}$ & $\begin{array}{l}761 \\
\pm 30\end{array}$ & $\begin{array}{r}1175 \\
\pm 43\end{array}$ & $\begin{array}{r}1103 \\
\pm 42\end{array}$ & $\begin{array}{r}1031 \\
\pm 41\end{array}$ & $\begin{array}{l}950 \\
\pm 42\end{array}$ & $\begin{array}{l}930 \\
\pm 42\end{array}$ & $\begin{array}{l}911 . \\
\pm 42 .\end{array}$ \\
\hline$\underset{3}{\text { Body }}$ & $\begin{array}{l}2269 . \\
2318 . \\
2368 . \\
2418 . \\
2467 . \\
2517 . \\
2566 . \\
2616 . \\
2665 . \\
2714 . \\
2764 . \\
2814 . \\
2863 . \\
2913 .\end{array}$ & $\begin{array}{l}1309 . \\
1369 . \\
1406 . \\
1409 . \\
1409 . \\
1406 . \\
1402 . \\
1392 . \\
1372 . \\
1348 . \\
1321 . \\
1295 . \\
1242 . \\
1171 .\end{array}$ & $\begin{array}{l}804 . \\
835 . \\
852 . \\
846 . \\
842 . \\
836 . \\
827 . \\
818 . \\
810 . \\
801 . \\
789 . \\
780 . \\
765 . \\
747 .\end{array}$ & $\begin{array}{l}1074 . \\
1126 . \\
1159 . \\
1165 . \\
1168 . \\
1170 . \\
1170 . \\
1166 . \\
1155 . \\
1141 . \\
1126 . \\
1110 . \\
1072 . \\
1017 .\end{array}$ & $\begin{array}{l}744 . \\
775 . \\
792 . \\
789 . \\
787 . \\
783 . \\
777 . \\
771 . \\
765 . \\
759 . \\
751 . \\
744 . \\
730 . \\
712 .\end{array}$ & $\begin{array}{l}1142 . \\
1184 . \\
1206 . \\
1199 \\
1189 \\
1177 \\
1162 . \\
1146 \\
1127 \\
1106 . \\
1082 . \\
1058 \\
1025 . \\
986\end{array}$ & $\begin{array}{r}1078 . \\
1119 . \\
1141 . \\
1135 . \\
1128 . \\
1118 . \\
1106 . \\
1093 . \\
1076 . \\
1058 . \\
1037 . \\
1017 . \\
986 . \\
949 .\end{array}$ & $\begin{array}{c}1014 . \\
1054 . \\
1076 . \\
1072 . \\
1067 . \\
1059 . \\
1049 . \\
1039 . \\
1026 . \\
1010 . \\
993 . \\
976 . \\
947 . \\
912 .\end{array}$ & $\begin{array}{r}945 . \\
983 . \\
1006 . \\
1004 . \\
1000 . \\
995 . \\
988 . \\
980 . \\
970 . \\
958 . \\
944 . \\
930 . \\
905 . \\
872 .\end{array}$ & $\begin{array}{l}928 . \\
966 . \\
989 . \\
987 . \\
984 . \\
979 . \\
973 . \\
966 . \\
956 . \\
945 . \\
932 . \\
919 . \\
895 . \\
864 .\end{array}$ & $\begin{array}{l}911 . \\
949 . \\
971 . \\
970 . \\
968 . \\
964 . \\
958 . \\
951 . \\
943 . \\
933 . \\
921 . \\
909 . \\
886 . \\
855 .\end{array}$ \\
\hline $\begin{array}{l}\text { Mean } \\
\text { Range/RMS }\end{array}$ & $\begin{array}{r}2591 . \\
693 .\end{array}$ & $\begin{array}{r}1347 \\
\pm 70\end{array}$ & $\begin{array}{l}811 \\
\pm 31\end{array}$ & $\begin{array}{r}1130 \\
\pm 45\end{array}$ & $\begin{array}{l}763 \\
\pm 23\end{array}$ & $\begin{array}{r}1128 \\
\pm 66\end{array}$ & $\begin{array}{c}1074 \\
\pm 57\end{array}$ & $\begin{array}{r}1021 \\
\pm 48\end{array}$ & $\begin{array}{l}963 . \\
\pm 39\end{array}$ & $\begin{array}{l}949 \\
\pm 36\end{array}$ & $\begin{array}{l}935 . \\
\pm 34 .\end{array}$ \\
\hline $\begin{array}{l}\text { Mean } \\
\text { Range/RMS }\end{array}$ & $\begin{array}{l}1801 . \\
2273 .\end{array}$ & $\begin{array}{l}1223 \\
\pm 174\end{array}$ & $\begin{array}{l}768 \\
\pm 96\end{array}$ & $\begin{array}{r}991 \\
\pm 158\end{array}$ & $\begin{array}{l}708 \\
\pm 92\end{array}$ & $\begin{array}{l}1072 \\
\pm 148\end{array}$ & $\begin{array}{l}1012 . \\
\pm 139\end{array}$ & $\begin{array}{r}951 \\
+131\end{array}$ & $\begin{array}{r}881 \\
\pm 127\end{array}$ & $\begin{array}{r}864 \\
\pm 127\end{array}$ & $\begin{array}{r}847 \\
\pm 126\end{array}$ \\
\hline
\end{tabular}

(a) Core height above core bed plate based on BOL metrology.

(b) Time and volume averaged.

(c) Root mean square deviation for gradient information.

(d) For sleeve temperatures see Table 3-3(a) 
TABLE 3-4 (a)

FTE-6 COMPARISON OF PREDICTED AND TIME-AVERAGED CHROMEL-ALUMEL THERMOCOUPLE TEMPERATURES

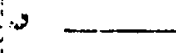

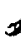

$$
\text { THF PMOCOUIPIE DATA }
$$

CDMPAPISUN OF TREUER ANO

TREVER Simulation $J$ EPOM REAL DAYS ATTEE FHOM EFP

.276 .12 .332 .52 .56 .00 .252 .00298 .00 $332.52-388.24 \ldots .55 .02 \ldots 298.00343 .00$ $398.140435 .12 \quad 40.98 \ldots 343.00 \ldots 385.40$ $435.12 \ldots 45.12 \ldots \ldots .00 \ldots 385.110325 .40 \ldots$ $435.12 \quad 580.99 .145 .87 \ldots 389.40 .499 .60$ $580.99 \quad 061.54 \quad 80.59 \quad 499.60 \quad 564.10$ $641.58 \quad 713.14 \quad 51.50 \quad 564.10610 .20 \ldots$ $713.14 \quad 811.87 \quad 99.73 \quad 010.20701 .20$

$811.87 \quad 811.87 \ldots .00 \quad 701.20 \quad 711.20$ $811.87 \quad 862.61 \quad 50.74 \ldots 7 n 1.207182 .00$ 862.01 .912 .6150 .00 .748 .00780 .00 $012.01 \quad 950.11 \quad 37.54 \quad 788.09818 .00$ $950.11 \quad 080.11 \ldots 30.00 \ldots 918.00 .835 .00 \ldots$ $980.111009 .11 \quad 29.09 \quad 835.00 .854 .00$ $1009.11+1056.11 .47 .00$. 954.00 BR०.7n $1056.11[1048.11 \quad 12.00$ 8ค9.70 897.30

TEMD. ERRTR TA....EA $(a)^{n}$ $555.0 \ldots .0 \quad .6$ QOR.O. . . . . . . $675.0 \ldots \ldots \ldots 4$ $692.0 \quad .0 \quad 1$ $63 n .0 \ldots \ldots .0 .81$ $640.0 \ldots .0 .10$ $592.0 \ldots \ldots 0.8$ $716.0 \ldots . .0 \quad 14$ $713.0 \ldots \ldots .0$ 717.0 649. $0 \ldots . .11$ $715.0 \ldots .0 .5$ $59 h .0 \ldots . . . n \quad 4$ $737.0 \ldots 0 \quad 3$ $714.0 \quad .0 \quad 14$ क71.0 . . 1 1 C/A MEASURED MEAN TEMP. TRANS. MEAN TRANS ERROP ...... OIFF ERROR CHANGE ERROR -

C/A CORRECTED TEMP: _ CORRECTED THERMOCOLPLE TEMP.

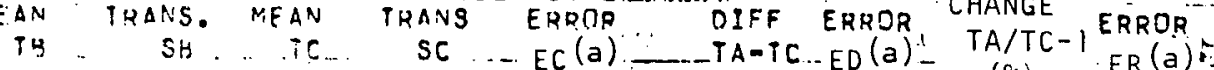

$053.3 \quad 42.1 \quad 653.3 \quad 42.1 \quad .0 \quad .098 .3 \quad .09 \quad-15$
(\%) ER $(a)=$ $653.3 \quad 42.1 \quad 653.3 \quad 42.1$ $.0 .098 .3 \quad .08$ $-15.1 \ldots . .0$

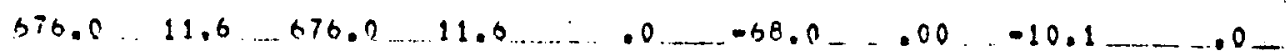

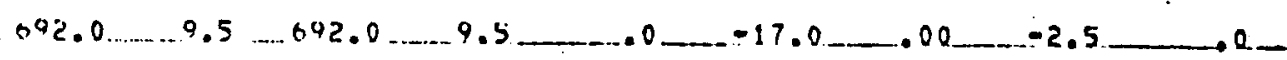

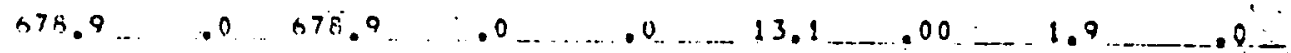

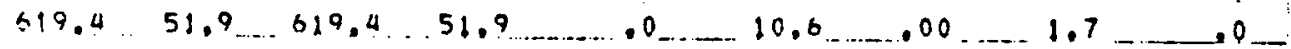

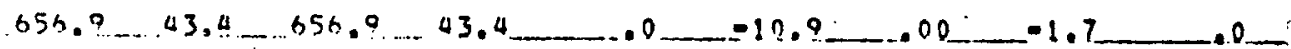
$650.3 \quad 10.2-650.3 \ldots 10.2 \ldots .00 .41 .7 \ldots .00 \quad 6.4 \ldots .0$

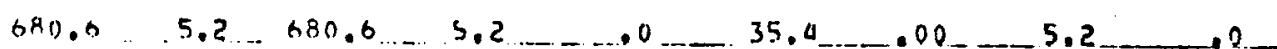

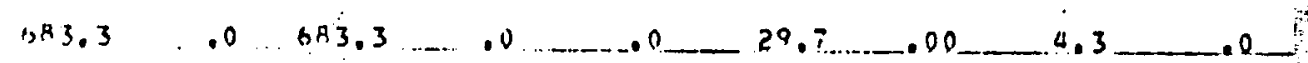

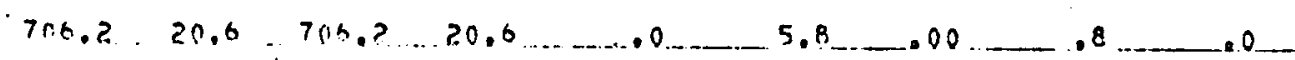

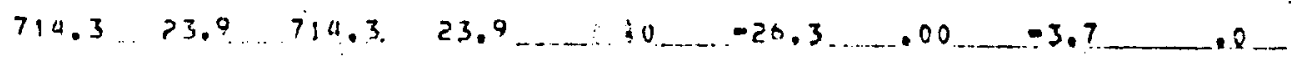

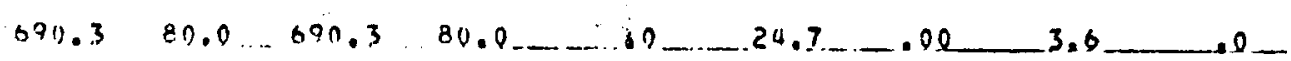
$586.0 .35 .1 .586 .0-35.1 \quad 00 \quad 10.0 \ldots 000+1.7 \quad 0$ $726.3 \cdot 7.3 \quad 726.3 \ldots 7.8 \ldots \ldots 0 \ldots 10.7 \ldots .00 \ldots 1.5 \ldots .0 .010$

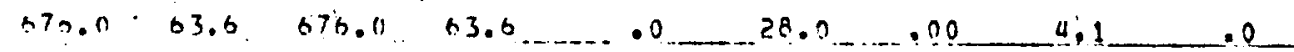
$641.5 \quad 1.9 \quad 649.5 \quad 1.9 \ldots \ldots .00 .029 .5 \ldots .00 \ldots 4.6 \ldots \ldots$ GRAND TOTAL

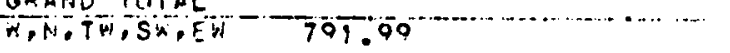
SH (1 SICSA) s6?.". 49.9 $.0 \quad 10 R$ $604.8 \quad 51.7$ GBA.B $51.7 \cdots \cdots \cdots$ $-2$ (a) NO ERROR STATEMENT AVAILABLE; STANDOFF PIN TEMPERATURE WAS $341^{\circ} \mathrm{C}$.
34.3

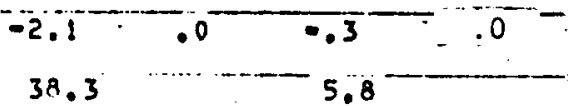

38.3

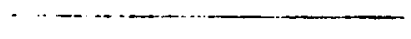


?

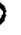

)

0

$\circ$

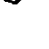

$0 \stackrel{\sim}{*}$

$\omega$

u

$\checkmark$

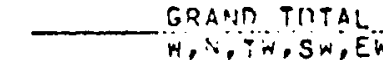

$\vartheta$

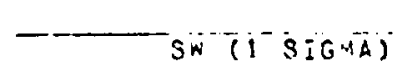

TABLE $3-4(\mathrm{~b})$
FTE-6 COMPARTSON OF PREDICTED

FIE- 6 COMPARISON OF PREDICTED AND TIME-AVERAGED TUNGSTEN-RHENIUM THERMOCOUPLE TEMPERATURES

\section{- THERMOCOUPLE DATA}

TREVER Simulation
NIRE MÉASIJRED

TEMP

$270.12 \ldots 32.52 \ldots 54.40 \ldots 252.40 .294 .00 \quad 053.0$

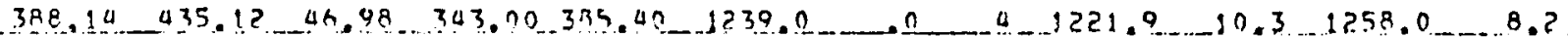

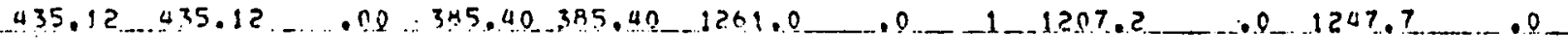

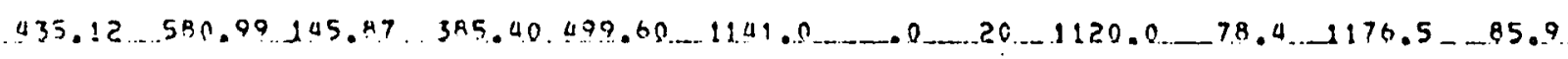

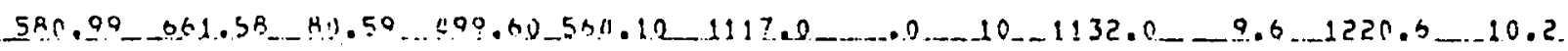$$
\text { 7. }
$$

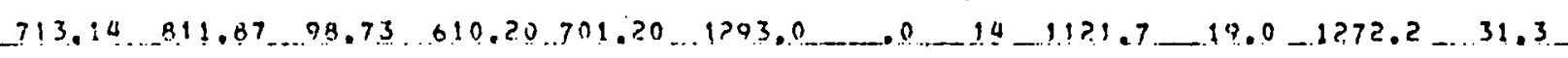

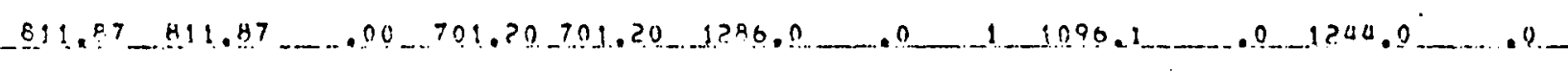

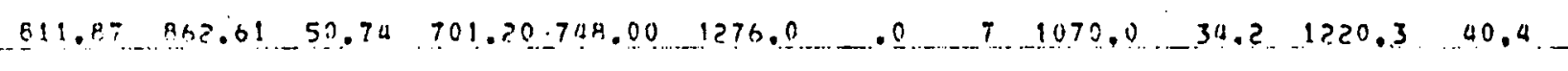

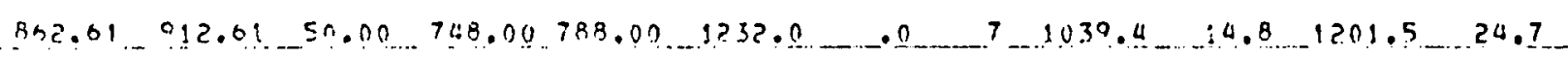

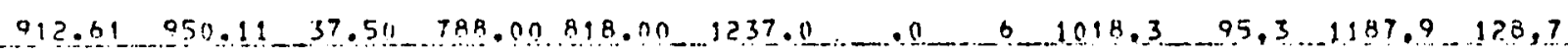

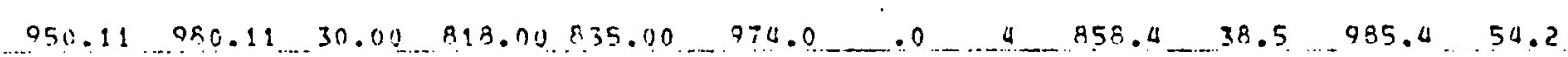

$980.11 .1009 .11 \ldots 20.00 .935 .00858 .00 \ldots 1268.0 \ldots \ldots .0 \quad 3 \quad 1060.7 \ldots 9.0 .1272 .2 \ldots 10.8$

$1009.111050 .11 .47 .00 .658 .00 .889 .70 .1202 .0 \quad .0 \quad 141021.5 \ldots 87.0 .1232 .9 \ldots 121.0 \ldots$

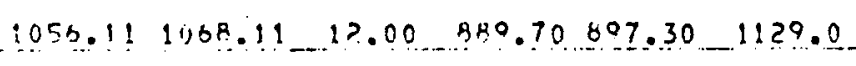
COMPARISON OF TREYER INE
CORPECTEC THERMOCCUPLE TEMF REL,

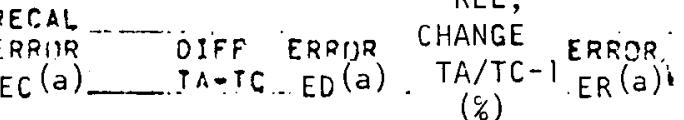

\begin{tabular}{c} 
OIFF ERRIJR CHANGE ERROR \\
EC (a)__ TA-TC ED (a) TA/TC-I ER (a) \\
\hline
\end{tabular}

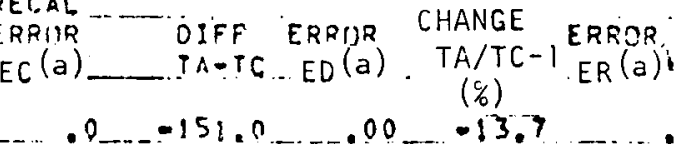

$.0 \ldots . . .74 .8 \ldots \ldots 00 \ldots . .6 .4 \ldots \ldots$

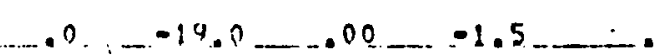
$\ldots .00_{-}+13.3 \ldots .00 \ldots 1.1 \ldots$ $\ldots 0 \ldots-35.5 \ldots \ldots .00 \ldots \ldots .0 .0 .0 . . . . .$. $.00-103.6 \ldots .00^{\circ} \quad-8.5$ $.0 \ldots \ldots-2.5 \ldots .00 \ldots \ldots . .2 \ldots$ $.0 \ldots+20.8 \ldots 00 \ldots$ $.0=42.0 \ldots-00 \ldots 3.4$ $\ldots .0 \ldots 5.7 \ldots .00 \ldots . . .6,6$. _. $3 n, 5 \ldots .00 \ldots 2.5$ $.0 \ldots 49,1 \ldots .00 \ldots .4 .1$ $\ldots 0 \ldots-11.4 \ldots . .00 \ldots \ldots-1.2 \ldots \ldots$ $.0 \ldots-4.2 \ldots .00 \ldots .3 \ldots$

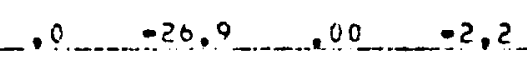
$.0 \ldots \ldots .0 .00 \ldots . .19 .1$ (a) NO ERROR Statement aVaILAble; Standoff PIN TEMPERATURE WAS $341^{\circ} \mathrm{C}$. 
FTE-6 AVERAGE NUCLIDE WEIGHTS ${ }^{\text {(a) }}$ TOR FUEL RODS, SPINE SAMPLES, AND TOTAL ELEMENT

\begin{tabular}{|c|c|c|c|c|c|c|c|c|c|c|c|c|c|}
\hline \multirow[b]{3}{*}{ Nuc11de } & \multirow{3}{*}{$\begin{array}{c}\text { A tomic Weight } \\
\text { (g/mol) }\end{array}$} & \multicolumn{8}{|c|}{ Nuclide Welghts Per Rod (g) } & \multicolumn{2}{|c|}{ Nuclide Welghts (g) } & \multicolumn{2}{|c|}{ Nuclide Kelghts (g) } \\
\hline & & \multicolumn{2}{|c|}{ Holes $1 \& 2$} & \multicolumn{2}{|c|}{ Holes $3 \& 4$} & \multicolumn{2}{|c|}{ Holes $5 \& 6$} & \multicolumn{2}{|c|}{ Holes $7 \& 8$} & \multirow{2}{*}{\multicolumn{2}{|c|}{\begin{tabular}{l|l}
\multicolumn{2}{c}{ Sp1ne Samples } \\
BOL & EOL
\end{tabular}}} & \multirow{2}{*}{\multicolumn{2}{|c|}{\begin{tabular}{l|l} 
Total Element \\
BOL
\end{tabular}}} \\
\hline & & BOL & EOL & $\mathrm{BOL}$ & EOL & BOL & EOL & BOL & EOL & & & & \\
\hline Th-232 & 232.03807 & 4.973 & 4.804 & 1.950 & 1.884 & 1.892 & 1.828 & 1.133 & 1.095 & 65.83 & 63.60 & 901.462 & 870.924 \\
\hline $\mathrm{Pa}-233$ & & 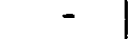 & 0.008824 & - & 0.003460 & - & 0.003357 & - & 0.002010 & - & 0.116811 & - & 1.601505 \\
\hline$u-233$ & 233.03965 & · & 0.099728 & - & 0.039105 & - & 0.037942 & - & 0.022721 & - & 1.320143 & - & 18.077802 \\
\hline U-234 (Bred) & 234.04098 & - & 0.008532 & - & 0.003345 & - & 0.003246 & - & 0.001944 & - & 0.109111 & - & 1.542739 \\
\hline U-235 (Bred) & 235.04394 & - & 0.000919 & - & 0.000360 & - & 0.000350 & - & 0.000209 & - & 0.012161 & - & 0.166553 \\
\hline $\mathrm{Th}_{0} / \mathrm{Th}_{1}$ & $232.038 / 232.064$ & 4.973 & 4.922 & 1.950 & 1.930 & 1.892 & 1.873 & 1.133 & 1.122 & 65.83 & 65.158 & 901.462 & 892.313 \\
\hline U-234（Fed) & 234.04098 & 0.004851 & 0.003439 & 0.004926 & 0.003492 & 0.004926 & 0.003492 & 0.004926 & 0.003492 & 0.019388 & 0.013743 & 1.649640 & 1.16935 \\
\hline U-235 (Ped) & 235.04394 & 0.610691 & 0.299515 & 0.610785 & 0.299561 & 0.610785 & 0.299561 & 0.610785 & 0.299561 & 2.440530 & 1.196965 & 207.651001 & 101.84300 \\
\hline$v-236$ & 236.04558 & 0.001770 & 0.061988 & 0.001797 & 0.062934 & 0.001797 & 0.062934 & 0.001797 & 0.062934 & 0.007074 & 0.217744 & 0.601883 & 21.07900 \\
\hline v-238 & 238.05082 & 0.038287 & 0.034015 & 0.038877 & 0.034539 & 0.038877 & 0.034539 & 0.038877 & 0.034539 & 0.153008 & 0.135935 & 13.018300 & 11.56570 \\
\hline $5 p-237$ & 237.04819 & - & 0.004054 & - & 0.004116 & - & 0.004116 & - & 0.004116 & - & 0.016203 & - & 1.37865 \\
\hline$x p-239$ & 239 & - & 0.000018 & - & 0.000018 & - & 0.000018 & - & 0.000018 & - & 0.000070 & - & 0.00598 \\
\hline$P u-238$ & 238.04958 & - & 0.000852 & - & 0.000865 & - & 0.000865 & - & 0.000865 & - & 0.003406 & - & 0.28975 \\
\hline Pu-239 & 239.05218 & - & 0.000983 & - & 0.000998 & - & 0.000998 & - & 0.000998 & - & 0.003929 & - & 0.33430 \\
\hline Pu -240 & 240.05383 & - & 0.000316 & - & 0.000321 & - & 0.000321 & - & 0.000321 & - & 0.001262 & - & 0.10734 \\
\hline$P u-241$ & 241.05877 & - & 0.000435 & - & 0.000435 & - & 0.000435 & - & 0.000435 & - & 0.001740 & - & 0.14805 \\
\hline $\begin{array}{l}u / U_{1} \\
x / x_{1}\end{array}$ & $\begin{array}{l}235.214 / 235.393 \\
232.408 / 232.318\end{array}$ & $\begin{array}{l}0.6556 \\
5.6286\end{array}$ & $\begin{array}{l}0.4058 \\
5.3278\end{array}$ & $\begin{array}{l}0.6557 \\
2.6057\end{array}$ & $\begin{array}{l}0.4073 \\
2.3373\end{array}$ & $\begin{array}{l}0.6557 \\
2.5477\end{array}$ & $\begin{array}{l}0.4073 \\
2.2803\end{array}$ & $\begin{array}{l}0.6557 \\
1.7887\end{array}$ & $\begin{array}{l}0.4073 \\
1.5293\end{array}$ & $\begin{array}{l}2.620 \\
68.45\end{array}$ & $\begin{array}{r}1.591 \\
66.749\end{array}$ & $\begin{array}{r}222.921 \\
1124.383\end{array}$ & $\begin{array}{r}137.921 \\
1030.234\end{array}$ \\
\hline
\end{tabular}

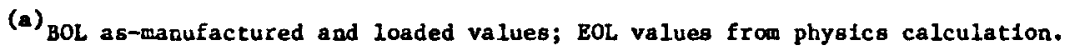


TABLE 3-6

FTE-6 PEAK AND MEAN BURNUP VALUES AND GRADIENTS

\begin{tabular}{|c|c|c|c|c|c|}
\hline $\begin{array}{r}\text { Body } \\
\text { No. }\end{array}$ & $\begin{array}{l}\text { Rod } \\
\text { No. }\end{array}$ & $\begin{array}{c}\text { Core Height } \\
(\mathrm{mm})\end{array}$ & $\begin{array}{c}\text { Fertile FIMA } \\
(\%)\end{array}$ & $\begin{array}{c}\text { Fissile FIMA } \\
(\%)\end{array}$ & $\begin{array}{c}\text { Total Element } \\
(\%)\end{array}$ \\
\hline \multirow[t]{3}{*}{1} & $\begin{array}{r}1 \\
2 \\
3 \\
4 \\
5 \\
6 \\
7 \\
8 \\
9 \\
10 \\
11 \\
12 \\
13 \\
14\end{array}$ & $\begin{array}{r}689 \\
738 \\
788 \\
837 \\
887 \\
936 \\
986 \\
1036 \\
1085 \\
1134 \\
1184 \\
1233 \\
1283 \\
1332\end{array}$ & $\begin{array}{l}0.14 \\
0.27 \\
0.39 \\
0.52 \\
0.65 \\
0.77 \\
0.90 \\
1.02 \\
1.13 \\
1.21 \\
1.29 \\
1.35 \\
1.41 \\
1.45\end{array}$ & $\begin{array}{l}20.08 \\
24.18 \\
27.89 \\
30.99 \\
33.83 \\
36.02 \\
38.05 \\
39.42 \\
40.73 \\
41.55 \\
42.37 \\
43.01 \\
43.65 \\
44.17\end{array}$ & $\begin{array}{l}4.05 \\
4.96 \\
5.78 \\
6.50 \\
7.16 \\
7.68 \\
8.19 \\
8.55 \\
8.90 \\
9.12 \\
9.35 \\
9.52 \\
9.69 \\
9.83\end{array}$ \\
\hline & \multicolumn{2}{|c|}{$\begin{array}{rr}\text { Mean } & 1011 \\
\text { Range/RMS } & 693\end{array}$} & $\begin{array}{r}0.89 \\
\pm 0.43 \\
\end{array}$ & $\begin{array}{r}36.14 \\
\pm 7.43 \\
\end{array}$ & $\begin{array}{r}7.81 \\
+1.79\end{array}$ \\
\hline & $\begin{array}{r}1 \\
2 \\
3 \\
4 \\
5 \\
6 \\
7 \\
8 \\
9 \\
10 \\
11\end{array}$ & $\begin{array}{l}1479 \\
1529 \\
1578 \\
1628 \\
1677 \\
1727 \\
1776 \\
1826 \\
1875 \\
1925 \\
1974\end{array}$ & $\begin{array}{l}1.49 \\
1.52 \\
1.55 \\
1.56 \\
1.56 \\
1.56 \\
1.54 \\
1.52 \\
1.49 \\
1.46 \\
1.42\end{array}$ & $\begin{array}{l}44.68 \\
45.05 \\
45.38 \\
45.53 \\
45.60 \\
45.54 \\
45.40 \\
45.14 \\
44.78 \\
44.40 \\
44.0\end{array}$ & $\begin{array}{r}9.96 \\
10.06 \\
10.15 \\
10.18 \\
10.20 \\
10.19 \\
10.14 \\
10.07 \\
9.98 \\
9.88 \\
9.77\end{array}$ \\
\hline
\end{tabular}


TABLE 3-6 (continued)

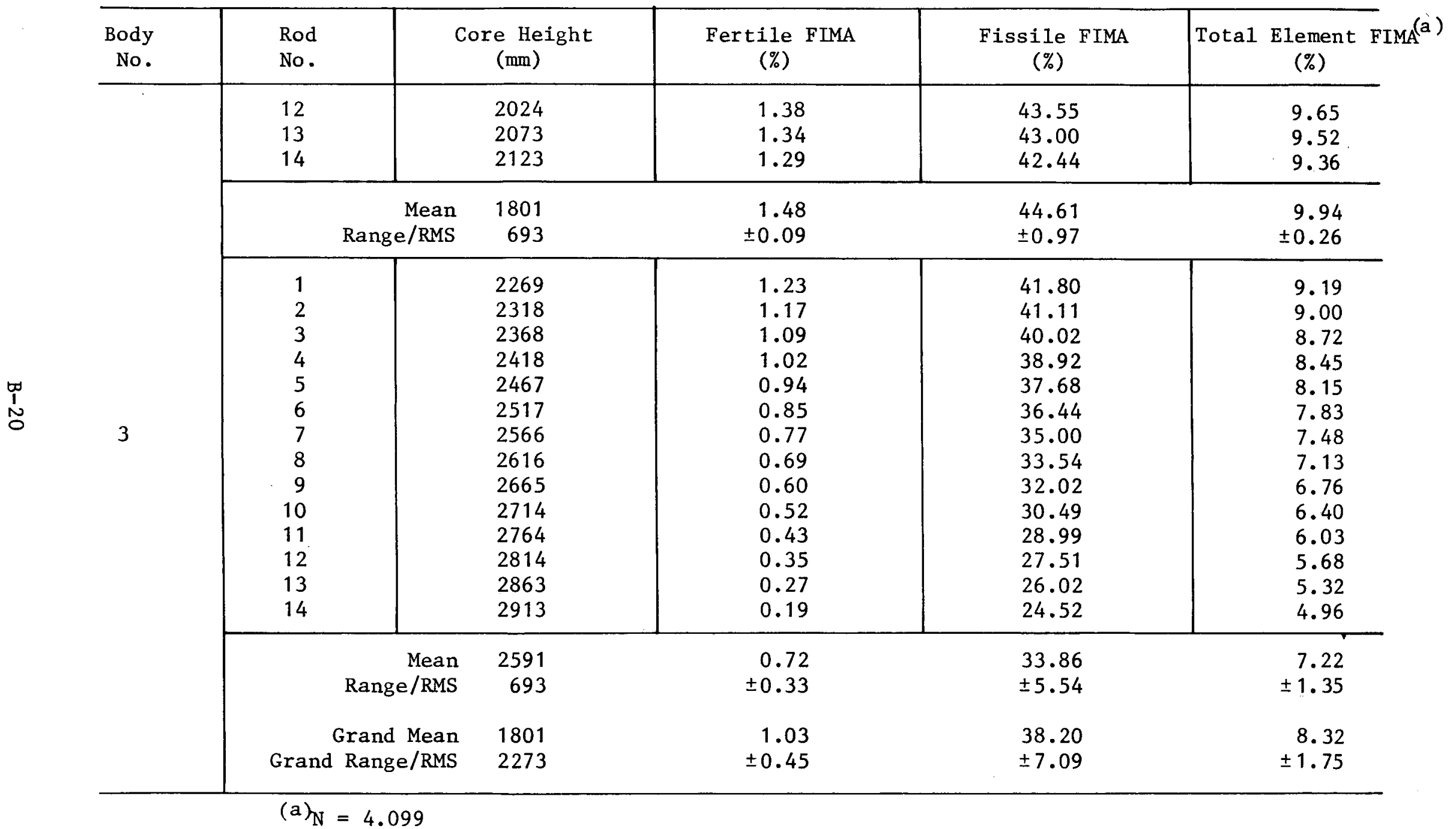


TABLE 3-7

NUCLEAR CONSTANTS AND UNCERTAINTY LIMITS USED IN BURNUP DETERMINATIONS

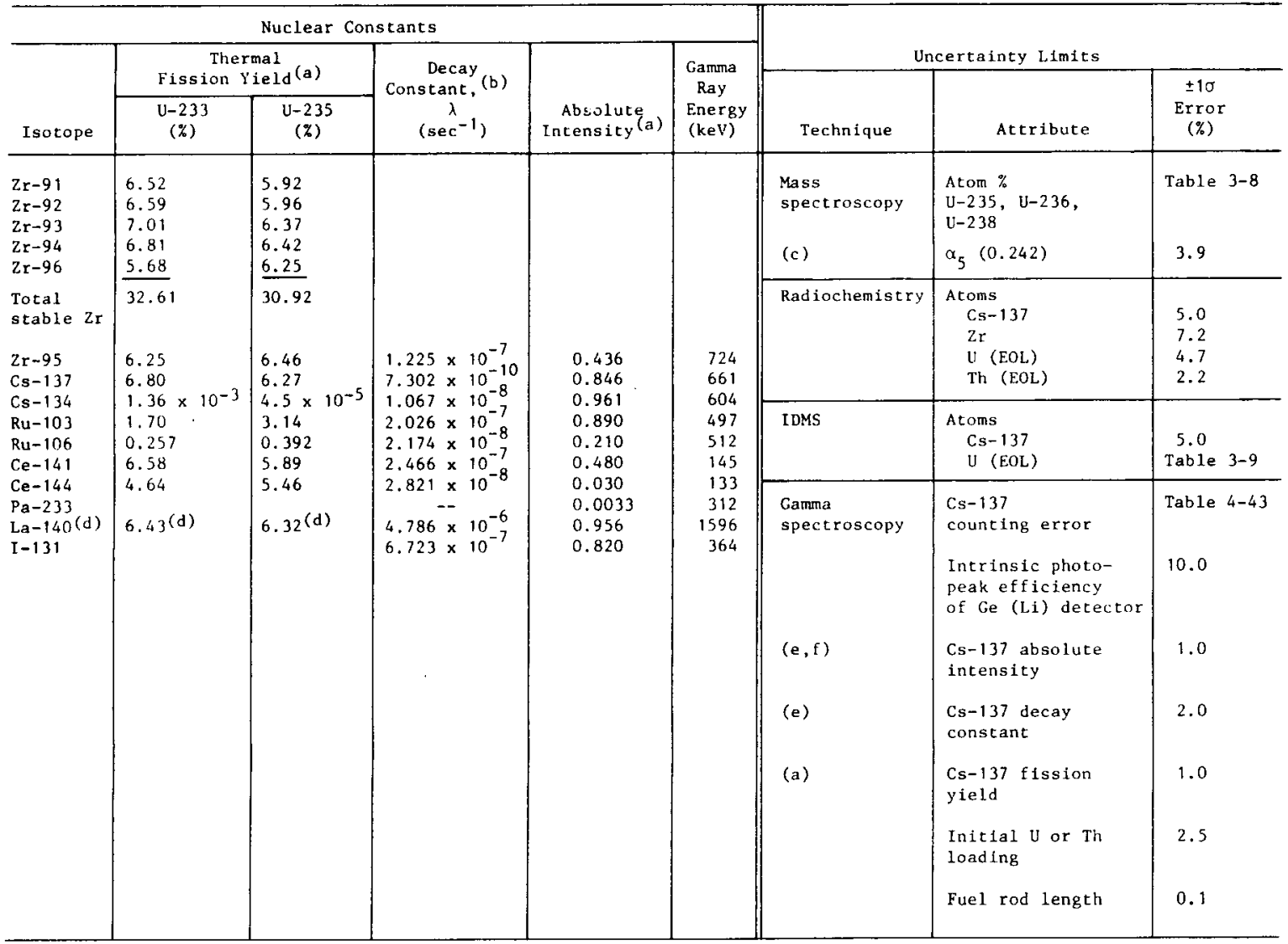

(a) Evaluated Nuclear Data File Library, ENDF/B, Version IV, Brookhaven National Laboratory.

(b) Meek, M. E., and B. F. Rider, "Compllation of Fission Product Yields," Vallecitos Nuclear Center Report NEDO- $12154-1,1974$.

(c) Wallroth, C. F., J. F. Holzgraf, D. D. Jensen, and L. R. Zumwalt, "Postirradiation Examination of Peach Bottom Fuel Test Element FTE-4," ERDA Report GA-A13452, General-Atomic Company, July 1977.

(d) Precursor Ba-140 is the direct fission yield isotope, measured through its daughter La-140. Direct yield La-140 is decayed at the time of gamma scan because of its shorter half life $\left[\lambda(\mathrm{La}-140)=4.786 \cdot 10^{-6} \mathrm{sec}^{-1}\right]$.

(e) Martin, H. J., and P. H. Blichert-Toft, Nuclear Data Tables, Vol. 8, October 1970.

(E) Merritt, Janet S., and J. G. U. Taylor, "Decay of Cestum-137 Determined by Absolute Counting Methods," Ana1. Chem. 37, 351 (1965). 
TABLE 3-8

FTE-6 MASS SPECTROMETRY AND IDMS RESULTS AND COMPARISONS WITH GAUGE/FEVER PREDICTIONS

\begin{tabular}{|c|c|c|c|c|c|c|c|c|c|c|c|c|c|}
\hline \multirow{3}{*}{$\begin{array}{c}\text { Particle } \\
\text { Batch } \\
\text { No. (a) }\end{array}$} & \multirow{3}{*}{$\begin{array}{l}\text { Splne } \\
\text { and } \\
\text { core } \\
\text { Location } \\
\text { (m) }\end{array}$} & \multirow{3}{*}{$\begin{array}{l}\text { Particle } \\
\text { I.D. }\end{array}$} & \multirow{3}{*}{$\begin{array}{l}\text { Analysis } \\
\text { Procedure }\end{array}$} & \multicolumn{10}{|c|}{ Relative Atomic Concentration (Atom $\%$ ) } \\
\hline & & & & \multicolumn{2}{|c|}{ U-233 } & \multicolumn{2}{|c|}{$\mathrm{v}-234$} & \multicolumn{2}{|c|}{$u-235$} & \multicolumn{2}{|c|}{$v-236$} & \multicolumn{2}{|c|}{$U-238$} \\
\hline & & & & $\mathrm{N}_{3}$ & $s_{3}(10)$ & $\mathrm{N}_{4}$ & $s_{4}(1 \sigma)$ & $\mathrm{N}_{5}$ & $S_{5}(10)$ & $N_{6}$ & $s_{6}(1 \sigma)$ & $\mathrm{N}_{8}$ & $s_{8}(1 \sigma)$ \\
\hline \multirow{3}{*}{$\begin{array}{l}\text { FLSSILE } \\
4000-302\end{array}$} & & & & & & & & & & & & & \\
\hline & $T S-6-5^{(b)}$ & $\begin{array}{l}\text { Archive } \\
\text { GAUGE/FEVER } \\
\text { C2055-2 } \\
\text { C2055-3 }\end{array}$ & $\begin{array}{l}\text { Mass Spec. } \\
\text { EOL (c) } \\
\text { Mass Spec. } \\
\text { Mass Spec. }\end{array}$ & $\begin{array}{l}-- \\
-- \\
--\end{array}$ & $\begin{array}{l}-- \\
\overline{-} \\
-\end{array}$ & $\begin{array}{l}0.782 \\
0.65 \\
0.893 \\
0.886\end{array}$ & $\begin{array}{c} \pm 0.010 \\
-- \\
\pm 0.006 \\
\pm 0.009\end{array}$ & $\begin{array}{l}93.202 \\
80.79 \\
78.21 \\
78.31\end{array}$ & $\begin{array}{c} \pm 0.015 \\
-. \\
\pm 0.07 \\
\pm 0.04\end{array}$ & $\begin{array}{l}0.2741 \\
10.93 \\
12.83 \\
12.79\end{array}$ & $\begin{array}{c} \pm 0.0027 \\
- \\
\pm 0.05 \\
\pm 0.04\end{array}$ & $\begin{array}{l}5.74 \\
7.65 \\
8.07 \\
8.02\end{array}$ & $\begin{array}{l} \pm 0.011 \\
- \\
\pm 0.06 \\
\pm 0.03\end{array}$ \\
\hline & \multicolumn{3}{|c|}{$\begin{array}{l}\text { Mean } \bar{N} \pm \mathrm{S}^{(\mathrm{d})} \\
\text { Calc/Meas }-1=\mathrm{z} \pm \mathrm{S}_{\mathrm{z}}(1 \sigma)^{\text {Mass Spec. }}\end{array}$} & - & - & $\begin{array}{r}0.890 \\
-0.270\end{array}$ & $\begin{array}{l} \pm 0.0059 \\
\pm 0.0048\end{array}$ & $\begin{array}{l}78.26 \\
0.032\end{array}$ & $\begin{array}{l} \pm 0.054 \\
\pm 0.0007\end{array}$ & $\begin{array}{l}12.81 \\
-0.147\end{array}$ & $\begin{array}{l} \pm 0.035 \\
\pm 0.0023\end{array}$ & $\begin{array}{l}8.05 \\
-0.050\end{array}$ & $\begin{array}{l} \pm 0.038 \\
\pm 0.0045\end{array}$ \\
\hline \multirow[t]{2}{*}{$4000-302$} & TS-6-5 & $\begin{array}{l}\mathrm{C} 2055-2 \\
\mathrm{C} 2055-3\end{array}$ & $\begin{array}{l}\text { IDMS } \\
\text { IDMS }\end{array}$ & $\begin{array}{l}-- \\
--\end{array}$ & -- & $\begin{array}{l}0.888 \\
0.883\end{array}$ & $\begin{array}{l} \pm 0.0077 \\
\pm 0.013\end{array}$ & $\begin{array}{l}78.137 \\
78.188\end{array}$ & $\begin{array}{l} \pm 0.115 \\
\pm 0.108\end{array}$ & $\begin{array}{l}12.877 \\
12.810\end{array}$ & $\begin{array}{l} \pm 0.089 \\
\pm 0.100\end{array}$ & $\begin{array}{l}8.164 \\
8.111\end{array}$ & $\begin{array}{l} \pm 0.082 \\
\pm 0.070\end{array}$ \\
\hline & $\begin{array}{l}\text { Mean } \bar{N} \pm S \\
\text { Calc/Meas }\end{array}$ & $1=z \pm S_{z}(10$ & IDMS & & & $\begin{array}{r}0.886 \\
-0.266\end{array}$ & $\begin{array}{l} \pm 0.0078 \\
\pm 0.0065\end{array}$ & $\begin{array}{r}78.163 \\
0.034\end{array}$ & $\begin{array}{l} \pm 0.081 \\
\pm 0.0011\end{array}$ & $\begin{array}{r}12.844 \\
-0.149\end{array}$ & $\begin{array}{l} \pm 0.071 \\
\pm 0.0047\end{array}$ & $\begin{array}{r}8.138 \\
-0.060\end{array}$ & $\begin{array}{l} \pm 0.057 \\
\pm 0.0066\end{array}$ \\
\hline \multicolumn{4}{|c|}{$\begin{array}{l}\text { Total Mean } \bar{N} \pm S \\
\text { Total Calc/Meas }-1=z+S_{z}(1 \sigma)\end{array}$} & & & $\begin{array}{r}0.888 \\
-0.268\end{array}$ & $\begin{array}{l} \pm 0.0051 \\
\pm 0.0042\end{array}$ & $\begin{array}{r}78.212 \\
0.033\end{array}$ & $\begin{array}{l} \pm 0.060 \\
\pm 0.00079\end{array}$ & $\begin{array}{l}12.827 \\
-0.148\end{array}$ & $\begin{array}{l} \pm 0.041 \\
\pm 0.027\end{array}$ & $\begin{array}{r}8.094 \\
-0.055\end{array}$ & $\begin{array}{l} \pm 0.046 \\
\pm 0.0054\end{array}$ \\
\hline \multicolumn{14}{|l|}{ FERTILE } \\
\hline \multirow[t]{2}{*}{$4000-242$} & TS-18-3 & $\begin{array}{l}\text { GAUGE/FEVER } \\
\text { C2054-3 } \\
\text { C2054-4 }\end{array}$ & $\begin{array}{l}\text { EOL } \\
\text { Mass Spec. } \\
\text { Mass Spec. }\end{array}$ & $\begin{array}{l}91.39 \\
92.66 \\
92.54\end{array}$ & $\begin{array}{l} \pm \overline{01} \\
\pm 0.04 \\
\pm 0.04\end{array}$ & $\begin{array}{l}7.54 \\
6.44 \\
6.51\end{array}$ & $\begin{array}{l}-- \\
\pm 0.01 \\
\pm 0.03\end{array}$ & $\begin{array}{l}1.07 \\
0.765 \\
0.818\end{array}$ & $\begin{array}{c}-- \\
\pm 0.008 \\
\pm 0.006\end{array}$ & $\begin{array}{l}-- \\
0.0370 \\
0.0346\end{array}$ & $\begin{array}{l}-- \\
\pm 0.004 \\
\pm 0.0003\end{array}$ & $\begin{array}{l}-- \\
0.0945 \\
0.0909\end{array}$ & $\begin{array}{c}-\overline{-} \\
\pm 0.0027 \\
\pm 0.0008\end{array}$ \\
\hline & \multicolumn{3}{|c|}{$\begin{array}{l}\text { Mean } \bar{N} \pm S \\
\text { Calc/Meas }-1=z \pm S_{z}(1 \sigma) \text { Mass Spec. }\end{array}$} & $\begin{array}{l}92.60 \\
-0.013\end{array}$ & $\begin{array}{l} \pm 0.047 \\
0.0005\end{array}$ & $\begin{aligned} & 6.48 \\
&+0.164\end{aligned}$ & $\begin{array}{l} \pm 0.040 \\
\pm 0.007\end{array}$ & $\begin{array}{l}0.792 \\
0.351\end{array}$ & $\begin{array}{l} \pm 0.086 \\
\pm 0.147\end{array}$ & $\begin{array}{l}0.0358 \\
--\end{array}$ & \pm 0.0022 & 0.0927 & \pm 0.0019 \\
\hline \multirow[t]{3}{*}{$4000-242$} & TS $-18-3$ & $\begin{array}{l}\text { C2054-3 } \\
\text { C2054-4 }\end{array}$ & $\begin{array}{l}\text { IDMS } \\
\text { IDMS }\end{array}$ & $\begin{array}{l}92.61 \\
92.54\end{array}$ & $\begin{array}{l} \pm 0.072 \\
\pm 0.087\end{array}$ & $\begin{array}{l}6.47 \\
6.54\end{array}$ & $\begin{array}{l} \pm 0.046 \\
\pm 0.060\end{array}$ & $\begin{array}{l}0.771 \\
0.825\end{array}$ & $\begin{array}{l} \pm 0.009 \\
\pm 0.008\end{array}$ & $\begin{array}{l}0.0378 \\
0.0403\end{array}$ & $\begin{array}{l} \pm 0.005 \\
\pm 0.0003\end{array}$ & $\begin{array}{l}0.0962 \\
0.0927\end{array}$ & $\begin{array}{l} \pm 0.003 \\
\pm 0.0007\end{array}$ \\
\hline & \multicolumn{3}{|c|}{$\begin{array}{l}\text { Mean } N \pm S^{(d)} \\
\text { Calc/Meas }-1=z \pm S_{2}(1 \sigma)\end{array}$} & $\begin{array}{l}92.58 \\
-0.013\end{array}$ & $\begin{array}{l} \pm 0.062 \\
\pm 0.0007\end{array}$ & $\begin{array}{l}6.51 \\
0.158\end{array}$ & $\begin{array}{l} \pm 0.045 \\
\pm 0.0080\end{array}$ & $\begin{array}{l}0.798 \\
0.341\end{array}$ & $\begin{array}{l} \pm 0.020 \\
\pm 0.034\end{array}$ & 0.0391 & \pm 0.0027 & 0.0945 & $\begin{array}{l} \pm 0.0020 \\
--\end{array}$ \\
\hline & \multicolumn{3}{|c|}{$\begin{array}{l}\text { otal Mean } \vec{N} \pm S(1 \sigma) \\
\text { otal Calc/Meas }-1=z \pm S_{z}(1 \sigma)\end{array}$} & $\begin{array}{l}92.59 \\
-0.013\end{array}$ & $\begin{array}{l} \pm 0.040 \\
\pm 0.0004\end{array}$ & $\begin{array}{l}6.50 \\
0.160\end{array}$ & $\begin{array}{l} \pm 0.032 \\
\pm 0.0057\end{array}$ & $\begin{array}{l}0.795 \\
0.346\end{array}$ & $\begin{array}{l} \pm 0.044 \\
\pm 0.074\end{array}$ & $\begin{array}{l}0.0375 \\
--\end{array}$ & $\begin{array}{c} \pm 0.0021 \\
--\end{array}$ & $\begin{array}{l}0.0936 \\
--\end{array}$ & $\begin{array}{c} \pm 0.0015 \\
--\end{array}$ \\
\hline
\end{tabular}

(a) Fuel materials branch data retrieval (Ref. 2 - 8).

(b) TS-6-5 the rmal stability type 2, crucible 6, hole 5 .

(c) AII GAUGE/FEVER atomic concentrations are calculated with mass spectrometry archive BOL concentrations.

${ }^{\text {(d) }} s_{x}=\left[\frac{1}{n^{2}} \sum s_{i}^{2}+\frac{1}{n^{2}} \sum\left(x_{i}-x\right)^{2}\right]^{1 / 2}$. 
TABLE 3-9

FTE-6 ISOTOPIC DILUTION MASS SPECTROMETRY RESULTS

\begin{tabular}{|c|c|c|c|c|c|c|c|c|c|c|c|c|c|c|c|}
\hline \multirow[b]{2}{*}{$\begin{array}{l}\text { Particle } \\
\text { Batch } \\
\text { No.(a) }\end{array}$} & \multirow[b]{2}{*}{$\begin{array}{l}\text { Spine } \\
\text { and Core } \\
\text { Location (b) } \\
\text { (mm) }\end{array}$} & \multicolumn{14}{|c|}{ Absolute Atomic Concentration } \\
\hline & & $\begin{array}{l}\text { Particle } \\
\text { I.D.(c) }\end{array}$ & $\begin{array}{c}\mathrm{U}-233 \\
(\mathrm{ppm})\end{array}$ & $\begin{array}{l}\text { Error } \\
(1 \sigma) \\
(\mathrm{ppm})\end{array}$ & $\begin{array}{l}\mathrm{U}-234 \\
(\mathrm{ppm})\end{array}$ & $\begin{array}{l}\text { Error } \\
(1 \sigma) \\
(\mathrm{ppm})\end{array}$ & $\begin{array}{l}U-235 \\
(p p m)\end{array}$ & $\begin{array}{l}\text { Error } \\
(1 \sigma) \\
(\mathrm{ppm})\end{array}$ & $\begin{array}{l}\mathrm{U}-236 \\
(\mathrm{ppm})\end{array}$ & $\begin{array}{l}\text { Error } \\
(1 \sigma) \\
(p r ; n)\end{array}$ & $\begin{array}{l}\mathrm{i}-238 \\
(\mathrm{ppm})\end{array}$ & $\begin{array}{l}\text { Error } \\
(1 \sigma) \\
(\mathrm{ppm})\end{array}$ & $\begin{array}{c}\text { Total U } \\
\text { (ppm) }\end{array}$ & $\begin{array}{c}\text { Error }(\mathrm{d}) \\
(1 \sigma) \\
(\mathrm{ppm})\end{array}$ & $\begin{array}{c}\text { Corrected Error } \\
(1 \sigma) \\
(\mathrm{ppm})\end{array}$ \\
\hline $4000-302$ & $\begin{array}{l}\text { TS 6-5 } \\
(2684)\end{array}$ & $\mathrm{C} 2055-2$ & - & -- & 0.00648 & \pm 0.00005 & 0.5704 & \pm 0.0029 & 0.0940 & \pm 0.0006 & 0.0596 & \pm 0.0006 & 0.730 & \pm 0.004 & \pm 0.003 \\
\hline $4000-302$ & $\begin{array}{l}\text { TS 6-5 } \\
(2684)\end{array}$ & $\mathrm{C} 2055-3$ & -- & -- & 0.00299 & \pm 0.00004 & 0.2649 & \pm 0.0017 & 0.0434 & \pm 0.0003 & 0.02748 & \pm 0.00021 & 0.3388 & \pm 0.0022 & \pm 0.0017 \\
\hline $4000-242$ & $\begin{array}{l}\text { TS 18-3 } \\
(2589)\end{array}$ & $\mathrm{C} 2054-3$ & 1.078 & \pm 0.006 & 0.0753 & \pm 0.0004 & 0.00898 & \pm 0.00010 & 0.00044 & \pm 0.00005 & 0.00112 & \pm 0.00003 & 1.164 & \pm 0.007 & \pm 0.006 \\
\hline $4000-242$ & TS $18-3$ & C2054-4 & 0.918 & \pm 0.007 & 0.0649 & \pm 0.0004 & 0.00818 & \pm 0.00006 & 0.00040 & \pm 0.00000 & 0.00092 & \pm 0.00001 & 0.992 & \pm 0.008 & \pm 0.007 \\
\hline
\end{tabular}

(a) Fuel Materials Branch data retrieval (Ref. 2-8).

(b) TS 6-5 thermal stability type II crucible 6 , hole 5 .

(c) C2055-2 hot-cell bottle C2055, particle 2 .

(d) From laboratory data sheets.

(e) $\mathrm{s}=\left(\Sigma \mathrm{s}_{\mathrm{i}}^{2}\right)^{1 / 2}$ 
TABLE $3-10$

FTE-6 COMPARISON OF IDMS AND RADIOCHEMISTRY RESULTS

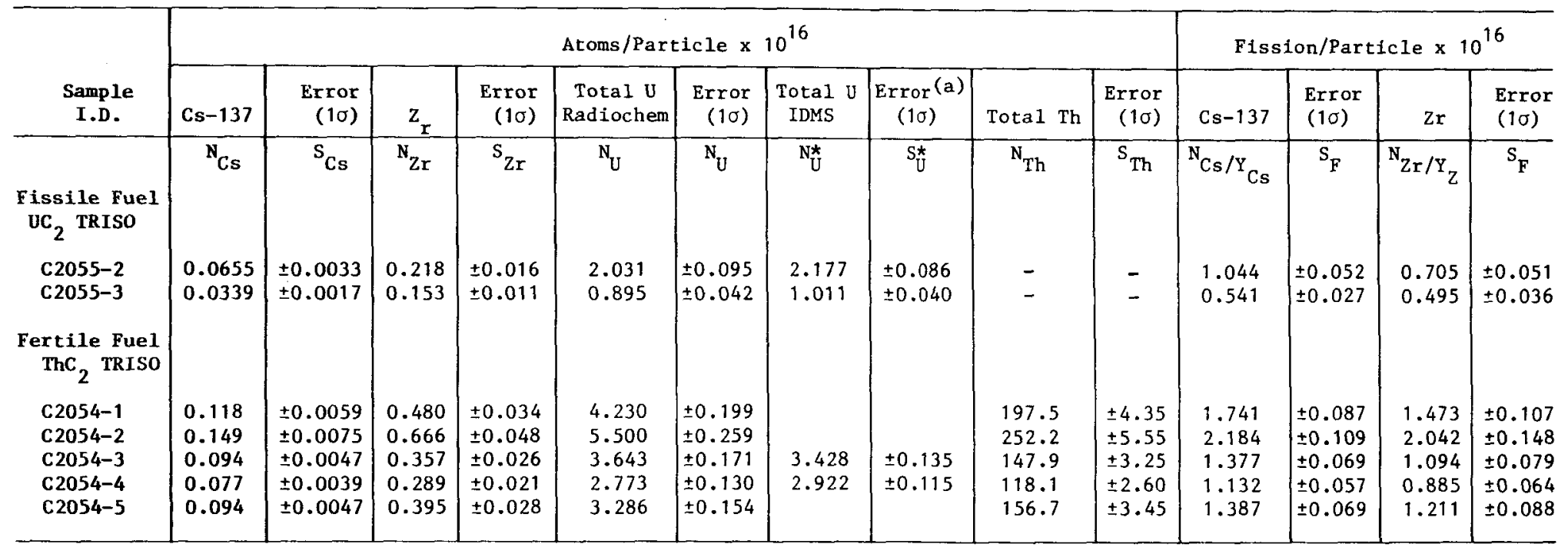

(a) Using laboratory-reported error on total uranium. 
TABLE 3-11

\begin{tabular}{|c|c|c|c|c|c|c|c|c|c|c|c|c|c|c|c|c|c|c|}
\hline & \multirow{2}{*}{$\begin{array}{c}\text { Core } \\
\text { and } \\
\text { Splne } \\
\text { Location } \\
(\mathrm{mm})\end{array}$} & \multirow[b]{2}{*}{$\begin{array}{c}\text { Particle } \\
\text { I.D. } \\
\text { No. }\end{array}$} & \multirow[b]{2}{*}{ Subject $\mathrm{s}$} & \multirow[b]{2}{*}{ symbo1 } & \multicolumn{4}{|c|}{$\begin{array}{c}\text { Mass spectrometry } \\
\text { FIMA }\end{array}$} & \multicolumn{4}{|c|}{$\begin{array}{l}\text { IDMS } \\
\text { FIMA }(c)\end{array}$} & \multicolumn{2}{|c|}{ Radiochemistry } & \multirow{2}{*}{$\begin{array}{c}\text { IDMS } \\
\begin{array}{c}\text { PIMA } \\
\text { Cs-137 } \\
\text { (atou } z)\end{array} \\
\end{array}$} & \multirow{2}{*}{$\begin{array}{c}\begin{array}{c}\text { Rad1o- } \\
\text { chem1stry }\end{array} \\
\begin{array}{c}\text { FIMA } \\
\text { Zr } \\
\text { (atod } x)\end{array}\end{array}$} & \multirow{2}{*}{$\begin{array}{l}\text { Selected }(d) \\
\text { Meagured } \\
\text { FIMA* } \\
\text { (atom } x) \\
\end{array}$} & \multirow{2}{*}{$\begin{array}{c}\text { Calculaced } \\
\text { GAJGEZ } \\
\text { FEVER } \\
\text { (atoo } z \text { ) } \\
\end{array}$} \\
\hline $\begin{array}{c}\text { Particle } \\
\text { Batch } \\
\text { No.(a) }\end{array}$ & & & & & $\begin{array}{c}\text { ASTM } \\
\text { EQ. 1 } \\
\text { (atom z) }\end{array}$ & $\begin{array}{c}\text { ASTM } \\
\text { EQ. 2 } \\
\text { (atom } x)\end{array}$ & $\begin{array}{c}\text { ASTM } \\
\text { EQ. } 3 \\
\text { (atom } z)\end{array}$ & $\begin{array}{c}\text { Mean } \\
\text { (atom } z \text { ) }\end{array}$ & $\begin{array}{c}\text { ASTM } \\
\text { EQ. } 1 \\
\text { (atom } x)\end{array}$ & $\begin{array}{c}\text { ASTM } \\
\text { EQ. 2 } \\
\text { (atom z) }\end{array}$ & $\begin{array}{c}\text { ASTM } \\
\text { EQ. } 3 \\
\text { (atom } 8 \text { ) }\end{array}$ & $\begin{array}{c}\text { Mean } \\
\text { (atom z) }\end{array}$ & $\begin{array}{c}\text { FIMA } \\
\text { Cs-137 } \\
(\text { atom z) }\end{array}$ & $\begin{array}{l}\text { FIMA } \\
\mathbf{Z r} \\
\text { (atom } z)\end{array}$ & & & & \\
\hline & \multicolumn{18}{|c|}{ Fissile Fuel Uc ${ }_{2}$ TRISO } \\
\hline $4000-302$ & $\begin{array}{l}\text { TS 6-5 } \\
\text { (2684) }\end{array}$ & $\begin{array}{l}\text { c2055-2 } \\
\text { c205s-3 } \\
\text { mean } \\
\text { (e) }\end{array}$ & $\begin{array}{l}\text { PIMA } \\
\text { Error (10) } \\
\text { FIMA } \\
\text { Error (10) } \\
\text { PIMA } \\
\text { Error (10) } \\
\text { Meas/Meast } 1 \\
\text { Error (10) } \\
\text { Calc/Meas-1 } \\
\text { Error (10) } \\
\end{array}$ & $\begin{array}{l}F_{5} \\
s_{5} \\
F_{5} \\
s_{5} \\
F_{5} \\
s_{p_{5}} \\
u^{*} \\
\text { su } \\
u \\
\text { su } \\
\end{array}$ & $\begin{array}{l}34.84 \\
\pm 0.15 \\
-0.0034 \\
\pm 0.0101 \\
-0.129 \\
\pm 0.004 \\
\end{array}$ & $\begin{array}{l}34.82 \\
\pm 0.85 \\
-0.0040 \\
\pm 0.0260 \\
-0.128 \\
\pm 0.021 \\
\end{array}$ & $\begin{array}{l}34.74 \\
\pm 0.28 \\
-0.0206 \\
\pm 0.0120 \\
-0.126 \\
\pm 0.007 \\
\end{array}$ & $\begin{array}{l}34.80 \\
\pm 0.31 \\
-0.0046 \\
\pm 0.0127 \\
-0.128 \\
\pm 0.008 \\
\end{array}$ & $\begin{array}{c}35.51 \\
\pm 0.50 \\
0.0157 \\
\pm 0.0171 \\
-0.145 \\
\pm 0.012 \\
\end{array}$ & $\begin{array}{l}34.61 \\
\pm 0.85 \\
-0.0100 \\
\pm 0.0259 \\
-0.123 \\
\pm 0.022 \\
\end{array}$ & $\begin{array}{l}35.23 \\
\pm 0.43 \\
0.0077 \\
\pm 0.0154 \\
-0.139 \\
\pm 0.011 \\
\end{array}$ & $\begin{array}{c}35.12 \\
\pm 0.52 \\
0.0046 \\
\pm 0.0175 \\
-0.136 \\
\pm 0.013 \\
\end{array}$ & \begin{tabular}{|c|}
33.95 \\
\pm 1.53 \\
37.67 \\
\pm 1.61 \\
35.81 \\
\pm 1.72 \\
0.0243 \\
\pm 0.0501 \\
-0.152 \\
\pm 0.041 \\
\end{tabular} & $\begin{array}{l}25.77 \\
\pm 1.65 \\
35.61 \\
\pm 1.98 \\
30.69 \\
\pm 3.71 \\
-0.122 \\
\pm 0.106 \\
-0.011 \\
\pm 0.119 \\
\end{array}$ & $\begin{array}{l}32.41 \\
\pm 1.39 \\
34.86 \\
\pm 1.45 \\
33.64 \\
\pm 1.32 \\
-0.038 \\
\pm 0.039 \\
-0.098 \\
\pm 0.035\end{array}$ & $\begin{array}{c}24.46 \\
\pm 1.52 \\
32.87 \\
\pm 1.83 \\
28.67 \\
\pm 3.20 \\
-0.180 \\
\pm 0.092 \\
0.0586 \\
\pm 0.118 \\
\end{array}$ & $\begin{array}{l}34.96 \\
\pm 0.32 \\
- \\
- \\
-0.132 \\
\pm 0.0079\end{array}$ & 30.35 \\
\hline & \multicolumn{18}{|c|}{ Fercile Fuel $\mathrm{ThO}_{2}$ TRISO } \\
\hline $4000-242$ & $\begin{array}{l}\text { TS } 18-3 \\
(2589)\end{array}$ & $\begin{array}{l}\text { C2054-1 } \\
\text { C2054-2 } \\
\text { C2054-3 } \\
\text { c2054-4 } \\
\text { C2054-5 } \\
\text { Mean } \\
\text { (e) }\end{array}$ & $\begin{array}{l}\text { FIMA } \\
\text { Error (10) } \\
\text { FIMA } \\
\text { Error (10) } \\
\text { FIMA } \\
\text { Error (10) } \\
\text { FIMA } \\
\text { Error (10) } \\
\text { FIMA } \\
\text { Error (10) } \\
\text { FIMA } \\
\text { Error (10) } \\
\text { Meas/Meas*-1 } \\
\text { Error (10) } \\
\text { Calc/meas-1 } \\
\text { Error (10) }\end{array}$ & \begin{tabular}{|l}
$F_{3}$ \\
$s_{3}$ \\
$F_{3}$ \\
$s_{3}$ \\
$F_{3}$ \\
$s_{3}$ \\
$F_{3}$ \\
$s_{3}$ \\
$F_{3}$ \\
$s_{3}$ \\
$F_{3}$ \\
$s_{F_{3}}$ \\
$u^{*}$ \\
$S u$ \\
$u$ \\
Su
\end{tabular} & $\begin{array}{l}- \\
- \\
-\end{array}$ & - & - & $\begin{array}{l}- \\
- \\
- \\
-\end{array}$ & - & $\begin{array}{l}- \\
- \\
- \\
-\end{array}$ & - & - & \begin{tabular}{|c|}
0.86 \\
\pm 0.047 \\
0.84 \\
\pm 0.045 \\
0.90 \\
\pm 0.049 \\
0.93 \\
\pm 0.050 \\
0.86 \\
\pm 0.046 \\
0.88 \\
\pm 0.026 \\
\\
+0.011 \\
\pm 0.093 \\
-0.159 \\
\pm 0.025
\end{tabular} & $\begin{array}{c}0.72 \\
\pm 0.055 \\
0.79 \\
\pm 0.059 \\
0.72 \\
\pm 0.054 \\
0.73 \\
\pm 0.054 \\
0.75 \\
\pm 0.057 \\
0.74 \\
\pm 0.028 \\
-0.149 \\
\pm 0.081 \\
0.000 \\
\pm 0.038\end{array}$ & $\begin{array}{c}0.84 \\
\pm 0.049 \\
0.90 \\
\pm 0.050 \\
- \\
0.87 \\
\pm 0.076 \\
-0.000 \\
\pm 0.000 \\
-0.149 \\
\pm 0.074\end{array}$ & $\begin{array}{c}0.79 \\
\pm 0.054 \\
0.72 \\
\pm 0.054 \\
- \\
0.76 \\
\pm 0.045 \\
\\
-0.126 \\
\pm 0.092 \\
-0.026 \\
\pm 0.058\end{array}$ & $\begin{array}{l}0.87 \\
\pm 0.076 \\
- \\
- \\
-0.149 \\
\pm 0.033\end{array}$ & 0.74 \\
\hline
\end{tabular}

(a) Fuel Material Branch Data retrieval number.

(b) TS 6-5: thermal stability type II cructble 6, hole 5.

(c) IDMS: 1sotope dilution mass spectroscopy.

(d) Fissile FIMA: average of mass spectrometry and IDMS; fert1le FIMA: IDMS with Cs-137 monitor.

(e) $s_{\overline{F s}_{s}}=\left\{\frac{1}{n^{2}} \sum s_{1}^{2}+\frac{1}{a^{2}} \sum\left(F_{s}-\bar{F}_{s}\right)^{2}\right\}^{1 / 2}$. 
TABLE $3-12$
FTE-6 COAPARISON BETWEEN GAMMA SPECTBOSCOPY AND GAUGE/FEYER CALCULATED FUEL ROD BURNUPS

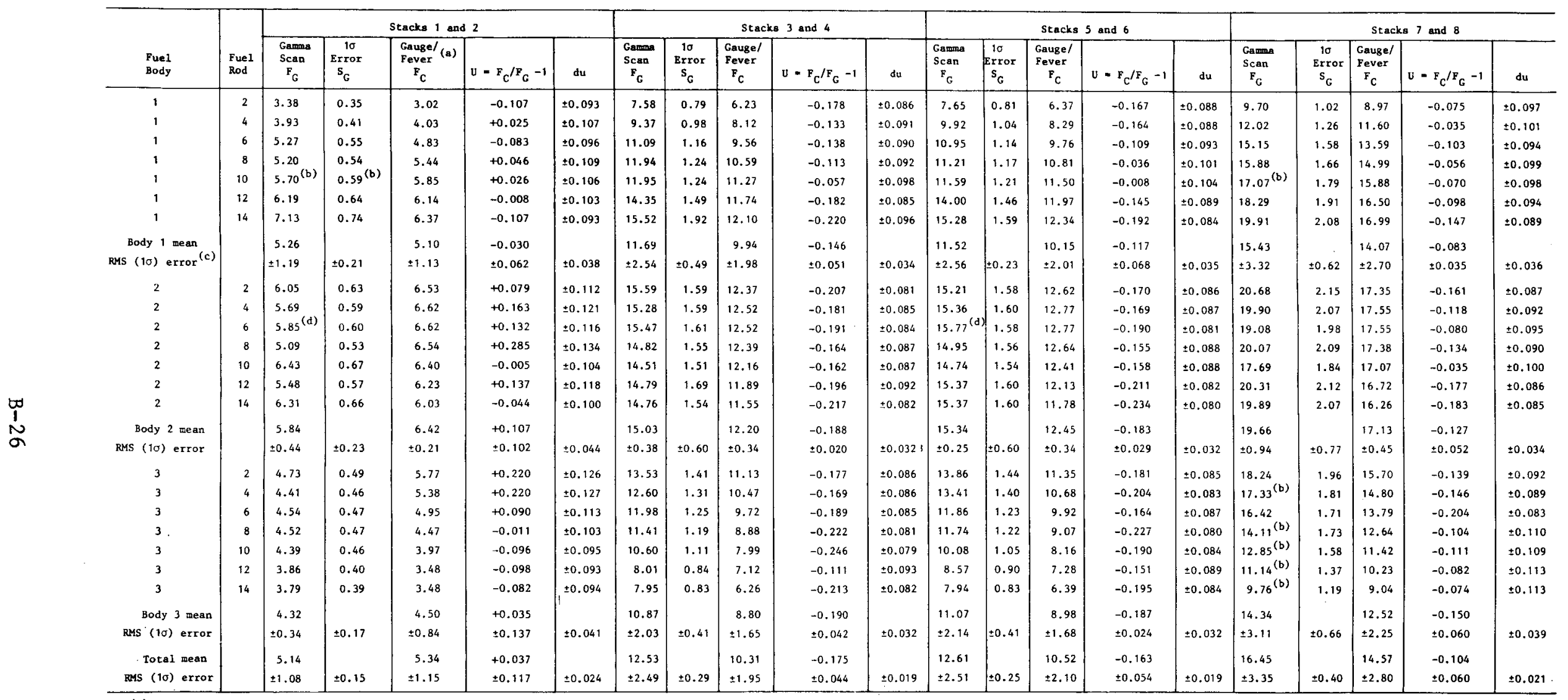

(a) 10 error not avallable for GAUGE/FEVER.

(b) Interpolated from ne1ghboring rods.

${ }^{(c)} s_{\mathrm{u}}^{2}=\frac{1}{\mathrm{a}} \sum\left[(\mathrm{F}-\mathrm{A})^{2}\right]$ for Rars.

$\mathrm{du}=\left\{\frac{1}{\mathrm{n}^{2}} \sum\left(\mathrm{du}_{1}\right)^{2}\right\}^{1 / 2}$

(d) ${ }_{\text {Rod }} 7$ substi iuted. 
TABLE 3-13

FTE-6 COMPOSITE BURNUP MEASUREMENTS AND COMPARISON WITH GAUGE/FEVER CALCULATIONS

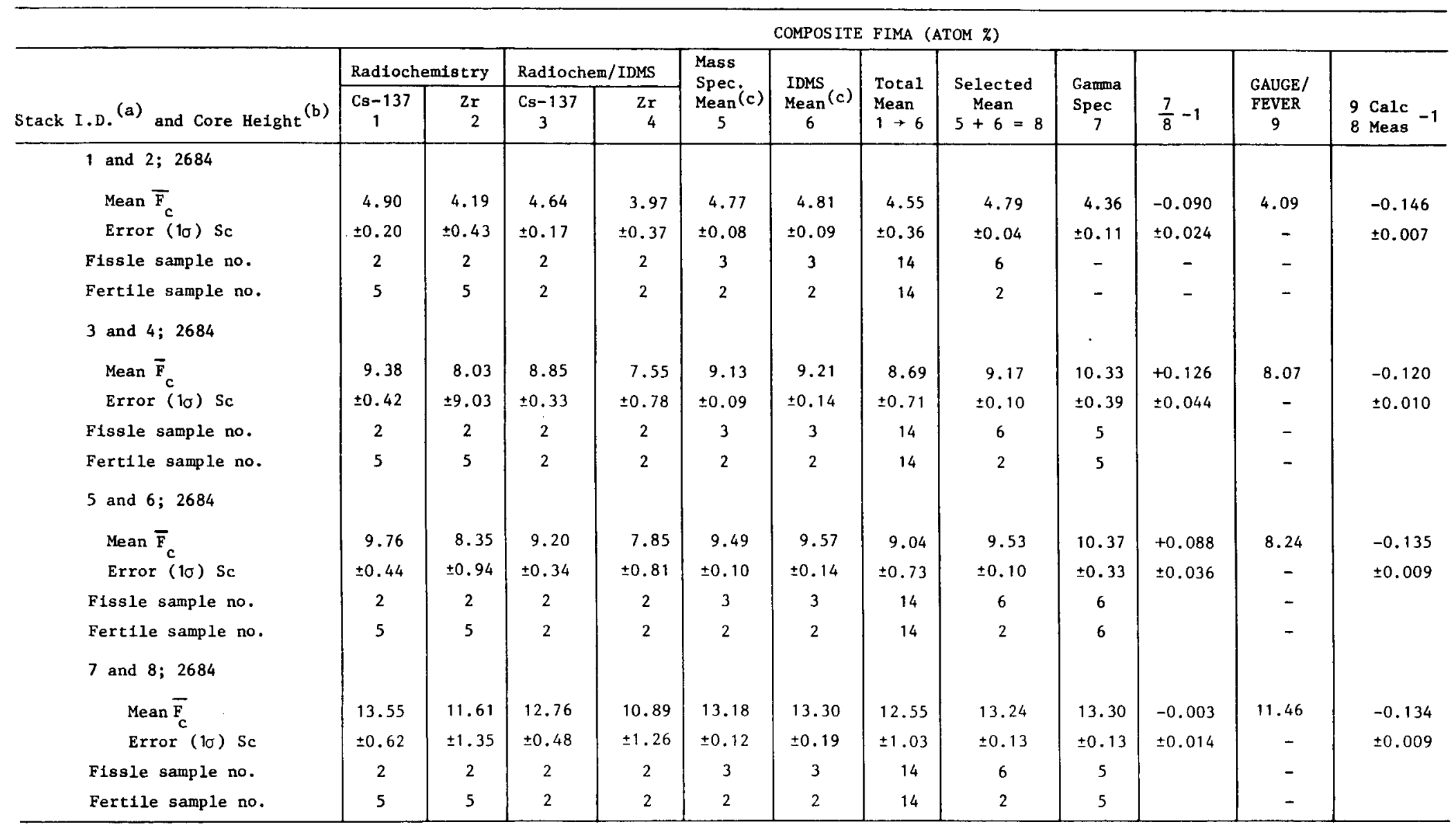

(a) Body-hole number

(b) "O" reference on drawing 11497.

(c) Calculated with radiochemistry/IDMS Cs-137 fertile.

(d) Not determined because of Cs-137 loss in these fuel rods. 
TABLE 3-14 (a)

FTE-6 STRESS SUMMARY FOR MIDPLANE OF BODY 1 (Axial Position 5)

\begin{tabular}{|c|c|c|c|c|c|c|c|c|c|c|}
\hline \multirow[b]{3}{*}{$\begin{array}{l}\text { Time } \\
\text { (h) }\end{array}$} & \multicolumn{6}{|c|}{ Operating Condition (a) } & \multicolumn{4}{|c|}{ Shutdown Condition (a) } \\
\hline & \multicolumn{2}{|c|}{ Temperature } & \multicolumn{2}{|c|}{ In-plane Stress } & \multicolumn{2}{|c|}{ Axial Stress } & \multicolumn{2}{|c|}{ In-plane Stress } & \multicolumn{2}{|c|}{ Axial Stress } \\
\hline & $\begin{array}{l}\text { Max } \\
\text { (K) }\end{array}$ & $\begin{array}{c}\text { Min } \\
(\mathrm{K})\end{array}$ & $\begin{array}{c}\operatorname{Max} \\
(\mathrm{MPa})\end{array}$ & $\underset{(\mathrm{MPa})}{\mathrm{Min}}$ & $\begin{array}{c}\operatorname{Max} \\
(\mathrm{MPa})\end{array}$ & $\begin{array}{c}\text { Min } \\
(\mathrm{MPa})\end{array}$ & $\begin{array}{c}\operatorname{Max} \\
(\mathrm{MPa})\end{array}$ & $\begin{array}{c}\text { Min } \\
(\mathrm{MPa})\end{array}$ & $\begin{array}{c}\operatorname{Max} \\
(\mathrm{MPa})\end{array}$ & $\begin{array}{c}\text { Min } \\
(\mathrm{MPa})\end{array}$ \\
\hline \multirow[t]{2}{*}{$\begin{array}{l}6624 \\
\text { (BOL) }\end{array}$} & 955 & 884 & 1.39 & -3.05 & 0.85 & -1.66 & 0.05 & -0.10 & 0.01 & -0.01 \\
\hline & $10^{(\mathrm{a})}$ & 297 & 288 & 359 & 297 & 10 & 288 & 100 & 288 & 359 \\
\hline \multirow[t]{2}{*}{7992} & 1066 & 947 & 1.07 & -2.43 & 1.15 & -1.95 & 3.15 & -1.29 & 2.00 & -0.97 \\
\hline & 10 & 297 & 288 & 359 & 297 & 20 & 359 & 358 & 9 & 297 \\
\hline \multirow[t]{2}{*}{9312} & 1206 & 1017 & 1.49 & -3.39 & 1.65 & -2.58 & 7.49 & -3.04 & 5.58 & -2.83 \\
\hline & 10 & 297 & 288 & 359 & 297 & 359 & 359 & 359 & 10 & 297 \\
\hline \multirow[t]{2}{*}{10440} & 1199 & 999 & 0.80 & -1.82 & 1.16 & -1.38 & 9.45 & -3.81 & 7.45 & -3.67 \\
\hline & 10 & 297 & 288 & 359 & 297 & 359 & 359 & 20 & 10 & 297 \\
\hline \multirow[t]{2}{*}{10441} & 1149 & 966 & 0.30 & -0.29 & -0.56 & -0.44 & 9.44 & -3.81 & 7.44 & -3.67 \\
\hline & 10 & 297 & 269 & 359 & 297 & 359 & 359 & 20 & 10 & 297 \\
\hline \multirow[t]{2}{*}{13944} & 1153 & 955 & 0.38 & -0.47 & 1.08 & -0.68 & 10.91 & -4.41 & 8.60 & -3.86 \\
\hline & 10 & 297 & 269 & 359 & 297 & 171 & 359 & 20 & 10 & 297 \\
\hline \multirow[t]{2}{*}{15888} & 1237 & 1003 & 0.23 & -0.26 & 1.08 & -0.62 & 13.79 & -5.56 & 11.64 & -5.59 \\
\hline & 10 & 297 & 269 & 320 & 9 & 211 & 359 & 20 & 10 & 297 \\
\hline \multirow[t]{2}{*}{17112} & 1285 & 1031 & 0.60 & -0.28 & 1.94 & -0.70 & 15.72 & -6.33 & 13.68 & -6.88 \\
\hline & 10 & 297 & 359 & 308 & 10 & 320 & 359 & 20 & 10 & 297 \\
\hline \multirow[t]{2}{*}{19488} & 1271 & 1027 & 2.43 & -1.07 & 3.97 & -1.33 & 16.33 & -6.56 & 14.92 & -7.67 \\
\hline & 10 & 297 & 359 & 288 & 20 & 272 & 359 & 359 & 20 & 297 \\
\hline
\end{tabular}


TABLE 3-14(a) (continued)

\begin{tabular}{|c|c|c|c|c|c|c|c|c|c|c|}
\hline & \multicolumn{6}{|c|}{ Operating Condition (a) } & \multicolumn{4}{|c|}{ Shutdown Condition (a) } \\
\hline & \multicolumn{2}{|c|}{ Temperature } & \multicolumn{2}{|c|}{ In-plane Stress } & \multicolumn{2}{|c|}{ Axial Stress } & \multicolumn{2}{|c|}{ In - plane Stress } & \multicolumn{2}{|c|}{ Axial Stress } \\
\hline $\begin{array}{r}\text { Time } \\
\text { (h) }\end{array}$ & $\begin{array}{l}\operatorname{Max} \\
(\mathrm{K})\end{array}$ & $\begin{array}{l}\text { Min } \\
(\mathrm{K})\end{array}$ & $\begin{array}{c}\operatorname{Max} \\
(\mathrm{MPa})\end{array}$ & $\begin{array}{c}\text { Min } \\
(\mathrm{MPa})\end{array}$ & $\begin{array}{c}\operatorname{Max} \\
(\mathrm{MPa})\end{array}$ & $\begin{array}{c}\mathrm{Min} \\
(\mathrm{MPa})\end{array}$ & $\begin{array}{c}\mathrm{Max} \\
(\mathrm{MPa})\end{array}$ & $\begin{array}{l}\mathrm{Min} \\
(\mathrm{MPa})\end{array}$ & $\begin{array}{c}\operatorname{Max} \\
(\mathrm{MPa})\end{array}$ & $\underset{\text { Min }}{M}$ \\
\hline 19489 & 1271 & 1027 & 2.43 & -1.07 & 3.97 & -1.33 & 16.33 & -6.56 & 14.92 & -7.67 \\
\hline & 10 & 297 & 359 & 288 & 20 & 272 & 359 & 359 & 20 & 297 \\
\hline 20712 & 1224 & 1008 & 3.65 & -1.60 & 5.13 & -1.77 & 15.30 & -6.14 & 14.38 & -7.34 \\
\hline & 10 & 297 & 359 & 288 & 20 & 272 & 359 & 359 & 20 & 297 \\
\hline 21912 & 1248 & 1024 & 3.13 & -1.37 & 4.93 & -1.71 & 15.60 & -6.26 & 14.74 & -7.62 \\
\hline \multirow{3}{*}{2800} & 10 & 297 & 359 & 288 & 20 & 289 & 359 & 359 & 20 & 297 \\
\hline & 1046 & 898 & 5.03 & -2.19 & 6.07 & -2.13 & 11.92 & -4.77 & 11.63 & -5.45 \\
\hline & 10 & 297 & 359 & 288 & 20 & 295 & 359 & 359 & 20 & 297 \\
\hline \multirow[t]{2}{*}{23520} & 1298 & 1042 & 1.12 & -0.47 & 3.70 & -1.16 & 16.36 & -6.59 & 15.34 & -7.92 \\
\hline & 10 & 297 & 359 & 288 & 20 & 318 & 359 & 359 & 20 & 297 \\
\hline \multirow[t]{2}{*}{24216} & 1240 & 1011 & 3.13 & -1.37 & 4.91 & -1.75 & 15.72 & -6.31 & 14.79 & -7.73 \\
\hline & 10 & 297 & 359 & 288 & 20 & 346 & 359 & 359 & 20 & 297 \\
\hline \multirow[t]{2}{*}{25344} & 1182 & 977 & 3.71 & -1.62 & 5.54 & -2.05 & 14.46 & -5.80 & 13.98 & -7.19 \\
\hline & 10 & 297 & 359 & 288 & 20 & 322 & 359 & 359 & 20 & 297 \\
\hline 25632 & 1182 & 977 & 3.45 & -1.50 & 5.50 & -1.99 & 14.33 & -5.75 & 13.98 & -7.15 \\
\hline EOL & 10 & 297 & 359 & 288 & 20 & 346 & 359 & 359 & 20 & 297 \\
\hline
\end{tabular}

(a) Number under each temperature and stress value at each time point refers to the finite element (see Fig. 3-34). 
TABI,E 3-14(b)

FTE-6 STRESS SUMMARY FOR MIDPLANE OF BODY 2 (Axia1 Position 15)

\begin{tabular}{|c|c|c|c|c|c|c|c|c|c|c|}
\hline \multirow[b]{3}{*}{$\begin{array}{l}\text { Time } \\
(h)\end{array}$} & \multicolumn{6}{|c|}{ Operating Condition (a) } & \multicolumn{4}{|c|}{ Shutdown Condition (a) } \\
\hline & \multicolumn{2}{|c|}{ Temperature } & \multicolumn{2}{|c|}{ In-plane Stress } & \multicolumn{2}{|c|}{ Axial Stress } & \multicolumn{2}{|c|}{ In-plane Stress } & \multicolumn{2}{|c|}{ Axial Stress } \\
\hline & $\begin{array}{c}\operatorname{Max} \\
(\mathrm{K})\end{array}$ & $\begin{array}{c}\text { Min } \\
(K)\end{array}$ & $\begin{array}{c}\text { Max } \\
\text { (MPa) }\end{array}$ & $\begin{array}{c}\text { Min } \\
(\mathrm{MPa})\end{array}$ & $\begin{array}{c}\operatorname{Max} \\
(\mathrm{MPa})\end{array}$ & $\begin{array}{c}\mathrm{Min} \\
(\mathrm{MPa})\end{array}$ & $\begin{array}{c}\text { Max } \\
(\mathrm{MPa})\end{array}$ & $\begin{array}{c}\text { Min } \\
(\mathrm{MPa})\end{array}$ & $\begin{array}{c}\operatorname{Max} \\
(\mathrm{MPa})\end{array}$ & $\begin{array}{c}\mathrm{Min} \\
(\mathrm{MPa})\end{array}$ \\
\hline \multirow[t]{2}{*}{6624} & 1157 & 1052 & 2.32 & -5.12 & 1.59 & -2.96 & 0.03 & -0.06 & 0.01 & -0.01 \\
\hline & $10^{(a)}$ & 297 & 288 & 359 & 297 & 10 & 288 & 100 & 288 & 359 \\
\hline \multirow[t]{2}{*}{7992} & 1318 & 1153 & 1.13 & -2.56 & 0.70 & -1.46 & 5.91 & -2.40 & 5.07 & -2.80 \\
\hline & 10 & 297 & 288 & 359 & 297 & 359 & 359 & 359 & 10 & 297 \\
\hline \multirow[t]{2}{*}{9312} & 1485 & 1251 & 0.15 & -0.13 & 1.11 & -0.61 & 12.23 & -4.93 & 11.17 & $-6 \cdot 44$ \\
\hline & 10 & 297 & 269 & 320 & 1 & 310 & 359 & 20 & 10 & 297 \\
\hline \multirow[t]{2}{*}{10440} & 1458 & 1225 & 1.90 & -0.83 & 2.87 & -1.65 & 13.40 & -5.39 & 12.60 & -7.40 \\
\hline & 10 & 297 & 359 & 288 & 20 & 297 & 359 & 359 & 20 & 297 \\
\hline \multirow[t]{2}{*}{10441} & 1394 & 1182 & 3.69 & -1.65 & 4.03 & -2.46 & 13.39 & -5.38 & 12.59 & -7.39 \\
\hline & 10 & 297 & 359 & 288 & 20 & 297 & 359 & 359 & 20 & 297 \\
\hline \multirow[t]{2}{*}{13944} & 1377 & 1153 & 4.13 & -1.80 & 5.61 & -3.11 & .13 .96 & -5.60 & 14.07 & -8.23 \\
\hline & 10 & 297 & 359 & 288 & 20 & 297 & 359 & 359 & 20 & 297 \\
\hline \multirow[t]{2}{*}{15888} & 1493 & 1226 & 2.52 & -1.08 & 4.62 & -2.47 & 15.01 & -6.03 & 15.05 & -9.03 \\
\hline & 10 & 297 & 359 & 288 & 20 & 297 & 359 & 359 & 20 & 297 \\
\hline \multirow[t]{2}{*}{17112} & 1570 & 1280 & 3.23 & -1.41 & 4.89 & -3.02 & 16.44 & -6.61 & 16.32 & -10.05 \\
\hline & 10 & 297 & 359 & 288 & 20 & 297 & 359 & 359 & 20 & 297 \\
\hline \multirow[t]{2}{*}{19488} & 1549 & 1276 & 5.33 & -2.33 & 6.64 & $-4 \cdot 34$ & 15.92 & -6.40 & 16.38 & -10.25 \\
\hline & 10 & 297 & 359 & 288 & 20 & 297 & 359 & 91 & 20 & 297 \\
\hline
\end{tabular}


TABLE 3-14(b) (continued)

\begin{tabular}{|c|c|c|c|c|c|c|c|c|c|c|}
\hline \multirow[b]{3}{*}{$\begin{array}{r}\text { Time } \\
\text { (h) }\end{array}$} & \multicolumn{6}{|c|}{ Operating Condition (a) } & \multicolumn{4}{|c|}{ Shutdown Condition (a) } \\
\hline & \multicolumn{2}{|c|}{ Temperature } & \multicolumn{2}{|c|}{ In-plane Stress } & \multicolumn{2}{|c|}{ Axial Stress } & \multicolumn{2}{|c|}{ In-plane Stress } & \multicolumn{2}{|c|}{ Axial Stress } \\
\hline & $\begin{array}{c}\operatorname{Max} \\
(\mathrm{K})\end{array}$ & $\begin{array}{l}\text { Min } \\
(\mathrm{K})\end{array}$ & $\begin{array}{c}\operatorname{Max} \\
(\mathrm{MPa})\end{array}$ & $\begin{array}{l}\text { Min } \\
(\mathrm{MPa})\end{array}$ & $\begin{array}{c}\operatorname{Max} \\
(\mathrm{MPa})\end{array}$ & $\begin{array}{c}\text { Min } \\
(\mathrm{MPa})\end{array}$ & $\begin{array}{c}\operatorname{Max} \\
(\mathrm{MPa})\end{array}$ & $\begin{array}{l}\text { Min } \\
(\mathrm{MPa})\end{array}$ & $\begin{array}{c}\operatorname{Max} \\
(\mathrm{MPa})\end{array}$ & $\begin{array}{l}\text { Min } \\
\text { (MPa) }\end{array}$ \\
\hline \multirow[t]{2}{*}{19489} & 1549 & 1276 & 5.32 & -2.33 & 6.64 & -4.34 & 15.92 & -6.39 & 16.37 & -10.25 \\
\hline & 10 & 297 & 359 & 288 & 20 & 297 & 359 & 91 & 20 & 297 \\
\hline \multirow[t]{2}{*}{20712} & 1501 & 1260 & 5.97 & -2.61 & 7.35 & -4.72 & 14.17 & -5.68 & 15.16 & -9.52 \\
\hline & 10 & 297 & 359 & 288 & 20 & 297 & 359 & 91 & 20 & 297 \\
\hline \multirow[t]{2}{*}{21912} & 1491 & 1259 & 5.81 & -2.54 & 7.38 & -4.70 & 13.26 & -5.31 & 14.56 & -9.13 \\
\hline & 10 & 297 & 359 & 288 & 20 & 297 & 359 & 91 & 20 & 297 \\
\hline \multirow[t]{2}{*}{22800} & 1222 & 1074 & 7.64 & -3.33 & 9.28 & -5.59 & 10.48 & -4.17 & 12.77 & -7.69 \\
\hline & 10 & 297 & 359 & 288 & 20 & 297 & 359 & 358 & 20 & 297 \\
\hline \multirow[t]{2}{*}{23520} & 1538 & 1272 & 4.58 & -1.99 & 6.99 & -4.43 & 14.05 & -5.63 & 15.57 & -9.71 \\
\hline & 10 & 297 & 359 & 288 & 20 & 297 & 359 & 91 & 20 & 297 \\
\hline \multirow[t]{2}{*}{24216} & 1470 & 1231 & 5.04 & -2.20 & 6.80 & -4.41 & 12.51 & -5.02 & 13.92 & -8.84 \\
\hline & 10 & 297 & 359 & 288 & 20 & 297 & 359 & 91 & 20 & 297 \\
\hline \multirow[t]{2}{*}{25344} & 1394 & 1181 & 5.48 & -2.39 & 7.54 & -4.82 & 11.13 & -4.44 & 13.20 & -8.26 \\
\hline & 10 & 297 & 359 & 288 & 20 & 297 & 359 & 10 & 20 & 297 \\
\hline \multirow[t]{2}{*}{25632} & 1393 & 1179 & 5.42 & -2.36 & 7.69 & -4.85 & 11.01 & -4.40 & 13.26 & -8.26 \\
\hline & 10 & 297 & 359 & 288 & 20 & 297 & 359 & 10 & 20 & 297 \\
\hline
\end{tabular}

(a) Number under each temperature and stress value at each time point refers to the finite element (see Fig. 3-34). 
TABLE 3-14(c)

FTE-6 STRESS SUMMARY FOR MIDPLANE OF BODY 3 (Axial Position 25)

\begin{tabular}{|c|c|c|c|c|c|c|c|c|c|c|}
\hline \multirow[b]{3}{*}{$\begin{array}{l}\text { Time } \\
(\mathrm{h})\end{array}$} & \multicolumn{6}{|c|}{ Operating Condition (a) } & \multicolumn{4}{|c|}{ Shutdown Condition (a) } \\
\hline & \multicolumn{2}{|c|}{ Temperature } & \multicolumn{2}{|c|}{ In-plane Stress } & \multicolumn{2}{|c|}{ Axial Stress } & \multicolumn{2}{|c|}{ In-plane Stress } & \multicolumn{2}{|c|}{ Axial Stress } \\
\hline & $\begin{array}{c}\operatorname{Max} \\
(\mathrm{K})\end{array}$ & $\begin{array}{l}\text { Min } \\
(\mathrm{K})\end{array}$ & $\begin{array}{c}\text { Max } \\
\text { (MPa) }\end{array}$ & $\begin{array}{l}\operatorname{Min} \\
(\mathrm{MPa})\end{array}$ & $\begin{array}{c}\operatorname{Max} \\
(\mathrm{MPa})\end{array}$ & $\begin{array}{l}\text { Min } \\
(\mathrm{MPa})\end{array}$ & $\begin{array}{c}\operatorname{Max} \\
(\mathrm{MPa})\end{array}$ & $\begin{array}{c}\mathrm{Min} \\
(\mathrm{MPa})\end{array}$ & $\begin{array}{c}\operatorname{Max} \\
(\mathrm{MPa})\end{array}$ & $\begin{array}{c}\text { Min } \\
(\mathrm{MPa})\end{array}$ \\
\hline \multirow[t]{2}{*}{6624} & 1127 & 1056 & 1.58 & -3.47 & 1.06 & -1.94 & 0.03 & -0.06 & 0.01 & -0.01 \\
\hline & $10^{(a)}$ & 297 & 288 & 359 & 297 & 10 & 288 & 100 & 288 & 359 \\
\hline \multirow[t]{2}{*}{7992} & 1261 & 1157 & 0.60 & -1.37 & 0.28 & -0.68 & 3.37 & -1.38 & 2.94 & -1.66 \\
\hline & 10 & 297 & 288 & 359 & 297 & 359 & 359 & 359 & 10 & 297 \\
\hline \multirow[t]{2}{*}{9312} & 1387 & 1247 & 0.27 & -0.61 & 0.53 & -0.19 & 6.68 & -2.70 & 6.20 & -3.56 \\
\hline & 10 & 297 & 288 & 359 & 1 & 310 & 359 & 20 & 10 & 297 \\
\hline \multirow[t]{2}{*}{10440} & 1354 & 1215 & 0.29 & -0.13 & 1.17 & -0.66 & 7.36 & -2.97 & 6.93 & -3.98 \\
\hline & 10 & 297 & 359 & 288 & 10 & 297 & 359 & 20 & 10 & 297 \\
\hline \multirow[t]{2}{*}{10441} & 1300 & 1174 & 1.32 & -0.58 & 1.87 & -1.13 & 7.36 & -2.97 & 6.93 & -3.98 \\
\hline & 10 & 297 & 359 & 288 & 10 & 297 & 359 & 20 & 10 & 297 \\
\hline \multirow[t]{2}{*}{13944} & 1339 & 1173 & 1.88 & -0.82 & 3.16 & -1.70 & 9.83 & -3.95 & 9.77 & -5.57 \\
\hline & 10 & 297 & 359 & 288 & 20 & 297 & 359 & 20 & 20 & 297 \\
\hline \multirow[t]{2}{*}{15888} & 1446 & 1246 & 1.41 & -0.61 & 2.87 & -1.54 & 11.28 & -4.54 & 11.11 & -6.54 \\
\hline & 10 & 297 & 359 & 288 & 20 & 297 & 359 & 20 & 20 & 297 \\
\hline \multirow[t]{2}{*}{17112} & 1525 & 1304 & 2.64 & -1.15 & 3.69 & -2.19 & 13.14 & -5.29 & 12.71 & -7.62 \\
\hline & 10 & 297 & 359 & 288 & 20 & 297 & 359 & 359 & 20 & 297 \\
\hline 19488 & 1515 & 1303 & 3.95 & -1.72 & 4.84 & -2.97 & 13.05 & -5.24 & 12.97 & -7.85 \\
\hline
\end{tabular}


TABLE 3-14(c) (continued)

\begin{tabular}{|c|c|c|c|c|c|c|c|c|c|c|}
\hline \multirow[b]{3}{*}{$\begin{array}{r}\text { Time } \\
\text { (h) }\end{array}$} & \multicolumn{6}{|c|}{ Operating Condition (a) } & \multicolumn{4}{|c|}{ Shutdown Condition (a) } \\
\hline & \multicolumn{2}{|c|}{ Temperature } & \multicolumn{2}{|c|}{ In-plane Stress } & \multicolumn{2}{|c|}{ Axial Stress } & \multicolumn{2}{|c|}{ In-plane Stress } & \multicolumn{2}{|c|}{ Axial Stress } \\
\hline & $\begin{array}{c}\operatorname{Max} \\
(\mathrm{K})\end{array}$ & $\begin{array}{l}\text { Min } \\
(\mathrm{K})\end{array}$ & $\begin{array}{c}\operatorname{Max} \\
(\mathrm{MPa})\end{array}$ & $\begin{array}{c}\mathrm{Min} \\
(\mathrm{MPa})\end{array}$ & $\begin{array}{c}\operatorname{Max} \\
(\mathrm{MPa})\end{array}$ & $\begin{array}{c}\text { Min } \\
(\mathrm{MPa})\end{array}$ & $\begin{array}{c}\mathrm{Max} \\
(\mathrm{MPa})\end{array}$ & $\begin{array}{c}\text { Min } \\
(\mathrm{MPa})\end{array}$ & $\begin{array}{c}\operatorname{Max} \\
(\mathrm{MPa})\end{array}$ & $\begin{array}{l}\text { Min } \\
(\mathrm{MPa})\end{array}$ \\
\hline & 10 & 297 & 359 & 288 & 20 & 297 & 359 & 359 & 20 & 297 \\
\hline 19489 & 1515 & 1303 & 3.95 & -1.72 & 4.84 & -2.97 & 13.06 & -5.24 & 12.97 & -7.85 \\
\hline & 10 & 297 & 359 & 288 & 20 & 297 & 359 & 359 & 20 & 297 \\
\hline 20712 & 1491 & 1304 & 4.76 & -2.07 & 5.56 & -3.36 & 11.98 & -4.80 & 12.27 & -7.40 \\
\hline & 10 & 297 & 359 & 288 & 20 & 297 & 359 & 91 & 20 & 297 \\
\hline 21912 & 1628 & 1401 & 2.31 & -0.99 & 3.51 & -2.32 & 12.42 & -4.98 & 12.79 & -7.67 \\
\hline & 10 & 297 & 359 & 288 & 359 & 297 & 359 & 359 & 20 & 297 \\
\hline 22800 & 1322 & 1181 & 5.65 & -2.46 & 6.42 & -3.83 & 9.85 & -3.92 & 10.79 & -6.37 \\
\hline & 10 & 297 & 359 & 288 & 20 & 297 & 359 & 10 & 20 & 297 \\
\hline 23520 & 1655 & 1406 & 2.96 & -1.29 & 4.31 & -2.84 & 13.91 & -5.58 & 14.37 & -8.61 \\
\hline & 10 & 297 & 359 & 288 & 20 & 297 & 359 & 359 & 20 & 297 \\
\hline 24216 & 1588 & 1367 & 4.72 & -2.07 & 5.26 & -3.39 & 13.20 & -5.29 & 13.47 & -8.16 \\
\hline & 10 & 297 & 359 & 288 & 20 & 297 & 359 & 359 & 20 & 297 \\
\hline 25344 & 1507 & 1312 & 4.49 & -1.96 & 5.51 & -3.59 & 11.42 & -4.57 & 12.04 & -7.37 \\
\hline & 10 & 297 & 359 & 288 & 20 & 297 & 359 & 91 & 20 & 297 \\
\hline 25632 & 1505 & 1311 & 4.25 & -1.85 & 5.41 & -3.53 & 11.21 & -4.49 & 11.91 & -7.38 \\
\hline & 10 & 297 & 359 & 288 & 20 & 297 & 359 & 91 & 20 & 297 \\
\hline
\end{tabular}

(a) Number under each temperature and stress value at each time point refers to the finite element. 
TABLE 3-15

H-327 GRAPHITE ULTIMATE TENSILE AND COMPRESSION STRENGTH A:D ELASTIC MODULUS

\begin{tabular}{|c|c|c|c|c|}
\hline \multirow[b]{3}{*}{ Block Position, Orientation } & \multicolumn{4}{|c|}{ U1timate Strength for $\mathrm{H}-327$ Graphite (Ref. 3-21) } \\
\hline & \multicolumn{2}{|c|}{ Mean } & \multicolumn{2}{|c|}{ Standard Deviation } \\
\hline & (psi) & $(\mathrm{MPa})$ & (psi) & $(\mathrm{MPa})$ \\
\hline \multicolumn{5}{|l|}{ U1timate Tensile Strength } \\
\hline End center, axial & 2180 & 15.031 & 435 & 3.000 \\
\hline End edge, axial (a) & 2395 & 16.514 & 480 & 3.310 \\
\hline Midlength center, axial & 1630 & 11.239 & 325 & 2.240 \\
\hline Midlength edge, axial ${ }^{(b)}$ & 2395 & 16.514 & 480 & 3.310 \\
\hline End center, radial & 1350 & 9.308 & 270 & 1.862 \\
\hline End edge, radial ${ }^{(a)}$ & 1350 & 9.308 & 270 & 1.862 \\
\hline Midlength center, radial & 940 & 6.481 & 190 & 1.310 \\
\hline Midlength edge, radial ${ }^{(b)}$ & 1290 & 8.895 & 260 & 1.793 \\
\hline U1timate compressive strength ${ }^{(b)}$ & $\geq 4000$ & $\geq 27.580$ & & \\
\hline
\end{tabular}

(a) End edge values obtained by taking midlength edge value for axial orientation and end center value for radial orientation. The standard deviation was calculated from $20 \%$ of the mean UTS value based upon characterization and QA test results.

(b) Used for unirradiated strength at room temperature in computing stress-tostreng th ratios.

\begin{tabular}{l|c|c|c|c}
\hline \multirow{2}{*}{ Pointwise Block Position, Orientation } & \multicolumn{3}{|c}{ Elastic Modulus Eor H-327 Graphite } \\
\cline { 2 - 5 } & \multicolumn{2}{|c|}{ Mean } & Stendard Deviation (Ref. 3-21) \\
\cline { 2 - 5 } & $\left(10^{6} \mathrm{psi}\right)$ & $\left(10^{3} \mathrm{MPa}\right)$ & $\left(10^{6} \mathrm{psi}\right)$ & $\left(10^{3} \mathrm{MPa}\right)$ \\
\hline End center, axia1 & 1.42 & 9.79 & 0.3 & 2.07 \\
End edge, axial (a) & 2.02 & 13.93 & 0.6 & 4.14 \\
Midlength center, axial & 1.42 & 9.79 & 0.4 & 2.76 \\
Midlength edge, axial (b) & 2.02 & 13.93 & 0.6 & 4.14 \\
End center, radial & 0.754 & 5.20 & 0.3 & 2.07 \\
End edge, radial & 0.669 & 4.61 & 0.3 & 2.07 \\
Midlength center, radia1 & 0.571 & 3.94 & 0.2 & 1.38 \\
Midlength edge, radial (b) & 0.774 & 5.34 & 0.3 & 2.07 \\
\hline
\end{tabular}

(a) End edge values obtained by taking midlength edge value for axial orientation and end center value for radial orientation.

(b) Average used in stress calculation. 
TABLE 3-16

FTE 6 IRRADIATION SUMMARY

\begin{tabular}{|c|c|c|c|c|c|c|c|}
\hline $\begin{array}{l}\text { Irradiation } \\
\text { Parameter }\end{array}$ & Peak & \pm Error & Mean & $\pm \mathrm{RMS}$ & \pm Error $(1 \sigma)$ & $\begin{array}{c}\text { Body } \\
\text { Sequence }\end{array}$ & $\begin{array}{l}\text { Referenced } \\
\text { Table }\end{array}$ \\
\hline Fluence $\left(10^{25} \mathrm{n} / \mathrm{r}\right.$ & & & & & & & \\
\hline $\begin{array}{l}\text { Fast } \\
(E \geq 29 \mathrm{fF})\end{array}$ & 2.88 & & 2.30 & \pm 0.59 & & $213 / 231$ & $3-2$ \\
\hline $\begin{array}{l}\text { Thermal } \\
(E<2.38 \mathrm{eV})\end{array}$ & 3.92 & & 2.99 & \pm 0.84 & & $231 / 231$ & $3-2$ \\
\hline $\begin{array}{l}\text { Temperature }\left({ }^{\circ} \mathrm{C}\right) \\
\text { (predicted) }\end{array}$ & & & & & & & \\
\hline Holes 1 and 2 & & & & & & & \\
\hline $\begin{array}{l}\text { Fuel avg } \\
\text { Fue1 max } \\
\text { Fue1 min }\end{array}$ & $\begin{array}{r}1161 \\
1429 \\
861\end{array}$ & & $\begin{array}{r}1024 \\
1242 \\
768\end{array}$ & $\begin{array}{l} \pm 141 \\
\pm 175 \\
\pm 96\end{array}$ & & $\begin{array}{l}231 / 231 \\
321 / 231 \\
231 / 231\end{array}$ & $\begin{array}{l}3-3(a) \\
3-3(a) \\
3-3(a)\end{array}$ \\
\hline $\begin{array}{l}\text { Temperature }\left({ }^{\circ} \mathrm{C}\right) \\
(\mathrm{T} / \mathrm{C}) \text { Comparison }\end{array}$ & & & & & & & \\
\hline $\begin{array}{l}\text { W/Re TREVER } \\
\text { W/Re readout } \\
\mathrm{C} / \mathrm{A} \text { TREVER } \\
\text { C/A readout }\end{array}$ & $\begin{array}{r}1293 \\
1336 \\
737 \\
758\end{array}$ & & $\begin{array}{r}1173 \\
1200 \\
663 \\
665\end{array}$ & $\begin{array}{l} \pm 98 \\
\pm 87 \\
\pm 50 \\
\pm 52\end{array}$ & & & $\begin{array}{l}3-4(b) \\
3-4(b) \\
3-4(a) \\
3-4(a)\end{array}$ \\
\hline $\begin{array}{l}\text { Burnup (\%) } \\
\text { (predicted) }\end{array}$ & & & & & & & \\
\hline $\begin{array}{l}\text { Fissile FIMA } \\
\text { Fertile FIMA } \\
\text { Mixed FIMA } \\
(\mathrm{N}=4.099)\end{array}$ & $\begin{array}{r}45.60 \\
1.56 \\
13.30\end{array}$ & & $\begin{array}{r}38.20 \\
1.03 \\
10.94\end{array}$ & $\begin{array}{l} \pm 7.09 \\
\pm 0.45 \\
\pm 1.75\end{array}$ & & $\begin{array}{l}213 / 231 \\
213 / 231 \\
213 / 231\end{array}$ & $\begin{array}{l}3-6 \\
3-6 \\
3-6\end{array}$ \\
\hline
\end{tabular}


TABLE 3-16 (continued)

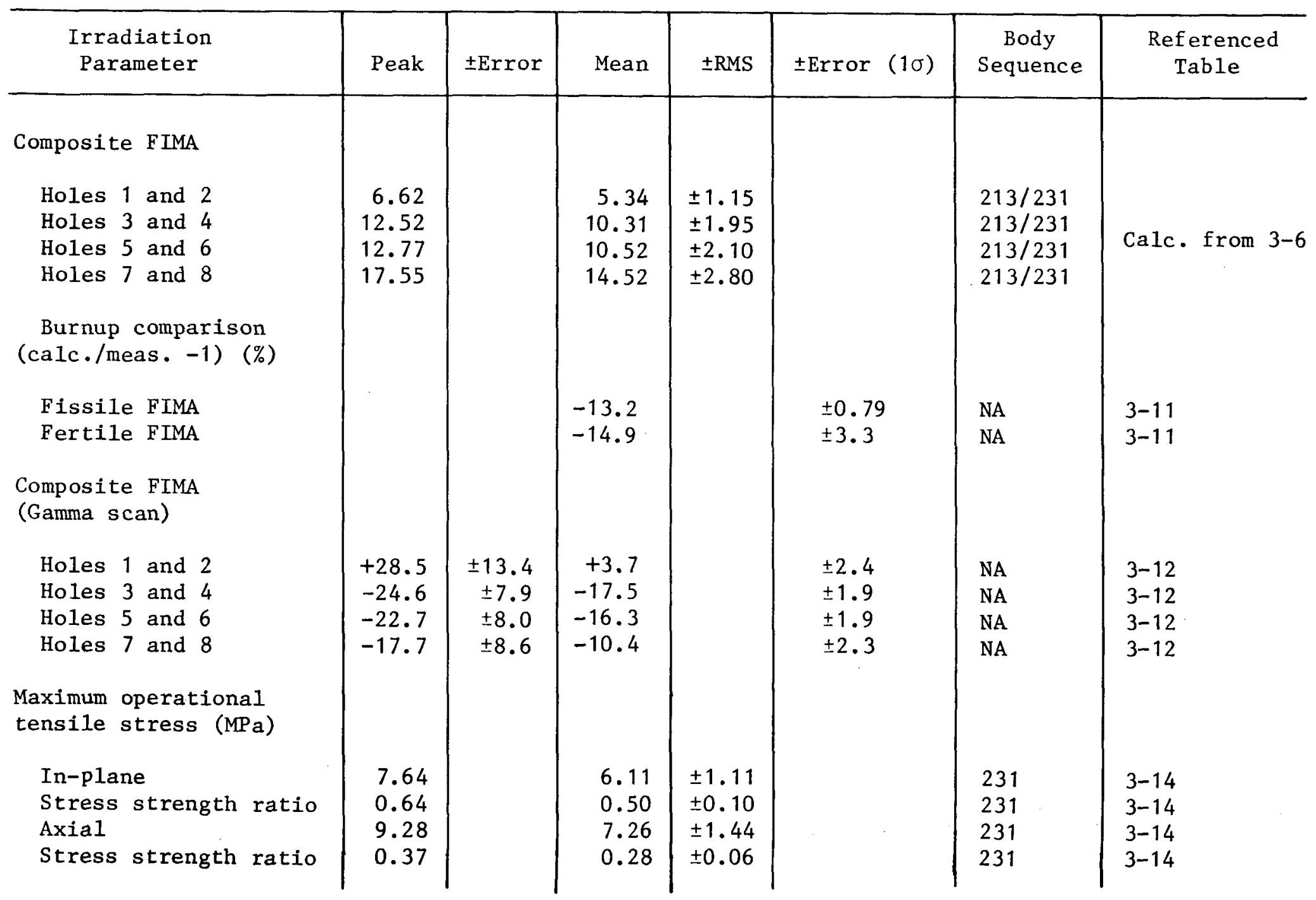


TABLE 3-16 (continued)

\begin{tabular}{|c|c|c|c|c|c|c|c|}
\hline $\begin{array}{l}\text { Irradiation } \\
\text { Parameter }\end{array}$ & Peak & \pm Error & Mean & $\pm \mathrm{RMS}$ & \pm Error $(1 \sigma)$ & $\begin{array}{c}\text { Body } \\
\text { Sequence }\end{array}$ & $\begin{array}{l}\text { Referenced } \\
\text { Table }\end{array}$ \\
\hline $\begin{array}{l}\text { Maximum shutdown } \\
\text { tensile stress (MPa) }\end{array}$ & & & & & & & \\
\hline $\begin{array}{l}\text { In-Plane } \\
\text { Stress-strength ratio } \\
\text { Axial } \\
\text { Stress-strength ratio }\end{array}$ & $\begin{array}{r}16.44 \\
1.53 \\
16.38 \\
0.73\end{array}$ & & $\begin{array}{r}15.57 \\
1.43 \\
15.36 \\
0.67\end{array}$ & $\begin{array}{l} \pm 1.17 \\
\pm 0.10 \\
\pm 0.82 \\
\pm 0.04\end{array}$ & & $\begin{array}{l}213 \\
213 \\
213 \\
213\end{array}$ & $\begin{array}{l}3-14 \\
3-14 \\
3-14 \\
3-14\end{array}$ \\
\hline $\begin{array}{l}\text { Fuel. body radial } \\
\text { strain }(\%)\end{array}$ & & - & & & & & \\
\hline $\begin{array}{l}\text { TREVER strain } \\
\text { Measured strain } \\
\text { Calc/meas }-1 \\
\text { Anisotropy }\end{array}$ & $\begin{array}{l}-0.50 \\
-0.40\end{array}$ & \pm 0.039 & $\begin{array}{r}-0.34 \\
-0.24 \\
53.40 \\
192.37\end{array}$ & $\begin{array}{r} \pm 0.15 \\
\pm 0.11 \\
\pm 81.46\end{array}$ & $\begin{array}{l} \pm 0.006 \\
\pm 10.98 \\
\pm 98.1\end{array}$ & $\begin{array}{l}231 \\
231 \\
123\end{array}$ & $\begin{array}{l}4-26 \\
4-26 \\
4-26 \\
4-25\end{array}$ \\
\hline $\begin{array}{l}\text { Fuel rod radial } \\
\text { strain }(\%)\end{array}$ & & & & & & & \\
\hline $\begin{array}{l}\text { Holes } 1 \text { and } 2 \\
\text { TREVER strain } \\
\text { Measured strain } \\
\text { Calc/meas }-1 \\
\text { Anisotropy }\end{array}$ & $\begin{array}{l}-3.70 \\
-3.58 \\
71.8\end{array}$ & $\begin{array}{l} \pm 0.15 \\
\pm 10.9\end{array}$ & $\begin{array}{l}-3 \cdot 50 \\
-2.76 \\
29.5 \\
92.1\end{array}$ & $\begin{array}{r} \pm 0.38 \\
\pm 0.48 \\
\pm 18.6\end{array}$ & $\begin{array}{l} \pm 0.019 \\
\pm 1.03 \\
\pm 4.3\end{array}$ & $\begin{array}{l}231 \\
231 \\
123 \\
123\end{array}$ & $\begin{array}{l}4-28 \\
4-32,4-28 \\
4-28 \\
4-32\end{array}$ \\
\hline Holes 3 and 4 & & & & & & & \\
\hline $\begin{array}{l}\text { TREVER strain } \\
\text { Measured strain } \\
\text { Calc/meas }-1 \\
\text { Anisotropy }\end{array}$ & $\begin{array}{r}-1.79 \\
-2.33 \\
+51.43\end{array}$ & $\begin{array}{l} \pm 0.085 \\
\pm 14.64\end{array}$ & $\begin{array}{l}-1.70 \\
-1.90 \\
-7.6 \\
94.2\end{array}$ & $\begin{array}{r} \pm 0.16 \\
\pm 0.38 \\
\pm 17.4\end{array}$ & $\begin{array}{l} \pm 0.018 \\
\pm 1.13 \\
\pm 6.3\end{array}$ & $\begin{array}{l}231 \\
231 \\
321 \\
321\end{array}$ & $\begin{array}{l}4-29 \\
4-29,4-33 \\
4-29 \\
4-33\end{array}$ \\
\hline
\end{tabular}


TABLE 3-16 (continued)

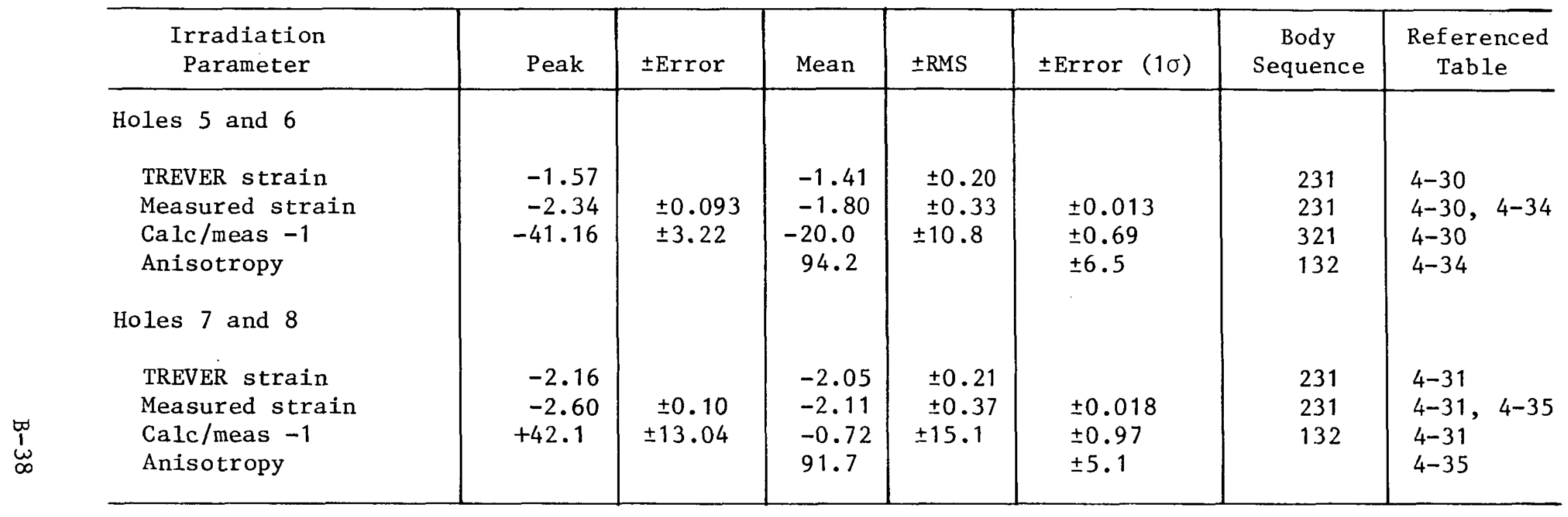


TABLE 4-1

FORMULA SUMPARY ON ERROR ANALYSIS

\begin{tabular}{|c|c|c|c|c|c|c|c|}
\hline \multirow[b]{2}{*}{ Error } & \multirow[b]{2}{*}{ Symbol } & & \multicolumn{4}{|c|}{ Analys1s Function } \\
\hline & & $\begin{array}{c}\text { Measur } \\
\text { Pretrradtaction }\end{array}$ & \begin{tabular}{|l|} 
rements \\
Posti rradtation \\
\end{tabular} & Difference & $\begin{array}{l}\text { Relative Change } \\
\text { (Radia1 Stratin) } \\
\end{array}$ & $\begin{array}{c}\begin{array}{c}\text { Average } \\
\text { (Ravdala and } \\
\text { axta1 stratn) }\end{array} \\
\end{array}$ & $\begin{array}{l}\text { Ratio } \\
\text { (Antstropy = Axta1 } \\
\text { Versus Radial Stratn) }\end{array}$ \\
\hline & & $x_{1}$ & $x_{2}$ & $x_{21}=x_{2}-x_{12}$ & $u=\frac{x_{2}}{x_{1}}-1$ & $v-\frac{1}{2}\left(\frac{x_{2}}{x_{1}}+\frac{y_{2}}{y_{1}}-2\right)$ & $x-\left(\frac{y_{2}}{y_{1}}-1\right)\left(\frac{x_{2}}{x_{1}}-1\right)^{-1}$ \\
\hline$\frac{\text { Measurtng System }}{\text { Precision }_{\text {pren }}}$ & e & $\begin{array}{l}e_{1}= \\
\pm e_{1}\end{array}$ & $\begin{array}{l}e_{2}= \\
\pm e_{2}\end{array}$ & $\begin{array}{l}e_{21}= \\
\pm\left(e_{1}+e_{2}\right)\end{array}$ & $\begin{array}{l}e_{u}-e_{x}- \\
=\frac{1}{z_{1}}\left(e_{1} \frac{\bar{x}_{2}}{\bar{x}_{1}}+e_{2}\right)\end{array}$ & $\begin{array}{l}e_{v}= \\
=\frac{1}{2}\left(e_{x}+e_{y}\right)\end{array}$ & $=\left(e_{y}+e_{x} \cdot \bar{y}\right)\left(\frac{\bar{x}_{2}}{e_{1}}-1\right)^{-1}$ \\
\hline $\begin{array}{l}\text { Standard Error, } \\
\text { Prectsion }\end{array}$ & P & $\mathrm{p}_{1}= \pm \frac{e_{1}}{\sqrt{12}}$ & $p_{2}= \pm \frac{e_{2}}{\sqrt{12}}$ & $=\left(p_{1}^{2}+p_{2}\right)^{1 / 2}$ & $\left\{\begin{array}{c}p_{u}= \pm \frac{1}{\bar{x}_{1}}\left[p_{1}\left(\frac{\bar{x}_{2}}{\bar{x}_{1}}\right)^{2}\right. \\
\left.\left.+p_{2}^{2}\right]\right]^{1 / 2}\end{array}\right.$ & $p_{y}= \pm \frac{1}{2}\left(p_{x}^{2}+p_{y}^{2}\right)$ & $\left\{\begin{array}{l}p_{w}- \pm\left(p_{y}^{2}\right. \\
\left.+p_{x}^{2} \overline{\bar{w}}^{2}\right)^{1 / 2}\left(\frac{\bar{x}_{2}}{\bar{x}_{1}}-1\right)^{-1}\end{array}\right.$ \\
\hline $\begin{array}{l}\text { Standard Error, } \\
\text { Cal thration at } 67 \% \\
\text { Confricence (10) }\end{array}$ & $r$ & $\begin{array}{l}r_{1}= \\
\pm\left[\sum\left(r_{1}\right)_{1}^{2}\right]^{1 / 2}\end{array}$ & $\begin{array}{l}r_{2}= \\
=\left[\begin{array}{l}\sum\left(r_{2}\right)_{1} \\
1\end{array}\right]^{1 / 2}\end{array}$ & $\begin{array}{l}r_{21}= \\
=\left(r_{1}^{2}+r_{2}^{2}\right)^{1 / 2}\end{array}$ & $\begin{array}{l}r_{u}=r_{x}= \\
=\frac{1}{x_{1}}\left[r_{1}^{2}\left(\frac{\bar{x}_{2}}{\bar{x}_{1}}\right)^{2}+r_{2}^{2}\right]^{1 / 2}\end{array}$ & $\begin{array}{l}r_{v}= \\
\pm \frac{1}{2}\left(r_{x}^{2}+r_{y}^{2}\right)^{1 / 2}\end{array}$ & $\begin{array}{l}r_{u}= \\
\pm\left(r_{y}^{2}+r_{x}^{2} \cdot \bar{w}^{2}\right)^{1 / 2}\left(\frac{\bar{x}_{2}}{\bar{x}_{1}}-1\right)^{-1}\end{array}$ \\
\hline 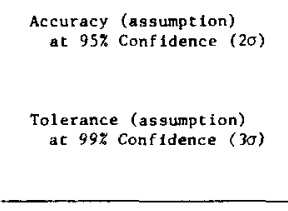 & a & $\begin{array}{l}a_{1}= \\
\pm\left(e_{1}+1.96 r_{1}\right) \\
\tau_{1}= \\
=3 r_{1} \\
\end{array}$ & $\begin{array}{l}\alpha_{2}= \\
=\left(e_{2}+1.96 r_{2}\right)\end{array}$ & $\begin{array}{l}a_{21} \\
=\left(e_{21}+1.96 r_{21}\right)\end{array}$ & $\begin{array}{l}a_{y}=a_{x}= \\
=\left(e_{x}+1.96 r_{x}\right)\end{array}$ & $\begin{array}{l}a_{v} \\
=\left(e_{v}+1.96 r_{v}\right)\end{array}$ & $\begin{array}{l}\alpha_{w}= \\
\pm\left(e_{w}+1.96 \Gamma_{w}\right)\end{array}$ \\
\hline $\begin{array}{l}\text { Sampie } \\
\text { Number of Measurements }\end{array}$ & $n$ & $\begin{array}{l}j=1 \\
n_{1}\end{array}$ & $\begin{array}{l}1=2 \\
n_{2}\end{array}$ & $n_{1} ; n_{2}$ & $n_{1}: n_{2}$ & $n_{x 1} ; n_{x 2} ; n_{y 1} ; n_{y 2}$ & $n_{x 1}: n_{x 2}: n_{y 1}: n_{y 2}$ \\
\hline $\begin{array}{l}\text { Sample Mean }{ }^{(a)} \\
\text { Column average }\end{array}$ & $x, u, v, w$ & $\overline{\mathbf{x}}_{1}$ & $\bar{x}_{2}$ & $\mathrm{x}_{21}^{\prime}=\overline{\Delta x}$ & $\vec{u}^{\prime} \cdot\left(\overline{\frac{\Delta x}{x_{1}}}\right)$ & $\nabla^{\prime}=\frac{1}{2}\left[\left(\frac{\overline{\Delta x}}{x_{1}}\right)+\left(\frac{\overline{\Delta y}}{y_{2}}\right)\right]$ & $\nabla=\left(\overline{\frac{\Delta y}{y_{1}}}\right)\left(\overline{\frac{\Delta x}{x_{1}}}\right)^{-1}$ \\
\hline LLne average & & & & & $\bar{u} \cdot \frac{\bar{x}_{2}}{\bar{x}_{1}}-1$ & $\bar{v}=\frac{1}{2}\left(\frac{\bar{x}_{2}}{\bar{x}_{1}}+\frac{\bar{y}_{2}}{\bar{y}_{1}}-2\right)$ & $\overline{\bar{\psi}}-\left(\frac{\bar{y}_{2}}{\bar{y}_{1}}-1\right)\left(\frac{\bar{x}_{2}}{\bar{x}_{1}}-1\right)^{-1}$ \\
\hline 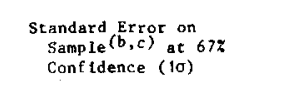 & $s$ & $\mathrm{~s}_{1}-$ & 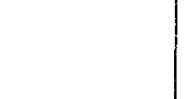 & $s_{3}$ from $\bar{x}_{21} ; s_{21}$ from $\bar{x}_{21}$ & $\begin{array}{l}s_{4} \text { from } \bar{u}^{\prime}: \\
s_{u}=s_{x} \text { from } \bar{u}\end{array}$ & $s_{\mathrm{v}}=$ & $s_{\mathrm{w}}=$ \\
\hline & & $=\left[\frac{1}{n_{j}-1} \varepsilon_{i}\right.$ & $\left.\left.n_{j} x_{j}^{2}\right)\right]^{1 / 2}$ & $s_{21}=:\left(s_{1}^{2}+s_{2}^{2}\right)^{1 / 2}$ & $\begin{array}{c}s_{u}= \pm \frac{1}{x_{1}}\left[s_{1}^{2}\left(\frac{\bar{x}_{2}}{\bar{x}_{1}}\right)^{2}\right. \\
\left.+s_{2}^{2}\right]^{1 / 2}\end{array}$ & $\pm \frac{1}{2}\left(s_{x}^{2}+s_{y}^{2}\right)^{1 / 2}$ & $=\left(s_{y}^{2}+s_{x}^{2} \bar{y}^{2}\right)^{1 / 2}\left(\frac{\bar{x}_{2}}{\bar{x}_{1}}-1\right)^{-1}$ \\
\hline $\begin{array}{l}\text { Comblned Standard Error } \\
\text { Co Mean }(C) \text { at } 677 \\
\text { Conf Idence }(10)\end{array}$ & $\varepsilon$ & $\begin{array}{l}\varepsilon_{j}- \\
\pm\left[r_{j}^{2}+\sum_{1}\right]\end{array}$ & $\left.\left(\begin{array}{l}s_{j}^{2} \\
n_{j}\end{array}\right)\right]^{1 / 2}$ & $\begin{array}{l}\varepsilon_{21}= \\
=\left(\varepsilon_{1}^{2}+\varepsilon_{2}^{2}\right)^{1 / 2}\end{array}$ & $\begin{array}{l}\varepsilon_{u}=\varepsilon_{x} \\
=\frac{1}{\bar{x}_{1}}\left[\varepsilon_{1}^{2}\left(\frac{\bar{x}_{2}}{\bar{x}_{1}}\right)^{2}+\varepsilon_{2}^{2}\right]^{1 / 2}\end{array}$ & $\begin{array}{l}\varepsilon_{v}= \\
=\frac{1}{2}\left(\varepsilon_{x}^{2}+\varepsilon_{y}^{2}\right)^{1 / 2}\end{array}$ & $\begin{array}{l}\varepsilon_{w} \cdot \\
=\left(\varepsilon_{y}^{2}+\varepsilon_{x}^{2} \bar{w}^{2}\right)^{1 / 2}\left(\frac{\bar{x}_{2}}{x_{1}}-1\right)^{-1}\end{array}$ \\
\hline 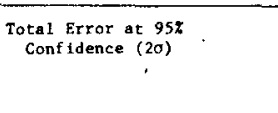 & d & $\begin{array}{l}\mathrm{dx}_{1} \\
=\left(1.96 \mathrm{E}_{1}\right)\end{array}$ & $\begin{array}{l}d x_{2} \\
=\left(\begin{array}{ll}1.96 \varepsilon_{2}\end{array}\right)\end{array}$ & $\begin{array}{l}\mathrm{dx} x_{21} \\
=\left(1.96 \mathrm{E}_{21}\right)\end{array}$ & $\begin{array}{l}d u \\
=\left(1.96 \varepsilon_{u}\right)\end{array}$ & $\begin{array}{l}d v \\
\pm\left(1.96 \varepsilon_{v}\right)\end{array}$ & $\begin{array}{l}d w \\
\pm\left(1.96 c_{w}\right)\end{array}$ \\
\hline
\end{tabular}

(a) Line average preferred over colums average.

(b) $s_{3}$ and $a_{4}$ from different averaging procedure; error progression is analog.

(c) Includes precision 11aits. Minimum standard errors on sample and sample mean

are, reapectively.

$$
\mathrm{s}_{\min }= \pm \frac{e}{\sqrt{12}} \text { and } \varepsilon_{\min }= \pm\left(\mathrm{r}^{2}+\frac{e^{2}}{12 \mathrm{n}}\right)^{1 / 2} \text {. }
$$


TABLE 4-2

ERROR ANALYSIS DEFAULT TABLE

\begin{tabular}{|c|c|c|c|c|c|c|c|c|c|c|c|c|}
\hline & Symbol & $\begin{array}{l}\text { Fue1 Rod } \\
\text { Diameter } \\
\text { (in.) }\end{array}$ & $\begin{array}{l}\text { Fuel Rod } \\
\text { Length } \\
\text { (in.) }\end{array}$ & $\begin{array}{l}\text { Fue1 Hole } \\
\text { Diam } \\
\text { (1n.) }\end{array}$ & $\begin{array}{l}\text { Spine Hole } \\
\text { Diam } \\
\text { (in.) }\end{array}$ & $\begin{array}{l}\text { Fue1 Body } \\
\text { Outer Diam } \\
\text { (in.) }\end{array}$ & $\begin{array}{c}\text { Sleeve } \\
\text { Inner Diam } \\
\text { (in.) }\end{array}$ & $\begin{array}{l}\text { Sleeve } \\
\text { Outer Diam } \\
\text { (in.) }\end{array}$ & $\begin{array}{l}\text { Fuel Stack } \\
\text { Length } \\
\text { (in.) }\end{array}$ & $\begin{array}{l}\text { Plenum } \\
\text { Length } \\
\text { (in.) }\end{array}$ & $\begin{array}{l}\text { Fuel Body } \\
\text { Length } \\
\text { (fn.) }\end{array}$ & $\begin{array}{l}\text { Element } \\
\text { Length } \\
\text { (in.) }\end{array}$ \\
\hline Specified value & Pre: $\mathrm{X}_{1}$ & .490 & 1.940 & .500 & 1.095 & 2.740 & 2.752 & 3.485 & 27.16 & 2.63 & 31.0 & 144.0 \\
\hline Tolerance & Pre: $\tau_{i}$ & \pm .003 & & \pm .001 & $+.01,-0$ & $+0,-.002$ & \pm .002 & \pm .005 & $+.25,-.16$ & \pm 0.02 & & \\
\hline \multirow[t]{2}{*}{ Actual values } & Pre: $\bar{x}_{1}$ & .4905 & 1.9490 & .4995 & & 2.740 & 2.7522 & 3.4868 & 27.2850 & 2.6042 & 31.1077 & 144.796 \\
\hline & Post: $\overline{\mathrm{x}}_{2}$ & .4795 & 1.9088 & & & 2.7336 & 2.7495 & 3.4805 & 26.6843 & 2.9002 & 30.9673 & 143.893 \\
\hline \multirow[t]{2}{*}{ Precision } & Pre: $e_{1}$ & \pm .0001 & \pm .0001 & \pm .0002 & \pm .0002 & \pm .0003 & \pm .0002 & \pm .0002 & \pm .006 & \pm .0003 & \pm .0006 & \pm .01125 \\
\hline & Post: $e_{2}$ & \pm .0001 & \pm .0001 & \pm .0002 & \pm .0002 & \pm .0003 & \pm .0003 & \pm .0003 & \pm .0006 & \pm .0003 & \pm .0006 & \pm .01125 \\
\hline \multirow[t]{2}{*}{ Eq. random error } & Pre: $r_{1}$ & \pm .0006 & \pm .0001 & \pm .00015 & \pm .00015 & \pm .00035 & \pm .00015 & \pm .00015 & \pm .007 & \pm .00035 & \pm .002 & \pm .01 \\
\hline & Post: $r_{2}$ & \pm .0001 & \pm .0001 & \pm .00015 & \pm .00015 & \pm .00035 & \pm .00015 & \pm .00035 & \pm .002 & \pm .00035 & \pm .002 & \pm .01 \\
\hline \multirow[t]{2}{*}{ Accuracy } & Pre: $\alpha_{1}$ & \pm .0013 & \pm .0003 & \pm .0005 & \pm .0005 & \pm .001 & \pm .0005 & \pm .0005 & \pm .020 & \pm .001 & \pm .0046 & \pm .03125 \\
\hline & Post: $\alpha_{2}$ & \pm .0003 & \pm .0003 & \pm .0005 & \pm .0005 & \pm .001 & \pm .0006 & \pm .0006 & \pm .0046 & \pm .001 & \pm .0046 & \pm .03125 \\
\hline \multirow{2}{*}{$\begin{array}{l}\text { Sample random } \\
\text { error }(1 \sigma)(\text { on } \\
\text { measurement) }(a)\end{array}$} & Pre: $S_{X_{1}}$ & & $\pm .0019^{(b)}$ & (c) & -- & 0 & $\pm .0009^{(\mathrm{d})}$ & $\pm .0013^{(\mathrm{d})}$ & (c) & (c) & $\pm .0091^{(\mathrm{b})}$ & (c) \\
\hline & Post: $\mathrm{s}_{\mathrm{X}_{2}}$ & & $\pm .0019^{(\mathrm{e})}$ & (c) & -- & \pm .0017 & $\pm .0008^{(e)}$ & $\pm .0007^{(e)}$ & (c) & (c) & \pm .0091 & (c) \\
\hline \multirow[t]{2}{*}{$\begin{array}{l}\text { Sample random } \\
\text { error }(10)(e)\end{array}$} & Pre: $S \bar{X}_{1}$ & \pm .0012 & \pm .0042 & -- & -- & 0 & \pm .0007 & \pm .001 & \pm .0340 & \pm .0298 & \pm .0070 & -- \\
\hline & Post: $s_{\bar{x}_{2}}$ & \pm .0034 & \pm .0096 & -- & -- & \pm .0033 & \pm .0087 & \pm .0053 & \pm .1256 & \pm .1146 & \pm .0834 & -- \\
\hline
\end{tabular}

(a) $s_{X}=\left[\frac{\sum_{j=1}^{m} s_{j}^{2}}{m}\right]^{1 / 2}$

(b) Postirradiation values used in absence of preirradiation data.

(c) Only 1 measurement made.

(d) From measurements on archive samples.

(e) From FTE-4 measurements.

$$
s_{\bar{x}}=\left\{\frac{1}{\sum_{j=1}^{m} n_{j}-1}\left[\sum_{j=1}^{m}\left(n_{j}-1\right)\left(s_{j}\right)^{2}+\sum_{j=1}^{m} n_{j}\left(x_{j}-\bar{x}\right)^{2}\right]\right\}^{1 / 2}
$$




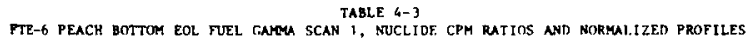

\begin{tabular}{|c|c|c|c|c|c|c|c|c|c|c|c|c|c|c|c|c|c|c|c|c|c|c|c|c|c|c|c|c|c|c|c|}
\hline \multicolumn{4}{|c|}{ Core Locat ion Parasecter } & \multicolumn{14}{|c|}{ Nucl1de CPM Ratios end 1 Sigan Count1ng Error Progressed } & \multicolumn{14}{|c|}{ 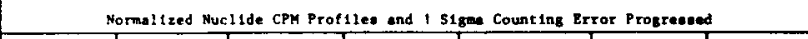 } \\
\hline \multirow{2}{*}{$\begin{array}{c}\text { CA } \\
\text { TaB No. } \\
\text { Spectrum } \\
\text { ID }\end{array}$} & \multirow{2}{*}{ 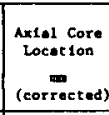 } & \multirow{2}{*}{ 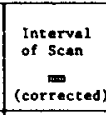 } & \multirow{2}{*}{$\begin{array}{c}\text { Puel } \\
\text { Body Puel } \\
\text { Stuck } \\
\text { m } \\
\text { (BoL Metrology) }\end{array}$} & \multicolumn{2}{|c|}{$\frac{\frac{S e-141}{2-95}}{2-95}$} & \multicolumn{2}{|c|}{$\frac{c_{e}-144}{2-95}$} & \multicolumn{2}{|c|}{\begin{tabular}{|l|}
$\frac{z-1311}{z-95}$ \\
\end{tabular}} & \multicolumn{2}{|c|}{$\frac{840-103}{2-95}$} & \multicolumn{2}{|c|}{$\frac{60-132}{2-95}$} & \multicolumn{2}{|c|}{$\frac{4-140}{z-95}$} & \multicolumn{2}{|c|}{ 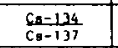 } & \multicolumn{2}{|c|}{\begin{tabular}{|l|}
$\frac{P_{A-2233}}{M_{\text {eson }}}$ \\
\end{tabular}} & \multicolumn{2}{|c|}{$\frac{\frac{c e-161}{\text { Sean }}}{\text { Ment }}$} & \multicolumn{2}{|c|}{$\frac{\frac{B u-103}{\text { Mean }}}{\text { Mean }}$} & \multicolumn{2}{|c|}{$\frac{6(x-1) 23}{\operatorname{menn}}$} & \multicolumn{2}{|c|}{ 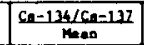 } & \multicolumn{2}{|l|}{$\frac{2 x-95}{4}$} & \multicolumn{2}{|l|}{$\frac{4-160}{1000}$} \\
\hline & & & & \begin{tabular}{|c|} 
Ret10 \\
$\frac{C P M}{C P M}$ \\
$C$
\end{tabular} & \begin{tabular}{|l|}
10 \\
$\frac{C P M}{C P M}$ \\
\end{tabular} & 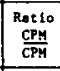 & \begin{tabular}{|l|}
10 \\
CPM \\
CPM \\
\end{tabular} & \begin{tabular}{|l} 
Ret10 \\
CPM \\
CPM \\
\end{tabular} & \begin{tabular}{|l|}
10 \\
$\frac{\mathrm{CPP}}{\mathrm{CPM}}$ \\
\end{tabular} & \begin{tabular}{|l} 
Retio \\
$\frac{P P M}{C P M}$ \\
$C P M$
\end{tabular} & $\begin{array}{l}10 \\
\frac{C P M}{C P M} \\
C P M\end{array}$ & \begin{tabular}{|c} 
Rat 10 \\
CPM \\
CPM \\
CPM
\end{tabular} & \begin{tabular}{|l|}
1 \\
$\frac{\mathrm{PP} M}{\mathrm{CPM}}$ \\
\end{tabular} & $\begin{array}{c}\text { Ratio } \\
\frac{C P M}{C^{P H P}}\end{array}$ & \begin{tabular}{|l|}
10 \\
$\frac{C P M}{C P M}$ \\
$C P M$ \\
\end{tabular} & $\begin{array}{l}\text { Ratio } \\
\frac{C P A}{C P H} \\
C P H\end{array}$ & $\begin{array}{l}10 \\
\frac{C P H}{C P A} \\
C P A\end{array}$ & \begin{tabular}{|c|} 
Rest \\
CPM \\
$P M$ \\
\end{tabular} & \begin{tabular}{|l|l}
10 \\
CPM \\
CPM
\end{tabular} & $\begin{array}{c}\text { Ret10 } \\
\frac{P P}{C P M} \\
C P M\end{array}$ & \begin{tabular}{|l|}
$\begin{array}{l}10 \\
P M \\
P M M \\
P P M\end{array}$ \\
\end{tabular} & \begin{tabular}{|c|} 
Ret10 \\
CPM \\
CPM \\
\end{tabular} & \begin{tabular}{|l|}
10 \\
$C P M$ \\
$C P M$ \\
\end{tabular} & \begin{tabular}{|c|} 
Ret10 10 \\
$\frac{C P M}{\mathrm{CPH}}$ \\
\end{tabular} & \begin{tabular}{|l|}
10 \\
$\mathrm{CP}$ \\
$\mathrm{CPM}$ \\
\end{tabular} & $\begin{array}{r}\text { Rat 1o } \\
\text { CPM } \\
\text { CPM }\end{array}$ & $\begin{array}{l}10 \\
\frac{\mathrm{CPM}}{\mathrm{CPM}} \\
\mathrm{CP}\end{array}$ & 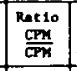 & \begin{tabular}{|l|}
$\begin{array}{l}\text { if } \\
\text { CPM } \\
\mathrm{CP}\end{array}$ \\
\end{tabular} & \begin{tabular}{|c|} 
necto \\
$\mathrm{CMM}$ \\
$\mathrm{CPM}$ \\
\end{tabular} & 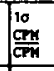 \\
\hline $\begin{array}{l}20 \\
20\end{array}$ & ${ }_{708}^{63 .}$ & 67: & & & & & & & & 1.211 & 63 & & & $\infty$ & .261 & & & .020 & & & & . & .006 & & & & & & & & \\
\hline $\begin{array}{l}203 \\
2003\end{array}$ & 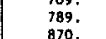 & 75. & & {$\left[\begin{array}{r}.903 \\
.975\end{array}\right.$} & . & $=$ & & 1.597 & {$\left[\begin{array}{ll}1.00 \\
264\end{array}\right.$} & 1.145 & .055 & . & . & 1.872 & .077 & $\begin{aligned} .598 \\
.598\end{aligned}$ & .101 & . & .008 & . 675 & $\begin{array}{l}.043 \\
.043\end{array}$ & .667 & . 026 & 然 & .111. & .3162 & . 060 & .635 & 019 & .667 & 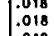 \\
\hline 205 & 934. & 78. & Body & 1.049 & .084 & .111 & $\mid .034$ & 1.116 & .163 & 1.1 .16 & .056 & . 0939 & . & 1.597 & .062 & .934 & .153 & s80 & 0.010 & $\begin{array}{r}.026 \\
1.046\end{array}$ & .077 & 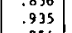 & 038 & 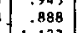 & .1170 & .877 & $\begin{array}{l}.716 \\
.133\end{array}$ & .913 & .025 & 年 .803193 & . 0192.019 \\
\hline $\begin{array}{l}206 \\
207\end{array}$ & $\begin{array}{l}1038.03 \\
1123 .\end{array}$ & . & & $\left|\begin{array}{l}1 \\
1.070 \\
i .251\end{array}\right|$ & $\begin{array}{l}.088 \\
.0202 \\
.09\end{array}$ & $\mid .073$ & $\mid \begin{array}{l}.034 \\
.029\end{array}$ & \begin{tabular}{|l}
1.1253 \\
1.8854
\end{tabular} & $\mid \begin{array}{l}.216 \\
.251\end{array}$ & $\mid \begin{array}{l}1.121 \\
1.303\end{array}$ & $\begin{array}{l}.064 \\
.061\end{array}$ & $\begin{array}{l}: 1169 \\
: 1115\end{array}$ & $\begin{array}{l}.0113 \\
.0129\end{array}$ & $\begin{array}{l}1.770 \\
1.840\end{array}$ & $\begin{array}{l}.005 \\
.072\end{array}$ & \begin{tabular}{|l}
.6899 \\
1.022
\end{tabular} & $\begin{array}{l}.107 \\
.145\end{array}$ & $\begin{array}{l}.643 \\
.686\end{array}$ & $\left\{\begin{array}{l}0.012 \\
.012\end{array}\right.$ & $\left.\begin{array}{l}1.083 \\
1.064\end{array}\right]$ & {$\left[\begin{array}{l}.081 \\
.080\end{array}\right]$} & 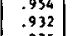 & $\begin{array}{l}.036 \\
.035 \\
.035\end{array}$ & $\begin{array}{l}1.123 \\
.900\end{array}$ & : 1230 & 要.960 & $\begin{array}{l}.072 \\
.138\end{array}$ & 年. 9890 & 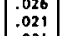 & 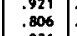 & 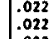 \\
\hline 209 & 1293. & 8. & & {$\left[\begin{array}{lll}10.042 \\
1.112\end{array}\right]$} & . 883 & .201 & $\mid \begin{array}{l}.043 \\
.042\end{array}$ & $\mid \begin{array}{l}1.833 \\
1.234\end{array}$ & $\mid \begin{array}{l}.209 \\
.123\end{array}$ & $\frac{1.209}{1.130}$ & $\begin{array}{l}.0059 \\
.068\end{array}$ & .1078 & .0148 & $\begin{array}{r}1.887 \\
1.808\end{array}$ & .069 & 1.265 & .200 & . 6040 & $\mid \begin{array}{l}011 \\
.011\end{array}$ & $\mid \begin{array}{l}1.998 \\
1.006\end{array}$ & \begin{tabular}{|l|}
.067 \\
.069
\end{tabular} & . 8980 & . 0.048 & $\begin{array}{l}.926 \\
\end{array}$ & .121 & 1.788 & .228 & 要8 880 & 告. .024 & 142 & 年. 0222 \\
\hline & & & $\begin{array}{l}1344 \\
1454\end{array}$ &.$\overline{774}$ & .064 & .723 &.$\overline{036}$ & $1 . \overline{416}$ & .023 & $1 . \overline{076}$ & .0 .052 & .1304 & $\ddot{o r t s s}$ & $1 . \overline{764}$ &.$\overline{060}$ & $1 . \overline{077}$ & . 151 & $\begin{array}{l}.-\overline{68} 2 \\
\end{array}$ &.$\overline{0 i 1}$ & $\overline{2}_{38}$ & .059 &.$\overline{864}$ &.$\overline{034}$ & 1.786 &.$\overline{141}$ & $1 . \overline{011}$ & ist & .875 & .024 & .866 & .022 \\
\hline $\begin{array}{l}212 \\
211\end{array}$ & $\begin{array}{l}51536 . \\
1627 .\end{array}$ & 86. & & $\left|\begin{array}{r}1.236 \\
.927\end{array}\right|$ & $\begin{array}{l}.0992 \\
.082\end{array}$ & .066 & .022 & $\mid \begin{array}{l}1.530 \\
1.488\end{array}$ & $\begin{array}{l}.239 \\
164\end{array}$ & {$\left[\begin{array}{l}1.163 \\
.943\end{array}\right.$} & $\begin{array}{l}.052 \\
.043\end{array}$ & $\begin{array}{l}.1336 \\
.06036\end{array}$ & $\begin{array}{l}.0884 \\
0.032\end{array}$ & \begin{tabular}{|l}
1.964 \\
1.629
\end{tabular} & $\begin{array}{l}.066 \\
0.055\end{array}$ & $\begin{array}{r}.957 \\
1.988\end{array}$ & .145 & $\mid \begin{array}{l}1.307 \\
1.659\end{array}$ & $\begin{array}{l}.014 \\
.016\end{array}$ & $\mid \begin{array}{l}1.372 \\
1,325\end{array}$ & \begin{tabular}{|l|}
.093 \\
.108
\end{tabular} & {$\left[\begin{array}{l}1.083 \\
1.136\end{array} \mid\right.$} & .0.039 .094 & $\begin{array}{l}1.428 \\
862\end{array}$ & $\begin{array}{r}181 \\
.121\end{array}$ & $\begin{array}{r}.899 \\
1.863\end{array}$ & $\begin{array}{l}130 \\
130\end{array}$ & 1.017 & \begin{tabular}{|l|}
.023 \\
0.12
\end{tabular} & 1. 121 & .025 \\
\hline 216 & 1718. & 86. & & .833 & .062 & -000 & .042 & .969 & 1.174 & $\therefore .042$ & .052 & 年. 0.0608 & .0087 & $\therefore .866$ & .058 & $\begin{array}{l}2.904 \\
2.029\end{array}$ & 323 & 1.503 & \begin{tabular}{|l|l|l}
3.015 \\
\end{tabular} & 1.131 & $\mid$\begin{tabular}{|l|}
.078 \\
.078
\end{tabular} & $\because .190$ & .051 & . 8048 & .109 & 1.905 & .559 & 1.244 & .029 & $\begin{array}{l}1.186 \\
80303\end{array}$ & .027 \\
\hline 211 & $\begin{array}{l}1809 . \\
1899\end{array}$ & 85. & & .921 & $\begin{array}{l}.067 \\
0.078\end{array}$ & . .095 & \begin{tabular}{|l|}
.027 \\
.029
\end{tabular} & .788 & 1.129 & \begin{tabular}{|l|l|}
1.012 \\
1.036
\end{tabular} & $\begin{array}{l}.051 \\
0.054\end{array}$ & $\begin{array}{l}.0730 \\
.01125\end{array}$ & .00894 & \begin{tabular}{|l|l}
1.7464 \\
1.821
\end{tabular} & .058 & 1.265 & $\begin{array}{l}.193 \\
102\end{array}$ & 1.533 & $\begin{array}{l}.015 \\
015\end{array}$ & $\left|\begin{array}{l}2.2020 \\
1.062\end{array}\right|$ & $\mid \begin{array}{l}.082 \\
.089\end{array}$ & $\begin{array}{r}.128 \\
984\end{array}$ & $\begin{array}{l}.0<8 \\
.043\end{array}$ & . 9 & $\begin{array}{r}.108 \\
140 \\
\end{array}$ & $\begin{array}{l}1.188 \\
1.294\end{array}$ & $\begin{array}{l}.221 \\
.238\end{array}$ & $\begin{array}{r}-216 \\
1035\end{array}$ & \begin{tabular}{|l|l|}
.030 \\
0.070
\end{tabular} & $\begin{array}{l}1.191 \\
1.058\end{array}$ & .026 \\
\hline 2 & $\begin{array}{l}1990 . \\
2080\end{array}$ & 86. & & .833 & .081 & \begin{tabular}{|l}
.0066 \\
.098
\end{tabular} & $\begin{array}{l}.028 \\
0.028\end{array}$ & .926 & $\mid .162$ & \begin{tabular}{|l|l}
1.078 \\
1.068
\end{tabular} & .061 & $\begin{array}{l}205 \\
3101 \\
1101\end{array}$ & $\left|\begin{array}{l}.0148 \\
0149\end{array}\right|$ & $\begin{array}{l}1.734 \\
1.670\end{array}$ & $\begin{array}{l}.070 \\
.063\end{array}$ & $\begin{array}{l}1.095 \\
1.221\end{array}$ & $\begin{array}{l}.156 \\
.196 \\
.191\end{array}$ & $\begin{array}{l}.713 \\
828\end{array}$ & \begin{tabular}{l|l}
.013 \\
.013
\end{tabular} & $\begin{array}{r}.913 \\
1.102\end{array}$ & $\begin{array}{l}.081 \\
.0990\end{array}$ & $\begin{array}{r}.994 \\
1.050\end{array}$ & $\begin{array}{l}.045 \\
.069\end{array}$ & $\mid \begin{array}{l}\mid .254 \\
1.221\end{array}$ & $\begin{array}{l}.143 \\
.135 \\
\end{array}$ & $\begin{array}{l}1.028 \\
1.145\end{array}$ & \begin{tabular}{|l}
.158 \\
.211
\end{tabular} & $\mid \begin{array}{l}1.005 \\
1.074\end{array}$ & $\left|\begin{array}{|c|}.032 \\
0030\end{array}\right|$ & $\begin{array}{l}978 \\
.004\end{array}$ & {$[.023$} \\
\hline $\begin{array}{l}1218 \\
219\end{array}$ & $\begin{array}{l}2160 . \\
2164 .\end{array}$ & 73. & 2130.6 & $\because \because 085$ & . 190 & -- & & $=$ & & 1.241 & .05 & is & .0284 & $\begin{array}{l}1.200 \\
\end{array}$ & .101 & .944 & 209 & .266 & $\begin{array}{l}0.007 \\
0\end{array}$ & .366 & .069 & .352 & .025 & $\begin{array}{lll} & 490 \\
\end{array}$ & .088 & .886 & .181 & .309 & 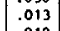 & .296 & .012 \\
\hline 22 & & & & $\mid \begin{array}{l}1.051 \\
\mid\end{array}$ & .158 & - & & בa & 8 & & .092 & م & נונ & (5.179 & .2964 & ד & 20 & . .404 & . 009 & & . .0688 & . 433 & . .0300 & $\because$ & & 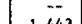 & 28. & & & & 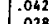 \\
\hline 22 & 24 & 90 & & .676 & .069 & .083 & .027 & 1.160 & 171 & .961 & .044 & .0972 & 0131 & 1.864 & .059 & $\because 1008$ & $\begin{array}{l}.169 \\
.69\end{array}$ & 1,728 & 017 & $\begin{array}{l}0.069 \\
1.013\end{array}$ & 0.098 & 1.213 & .046 & 386 & 176 & .946 & 157 & & & & .029 \\
\hline 32 & & & & .544 & .059 & - & 036 & .925 & .141 & $\begin{array}{r}.990 \\
990\end{array}$ & $\begin{array}{r}.046 \\
.048\end{array}$ & . 0736 & . 060 & 1.813 & .059 & 1.329 & .229 & 1.990 & \begin{tabular}{|c|c|}
0.018 \\
0.014
\end{tabular} & $\begin{array}{l}1.769 \\
\end{array}$ & .079 & 1.176 & .004 & . 988 & $\begin{array}{r}135 \\
.138\end{array}$ & 1.248 & $\begin{array}{l}.272 \\
110\end{array}$ & & & & 004 \\
\hline 22 & $\begin{array}{l}26 \\
27\end{array}$ & & & 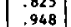 & .083 &.- & .036 & $1-4 / 7$ & 212 & $\begin{array}{l}1.043 \\
1.216\end{array}$ & .057 & . 0988 & .0131 & $\begin{array}{l}1.648 \\
1.753\end{array}$ & .065 & .785 & .139 & $\begin{array}{l}.053 \\
.850\end{array}$ & \begin{tabular}{l|l}
0.014 \\
0
\end{tabular} & . 9896 & 0.017 & $\begin{array}{l}0.024 \\
1.027\end{array}$ & .039 & 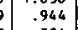 & $\mid \begin{array}{l}.186 \\
.119 \\
\end{array}$ & . & 100 & 923 & 235 & 908 & 等. .02642 \\
\hline 228 & $\begin{array}{l}27 \\
28\end{array}$ & 78 & & $\begin{array}{r}.939 \\
\quad .006\end{array}$ & $\begin{array}{l}.088 \\
.04\end{array}$ & $\begin{array}{l}.156 \\
1124\end{array}$ & $\begin{array}{l}.056 \\
.035\end{array}$ & $\mid \begin{array}{l}.985 \\
1.370\end{array}$ & $\begin{array}{l}.210 \\
.238\end{array}$ & $\begin{array}{l}1.262 \\
1.158\end{array}$ & $\begin{array}{l}.068 \\
.064\end{array}$ & $\begin{array}{l}.014 \\
.0959\end{array}$ & $\begin{array}{l}.0163 \\
.0139\end{array}$ & \begin{tabular}{|l}
1.840 \\
1.629
\end{tabular} & $\begin{array}{l}.0777 \\
.067\end{array}$ & $\begin{array}{l}7399 \\
.254\end{array}$ & $\begin{array}{l}148 \\
124\end{array}$ & $\begin{array}{l}.657 \\
.535\end{array}$ & \begin{tabular}{l|l}
7 \\
$\vdots$ \\
\end{tabular} & $\begin{array}{l}1796 \\
196\end{array}$ & .067 & 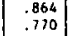 & & $\begin{array}{l}.784 \\
7220\end{array}$ & & $\begin{array}{l}.6964 \\
.426\end{array}$ & $\begin{array}{l}.03 \\
.055\end{array}$ & . & 11 & 63 & .020 \\
\hline Bod & Mean & 546. & & 1.089 & .083 & .121 & .040 & 1.425 & .219 & 1.197 & .059 & .1124 & .0146 & 1.816 & .071 & .785 & .131 & .552 & \begin{tabular}{l|l}
2.010 \\
\end{tabular} & .933 & \begin{tabular}{|l}
.067 \\
\end{tabular} & .860 & .034 & 891 & .113 & .737 & .098 & .786 & .023 & .797 & .021 \\
\hline & RMS & 597. & & . & .078 & . 0.088 & .030 & \begin{tabular}{|l}
.290 \\
1.1230
\end{tabular} & .185 & $\begin{array}{r}.066 \\
1.049\end{array}$ & \begin{tabular}{|l|l}
.053 \\
\end{tabular} & $\begin{array}{l}.0142 \\
.09662\end{array}$ & .0124 & $\begin{array}{r}.103 \\
1.776\end{array}$ & .063 & $\begin{array}{r}.260 \\
1.418\end{array}$ & .228 & \begin{tabular}{|l}
.128 \\
1.235
\end{tabular} & \begin{tabular}{l|l}
8 & 014 \\
5 & 01
\end{tabular} & $\mid \begin{array}{r}150 \\
\therefore 160\end{array}$ & . & $\begin{array}{l}.144 \\
1.081\end{array}$ & .045 & \begin{tabular}{|l|}
.096 \\
\end{tabular} & .138 & $\begin{array}{r}.189 \\
1.331\end{array}$ & .288 & $\begin{array}{l}\because 116 \\
1.128\end{array}$ & .030 & $\begin{array}{l}.105 \\
122 \\
\end{array}$ & .025 \\
\hline & & 596. & & 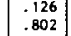 & .073 & $\begin{array}{l}.017 \\
.120\end{array}$ & .033 & $\mid \begin{array}{l}.296 \\
\therefore .108\end{array}$ & .190 & $\begin{array}{l}.062 \\
1.088\end{array}$ & .052 & $\begin{array}{l}.0279 \\
.0903\end{array}$ & .0128 & \begin{tabular}{|l|}
.108 \\
$\therefore .763$
\end{tabular} & .064 & $\begin{array}{l}.392 \\
.951\end{array}$ & .170 & $\mid \begin{array}{l}3238 \\
1.213\end{array}$ & $\begin{array}{l}8.015 \\
\end{array}$ & 年. & .081 & $\begin{array}{r}.070 \\
1.059\end{array}$ & .042 & 394 & .137 & $\begin{array}{l}.599 \\
.893\end{array}$ & .149 & 菌, 1168 & .028 & $\begin{array}{l}108 \\
081\end{array}$ & .025 \\
\hline & & & & .161 & & .026 & & .201 & & & & & & .085 & & & & .534 & & & & .180 & & & & .29 & & 275 & & & \\
\hline Tntal Element & $\begin{array}{l}\text { Grand Mean } \\
\text { RHS }\end{array}$ & 1739. & & $\begin{array}{l}.946 \\
.173\end{array}$ & .078 & $\begin{array}{l}.108 \\
.029\end{array}$ & .034 & $\begin{array}{l}1.256 \\
.298\end{array}$ & .195 & $\begin{array}{l}1.111 \\
104\end{array}$ & $\begin{array}{l}.054 \\
.012\end{array}$ & $\begin{array}{l}.0991 \\
.0214\end{array}$ & .0132 & $\begin{array}{r}1.781 \\
.101\end{array}$ & .066 & $\begin{array}{l}1.065 \\
.433\end{array}$ & .183 & $\begin{array}{r}1.000 \\
.487\end{array}$ & \begin{tabular}{l|l}
0.013 \\
\end{tabular} & $\begin{array}{r}1.000 \\
.179\end{array}$ & .019 & \begin{tabular}{|c|}
1.000 \\
.163
\end{tabular} \mid & .040 & \begin{tabular}{|l|l|}
0 & 1.000 \\
.210
\end{tabular} & .130 & $\begin{array}{r}1.000 \\
.431\end{array}$ & .178 & $\begin{array}{l}1.000 \\
.240\end{array}$ & .027 & $\begin{array}{l}1.000 \\
.242\end{array}$ & .024 \\
\hline
\end{tabular}


FTE-6 PEACH BOTTOM EOL FUEL GABMPA SCAN 1, NUCLIDE CPM TABULAT TON

\begin{tabular}{|c|c|c|c|c|c|c|c|c|c|c|c|c|c|c|c|c|c|c|c|c|c|}
\hline \multirow{3}{*}{$\begin{array}{c}\text { Spectrum } \\
\text { ID } \\
\text { GA } \\
\text { Tag No. } \\
\end{array}$} & \multicolumn{2}{|c|}{\begin{tabular}{|c|} 
Core Location Parameters \\
\end{tabular}} & \multirow[b]{3}{*}{$\begin{array}{l}\text { Analysio } \\
\text { Parameter }\end{array}$} & \multicolumn{18}{|c|}{ Nuc11de CPM Decayed Back to October 31, 1974, 15.35 Hr, and 2-51 gma Counting Error } \\
\hline & \multirow{2}{*}{$\begin{array}{c}\text { Center Point } \\
\text { Axtal Core } \\
\text { Location } \\
\text { mas } \\
\text { (corrected) } \\
\end{array}$} & \multirow{2}{*}{\begin{tabular}{|c|} 
Interval \\
of Scans \\
mm \\
(corrected) \\
\end{tabular}} & & \multicolumn{2}{|c|}{$\begin{array}{l}\text { Ge-144 } \\
133 \mathrm{KeV} \\
\end{array}$} & \multicolumn{2}{|c|}{$\begin{array}{l}\mathrm{Ce}-141 \\
145 \mathrm{KeV} \\
\end{array}$} & \multicolumn{2}{|c|}{$\begin{array}{l}\text { Pu-233 } \\
312 \mathrm{KeV}\end{array}$} & \multicolumn{2}{|c|}{$\begin{array}{r}I-131 \\
364 \mathrm{KeV}\end{array}$} & \multicolumn{2}{|c|}{$\begin{array}{l}\text { Ru-103 } \\
497 \mathrm{KeV}\end{array}$} & \multicolumn{2}{|c|}{$\begin{array}{l}C_{s-137} \\
661 \mathrm{KeV}\end{array}$} & \multicolumn{2}{|c|}{$\begin{array}{c}2 \mathbf{r}-95 \\
724 \mathrm{KeV}\end{array}$} & \multicolumn{2}{|c|}{$\begin{array}{l}\mathrm{Cs}-134 \\
796 \mathrm{KeV}\end{array}$} & \multicolumn{2}{|c|}{$\begin{array}{l}\text { La-140 } \\
1596 \mathrm{KeV}\end{array}$} \\
\hline & & & & $\begin{array}{c}\mathrm{CPM} \\
\left(\infty \mathrm{AIn}{ }^{-1}\right)\end{array}$ & $\begin{array}{l}2 \sigma \\
(z)\end{array}$ & ${ }_{\left(\pi+n^{-1}\right)}^{C P M}$ & $\begin{array}{l}2 \sigma \\
(x) \\
\end{array}$ & $\begin{array}{c}\mathrm{CP}^{\mathrm{M}}-1 \\
\left(\mathrm{mIn}^{-1}\right)\end{array}$ & $\begin{array}{l}2 \sigma \\
(z) \\
\end{array}$ & $\begin{array}{c}\mathrm{CPM}_{4} \\
\left(\mathrm{~min}^{-1}\right)\end{array}$ & $\begin{array}{l}2 \sigma \\
(x) \\
\end{array}$ & $\underset{(m i n)}{\left.\mathrm{CPM}_{1}\right)}$ & $\begin{array}{l}20 \\
(z) \\
\end{array}$ & $\left(\begin{array}{c}\mathrm{CPM} \\
\left(\operatorname{con}{ }^{-1}\right)\end{array}\right.$ & $\begin{array}{l}20 \\
(z)\end{array}$ & $\begin{array}{c}\mathrm{CPM}^{\mathrm{C}} \\
(\mathrm{min}\end{array}$ & $\begin{array}{ll}20 \\
(x)\end{array}$ & ${ }_{\left(\cos n^{-1}\right)}$ & $\begin{array}{l}2 \sigma \\
(\mathrm{z})\end{array}$ & $\underset{\left(\min ^{-1}\right)}{C P M}$ & $\begin{array}{l}2 \sigma \\
(x)\end{array}$ \\
\hline $202-208$ & $\begin{array}{l}\text { Body } 1 \\
960\end{array}$ & $\$ 46$ & $\begin{array}{l}\text { Mean } \\
\text { RMS }\end{array}$ & $\begin{array}{l}703 \\
181\end{array}$ & 60.0 & $\begin{array}{r}5855 \\
947\end{array}$ & 13.9 & $\begin{array}{r}26210 \\
6089\end{array}$ & 3.62 & $\begin{array}{l}7622 \\
1702\end{array}$ & 30.1 & $\begin{array}{r}6413 \\
849\end{array}$ & 7.89 & $\begin{array}{r}589 \\
97\end{array}$ & 25.2 & $\begin{array}{r}5377 \\
797\end{array}$ & 5.71 & $\begin{array}{l}522 \\
200\end{array}$ & 21.6 & $\begin{array}{l}9717 \\
1286\end{array}$ & 5.20 \\
\hline $212-218$ & $\begin{array}{l}\text { Body } 2 \\
1809\end{array}$ & 597 & $\begin{array}{l}\text { Mean } \\
\text { RMS }\end{array}$ & $\begin{array}{l}661 \\
124\end{array}$ & 68.9 & $\begin{array}{r}7282 \\
920\end{array}$ & 15.3 & $\begin{array}{l}58633 \\
15563\end{array}$ & 2.54 & $\begin{array}{l}8757 \\
2326\end{array}$ & 31.9 & $\begin{array}{r}8065 \\
528\end{array}$ & 8.34 & $\begin{array}{l}725 \\
147\end{array}$ & 25.4 & $\begin{array}{r}7722 \\
792\end{array}$ & 5.30 & $\begin{array}{l}978 \\
118\end{array}$ & 15.4 & $\begin{array}{r}13679 \\
1320\end{array}$ & 4.54 \\
\hline & Total Element & 1739 & $\begin{array}{l}\text { Grand Mean } \\
\text { RMS }\end{array}$ & $\begin{array}{l}706 \\
144\end{array}$ & 64.3 & $\begin{array}{l}6275 \\
1129\end{array}$ & 15.8 & $\begin{array}{l}47470 \\
23100\end{array}$ & 3.02 & 8338 & 31.3 & $\begin{array}{l}7460 \\
1221\end{array}$ & 8.10 & $\begin{array}{l}661 \\
140\end{array}$ & 26.2 & $\begin{array}{l}6845 \\
1642\end{array}$ & 5.41 & $\begin{array}{l}717 \\
293\end{array}$ & 21.6 & $\begin{array}{l}12193 \\
2954\end{array}$ & 4.83 \\
\hline
\end{tabular}


TABLE $4-5$

FTE-6 FUEL STACK 1 GAMMA SCANS, NUCLIDE CPM TABULATION

\begin{tabular}{|c|c|c|c|c|c|c|c|c|c|c|}
\hline \multirow[b]{3}{*}{ Fue1 Body } & \multicolumn{10}{|c|}{$\begin{array}{c}\text { Nuclide CPM Decayed Back to October } 31,1974,1535 \mathrm{Hr} \text {, } \\
\text { and } 2 \text { Sigma Counting Errors }\end{array}$} \\
\hline & \multicolumn{2}{|c|}{$\begin{array}{l}\mathrm{Ru}-106 \\
511.9\end{array}$} & \multicolumn{2}{|c|}{$\begin{array}{l}C s-134 \\
604.7\end{array}$} & \multicolumn{2}{|c|}{$\begin{array}{l}\text { Cs }-137 \\
661.6\end{array}$} & \multicolumn{2}{|c|}{$\begin{array}{l}\mathrm{Ce}-144 \\
696.5\end{array}$} & \multicolumn{2}{|c|}{$\begin{array}{l}\mathrm{Zr}-95 \\
724.2\end{array}$} \\
\hline & $\underset{\left(\min ^{-1}\right)}{\mathrm{CPM}}$ & $\begin{array}{l}2 \sigma \\
(\%)\end{array}$ & $\underset{\left(\min ^{-1}\right)}{\mathrm{CPM}}$ & $\begin{array}{l}2 \sigma \\
(\%)\end{array}$ & $\begin{array}{c}\mathrm{CPM} \\
\left(\min ^{-1}\right)\end{array}$ & $\begin{array}{l}2 \sigma \\
(\%)\end{array}$ & $\underset{\left(\min ^{-1}\right)}{\mathrm{CPM}}$ & $\begin{array}{l}2 \sigma \\
(\%)\end{array}$ & $\underset{\left(\min ^{-1}\right)}{\mathrm{CPM}}$ & $\begin{array}{l}2 \sigma \\
(\%)\end{array}$ \\
\hline $\begin{array}{l}\text { Body } 1 \\
\text { Mean } \\
\text { RMS }\end{array}$ & $\begin{array}{l}320.0 \\
\pm 62.2\end{array}$ & 15.61 & $\begin{array}{l}1545.7 \\
\pm 706.8\end{array}$ & 2.42 & $\begin{array}{r}1283.0 \\
\pm 3149.4\end{array}$ & 1.85 & $\begin{array}{l}229.3 \\
\pm 50.0\end{array}$ & 15.89 & $\begin{array}{l}10708.7 \\
\pm 3023.1\end{array}$ & 22.97 \\
\hline $\begin{array}{l}\text { Body } 2 \\
\text { Mean } \\
\text { RMS/Error }\end{array}$ & $\begin{array}{l}482.3 \\
\pm 22.7\end{array}$ & 10.99 & $\begin{array}{r}2345.9 \\
\pm 91.9\end{array}$ & 1.90 & $\begin{array}{r}1457.6 \\
\pm 110.4\end{array}$ & 1.78 & $\begin{array}{l}307.0 \\
\pm 13.9\end{array}$ & 11.92 & $\begin{array}{r}15567.9 \\
\pm 477.9\end{array}$ & 13.03 \\
\hline $\begin{array}{l}\text { Body } 3 \\
\text { Mean } \\
\text { RMS/Error }\end{array}$ & $\begin{array}{r}359.9 \\
\pm 66.3\end{array}$ & 13.56 & $\begin{array}{r}1282.6 \\
\pm 341.6\end{array}$ & 2.95 & $\begin{array}{r}1075.9 \\
\pm 86.4\end{array}$ & 2.36 & $\begin{array}{l}232.4 \\
\pm 50.4\end{array}$ & 15.64 & $\begin{array}{r}12053.0 \\
\pm 1856.5\end{array}$ & 17.97 \\
\hline $\begin{array}{l}\text { Total Element } \\
\text { Mean } \\
\text { RMS/Error }\end{array}$ & $\begin{array}{l}393.25 \\
\pm 89.2\end{array}$ & 13.41 & $\begin{array}{l}1733.65 \\
\pm 645.8\end{array}$ & 2.45 & $\begin{array}{l}1269.85 \\
\pm 248.3\end{array}$ & 1.91 & $\begin{array}{l}257.62 \\
\pm 55.0\end{array}$ & 12.79 & $\begin{array}{l}12880.0 \\
\pm 2871.2\end{array}$ & 18.19 \\
\hline
\end{tabular}




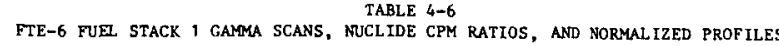

\begin{tabular}{|c|c|c|c|c|c|c|c|c|c|c|c|c|c|c|c|c|c|c|c|c|c|c|}
\hline EOL & ocation & Parame & & & & Nuc $11 \mathrm{c}$ & e CPM Rat 1 & os and & -Sigma Cou & inting $E$ & & & & & nalized Nu & clide $\mathrm{C}$ & Profiles & and $1-s$ & 1 gma Cour & $\operatorname{lng}$ Error & & \\
\hline $\begin{array}{l}\text { Fuel Body } \\
\text { Fole - Rod }\end{array}$ & $\begin{array}{c}\text { Cont } \\
\text { Sce } \\
\text { Core }\end{array}$ & $\begin{array}{l}\text { nuous } \\
\text { n } \\
\text { elght }\end{array}$ & \begin{tabular}{|c|} 
Fuel \\
Sce \\
Core
\end{tabular} & & $\frac{C_{e-1}}{Z_{r-1}}$ & & $\frac{R u-1}{2 r-9}$ & & $\frac{C_{s-1}}{2 r-9}$ & & $\frac{c_{s-}}{c_{s-}}$ & & & $\frac{144}{n+1}$ & $\frac{\mathrm{Ru}-1}{\mathrm{Mes}}$ & & $\frac{\mathrm{Cs}-1}{\mathrm{Me}}$ & & $\frac{\mathrm{Cs}-134}{\mathrm{M}}$ & $\frac{8-137}{n}$ & $\frac{\mathrm{zr}_{\mathrm{r}} \text { Mea }}{2}$ & \\
\hline $1 \equiv 1$ & $\begin{array}{c}x \\
\text { (man) } \\
\end{array}$ & $\begin{array}{c}\Delta x \\
(m x)\end{array}$ & $\begin{array}{c}x \\
\text { (nam) }\end{array}$ & $\begin{array}{c}\Delta \mathrm{X} \\
\text { (min) }\end{array}$ & Ratio & 10 & Rat10 & 10 & Rat 10 & 10 & Rat io & 10 & Ratio & 10 & Ratio & 10 & Rat 10 & 10 & Ratio & 10 & Rat1o & 10 \\
\hline $1-\mathrm{I}-2$ & $\begin{array}{l}703 \\
789\end{array}$ & $\begin{array}{l}75 \\
75\end{array}$ & 737 & 49 & \begin{tabular}{|l|}
0.035 \\
\end{tabular} & 0.009 & 0.050 & 0.018 & 0.178 & 0.041 & 0.702 & 0.017 & 0.637 & 0.061 & 0.595 & 0.044 & 0.653 & 0.008 & 0.532 & 0.013 & 0.361 & 0.083 \\
\hline $1-\mathrm{I}-4$ & 870 & 77 & 834 & 49 & 0.019 & 0.002 & 0.028 & 0.003 & 0.105 & 0.009 & 0.960 & 0.013 & 0.672 & 0.066 & 0.669 & 0.054 & 0.767 & 0.009 & 0.728 & 0.010 & 0.721 & 0.059 \\
\hline $1-\mathrm{I}-6$ & 954 & 78 & 931 & 49 & 0.018 & 0.002 & 0.027 & 0.003 & 0.103 & 0.008 & 1.183 & 0.018 & 0.873 & 0.067 & 0.870 & 0.061 & 1.027 & 0.009 & 0.897 & 0.013 & \begin{tabular}{|l|l|}
0.981 \\
\end{tabular} & 0.072 \\
\hline $1-1-8$ & 1038 & 80 & 1022 & 49 & 0.021 & 0.002 & 0.030 & 0.003 & 0.113 & 0.009 & 1.202 & 0.017 & 0.947 & 0.072 & 0.877 & 0.058 & 1.015 & 0.009 & 0.912 & 0.013 & \begin{tabular}{|l|l|}
0.884 \\
\end{tabular} & 0.071 \\
\hline $1-t-10$ & 1123 & 80 & 1126 & 49 & - & - & - & -- & - & -- & -- & -- & -- & -- & -- & - & -- & -- & - & - & -- & - \\
\hline $1-\mathrm{t}-12$ & 1208 & 81 & 1226 & 49 & 0.021 & 0.002 & 0.024 & 0.003 & 0.122 & 0.010 & 1.203 & 0.015 & 1.009 & 0.071 & 0.786 & 0.087 & 1.210 & 0.010 & 0.912 & 0.011 & 0.981 & 0.077 \\
\hline $1-\mathrm{I}-14$ & 1293 & 78 & 1320 & 49 & 0.023 & 0.002 & 0.035 & 0.003 & 0.129 & 0.009 & 1.597 & 0.017 & 1.203 & 0.076 & 1.086 & 0.072 & 1.390 & 0.010 & 1.211 & 0.012 & 1.060 & 0.071 \\
\hline $\begin{array}{c}\text { Body } 1 \\
\text { Mean } \\
\text { RMS }\end{array}$ & & & & & $\begin{array}{l}0.023 \\
0.005\end{array}$ & $\mid 0.004$ & $\begin{array}{l}0.032 \\
0.008\end{array}$ & 0.008 & $\begin{array}{l}0.125 \\
0.025\end{array}$ & 0.019 & $\begin{array}{l}1.141 \\
0.272\end{array}$ & 0.016 & $\begin{array}{l}0.890 \\
0.194\end{array}$ & 0.069 & $\begin{array}{l}0.814 \\
0.158\end{array}$ & 0.064 & $\begin{array}{l}1.010 \\
0.248\end{array}$ & 0.009 & $\begin{array}{l}0.865 \\
0.226\end{array}$ & 0.012 & \begin{tabular}{|l|}
0.831 \\
0.235
\end{tabular} & 0.073 \\
\hline $2-I-2$ & 1536 & 84 & 1527 & 48 & 0.019 & 0.002 & 0.030 & 0.003 & 0.099 & \begin{tabular}{|l|}
0.007 \\
\end{tabular} & 1.636 & 0.022 & 1.130 & 0.067 & 1.157 & 0.076 & 1.184 & 0.011 & 1.241 & 0.016 & 1.178 & 0.085 \\
\hline $2-\mathrm{I}-4$ & 1627 & 86 & 1624 & 48 & 0.019 & 0.002 & 0.031 & 0.003 & 0.088 & 0.005 & 1.611 & 0.020 & 1.192 & 0.074 & 1.284 & 0.065 & 1.115 & 0.010 & 1.254 & 0.015 & 1.247 & 0.070 \\
\hline $2-I-7$ & 1718 & 85 & 1769 & 48 & 0.021 & 0.002 & 0.031 & 0.003 & 0.092 & 0.005 & 1.654 & 0.022 & 1.300 & 0.082 & 1.266 & 0.072 & 1.148 & 0.011 & 1.255 & 0.016 & 1.235 & 0.070 \\
\hline $2-I-8$ & 1809 & 85 & 1817 & 48 & 0.020 & 0.002 & 0.032 & 0.003 & 0.085 & 0.006 & 1.588 & 0.023 & 1.134 & 0.072 & 1.193 & 0.068 & 0.997 & 0.010 & 1.204 & 0.017 & 1.153 & 0.081 \\
\hline $2-I-10$ & 1893 & 85 & 1913 & 48 & 0.020 & 0.002 & 0.033 & 0.003 & 0.101 & 0.007 & 1.600 & 0.019 & 1.215 & 0.071 & 1.317 & 0.062 & 0.254 & 0.010 & 1.214 & 0.014 & 1.225 & 0.089 \\
\hline $2-\mathrm{I}-12$ & 1990 & 86 & 2010 & 48 & 0.019 & 1.002 & 0.1129 & 0.003 & 0.084 & 0.006 & 1.601 & 0.023 & 1.172 & 0.075 & 1.193 & 0.066 & 1.071 & 0.010 & 1.214 & 0.017 & 1.251 & 0.086 \\
\hline $2-\mathrm{I}-14$ & 2080 & 85 & 2106 & 48 & 0.020 & 0.002 & 0.031 & 0.003 & 0.104 & 0.007 & 1.610 & 0.021 & 1.200 & 0.067 & 1.175 & 0.069 & 1.237 & 0.011 & 1.221 & 0.015 & 1.173 & 0.078 \\
\hline $\begin{array}{c}\text { Body } 2 \\
\text { Mean } \\
\text { RMS }\end{array}$ & & & & & $\begin{array}{c}0 \\
0.020 \\
0.0007\end{array}$ & 0.002 & $\begin{array}{l}0.031 \\
0.0012\end{array}$ & 0.003 & $\begin{array}{l}0.093 \\
0.0075\end{array}$ & 0.006 & $\begin{array}{l}1.614 \\
0.021 \\
\end{array}$ & 0.021 & $\begin{array}{l}1.192 \\
0.053\end{array}$ & 0.073 & $\begin{array}{l}1.226 \\
0.057\end{array}$ & 0.068 & $\begin{array}{l}1.144 \\
0.085\end{array}$ & 0.010 & $\begin{array}{l}1.229 \\
0.019\end{array}$ & 0.0157 & $\begin{array}{l}1.209 \\
0.037\end{array}$ & 0.080 \\
\hline $3-1-2$ & 2332 & 90 & 2310 & 48 & 0.020 & 0.002 & 0.031 & 0.003 & 0.082 & 0.007 & 1.483 & 0.024 & 1.106 & 0.082 & 1.139 & 0.071 & 0.932 & $0 . \overline{010}$ & 1.125 & 0.018 & 1,116 & 0.088 \\
\hline $3-\mathrm{I}-4$ & 2428 & 90 & 2407 & 48 & 0.021 & 0.002 & 0.031 & 0.003 & 0.076 & 0.006 & 1.380 & 0.024 & 1.180 & 0.076 & 1.127 & 0.075 & 0.866 & 0.009 & 1.047 & 0.018 & 1.126 & 0.087 \\
\hline $3-I-6$ & 2533 & 90 & 2504 & 48 & 0.019 & 0.002 & 0.030 & 0.003 & 0.088 & 0.007 & 1.254 & 0.022 & 0.970 & 0.070 & 0.971 & 0.069 & 0.891 & 0.010 & 0.951 & 0.017 & 1.000 & 0.084 \\
\hline $3-\mathrm{I}-8$ & 2615 & 84 & 2600 & 48 & 0.020 & 0.002 & 0.031 & 0.003 & 0.093 & 0.008 & 1.292 & 0.021 & 0.963 & 0.066 & 0.958 & 0.056 & 0.887 & 0.009 & 0.980 & 0.016 & 0.938 & 0.085 \\
\hline $3-\mathbf{I}-10$ & 2704 & 84 & 2697 & 48 & 0.019 & 0.002 & 0.029 & 0.003 & 0.104 & 0.010 & 1.126 & 0.019 & 0.788 & 0.068 & 0.762 & 0.053 & 0.862 & 0.008 & 0.854 & 0.014 & 0.814 & 0.077 \\
\hline $3-1-12$ & 2791 & 80 & 2794 & 48 & 0.019 & 0.003 & 0.031 & 0.004 & 0.103 & 0.011 & 0.908 & 0.020 & 0.691 & 0.064 & 0.740 & 0.055 & 0.754 & 0.009 & 0.689 & 0.015 & 0.720 & 0.079 \\
\hline $3-I-14$ & 2875 & 78 & 2890 & 48 & 0.015 & 0.002 & 0.026 & 0.003 & 0.087 & 0.009 & 0.777 & 0.017 & 0.617 & 0.059 & 0.707 & 0.056 & 0.738 & 0.008 & 0.589 & 0.013 & 0.835 & 0.086 \\
\hline $\begin{array}{c}\text { Body } 3 \\
\text { Mean } \\
\text { RMS }\end{array}$ & & & & & $\begin{array}{l}0.019 \\
0.002\end{array}$ & 0.002 & $\begin{array}{l}0.030 \\
0.002\end{array}$ & 0.003 & $\begin{array}{l}0.090 \\
0.010\end{array}$ & 0.008 & $\begin{array}{l}1.174 \\
1.236\end{array}$ & 0.021 & $\begin{array}{l}0.902 \\
0.195\end{array}$ & 0.070 & $\begin{array}{l}0.915 \\
0.168\end{array}$ & 0.063 & $\begin{array}{l}0.847 \\
0.067\end{array}$ & 0.009 & $\begin{array}{l}0.891 \\
0.179\end{array}$ & 0.0159 & $\begin{array}{l}0.936 \\
0.144\end{array}$ & 0.084 \\
\hline $\begin{array}{l}\text { Total } \\
\text { Element } \\
\text { Mean } \\
\text { RMS }\end{array}$ & & & & & $\begin{array}{r}0.0204 \\
\pm 0.0037\end{array}$ & 0.003 & $\begin{array}{c}0.031 \\
\pm 0.0050\end{array}$ & 0.005 & $\begin{array}{r}0.1018 \\
\pm 0.0218\end{array}$ & 0.011 & $\begin{array}{r}1.3184 \\
\pm 0.2984\end{array}$ & 0.020 & $\begin{array}{c}1.000 \\
: 0.2133\end{array}$ & 0.070 & $\begin{array}{c}0.994 \\
\pm 0.2222\end{array}$ & 0.065 & $\begin{array}{c}1.000 \\
\pm 0.1949\end{array}$ & 0.009 & $\begin{array}{r}1.000 \\
+0.228\end{array}$ & 0.0147 & $\begin{array}{c}1.000 \\
\pm 0.2227\end{array}$ & 0.080 \\
\hline
\end{tabular}




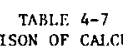

PTE-6 PUEL GATA SCA: COIPARISON OF CAICLLLATED AND, MEASURED EOI. PONER PROFILES FOR PEACH BOTTMM SCAN 1 z $\mathbf{r}-95+$ La-140

\begin{tabular}{|c|c|c|c|c|c|c|c|c|c|c|c|c|c|c|c|c|}
\hline \multirow{4}{*}{$\begin{array}{c}\text { For Location Parameter } \\
\text { Fue1 Body }\end{array}$} & \multicolumn{8}{|c|}{ Peach Bottom Nornalized Nuc11de CPM Profile Comparison } & \multicolumn{8}{|c|}{ Calculated vs. Measured EOL Power Profile Comparison } \\
\hline & \multirow{2}{*}{\multicolumn{2}{|c|}{$\frac{z_{r}-95}{\text { Mean }}$}} & \multirow{2}{*}{\multicolumn{2}{|c|}{$\frac{\mathrm{La}-140}{\text { Mean }}$}} & \multirow{2}{*}{\multicolumn{2}{|c|}{$\frac{\text { Re1. Diff. }}{Z=C / M-1}$}} & \multicolumn{2}{|c|}{ Compartson } & \multirow{2}{*}{\multicolumn{2}{|c|}{$\frac{\text { Power EOL }}{\text { Mean }}$}} & \multirow{2}{*}{\multicolumn{2}{|c|}{$\frac{2 \mathrm{x}-95+\mathrm{La}-140}{\text { Mean }}$}} & \multirow{2}{*}{\multicolumn{2}{|c|}{$\frac{\text { Re1. DIff. }}{2=C M / 1}$}} & \multicolumn{2}{|c|}{ Comparison } \\
\hline & & & & & & & \multirow{2}{*}{$\begin{array}{c}\text { Test } 1 \\
\mathrm{D}=7 / \mathrm{S}_{z} \\
(-)\end{array}$} & \multirow{2}{*}{$\begin{array}{c}D^{2} \\
(-)\end{array}$} & & & & & & & Test 1 & Test 2 \\
\hline & $\begin{array}{c}\mathrm{C} \\
\frac{\mathrm{CPM}}{\mathrm{CPM}}\end{array}$ & $\begin{array}{r}s_{c} \\
\frac{C P M}{C P M}\end{array}$ & $\begin{array}{c}M \\
\frac{\mathrm{CPM}}{\mathrm{CPM}}\end{array}$ & $\begin{array}{r}\mathrm{s}_{\mathrm{m}} \\
\frac{\mathrm{CPM}}{\mathrm{CPM}}\end{array}$ & $\begin{array}{l}z \\
(z)\end{array}$ & $\begin{array}{l}s_{z} \\
(\%)\end{array}$ & & & $\begin{array}{c}\mathrm{C} \\
\frac{\mathrm{Rw}}{\mathrm{Rw}} \\
\end{array}$ & $\begin{array}{l}S_{c} \\
\frac{R w}{R w}\end{array}$ & $\begin{array}{c}c \\
\frac{C P M}{C P M}\end{array}$ & $\begin{array}{l}S_{m} \\
\frac{C P M}{C P M}\end{array}$ & $\begin{array}{l}z \\
(z)\end{array}$ & $\begin{array}{l}s_{z} \\
(z)\end{array}$ & $\begin{array}{c}D=z / S_{2} \\
(-)\end{array}$ & $\begin{array}{l}D^{2} \\
(-)\end{array}$ \\
\hline Body 1 & & & & & & & & & & & & & & & & \\
\hline Mean & 0.786 & 0.023 & 0.797 & 0.021 & -1.48 & 3.81 & -0.49 & 2.5 .5 & 0.825 & & 0.792 & 0.047 & 2.99 & 2.98 & 0.21 & 41.33 \\
\hline $\begin{array}{l}\text { RMS/error/test } \\
\text { Est result/blas est1mate (2o) }\end{array}$ & 0.116 & 0.003 & 0.105 & 0.008 & $\begin{array}{l}5.54 \\
b=0\end{array}$ & 1.44 & $\begin{array}{l}1.31 \cdot 1.96 \\
\text { No dif ference }\end{array}$ & $\begin{array}{l}\cdot 2 . m \\
\text { Small difference }\end{array}$ & 0.195 & & 0.111 & 0.018 & $\begin{array}{l}14.52 \\
b=0\end{array}$ & 1.13 & $\begin{array}{l}0.57: 1.96 \\
\text { No difference }\end{array}$ & $\begin{array}{l}>2.01 \\
\text { Difference }\end{array}$ \\
\hline Body 2 & & & & & & & & & & & & & & & & \\
\hline Mean & 1.128 & 0.030 & 1.122 & 0.025 & 1.61 & 3.53 & 0.29 & 4.02 & 1.188 & & 1.125 & 0.020 & 6.92 & 3.11 & 2.42 & 18.20 \\
\hline $\begin{array}{l}\text { RMS/error/test } \\
\text { Test result/bias estimate (2o) }\end{array}$ & 0.116 & 0.011 & 0.108 & 0.009 & $\begin{array}{l}6.60 \\
b=0\end{array}$ & 1.33 & $\begin{array}{l}n .75<1.96 \\
\text { No difference }\end{array}$ & $\begin{array}{l}>2.01 \\
\text { Some difference }\end{array}$ & 0.015 & & 0.112 & 0.008 & $\begin{array}{l}8.87 \\
i=6.9\end{array}$ & .38 & $\begin{array}{l}6.40>1.96 \\
\text { some difference }\end{array}$ & $\begin{array}{l}>2.01 \\
\text { Some difference }\end{array}$ \\
\hline Body 3 & & & & & & & & & & & & & & & & \\
\hline Mean & 1.086 & 0.028 & 1.081 & 0.025 & 1.25 & 3.57 & 0.22 & 1.81 & 0.877 & & 1.084 & 0.019 & -16.92 & 2.18 & -11.03 & 178.75 \\
\hline $\begin{array}{l}\text { RMS/error/cest } \\
\text { Test result/blas estimate ( } 20 \text { ) }\end{array}$ & 0.275 & 0.011 & 0.301 & 0.003 & $\begin{array}{l}5.03 \\
\mathrm{~b}=0\end{array}$ & 1.35 & $\begin{array}{l}0.57: 1.96 \\
\text { No difference }\end{array}$ & $\begin{array}{l}-2.01 \\
\text { No difference }\end{array}$ & 0.155 & & 0.288 & 0.007 & $\begin{array}{l}8.40 \\
\hat{b}=-16 .\end{array}$ & $\begin{array}{l}0.83 \\
\pm 1.6\end{array}$ & $\begin{array}{l}29.19>1.96 \\
\text { Large dif ference }\end{array}$ & $\begin{array}{l}>>2.01 \\
\text { Large difference }\end{array}$ \\
\hline Total element grand mean & 1.000 & 0.027 & 1.000 & 0.024 & 0.46 & 3.64 & 0.007 & 2.79 & $0.963^{(a)}$ & & 1.000 & 0.018 & -2.34 & 2.79 & -2.80 & 79.42 \\
\hline $\begin{array}{l}\text { RMS/error/test } \\
\text { Test results/bias estimate (2o) }\end{array}$ & 0.240 & 0.002 & 0.242 & 0.005 & $\begin{array}{l}6.05 \\
\dot{b}=0\end{array}$ & 0.79 & $\begin{array}{l}0.01<1.96 \\
\text { No difference }\end{array}$ & $\begin{array}{l}\$ 1.55 \\
\text { No difference }\end{array}$ & 0.216 & & 0.241 & 0.004 & $\begin{array}{l}36.30 \\
b=-2.3\end{array}$ & $\begin{array}{c}0.61 \\
1.2^{(a)}\end{array}$ & $\begin{array}{l}12.8>1.96 \\
\text { Some difference }\end{array}$ & $\begin{array}{l}\gg>1.55 \\
\text { Large difference }\end{array}$ \\
\hline
\end{tabular}

(a) $\hat{b}$ because of lack of uniform sampling; normalization to 1.000 w111 give $\hat{b}=0$. 
TABLE 4-8

MEASURED EOL POWER PROFILES FOR STACK 1 FUEL RODS

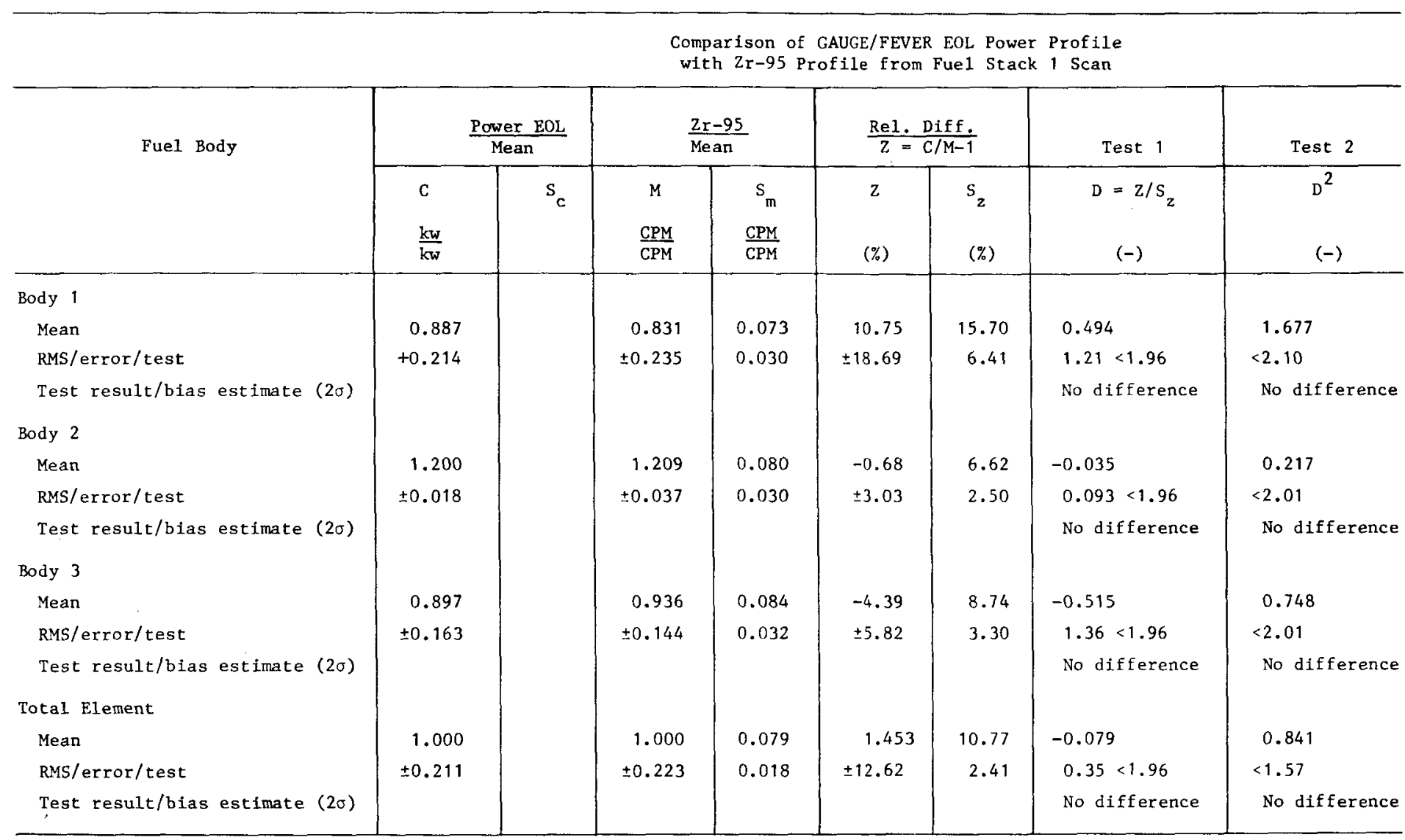


FUEL GAMPA SCAN COKPARISON OF TABLE $4-9$

TABLE $4-9$
C $-137 / 2 r-95$ PROFILES FOR CESIUM MOBILITY

\begin{tabular}{|c|c|c|c|c|c|c|c|c|c|c|c|c|c|c|c|c|}
\hline \multirow{4}{*}{ Puel Body } & \multicolumn{8}{|c|}{ Peach Bottom Nuc11de CPM Rat10 Compar1son for Cesium Mobility } & \multicolumn{8}{|c|}{ Fuel Rod Nuc11de CPM Rat1o Comparison for Cestum Mobility } \\
\hline & \multirow{2}{*}{\multicolumn{2}{|c|}{$\frac{\left(\frac{C a-137}{\mathrm{Zr}-95}\right)_{\text {P.A. }}}{}$}} & \multirow{2}{*}{\multicolumn{2}{|c|}{$\left(\frac{c_{s-137}}{2 r-95}\right)_{\text {unfalled }}$}} & \multirow{2}{*}{\multicolumn{2}{|c|}{$\frac{\text { Rel. Diff. }}{Z=C / M-1}$}} & \multicolumn{2}{|c|}{ Comparison } & \multirow{2}{*}{\multicolumn{2}{|c|}{$\left(\frac{C_{8-137}}{2 r-95}\right)_{I}$}} & \multirow{2}{*}{\multicolumn{2}{|c|}{$\left(\frac{C_{B}-137}{2 r-95}\right)_{\text {unf alled }}$}} & \multirow{2}{*}{\multicolumn{2}{|c|}{$\frac{\text { Re1. Diff }}{2-\cos }$}} & \multicolumn{2}{|c|}{ Comparison } \\
\hline & & & & & & & \multirow{2}{*}{$\begin{array}{c}\text { Test } 1 \\
D=2 / S_{2} \\
(-)\end{array}$} & \multirow{2}{*}{$\begin{array}{c}\text { Test } 2 \\
D^{2} \\
(-)\end{array}$} & & & & & & & \multirow{2}{*}{$\begin{array}{c}\text { Test } 1 \\
D=z / S z \\
(-)\end{array}$} & \multirow{2}{*}{$\begin{array}{c}\text { Test } 2 \\
\mathrm{D}^{2} \\
(-)\end{array}$} \\
\hline & $\begin{array}{c}\mathrm{C} \\
\mathrm{CPM} \\
\mathrm{CPM}\end{array}$ & $s_{c}$ & $\begin{array}{c}\mathrm{M} \\
\mathrm{CPM} \\
\mathrm{CPM} \\
\end{array}$ & $s_{\mathrm{m}}$ & $\begin{array}{l}z \\
(z) \\
\end{array}$ & $\begin{array}{l}s_{z} \\
(x) \\
\end{array}$ & & & $\begin{array}{c}c \\
\frac{C P M}{C P M}\end{array}$ & $\begin{array}{l}s_{c} \\
\frac{c P M}{C P M}\end{array}$ & $\frac{M}{C P M}$ & $\begin{array}{l}s_{\mathrm{w}} \\
\frac{\mathrm{cms}}{\mathrm{CPM}}\end{array}$ & (z) & $\begin{array}{l}s_{z} \\
(z)\end{array}$ & & \\
\hline Body 1 & & & & & & & & & & & & & & & & \\
\hline Mean & 0.1124 & 0.0194 & 0.1142 & 0.0080 & -1.59 & 13.26 & -0.22 & 0.93 & 0.1144 & 0.0079 & 0.1142 & 0.0080 & 0.19 & 8.12 & -0.04 & 1.32 \\
\hline RMS/error/test & 0.0142 & 0.0039 & 0.1142 & 0.0080 & 12.40 & 5.41 & $0.54<1.96$ & $<2.10$ & 0.0099 & 0.0035 & 0.1142 & 0.0080 & 8.69 & 3.63 & $0.09<1.96$ & $<2.21$ \\
\hline $\begin{array}{l}\text { Test result/bias eat1mate }(20) \\
\text { Body } 2\end{array}$ & \multicolumn{2}{|c|}{$\begin{array}{r}1.13<2.10 \text { No dif- } \\
\text { ference }\end{array}$} & & & $\hat{b}=0$ & & No difference & No difference & \multicolumn{2}{|c|}{$\begin{array}{r}1.88<2.10 \text { No Dif- } \\
\text { ference }\end{array}$} & 0.1142 & 0.0080 & $\hat{b}=0$ & & No $d \mathbf{1 f}$ ference & No difference \\
\hline Mean & 0.0966 & 0.0124 & 0.1142 & 0.0080 & -15.42 & 11.83 & -1.95 & 10.37 & 0.0934 & 0.0063 & 0.1142 & 0.0080 & -18.20 & 5.92 & -3.26 & 12.63 \\
\hline RMS/error/test & 0.0279 & 0.0047 & 0.1142 & 0.0080 & 24.44 & 4.47 & $5.16>1.96$ & $>2.01$ & 0.0074 & 0.0024 & 0.1142 & 0.0080 & 6.43 & 2.24 & $8.63>1.96$ & $>2.01$ \\
\hline $\begin{array}{l}\text { Test result/blas est1mate (20) } \\
\text { Body } 3\end{array}$ & \multicolumn{2}{|c|}{$\begin{array}{c}5.91>2.10 \text { Differ- } \\
\text { ence }\end{array}$} & 0.1142 & 0.0080 & \multicolumn{2}{|c|}{$\hat{b}=15.4 \pm 8.8$} & Difference & Difference & \multicolumn{2}{|c|}{$\begin{array}{r}1.51<2.10 \text { No D1f- } \\
\text { ference }\end{array}$} & 0.1142 & 0.0080 & \multicolumn{2}{|c|}{$\hat{b}=-18.2 \pm 4.4$} & Dif ference & DIfference \\
\hline Mean & 0.0903 & 0.128 & 0.1142 & 0.0080 & -20.83 & 11.67 & -1.90 & 4.93 & 0.906 & 0.0085 & 0.1142 & 0.0080 & -20.72 & 7.72 & -1.22 & 12.93 \\
\hline PAs/error/Lest. & 0.0108 & 0.0048 & 0.1142 & 0.0080 & 9.45 & 4.41 & $5.02>1.96$ & $>2.01$ & 0.0097 & 0.0032 & 0.1142 & 0.0080 & 8.48 & 2.92 & $3.23>1.96$ & 22.01 \\
\hline $\begin{array}{l}\text { Test result/blas est1mate (2o) } \\
\text { Total Element }\end{array}$ & \multicolumn{2}{|c|}{$\begin{array}{c}0.83<2.10 \text { Differ- } \\
\text { ence }\end{array}$} & 0.1142 & 0.0080 & \multicolumn{2}{|c|}{$\hat{b}=20.9 \pm 8.6$} & Difference & Dif ference & \multicolumn{2}{|c|}{$\begin{array}{r}1.52<2.10 \text { No Dif- } \\
\text { ference }\end{array}$} & 0.1142 & 0.0080 & \multicolumn{2}{|c|}{$\hat{b}=20.7 \pm 5.7$} & D1fference & Difference \\
\hline mean & 0.0991 & 0.0132 & 0.1142 & 0.0189 & -13.20 & 12.22 & -1.41 & 5.41 & 0.0979 & 0.0076 & 0.1142 & 0.0189 & -14.29 & 7.23 & -1.66 & 9.76 \\
\hline RMS/error/test & 0.0214 & 0.0030 & 0.0080 & 0.0030 & 18.70 & 2.73 & $6.32>1.96$ & $>1.57$ & 0.0134 & 0.0017 & 0.0080 & 0.0080 & 11.73 & 1.66 & $7.24>1.96$ & $>1.57$ \\
\hline Yest result/blas estimate $(2 \sigma)$ & \multicolumn{2}{|c|}{$\begin{array}{c}2.76>1.57 \begin{array}{c}\text { Differ- } \\
\text { ence }\end{array} \\
.\end{array}$} & & & \multicolumn{2}{|c|}{$\hat{b}=-13.2 \pm 5.4$} & Difference & Difference & $3.27>1.5$ & $\begin{array}{l}\text { Differ- } \\
\text { ence }\end{array}$ & $0.18<1.36$ & $\begin{array}{l}\text { No Differ- } \\
\text { ence }\end{array}$ & \multicolumn{2}{|c|}{$\hat{b}=-14.3 \pm 3.2$} & Dif ference & Difference \\
\hline
\end{tabular}


TABLE 4-10

FTE-6 FUEL GAMRA SCAN COMPARISON OF Cs-137/2r-95 PROFILES FROM TWO DIFFERENT SCANNING METHODS

\begin{tabular}{|c|c|c|c|c|c|c|c|c|}
\hline \multirow[t]{4}{*}{ Fue1 Body } & \multicolumn{8}{|c|}{$\begin{array}{l}\text { Nuclide CPM Ratio Comparison from Continuous Scans at } \\
\text { Peach Bottom and Fuel Rod Scans (III) }\end{array}$} \\
\hline & \multirow{2}{*}{\multicolumn{2}{|c|}{$\left(\frac{\mathrm{Cs}-137}{2 r-95}\right)_{\text {P.B. }}$}} & \multirow{2}{*}{\multicolumn{2}{|c|}{$\left(\frac{\operatorname{Cs}-137}{Z_{r}-95}\right)_{I}$}} & \multirow{2}{*}{\multicolumn{2}{|c|}{$\begin{aligned} \text { Re1. Diff. } & \text { D } \\
Z & =\text { CM-1 }\end{aligned}$}} & \multicolumn{2}{|c|}{ Comparison } \\
\hline & & & & & & & \multirow{2}{*}{$\begin{array}{c}\text { Test } 1 \\
=z / S_{z} \\
(-)\end{array}$} & \multirow{2}{*}{$\frac{\text { Test } 2}{\mathrm{D}^{2}}$} \\
\hline & $\begin{array}{c}\mathrm{C} \\
\frac{\mathrm{CPM}}{\mathrm{CPM}}\end{array}$ & $\begin{array}{l}s_{c} \\
\frac{C P M}{C P M}\end{array}$ & $\begin{array}{c}\mathrm{M} \\
\frac{\mathrm{CPM}}{\mathrm{CPM}}\end{array}$ & $\begin{array}{l}\mathrm{s}_{\mathrm{m}} \\
\frac{\mathrm{CPM}}{\mathrm{CPM}}\end{array}$ & $\begin{array}{c}z \\
(\%)\end{array}$ & $\begin{array}{l}s_{z} \\
(\%)\end{array}$ & & \\
\hline \multicolumn{9}{|l|}{ Body 1} \\
\hline Mean & 0.1107 & 0.0194 & 0.1144 & 0.0288 & -2.62 & 14.88 & -0.29 & 0.68 \\
\hline RMS/error/test & 0.0099 & 0.0064 & 0.0099 & 0.0039 & 11.93 & 6.65 & $0.65<1.96$ & $<2.21$ \\
\hline $\begin{array}{l}\text { Test result/bias estimate }(2 \sigma) \\
\text { Body } 2\end{array}$ & \multicolumn{2}{|c|}{ No $d i f f e r e n c e$} & \multicolumn{2}{|c|}{$1.52<2.21$ No difference } & \multicolumn{2}{|c|}{ No difference } & No difference & No difference \\
\hline Mean & 0.0966 & 0.0124 & 0.0934 & 0.0063 & 2.94 & 15.82 & -0.25 & 3.55 \\
\hline RMS/error/test & 0.0279 & 0.0047 & 0.0074 & 0.0034 & 27.78 & 5.98 & $0.65<1.96$ & $>2.01$ \\
\hline $\begin{array}{l}\text { Test result/bias estimate }(2 \sigma) \\
\text { Body } 3\end{array}$ & \multicolumn{2}{|c|}{$5.91>2.10$ Difference } & \multicolumn{2}{|c|}{$1.51<2.10$ No difference } & \multicolumn{2}{|c|}{$3.60>2.12$ Difference } & No difference & Difference \\
\hline Mean & 0.0903 & 0.0128 & 0.0906 & 0.0085 & 0.33 & 17.17 & -0.07 & 0.60 \\
\hline RMS/error/test & 0.0108 & 0.0048 & 0.0097 & 0.0032 & 13.64 & 6.49 & $0.18<1.96$ & $<2.01$ \\
\hline $\begin{array}{l}\text { Test result/bias estimate }(2 \sigma) \\
\text { Total Element }\end{array}$ & \multicolumn{2}{|c|}{ No difference } & \multicolumn{2}{|c|}{$1.52<2.10$ Ho difference } & \multicolumn{2}{|c|}{ No difference } & No difference & No difference \\
\hline Mean & 0.0980 & 0.0135 & 0.0979 & 0.0078 & 0.51 & 16.10 & -0.19 & 1.71 \\
\hline RMS/error/test & 0.0205 & 0.0031 & 0.0134 & 0.0018 & 19.88 & 3.69 & $0.84<1.96$ & $>1.57$ \\
\hline Test result/bias estimate $(2 \sigma)$ & \multicolumn{2}{|c|}{$2.42>1.57$ Difference } & \multicolumn{2}{|c|}{$3.11>1.59$ Difference } & \multicolumn{2}{|c|}{$1.60>1.59$ Difference } & No difference & Small difference \\
\hline
\end{tabular}




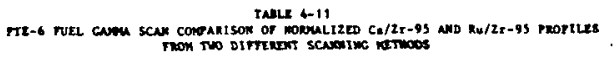

\begin{tabular}{|c|c|c|c|c|c|c|c|c|c|c|c|c|c|c|c|c|}
\hline \multirow{4}{*}{ 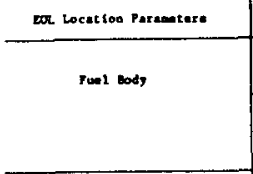 } & \multicolumn{8}{|c|}{ 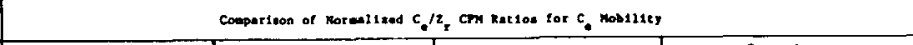 } & \multicolumn{8}{|c|}{ 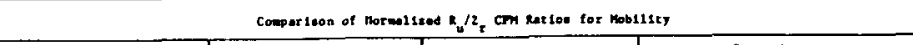 } \\
\hline & \multirow{2}{*}{\multicolumn{2}{|c|}{ 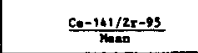 }} & \multirow{2}{*}{\multicolumn{2}{|c|}{$\frac{6=-1,4 / 2 x-95}{\operatorname{lngas}}$}} & \multirow{2}{*}{\multicolumn{2}{|c|}{$\begin{array}{c}\text { nel1: } \\
2\end{array}$}} & \multicolumn{2}{|c|}{$\cos a x+1000$} & \multirow{2}{*}{\multicolumn{2}{|c|}{$\frac{20-103 / 2 x-12}{\operatorname{mos}}$}} & \multirow{2}{*}{\multicolumn{2}{|c|}{ 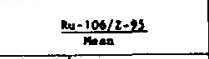 }} & \multirow{2}{*}{\multicolumn{2}{|c|}{$\begin{array}{l}\text { noll } \\
\text { Z }\end{array}$}} & \multicolumn{2}{|c|}{ cologertions } \\
\hline & & & & & & & \multirow{2}{*}{$\begin{array}{c}2.0+1 \\
0-2 / s \\
(-1\end{array}$} & \multirow{2}{*}{$\begin{array}{r}\frac{\text { Tate }^{2}}{D^{2}} \\
(-1 \\
\end{array}$} & & & & & & & \multirow{2}{*}{$\begin{array}{c}\text { reas } 1 \\
0=z / s_{R} \\
(-)\end{array}$} & \multirow{2}{*}{$\begin{array}{l}\operatorname{tane} 2 \\
0^{2} \\
\leftrightarrow\end{array}$} \\
\hline & $\begin{array}{c}c \\
\mathrm{~cm} \\
\mathrm{~cm} \\
\mathrm{~cm}\end{array}$ & 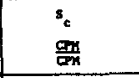 & $\frac{\mathrm{cm}}{\mathrm{cm}}$ & $\begin{array}{l}s_{0} \\
\frac{\mathrm{cm}}{\mathrm{d} m}\end{array}$ & $\begin{array}{c}\mathbf{z} \\
(\boldsymbol{x})\end{array}$ & $\begin{array}{l}38 \\
\text { (x) }\end{array}$ & & & $\begin{array}{c}\mathrm{c} \\
\mathrm{cm} \\
\mathrm{cm}\end{array}$ & $\begin{array}{l}s_{c} \\
\frac{c m}{c m}\end{array}$ & $\begin{array}{c}n \\
\frac{\mathrm{cm}}{\mathrm{cm}}\end{array}$ & s. & $\begin{array}{c}2 \\
\text { (3) } \\
\end{array}$ & $\begin{array}{l}\text { s: } \\
\text { (x) }\end{array}$ & & \\
\hline 2001 & & & & & & & & & & & & & & & & \\
\hline 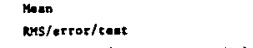 & $\left|\begin{array}{l}1.134 \\
0.081\end{array}\right|$ & $\begin{array}{l}0.087 \\
0.036\end{array}$ & $\begin{array}{l}1 \cdots 11 \\
0.278\end{array}$ & $\begin{array}{l}0.201 \\
0.082\end{array}$ & $\begin{array}{r}7.33 \\
20.53\end{array}$ & $\begin{array}{r}13.26 \\
6.83\end{array}$ & $\begin{array}{l}0.306 \\
1.24\end{array}=1.96$ & \begin{tabular}{|l}
1.630 \\
.2 .10
\end{tabular} & $\begin{array}{l}1.070 \\
0.059\end{array}$ & $\begin{array}{l}0.033 \\
0.023\end{array}$ & $0.2 m$ & $\begin{array}{ll}0.181 \\
0.014\end{array}$ & 22.60 & $\begin{array}{l}10.02 \\
3.9\end{array}$ & $0.963 \times 1.96$ & $=2.10$ \\
\hline Teex result/ & $0.99<2$ & .01 Ho Differsence & 2.3032 & $\begin{array}{l}21 \text { sant1 } \\
\text { diffreance. }\end{array}$ & 2.18 & 21 No ditserence & No ditlerence & No ditference & & no difference & $2.21 \cdot 2$ & 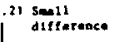 & $2.94 \cdot 2$ & 21 Differences & tho difterence & 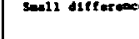 \\
\hline $\begin{array}{c}\text { sody } 2 \\
\text { nean }\end{array}$ & 1.002 & 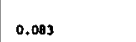 & 0.980 & 0.098 & 3.97 & 13.81 & 0.136 & & 0.946 & & 1.0 & & -3.327 & 10.43 & -0.600 & 1.024 \\
\hline 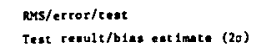 & $\mid \begin{array}{l}0.133 \\
3.00,2 .\end{array}$ & $\begin{array}{l}0.031 \\
10 \text { Ditserence }\end{array}$ & $\begin{array}{l}0.022 \\
0.21<2\end{array}$ & $\begin{array}{l}0.037 \\
10 \text { No difference }\end{array}$ & $\begin{array}{l}15.96 \\
1.36 .2\end{array}$ & $\begin{array}{l}\text { S.22 } \\
.10 \text { No difference }\end{array}$ & $\begin{array}{l}0.36010 .96 \\
\text { No ditterences }\end{array}$ & \begin{tabular}{|l}
-2.01 \\
No ditterences
\end{tabular} & $\begin{array}{l}0.035 \\
1.57 .2\end{array}$ & $\begin{array}{l}0.018 \\
10 \text { :o Difference. }\end{array}$ & $\begin{array}{l}0.039 \\
0.19 \cdot 2\end{array}$ & $\begin{array}{l}0.037 \\
\text {, } 010 \text { Ho dif torence }\end{array}$ & $\begin{array}{ll}8.02 \\
0.69 & <2\end{array}$ & $\begin{array}{l}30.06 \\
10 \text { Ho Alf terence }\end{array}$ & No difterences & no ostrerence \\
\hline $\begin{array}{c}\text { Dody } 3 \\
\text { Mean } \\
\text { sons }\end{array}$ & 0.848 & 0.082 & 0.931 & 0.10 & -6.90 & $\begin{array}{c}15.15 \\
5.13\end{array}$ & $\begin{array}{l}-1.108 \\
3.143 \times 1.96\end{array}$ & $\begin{array}{l}3.607 \\
2.01\end{array}$ & $\left|\begin{array}{l}0.980 \\
0.101\end{array}\right|$ & $\begin{array}{l}0.069 \\
0.018\end{array}$ & $\begin{array}{l}0.963 \\
0.056\end{array}$ & $\begin{array}{l}0.102 \\
0.039\end{array}$ & \begin{tabular}{|c|c|}
2.373 \\
14.20
\end{tabular} & $\begin{array}{l}12.33 \\
.1 .66\end{array}$ & $\begin{array}{l}-0.062 \\
0.111 \times 1.96\end{array}$ & \begin{tabular}{|l}
1.203 \\
$<.01$ \\
$<2.01$
\end{tabular} \\
\hline 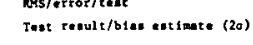 & $\left|\begin{array}{l}0.170 \\
5.01>2\end{array}\right|$ & $\begin{array}{l}0.031 \\
\text { 2. } 10 \text { D15ference }\end{array}$ & $\begin{array}{l}.0 .079 \\
0.79>2\end{array}$ & io No difterence & $3.65>2$. & 10 ditference & Difference & wat ferences & $4.96 \times 2$. & 10 difterence & 0.33 .2 & ierence. & 1.55 & & Ho stifterence & No sifteresces \\
\hline Total E1emont & & & & & & & & & & & & & & & & \\
\hline ens & $\begin{array}{l}1.008 \\
0.182\end{array}$ & $\mid \begin{array}{l}0.064 \\
0.019\end{array}$ & $\begin{array}{l}0.097 \\
0.1 m\end{array}$ & $\mid \begin{array}{l}0.393 \\
0.031\end{array}$ & $\begin{array}{l}1.173 \\
22.63\end{array}$ & \begin{tabular}{|l|l}
16.73 \\
3.29
\end{tabular} & $\begin{array}{r}-0.216 \\
0.966 \cdot 1.96\end{array}$ & $\mid \begin{array}{l}2.620 \\
1.57\end{array}$ & $\begin{array}{l}1.001 \\
0.091\end{array}$ & $\mid \begin{array}{l}0.058 \\
0.013\end{array}$ & $\begin{array}{l}0.6 \\
0.16 n\end{array}$ & $\left|\begin{array}{l}0.150 \\
0.029\end{array}\right|$ & \begin{tabular}{|l}
1.107 \\
16.61
\end{tabular} & & $0.286 \times 1.16$ & \\
\hline & 4.92 & .36 difference. & 1.11 & ou no difference & 2.65 & a3 Difference & No difference & \begin{tabular}{|l|} 
Difference \\
\end{tabular} & $2.5 \mathrm{~A}$ & erence & & ence & & & & areasen \\
\hline
\end{tabular}


TABLE 4-12
FTE-6 FUEL GAMMA SCAN COMPARI SON

FOR STACK 3 RED THERMAL, FLUENCE PROFILES

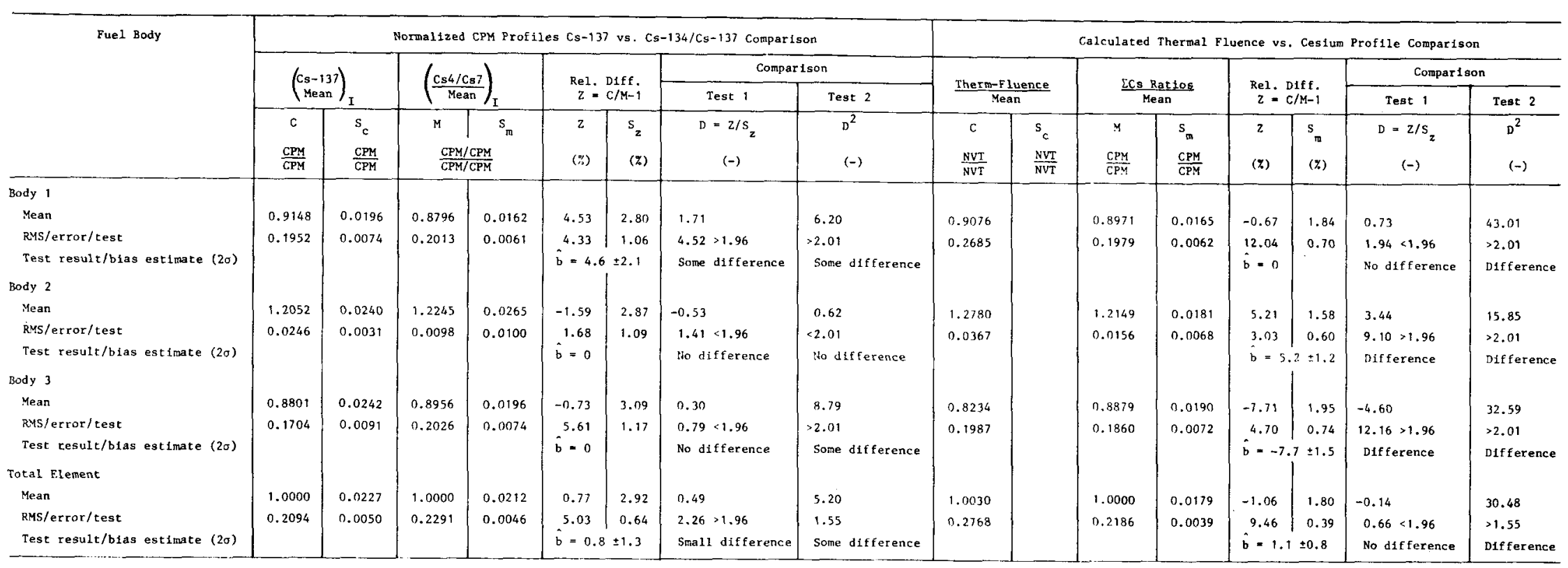


TABLE $4-13$ FTE-6 COMPARISON OF ACCUMULATIVE AND COMPOSITE FUEL STACK LENGTHS

DERIVED FROM METROLOCY AND GMMMA SCANIINTA

\begin{tabular}{|c|c|c|c|c|c|c|c|c|c|c|c|c|c|}
\hline \multirow[t]{2}{*}{ Fuel Body } & \multicolumn{3}{|c|}{ Accumulative Fuel stack Length ${ }^{(a)}$} & \multicolumn{3}{|c|}{ Composite Fuel Stack Length } & \multicolumn{2}{|c|}{ Absolute Difference } & \multicolumn{2}{|c|}{ Relative Difference } & \multirow{2}{*}{$\begin{array}{c}\text { Test } 1 \\
D=z / s_{z} \\
--\end{array}$} & \multirow{2}{*}{$\frac{\text { Test } 2}{\mathrm{D}^{2}}$} & \multirow[t]{2}{*}{ Test Results } \\
\hline & $\begin{array}{l}\bar{x}_{1} \\
\text { (in.) }\end{array}$ & $\begin{array}{c}\varepsilon x_{1} \\
(\ln .)\end{array}$ & $\begin{array}{l}E_{\bar{x}_{1}} \\
(\mathrm{in})\end{array}$ & $\begin{array}{l}x_{2} \\
(1 \mathrm{n})\end{array}$ & $\begin{array}{l}\varepsilon_{\bar{x}_{2}} \\
(\text { in.) }\end{array}$ & $\begin{array}{l}\varepsilon_{\bar{x}_{2}} \\
\left(\ln _{2}\right)\end{array}$ & $\begin{aligned} \mathrm{d}= & \overline{\mathrm{x}}_{2}-\bar{x}_{1} \\
& (\text { in. })\end{aligned}$ & $\begin{array}{c}s_{d} \\
\left(1 n_{0}\right)\end{array}$ & $\begin{array}{c}z=x_{2} / x_{1}-1 \\
(x)\end{array}$ & $\begin{array}{l}s_{z} \\
(z)\end{array}$ & & & \\
\hline BOL & & & & & & & & & & & & & \\
\hline 1 & 27.2849 & & 0.0127 & 27.1925 & & 0.0085 & 0.0924 & 0.0153 & 0.34 & 0.06 & 6.04 & 36.48 & significant \\
\hline 2 & 27.2842 & & 0.0130 & 27.2028 & & 0.0101 & 0.0814 & 0.0165 & 0.30 & 0.06 & 4.94 & 24.39 & significant \\
\hline 3 & 27.2857 & & 0.0171 & 27.2039 & & 0.0136 & 0.0818 & 0.0218 & 0.30 & 0.08 & 3.74 & 13.98 & significant \\
\hline Total element & 27.2850 & & 0.0099 & 27.1997 & & 0.0092 & 0.0853 & 0.0135 & 0.31 & 0.05 & 6.30 & 39.72 & significant \\
\hline $1,2,63$ mean & & & & & & & & & & & $4.91=1.96$ & $24.95=2.60$ & \\
\hline $\begin{array}{l}\text { Test results } \\
\text { EOL }\end{array}$ & & & & & & & $b=0.0061 \pm 0.0019$ & u) per rod & & & signif icant & significant & \\
\hline 1 & 26.8187 & & 0.0272 & 26.7653 & & 0.0379 & 0.0534 & 0.0467 & 0.20 & 0.17 & 1.14 & 1.31 & not significant \\
\hline 2 & 26.6866 & & 0.0281 & 26.6527 & & 0.0418 & 0.0339 & 0.0504 & 0.13 & 0.19 & 0.67 & 0.45 & not significant \\
\hline 3 & 26.6701 & & 0.0301 & 26.6349 & & 0.0433 & 0.0352 & 0.0527 & 0.13 & 0.20 & 0.67 & 0.44 & not significant \\
\hline Tutal elemenc & 26.7252 & & 0.0232 & 26.6843 & & 0.0257 & 0.0409 & 0.0346 & 0.15 & 0.13 & 1.18 & 1. 39 & not significant \\
\hline $1,2,83$ mean & & & & & & & & & & & $0.83 \div 1.96$ & $0.73<2.60$ & \\
\hline Test results & & & & & & & $\hat{b}=0.0029: 0.0048$ & c) per rod & & & not significant & nut significant & \\
\hline EOL & Compos & Fuel st & Length & Peach & ot tom o & scan & & & & & & & \\
\hline 1 & 26.7653 & 0.1070 & & 26.6678 & & $0.1296^{(6)}$ & 0.0975 & 0.1681 & 0.37 & 0.63 & 0.58 & 0.33 & not significant \\
\hline 2 & 26.6527 & 0.1181 & & 26.7290 & & $0.1092^{\text {(b) }}$ & -0.0763 & 0.1608 & -0.29 & 0.60 & -0.48 & 0.22 & not significant \\
\hline 3 & 26.6349 & 0.1224 & & 26.6546 & & $0.0874^{(b)}$ & -0.0197 & 0.1504 & -0.07 & 0.56 & -0.13 & 0.02 & not significant \\
\hline Total element & 26.6843 & 0.1256 & & 26.6838 & & 0.0562 & 0.0005 & 0.1376 & $<0.01$ & 0.52 & $<0.01$ & $<0.01$ & not significant \\
\hline $\begin{array}{l}1,2,83 \text { mean } \\
\text { Test resultas }\end{array}$ & & & & & & & & & & & $\begin{array}{c}-0.01 \\
\text { not s1gnificant }\end{array}$ & $\begin{array}{c}0.19 \times 2.60 \\
\text { not significant }\end{array}$ & \\
\hline
\end{tabular}

(a) Average standard deviation for fuel rod length $\bar{s}_{1}=1.347$ mil (see table $s_{-2}$ )

Average standard deviation for accumulative fuel stack $\bar{s}_{j}=\sqrt{14} \cdot \bar{s}_{1}=5.040 \mathrm{mil}$

(b) $s_{\bar{x}}$ from three single-channel analyzer strip charts (see Fíg, 4-5). 
TABLE 4-14

FTE-6 THERMOCOUPLE RESISTANCE DATA

\begin{tabular}{|c|c|c|c|c|c|c|}
\hline \multirow[b]{2}{*}{$\begin{array}{l}\text { Thermocouple Type } \\
\text { (Accuracy: } \pm 0.2 \Omega \text { ) }\end{array}$} & \multicolumn{2}{|c|}{$\begin{array}{l}\text { + to Ground } \\
\text { Circuit } \\
(\Omega)\end{array}$} & \multicolumn{2}{|c|}{$\begin{array}{l}\text { - to Ground } \\
\text { Circuit } \\
(\Omega)\end{array}$} & \multicolumn{2}{|c|}{ Loop $(\Omega)$} \\
\hline & $\begin{array}{l}\text { Pre- } \\
\text { Irrad. }\end{array}$ & $\begin{array}{l}\text { Post- } \\
\text { Irrad. }\end{array}$ & $\begin{array}{l}\text { Pre- } \\
\text { Irrad. }\end{array}$ & $\begin{array}{l}\text { Post- } \\
\text { Irrad. }\end{array}$ & $\begin{array}{l}\text { Pre- } \\
\text { Irrad. }\end{array}$ & $\begin{array}{l}\text { Post- } \\
\text { Irrad. }\end{array}$ \\
\hline $\begin{array}{l}\text { Thermocouple A } \\
\mathrm{W}-3 \% \operatorname{Re} / \mathrm{W}-25 \% \operatorname{Re}\end{array}$ & $\mathrm{NA}^{(a)}$ & 6 & NA & 15 & NA & 20 \\
\hline $\begin{array}{l}\text { Thermocouple B } \\
\text { C/A }\end{array}$ & $\mathrm{NA}$ & 35 & NA & 15 & NA & 50 \\
\hline
\end{tabular}

(a) $\mathrm{NA}=$ not available. 
TABLE $4-15$
FTE-6 DISTANCE d - TOP OF SPINE SAMPLE TO EDGE OF HOLE

\begin{tabular}{|c|c|c|c|c|c|c|}
\hline $\begin{array}{l}\text { Body } \\
\text { No. }\end{array}$ & $\begin{array}{l}\text { Spine Stack } \\
\text { Length, L } \\
\text { (in.) }\end{array}$ & $\begin{array}{c}\text { Pre-Irrad. } \\
\mathrm{d}_{1} \\
\text { (in.) }\end{array}$ & $\begin{array}{c}\text { Post-Irrad. } \\
d_{2} \\
\text { (in.) }\end{array}$ & $\begin{array}{c}\Delta \mathrm{d} \\
(\mathrm{in} .)\end{array}$ & $\begin{array}{l}\Delta G^{(b)} \\
\text { (in.) }\end{array}$ & 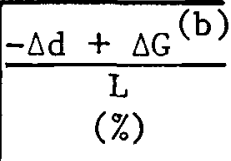 \\
\hline 1 & 28.530 & 2.081 & 2.356 & 0.275 & -0.029 & -1.065 \\
\hline 2 & 28.480 & 2.063 & 2.245 & 0.182 & -0.2235 & -1.424 \\
\hline 3 & 28.550 & 2.073 & 2.795 & 0.722 & -0.1635 & -3.101 \\
\hline
\end{tabular}

(a) Accuracy: \pm 0.001 in.

(b) $\Delta G \equiv$ length of graphite fuel body. 
TABLE 4-16

FTE-6 FUEL STACK LENGTH

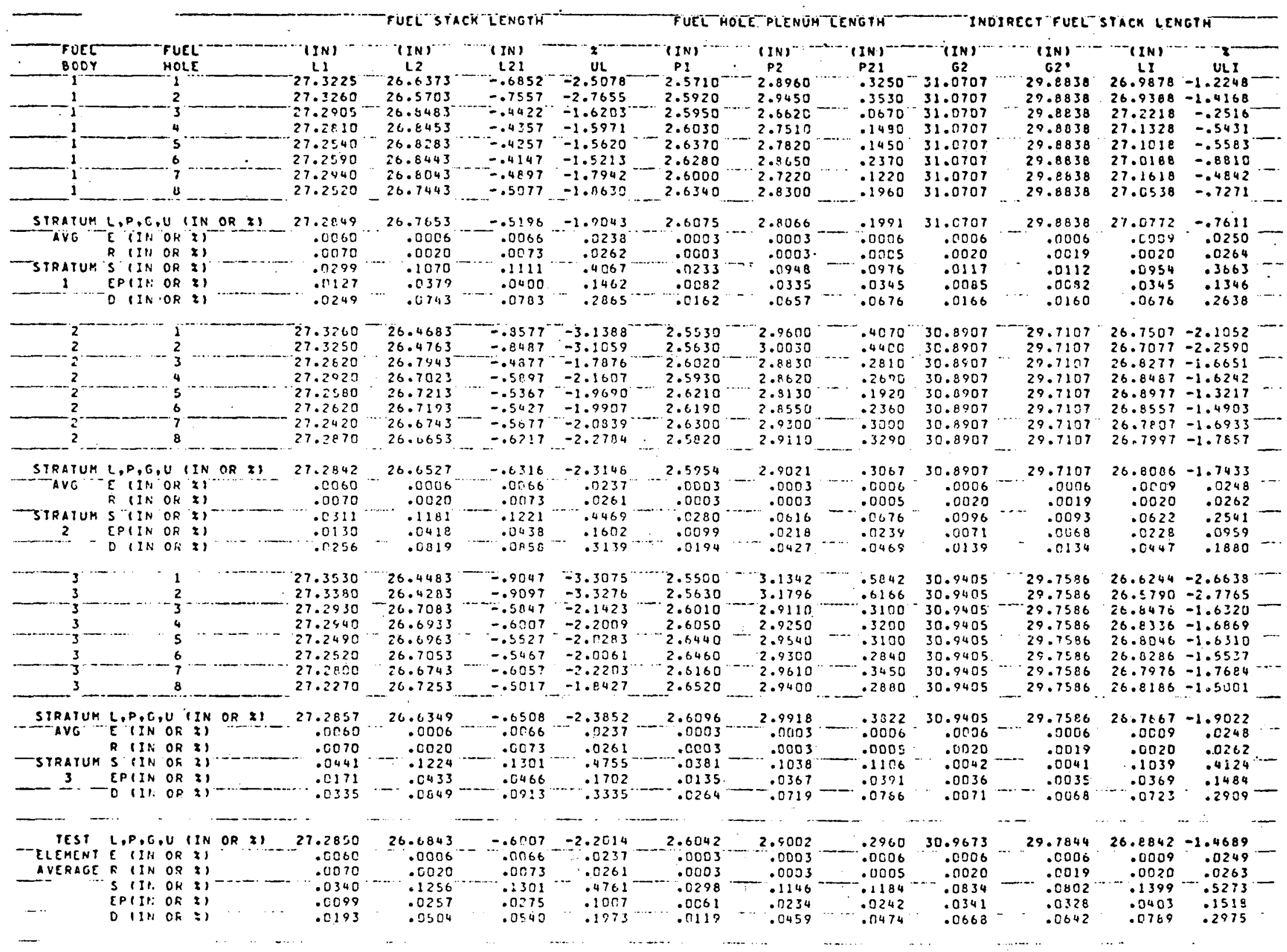


TABLE $\quad 4-17$

FTE-6 FUEL ROD REMOVAL SUMMARY

\begin{tabular}{|c|c|c|c|c|}
\hline \multirow[b]{2}{*}{ Body } & \multirow[b]{2}{*}{ Hole } & \multicolumn{2}{|c|}{ Force (1b) } & \multirow[b]{2}{*}{ Visual Examination } \\
\hline & & Initial & Sustaining & \\
\hline \multirow[t]{8}{*}{1} & 1 & 5 & 0 & $\begin{array}{l}\text { Several rods had cracked end caps and } \\
\text { surface cracks. }\end{array}$ \\
\hline & 2 & 30 & 0 & $\begin{array}{l}\text { Several rods had cracked end caps and } \\
\text { surface cracks. }\end{array}$ \\
\hline & 3 & 25 & 10 & $\begin{array}{l}\text { About half of the rods had cracked end } \\
\text { caps. }\end{array}$ \\
\hline & 4 & 15 & 10 & Three rods had cracked end caps. \\
\hline & 5 & 10 & $<10$ & Two rods had cracked end caps. \\
\hline & 6 & 10 & $<10$ & $\begin{array}{l}\text { Five rods had cracked end caps and surface } \\
\text { cracks. }\end{array}$ \\
\hline & 7 & 12 & $<12$ & Good general condition. \\
\hline & 8 & 10 & $<10$ & $\begin{array}{l}\text { Several rods had cracked end caps and } \\
\text { surface cracks. }\end{array}$ \\
\hline \multirow[t]{8}{*}{2} & 1 & 25 & 25 & $\begin{array}{l}\text { Majority of rods had cracked end caps and } \\
\text { surface cracks. }\end{array}$ \\
\hline & 2 & 30 & 5 & $\begin{array}{l}\text { Majority of rods had cracked end caps and } \\
\text { surface cracks. }\end{array}$ \\
\hline & 3 & 25 & 0 & $\begin{array}{l}\text { Several rods at top end of body had sur- } \\
\text { face cracks. }\end{array}$ \\
\hline & 4 & 20 & 0 & $\begin{array}{l}\text { Several rods at top end of body had sur- } \\
\text { face cracks. }\end{array}$ \\
\hline & 5 & 25 & 15 & $\begin{array}{l}\text { Several rods had cracked end caps and } \\
\text { surface cracks. }\end{array}$ \\
\hline & 6 & 10 & 5 & Several rods had cracked end caps. \\
\hline & 7 & 20 & 10 & $\begin{array}{l}\text { Several rods were broken in half and had } \\
\text { cracked end caps. }\end{array}$ \\
\hline & 8 & 30 & 10 & Several rods had cracked end caps. \\
\hline \multirow[t]{8}{*}{3} & 1 & 15 & 5 & $\begin{array}{l}\text { Several rods had cracked end caps and } \\
\text { exhibited bowing. }\end{array}$ \\
\hline & 2 & 25 & 15 & $\begin{array}{l}\text { Several rods had cracked end caps and } \\
\text { exhibited bowing at lower end of body. }\end{array}$ \\
\hline & 3 & 40 & 30 & Majority of rods had cracked end caps. \\
\hline & 4 & 50 & 30 & $\begin{array}{l}\text { Rods at lower end of body had cracked end } \\
\text { caps. }\end{array}$ \\
\hline & 5 & 35 & 20 & $\begin{array}{l}\text { Rods at lower end of body had cracked end } \\
\text { caps. }\end{array}$ \\
\hline & 6 & 0 & 0 & $\begin{array}{l}\text { Rods at lower end of body had cracked end } \\
\text { caps. }\end{array}$ \\
\hline & 7 & 25 & 10 & $\begin{array}{l}\text { Rods at lower end of body had cracked end } \\
\text { caps. }\end{array}$ \\
\hline & 8 & 10 & 5 & Rods at lower end were cracked in half. \\
\hline
\end{tabular}


TABLE 4-18

FTE-6 TOTAL ELEMENT LENGTH AND FUEL BODY WEIGHT

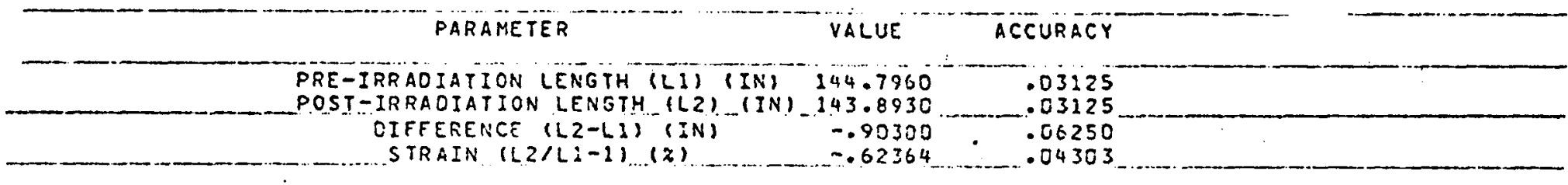

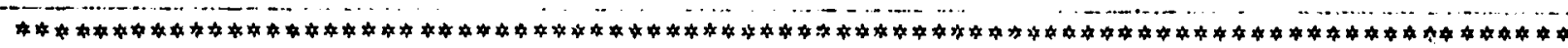

FUEL BOCY WEIGHT, TEST ELEMENT FTE-6

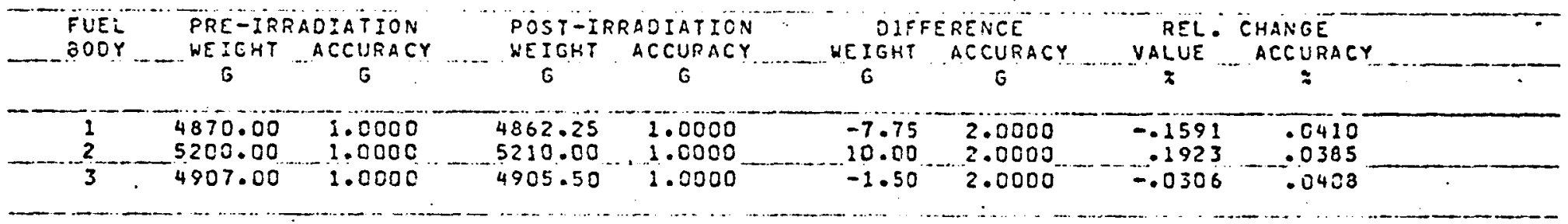


TABLE 4-19

FTE-6 SLEEVE DIAMETER

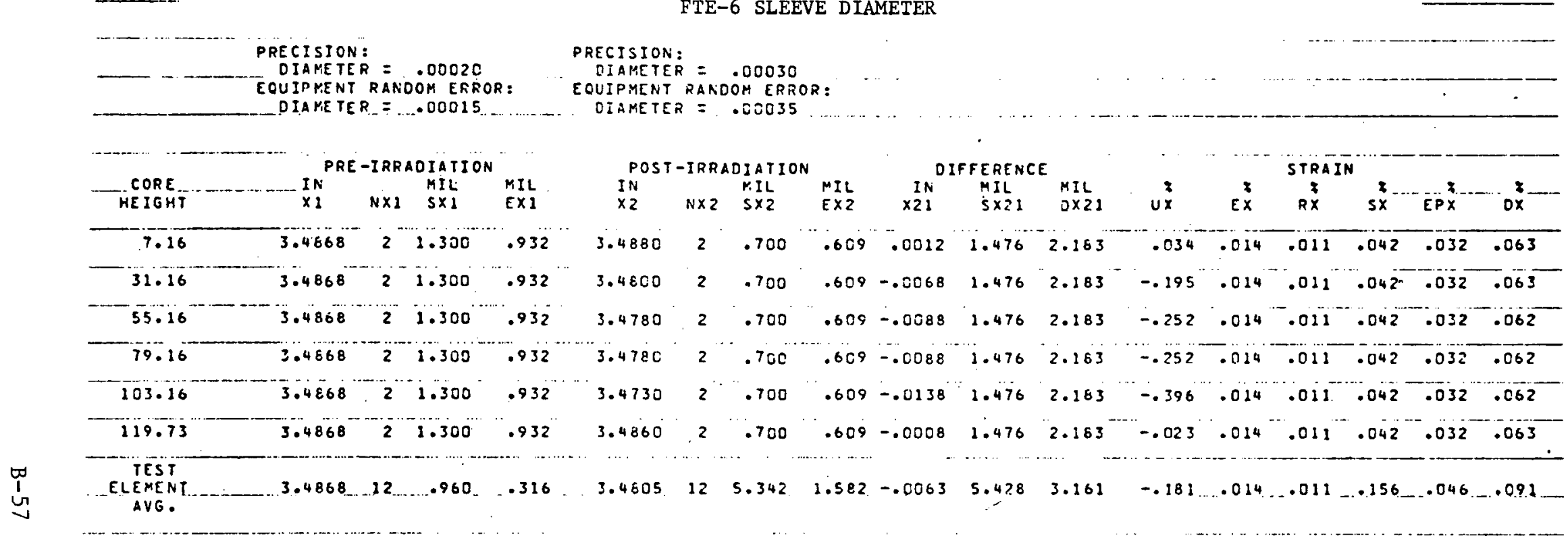

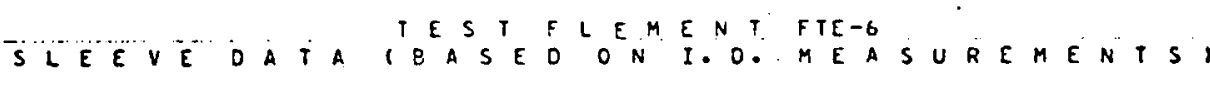

PRECISION:

EOUIPMENT RANOOM ERROR: -.... EOUIPMENT PANDOM ERROR:

OIAMETER $=$. .OOU15 $\quad$ DIAMETER $=.00035$

\begin{tabular}{|c|c|c|c|c|c|c|c|c|c|c|c|c|c|c|c|c|c|}
\hline & PRE & -IRRA & DIAIC & & POST & -IRRA & DIATION & & & FFERENC & & & & STRA & & & \\
\hline $\begin{array}{l}\text { CORE } \\
\text { HEIGHT }\end{array}$ & $\begin{array}{ll}\text { In } \\
\times 1\end{array}$ & $N \times 2$ & $\begin{array}{l}M I L \\
S \times 1\end{array}$ & $\begin{array}{l}\text { MIL } \\
\text { EXI }\end{array}$ & $\begin{array}{l}\text { IN } \\
\times 2\end{array}$ & $N \times 2$ & $\begin{array}{l}M 1 L \\
S \times 2\end{array}$ & $\begin{array}{l}\text { MIL } \\
\text { EX? }\end{array}$ & $\begin{array}{r}\text { IN } \\
\times 21\end{array}$ & $\begin{array}{l}\text { HIL } \\
5 \times 21\end{array}$ & $\begin{array}{l}M I L \\
0 \times 21\end{array}$ & $u^{2}$ & $E x$ & $8 x$ & $5 x^{x}$ & EPX & $-0 x^{8}$ \\
\hline 24.75 & 2.7522 & 2 & .900 & .655 & 2.7490 & 2 & .800 & .668 & -.0032 & 1.204 & 1.834 & -.116 & .018 & .014 & .044 & .034 & .057 \\
\hline 129.73 & 2.7522 & 2 & .900 & .655 & 2.7500 & 2 & .300 & .668 & -.0022 & 2.204 & 1.334 & -.080 & .018 & .014 & .044 & .034 & .067 \\
\hline $\begin{array}{c}\text { TEST } \\
\text { ELLEMENT } \\
\text { AVG. }\end{array}$ & 2.7522 & $\ldots$ & .0735 &. .398 & 2.7495 & 4 & .872 & .561 & -.0027 & 1.140 & 1.348 & $\because 098$ & $\ldots .018$ & .014 & .041 & .025 & .049 \\
\hline
\end{tabular}


TABLE $\quad 4-20$

FTE-6 FUEL BODY BOW

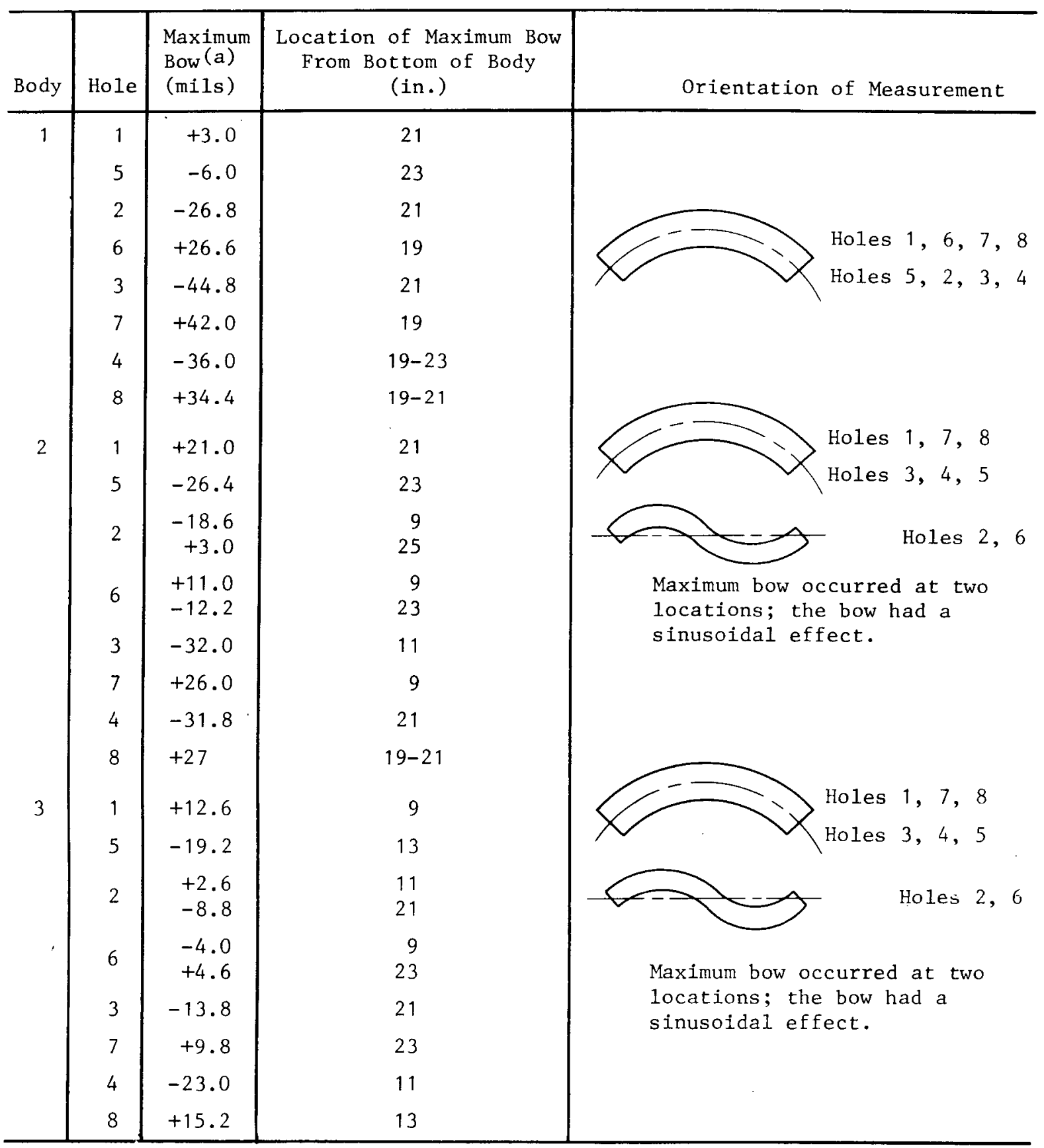

(a) Accuracy: $\pm 1 \mathrm{mil}$. 
TABI.P. $4-21$

FTE-6 FUEL BODY BOW ANALYSIS

\begin{tabular}{|c|c|c|c|c|c|c|c|c|c|c|c|c|c|}
\hline Fuel Body & I.D. & \begin{tabular}{|c|} 
Fast \\
Fluence \\
$10^{25} \mathrm{n} / \mathrm{m}^{2}$ \\
\end{tabular} & $\begin{array}{l}\text { Time Averaged } \\
\text { Irr. Temp. } \\
{ }^{\circ} \mathrm{C}\end{array}$ & $\begin{array}{c}\text { Maxi } \\
\text { Location } \\
\text { Hole }\end{array}$ & $\begin{array}{c}\text { Abs. Amount } \\
\text { m11 }\end{array}$ & $\begin{array}{c}\text { Diametrical } \\
\text { Gap } \\
\text { mil } \\
\end{array}$ & $\begin{array}{c}\text { Restraint } \\
\text { Bow } \\
\text { n11 }\end{array}$ & $\begin{array}{c}\text { UTS } \\
\text { (measured) } \\
\text { MPa }\end{array}$ & $\begin{array}{c}E / \mathrm{E}_{\mathrm{o}}{ }^{(a)} \\
\text { (calculated) }\end{array}$ & \begin{tabular}{|l} 
Restraint \\
Stress \\
$\mathrm{MPa}$ \\
\end{tabular} & $\begin{array}{c}\text { Fiber Stress }(b) \\
\text { Stress Factor }\end{array}$ & \begin{tabular}{|c|} 
Max. Rending \\
Stress \\
$: \mathrm{Pa}$ \\
\end{tabular} & $\begin{array}{l}\text { Fiber Stress }(c) \\
\text { Stress Factor }\end{array}$ \\
\hline $\begin{array}{l}\text { Eody } 1 \\
\text { Yeas } \\
\text { SLis.D. }\end{array}$ & $25: 3-44-3$ & $\begin{array}{l}2.101 \\
0.569\end{array}$ & $\begin{array}{l}730 \\
106\end{array}$ & 387 & $\begin{array}{r}43.40 \\
1.98\end{array}$ & $\begin{array}{l}9.54 \\
1.144\end{array}$ & $\begin{array}{r}33.86 \\
2.45\end{array}$ & 14.82 & 1.90 & $\begin{array}{r}11.33 \\
0.82\end{array}$ & $\begin{array}{l}0.50 \\
0.04\end{array}$ & $\begin{array}{l}9.68 \\
0.70\end{array}$ & $\begin{array}{l}0.42 \\
0.02\end{array}$ \\
\hline $\begin{array}{l}\text { Body } 2 \\
\text { Yeun } \\
\text { Res/S.D. }\end{array}$ & $738-43-10$ & $\begin{array}{l}2.828 \\
0.052\end{array}$ & $\begin{array}{r}950 \\
42\end{array}$ & 367,458 & $\begin{array}{r}29.20 \\
3.15\end{array}$ & $\begin{array}{r}13.86 \\
2.96\end{array}$ & $\begin{array}{r}15.34 \\
4.32\end{array}$ & 12.62 & 1.76 & $\begin{array}{l}4.79 \\
1.35\end{array}$ & $\begin{array}{l}0.26 \\
0.07\end{array}$ & $\begin{array}{l}6.08 \\
1.71\end{array}$ & $\begin{array}{l}0.33 \\
0.09\end{array}$ \\
\hline $\begin{array}{l}\text { Body } 3 \\
\text { Yeun } \\
\text { Res's.d. }\end{array}$ & $733-37-2$ & $\begin{array}{l}1.983 \\
0.587\end{array}$ & $\begin{array}{r}963 \\
39\end{array}$ & 458 & $\begin{array}{r}19.10 \\
5.52\end{array}$ & $\begin{array}{l}8.60 \\
3.50\end{array}$ & $\begin{array}{r}10.50 \\
6.54\end{array}$ & 12.62 & 1.75 & $\begin{array}{l}3.25 \\
2.02\end{array}$ & $\begin{array}{l}0.18 \\
0.11\end{array}$ & $\begin{array}{l}3.94 \\
2.45\end{array}$ & $\begin{array}{l}0.21 \\
0.13\end{array}$ \\
\hline Reference & & Table 3-2 & Table 3-3 & & Table 4-20 & Table $4-22$ & & GLCC QC Records & Ref. 3-21 & & & & \\
\hline
\end{tabular}

${ }^{(a)} E_{E_{0}}=10.3 \cdot 10^{3}$ MPa; stress factor $S=\sigma / U T S$, with UTS $=U_{T S}\left(E / E_{o}\right)^{0.67}$ for axial direction (nominal UTS $S_{0}=11.24$ MPa; see Table 3-15).

(b) Restraint stress; no deflection of sleeve (three-point bending case applfed to restraint bow).

(c) Maximum bending stress; fiber stress which led to the deformation of the fuel body (four-point bending case applied to maximum bow). 
TABLE 4-22

FTE-6 FUEL BODY $\uparrow$ DIMENSIONAL CHANGES

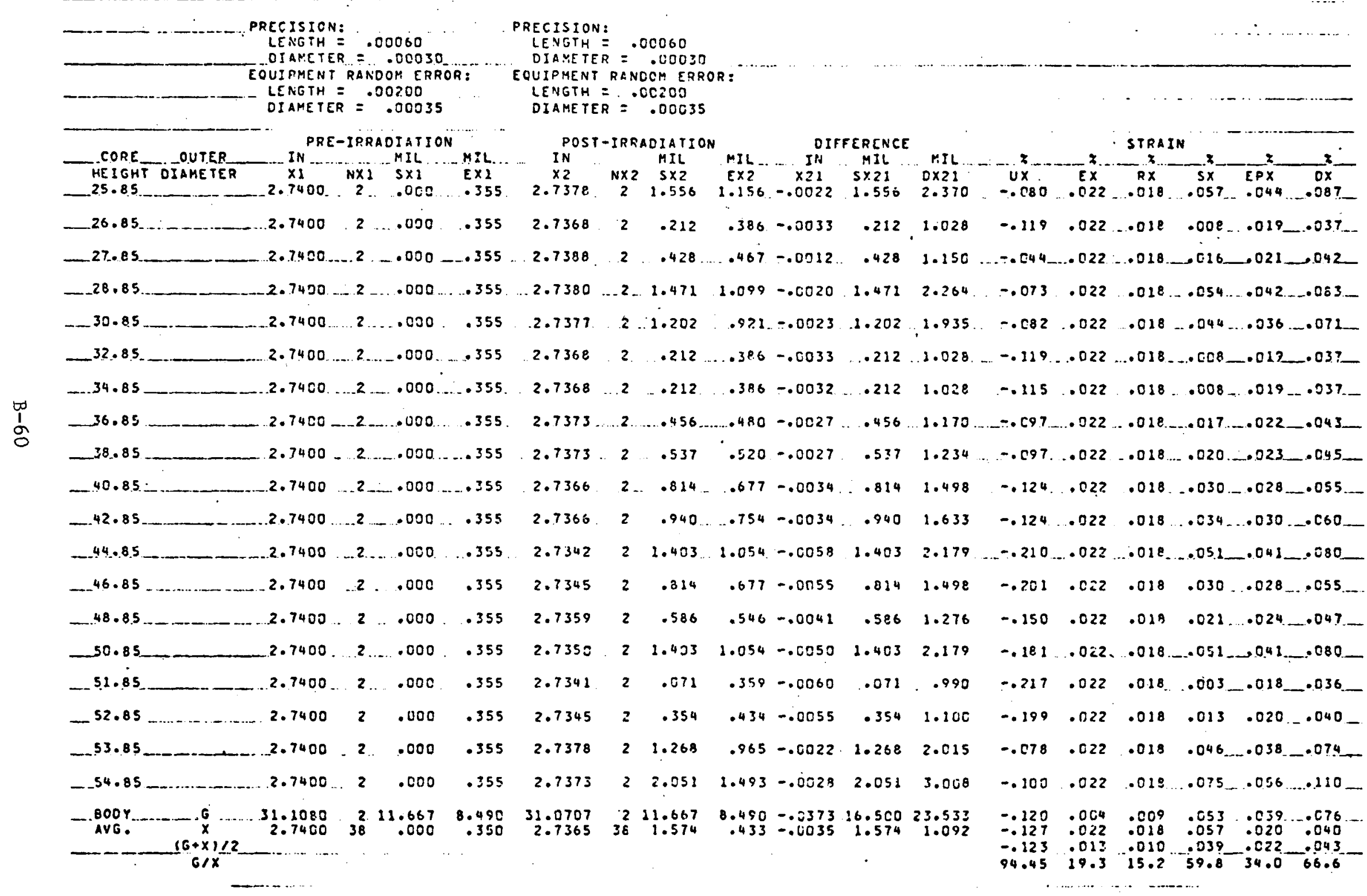


TABLE 4-23

FTE-6 FUEL BODY 2 DIMENSIONAL CHANGES

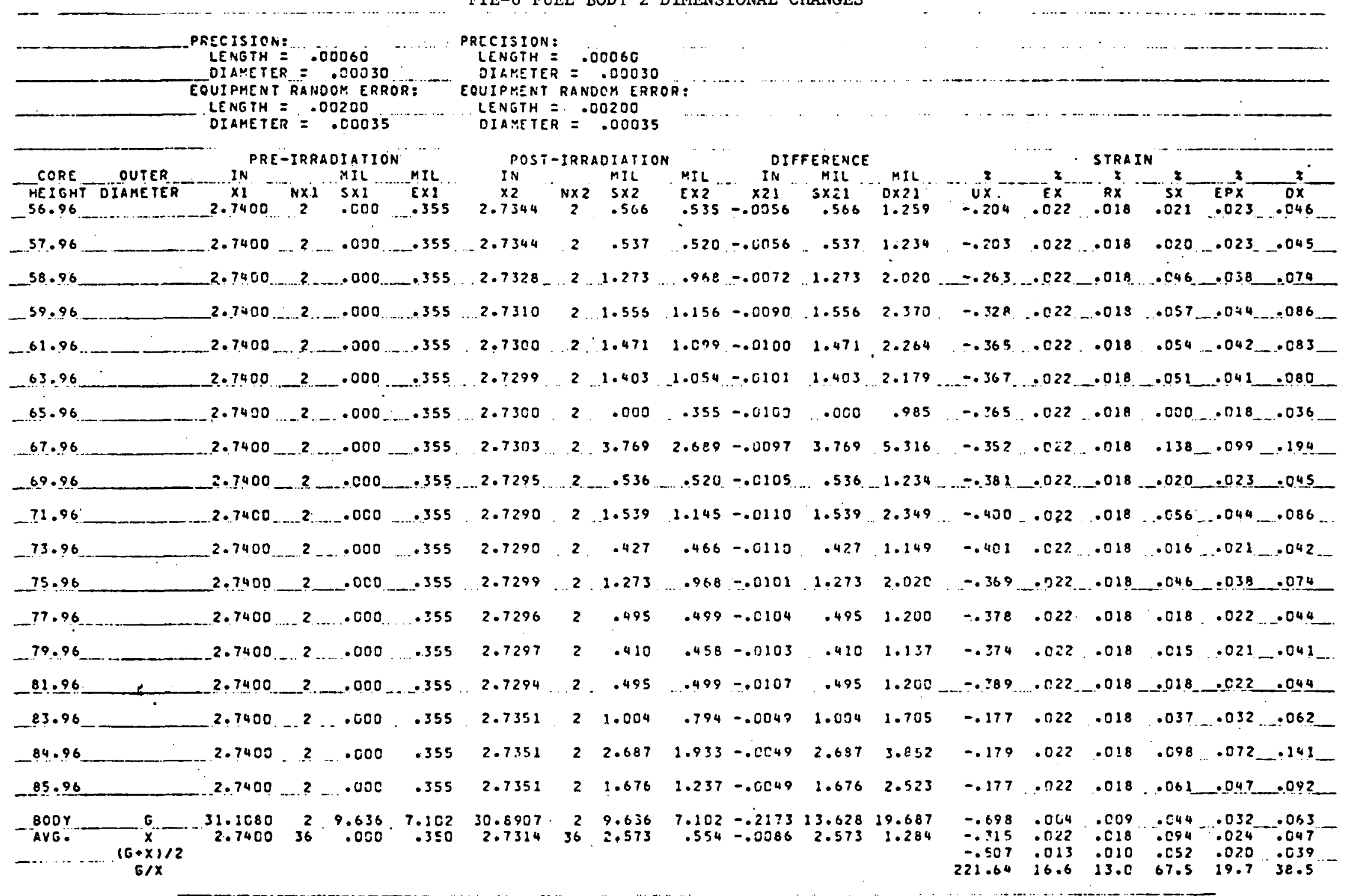


TABLE 4-24

FTE-6 FUEL BODY 3 DIMENSIONAL CHANGES

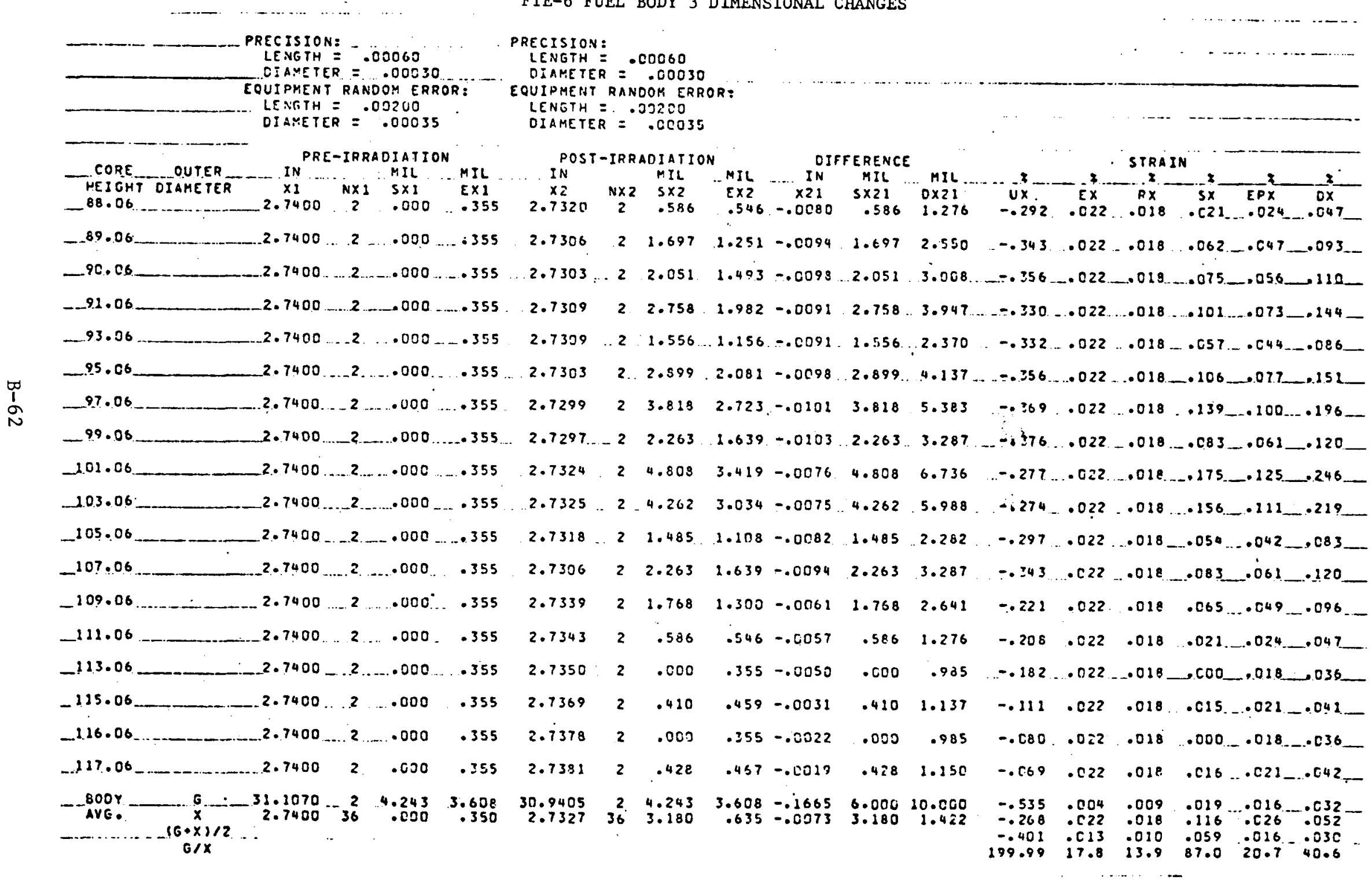


TABLE 4-25

FTE-6 FUEL BODY STRAIN SUMMARY

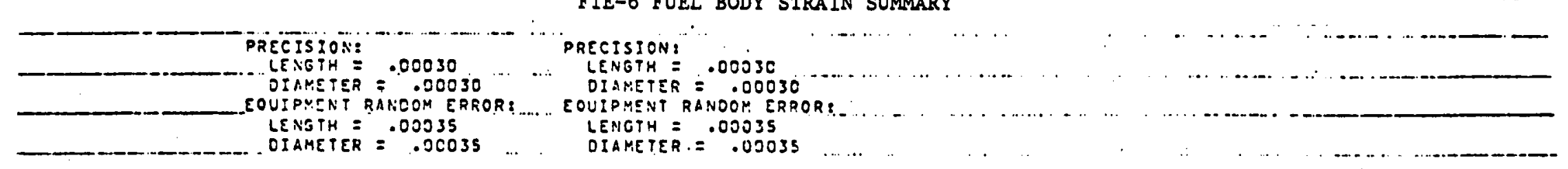

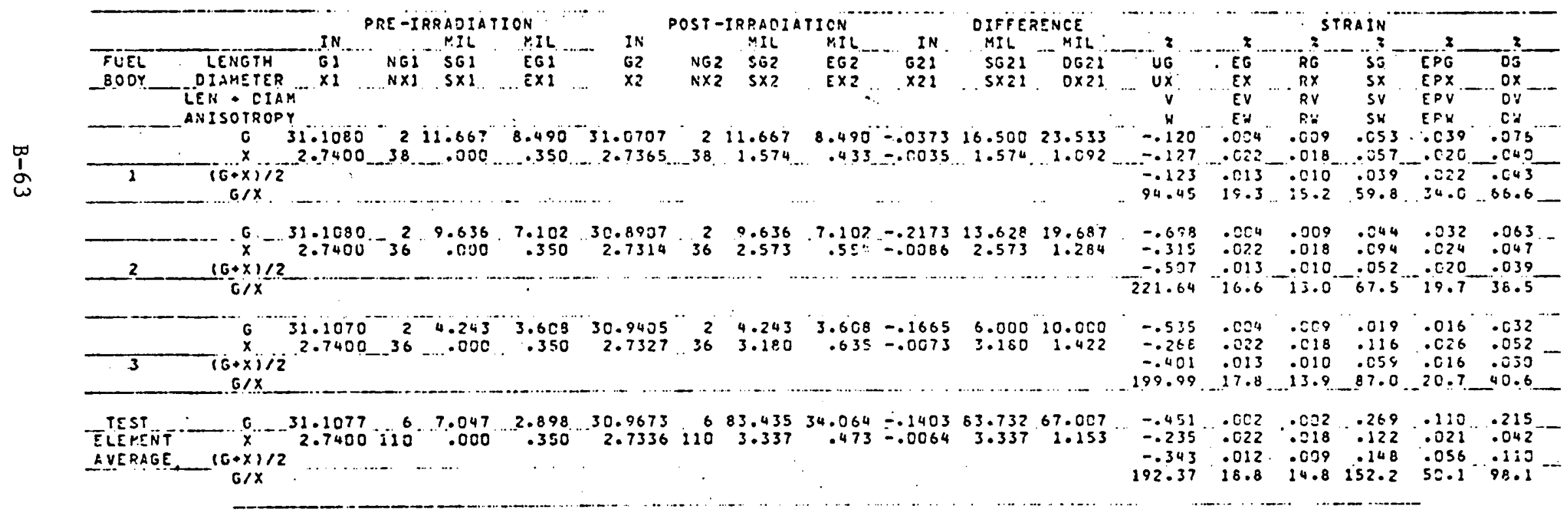


TABLE $\quad 4-26$

FTE-6 COMPARISON OF MEASURED AND CALCULATED FUEL BODY STRAIN

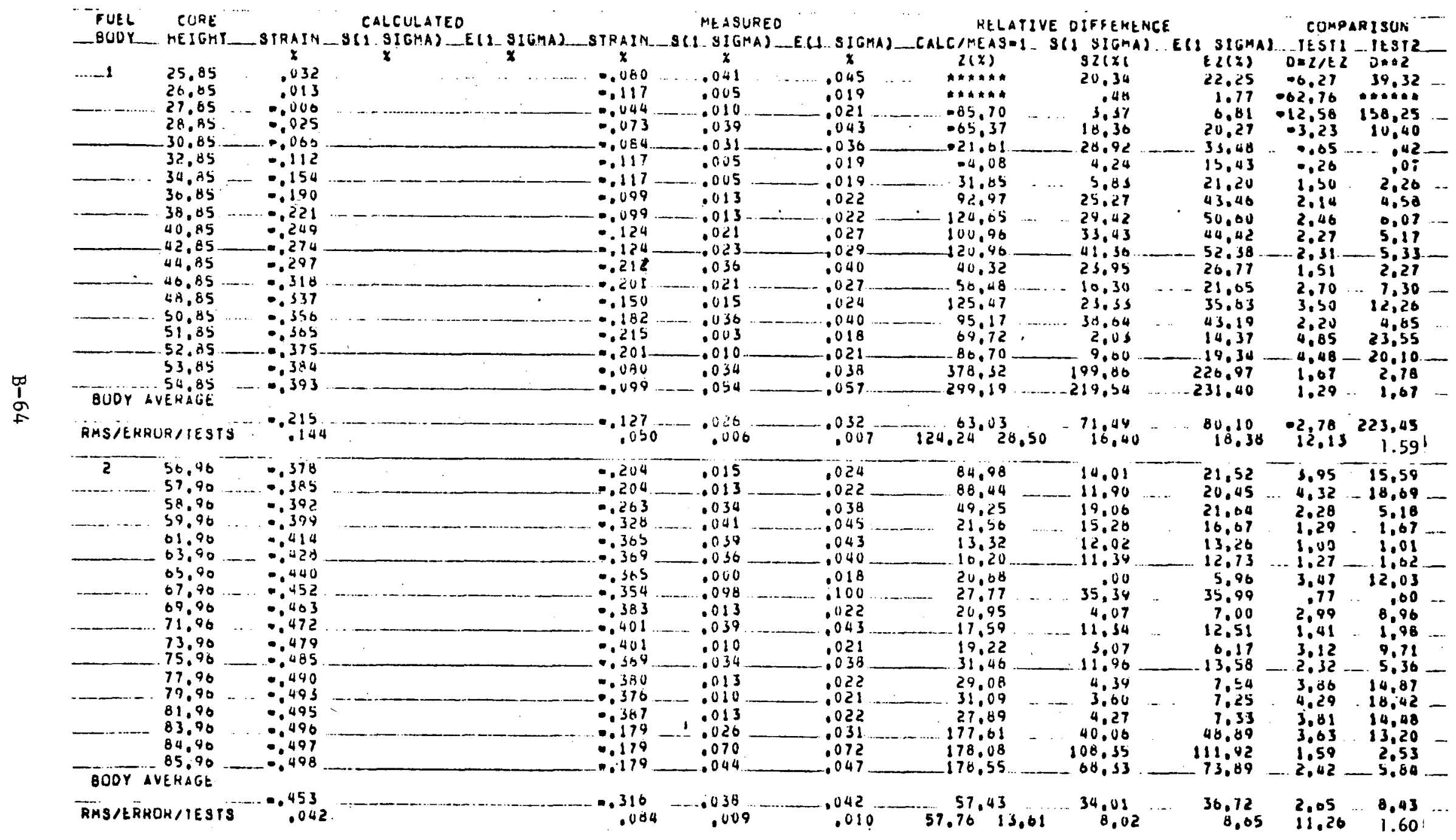


TABLE 4-26 (Continued)

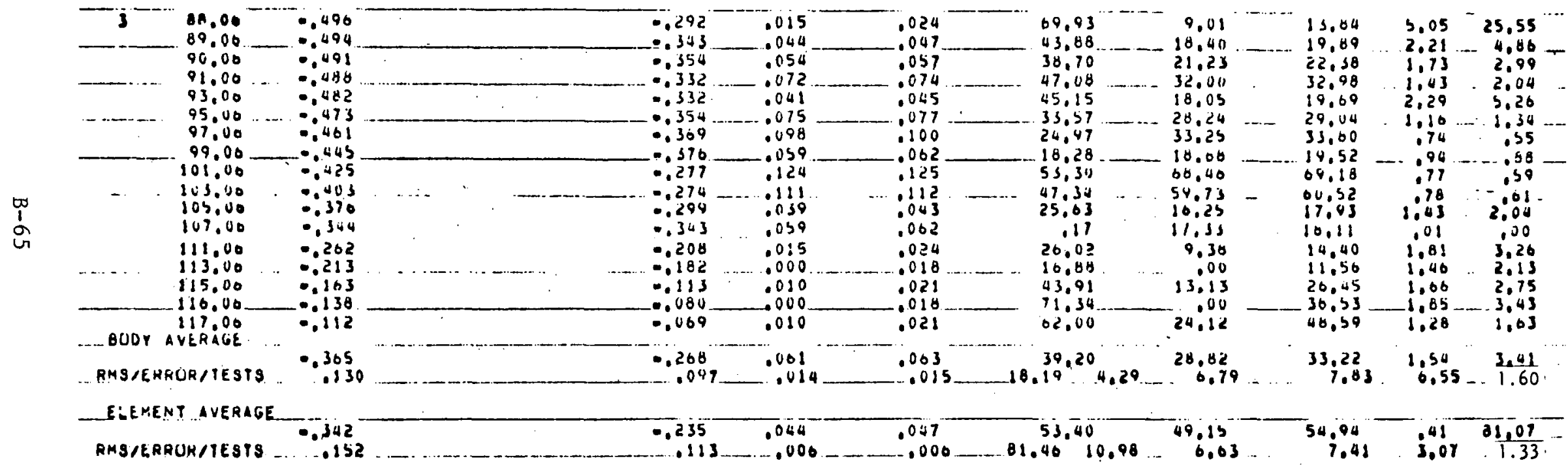


TABLE 4-27

FTE-6 SLEEVE FUEL BODY GAPS

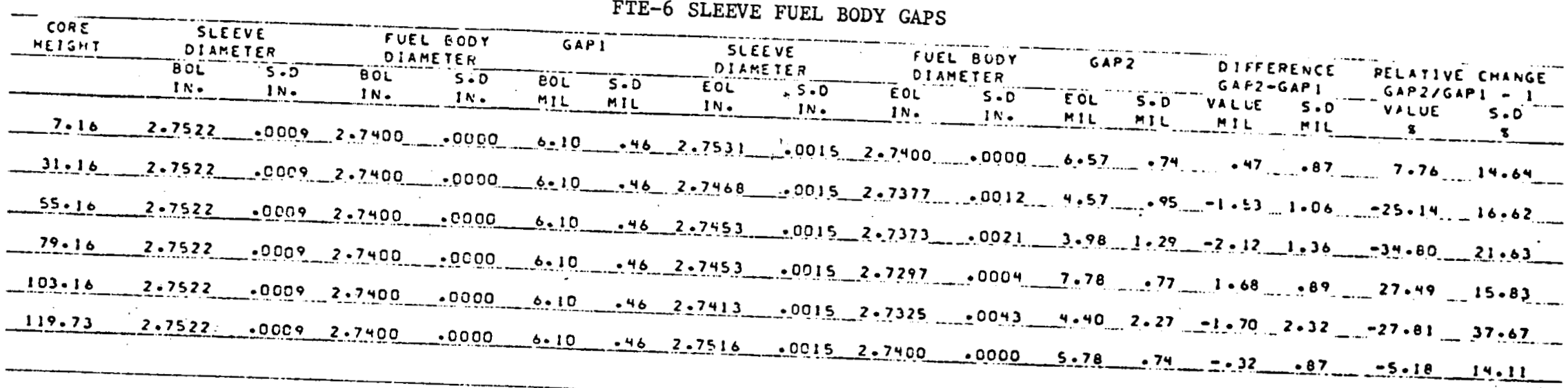

EOL SLEeVe i.D. DIA. = BOL SLEeve 1.D.* EOL SLEEVE 0.D./BOL SLEEVE O.D.

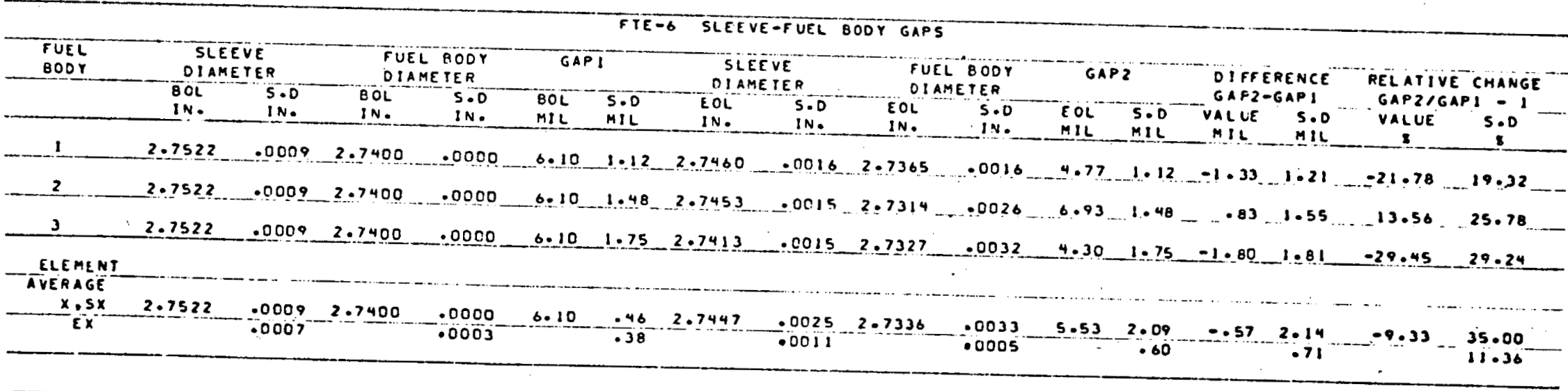

EOL SLEEVE I.D. DIA. = BOL SLEEVE I.D.* EOL SLEEVE 0.0./BOL SLEEVE O.D. 
TABLE 4-28

FTE-6 COMPARISON OF MEASURED AND CALCULATED FUEL ROD STRAIN

HOLES 1 AND $2 \mathrm{UO}_{2}$ TRISO (26.2\% FUEL VOL.) AND ThO 2 BISO (73.8\% FUEL VOL.) FUEL

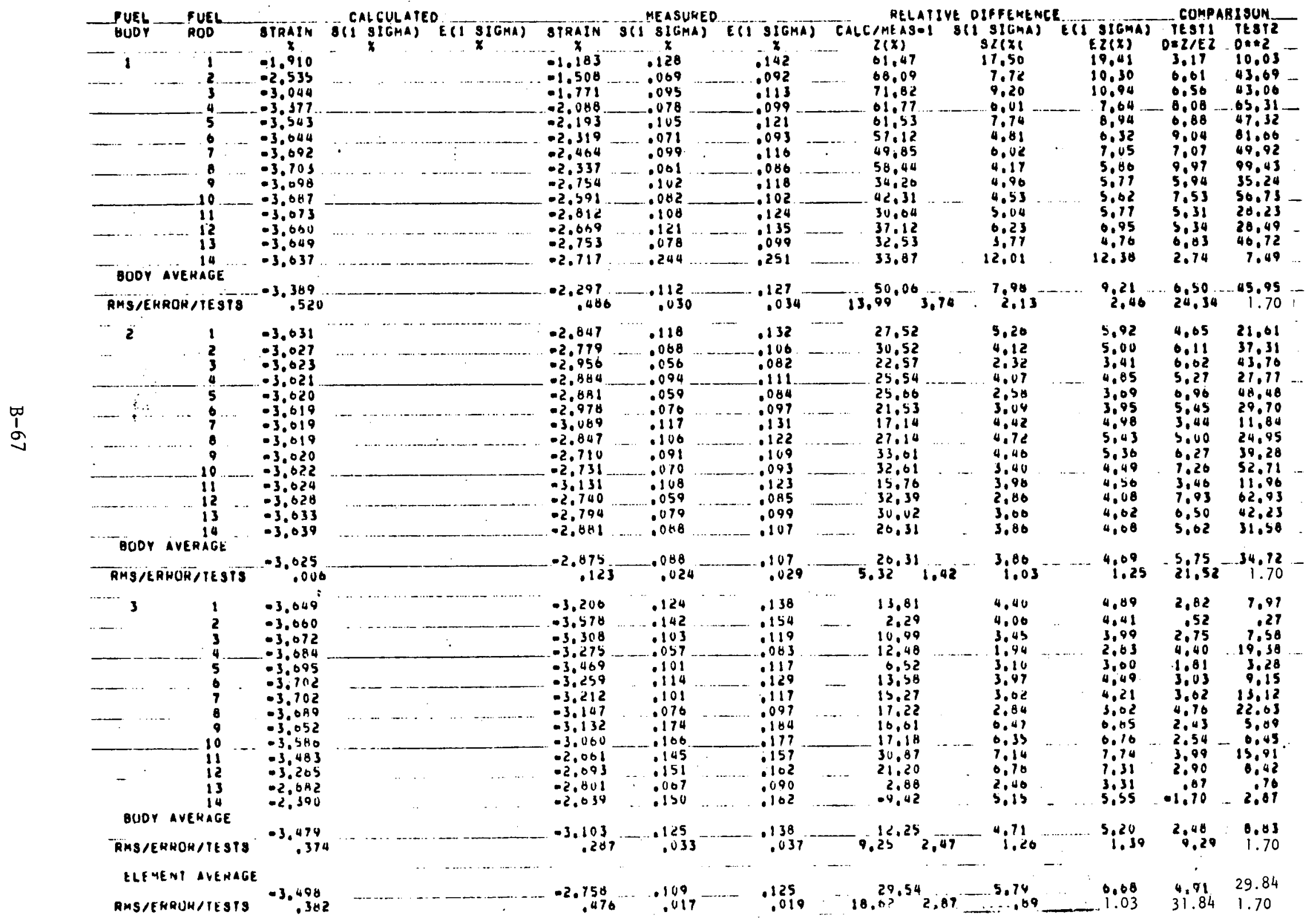


TABLE $4-29$

FTE-6 COMPARISON OF MEASURED AND CALCUIATED FUEL ROD STRAIN

HOLES 3 AND 4 (Th,U) $C_{2}$ TRISO (92.5\% FUEL VOL.) AND ThC 2 BISO (7.5\% FUEL VOL.) FUEL

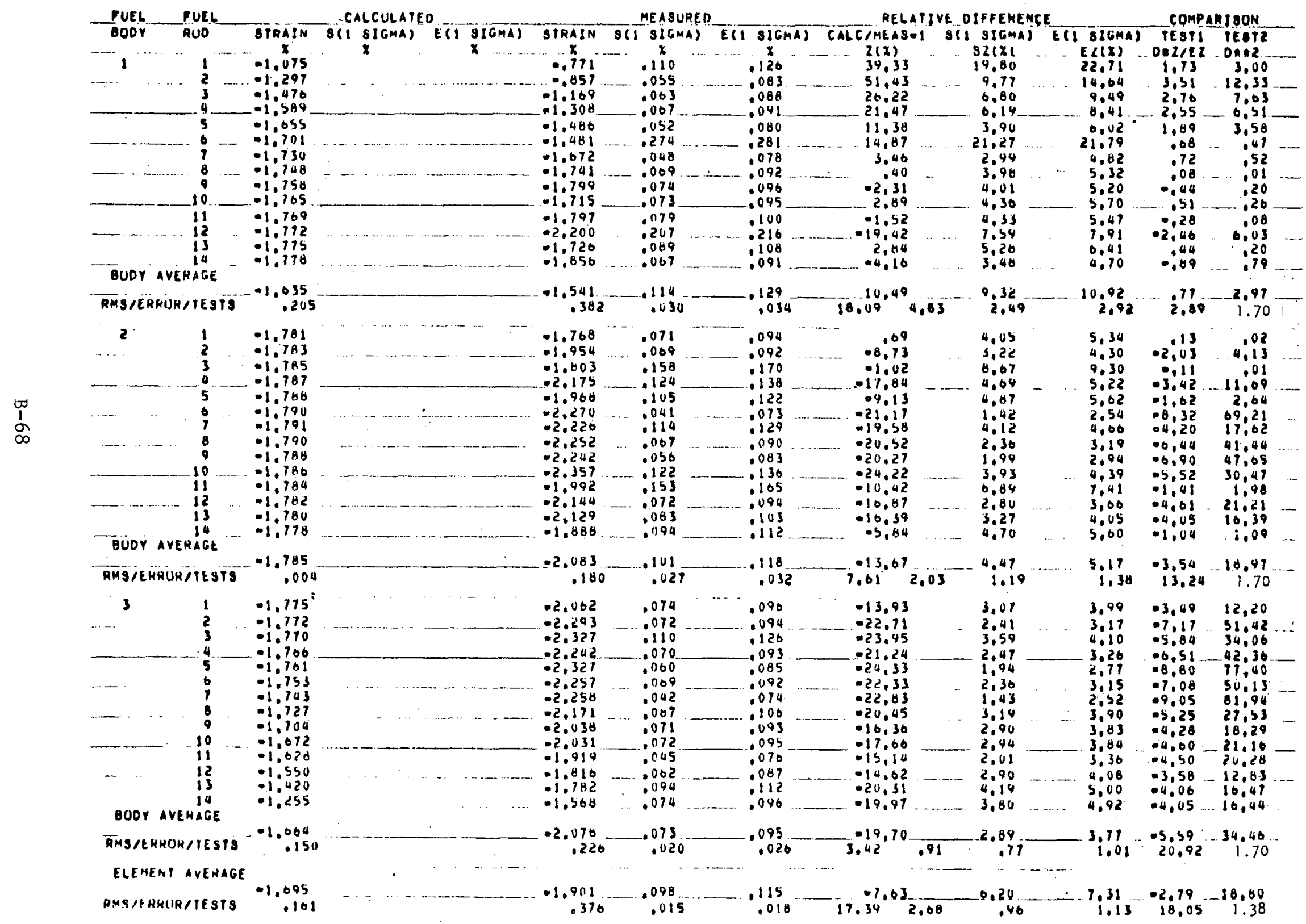

O 
TABLE $4-30$

FTE-6 COMPARISON OF MEASURED AND CALCULATED FUEL ROD STRAIN

HOLES 5 AND 6 (Th,U) $C_{2}$ TRISO (92.7\% FUEL VOL.) AND $\mathrm{ThC}_{2}$ TRISO (7.3\% FUEL VOL.) FUEL

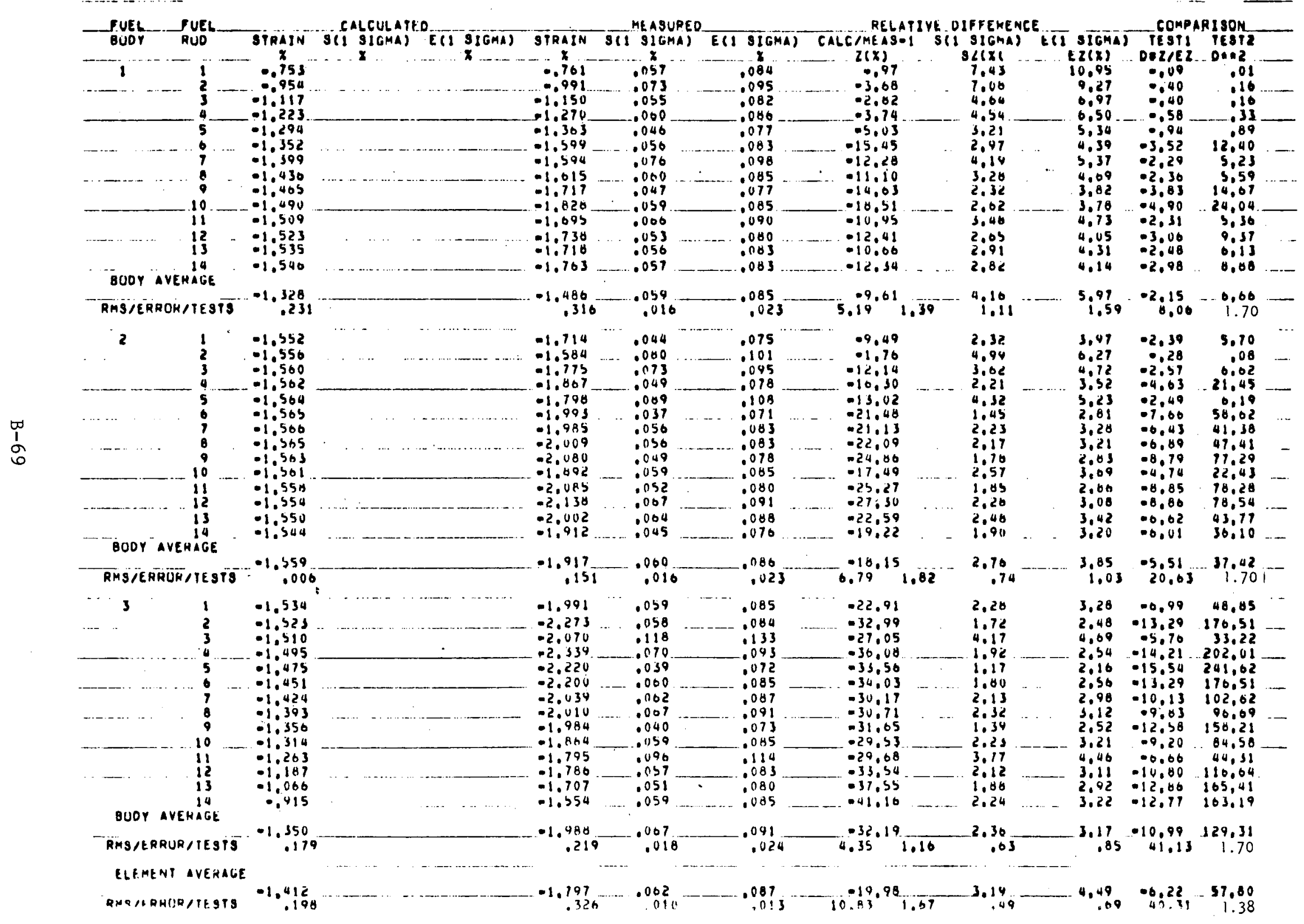


TABLE 4-31

FTE-6 COMPARISON OF MEASURED AND CALCULATED FUEL ROD STRAIN

HOLES 7 AND 8 UC $_{2}$ TRISO (80.2\% FUEL VOL.) AND ThC 2 BISO (19.8\% FUEL VOL.) FUEL

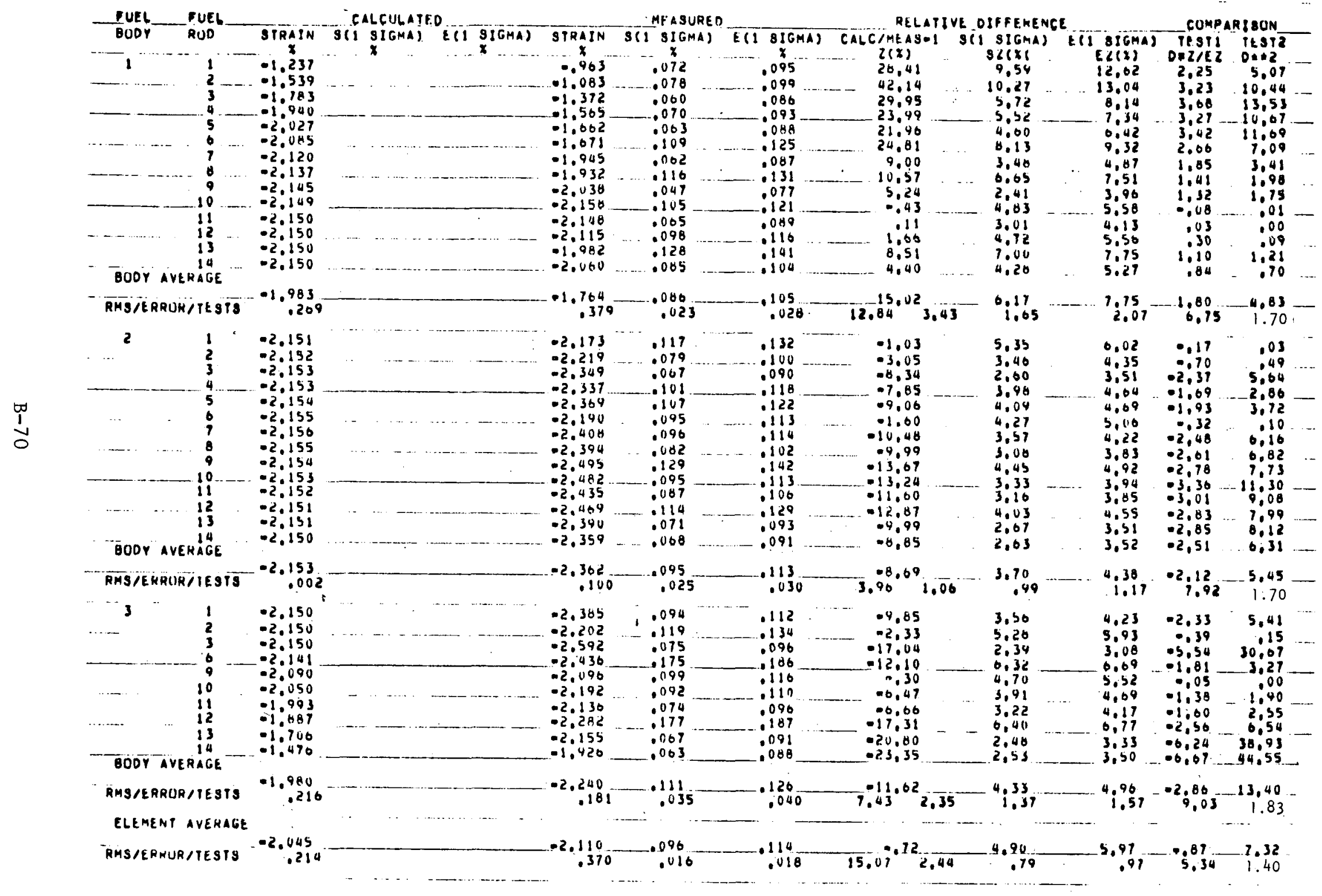


TABLE 4-32

FTE-6 HOLE 1 AND 2 FUEL ROD STRAIN SUMMARY

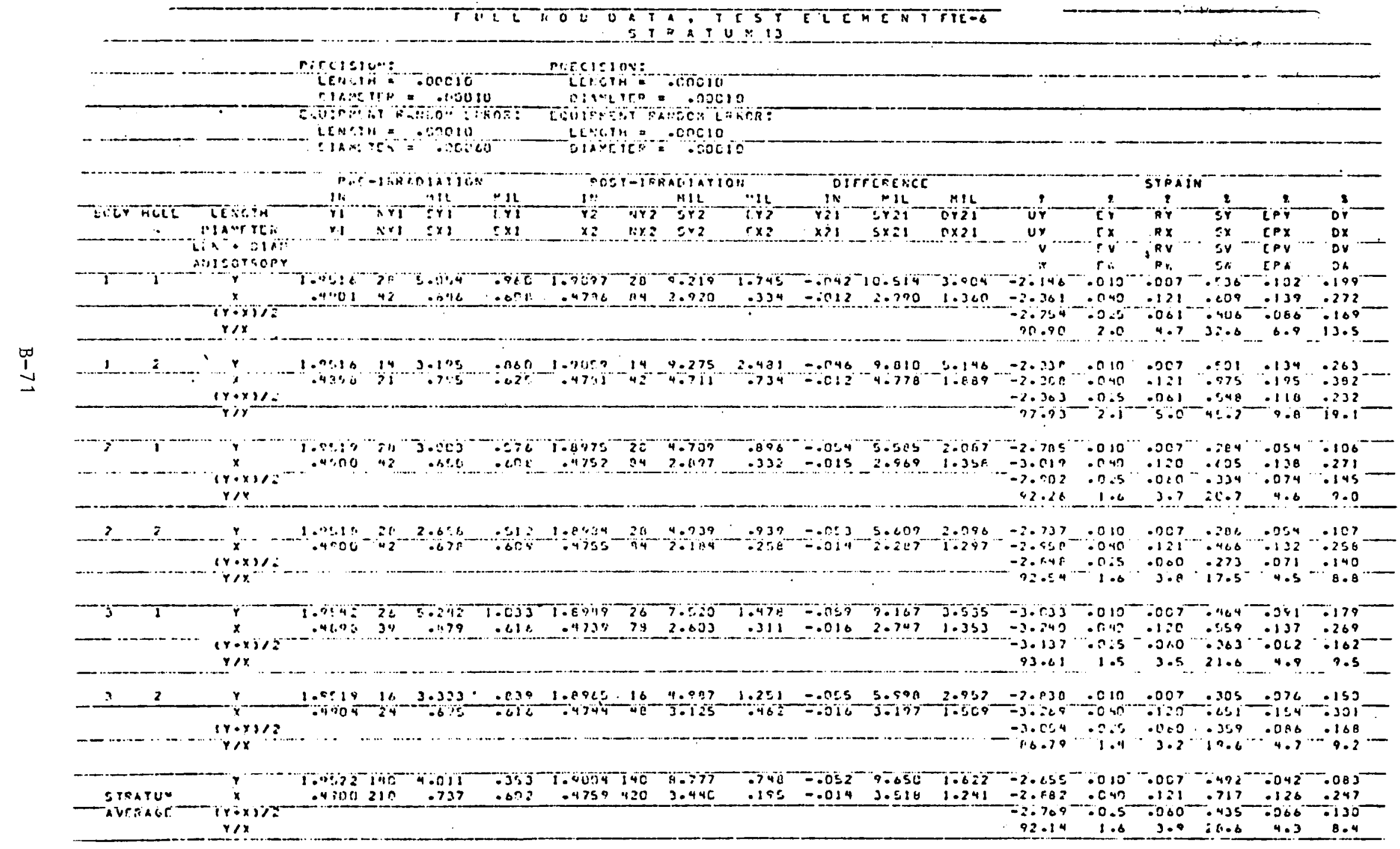


TABLE 4-33

FTE-6 HOLE 3 AND 4 FUEL ROD STRAIN SUMMARY

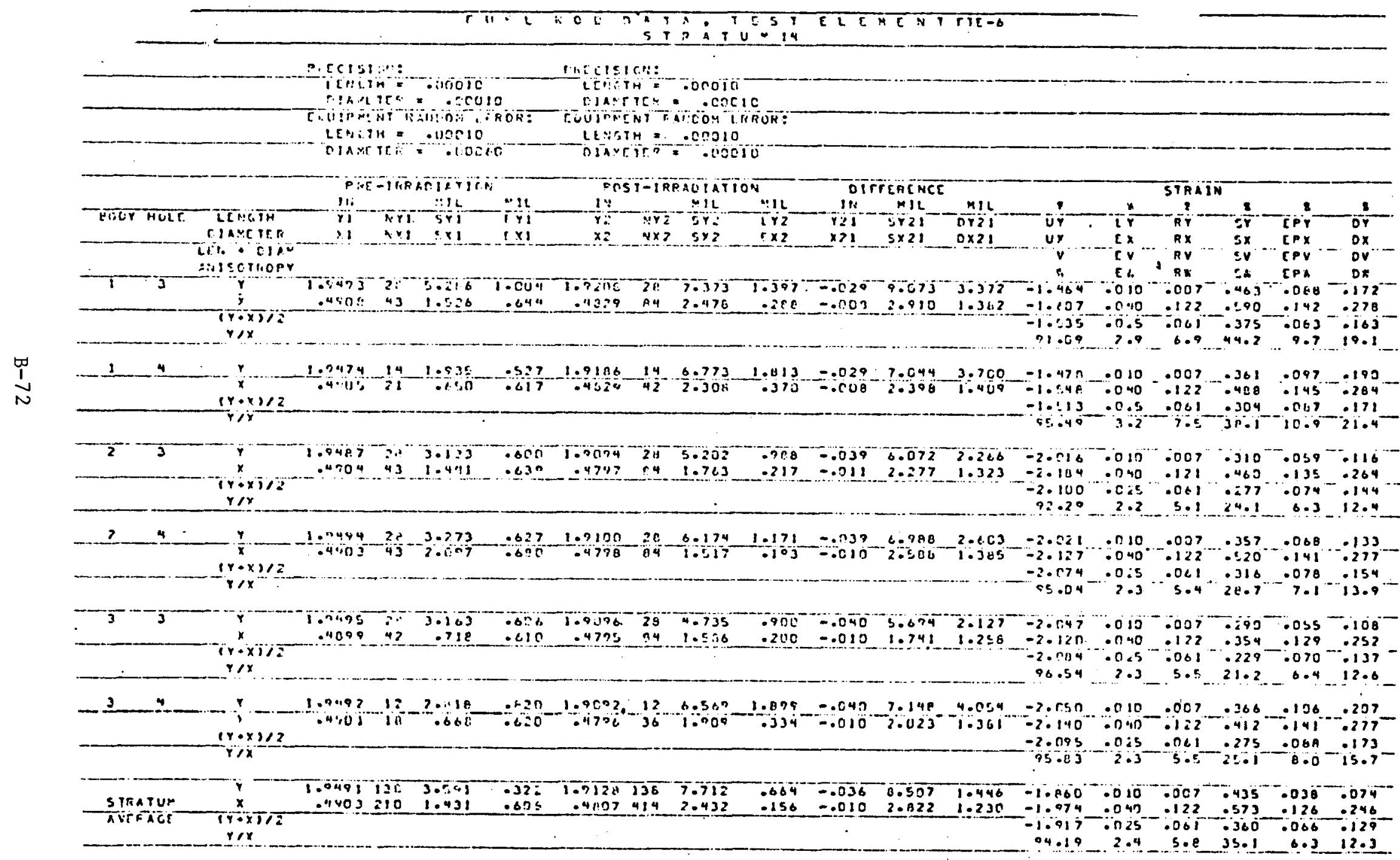


TABLE 4-34

FTE-6 HOLE 5 AND 6 FUEL ROD STRAIN SUMMARY

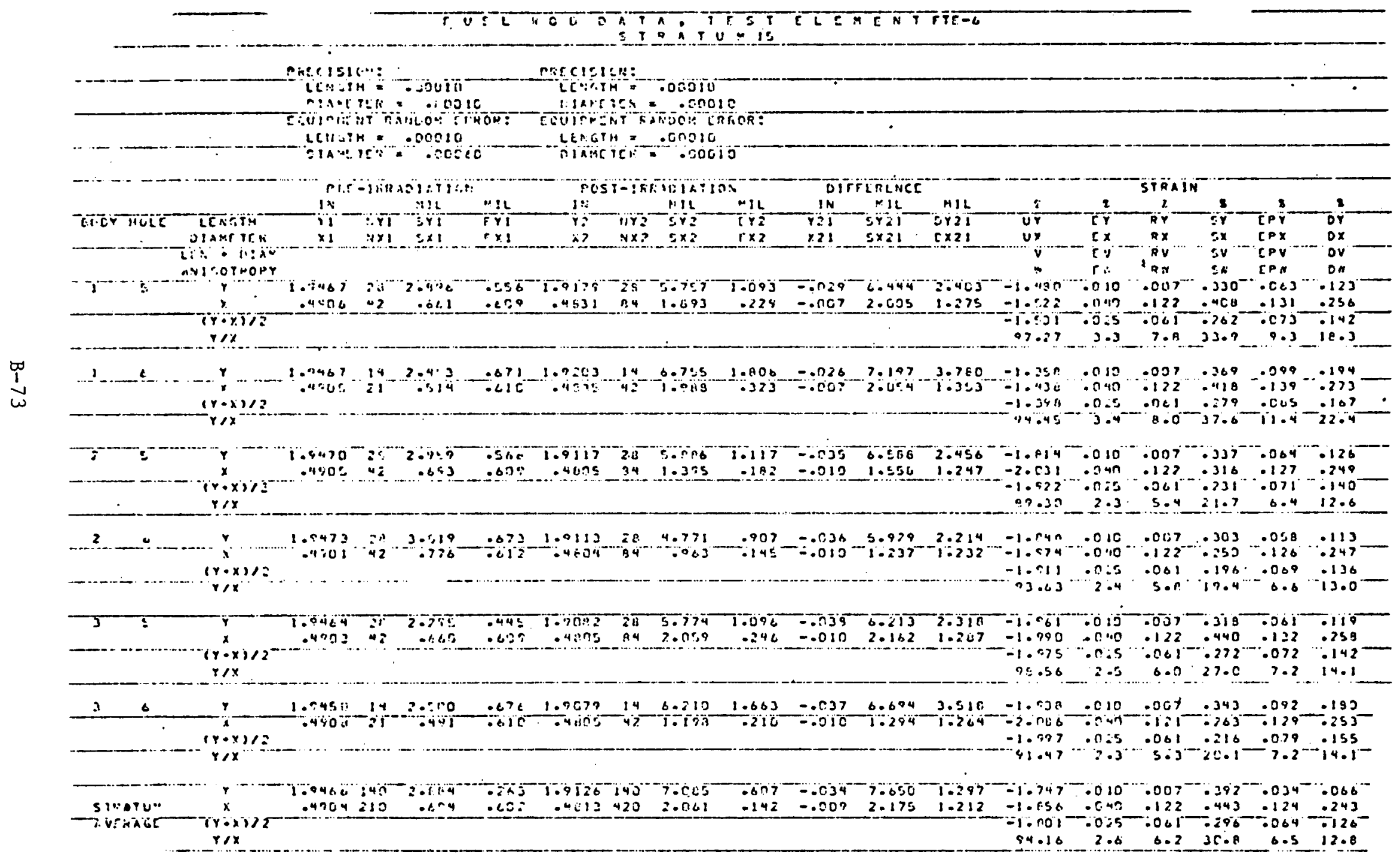


TABLE 4-35

FTE-6 HOLE 7 AND 8 AND TOTAL ELEMENT STRAIN SUMMARY

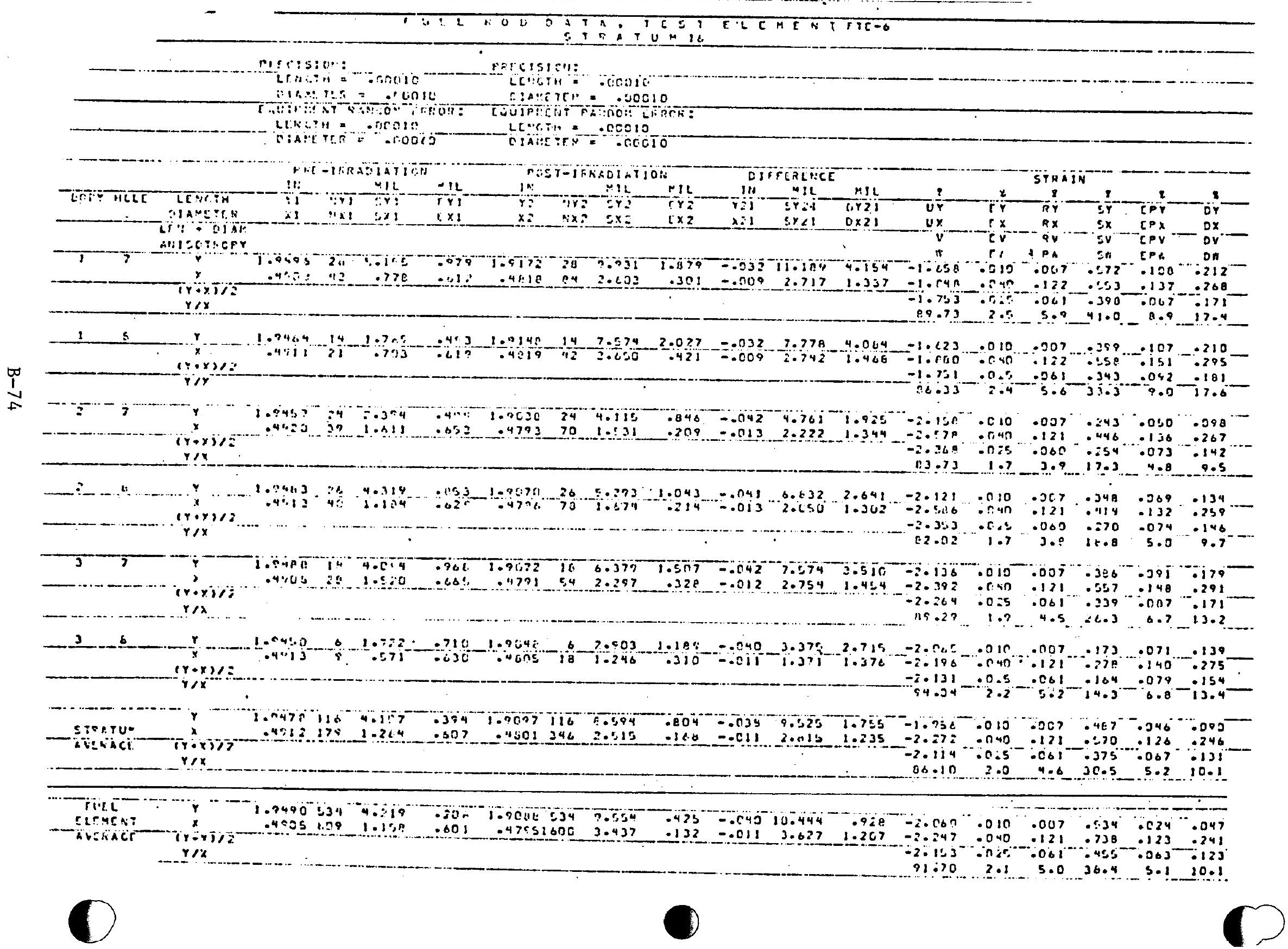


TABLE 4-36

FTE-6 COMPARISON OF MEASURED AND CALCULATED FUEL ROD - FUEL HOLE GAPS, HOLES 1 AND 2

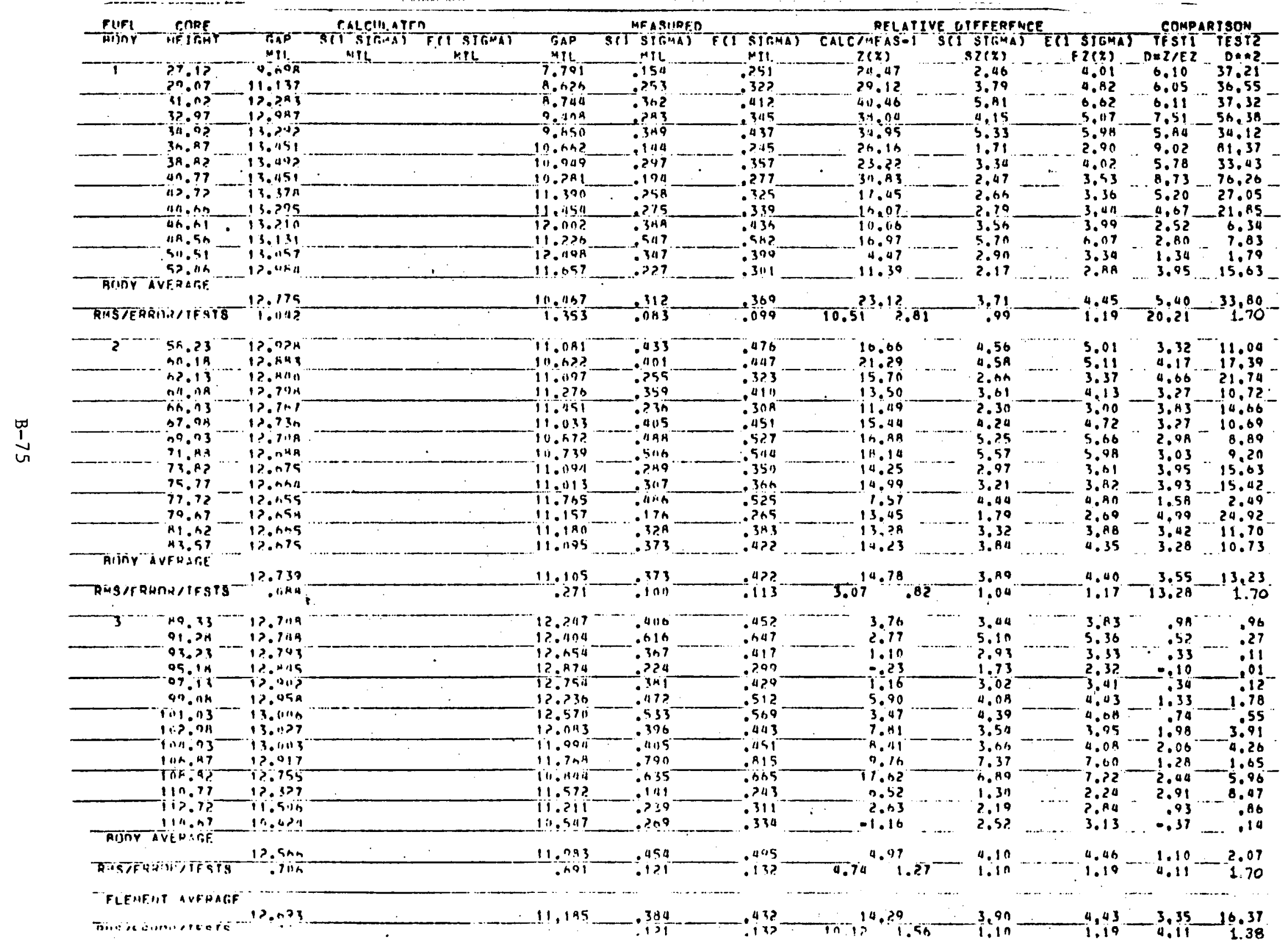


TABLE 4-37

FTE-6 COMPARISON OF MEASURED AND CALCULATED FUEL ROD - FUEL HOLE GAPS, HOLES 3 AND 4

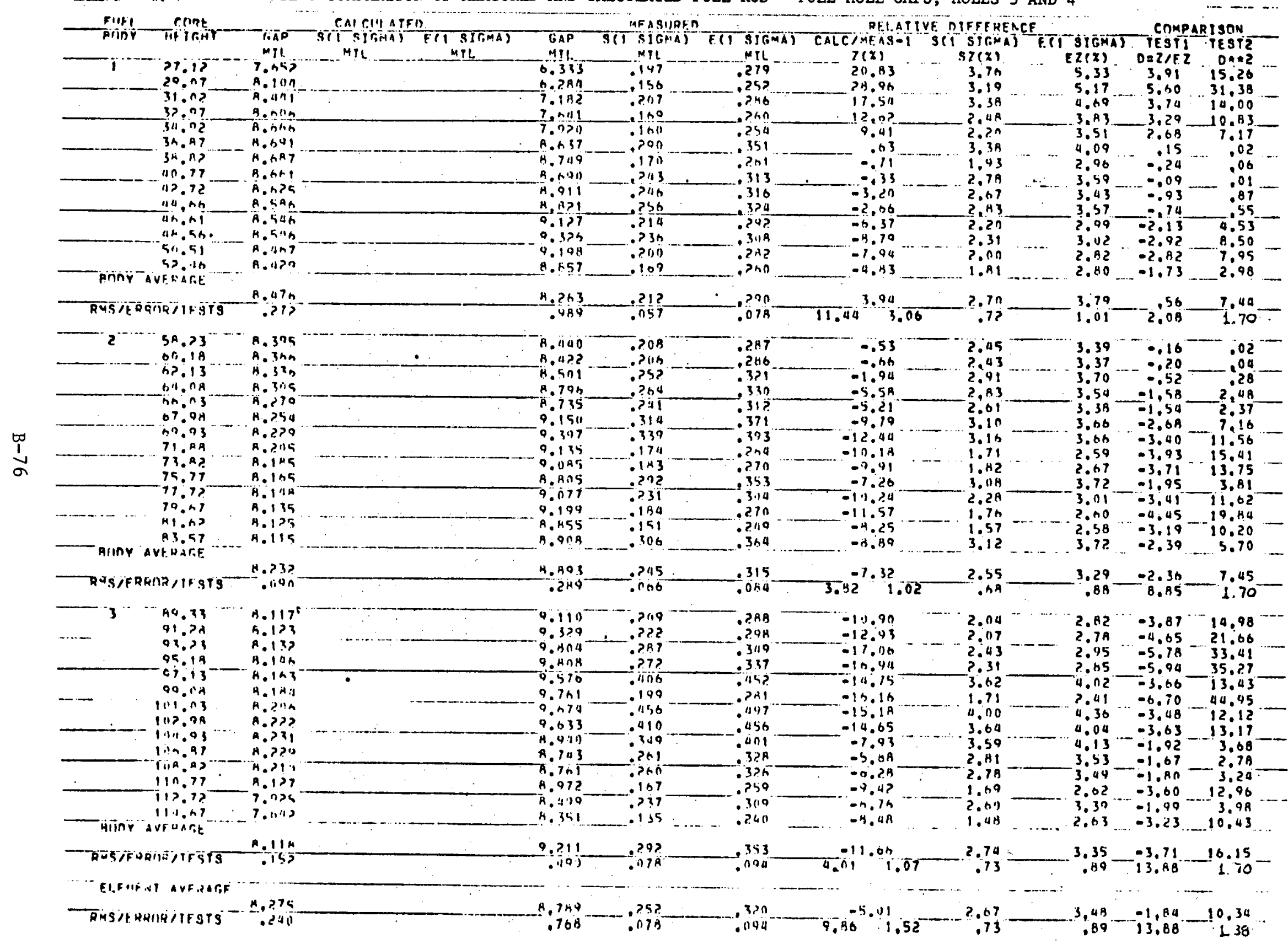


TABLE $4-38$

FTE-6 COMPARISON OF MEASURED AND CALCULATED FUEL ROD - FUEL HOLE GAPS, HOLES 5 AND 6

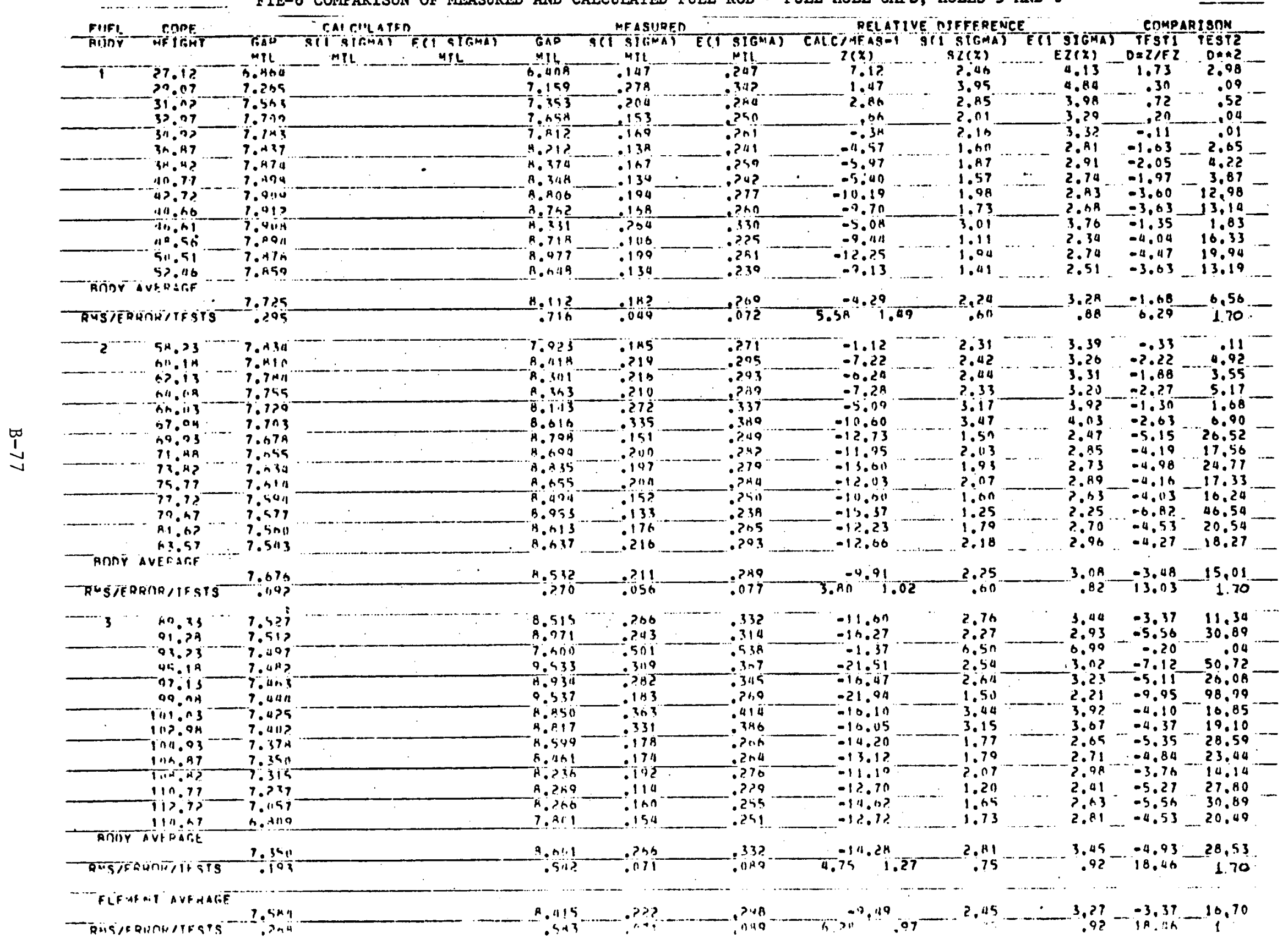


TABLE 4-39

FTE-6 COMPARISON OF MEASURED AND CALCULATED' FUEL ROD - FUEL HOLE GAPS, HOLES 7 AND 8

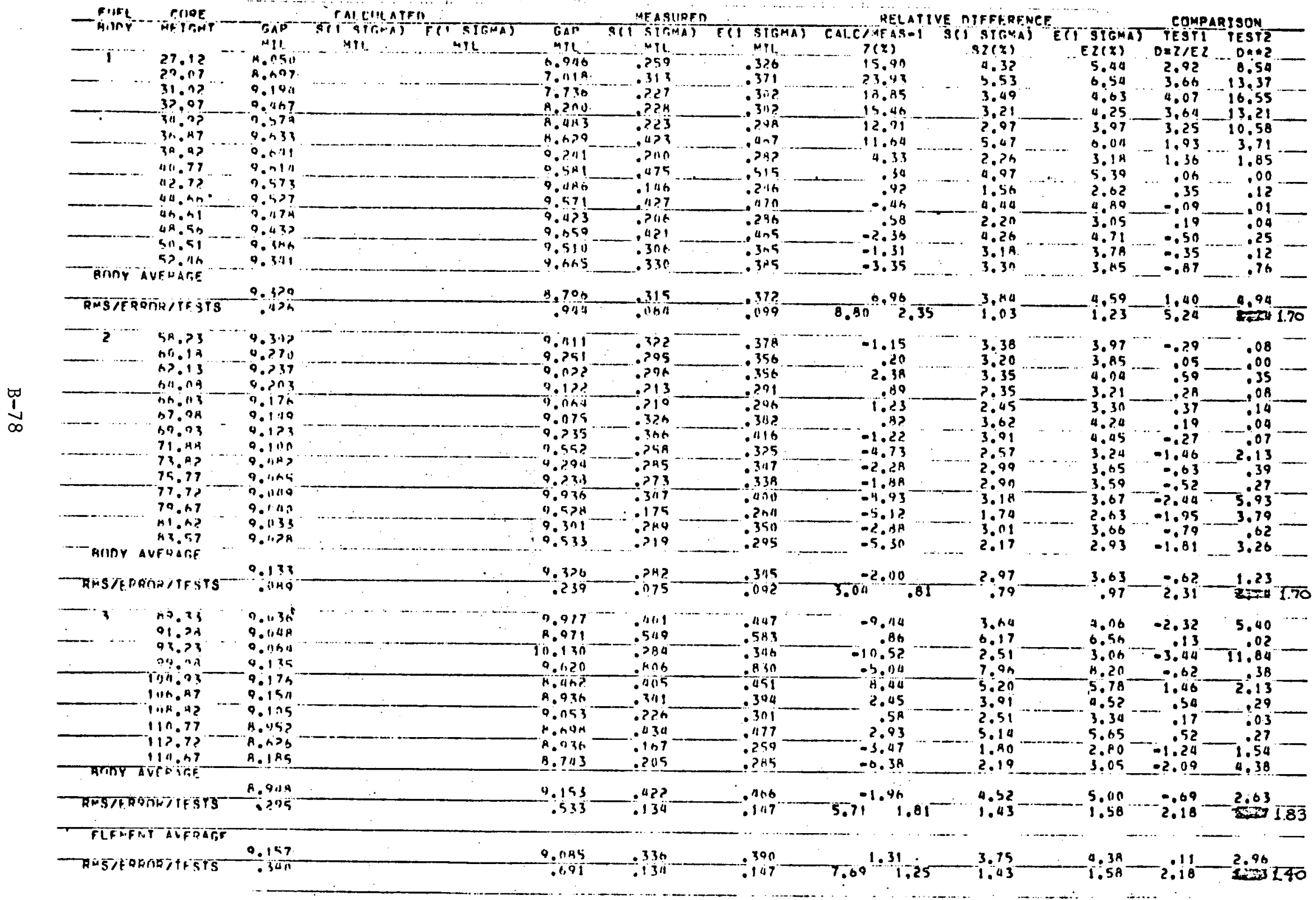


TABLE $\quad 4-40$

FTE-6 FUEL ROD BOW

\begin{tabular}{c|c|c}
\hline Rod & $\begin{array}{c}\text { Mean } \\
\text { Core Height } \\
(\mathrm{mm})\end{array}$ & $\begin{array}{c}\text { Bow } \\
\text { (mm) }\end{array}$ \\
\hline $2-1-10$ & 1925 & 0.53 \\
$2-1-11$ & 1974 & 0.45 \\
$2-2-5$ & 1677 & 0.39 \\
$2-2-10$ & 1925 & 0.42 \\
$2-2-13$ & 2073 & 0.42 \\
$3-1-1$ & 2269 & 0.55 \\
$3-1-2$ & 2318 & 0.46 \\
$3-1-3$ & 2368 & 0.45 \\
$3-1-4$ & 2418 & 0.57 \\
$3-1-5$ & 2467 & 0.59 \\
$3-1-6$ & 2517 & 0.53 \\
$3-1-7$ & 2566 & 0.44 \\
$3-1-12$ & 2814 & 0.54 \\
$3-1-13$ & 2863 & 0.50 \\
$3-2-3$ & 2368 & 0.40 \\
$3-2-2$ & 2566 & 0.28 \\
$3-2-13$ & 2863 & 0.45 \\
\hline
\end{tabular}




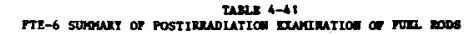

\begin{tabular}{|c|c|c|c|c|c|c|c|c|c|c|c|c|c|c|c|c|c|c|c|c|c|}
\hline \multirow{4}{*}{ 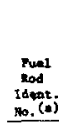 } & & & \multirow{2}{*}{\multicolumn{3}{|c|}{ 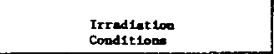 }} & \multirow{4}{*}{ 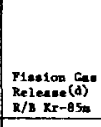 } & \multirow{3}{*}{\multicolumn{2}{|c|}{ 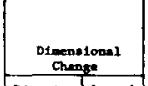 }} & \multirow{4}{*}{ 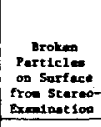 } & \multicolumn{12}{|c|}{ 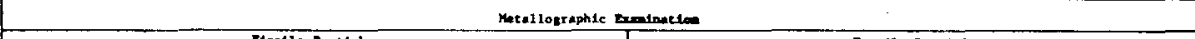 } \\
\hline & \multirow{2}{*}{\multicolumn{2}{|c|}{ neal trpese }} & & & & & & & & \multirow{2}{*}{\multicolumn{6}{|c|}{\begin{tabular}{c|c|c|} 
PAstile Pert tele \\
952
\end{tabular}}} & \multicolumn{6}{|c|}{ Port 11. Particle } \\
\hline & & & \multirow{2}{*}{ 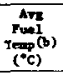 } & \multirow{2}{*}{ 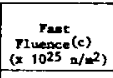 } & \multirow{2}{*}{ 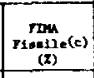 } & & & & & $\begin{array}{l}\text { opx } \\
\text { zatiure }\end{array}$ & & Palure & & 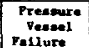 & 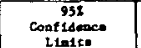 & $\mid \begin{array}{l}027 x \\
\text { pallure }\end{array}$ & 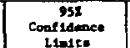 & & 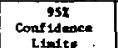 & 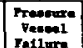 & 332 \\
\hline & Pasolle Part1c1e & Pertule Particle & & & & & $\frac{D^{\text {Dimenter }}}{(z)}$ & 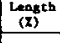 & & 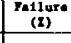 & 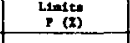 & $\begin{array}{c}\begin{array}{c}\text { Painure } \\
\text { (z) }\end{array} \\
\end{array}$ & $\begin{array}{c}\substack{\text { Lantece } \\
P(x)} \\
\end{array}$ & 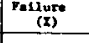 & 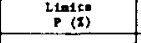 & \begin{tabular}{|c|} 
patiure \\
$(x)$
\end{tabular} & 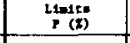 & 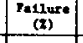 & 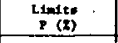 & \begin{tabular}{|c|c|}
$\substack{\text { misluming } \\
(\alpha)}$ \\
$(\alpha)$
\end{tabular} & 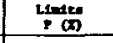 \\
\hline $2-1-7$ & 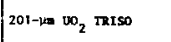 & $410-1=\pi \infty 0_{2} \mathrm{BrsO}$ & 1128 & 2.86 & 45.4 & $\therefore .1 \times 10^{-3}$ & -2.28 & -3.03 & 4 & 22.8 & $16.1 \leq 7 \leq 31.3$ & 22.8 & $16.1 \leq \geqslant \leq 31.3$ & 21.1 & $16.6 \leq P \leq 29.4$ & $0.4^{(t)}$ & $0.1 \leq r \leq 2.1$ & & & 0.4 & $0.1 \leq P \leq 2.1$ \\
\hline $2-2-7$ & 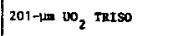 & $410-1=\mathrm{TrO}_{2} \mathrm{BrSO}$ & 1128 & 2.84 & 45.4 & $1.2 \times 10^{-3}$ & -2.62 & $\mid-3.43$ & 4 & 13.2 & $8.5 \leq P \leq 20.0$ & 19.9 & $14.0 \leq P \leq 27.3$ & 8.1 & $4.6 \leq 7 \leq 13.8$ & 0.0 & $0.0 \leq \mathrm{P} \leq 1.8$ & & & 0.0 & $0.0 \leq x \leq 1.0$ \\
\hline 2-3-7 & 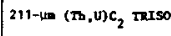 & 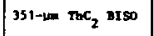 & 1117 & 2.84 & 45.4 & $1.8 \times 10^{-4}$ & -1.82 & $\mid-1.70$ & s & 0.7 & $0.3 \leq p \leq 1.7$ & 0.0 & $0.0 \leq P \leq 0.6$ & 0.0 & $0.0 \leq P \leq 0.6$ & 0.0 & $0.0 \leq x \leq 5.7$ & & & 0.0 & $0.0 \leq 1 \leq 5.7$ \\
\hline $2-4-7$ & $211-H=(\pi, y) C_{2}$ TrRso & 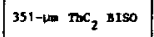 & 1117 & 2.84 & 45.4 & $1.1 \times 10^{-4}$ & -1.73 & $-2,37$ & 。 & & & & & & & & & & & & \\
\hline 2-s-9 & $211-\mathrm{im}(\mathrm{Th}, 0) \mathrm{C}_{2} \mathrm{TR} / \mathrm{so}$ & $360-5=\mathrm{TrC}_{2} \mathrm{TRMSO}$ & 112 & 2.84 & 45.4 & $2.3 \times 10^{-5}$ & $\mid-1,62$ & -1.86 & . & & & & & & & & & & & & \\
\hline $2-6-7$ & $211-1 \mathrm{H}(\mathrm{m}, \mathrm{n}, \mathrm{c}) \mathrm{C}_{2} \mathrm{Tarso}$ & $360-\ln =\mathrm{me}_{2} \mathrm{TRRSO}$ & 1112 & 2.84 & 45.4 & $1.1 \times 10^{-4} \mid$ & -1.55 & -1.70 & - & 1.4 & $0.7 \leq P \leq 2.7$ & 0.3 & $0.1 \leq P \leq 1.2$ & 0.2 & $0.0 \leq P \leq 1.0$ & 8.7 & $2.5 \leq r \leq 21.0$ & 0.0 & $0.0 \leq P \leq 7.5$ & 0.0 & $0.0 \leq 1 \leq 7.5$ \\
\hline $2-7-8$ & $99-10 \mathrm{uc} c_{2} \mathrm{Tz2150}$ & 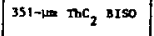 & 1128 & 2.83 & 45.2 & $1.7 \times 10^{-4}$ & -2.01 & -2.22 & 3 & 1.7 & $0.8 \leq P \leq 3.5$ & 2.2 & $1.2 \leq P \leq 4.1$ & 0.8 & $0.2 \leq P \leq 2.1$ & 0.0 & $0.0 \leq P \leq 2.2$ & & & 0.0 & $0.0 \leq P \leq 2.2$ \\
\hline $2-8-7$ & $99-\mathrm{W}=\mathrm{oc} \mathrm{C}_{2}$ тRISO & 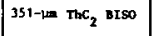 & 1821 & 2.84 & 65.4 & $2.6 \times 10^{-6}$ & -1.84 & -2.37 & 1 & & & & & & & & & & & & \\
\hline
\end{tabular}

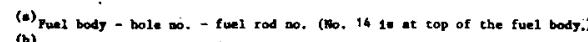

(b) Calculated by treva code.

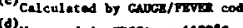

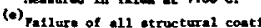

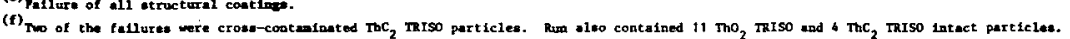


TABLE 4-42
FTE-6 FISSION GAS RELEASE SUIMARY

\begin{tabular}{|c|c|c|c|c|c|c|c|c|c|c|c|c|c|c|}
\hline \multirow[b]{3}{*}{$\begin{array}{l}\text { Fuel } \\
\text { Rod } \\
\text { I.D. }\end{array}$} & \multirow{2}{*}{\multicolumn{2}{|c|}{$\begin{array}{l}\text { Fraction of } \\
\text { Total Thor 1um }\end{array}$}} & \multirow{3}{*}{$\begin{array}{c}\text { Defective } \\
\text { Fissile } \\
\text { Particles }(a) \\
\text { C } \\
(z) \\
\end{array}$} & \multirow{2}{*}{\multicolumn{2}{|c|}{$\begin{array}{l}\text { Praction of } \\
\text { Fiationg (b) }\end{array}$}} & \multirow[b]{3}{*}{$\begin{array}{c}\underset{\text { Thortum }}{\text { Contamination }} \\
\text { Co } \\
\text { (g Th/g Th) } \\
\end{array}$} & \multirow[b]{3}{*}{\begin{tabular}{|c}
$\begin{array}{c}\text { Defective } \\
\mathrm{U}-235 \\
\mathrm{H}-\mathrm{CE}(\mathrm{R} / \mathrm{B})_{\mathrm{f}}\end{array}$ \\
\end{tabular}} & \multicolumn{3}{|c|}{ Calculated FGR for $\mathrm{Kr}-8 \mathrm{~s} \mathrm{~m}$} & \multirow[b]{3}{*}{$\begin{array}{l}\text { Expected FGR } \\
(R / B) K r-B S m \\
L=H+I+J+K \\
L\end{array}$} & \multirow[b]{3}{*}{ 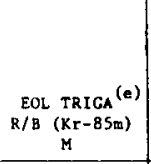 } & \multirow[b]{3}{*}{$\begin{array}{c}\Delta R / B \\
N=M-L\end{array}$} & \multirow[b]{3}{*}{ 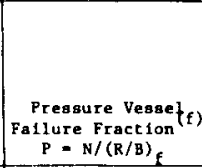 } \\
\hline & & & & & & & & \multirow[b]{2}{*}{$\begin{array}{c}\text { Fissile } \\
\text { Th } \\
\mathrm{I}=\mathrm{ACF}(\mathrm{R} / \mathrm{B}) \mathrm{E} \\
\end{array}$} & \multirow[b]{2}{*}{$\begin{array}{c}\mathrm{J} \\
\mathrm{J}\end{array}$} & \multirow[b]{2}{*}{$\begin{array}{l}\text { Thorium Cont }{ }^{(d)} \\
K=F G(R / B)\end{array}$} & & & & \\
\hline & \begin{tabular}{|c}
$\begin{array}{c}\text { Fisalle } \\
\mathbf{A} \\
(\mathrm{g} \mathrm{Th} / \mathrm{g} \mathrm{Th})\end{array}$ \\
\end{tabular} & \begin{tabular}{|c|}
$\begin{array}{c}\text { Fert1le } \\
\mathrm{B} \\
(\mathrm{B} \text { Th } / \mathrm{g} \text { Th }\end{array}$ \\
\end{tabular} & & $\underset{E}{U-235}$ & $\underset{F}{U-233}$ & & & & & & & & & \\
\hline $1-1-12$ & 0.000 & 1.000 & 0.10 & 0.56 & 0.44 & $5 \times 10^{-6}$ & $3.0 \times 10^{-6}$ & 0 & 0 & $6.6 \times 10^{-7}$ & $3.7 \times 10^{-6}$ & $2.4 \times 10^{-4}$ & $2.4 \times 10^{-4}$ & 4.5 \\
\hline $2-1-7$ & 0.000 & 1.000 & 0.10 & 0.52 & 0.48 & $5 \times 10^{-6}$ & $2.8 \times 10^{-6}$ & 0 & 0 & $7.2 \times 10^{-7}$ & $3.5 \times 10^{-6}$ & $1.1 \times 10^{-3}$ & $1.1 \times 10^{-3}$ & 20.8 \\
\hline $2-2-7$ & 0.000 & 1.000 & 0.10 & 0.52 & 0.48 & $5 \times 10^{-6}$ & $2.8 \times 10^{-6}$ & 0 & 0 & $1.2 \times 10^{-7}$ & $3.5 \times 10^{-6}$ & $1.2 \times 10^{-3}$ & $1.2 \times 10^{-3}$ & 22.6 \\
\hline $2-3-7$ & 0.777 & 0.223 & 0.07 & 0.74 & 0.26 & $1.5 \times 10^{-4}$ & $2.7 \times 10^{-6}$ & $7.5 \times 10^{-7}$ & 0 & $1.2 \times 10^{-5}$ & $1.5 \times 10^{-5}$ & $1.8 \times 10^{-4}$ & $1.7 \times 10^{-4}$ & 3.2 \\
\hline $2-4-7$ & 0.777 & 0.223 & 0.07 & 0.74 & 0.26 & $1.5 \times 10^{-4}$ & $2.7 \times 10^{-6}$ & $7.5 \times 10^{-7}$ & 0 & $1.2 \times 10^{-5}$ & $1.5 \times 10^{-5}$ & $1.1 \times 10^{-4}$ & $9.5 \times 10^{-5}$ & 1.8 \\
\hline $2-5-7$ & 0.801 & 0.199 & 0.74 & 0.14 & 0.26 & $3.7 \times 10^{-5}$ & $2.9 \times 10^{-5}$ & $8.2 \times 10^{-6}$ & 0 & $2.9 \times 10^{-6}$ & $4.0 \times 10^{-5}$ & $2.3 \times 10^{-5}$ & $-1.7 \times 10^{-5}$ & 0 \\
\hline $2-6-7$ & 0.801 & 0.199 & 0.74 & 0.74 & 0.26 & $3.7 \times 10^{-5}$ & $2.9 \times 10^{-5}$ & 8. $2 \times 10^{-6}$ & 0 & $2.9 \times 10^{-6}$ & $4.0 \times 10^{-5}$ & $2.3 \times 10^{-5}$ & $4.7 \times 10^{-5}$ & 0 \\
\hline $2-7-8$ & 0.000 & 1.000 & 0.50 & 0.81 & 0.19 & $2.5 \times 10^{-5}$ & $2.1 \times 10^{-5}$ & 0 & 0 & $1.4 \times 10^{-6}$ & $2.2 \times 10^{-5}$ & $1.7 \times 10^{-4}$ & $1.5 \times 10^{-4}$ & 2.8 \\
\hline $2-8-7$ & 0.000 & 1.000 & 0.50 & 0.81 & 0.19 & $2.5 \times 10^{-5}$ & $2.1 \times 10^{-5}$ & 0 & 0 & $1.4 \times 10^{-6}$ & $2.2 \times 10^{-5}$ & $1.7 \times 10^{-4}$ & $1.5 \times 10^{-4}$ & 2.8 \\
\hline
\end{tabular}

(a) calculated from nominal preirradiation fission gas release measurenent.

(b) GAUGE/FEvER calculated.

(c) Nom1nal preirradiation hydrolysis measurement.

(d) $(\mathrm{R} / \mathrm{B})_{\mathrm{C}}=0.3$

(e) ${ }_{\mathrm{At}} 1100^{\circ} \mathrm{C}$.

${ }^{(f)}(R / B) F$ for constralned geometry equal to 0.0053 . 
TABLE 4-43

FTE-6 FUEL ROD GAMMA SCAN SUMMARY

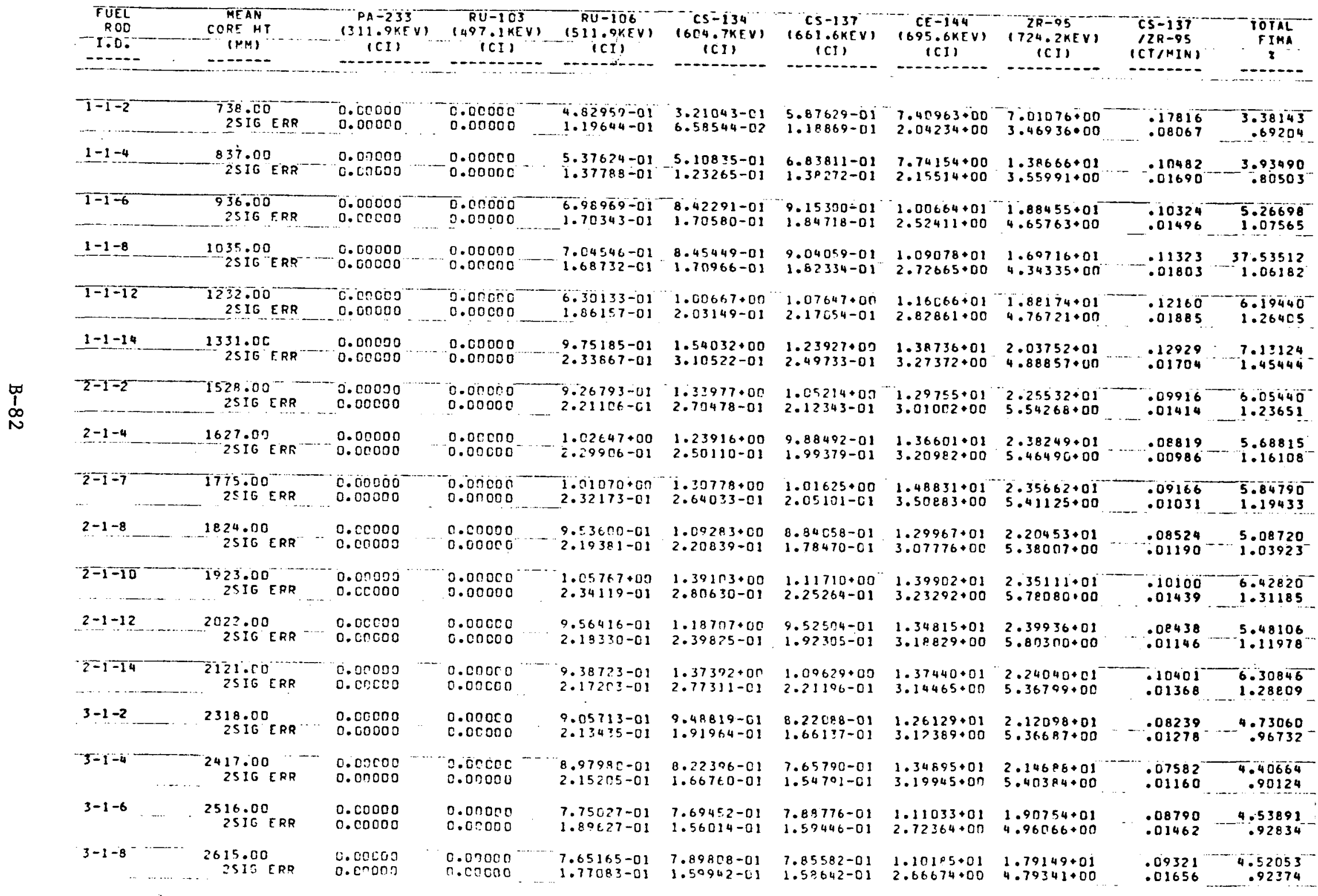


TABLE 4-43 (Continued)

\begin{tabular}{|c|c|c|c|c|c|c|c|c|c|c|}
\hline $\begin{array}{c}\text { TUEL } \\
\text { ROD } \\
\text { TOO: }\end{array}$ & $\begin{array}{l}\text { MEAN } \\
\text { CORE HT } \\
\text { (PM) }\end{array}$ & $\begin{array}{c}P A-233 \\
(311.9 K E V) \\
(C I)\end{array}$ & $\begin{array}{c}R U=103 \\
(497.1 \text { KEVI } \\
\text { (CI) }\end{array}$ & $\begin{array}{c}R Q-106 \\
\text { (511.9KEY) } \\
\text { (CI) }\end{array}$ & $\begin{array}{c}\text { CS-i34 } \\
(604.7 K E V) \\
(C I)\end{array}$ & $\begin{array}{l}\text { CS-137 } \\
(66) .6 K E V) \\
(C I)\end{array}$ & $\begin{array}{l}\text { CE-144 } \\
(695.6 K E V) \\
(C 1)\end{array}$ & $\begin{array}{c}2 R=95 \\
1724.2 K E V) \\
(C 1)\end{array}$ & $\begin{array}{l}C S E-i 3 j \\
12 R-95 \\
1 C T I M I N I \\
\end{array}$ & $\begin{array}{l}\text { TotiaL } \\
\text { FIMA }\end{array}$ \\
\hline $3-1-10$ & $\begin{array}{r}2713006 \\
2516 \text { ERR }\end{array}$ & $\begin{array}{l}0.00000^{\circ} \\
0.00000\end{array}$ & $\begin{array}{l}0.00000 \\
0.00000\end{array}$ & $\begin{array}{l}6.09078-01 \\
1.47900-01\end{array}$ & $\begin{array}{l}6.68511-01 \\
1.35512-01\end{array}$ & $\begin{array}{l}7.62821-01 \\
1.54031-01\end{array}$ & $\begin{array}{l}9.02207+00 \\
2.37221+00\end{array}$ & $\begin{array}{l}1.55432+01 \\
4.24193+00\end{array}$ & $\begin{array}{l}.10432 \\
.01936\end{array}$ & $\begin{array}{r}4 . \overline{38956} \\
.89690\end{array}$ \\
\hline $3-1-12$ & $\begin{array}{l}281 ? .00 \\
25 I G E R R\end{array}$ & $\begin{array}{l}\text { C. } 00000 \\
0.00000\end{array}$ & $\begin{array}{l}0.0 \operatorname{ccc} 0 \\
0.00000\end{array}$ & $\begin{array}{l}5.93801-01 \\
1.46999-01\end{array}$ & $\begin{array}{l}4.73929-01 \\
9.68382-02\end{array}$ & $\begin{array}{l}6.70677-01 \\
1.35683-01\end{array}$ & $\begin{array}{l}7.951 C 8+00 \\
2.15616+00\end{array}$ & $\begin{array}{l}1.38225+01 \\
4.06229+00\end{array}$ & $\begin{array}{l}.10314 \\
.02224\end{array}$ & $\begin{array}{l}33 \\
93^{-}\end{array}$ \\
\hline $3-1-14$ & $\begin{array}{r}2911000 \\
25 I G \text { ERR }\end{array}$ & $\begin{array}{l}0.00000 \\
0.00000\end{array}$ & $\begin{array}{l}c .00000 \\
0.00000\end{array}$ & $\begin{array}{l}5.69211-01 \\
1.45005-01\end{array}$ & $\begin{array}{l}3.98398-01 \\
8.14752-02\end{array}$ & $\begin{array}{l}6.58904-01 \\
1.33159-01\end{array}$ & $\begin{array}{l}7.12663+00 \\
1.95171+00\end{array}$ & $\begin{array}{l}1.65804+01 \\
4.58586+00\end{array}$ & $\begin{array}{l}.087710 \\
.01771 .\end{array}$ & $\begin{array}{r}3.79158 \\
.07531\end{array}$ \\
\hline $1-2-6$ & $\begin{array}{l}936^{\circ} .00 \\
25 I G^{\circ} E R R\end{array}$ & $\begin{array}{l}0.00000 \\
0.00000\end{array}$ & $\begin{array}{l}0.00000 \\
0.00000\end{array}$ & $\begin{array}{l}7.10639-01 \\
1.63383-01\end{array}$ & $\begin{array}{l}7.71765-01 \\
1.564 \geq 3-01\end{array}$ & $\begin{array}{l}8.51057-01 \\
1.71897-01\end{array}$ & $\begin{array}{l}9.19035+00 \\
2.42120+00\end{array}$ & $\begin{array}{l}1.62303+01 \\
4.48140+00\end{array}$ & $\begin{array}{l}.11146 \\
.02121\end{array}$ & $\begin{array}{l}4.71 .893 \\
1.00091\end{array}$ \\
\hline $2-2-1$ & $\begin{array}{r}1479.00 \\
2516 \text { ERR }\end{array}$ & $\begin{array}{l}0.00000 \\
c .00030\end{array}$ & $\begin{array}{l}0.000 \\
0.000\end{array}$ & $\begin{array}{l}9.19 \\
2.26\end{array}$ & $\begin{array}{l}1.40023+0.0 \\
2.63048-01\end{array}$ & $\begin{array}{l}1.09873+00 \\
2.21965-01\end{array}$ & $\begin{array}{l}1.27330 .01 \\
2.87764+00\end{array}$ & $\begin{array}{l}2.06258 \cdot 01 \\
4.19621+00\end{array}$ & & $\begin{array}{l}6.32249 \\
1.29242\end{array}$ \\
\hline $2-2$ & $\begin{array}{l}1528 . C 0 \\
2516 E R R^{-}\end{array}$ & $\begin{array}{l}0.00000 \\
0.00000 .\end{array}$ & $\begin{array}{l}0.00000 \\
0.00000\end{array}$ & $\begin{array}{l}9.65650-01 \\
2.30946-01\end{array}$ & $\begin{array}{l}1.39 \\
2.82\end{array}$ & $\begin{array}{l}1.1 \\
2.2\end{array}$ & $\begin{array}{l}1.2 \times C \\
2.8 \times 5\end{array}$ & $\begin{array}{l}2.17 \\
4.43\end{array}$ & $\begin{array}{l}.10853 \\
.00404\end{array}$ & $\begin{array}{l}6.49295 \\
1.30928\end{array}$ \\
\hline $2-2-3$ & $\begin{array}{r}1578.00 \\
2516 \text { CRR }\end{array}$ & $\begin{array}{l}0.0 n 000 \\
0.00000\end{array}$ & $\begin{array}{l}0.02000 \\
0.00000\end{array}$ & $\begin{array}{l}9.77174-01 \\
2.36498-01\end{array}$ & $\begin{array}{l}1.35904+0 r \\
2.74780-01\end{array}$ & $\begin{array}{l}1.06542 .00 \\
2.15401-01\end{array}$ & $\begin{array}{l}1.32520+01 \\
2.96652+00\end{array}$ & $\begin{array}{l}2.20311+01 \\
4.48247+03\end{array}$ & $\begin{array}{l}-10279^{\circ} \\
.05393\end{array}$ & $\begin{array}{l}6.13080^{-1} \\
1.25411 \ldots\end{array}$ \\
\hline $2-2-4$ & $\begin{array}{l}1627.00 \\
2510^{\circ} E R R\end{array}$ & $\begin{array}{l}0.00000 \\
0.00000\end{array}$ & $\begin{array}{l}0.00000 \\
0.00000\end{array}$ & $\begin{array}{l}9.70538-01 \\
2.30813-01\end{array}$ & $\begin{array}{l}1.18307+00 \\
2.39379-01\end{array}$ & $\begin{array}{l}9.53754-01 \\
1.92 E 98-01\end{array}$ & $\begin{array}{l}1.24082+01 \\
2.82725+00\end{array}$ & $\begin{array}{l}2.07 \\
4.21\end{array}$ & & $\begin{array}{l}5.48826 \\
1.12305\end{array}$ \\
\hline & $\begin{array}{r}1676.00 \\
2510 \text { ERR }\end{array}$ & $\begin{array}{l}0.00000 \\
0.00000\end{array}$ & $\begin{array}{l}0.00 \\
0.00\end{array}$ & $\begin{array}{l}9.476 \\
2.232\end{array}$ & $\begin{array}{l}1.08 \\
2.19\end{array}$ & $\begin{array}{l}6.79 \\
1.77\end{array}$ & $\begin{array}{l}1.29 \\
2.94\end{array}$ & $\begin{array}{l}2.11 \\
4.30\end{array}$ & & \\
\hline $2-2-$ & $\begin{array}{l}1726.00 \\
2516 \text { ERR }\end{array}$ & $\begin{array}{l}0.00000 \\
0.00000\end{array}$ & $\begin{array}{r}0.00000 \\
0.00000\end{array}$ & $\frac{1}{2.01626+00}$ & $\begin{array}{l}1.26 \\
2.55\end{array}$ & $\begin{array}{l}1.00 \\
2.0\end{array}$ & $\begin{array}{l}1.47 \\
3.28\end{array}$ & $\begin{array}{l}2.36 \\
4.81\end{array}$ & $\begin{array}{l}.09054 \\
.00333\end{array}$ & $\begin{array}{l}5.80792 \\
1.18841\end{array}$ \\
\hline $2-2-7$ & $\begin{array}{l}1715.00 \\
2516 \text { ERR }\end{array}$ & $\begin{array}{l}0.00<00 \\
0.00000\end{array}$ & $\begin{array}{l}0.00300 \\
0.00000\end{array}$ & $\begin{array}{l}1.00941+00 \\
2.29524-01\end{array}$ & $\begin{array}{l}1.33756+00 \\
2.700 ? 0-01\end{array}$ & $\begin{array}{l}1.04002+00 \\
2.07888-01\end{array}$ & $\begin{array}{l}1.40816+01 \\
3.30305000\end{array}$ & $\begin{array}{l}2.20034 \div 01 \\
4.98891+00\end{array}$ & $\begin{array}{l}10047 \\
.01069\end{array}$ & $\begin{array}{l}5.76669 \\
1.22222\end{array}$ \\
\hline $2-2-8$ & $\begin{array}{l}1824.00 \\
25 I G E R R\end{array}$ & $\begin{array}{l}0.00000 \\
0.00000\end{array}$ & $\begin{array}{l}0.00000 \\
0.00000\end{array}$ & $\begin{array}{l}9.92807-01 \\
2.27109-01\end{array}$ & $\begin{array}{l}1.13696+00 \\
2.29817-01\end{array}$ & $\begin{array}{l}9.03436-01 \\
1.82468-01\end{array}$ & $\begin{array}{l}1.26480 .01 \\
3.03184+00\end{array}$ & $\begin{array}{l}2.41225+01 \\
5.93947+00\end{array}$ & .07961 & $\begin{array}{l}5.00936 \\
1.06246\end{array}$ \\
\hline $2-2-9$ & $\begin{array}{l}1874.00 \\
\text { TSIG ERR }\end{array}$ & $\begin{array}{l}0.00000 \\
0.00000\end{array}$ & $\begin{array}{l}0.00000 \\
\text { C.05000 }\end{array}$ & $\begin{array}{l}9.49149-01 \\
2.23820-01\end{array}$ & $\begin{array}{l}1.12794+0 C \\
2.28512-01\end{array}$ & $\begin{array}{l}9.21972-01 \\
1.86629-01\end{array}$ & $\begin{array}{l}1.30194+01 \\
2.94175+00\end{array}$ & $\begin{array}{l}2.19915+01 \\
4.47072+00\end{array}$ & $\begin{array}{l}.08911 \\
.00345\end{array}$ & $\begin{array}{l}5.30537 \\
1.00646\end{array}$ \\
\hline $2-2-10$ & $\begin{array}{l}1923.00 \\
25 I 6 \text { ERR }\end{array}$ & $\begin{array}{l}0.00000 \\
0.00000\end{array}$ & $\begin{array}{l}0.00000 \\
0.00000\end{array}$ & $\begin{array}{l}9.49848-01 \\
2.26504-01\end{array}$ & $\begin{array}{l}1.281 \times 0+00 \\
2.59217-01\end{array}$ & $\begin{array}{l}1.02206+00 \\
2.05646-01\end{array}$ & $\begin{array}{l}1.28059+01 \\
2.93553+00\end{array}$ & $\begin{array}{l}2.17651 .01 \\
4.42568+00\end{array}$ & $\begin{array}{l}.09982 \\
.00376\end{array}$ & $\begin{array}{l}5.88130 \\
1.20313-\end{array}$ \\
\hline $3-2-6$ & $\begin{array}{l}2516.00 \\
25 \text { IG ERR }\end{array}$ & $\begin{array}{l}0.00000 \\
0.00000\end{array}$ & $\begin{array}{l}0.00000 \\
0.00000\end{array}$ & $\begin{array}{l}9.45274-01 \\
2.13242-01\end{array}$ & $\begin{array}{l}8.657 \geq 1-01 \\
1.75079-0.1\end{array}$ & $\begin{array}{l}8.13717-01 \\
1.64247-01\end{array}$ & $\begin{array}{l}1.14166+01 \\
2.69456+00\end{array}$ & $\begin{array}{l}2.14577 .01 \\
5.16121 .00\end{array}$ & $\begin{array}{l}.08061 \\
.01075\end{array}$ & $\begin{array}{r}4.68235 \\
.95641\end{array}$ \\
\hline
\end{tabular}


TABLE 4-43 (Continued)

\begin{tabular}{|c|c|c|c|c|c|c|c|c|c|}
\hline $\begin{array}{r}\text { FUEL } \\
\text { POD } \\
1.0 .- \\
\hdashline\end{array}$ & $\begin{array}{l}\text { MEAN } \\
\text { CORE } \\
\text { MY } \\
\end{array}$ & $\begin{array}{c}P A-233 \\
(311.9 K E V) \\
(C 1)\end{array}$ & $\begin{array}{r}R U-103 \\
(497.1 \mathrm{KEV}) \\
\text { (CI) }\end{array}$ & $\begin{array}{l}\text { RU-106 } \\
\text { (5) } 11.9 \mathrm{KEV}) \\
\text { (CI) }\end{array}$ & $\begin{array}{c}C S=134 \\
(604.7 \times E V) \\
(C 1) \\
(C 1)\end{array}$ & 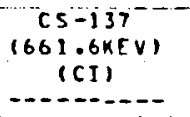 & $\begin{array}{c}C E-144 \\
1695.6 k 2 \mathrm{KV}) \\
(C 1)\end{array}$ & $\begin{array}{l}2 \mathrm{R}=95 \\
(724.2 \mathrm{KEV}) \\
(\mathrm{C} 1)\end{array}$ & 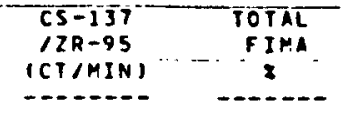 \\
\hline$\sqrt{1-3}-2$ & $\begin{array}{r}73800 \\
22510 \text { ERR }\end{array}$ & $\begin{array}{l}0.00000 \\
0.00000\end{array}$ & $\begin{array}{l}0.00000 \\
0.00000\end{array}$ & $\begin{array}{l}9.76238-01 \\
2.42121-01\end{array}$ & $\begin{array}{l}3.65173-01 \\
7.47713-02\end{array}$ & $\begin{array}{l}6.08894-\bar{C} 1- \\
1.23110-01\end{array}$ & $\begin{array}{l}6.28 \\
1.77\end{array}$ & $\begin{array}{r}1.295 \\
3.553\end{array}$ & $\begin{array}{l}7.58170 \\
1.55097\end{array}$ \\
\hline $1-3-4$ & $-8 \frac{37}{25}=00$ & $\begin{array}{l}1.89428+05 \\
3.80765+04\end{array}$ & $\begin{array}{l}0.00000 \\
0.00000\end{array}$ & $\begin{array}{l}6.31562=01 \\
1.55467=01\end{array}$ & $\begin{array}{l}5.82511-01 \\
-1.18582-01\end{array}$ & $\begin{array}{l}7.52788-01 \\
1.52171-01\end{array}$ & $\begin{array}{l}1.80723+00 \\
1.43435+00\end{array}$ & $\begin{array}{l}1.37232+01 \\
-3.78257+00\end{array}$ & 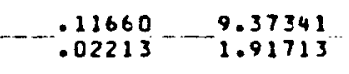 \\
\hline $1-3-6$ & $\begin{array}{l}936.00 \\
2 S I G E P R\end{array}$ & $\begin{array}{l}0.00000 \\
0.00000\end{array}$ & $\begin{array}{l}0.00000 \\
0.00000\end{array}$ & $\begin{array}{l}7.18956-01 \\
1.77064-01\end{array}$ & $\begin{array}{l}8.33769-01 \\
1.890 \geq 2-01\end{array}$ & $\begin{array}{l}8.90473-01 \\
1.79841-01\end{array}$ & $\begin{array}{l}1.01305+01 \\
2.55296+00\end{array}$ & $\begin{array}{l}1.65239 \cdot 01 \\
4.49619 \cdot 00\end{array}$ & $\begin{array}{r}11455 \quad 11.08780 \\
002113 \\
2.26592\end{array}$ \\
\hline $1-3-8$ & $\begin{array}{l}1034.00 \\
2516^{\circ} \text { ERR }\end{array}$ & $\begin{aligned} 0.00000 \\
0.00000\end{aligned}$ & $\begin{array}{l}0.00000 \\
0.00000\end{array}$ & $\begin{array}{l}8.52703-01 \\
2.04030-01\end{array}$ & $\begin{array}{r}9.33016-01 \\
1.33821-01\end{array}$ & $\begin{array}{l}9.59010-01 \\
1.93523-01\end{array}$ & $\begin{array}{l}1.06600+01 \\
2.68383+00\end{array}$ & $\begin{array}{l}1.76158+01 \\
4.58446+00\end{array}$ & $\begin{array}{r}11572 \\
-01924\end{array}-\frac{11.94120}{2.43849}$ \\
\hline $1=3-10$ & $\begin{array}{l}1133.00 \\
2516 \text { ERR }\end{array}$ & $\begin{array}{l}0.00000^{\circ} \\
0.00000\end{array}$ & $\begin{array}{l}0.00000 \\
0.00000\end{array}$ & $\begin{array}{l}7.64550-01 \\
1.99263-01\end{array}$ & $\begin{array}{l}1.01181+00 \\
2.04685-01\end{array}$ & $\begin{array}{l}9.50966-01 \\
1.93830-01\end{array}$ & $\begin{array}{l}9.26806+00 \\
2.09371+00\end{array}$ & $\begin{array}{l}1.7 P 440 * 01- \\
4.60665+00\end{array}$ & $\begin{array}{r}.11435 \\
.011 .95311 \\
.01865 \\
2.44223\end{array}$ \\
\hline $1-3-12$ & $\begin{array}{l}1232.00 \\
-2516 \mathrm{ERR}\end{array}$ & $\begin{array}{l}0.00000 \\
0.00000\end{array}$ & $\begin{array}{l}0.00000 \\
0.00000\end{array}$ & $\begin{array}{l}9.14201-01 \\
2.07918-01\end{array}$ & $\begin{array}{l}1.41715+00 \\
2.26181-01\end{array}$ & $\begin{array}{l}1.15218+00 \\
2.32474-01\end{array}$ & $\begin{array}{l}1.15128 \cdot 01 \\
2.95491+00\end{array}$ & $\begin{array}{r}2.06951 .01 \\
-5.27456 .00\end{array}$ & $\begin{array}{r}14.34653 \\
2.92934\end{array}$ \\
\hline $1-3-14$ & $\begin{array}{r}1331000 \\
2516 \text { ERR }\end{array}$ & $\begin{array}{l}0.00000 \\
0.00000\end{array}$ & $\begin{array}{l}0.00000 \\
0.00000\end{array}$ & $\begin{array}{l}1.07091+000 \\
2.52757-01\end{array}$ & $\begin{array}{l}-1.64125+00 \\
3.31151-01\end{array}$ & $\begin{array}{l}1.24615 \cdot 000 \\
2.51327-01\end{array}$ & $\begin{array}{l}1.42540 \cdot 01 \\
3.34570+00\end{array}$ & $\begin{array}{l}2.224290 .01 \\
5.47411 .00\end{array}$ & $\begin{array}{r}.11909 \\
001702 \quad 35151651 \\
016703\end{array}$ \\
\hline $2-3-2$ & $\begin{array}{l}1528.00 \\
2516^{\circ} \text { ERR }\end{array}$ & $\begin{array}{l}0.00000 \\
0.00000\end{array}$ & $\begin{array}{l}0.00000 \\
0.00000^{\circ}\end{array}$ & $\begin{array}{l}\frac{1}{2.04853+00} 2.041989=01 \\
2.49\end{array}$ & $\begin{array}{r}1.68307+00 \\
-3.39532-01\end{array}$ & $\begin{array}{l}1.22832+00 \\
2.47692-01\end{array}$ & $\begin{array}{l}1.34753+01 \\
3.22284 \div 00\end{array}$ & $\begin{array}{l}2.27419+01 \\
5.46825+00\end{array}$ & $\begin{array}{rr}11481 & 15.29454 \\
.01527 & 3.12127\end{array}$ \\
\hline $2-3-4$ & $\begin{array}{l}1627.00 \\
2516 \text { ERR }\end{array}$ & $\begin{array}{l}0.00000^{-} \\
0.00000\end{array}$ & $\begin{array}{l}0.00000 \\
0.00000\end{array}$ & $\begin{array}{l}1.020027+00 \\
2.31235-01\end{array}$ & $\begin{array}{l}1.71589+00 \\
3.46053-01\end{array}$ & $\begin{array}{l}1.226760+00 \\
2.47459-01\end{array}$ & $\begin{array}{l}1.37855+01 \\
3.17952+00\end{array}$ & $\begin{array}{l}2.29014+01 \\
5.64013+00\end{array}$ & $\begin{array}{r}.11386 \\
\therefore 01632 \\
\therefore\end{array}$ \\
\hline $2-3-7$ & $\begin{array}{l}1775.00 \\
2516 \text { ERR }\end{array}$ & $\begin{array}{l}0.00000 \\
0.00000\end{array}$ & $\begin{array}{l}0.00000 \\
0.00000\end{array}$ & $\begin{array}{l}1.07117+00 \\
2.442222-01\end{array}$ & $\begin{array}{l}1.70895+00 \\
3.44504-01\end{array}$ & $\begin{array}{l}1.24276+00 \\
2.50615-01\end{array}$ & $\begin{array}{l}1.31420+01 \\
3.06253+00\end{array}$ & $\begin{array}{l}2.22 \\
5.06\end{array}$ & $\begin{array}{r}.11882 \\
-.01291\end{array}$ \\
\hline $2-3=8$ & $\begin{array}{r}1824.00 \\
2516 \text { ERR }\end{array}$ & $\begin{array}{l}0.00000^{-} \\
0.00000\end{array}$ & $\begin{array}{l}0.000000 \\
0.00000\end{array}$ & $\begin{array}{l}1.07378+00 \\
2.47712-01\end{array}$ & $\begin{array}{l}1.63463 .00 \\
3.29787-01\end{array}$ & $\begin{array}{l}1.19048+00 \\
2.40140-01\end{array}$ & $\begin{array}{l}1.20210+01 \\
2.90468 \cdot 00\end{array}$ & $\begin{array}{l}2.04571+01 \\
5.29798 \cdot 00\end{array}$ & 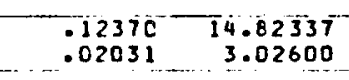 \\
\hline $2-3-30$ & $\begin{array}{l}1923.00 \\
2510^{\circ} \text { ERR }\end{array}$ & $\begin{array}{l}0.00000 \\
0.00000\end{array}$ & $\begin{array}{l}0.00000 \\
0.00000\end{array}$ & $\begin{array}{l}1.05998+00 \\
2.45975-01\end{array}$ & $\begin{array}{l}1.58065+00 \\
3.18965-01\end{array}$ & $\begin{array}{l}1.16497 \times 00 \\
2.35063-01\end{array}$ & $\begin{array}{l}1.32324+01 \\
3.12799+00\end{array}$ & $\begin{array}{l}2 \cdot 10058 \times 01 \\
5: 358<2+00\end{array}$ & $\begin{array}{rr}.11789 & 14.50569 \\
.01864 & 2.96195\end{array}$ \\
\hline $2-3-12$ & $\begin{array}{r}2022.00 \\
251 G E P R\end{array}$ & $\begin{array}{l}0.00000 \\
\text { c. } 00000\end{array}$ & $\begin{array}{l}0.00000 \\
0.00000\end{array}$ & $\begin{array}{l}8+00 \\
4-01\end{array}$ & $\begin{array}{l}1.59 \\
3.72\end{array}$ & $\begin{array}{l}1.38752+00 \\
2.39542-01\end{array}$ & $\begin{array}{l}1.32862+01 \\
3.16542+00\end{array}$ & $\begin{array}{l}2.26104 * 01 \\
5.71441 * 00\end{array}$ & $\begin{array}{r}111164 \\
001721 \\
0\end{array}$ \\
\hline $2-3-14$ & $\begin{array}{l}2121.00^{\circ} \\
=2510^{\circ} \mathrm{ERR}^{-}\end{array}$ & $\begin{array}{l}0.00000 \\
0.00000\end{array}$ & $\begin{array}{l}0.00000 \\
0.00000\end{array}$ & $\begin{array}{l}1.10754+00 \\
2.50658-01\end{array}$ & $\begin{array}{r}1.63767+00 \\
-3.30161-01\end{array}$ & $\begin{array}{l}1.18559+00 \\
2.38985-01\end{array}$ & $\begin{array}{l}1.21449+01 \\
2.87155+00\end{array}$ & $\begin{array}{l}2.25475+01 \\
5.54127+00\end{array}$ & $\begin{array}{rr}11177 & 14.76252 \\
.01589 & 3.01165\end{array}$ \\
\hline $3-3=2$ & $\begin{array}{r}2319.00 \\
2516 E R R\end{array}$ & $\begin{array}{l}0.00000 \\
0.00000\end{array}$ & $\begin{array}{l}0.00000 \\
0.00000\end{array}$ & $\begin{array}{r}9.82537-01 \\
2.31539-01\end{array}$ & $\begin{array}{l}-1.38015+00^{-1} \\
2.78697-01\end{array}$ & $\begin{array}{l}1.08670+00 \\
2.19289-01\end{array}$ & $\begin{array}{l}1.27440+01 \\
3.04725+00\end{array}$ & $\begin{array}{r}2.16242+01 \\
5.46778+00\end{array}$ & 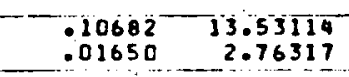 \\
\hline $3-3-4$ & $\begin{array}{l}2417.00 \\
2516 \text { ERR }\end{array}$ & $\begin{array}{l}0.00000 \\
0.00000\end{array}$ & $\begin{array}{l}0.00000 \\
0.00000\end{array}$ & $\begin{array}{l}9.06672-01 \\
2.08102-01\end{array}$ & $\begin{array}{l}1.30406+00 \\
2.63307-01\end{array}$ & $\begin{array}{l}1.01219+00 \\
2.04263-01\end{array}$ & $\begin{array}{l}1.11111+01 \\
2.68790+00\end{array}$ & $\begin{array}{r}2.25074+01 \\
5.43105+00\end{array}$ & $\begin{array}{rr}.09559 & 12.60341 \\
.01288 & 2.57382\end{array}$ \\
\hline $3-3-6$ & $\begin{array}{r}2516.00 \\
251 G \text { ERR }\end{array}$ & $\begin{array}{l}0.00000 \\
0.00000\end{array}$ & $\begin{array}{l}0.00000 \\
0.00000\end{array}$ & $\begin{array}{l}8.84761-01 \\
2.04063-01\end{array}$ & $\begin{array}{l}1.10406+00 \\
2.23133-01\end{array}$ & $\begin{array}{l}9.62238-01 \\
1.94234-01\end{array}$ & $\begin{array}{l}2.13428+01 \\
2.67595+00\end{array}$ & $\begin{array}{r}1.83258+01 \\
4.68087+00\end{array}$ & 0.01161 \\
\hline
\end{tabular}


TABLE 4-43 (Continued)

\begin{tabular}{|c|c|c|c|c|c|c|c|c|c|c|}
\hline $\begin{array}{l}\text { TuEt } \\
\text { RoD } \\
\text { rod }\end{array}$ & $\begin{array}{l}\text { METT } \\
\text { CORE HT } \\
\text { (RM) }\end{array}$ & $\begin{array}{l}P A=233 \\
\text { (3)1.9kEV) } \\
\text { (CII }\end{array}$ & 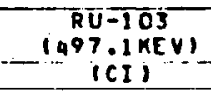 & $\begin{array}{c}\text { RU }-106 \\
\text { (51) } 9 \times 1 \mathrm{KV} \\
\text { (CI) }\end{array}$ & 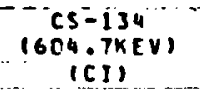 & $\begin{array}{c}\text { CS-137 } \\
\text { (661.6KEV } \\
\text { ICII }\end{array}$ & $\begin{array}{l}\text { CE } \\
\text { 1695.6KEV } \\
\text { ICII }\end{array}$ & 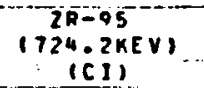 & $\begin{array}{l}(5-133 \\
228-95 \\
(C T M I N)\end{array}$ & $\begin{array}{c}\text { TojaL } \\
\text { rima } \\
8\end{array}$ \\
\hline $3-3-8$ & $\begin{array}{l}26 \overline{15.00} \\
2516 \quad \text { ERR }\end{array}$ & $\begin{array}{l}0.000000^{-0} \\
0.00000 .\end{array}$ & $\begin{array}{l}0.00000 \\
0.00006\end{array}$ & $\begin{array}{l}8.3484901 \\
1.95612-01\end{array}$ & $\begin{array}{l}9.56887-01^{-} \\
9.93511-01\end{array}$ & $\begin{array}{l}9.16391-01 \\
1.850400-01\end{array}$ & $\begin{array}{l}1.1026701 \\
2.74191000\end{array}$ & $\begin{array}{l}1.75924001 \\
4.60191+00\end{array}$ & $\begin{array}{l}.11072 \\
.01866\end{array}$ & $\begin{array}{r}1 \longdiv { 1 . 4 1 0 5 3 } \\
2.33147\end{array}$ \\
\hline $3-3-10$ & $\begin{array}{l}2713.00 \\
251 G^{\circ} \text { ERR }\end{array}$ & $\begin{array}{l}0 . \operatorname{cecos} \\
0 . \operatorname{crcos0}\end{array}$ & $\begin{array}{l}0.0000 \mathrm{c} \\
0.00000^{\circ}\end{array}$ & $\begin{array}{r}7.85096-01 \\
-\because .82478-01\end{array}$ & $\begin{array}{l}8.03258-01 \\
1.62625-01\end{array}$ & $\begin{array}{l}8.51036-01 \\
1.71868-01\end{array}$ & $\begin{array}{l}1.01596 \times 01 \\
2.51448 \times 00\end{array}$ & $\begin{array}{l}1.76807+01 \\
4.52703000\end{array}$ & $\begin{array}{l}.10231 \\
.01635\end{array}$ & $\begin{array}{r}10.59675 \\
2.16547^{-}\end{array}$ \\
\hline$-3-3-12$ & $\begin{array}{r}2816.00 \\
2516 \text { ERR }\end{array}$ & $\begin{array}{l}0.00000 \\
0.00000\end{array}$ & & $\begin{array}{l}5.66603-01 \\
1.39723-01\end{array}$ & $\begin{array}{l}4.80263-01 \\
9.788 ? 3-02\end{array}$ & $\begin{array}{l}6.43238-01 \\
1.30089-01\end{array}$ & $\begin{array}{l}7.82223 \cdot 00 \\
2.01794+00\end{array}$ & $\begin{array}{l}1.28931+01 \\
3.66048+00\end{array}$ & $\begin{array}{l}.10605 \\
.02139\end{array}$ & \\
\hline $3-3-14$ & $\begin{array}{l}2911.00 \\
\quad 2 S I G E R R\end{array}$ & $\begin{array}{l}0.00000 \\
0.00000\end{array}$ & $\begin{array}{l}0.0 n 000 \\
0.00000\end{array}$ & $\begin{array}{l}5.32058-01 \\
1.36329-01\end{array}$ & $\begin{array}{r}4.05193-01 \\
-8.32390-02\end{array}$ & $\begin{array}{l}6.38501-01 \\
1.29188-01\end{array}$ & $\begin{array}{l}8.27438+00 \\
2.21996+00\end{array}$ & $\begin{array}{l}1.03747+01 \\
3.12707 .00\end{array}$ & $\begin{array}{l}.13082 \\
-.02954\end{array}$ & $\begin{array}{r}7.95036 \\
3.62744^{-}\end{array}$ \\
\hline $1-4-6$ & $\begin{array}{l}936.02 \\
2 S 16 \quad R R\end{array}$ & $\begin{array}{l}\text { c. } 0000000^{-} \\
0.07000\end{array}$ & $\begin{array}{l}0.000000 \\
0.00000\end{array}$ & $\begin{array}{l}7.90531-01 \\
1.83031-01\end{array}$ & $\begin{array}{l}8.10272-01 \\
1.65979-01\end{array}$ & $\begin{array}{l}8.82381-01 \\
1.78198-01\end{array}$ & $\begin{array}{l}9.37366+00 \\
2.29347 * 00\end{array}$ & $\begin{array}{l}1.56165 \cdot 01 \\
4.27992 * 00\end{array}$ & $\begin{array}{r}.12010 \\
.02250\end{array}$ & $\begin{array}{r}10.98705 \\
2.24523\end{array}$ \\
\hline $2-4-7$ & $\begin{array}{l}1775.00 \\
-250^{2}=R_{R}\end{array}$ & $\begin{array}{r}0.00000 \\
0.00000\end{array}$ & $\begin{array}{r}0.00000 \\
0.00000\end{array}$ & $\begin{array}{r}1.07888 \cdot 00 \\
-2.40142-01\end{array}$ & $\begin{array}{l}1.69173+00 \\
3.41009-01\end{array}$ & $\begin{array}{l}1.21266 \cdot 00 \\
2.44553-01\end{array}$ & $\begin{array}{l}1.37321+01 \\
3.67180+00\end{array}$ & $\begin{array}{l}2.09612 \cdot 01 \\
4.822250 .00\end{array}$ & $\begin{array}{l}.12297 \\
001391\end{array}$ & $\begin{array}{r}15.09953 \\
3.08169\end{array}$ \\
\hline $2-4-8$ & $\begin{array}{r}1824000 \\
2516 \text { ERR }\end{array}$ & $\begin{array}{l}0.000000 \\
\text { c.coodo }\end{array}$ & $\begin{array}{l}C .0 n c 000 \\
0.00000\end{array}$ & $\begin{array}{l}1.02177+00 \\
2.46780-01\end{array}$ & $\begin{array}{l}1.59363+0 n \\
3.21760-01\end{array}$ & $\begin{array}{l}1.15894 \cdot 00 \\
2.339=0-01\end{array}$ & $\begin{array}{l}1.17 E 87+01 \\
3.08979+00\end{array}$ & $\begin{array}{l}2.04895 \% 01 \\
5.51351 .00\end{array}$ & $\begin{array}{l}.12023 \\
: 02162\end{array}$ & $\begin{array}{r}14.43070^{-} \\
2.94758\end{array}$ \\
\hline $3-4-$ & $\begin{array}{l}2516.00 \\
25 \text { IG ERR }\end{array}$ & $\begin{array}{l}0.00000 \\
0.000000\end{array}$ & $\begin{array}{l}0.00000 \\
0.00000\end{array}$ & $\begin{array}{r}8.98527-01 \\
1.97848-61\end{array}$ & $\begin{array}{l}1.20921+00 \\
2.440 \geq 2-01\end{array}$ & $\begin{array}{l}1.02421+00 \\
2.06533-01\end{array}$ & $\begin{array}{l}1.15808+01 \\
2.74614+00\end{array}$ & $\begin{array}{l}1.90359 \cdot 01 \\
4.55593 \times 00\end{array}$ & $\begin{array}{l}.11437 \\
001497\end{array}$ & $\begin{array}{r}12.75302 \\
2.60260^{\circ}\end{array}$ \\
\hline
\end{tabular}


TABLE 4-43 (Continued)

\begin{tabular}{|c|c|c|c|c|c|c|c|c|c|c|}
\hline $\begin{array}{r}\text { FUËL } \\
R O D \\
I: 0 . \\
\end{array}$ & $\begin{array}{l}\text { MFAN } \\
\text { COPE HT } \\
\text { (MM) }\end{array}$ & $\begin{array}{c}P A-233 \\
(311.9 K E V) \\
(C I)\end{array}$ & $\begin{array}{c}R \mathrm{U}-103 \\
(497.1 \mathrm{KEV}) \\
\text { (CI) }\end{array}$ & $\begin{array}{l}\text { PU-106 } \\
\text { (511.9KEV) } \\
\text { (CI) }\end{array}$ & $\begin{array}{c}\text { CS-134 } \\
1604.7 \mathrm{KEV}, \\
(\mathrm{CI})\end{array}$ & $\begin{array}{c}\operatorname{cs}-137 \\
(661.6 \mathrm{kEV}) \\
(\mathrm{CI})\end{array}$ & $\begin{array}{c}\text { CE }-144 \\
(695.6 \mathrm{KTV}) \\
(C \mathrm{~N})\end{array}$ & $\begin{array}{l}2 R-95 \\
1724.2 K E V) \\
\text { ICI }\end{array}$ & $\begin{array}{l}\text { CS-137 } \\
\text { (ZR-95 } \\
(C T / M I N)\end{array}$ & $\begin{array}{c}\text { POTAL } \\
\text { FIHA } \\
8\end{array}$ \\
\hline $1-5-2$ & $\begin{array}{r}738.00 \\
2516 \text { ERR }\end{array}$ & $\begin{array}{l}0.00000 \\
0.00000\end{array}$ & $\begin{array}{l}0.00000 \\
c .00000\end{array}$ & $\begin{array}{l}4.95754-01 \\
1.25928-01\end{array}$ & $\begin{array}{l}3.63268-01 \\
7.45596-02\end{array}$ & $\begin{array}{l}6.00887-01 \\
1.23330-01\end{array}$ & $\begin{array}{l}6.78274 \cdot 00 \\
1.82561 \cdot 00\end{array}$ & $\begin{array}{l}1.23623001 \\
3.87769000\end{array}$ & $\begin{array}{l}.10332 \\
.02525\end{array}$ & $\begin{array}{l}7.65289 \\
1.58704\end{array}$ \\
\hline $1-5-4$ & $\begin{array}{l}937.00 \\
2516 \text { ERR }\end{array}$ & $\begin{array}{l}c .00000 \\
0.00000\end{array}$ & $\begin{array}{l}0.000 n 0 \\
0.000 r 0\end{array}$ & $\begin{array}{l}5.99490-C 1 \\
1.49462-01\end{array}$ & $\begin{array}{l}6.31526-01 \\
1.28310-01\end{array}$ & $\begin{array}{l}7.82511-01 \\
1.58139-01\end{array}$ & $\begin{array}{l}7.89183+00 \\
2.34304+00\end{array}$ & $\begin{array}{l}1.04506+01 \\
3.23492+00\end{array}$ & $\begin{array}{l}.15916 \\
.03761\end{array}$ & $\begin{array}{l}9.96606 \\
2.03787\end{array}$ \\
\hline $1-5-6$ & $\begin{array}{r}936.00 \\
2516 \text { ERR }\end{array}$ & $\begin{array}{l}0.00000 \\
0.00000\end{array}$ & $\begin{array}{l}0.00000 \\
0.00000\end{array}$ & $\begin{array}{l}7.42280-01 \\
1.86160-01\end{array}$ & $\begin{array}{l}8.08980-01 \\
1.63914-01\end{array}$ & $\begin{array}{l}8.59683-01 \\
1.73673-01\end{array}$ & $\begin{array}{l}9.61399+00 \\
2.43269+00\end{array}$ & $\begin{array}{l}1.76353 \% 01 \\
4.50666+00\end{array}$ & $\begin{array}{l}.10362 \\
.01648\end{array}$ & $\begin{array}{r}10.94892 \\
2.23813\end{array}$ \\
\hline $1-5-8$ & $-\frac{1034.00}{2516^{\circ} \text { ERR }}$ & $\begin{array}{l}5.50000 \\
0.00000\end{array}$ & $\begin{array}{l}0.00000 \\
0.00000\end{array}$ & $\begin{array}{r}7.72570-01 \\
-3.87541-01\end{array}$ & $\begin{array}{l}8.67118-01 \\
1.75651-01\end{array}$ & $\begin{array}{l}8.79975-01 \\
1.77712-01\end{array}$ & $\begin{array}{l}9.40057+00 \\
2.41454+00\end{array}$ & $\begin{array}{l}1.65808+01 \\
4.51728+00\end{array}$ & $\begin{array}{l}.11221 \\
.02086\end{array}$ & $\begin{array}{r}11.20735 \\
2.29025\end{array}$ \\
\hline $1-5-10$ & $\begin{array}{r}1133.00 \\
2 S I G E R R\end{array}$ & $\begin{array}{l}0.00000 \\
0.00000\end{array}$ & $\begin{array}{l}0.00000 \\
0.00000\end{array}$ & $\begin{array}{l}7.38034-01 \\
1.80408-01\end{array}$ & $\begin{array}{l}9.65442-01 \\
1.95338-01\end{array}$ & $\begin{array}{l}9.09684-01 \\
1.83712-01\end{array}$ & $\begin{array}{l}1.02775+01 \\
2.66674+00\end{array}$ & $\begin{array}{l}1.66499+01 \\
4.49362+00\end{array}$ & $\begin{array}{l}.11613 \\
.02104\end{array}$ & $\begin{array}{r}11.58573 \\
2.36757\end{array}$ \\
\hline $1-5-12$ & $\begin{array}{r}1232.00 \\
2516 \mathrm{ERR}\end{array}$ & $\begin{array}{l}0.00000 \\
0.00000\end{array}$ & $\begin{array}{l}0.00000 \\
0.00000\end{array}$ & $\begin{array}{r}9.74795-01 \\
2.32874-01\end{array}$ & $\begin{array}{l}1.35982+00 \\
2.74539-01\end{array}$ & $\begin{array}{l}1.09950 * 00 \\
2.21753-01\end{array}$ & $\begin{array}{l}1.13897+01 \\
2.84508 \cdot 00\end{array}$ & $\begin{array}{l}2.14343+01 \\
5.50807+00\end{array}$ & $\begin{array}{r}.10899 \\
.01755\end{array}$ & $\begin{array}{r}13.99688 \\
2.85805\end{array}$ \\
\hline $1-5-14$ & $\begin{array}{l}1331.00 \\
2516 \text { ERR }\end{array}$ & $\begin{array}{l}0.00000 \\
0.00000\end{array}$ & $\begin{array}{l}0.00000 \\
0.00000\end{array}$ & $\begin{array}{l}9.64805-01 \\
2.25666-01\end{array}$ & $\begin{array}{l}1.55759+00 \\
3.14354-01\end{array}$ & $\begin{array}{l}1.19993+00 \\
2.42035-01\end{array}$ & $\begin{array}{l}1.33012+01 \\
3.24536 \cdot 00\end{array}$ & $\begin{array}{l}2.24607+01 \\
5.70803+00\end{array}$ & $\begin{array}{l}111356 \\
.01776\end{array}$ & $\begin{array}{r}15.28228 \\
3.11957\end{array}$ \\
\hline $2-5-2$ & $\begin{array}{r}1528.00 \\
2516 " 6 R R\end{array}$ & $\begin{array}{l}\text { C.00000 } \\
0.00000\end{array}$ & $\begin{array}{l}0.00000 \\
0.00000\end{array}$ & $\begin{array}{l}1.02323+00 \\
2.26839-01\end{array}$ & $\begin{array}{l}1.61439+00 \\
3.25490-01\end{array}$ & $\begin{array}{l}1.19455+00 \\
2.40316-01\end{array}$ & $\begin{array}{l}1.32769+01 \\
3.15823+00\end{array}$ & $\begin{array}{l}2.16535+01 \\
5.26609000\end{array}$ & $\begin{array}{l}011726 \\
01615\end{array}$ & $\begin{array}{r}15.21373 \\
3.10401\end{array}$ \\
\hline 2 & $\begin{array}{c}1627.00 \\
2516 E R R\end{array}$ & $\begin{array}{l}0.00000 \\
0.00000\end{array}$ & $\begin{array}{l}0.00000 \\
0.00000\end{array}$ & $\begin{array}{l}1.03201+00 \\
2.291 \geq 4-01\end{array}$ & $\begin{array}{l}1.67025+00 \\
3.36642-01\end{array}$ & $\begin{array}{l}1.20606+00 \\
2.43084-01\end{array}$ & $\begin{array}{l}1.40299+01 \\
3.22794+00\end{array}$ & $\begin{array}{l}2.14566+01 \\
5.20979+00\end{array}$ & $\begin{array}{l}.11948 \\
.01637\end{array}$ & $\begin{array}{r}15.36040 \\
3.13330\end{array}$ \\
\hline $2-5$ & $\begin{array}{l}1775.00 \\
2 S I G \text { ERR }\end{array}$ & $\begin{array}{l}0.00000 \\
0.00000\end{array}$ & $\begin{array}{l}0.00000 \\
0.00000\end{array}$ & $\begin{array}{l}1.05098+00 \\
2.38977-01\end{array}$ & $\begin{array}{l}1.72150+00 \\
3.47213=01\end{array}$ & $\begin{array}{l}1.23836+00 \\
2.49726-01\end{array}$ & $\begin{array}{l}1.31403+01 \\
3.06946+00\end{array}$ & $\begin{array}{l}2.08 \\
4.88\end{array}$ & $\begin{array}{l}.12643 \\
.01541\end{array}$ & $\begin{array}{r}15.77169 \\
3.21876\end{array}$ \\
\hline $2-5-8$ & $\begin{array}{r}1824.00 \\
2 S I G E R R\end{array}$ & $\begin{array}{l}0.00000 \\
0.00000\end{array}$ & $\begin{array}{l}0 . \operatorname{cococ} \\
0.070000\end{array}$ & $\begin{array}{l}9.01728-01 \\
2.16300-01\end{array}$ & $\begin{array}{l}1.61498+00 \\
3.25975-01\end{array}$ & $\begin{array}{l}1.17346+00 \\
2.36839-01\end{array}$ & $\begin{array}{l}1.26225+01 \\
3.24917+00\end{array}$ & $\begin{array}{l}1.90947+01 \\
5.16621+00\end{array}$ & $\begin{array}{l}.13063 \\
.02378\end{array}$ & $\begin{array}{r}14.94515 \\
3.05241\end{array}$ \\
\hline $2-5-10$ & $\begin{array}{l}1923.00 \\
25 I G E R R\end{array}$ & $\begin{array}{l}0.00000 \\
0.00000\end{array}$ & $\begin{array}{l}0.00000 \\
0.00000\end{array}$ & $\begin{array}{l}1.05028+00 \\
2.41777-01\end{array}$ & $\begin{array}{l}1.626[1+00 \\
3.28063-01\end{array}$ & $\begin{array}{l}1.15757+00 \\
2.33521-01\end{array}$ & $\begin{array}{l}1.28230+01 \\
3.07239+00\end{array}$ & $\begin{array}{l}2.05397+01 \\
5.33627+00\end{array}$ & $\begin{array}{l}.11980 \\
.01982\end{array}$ & $\begin{array}{r}14.74404 \\
-3.00981\end{array}$ \\
\hline $2-5-12$ & $\begin{array}{l}2022.00 \\
2516 E R R\end{array}$ & $\begin{array}{l}0.00000 \\
0.00000\end{array}$ & $\begin{array}{l}0.00000 \\
0.00000\end{array}$ & $\begin{array}{l}1.07183+00 \\
2.36674-01\end{array}$ & $\begin{array}{l}1.66468+0 R \\
3.35555-01\end{array}$ & $\begin{array}{l}1.20691+00 \\
2.43210-01\end{array}$ & $\begin{array}{l}1.32540+01 \\
3.05303+00\end{array}$ & $\begin{array}{l}2.35874 \cdot 01 \\
5.80092+00\end{array}$ & $\begin{array}{l}.10876 \\
.01549\end{array}$ & $\begin{array}{r}15.37115 \\
3.13499\end{array}$ \\
\hline $2-5-14$ & $\begin{array}{l}2121.00 \\
2 S 16 \text { ERR }\end{array}$ & $\begin{array}{l}0.00000 \\
0.00000\end{array}$ & $\begin{array}{l}0.00000 \\
0.00000\end{array}$ & $\begin{array}{r}9.99139-01 \\
2.30935-01\end{array}$ & $\begin{array}{l}1.66296+00 \\
3.35406-01\end{array}$ & $\begin{array}{l}1.20664+00 \\
2.43339-01\end{array}$ & $\begin{array}{l}1.28183+01 \\
3.15540+00\end{array}$ & $\begin{array}{l}2.15456+01 \\
5.43096+00\end{array}$ & $\begin{array}{l}.11904 \\
.01821\end{array}$ & $\begin{array}{r}15.36774 \\
3.13643\end{array}$ \\
\hline $3-5-2$ & $\begin{array}{l}2318.00 \\
251 G E R R\end{array}$ & $\begin{array}{l}0.00000 \\
0.00000\end{array}$ & $\begin{array}{l}0.00000 \\
0.00000\end{array}$ & $\begin{array}{l}9.08700-01 \\
2.17084-01\end{array}$ & $\begin{array}{l}1.39784+00 \\
2.82216-01\end{array}$ & $\begin{array}{r}1.08810+00 \\
2.19554-01\end{array}$ & $\begin{array}{l}1.35587 .01 \\
3.29898+00\end{array}$ & $\begin{array}{l}2.14005+01^{-} \\
5.34190+00\end{array}$ & $\begin{array}{l}.10808 \\
.01611\end{array}$ & $\begin{array}{r}13.85807 \\
2.82971\end{array}$ \\
\hline $3-5-4$ & $\begin{array}{l}2417.00 \\
2 \leq I G \quad E R R\end{array}$ & $\begin{array}{l}0.00000 \\
0.00000\end{array}$ & $\begin{array}{l}0.00 \pi c 0 \\
0.0 r 050\end{array}$ & $\begin{array}{l}9.21305-01 \\
2.22874-01\end{array}$ & $\begin{array}{l}1.28250+00 \\
2.59026-01\end{array}$ & $\begin{array}{l}1.05325+00 \\
2.12533-01\end{array}$ & $\begin{array}{l}1.27447+01 \\
3.06758+00\end{array}$ & $\begin{array}{l}1.98715+01 \\
5.10575+00\end{array}$ & $\begin{array}{l}.11265 \\
.01814\end{array}$ & $-\begin{array}{r}13.41203 \\
2.73918\end{array}$ \\
\hline $3-5-6$ & $\begin{array}{l}2516.00 \\
2516 E R R\end{array}$ & $\begin{array}{l}0.00000 \\
0.65000\end{array}$ & $\begin{array}{l}0 . \operatorname{lognn} \\
0.0 \operatorname{con} 0\end{array}$ & $\begin{array}{l}2.14352-01 \\
1.97546-01\end{array}$ & $\begin{array}{l}1.06261+0 R \\
2.14845-01\end{array}$ & $\begin{array}{l}9.31575-C 1 \\
1 . E 8115-01\end{array}$ & $\begin{array}{l}1.02654+01 \\
2.58921+00\end{array}$ & $\begin{array}{l}1.74520+01 \\
4.58310+00\end{array}$ & $\begin{array}{l}.11346 \\
.01930\end{array}$ & $\begin{array}{r}11.86454 \\
2.42434\end{array}$ \\
\hline
\end{tabular}




\begin{tabular}{|c|c|c|c|c|c|c|c|c|c|c|}
\hline $\begin{array}{r}\text { TUEL } \\
\text { ROD } \\
\text { I:O:- }\end{array}$ & $\begin{array}{l}\text { MEAM } \\
\text { CORE HY } \\
\text { IPYHI }\end{array}$ & $\begin{array}{c}P A=233 \\
311.9 \times 5 V 1 \\
\text { ICI }\end{array}$ & $\begin{array}{c}R 0=103 \\
(497.1 \mathrm{KEV}) \\
(\mathrm{CI}) \\
\end{array}$ & $\begin{array}{l}\text { RU-io6 } \\
\text { (511.9KEV! } \\
\text { ICI) }\end{array}$ & $\begin{array}{c}\text { CS-134 } \\
(604 \text { CKEV) } \\
(C I)\end{array}$ & $\begin{array}{c}(5-137 \\
(661.6 K(V) \\
(C 1)\end{array}$ & $\begin{array}{l}C E=144 \\
(695.6 K E V) \\
\text { (CI) }\end{array}$ & $\begin{array}{c}7 R=95 \\
(724 \cdot 2 K(V) \\
(C 1) \\
\end{array}$ & 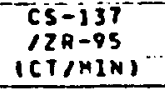 & $\begin{array}{l}\text { iofiat } \\
\text { fima } \\
\text { I }\end{array}$ \\
\hline $3=5-8$ & $\begin{array}{l}2 E 15.00 \\
2 S I G \text { EPR }\end{array}$ & $\begin{array}{l}\text { c. } 00000 \\
\text { c. } 00000\end{array}$ & $\begin{array}{l}0.00000^{-} \\
0.00000\end{array}$ & $\begin{array}{l}8.09800-01 \\
1.88751-01\end{array}$ & $\begin{array}{l}9.80508-01 \\
1.98299-01\end{array}$ & $\begin{array}{l}9.21447-01 \\
1.85975-01\end{array}$ & $\begin{array}{l}1.02065401 \\
2.55498 \times 00\end{array}$ & $\begin{array}{l}1.80398+01 \\
4.75375+00\end{array}$ & $\begin{array}{l}.10857 \\
.01861\end{array}$ & $\begin{array}{r}11.73554 \\
2.39688\end{array}$ \\
\hline $3-5-10$ & $\begin{array}{l}2713.00 \\
2 S I C E R R\end{array}$ & $\begin{array}{l}0.00000 \\
0.00000\end{array}$ & $\begin{array}{l}0.00000 \\
0.00000\end{array}$ & $\begin{array}{r}6.72150-01 \\
1.66828-01\end{array}$ & $\begin{array}{l}7.26791-01 \\
1.47412-01\end{array}$ & $\begin{array}{l}7.91770-01 \\
1.60018-01\end{array}$ & $\begin{array}{l}9.37556+00 \\
2.43282+00\end{array}$ & $\begin{array}{l}1.70318+01 \\
4.51248+00\end{array}$ & $\begin{array}{l}.09881 \\
.01718\end{array}$ & $\begin{array}{r}10.08398 \\
2.06208\end{array}$ \\
\hline $3-5-12$ & $\begin{array}{r}281 ? 000 \\
2 S I G E R R\end{array}$ & $\begin{array}{l}0.00000 \\
0.00000\end{array}$ & $\begin{array}{l}0.00000 \\
0.00000\end{array}$ & $\begin{array}{l}6.47322-01 \\
1.59834-01\end{array}$ & $\begin{array}{l}4.91650-01 \\
1.00270-01\end{array}$ & $\begin{array}{l}6.72582-01 \\
1.36038-01\end{array}$ & $\begin{array}{l}7.71294 \cdot 30 \\
2.01660+00\end{array}$ & $\begin{array}{l}1.702=7+01 \\
4.510 \times 3+00\end{array}$ & $\begin{array}{l}.08397 \\
.01462\end{array}$ & $\begin{array}{l}8.56600 \\
1.75293\end{array}$ \\
\hline $3-5-14$ & $\begin{array}{l}2911.00 \\
2516 E R R\end{array}$ & $\begin{array}{r}0.00000 \\
0.00000\end{array}$ & $\begin{array}{l}0.00000 \\
0.00000\end{array}$ & $\begin{array}{l}5.16054-01 \\
1.27392-01\end{array}$ & $\begin{array}{r}3.97838-01 \\
8.15912-02\end{array}$ & $\begin{array}{l}0.23500-01 \\
1.25211-01\end{array}$ & $\begin{array}{l}7.11110+00 \\
1.96609+00\end{array}$ & $\begin{array}{l}1.26273+01 \\
3.71103+00\end{array}$ & $\begin{array}{l}.10496 \\
.02264\end{array}$ & $\begin{array}{l}7.94089 \\
1.62618\end{array}$ \\
\hline $1-6-6$ & $\begin{array}{r}936: 00 \\
2516 \leq R R\end{array}$ & $\begin{array}{l}0.00000 \\
0.00000\end{array}$ & $\begin{array}{l}0.00000 \\
0.00000\end{array}$ & $\begin{array}{l}6.64951-01 \\
1.62771-01\end{array}$ & $\begin{array}{l}7.90111-01 \\
1.00061-01\end{array}$ & $\begin{array}{l}8.39251-01 \\
1.60512-01\end{array}$ & $\begin{array}{l}9.41873+00 \\
2.34216+00\end{array}$ & $\begin{array}{l}1.39896+01 \\
3.89756+00\end{array}$ & $\begin{array}{r}.12844 \\
.02528\end{array}$ & $\begin{array}{r}10.68870 \\
2.18455\end{array}$ \\
\hline $2-6-7$ & $\begin{array}{l}1775.00 \\
2510^{-6 R E}\end{array}$ & $\begin{array}{l}0.00000 \\
0.00000\end{array}$ & $\begin{array}{l}\text { C.00000 } \\
0.00000\end{array}$ & $\begin{array}{l}1.04557+00 \\
2.41526-01\end{array}$ & $\begin{array}{l}1.6261890 n \\
3.28027-61\end{array}$ & $\begin{array}{l}1.17958000 \\
2.37931-01\end{array}$ & $\begin{array}{l}1.44344+C 1 \\
3.31722+00\end{array}$ & $\begin{array}{l}1.99446 \cdot 01 \\
4.81266 \cdot 00\end{array}$ & $\begin{array}{r}-2571 \\
-01692\end{array}$ & $\begin{array}{r}15.02316 \\
3.06666\end{array}$ \\
\hline $2-6-8$ & $\begin{array}{r}1824.00 \\
2516 \quad \text { ERR }\end{array}$ & $\begin{array}{l}\text { a.conón } \\
0.0 n n n a\end{array}$ & $\begin{array}{l}0.00000 \\
0.000 n 0\end{array}$ & $\begin{array}{l}1.07978+00 \\
2.45879-01\end{array}$ & $\begin{array}{l}1.60504+00 \\
3.27710-01\end{array}$ & $\begin{array}{l}1.16741+0 ? \\
2.35487-01\end{array}$ & $\begin{array}{l}1.40836 .01 \\
3.34185 \cdot 00\end{array}$ & $\begin{array}{l}2.17409 \cdot 01 \\
5.44626 \cdot 00\end{array}$ & $\begin{array}{l}.11414 \\
.01717\end{array}$ & $\begin{array}{r}14.86817 \\
3.03515\end{array}$ \\
\hline $3-6-6$ & $\begin{array}{l}2516.00 \\
2510 \text { ERR }\end{array}$ & $\begin{array}{l}\text { o. c0000 } \\
\text { c. onono }\end{array}$ & $\begin{array}{l}0.00000 \\
0.00000\end{array}$ & $\begin{array}{l}8.79236-01 \\
2.05293-01\end{array}$ & $\begin{array}{l}1.10118+00 \\
2.225 \times 1-01\end{array}$ & $\begin{array}{l}9.49440-01 \\
1.91660-01\end{array}$ & $\begin{array}{l}1.17273+01 \\
2.88479 \cdot 00\end{array}$ & $\begin{array}{l}1.80453+01 \\
4.61145+00\end{array}$ & $\begin{array}{l}11184 \\
.01777\end{array}$ & $\begin{array}{r}12.09207 \\
2.47010\end{array}$ \\
\hline
\end{tabular}


TABLE 4-43 (Continued)

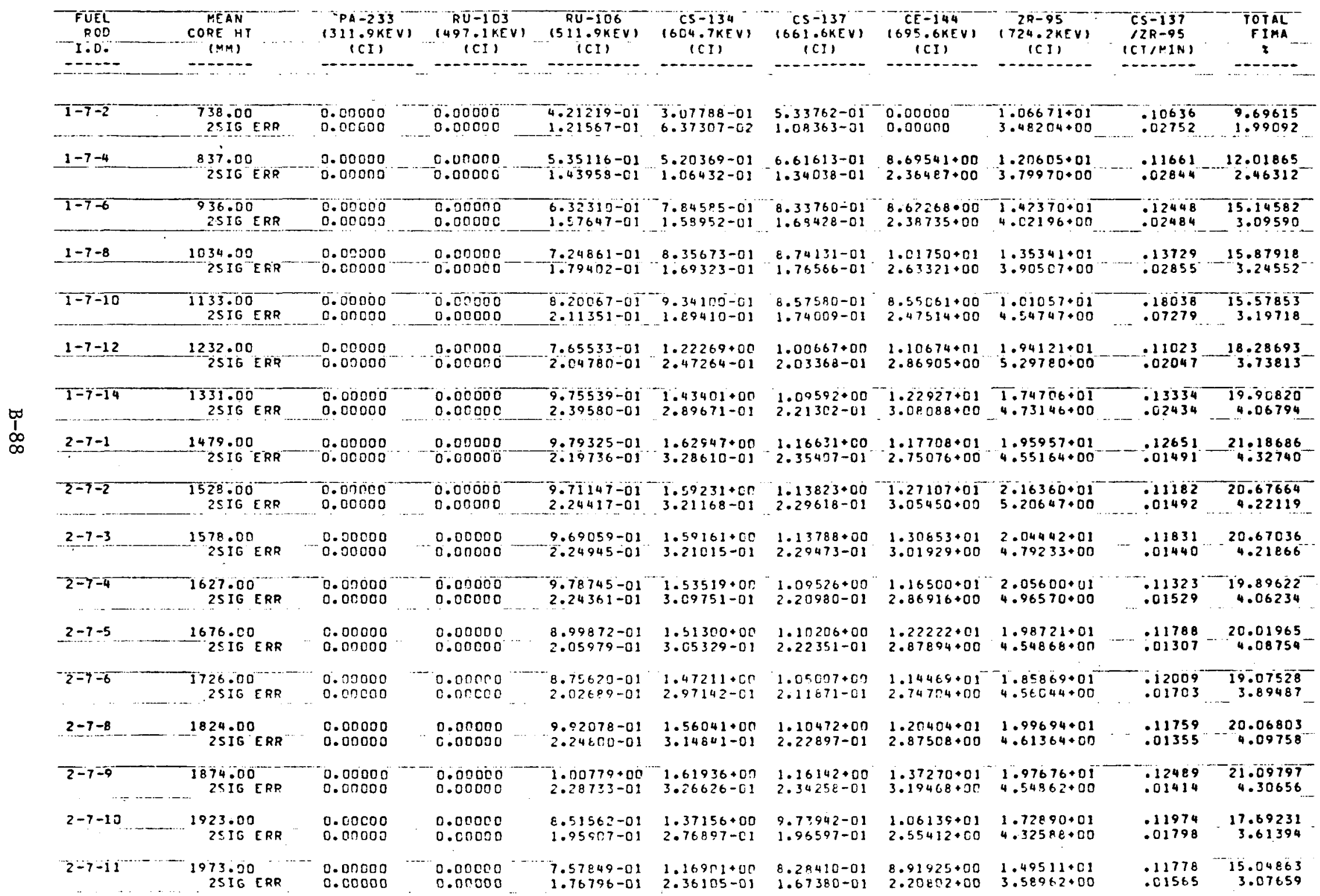


TABLE 4-43 (Continued)

\begin{tabular}{|c|c|c|c|c|c|c|c|c|c|c|}
\hline $\begin{array}{l}\text { TUEL } \\
\text { ROD } \\
\text { T.O- }\end{array}$ & $\begin{array}{l}\text { AEAN } \\
\text { CORE HT } \\
\text { (NM) }\end{array}$ & $\begin{array}{c}\text { PA } 2335 \\
(311.9 K E V) \\
(C I)\end{array}$ & $\begin{array}{l}\text { R0 } \\
\text { 497.103 } \\
\text { (CI) }\end{array}$ & $\begin{array}{l}\text { RU-106 } \\
\text { (51).9KEV) } \\
\text { ICI) }\end{array}$ & $\begin{array}{l}\text { CS-i34 } \\
\text { (604 RTKEV) } \\
\text { (CI) }\end{array}$ & $\begin{array}{c}(5-137 \\
(661.6 x(y) \\
(C))\end{array}$ & $\begin{array}{l}C E=144 \\
(695.6 K E V) \\
(C 1)\end{array}$ & $\begin{array}{c}2 R-05 \\
(724+2 K E V) \\
\text { (CI) }\end{array}$ & $\begin{array}{l}C S-13 T \\
\mid Z R-9 S \\
|C T / M I N|\end{array}$ & $\begin{array}{l}\text { TódiL } \\
\text { FIMA }\end{array}$ \\
\hline $2-7-12$ & $\begin{array}{l}2022.00 \\
2 S 1 G E R R\end{array}$ & $\begin{array}{l}0.00000 \\
0.00000\end{array}$ & $\begin{array}{l}0.00000 \\
0.00000\end{array}$ & $\begin{array}{l}9.6 \geq 120-01 \\
2.18954-01\end{array}$ & $\begin{array}{l}1.48 B 17+00 \\
3.00265-01\end{array}$ & $\begin{array}{l}1.11804+00 \\
2.25633-01\end{array}$ & $\begin{array}{l}1.15041+01 \\
2.75637+00\end{array}$ & $\begin{array}{l}1.89046+01 \\
4.8497 \varepsilon+00\end{array}$ & $\begin{array}{l}.12571 \\
.02017\end{array}$ & $\begin{array}{r}20.30993 \\
4.14779\end{array}$ \\
\hline $2-7-13$ & $\begin{array}{l}2071.00 \\
2510 \text { ERP }\end{array}$ & $\begin{array}{l}0.00050 \\
0.00000\end{array}$ & $\begin{array}{l}0.00000 \\
0.00000\end{array}$ & $\begin{array}{l}9.52588-01 \\
2.23493-01\end{array}$ & $\begin{array}{l}1.48123+00 \\
2.98827-61\end{array}$ & $\begin{array}{l}1.06975 \cdot 00 \\
2.15831-01\end{array}$ & $\begin{array}{l}1.12971+01 \\
2.64063+00\end{array}$ & $\begin{array}{l}2.08073+01 \\
4.71963+00\end{array}$ & $\begin{array}{l}.10928 \\
.01164\end{array}$ & $\begin{array}{r}19.43268 \\
3.96769\end{array}$ \\
\hline $2-7-14$ & $\begin{array}{r}2121.000 \\
251 G E R R\end{array}$ & $\begin{array}{l}0.00000 \\
0.00000\end{array}$ & $\begin{array}{l}0.00000 \\
0.00000\end{array}$ & $\begin{array}{l}1.00 \\
2.35\end{array}$ & $\begin{array}{l}1.54209+00 \\
3.11213-01\end{array}$ & $\begin{array}{l}1.09490+00 \\
2.20906-01\end{array}$ & $\begin{array}{l}7.50150 .01 \\
1.51848 .01\end{array}$ & $\begin{array}{l}1.94664+01 \\
4.82359000\end{array}$ & $\begin{array}{r}.11956 \\
.01745\end{array}$ & $\begin{array}{r}19.88958 \\
4.06098\end{array}$ \\
\hline $3-7-2$ & $\begin{array}{l}2319.00 \\
2 S 10 \text { ERR }\end{array}$ & $\begin{array}{l}0.00000 \\
0.00000\end{array}$ & $\begin{array}{l}0.00000 \\
0.00000\end{array}$ & $\begin{array}{l}9.49548-01 \\
2.37544-01\end{array}$ & $\begin{array}{l}1.30813+00 \\
2.64204-01\end{array}$ & $\begin{array}{l}1.00424+00 \\
2.02907-01\end{array}$ & $\begin{array}{l}1.32033+01 \\
3.25857+00\end{array}$ & $\begin{array}{l}1.89037+01 \\
5.17955000\end{array}$ & $\begin{array}{l}.11292 \\
.02115\end{array}$ & $\begin{array}{r}18 \\
3 .\end{array}$ \\
\hline $3-7-6$ & $\begin{array}{r}2516.00 \\
251 G E R P\end{array}$ & $\begin{array}{l}0.0 n 000 \\
0.0 n 000\end{array}$ & $\begin{array}{l}0.00000 \\
0.00000\end{array}$ & $\begin{array}{l}8.16800-01 \\
2.03210-01\end{array}$ & $\begin{array}{l}1.05613+00 \\
2.13811-01\end{array}$ & $\begin{array}{l}9.03832-01 \\
1.82742-01\end{array}$ & $\begin{array}{l}9.54209+00 \\
2.56577+00\end{array}$ & $\begin{array}{l}9.32642+00 \\
5.32931+00\end{array}$ & $\begin{array}{l}.20599 \\
.11028\end{array}$ & $\begin{array}{r}16.4 \\
3.3\end{array}$ \\
\hline $3-7-9$ & $\begin{array}{l}2664.00 \\
2 \$ 16 \text { ERR }\end{array}$ & $\begin{array}{l}0.00000 \\
0.00000 .\end{array}$ & $\begin{array}{l}0.00000 \\
0.00000\end{array}$ & $\begin{array}{l}0.55988-01 \\
1.67880-01\end{array}$ & $\begin{array}{l}7.70446-01 \\
1.56350-C 1\end{array}$ & $\begin{array}{l}7.64041-01 \\
1.54546-01\end{array}$ & $\begin{array}{l}8.37465+00 \\
2.35243+00\end{array}$ & $\begin{array}{l}1.54225+01 \\
4.15620 \cdot 00\end{array}$ & $\begin{array}{l}.10530 \\
.01905\end{array}$ & 31 \\
\hline $3-7-11$ & $\begin{array}{r}2763000 \\
2516 \text { ERR }\end{array}$ & $\begin{array}{l}0.000 n 0 \\
0.00000\end{array}$ & & $\begin{array}{l}01 \\
01\end{array}$ & $\begin{array}{l}5-C 1 \\
1-C 1\end{array}$ & & $\begin{array}{l}8.0 \\
2.3\end{array}$ & $\begin{array}{l}1.4 \\
4.0\end{array}$ & & \\
\hline $3-7-13$ & $\begin{array}{r}2861.00 \\
25 I G^{\prime \prime E R P}\end{array}$ & $\begin{array}{l}0.05000 \\
0.00000\end{array}$ & $\begin{array}{l}0.00000 \\
0.00000\end{array}$ & $\begin{array}{l}5.04493-01 \\
1.31421-01 .\end{array}$ & $\begin{array}{l}4.08 \\
8.38\end{array}$ & & $\begin{array}{l}7.64 \\
3.17\end{array}$ & 9.9 & & $\begin{array}{l}07 \\
55\end{array}$ \\
\hline$T-8-6$ & $\begin{array}{r}96.00 \\
2516 \cdot E R P\end{array}$ & $\begin{array}{l}0.00000 \\
0.00000\end{array}$ & $\begin{array}{l}0.00000 \\
0.00000\end{array}$ & $\begin{array}{l}6.32817-01 \\
1.5 n 227-01\end{array}$ & $\begin{array}{l}7.25787-01 \\
1.47199-01\end{array}$ & $\begin{array}{l}7.72585-01 \\
1.56198-01\end{array}$ & $\begin{array}{l}8.87653+00 \\
2.30053+00\end{array}$ & $\begin{array}{r}1.5288440 \\
4.20460 \cdot 0\end{array}$ & & $\begin{array}{l}4.04180 \\
2.87101\end{array}$ \\
\hline $2-8-1$ & $\begin{array}{l}1479.80 \\
2516 \text { ERR }\end{array}$ & $\begin{array}{l}0.00000 \\
0.00000\end{array}$ & $\begin{array}{l}0.00000 \\
0.00000\end{array}$ & $\begin{array}{l}1.01645+00 \\
2.33496-01\end{array}$ & $\begin{array}{l}1.650 \\
3.3288\end{array}$ & $\begin{array}{l}49 \cdot 00 \\
26-01\end{array}$ & $\begin{array}{l}01 \\
00\end{array}$ & $\begin{array}{l}1.99314 .6 \\
5.12310 .1\end{array}$ & & $\begin{array}{r}21.55348 \\
4.39987\end{array}$ \\
\hline $\begin{array}{l}2-8-2 \\
-\cdots\end{array}$ & $\begin{array}{c}1528.60 \\
2515 \mathrm{ERR}\end{array}$ & $\begin{array}{l}0.00000 \\
0.00000\end{array}$ & $\begin{array}{l}0.00000 \\
0.00000\end{array}$ & $\begin{array}{l}9.28384-01 \\
2.28791-01\end{array}$ & $\begin{array}{l}1.63420+00 \\
3.27605-01\end{array}$ & $\begin{array}{l}492+00 \\
979-01\end{array}$ & $\begin{array}{r}1.27 \\
3.02\end{array}$ & $\begin{array}{l}2.015540010 \\
5.04750 .00\end{array}$ & $\begin{array}{l}.12496 \\
.01878\end{array}$ & $\begin{array}{r}21.5 \overline{2495} \\
4.39339\end{array}$ \\
\hline $2-8-3$ & $\begin{array}{l}1578.40 \\
2 S 1 G \text { ERR }\end{array}$ & $\begin{array}{l}0.00003 \\
0.00000\end{array}$ & $\begin{array}{l}0.00000 \\
0.00000\end{array}$ & $\begin{array}{r}9.24710-01 \\
-2.15072-01\end{array}$ & $\begin{array}{l}1.62690+00 \\
3.281 \times 7-01\end{array}$ & $\begin{array}{l}1.17219+00 \\
2.35421-01\end{array}$ & $\begin{array}{l}1.23779+01 \\
2.95167+00\end{array}$ & $\begin{array}{l}2.21787+01 \\
5.48014+00\end{array}$ & $\begin{array}{l}.11234 \\
.01625\end{array}$ & $\begin{array}{r}21.29361 \\
4.34633\end{array}$ \\
\hline $\begin{array}{l}2-8=4 \\
-2\end{array}$ & $\begin{array}{c}1627.20^{\circ} \\
2516 \text { ERR }\end{array}$ & $\begin{array}{l}0.00005 \\
0.00000\end{array}$ & $\begin{array}{l}0.00000 \\
0.00000\end{array}$ & $\begin{array}{l}1.03351+00 \\
2.40477-01\end{array}$ & $\begin{array}{l}1.56053+00 \\
3.14651-01\end{array}$ & $\begin{array}{l}1.11616+00 \\
2.25177-01\end{array}$ & $\begin{array}{l}1.21409+01 \\
2.95334+00\end{array}$ & $\begin{array}{l}1.87815+01 \\
4.64730+00\end{array}$ & $\begin{array}{l}.12632 \\
.01836\end{array}$ & $\begin{array}{l}7580^{\circ} \\
3954\end{array}$ \\
\hline $2-8-5$ & $\begin{array}{c}1676.00 \\
2516 \text { ERR }\end{array}$ & $\begin{array}{l}0.00000 \\
0.00000\end{array}$ & $\begin{array}{l}0.00000 \\
0.00000\end{array}$ & $\begin{array}{r}9.28650-01 \\
2.15612-01\end{array}$ & $\begin{array}{l}1.55370+0 r \\
3.13500-01\end{array}$ & & $\begin{array}{l}1.23137+01 \\
2.92581+00\end{array}$ & $\begin{array}{l}1.64178+01 \\
4.79902+00\end{array}$ & $\begin{array}{l}.12705 \\
.02118\end{array}$ & $\begin{array}{r}19.99728 \\
4.08281\end{array}$ \\
\hline $\begin{array}{l}2=8=6 \\
\cdots \quad \cdots\end{array}$ & $\begin{array}{r}\text { T726:00 } \\
-. \quad 25 I 6 \text { EPD }\end{array}$ & $\begin{array}{l}-0.00000 " \\
0.00000\end{array}$ & $\begin{array}{l}0.0 n 000 \\
\text { c.oneso }\end{array}$ & $\begin{array}{l}9.40003-01 \\
2.16299-01\end{array}$ & $\begin{array}{l}1.57308+0 r \\
3.17375-01\end{array}$ & $\begin{array}{l}1.11837+00 \\
2.25549-01\end{array}$ & $\begin{array}{l}1.25428+01 \\
2.90153+00\end{array}$ & $\begin{array}{l}1.81495 \times 01 \\
4.55829+00\end{array}$ & $\begin{array}{l}.13099 \\
.01984\end{array}$ & $\begin{array}{r}20.31602 \\
4.14649\end{array}$ \\
\hline $2-8-8$ & $\begin{array}{r}1824.60 \\
2516^{\circ} E R R\end{array}$ & $\begin{array}{l}0.00000 \\
0.05000\end{array}$ & $\begin{array}{l}0.00000 \\
0.00000\end{array}$ & $\begin{array}{r}8.87415-01 \\
2.07519-01\end{array}$ & $\begin{array}{l}1.57827+00 \\
3.18443-01\end{array}$ & $\begin{array}{l}1.11938+00 \\
2.25836-01\end{array}$ & $\begin{array}{l}1.25777+01 \\
2.99595+00\end{array}$ & $\begin{array}{l}1.98396+01 \\
4.80716+00\end{array}$ & $\begin{array}{l}.11993 \\
.01636\end{array}$ & $\begin{array}{r}20.33437 \\
4.15164\end{array}$ \\
\hline $2-8=9$ & $\begin{array}{l}1374.00 \\
2 S: 16 \text { ERR }\end{array}$ & $\begin{array}{l}0.50000 \\
0.00000\end{array}$ & $\begin{array}{l}0.05000 \\
0.00000\end{array}$ & $\begin{array}{l}1.01368+00 \\
2.33006-01\end{array}$ & $\begin{array}{l}1.61734+00 \\
3.26257-01\end{array}$ & $\begin{array}{l}1.16399+00 \\
2.34796-01\end{array}$ & $\begin{array}{l}1.37176+01 \\
3.24411+00\end{array}$ & $\begin{array}{l}2.00118+01 \\
5.21689+00\end{array}$ & $\begin{array}{l}.12364 \\
.02063\end{array}$ & $\begin{array}{l}1.14465 \\
4.31641\end{array}$ \\
\hline
\end{tabular}


TABLE 4-43 (Continued)

\begin{tabular}{|c|c|c|c|c|c|c|c|c|c|c|}
\hline $\begin{array}{l}T U E L L \\
R 00 \\
1.00\end{array}$ & $\begin{array}{l}\text { MEAN } \\
\text { CORE HT } \\
\text { IPMI }\end{array}$ & $\begin{array}{c}\text { PA }=233 \\
(311.9 \mathrm{KEV}) \\
\text { (CI) }\end{array}$ & $\begin{array}{l}R U-103 \\
\text { (497.1KEVI } \\
\text { (CI) }\end{array}$ & $\begin{array}{l}R U-106 \\
\text { (51).OKEVI } \\
\text { ICI) }\end{array}$ & $\begin{array}{c}\text { CS-134 } \\
\text { (6C4.PKEVI } \\
\text { (CI) }\end{array}$ & $\begin{array}{c}\operatorname{cs}-137 \\
(661.6 K 5 V) \\
(C 1)\end{array}$ & $\begin{array}{l}C E-144 \\
\text { (695.6KEV) } \\
\text { (CI) }\end{array}$ & $\begin{array}{l}2 R-95 \\
(724.2 K E V) \\
\text { (CI) }\end{array}$ & $\begin{array}{l}C S-13 i \\
(2 R-95 \\
(C T / A I N)\end{array}$ & $\begin{array}{l}\text { FoTAL } \\
\text { FIMA }\end{array}$ \\
\hline $2-8-10$ & $\begin{array}{l}1923.00 \\
2516 \mathrm{E} \approx \mathrm{R}\end{array}$ & $\begin{array}{l}0.00000 \\
0.00000\end{array}$ & $\begin{array}{l}0.00000 \\
0.00000\end{array}$ & $\begin{array}{l}9.10384-01 \\
2.07263-01\end{array}$ & $\begin{array}{l}3.49161+00 \\
3.00895-01\end{array}$ & $\begin{array}{l}1.05533+00 \\
2.12887-01\end{array}$ & $\begin{array}{l}1.14199+01 \\
2.81117+00\end{array}$ & $\begin{array}{l}1.71019+01 \\
4.28826+00\end{array}$ & $\begin{array}{l}.13117 \\
.01979\end{array}$ & $\begin{array}{r}19.17079 \\
3.91362\end{array}$ \\
\hline $2-8-11$ & $\begin{array}{l}1973.00 \\
2 S 1 G \text { ERR }\end{array}$ & $\begin{array}{l}0.05000 \\
0.00030\end{array}$ & $\begin{array}{l}\text { c.02000 } \\
0.00000\end{array}$ & $\begin{array}{l}9.77219-01 \\
2.25547-01\end{array}$ & $\begin{array}{l}1.5910 .7+00 \\
3.21040-01\end{array}$ & $\begin{array}{l}1.14489+00 \\
2.30952-01\end{array}$ & $\begin{array}{l}1.23261+01 \\
2.97497+00\end{array}$ & $\begin{array}{l}2.218 .97+01 \\
5.51661+00\end{array}$ & $\begin{array}{r}.10467 \\
.01615\end{array}$ & $\begin{array}{r}20.79765 \\
4.24574\end{array}$ \\
\hline $2-8-12$ & $\begin{array}{l}2022.00 \\
2516 \text { ERR }\end{array}$ & $\begin{array}{l}0.00000 \\
0.00300\end{array}$ & $\begin{array}{l}0.09000 \\
0.0000 \mathrm{C}\end{array}$ & $\begin{array}{l}8.67417-01 \\
1.96672-01\end{array}$ & $\begin{array}{l}1.49581+0 C \\
2.99828-01\end{array}$ & $\begin{array}{l}1.08388+00 \\
2.19622-01\end{array}$ & $\begin{array}{l}1.17611+01 \\
2.72948+00\end{array}$ & $\begin{array}{l}2.00659+01 \\
4.83529+00\end{array}$ & $\begin{array}{l}.11482 \\
.01539\end{array}$ & $\begin{array}{r}19.68937 \\
4.02010\end{array}$ \\
\hline $2-8-13$ & $\begin{array}{l}2071.00 \\
25 I G \text { ERR }\end{array}$ & $\begin{array}{l}0.00000 \\
0.00000\end{array}$ & $\begin{array}{l}0.00000 \\
0.00000\end{array}$ & $\begin{array}{l}7.09553-01 \\
1.71847-01\end{array}$ & $\begin{array}{l}7.57472-01 \\
1.53605-01\end{array}$ & $\begin{array}{l}7.94795-01 \\
1.60597-01\end{array}$ & $\begin{array}{l}1.00872+01 \\
2.46404+00\end{array}$ & $\begin{array}{l}1.88724+01 \\
4.86270+00\end{array}$ & $\begin{array}{l}.08952 \\
.01455\end{array}$ & $\begin{array}{r}14.43799 \\
2.95188\end{array}$ \\
\hline$\overline{2-8-14}$ & $\begin{array}{r}2121.0 D \\
2516 \text { ERR }\end{array}$ & $\begin{array}{l}0.00000 \\
0.00000\end{array}$ & $\begin{array}{l}0.00000^{-} \\
0.00000\end{array}$ & $\begin{array}{l}6.79137-01 \\
1.58814-01\end{array}$ & $\begin{array}{l}1.49473+00 \\
3.01525-01\end{array}$ & $\begin{array}{l}1.06506+000 \\
2.14885-01\end{array}$ & $\begin{array}{l}1.15978+01 \\
2.78517+00\end{array}$ & $\begin{array}{l}1.90226 \div 01 \\
4.56947+00\end{array}$ & $\begin{array}{l}.11900 \\
.01579\end{array}$ & $\begin{array}{r}19.34745 \\
3.95029\end{array}$ \\
\hline $3-8-6$ & $\begin{array}{l}2516.00 \\
25 I G \text { ERR }\end{array}$ & $\begin{array}{l}0.00000 \\
0.00000\end{array}$ & $\begin{array}{l}0.00000 \\
0.00000\end{array}$ & $\begin{array}{l}7.91693-01 \\
1.84933-01\end{array}$ & $\begin{array}{r}1.03179+00 \\
2.08505-01\end{array}$ & $\begin{array}{l}8.87182-01 \\
1.79084-01\end{array}$ & $\begin{array}{l}1.04967+01 \\
2.59607+00\end{array}$ & $\begin{array}{l}1.57299+01 \\
4.03241+00\end{array}$ & $\begin{array}{r}.11989 \\
.01920\end{array}$ & $\begin{array}{l}16.11626 \\
3.29201^{-}\end{array}$ \\
\hline
\end{tabular}


TABLE $4-44$

FTE-6 TREVER-CALCULATED TEMPERATURES USED AS INPUT

TO THE FIPERQ FITS OF THE Cs-137 PROFILE IN FUEL BODY 2 GRAPHITE WEB(a)

\begin{tabular}{c|c|c}
\hline $\begin{array}{c}\text { Time } \\
\text { real time, } \\
\text { days) }\end{array}$ & $\begin{array}{c}\text { Inner Boundary } \\
\text { Temperature } \\
(\mathrm{K})\end{array}$ & $\begin{array}{c}\text { Outer Boundary } \\
\text { Temperature } \\
(\mathrm{K})\end{array}$ \\
\hline 57 & 1043 & 1028 \\
112 & 1143 & 1128 \\
169 & 1253 & 1218 \\
305 & 1268 & 1223 \\
386 & 1173 & 1238 \\
437 & 1253 & 1203 \\
536 & 1313 & 1263 \\
588 & 1313 & 1268 \\
637 & 1293 & 1253 \\
674 & 1333 & 1293 \\
704 & 1118 & 1093 \\
733 & 1343 & 1303 \\
780 & 1303 & 1258 \\
792 & 1243 & 1208 \\
\hline
\end{tabular}

(a) TREVER core location: $2113 \mathrm{~mm}$. 
TABLE $4-45$

KMC DATA FOR KERNEL TYPES USED IN PEACH BOTTOM TEST ELEMENTS

\begin{tabular}{|c|c|c|c|c|}
\hline \multirow[b]{2}{*}{ Kernel Type } & \multirow{2}{*}{$\mathrm{cm}^{2}{ }^{\mathrm{KMC}}{ }^{\circ} \mathrm{K} / \mathrm{sec}$} & \multicolumn{2}{|c|}{ Correlation Coefficients (b) } & \multirow{2}{*}{$\begin{array}{c}\text { Source } \\
\text { of } \\
\text { Data }\end{array}$} \\
\hline & & $\mathrm{C}_{5 \%}$ & $\mathrm{C}_{95}$ & \\
\hline $\mathrm{UO}_{2}$ & $1.70 \times 10^{-3} e^{\frac{-22,100}{R T}}$ & 0.31 & 3.2 & c \\
\hline $\mathrm{UC}_{2}$ & $6.2 \times 10^{3} e^{\frac{-74,387}{R T}}$ & 0.35 & 2.85 & $d$ \\
\hline $4 / 1(\mathrm{Th} / \mathrm{U}) \mathrm{O}_{2}$ & $0.151 e^{\frac{-36000}{R T}}$ & 0.17 & 6.0 & c \\
\hline $4 / 1(\mathrm{Th} / \mathrm{U}) \mathrm{C}_{2}$ & $1.59 \times 10^{2} \mathrm{e}^{\frac{-62,300}{\mathrm{RT}}}$ & 0.28 & 3.59 & e \\
\hline $\mathrm{ThC}_{2}$ & $2.204 \times 10^{4} e^{\frac{-75,700}{R T}}$ & 0.30 & 3.32 & $\mathrm{f}$ \\
\hline $\mathrm{ThO}_{2}$ & $103.6 e^{\frac{-60,816}{R T}}$ & 0.18 & 5.61 & d \\
\hline
\end{tabular}

(a) most probable value $=\mathrm{KMC}_{50 \%} ; \mathrm{R}=1.9869 \mathrm{cal} / \mathrm{mole}{ }^{\circ} \mathrm{K}$.

(b) $\mathrm{KMC}$ at the $5 \%$ confidence level $=\mathrm{C}_{5 \%} \times \mathrm{KMC}_{50 \%}$, $\mathrm{KMC}$ at the $95 \%$ confidence leve $1=\mathrm{C}_{95 \%} \times \mathrm{KMC}_{50}$.

(c) Lindemer, T. B. and R. L. Pearson, "Kernel Migration for HTGR Fuels from the Th-U-C-O-N System," ORNL/TM-5207, submitted for publication in the J. Am. Cer. Soc.

(d) Fuel Design Data Manual, Issue B; Document No. 901866.

(e) Sims, J. R., et. a1., "Kernel Migration in Unirradiated TRISO Coated (Th/U)C $\mathrm{C}_{2}$ Fuel Particles," GA-A13825, to be published.

(f) Sims, J. R., Jr., "Kernel Migration Measurements on Unirradiated BISO and TRISO Coated ThC, Fuel Particles," General Atomic unpublished data, November 17, 1975. 
TABLE 4-46

FTE- 6 UO 2 FUEL PERFORMANCE PREDICTIONS

\begin{tabular}{|c|c|c|c|c|c|c|c|c|c|c|c|c|c|c|c|}
\hline \multirow[b]{2}{*}{$\begin{array}{r}\text { Trever } \\
\text { Point }\end{array}$} & \multirow{2}{*}{$\begin{array}{c}\text { Trever } \\
\text { Poine } \\
\text { Location } \\
(\mathrm{mm})\end{array}$} & \multirow{2}{*}{\multicolumn{2}{|c|}{\begin{tabular}{|c|c|}
\multicolumn{2}{c|}{ Pred1cted Fue1 Fa1lure } \\
$\begin{array}{c}\text { Buffer }(\mathrm{a}) \\
(\%)\end{array}$ & $\begin{array}{c}\text { Buffer }+ \text { IPyc } \\
(\%)\end{array}$ \\
\end{tabular}}} & \multirow{2}{*}{$\begin{array}{l}\text { Measured Fue } 1 \\
\text { Failure } \\
(\%)\end{array}$} & \multicolumn{10}{|c|}{ Trever Segment Kernel Migration $(\mu \mathrm{m})$} & \multirow{2}{*}{\begin{tabular}{|l} 
Vo1. Wt \\
Average \\
\end{tabular}} \\
\hline & & & & & 1 & 2 & 3 & 4 & 5 & 6 & 7 & 8 & 9 & 10 & \\
\hline 1 & 698.9 & 0.0 & 0.0 & 0.0 & 0.08 & 0.24 & 0.38 & 0.51 & 0.62 & 0.71 & 0.77 & 0.80 & 0.80 & 0.78 & 0.63 \\
\hline 2 & 768.2 & 0.0 & 0.0 & 0.0 & 0.20 & 0.61 & 0.99 & 1.32 & 1.60 & 1.82 & 1.96 & 2.03 & 2.03 & 1.95 & 1.60 \\
\hline 3 & 837.5 & 0.0 & 0.0 & 0.0 & 0.51 & 1.50 & 2.43 & 3.26 & 3.96 & 4.49 & 4.85 & 5.02 & 5.00 & 4.82 & 3.95 \\
\hline 4 & 906.8 & 0.0 & 0.0 & 0.0 & 0.92 & 2.73 & 4.42 & 5.94 & 7.22 & 8.21 & 8.88 & 9.21 & 9.20 & 8.87 & 7.22 \\
\hline 5 & 976.1 & 0.0 & 0.0 & 0.0 & 1.35 & 3.99 & 6.47 & 8.69 & 10.57 & 12.04 & 13.04 & 13.57 & 13.59 & 13.14 & 10.61 \\
\hline 6 & 1045.4 & 0.0 & 0.0 & 0.5 & 1.81 & 5.36 & 8.70 & 11.71 & 14.26 & 16.27 & 17.67 & 18.41 & 18.49 & 17.93 & 14.36 \\
\hline 7 & 1114.7 & 0.0 & 0.0 & 3.2 & 2.33 & 6.89 & 11.19 & 15.07 & 18.39 & 21.01 & 22.86 & 23.88 & 24.04 & 23.39 & 18.57 \\
\hline 8 & 1184.0 & 1.99 & 0.0 & 5.0 & 2.82 & 8.36 & 13.59 & 18.32 & 22.37 & 25.60 & 27.91 & 29.21 & 29.49 & 28.77 & 22.66 \\
\hline 9 & 1253.0 & 7.77 & 0.0 & 6.0 & 3.28 & 9.73 & 15.82 & 21.34 & 26.10 & 29.92 & 32.67 & 34.26 & 34.68 & 33.93 & 26.52 \\
\hline 10 & 1322.6 & 20.53 & 0.0 & 4.5 & 3.77 & 11.19 & 18.21 & 24.59 & 30.11 & 34.56 & 37.81 & 39.75 & 40.34 & 39.60 & 30.69 \\
\hline 11 & 1489.0 & 31.22 & 0.0 & 17.5 & 4.10 & 12.18 & 19.83 & 26.79 & 32.82 & 37.71 & 41.30 & 43.48 & 44.19 & 43.45 & 33.52 \\
\hline 12 & 1558.4 & 38.79 & 0.0 & 20.5 & 4.44 & 13.18 & 21.47 & 29.03 & 35.60 & 40.97 & 44.95 & 47.42 & 48.31 & 47.65 & 36.47 \\
\hline 13 & 1627.7 & 41.00 & 1.47 & 22.5 & 4.80 & 14.25 & 23.22 & 31.42 & 38.58 & 44.45 & 48.85 & 51.63 & 52.74 & 52.16 & 39.63 \\
\hline 14 & 1697.0 & 42.02 & 3.38 & 24.0 & 5.05 & 15.00 & 24.45 & 33.11 & 40.69 & 46.94 & 51.66 & 54.71 & 56.01 & 55.55 & 41.91 \\
\hline 15 & 1766.3 & 46.39 & 6.34 & 24.5 & 5.29 & 15.70 & 25.61 & 34.71 & 42.69 & 49.32 & 54.36 & 57.68 & 59.19 & 58.85 & 44.10 \\
\hline 16 & 1835.6 & 50.47 & 10.05 & 25.0 & 5.45 & 16.18 & 26.41 & 35.82 & 44.10 & 51.0 & 56.30 & 59.84 & 61.53 & 61.35 & 45.66 \\
\hline 17 & 1904.9 & 52.93 & 12.42 & 25.0 & 5.55 & 16.49 & 26.92 & 36.53 & 45.02 & 52.12 & 57.63 & 61.37 & 63.24 & 63.23 & 46.74 \\
\hline 18 & 1974.2 & 54.52 & 14.29 & 23.5 & 5.61 & 16.67 & 27.23 & 36.97 & 45.60 & 52.86 & 58.53 & 62.43 & 64.48 & 64.63 & 47.47 \\
\hline 19 & 2043.5 & 54.82 & 15.10 & 21.0 & 5.60 & 16.65 & 27.20 & 36.96 & 45.63 & 52.95 & 58.72 & 62.75 & 64.94 & 65.26 & 47.62 \\
\hline 20 & 2112.8 & 54.99 & 14.92 & 18.0 & 5.55 & 16.49 & 26.96 & 36.65 & 45.29 & 52.62 & 58.42 & 62.55 & 64.88 & 65.36 & 47.39 \\
\hline 21 & 2279.0 & 51.47 & 12.61 & 23.0 & 5.37 & 15.98 & 26.14 & 35.56 & 43.98 & 51.15 & 56.88 & 61.00 & 63.41 & 64.06 & 46.13 \\
\hline 22 & 2348.3 & 47.05 & 8.03 & 27.5 & 5.11 & 15.21 & 24.88 & 33.86 & 41.91 & 48.80 & 54.34 & 58.38 & 60.80 & 61.57 & 44.68 \\
\hline 23 & 2417.6 & 41.86 & 4.45 & 33.0 & 4.78 & 14.21 & 23.26 & 31.68 & 39.25 & 45.75 & 51.02 & 54.91 & 57.31 & 58.19 & 41.38 \\
\hline 24 & 2486.9 & 36.33 & 1.65 & 27.5 & 4.39 & 13.07 & 21.40 & 29.17 & 36.17 & 42.21 & 47.13 & 50.80 & 53.14 & 54.09 & 38.23 \\
\hline 25 & 2556.2 & 33.04 & 0.0 & 21.5 & 3.91 & 11.62 & 19.04 & 25.97 & 32.22 & 37.65 & 42.10 & 45.48 & 47.68 & 48.67 & 34.16 \\
\hline 26 & 2625.5 & 20.60 & 0.0 & 15.0 & 3.40 & 10.13 & 16.61 & 22.66 & 28.15 & 32.94 & 36.89 & 39.92 & 41.96 & 42.95 & 29.93 \\
\hline 27 & 2694.8 & 7.11 & 0.0 & 6.5 & 2.82 & 8.39 & 13.76 & 18.80 & 23.37 & 27.38 & 30.72 & 33.31 & 35.10 & 36.05 & 24.93 \\
\hline 28 & 2764.1 & 0.72 & 0.0 & 1.5 & 2.22 & 6.61 & 10.84 & 14.82 & 18.45 & 21.65 & 24.34 & 26.46 & 27.97 & 28.83 & 19.75 \\
\hline 29 & 2833.4 & 0.0 & 0.0 & 0.0 & 1.71 & 5.10 & 8.37 & 11.46 & 14.28 & 16.79 & 18.92 & 20.62 & 21.62 & 22.64 & 15.35 \\
\hline \multirow[t]{2}{*}{30} & 2902.7 & 0.0 & 0.0 & 0.0 & 1.61 & 4.79 & 7.87 & 10.77 & 13.44 & 15.80 & 17.81 & 19.43 & 20.63 & 21.38 & 14.46 \\
\hline & & & \multicolumn{2}{|c|}{ Segment Volume $\%$} & $\overline{2.02}$ & $\overline{5.99}$ & $\overline{10.03}$ & $\overline{14.64}$ & $\overline{14.01}$ & $\overrightarrow{13.47}$ & $\overline{12.79}$ & $\overline{11.67}$ & $\overline{9.71}$ & $\overline{5.68}$ & $\overline{100.00}$ \\
\hline
\end{tabular}

(a) The average buffer thickness was $53.38 \mu \mathrm{m}$, with a standard deviation of $8.685 \mu \mathrm{m}$.

(b) The average inner pyrocarbon thickness was $26.82 \mu \mathrm{m}$, with a standard deviation of $5.168 \mu \mathrm{m}$. 
TABLE $4-47$
FTE-6 Postirradiation Data for the TYPE 11 THERMal STABILITY SPINE SAMPLES
(a)

\begin{tabular}{|c|c|c|c|c|c|c|c|c|c|c|c|c|c|c|c|c|c|c|}
\hline \multirow[b]{3}{*}{$\underset{\substack{\text { FMB } \\
\text { Number }}}{(b)}$} & \multirow[b]{3}{*}{$\begin{array}{c}\text { Particle } \\
\text { Type }\end{array}$} & \multirow[b]{3}{*}{ Locatton ${ }^{(c)}$} & \multirow{3}{*}{$\begin{array}{c}\text { EOL } \\
\text { Avg } \\
\text { Fuel } \\
\text { Tempid }(d) \\
\left({ }^{\circ} \mathrm{C}\right)\end{array}$} & \multirow{2}{*}{\multicolumn{2}{|c|}{$\begin{array}{l}\text { Number of Particles } \\
\text { in Sample }\end{array}$}} & \multirow{2}{*}{\multicolumn{2}{|c|}{$\begin{array}{c}\text { Stereo } \\
\text { Examination }\end{array}$}} & \multicolumn{11}{|c|}{ Metallography Examination ${ }^{(f)}$} \\
\hline & & & & & & & & \multirow[b]{2}{*}{$\begin{array}{l}\text { Fission Gas } \\
\text { Release (e) } \\
\text { R/B Kr-85m }\end{array}$} & \multirow[b]{2}{*}{$\left|\begin{array}{c}\text { OPyC } \\
\text { Failure } \\
(\%)\end{array}\right|$} & \multirow{2}{*}{$\begin{array}{c}95 \% \\
\text { Confidence } \\
\text { Limits } \\
\mathrm{P}(\%)\end{array}$} & \multirow[b]{2}{*}{$\begin{array}{c}\text { sic } \\
\text { Failure } \\
(z)\end{array}$} & \multirow{2}{*}{$\begin{array}{c}95 \% \\
\text { Confidence } \\
\text { Lim1ts } \\
P(\%)\end{array}$} & \multirow{2}{*}{\begin{tabular}{|c|} 
Pressure \\
Vesse1 \\
Fallure \\
$(\%)$
\end{tabular}} & \multirow{2}{*}{$\begin{array}{c}95 x \\
\text { Confidence } \\
\text { Limits } \\
p(\%)\end{array}$} & \multirow{2}{*}{$\begin{array}{c}\text { Mixed } \\
\text { Fission } \\
\text { Product } \\
\text { in IPyC } \\
\text { (\%) } \\
\end{array}$} & \multirow{2}{*}{$\begin{array}{l}95 \% \\
\text { Confidence } \\
\text { Lim1ts } \\
\text { P (\%) }\end{array}$} & \multirow[b]{2}{*}{$\begin{array}{c}S i c \\
\text { Attack } \\
(\%)\end{array}$} & \multirow{2}{*}{$\begin{array}{c}95 \pi \\
\text { Confidence } \\
\text { Limits } \\
(z)\end{array}$} \\
\hline & & & & $\begin{array}{l}\begin{array}{l}\text { Stereo } \\
\text { Exam. }\end{array} \\
\end{array}$ & Metallography & $\begin{array}{c}\text { OPyC } \\
\text { Faylure } \\
(\%)\end{array}$ & $\begin{array}{c}\text { Sid } \\
\text { Failure } \\
(\%) \\
\end{array}$ & & & & & & & & & & & \\
\hline $4000-242$ & TRISO $\mathrm{ThC}_{2}$ & TS $18-3$ & 1140 & 962 & 63 & $<1 \%(g)$ & & $1.15 \times 10^{-4}$ & 0 & $0 \leq \mathrm{P} \leq 5.7$ & 1.6 & $0 \leq P \leq 5.7$ & 0 & $0 \leq P \leq 5.7$ & 0 & $0 \leq P<5.7$ & 0 & $0 \leq P \leq 5.7$ \\
\hline $400-302$ & TRISO $\mathrm{UC}_{2}$ & TS $6-5$ & 1090 & 4053 & 185 & 0 & -- & $5.90 \times 10^{-5}$ & 0 & $0 \leq \mathrm{P} \leq 2.0$ & 0 & $0 \leq \mathrm{P} \leq 2.0$ & 0 & $0 \leq P \leq 2.0$ & 100 & $97.9 \leq \mathrm{P} \leq 100$ & 2.7 & $1.2 \leq \mathrm{P} \leq 6.2$ \\
\hline
\end{tabular}

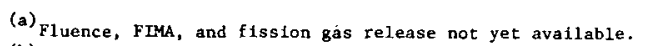

(b) Fuel Materials Branch data retrleval number.

(c) TS 15-6 (thermal stability type II crucible No. 15, hole 6).

(d) Calculated by TREVER

(e) Measured in TRICA at $1100^{\circ} \mathrm{C}$.

(f) Approximately 10 to $20 \%$ of the sample is examined.

(g) One cracked doublet and four coated fragments.

TABLE $4-48$

FTE-6 PREIRRADIATION DATA FOR THE TYPE II THERMAL STABILITY SPINE SAMPLES

\begin{tabular}{|c|c|c|c|c|c|c|c|c|c|c|c|c|c|c|c|c|}
\hline \multirow{4}{*}{$\begin{array}{c}\text { FMB } \\
\text { No. (a) }\end{array}$} & \multirow{2}{*}{\multicolumn{3}{|c|}{ Kerne1 }} & \multirow{4}{*}{$\begin{array}{l}\text { Coating } \\
\text { Type }\end{array}$} & \multirow{2}{*}{\multicolumn{8}{|c|}{ As-Manufactured Coating Parameters }} & \multirow{4}{*}{$\begin{array}{c}\text { Density } \\
\left(\mathrm{Mg} / \mathrm{m}^{3}\right)\end{array}$} & \multirow[b]{4}{*}{$\begin{array}{c}\text { Diameter } \\
(\mu \mathrm{m})\end{array}$} & \multirow{3}{*}{\multicolumn{2}{|c|}{$\begin{array}{l}\text { arameters } \\
\begin{array}{c}\text { Heavy Metal } \\
\text { Content }\end{array}\end{array}$}} \\
\hline & & & & & & & & & & & & & & & & \\
\hline & \multirow[b]{2}{*}{ Type } & \multirow{2}{*}{$\begin{array}{l}\text { Density } \\
(\mathrm{Mg} / \mathrm{m} 3)\end{array}$} & \multirow{2}{*}{\begin{tabular}{|c|} 
Nominal \\
Diameter \\
$(\mu \mathrm{m})$
\end{tabular}} & & \multicolumn{5}{|c|}{ Nominal Thickness $(\mu \mathrm{m})$} & \multirow{2}{*}{$\begin{array}{c}\text { OPyC } \\
\text { Density } \\
\left(\mathrm{Mg} / \mathrm{m}^{3}\right)\end{array}$} & \multirow[b]{2}{*}{$\begin{array}{c}\text { OPyC } \\
\text { OPTAF(b) }\end{array}$} & \multirow{2}{*}{$\begin{array}{c}\text { SiC } \\
\text { Density } \\
\left(\mathrm{Mg} / \mathrm{m}^{3}\right)\end{array}$} & & & & \\
\hline & & & & & Buffer & IPyC & $\mathrm{SiC}$ & OPyC & Total & & & & & & \begin{tabular}{|c|}
$U$ \\
$(w t \%)$
\end{tabular} & $\begin{array}{c}\mathrm{Th} \\
\text { (wt \%) }\end{array}$ \\
\hline $4000-242$ & $\mathrm{ThC}_{2}$ & 8.86 & 384 & TRISO & 46 & 28 & 23 & 42 & 133 & 1.71 & 1.04 & 3.19 & 3.30 & 650 & -- & 48.71 \\
\hline $4000-302$ & $\mathrm{UC}_{2}$ & 10.43 & 100 & TRISO & 50 & 18 & 20 & 36 & 122 & 1.71 & 1.05 & 3.19 & 2.05 & 344 & 11.57 & -- \\
\hline
\end{tabular}

(a) Fuel Materials Branch data retrieval number.

(b) BAF disc OPTAF.

(c) Density in air. 
TABLE 4-49

SUMMARY DATA ON FTE-6 DIFFUSION SPINE SAMPLES

\begin{tabular}{|c|c|c|c|c|c|c|c|c|c|c|c|c|}
\hline \multirow[b]{3}{*}{$\begin{array}{c}\text { Crucible } \\
\text { Number }\end{array}$} & \multirow[b]{3}{*}{ Isotope } & \multicolumn{4}{|c|}{ Sample Temperature (a) } & \multirow{3}{*}{\begin{tabular}{|c|}
$\begin{array}{c}\text { Mean Active } \\
\text { Core Height } \\
(\mathrm{mm})\end{array}$ \\
\end{tabular}} & \multirow{2}{*}{\multicolumn{2}{|c|}{$\begin{array}{l}\text { Neutron Fluence } \\
\left(\mathrm{n} / \mathrm{m}^{2}\right)\end{array}$}} & \multicolumn{4}{|c|}{ Matrix Loading $(\mu \mathrm{g} / \mathrm{mg})$} \\
\hline & & \multirow{2}{*}{$\begin{array}{l}\text { RMS } \\
\text { Min } \\
(\mathrm{K})\end{array}$} & \multicolumn{2}{|c|}{$\begin{array}{c}\text { Activation-Energy } \\
\text { Weighted }\end{array}$} & \multirow{2}{*}{$\begin{array}{l}\text { RMS } \\
\operatorname{Max} \\
(\mathrm{K}) \\
\end{array}$} & & & & \multirow[b]{2}{*}{$\begin{array}{l}\text { Element } \\
\text { Loading } \\
\end{array}$} & \multirow[b]{2}{*}{ Isotope } & \multirow[b]{2}{*}{$\begin{array}{l}\text { Isotope } \\
\text { Loading }\end{array}$} & \multirow{2}{*}{$\begin{array}{l}\text { Uncertainty } \\
\text { of Isotope } \\
\text { Loading (d) } \\
(\%) \\
\end{array}$} \\
\hline & & & $(\mathrm{k})$ & ${ }^{10^{4}}(\mathrm{~K})^{\mathrm{T}}$ & & & $\begin{array}{c}\text { Thermal (b) } \\
\left(\times 10^{25}\right)\end{array}$ & $\begin{array}{l}\text { Fast (c) } \\
\left(\times 10^{25}\right) \\
\end{array}$ & & & & \\
\hline 13 & $\begin{array}{l}\mathrm{Sr} \\
(\mathrm{Cs})\end{array}$ & $\begin{array}{l}1192 \\
1159\end{array}$ & $\begin{array}{l}1226 \\
1208\end{array}$ & $\begin{array}{l}8.16 \\
8.28\end{array}$ & $\begin{array}{l}1260 \\
1257\end{array}$ & 927.1 & 2.37 & 2.04 & $\begin{array}{l}1.59^{(\mathrm{e})} \\
0\end{array}$ & $\begin{array}{l}\mathrm{Sr}-85 \\
(\mathrm{Cs}-134)\end{array}$ & {$[3.32(-3)]$} & $(1.32)$ \\
\hline 17 & $\begin{array}{l}\mathrm{Sr} \\
(\mathrm{Cs})\end{array}$ & $\begin{array}{l}1345 \\
1314\end{array}$ & $\begin{array}{l}1385 \\
1367\end{array}$ & $\begin{array}{l}7.22 \\
7.32\end{array}$ & $\begin{array}{l}1425 \\
1420\end{array}$ & 1216.2 & 3.46 & 2.63 & $\begin{array}{l}0.830^{(\mathrm{e})} \\
0\end{array}$ & $\begin{array}{l}\mathrm{Sr}-85 \\
(\mathrm{Cs}-134)\end{array}$ & {$\left[\begin{array}{c}(f) \\
{[1.31(-3)]}\end{array}\right.$} & $(--)$ \\
\hline 19 & $\begin{array}{l}\mathrm{Sr} \\
(\mathrm{Cs})\end{array}$ & $\begin{array}{l}1345 \\
1314\end{array}$ & $\begin{array}{l}1385 \\
1367\end{array}$ & $\begin{array}{l}7.22 \\
7.32\end{array}$ & $\begin{array}{l}1425 \\
1420\end{array}$ & 1280.2 & 3.59 & 2.70 & $1.59^{(\mathrm{e})}$ & \begin{tabular}{|l} 
Sr-85 \\
$(\mathrm{Cs}-134)$
\end{tabular} & $\begin{array}{c}(f) \\
{[1.78(-3)]}\end{array}$ & $(1.60)$ \\
\hline 23 & $\mathrm{Sm}$ & 1068 & 1100 & 9.09 & 1132 & 799.1 & 1.74 & 1.59 & 1.02 & Sm-152 & $(\mathrm{g})$ & -- \\
\hline 26 & Sm & 1330 & 1370 & 7.30 & 1410 & 1312.2 & 3.63 & 2.72 & $5.92(e)$ & $\mathrm{Sm}-152$ & (g) & -- \\
\hline 33 & $\begin{array}{l}\mathrm{Ba} \\
(\mathrm{Cs})\end{array}$ & $\begin{array}{l}1358 \\
1326\end{array}$ & $\begin{array}{l}1398 \\
1380\end{array}$ & $\begin{array}{l}7.15 \\
7.25\end{array}$ & $\begin{array}{l}1438 \\
1434\end{array}$ & 1248.2 & 3.54 & 2.67 & $\begin{array}{l}0.065^{(\mathrm{e})} \\
0\end{array}$ & $\begin{array}{l}\mathrm{Ba}-133 \\
(\mathrm{Cs}-134)\end{array}$ & {$\left[\begin{array}{c}(f) \\
{[1.22(-3)]}\end{array}\right.$} & $\overline{(3.13)}$ \\
\hline 40 & $\begin{array}{l}\mathrm{Cs} \\
\mathrm{Sr} \\
\mathrm{Ba} \\
\mathrm{Sm}\end{array}$ & $\begin{array}{l}1075 \\
1108 \\
1108 \\
1108\end{array}$ & $\begin{array}{l}1122 \\
1140 \\
1140 \\
1140\end{array}$ & $\begin{array}{l}8.91 \\
8.77 \\
8.77 \\
8.77\end{array}$ & $\begin{array}{l}1169 \\
1172 \\
1172 \\
1172\end{array}$ & 831.1 & 1.90 & 1.70 & $\left.\begin{array}{l}0.921 \\
0.46 \\
0.62 \\
6.42\end{array}\right\}^{(e)}$ & $\begin{array}{l}\mathrm{Cs}-134 \\
\mathrm{Sr}-85 \\
\mathrm{Ba}-133 \\
\mathrm{Sm}-152 / \mathrm{Eu}-154\end{array}$ & $\begin{array}{c}6.85(-2) \\
(f) \\
(f) \\
2.33(-2)\end{array}$ & $\begin{array}{l}0.77 \\
-- \\
-- \\
2.08\end{array}$ \\
\hline 51 & $\left.\begin{array}{l}\mathrm{Cs} \\
\mathrm{Sr} \\
\mathrm{Ba} \\
\mathrm{Sm}\end{array}\right\}^{(\mathrm{h})}$ & $\begin{array}{l}1106 \\
1139 \\
1139 \\
1139\end{array}$ & $\begin{array}{l}1153 \\
1171 \\
1171 \\
1171\end{array}$ & $\begin{array}{l}8.67 \\
8.54 \\
8.54 \\
8.54\end{array}$ & $\begin{array}{l}1200 \\
1203 \\
1203 \\
1203\end{array}$ & 863.1 & 2.06 & 1.82 & $\begin{array}{l}2.24 \\
0.535 \\
0.761 \\
1.16\end{array}$ & $\begin{array}{l}\mathrm{Cs}-134 \\
\mathrm{Sr}-85 \\
\mathrm{Ba}-133 \\
\mathrm{Sm}-152 / \mathrm{Eu}-154\end{array}$ & (g) & -- \\
\hline 60 & $\left.\begin{array}{l}\mathrm{Cs} \\
\mathrm{Sr} \\
\mathrm{Ba} \\
\mathrm{Sm}\end{array}\right\}(\mathrm{i})$ & $\begin{array}{l}1136 \\
1169 \\
1169 \\
1169\end{array}$ & $\begin{array}{l}1184 \\
1202 \\
1202 \\
1202\end{array}$ & $\begin{array}{l}8.45 \\
8.32 \\
8.32 \\
8.32\end{array}$ & $\begin{array}{l}1232 \\
1235 \\
1235 \\
1235\end{array}$ & 895.1 & 2.22 & 1.95 & $\left.\begin{array}{l}0.508 \\
1.36 \\
0.844 \\
1.60\end{array}\right\}(j)$ & $\begin{array}{l}\text { Cs-134 } \\
\text { Sr-85 } \\
\mathrm{Ba}-133 \\
\mathrm{Sm}-152 / \mathrm{Eu}-154\end{array}$ & $\begin{array}{c}3.99(-2) \\
\text { (f) } \\
\text { (f) } \\
1.15(-1)\end{array}$ & $\begin{array}{l}1.65 \\
-- \\
-- \\
1.58\end{array}$ \\
\hline
\end{tabular}

(a) For Cs, $Q=143,200$ joules/mole; for $\mathrm{Sr}$, Ba, $\mathrm{Eu}(\mathrm{Sm}), Q=267,500$ joules/mole. (See Table 2-6(b).)

(b) Thermal flux $0<E<0.38$ aJ.

(c) Fast flux E>28.8 fJ.

(d) Uncertainty determined from gamma-counting measurements.

(e) Furfuryl alcohol/resin coke matrix.

(f) Undetectable above background.

(g) Not analyzed.

${ }^{(h)} \mathrm{Cs}, \mathrm{Sr}, \mathrm{Ba}, \mathrm{Sm}+1 \% \mathrm{MgF}_{2}$.

(i) $\mathrm{Cs}, \mathrm{Sr}, \mathrm{Ba}, \mathrm{Sm}+0.5 \% \mathrm{~s}$.

(j) Calcined petroleum coke containing approximately $0.5 \%$ sulfur. 
TABLE 4-50

NUCLEAR TRANSFORMATIONS OF METAL DIFFUSANTS

\begin{tabular}{l|c|c|c}
\hline Loaded Species & $\begin{array}{c}\text { Isotopic Conc. } \\
(\%)\end{array}$ & Nuclear Reaction & $\begin{array}{c}\text { Product } \\
\text { Species }\end{array}$ \\
\hline $\mathrm{Cs}-133$ & 100 & $\mathrm{n}, \gamma$ & $\mathrm{Cs}-134$ \\
$\mathrm{Sr}-84$ & 82 (enriched) & $\mathrm{n}, \gamma$ & $\mathrm{Sr}-85$ \\
$\mathrm{Sm}-152$ & 99 (enriched) & $\mathrm{n}, \gamma$ Sm-153 B Eu-153 $\mathrm{n}, \gamma$ & $\mathrm{Eu}-154$ \\
$\mathrm{Ba}$ (natural) & $\mathrm{Ba}-133$ tagged & none & $\mathrm{Ba}-133$ \\
\hline
\end{tabular}


TABLE $\quad 4-51$

FTE-6 DIFFUSION COEFFICIENTS FOR STRONTIUM AND EUROPIUM DERIVED FROM DIFFUSION SAMPLE ANALYSIS

\begin{tabular}{|c|c|c|c|c|c|c|c|}
\hline $\begin{array}{l}\text { Crucible } \\
\text { Number (a) }\end{array}$ & Isotope & $\begin{array}{c}\text { Activation } \\
\text { Energy-Weighted } \\
\text { Temperature (b) } \\
\text { (K) }\end{array}$ & $\frac{10^{4}}{T(K)}$ & $\begin{array}{c}\mathrm{C}_{\mathrm{m}} \\
(\mu \mathrm{g} / \mathrm{mg})\end{array}$ & $\begin{array}{c}C_{s}^{o} \\
(\mu g / m g)\end{array}$ & $\begin{array}{c}D \cdot t^{(c)} \\
\left(m^{2}\right)\end{array}$ & $\left(\mathrm{m}^{2} / \mathrm{s}\right)$ \\
\hline $13(\mathrm{P})$ & Sr-85 & 1226 & 8.16 & (d) & $1.5(-4)$ & $5.4(-8)$ & $79(-16)$ \\
\hline $13(W)$ & $S r-85$ & 1226 & 8.16 & (d) & $1.9(-4)$ & $6.0(-8)$ & $8.8(-16)$ \\
\hline $17(\mathrm{P})$ & $\mathrm{Sr}-85$ & 1385 & 7.22 & (d) & $1.9(-4)$ & $4.3(-7)$ & $6.3(-15)$ \\
\hline $19(P)$ & $\mathrm{Sr}-85$ & 1385 & 7.22 & (d) & $7.5(-5)$ & $3.2(-6)$ & $4.7(-14)$ \\
\hline $19(W)$ & $\mathrm{Sr}-85$ & 1385 & 7.22 & (d) & $7.1(-5)$ & $4.2(-6)$ & $6.1(-14)$ \\
\hline $40(P)(e, f)$ & $\mathrm{Eu}-154$ & 1140 & 8.77 & $2.3(-2)$ & $1.7(-2)$ & $3.9(-9)$ & $5.7(-17)$ \\
\hline $40(W)$ & $\mathrm{Eu}-154$ & 1140 & 8.77 & $2.3(-2)$ & $1.1(-2)$ & $3.0(-9)$ & $4.4(-17)$ \\
\hline $60(P)(e, f)$ & Eu-154 & 1202 & 8.32 & $1.2(-1)$ & (d) & -- & -- \\
\hline $60(W)$ & $\mathrm{Eu}-154$ & 1202 & 8.32 & $1.2(-1)$ & (d) & -- & -- \\
\hline
\end{tabular}

(a) $\mathrm{P}=$ center post; $\mathrm{W}=$ crucible wall.

(b) Temperature weighted assuming $Q=267,500$ joules/mole for both strontium and europium; see Table 2-6(b).

(c) $t=\operatorname{Real}$ time $[6.84(7) \mathrm{s}]$.

(d) Undetectable above background.

(e) Although Ba-133 was introduced in the matrices of crucibles 33 , 40, and 60 , no counts were detected above background in either the matrix material or the graphite samples for any of these crucibles.

(f) Strontium was undetectable above background, although it was initially present in the matrix material. 
TABLE 4-52

FTE-6 CESIUM DIFFUSION COEFFICIENTS AND PARTITION FACTORS DERIVED FROM DIFFUSION SAMPLE ANALYSIS

\begin{tabular}{|c|c|c|c|c|c|c|c|c|c|}
\hline \multirow[b]{2}{*}{$\begin{array}{c}\text { Crucible } \\
\text { Number }\end{array}$} & \multicolumn{2}{|c|}{$\begin{array}{c}\text { Activation } \\
\text { Energy-Weighted } \\
\text { Temperature (a) }\end{array}$} & \multirow[b]{2}{*}{$\begin{array}{c}\mathrm{C}_{\mathrm{m}} \\
(\mu \mathrm{g} / \mathrm{mg})\end{array}$} & \multirow[b]{2}{*}{$\begin{array}{c}\mathrm{J} \\
\left(\mu \mathrm{g} / \mathrm{mm}^{2}-\mathrm{s}\right)\end{array}$} & \multirow[b]{2}{*}{$\begin{array}{l}\mathrm{K} / \mathrm{D} \\
\left(\mathrm{m}^{-2}\right)\end{array}$} & \multirow[b]{2}{*}{$\begin{array}{c}\mathrm{C}_{\ell} \\
(\mu \mathrm{g} / \mathrm{mg})\end{array}$} & \multirow[b]{2}{*}{$\begin{array}{r}D_{\text {eff }} \\
\left(\mathrm{m}^{2} / \mathrm{s}\right)\end{array}$} & \multirow{2}{*}{$\begin{array}{c}\mathrm{C}_{\mathrm{g}}{ }^{(\mathrm{c})} \\
(\mu \mathrm{g} / \mathrm{mg})\end{array}$} & \multirow[b]{2}{*}{$\begin{array}{c}\phi \\
\left(C_{m} / C_{g}\right) \\
\end{array}$} \\
\hline & $(\mathrm{K})$ & $\frac{10^{4}}{\mathrm{~T}(\mathrm{~K})}$ & & & & & & & \\
\hline 13 & 1208 & 8.28 & $3.32(-3)$ & $6.9(-11)$ & -- & $4.0(-4)$ & $7.6(-13)$ & $1.5(-5)$ & 221 \\
\hline 17 & 1367 & 7.32 & $1.31(-3)$ & $2.7(-11)$ & -- & $5.6(-4)$ & $2.1(-13)$ & $3.0(-5)$ & 44 \\
\hline 19 & 1367 & 7.32 & $1.78(-3)$ & $3.7(-11)$ & -- & $4.2(-4)$ & $3.9(-13)$ & $3.0(-5)$ & 59 \\
\hline 33 & 1380 & 7.25 & $1.22(-3)$ & $2.5(-11)$ & -- & $4.2(-4)$ & $2.6(-13)$ & $3.0(-5)$ & 41 \\
\hline $40(P)(c)$ & 1122 & 8.91 & $6.85(-2)$ & -- & $5.4(+4)$ & -- & $2.7(-13)$ & $3.0(-4)$ & 228 \\
\hline $40(W)$ & & & & & $2.5(+5)$ & -- & $5.8(-14)$ & & \\
\hline $60(P)$ & 1184 & 8.45 & $3.99(-2)$ & -- & $3.9(+4)$ & -- & $3.8(-13)$ & $1.0(-3)$ & 40 \\
\hline $60(\mathrm{~W})$ & & & & & $4.0(+4)$ & -- & $3.7(-13)$ & & \\
\hline
\end{tabular}

(a) Derived from CPROFIT analysis: $D_{\text {eff }}=[(\mathrm{K} / \mathrm{D})(\mathrm{t})]^{-1}$, where $t=$ real time $[6.84(7) \mathrm{s}]$.

(b) Extrapolated concentration of cesium-134 in crucible wall graphite adjacent to the matrix material.

(c) $\phi=$ partition factor.

(d) $P=$ center post; $W=$ crucible wall. 